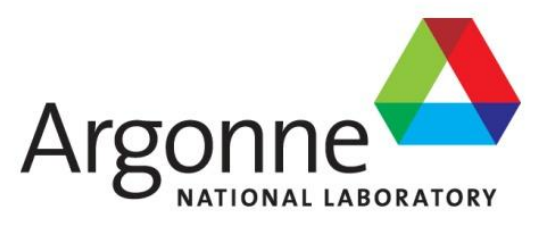

\title{
Derived Concentration Guideline Levels for Argonne National Laboratory's Building 310 Area
}

Environmental Science Division 


\section{About Argonne National Laboratory}

Argonne is a U.S. Department of Energy laboratory managed by UChicago Argonne, LLC under contract DE-AC02-06CH11357. The Laboratory's main facility is outside Chicago,

at 9700 South Cass Avenue, Argonne, Illinois 60439. For information about Argonne

and its pioneering science and technology programs, see www.anl.gov.

\section{Availability of This Report}

This report is available, at no cost, at http://www.osti.gov/bridge. It is also available

on paper to the U.S. Department of Energy and its contractors, for a processing fee, from:

\section{U.S. Department of Energy}

Office of Scientific and Technical Information

P.O. Box 62

Oak Ridge, TN 37831-0062

phone (865) 576-8401

fax (865) 576-5728

reports@adonis.osti.gov

\section{Disclaimer}

This report was prepared as an account of work sponsored by an agency of the United States Government. Neither the United States Government nor any agency thereof, nor UChicago Argonne, LLC, nor any of their employees or officers, makes any warranty, express or implied, or assumes any legal liability or responsibility for the accuracy, completeness, or usefulness of any information, apparatus, product, or process disclosed, or represents that its use would not infringe privately owned rights. Reference herein to any specific commercial product, process, or service by trade name, trademark, manufacturer, or otherwise, does not necessarily constitute or imply its endorsement, recommendation, or favoring by the United States Government or any agency thereof. The views and opinions of document authors expressed herein do not necessarily state or reflect those of the United States Government or any agency thereof, Argonne National Laboratory, or UChicago Argonne, LLC. 


\section{Derived Concentration Guideline Levels for Argonne National Laboratory's Building 310 Area}

prepared by

S. Kamboj and C. Yu

Environmental Science Division, Argonne National Laboratory

June 2011 



\section{CONTENTS}

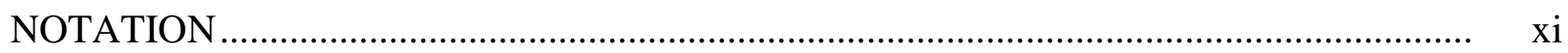

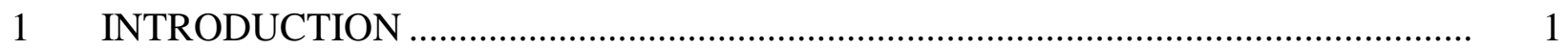

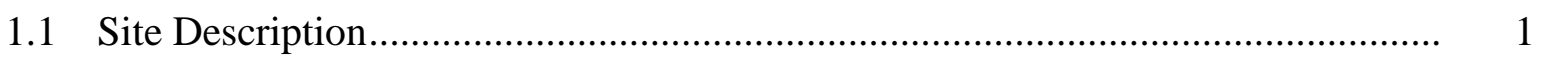

1.2 Building History .............................................................................................. 1

1.3 Derivation of Guideline Levels........................................................................ 5

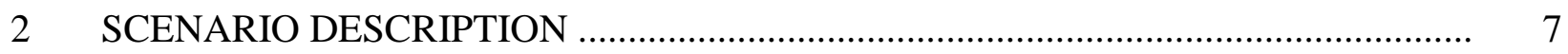

$2.1 \quad$ Land Use Considerations .................................................................................. 7

2.2 Exposure Scenarios ..................................................................................... 7

2.3 Pathways and Key Parameters ............................................................................. 8

3 DOSE-TO-SOURCE CONCENTRATION RATIOS ……………............................ 13

$3.1 \quad$ Probabilistic Analysis ............................................................................... 13

3.2 Deterministic Analysis ................................................................................... 32

$4 \quad$ DERIVED CONCENTRATION GUIDELINE LEVELS ............................................ 39

4.1 DCGLs Based on Probabilistic Analysis ……………......................................... 39

4.2 DCGLs Based on Deterministic Analysis............................................................. 39

4.3 Summary DCGLs for Building 310 Area …………………………………….... 39

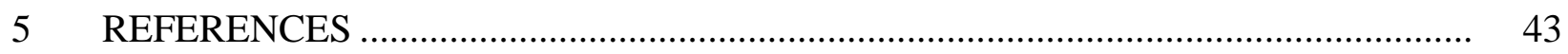

APPENDIX A: Parameters Used for Probabilistic Analysis ................................................. 45

APPENDIX B: Parameters Used for Deterministic Analysis................................................ 71

APPENDIX C: Detailed Probabilistic Analysis Results ....................................................... 91

\section{TABLES}

1 Screening Analysis for Radionuclides Present in Building 310 Soils .......................... 4

2 Summary of Applicable Exposure Pathways for Different Scenarios

Considered for Argonne's Building 310 Area .......................................................... 10 


\section{TABLES (Cont.)}

$3 \quad$ List of Key Parameters for Applicable Exposure Pathways ....................................... 11

4 Total Peak DSR Percentiles of Am-241 for Different Exposure Scenarios for the Building 310 Area ................................................................................. 17

5 Total Peak DSR Percentiles of C-14 for Different Exposure Scenarios for the Building 310 Area ............................................................................... 18

6 Total Peak DSR Percentiles of Co-60 for Different Exposure Scenarios for the Building 310 Area ................................................................................. 19

7 Total Peak DSR Percentiles of Cs-137 for Different Exposure Scenarios for the Building 310 Area ..................................................................................... 20

8 Total Peak DSR Percentiles of I-129 for Different Exposure Scenarios for the Building 310 Area ......................................................................................

9 Total Peak DSR Percentiles of Np-237 for Different Exposure Scenarios for the Building 310 Area

10 Total Peak DSR Percentiles of Pu-238 for Different Exposure Scenarios for the Building 310 Area

11 Total Peak DSR Percentiles of Pu-239 for Different Exposure Scenarios for the Building 310 Area.

12 Total Peak DSR Percentiles of Pu-240 for Different Exposure Scenarios for the Building 310 Area

13 Total Peak DSR Percentiles of Sr-90 for Different Exposure Scenarios for the Building 310 Area

14 Total Peak DSR Percentiles of Tc-99 for Different Exposure Scenarios for the Building 310 Area

15 Total Peak DSR Percentiles of U-234 for Different Exposure Scenarios for the Building 310 Area

16 Total Peak DSR Percentiles of U-235 for Different Exposure Scenarios for the Building 310 Area

17 Total Peak DSR Percentiles of U-238 for Different Exposure Scenarios for the Building 310 Area 


\section{TABLES (Cont.)}

18 DSRs at Peak of the Means, Mean of the Peaks, and 95th Percentile of the Peaks for Different Scenarios

19 Maximum DSRs for the Industrial Scenario for the Building 310 Area ..................... 34

20 Maximum DSRs for the Offsite Resident Scenario for the Building 310 Area............ 35

21 Maximum DSRs for the Recreational Scenario for the Building 310 Area ................ 36

22 Maximum DSRs for the Onsite Resident Scenario for the Building 310 Area ........... 37

23 Total DSRs for Different Scenarios for the Building 310 Area................................... 38

24 DCGLs from Using Probabilistic Analysis at a 25-mrem/yr Dose Limit for the Building 310 Area ..................................................................................... 40

25 DCGLs from Using Deterministic Analysis at a 25-mrem/yr Dose Limit for the Building 310 Area ........................................................................................ 41

26 Summary of DCGLs for the Building 310 Area .................................................... 41

A.1 Parameter Values and Distributions Used in the Probabilistic Analysis of Different Scenarios

A.2 Parameter Distributions Used in the Probabilistic Analysis for the Contaminated Zone and Unsaturated Zone Kd Values.

A.3 Parameter Distributions Used in the Probabilistic Analysis for the Saturated Zone Kd Values .....

A.4 Parameter Distributions Used in the Probabilistic Analysis for the Plant Transfer Factor

A.5 Parameter Distributions Used in the Probabilistic Analysis for the Meat Transfer Factor.

A.6 Parameter Distribution Used in the Probabilistic Analysis for the Milk Transfer Factor

A.7 Parameter Values and Distributions Used in the Probabilistic Analysis for the Fish and Crustacea Transfer Factors

A.8 Notations Used in Input Parameter Table A.1 


\section{TABLES (Cont.)}

A.9 Parameter Correlations for Probabilistic Analysis of Different Scenarios

A.10 Additional Input Parameters Used in the RESRAD-OFFSITE

Probabilistic Analysis

B.1 Parameter Values Used in the Deterministic Analysis of Different Scenarios

B.2 Parameter Values Used in the Deterministic Analysis for the Kd, Plant, Meat, and Milk Transfer Factors

B.3 Parameter Values Used in the Deterministic Analysis for the Fish and Crustacea Transfer Factors

B.4 Additional Input Parameters Used in the RESRAD-OFFSITE

Deterministic Analysis

C.1 Peak DSR Percentiles for Different Exposure Pathways for Am-241

in Industrial Use Scenario.

C.2 Peak DSR Percentiles for Different Exposure Pathways for C-14 in Industrial Use Scenario

C.3 Peak DSR Percentiles for Different Exposure Pathways for Co-60 in Industrial Use Scenario.

C.4 Peak DSR Percentiles for Different Exposure Pathways for Cs-137 in Industrial Use Scenario.

C.5 Peak DSR Percentiles for Different Exposure Pathways for I-129 in Industrial Use Scenario.

C.6 Peak DSR Percentiles for Different Exposure Pathways for Np-237 in Industrial Use Scenario.

C.7 Peak DSR Percentiles for Different Exposure Pathways for Pu-238

in Industrial Use Scenario.

C.8 Peak DSR Percentiles for Different Exposure Pathways for Pu-239

in Industrial Use Scenario.

C.9 Peak DSR Percentiles for Different Exposure Pathways for Pu-240

in Industrial Use Scenario 


\section{TABLES (Cont.)}

C.10 Peak DSR Percentiles for Different Exposure Pathways for Sr-90

in Industrial Use Scenario.

C.11 Peak DSR Percentiles for Different Exposure Pathways for Tc-99

in Industrial Use Scenario....

C.12 Peak DSR Percentiles for Different Exposure Pathways for U-234

in Industrial Use Scenario

C.13 Peak DSR Percentiles for Different Exposure Pathways for U-235

in Industrial Use Scenario.

C.14 Peak DSR Percentiles for Different Exposure Pathways for U-238

in Industrial Use Scenario.

C.15 Peak DSR Percentiles for Different Exposure Pathways for Am-241

in Offsite Resident Scenario

C.16 Peak DSR Percentiles for Different Exposure Pathways for C-14

in Offsite Resident Scenario

C.17 Peak DSR Percentiles for Different Exposure Pathways for Co-60

in Offsite Resident Scenario

C.18 Peak DSR Percentiles for Different Exposure Pathways for Cs-137

in Offsite Resident Scenario

C.19 Peak DSR Percentiles for Different Exposure Pathways for I-129

in Offsite Resident Scenario

C.20 Peak DSR Percentiles for Different Exposure Pathways for Np-237

in Offsite Resident Scenario

C.21 Peak DSR Percentiles for Different Exposure Pathways for Pu-238

in Offsite Resident Scenario

C.22 Peak DSR Percentiles for Different Exposure Pathways for Pu-239

in Offsite Resident Scenario

C.23 Peak DSR Percentiles for Different Exposure Pathways for Pu-240

in Offsite Resident Scenario 


\section{TABLES (Cont.)}

C.24 Peak DSR Percentiles for Different Exposure Pathways for Sr-90 in

Offsite Resident Scenario

C.25 Peak DSR Percentiles for Different Exposure Pathways for Tc-99

in Offsite Resident Scenario

C.26 Peak DSR Percentiles for Different Exposure Pathways for U-234

in Offsite Resident Scenario

C.27 Peak DSR Percentiles for Different Exposure Pathways for U-235

in Offsite Resident Scenario

C.28 Peak DSR Percentiles for Different Exposure Pathways for U-238

in Offsite Resident Scenario

C.29 Peak DSR Percentiles for Different Exposure Pathways for Am-241

in Recreational Use Scenario

C.30 Peak DSR Percentiles for Different Exposure Pathways for C-14

in Recreational Use Scenario

C.31 Peak DSR Percentiles for Different Exposure Pathways for Co-60

in Recreational Use Scenario

C.32 Peak DSR Percentiles for Different Exposure Pathways for Cs-137

in Recreational Use Scenario

C.33 Peak DSR Percentiles for Different Exposure Pathways for I-129

in Recreational Use Scenario

C.34 Peak DSR Percentiles for Different Exposure Pathways for Np-237

in Recreationa Use Scenario

C.35 Peak DSR Percentiles for Different Exposure Pathways for Pu-238

in Recreational Use Scenario

C.36 Peak DSR Percentiles for Different Exposure Pathways for Pu-239

in Recreational Use Scenario

C.37 Peak DSR Percentiles for Different Exposure Pathways for Pu-240

in Recreational Use Scenario 


\section{TABLES (Cont.)}

C.38 Peak DSR Percentiles for Different Exposure Pathways for Sr-90 in Recreational Use Scenario

C.39 Peak DSR Percentiles for Different Exposure Pathways for Tc-99 in Recreational Use Scenario

C.40 Peak DSR Percentiles for Different Exposure Pathways for U-234 in Recreational Use Scenario

C.41 Peak DSR Percentiles for Different Exposure Pathways for U-235 in Recreational Use Scenario

C.42 Peak DSR Percentiles for Different Exposure Pathways for U-238 in Recreational Use Scenario

C.43 Peak DSR Percentiles for Different Exposure Pathways for Am-241 in Onsite Resident Scenario

C.44 Peak DSR Percentiles for Different Exposure Pathways for C-14 in Onsite Resident Scenario

C.45 Peak DSR Percentiles for Different Exposure Pathways for Co-60 in Onsite Resident Scenario

C.46 Peak DSR Percentiles for Different Exposure Pathways for Cs-137 in Onsite Resident Scenario

C.47 Peak DSR Percentiles for Different Exposure Pathways for I-129 in Onsite Resident Scenario

C.48 Peak DSR Percentiles for Different Exposure Pathways for Np-237 in Onsite Resident Scenario

C.49 Peak DSR Percentiles for Different Exposure Pathways for Pu-238 in Onsite Resident Scenario

C.50 Peak DSR Percentiles for Different Exposure Pathways for Pu-239 in Onsite Resident Scenario

C.51 Peak DSR Percentiles for Different Exposure Pathways for Pu-240 in Onsite Resident Scenario 


\section{TABLES (Cont.)}

C.52 Peak DSR Percentiles for Different Exposure Pathways for Sr-90 in Onsite Resident Scenario

C.53 Peak DSR Percentiles for Different Exposure Pathways for Tc-99 in Onsite Resident Scenario

C.54 Peak DSR Percentiles for Different Exposure Pathways for U-234 in Onsite Resident Scenario

C.55 Peak DSR Percentiles for Different Exposure Pathways for U-235

in Onsite Resident Scenario

C.56 Peak DSR Percentiles for Different Exposure Pathways for U-238 in Onsite Resident Scenario 


\section{NOTATION}

\section{ACRONYMS AND ABBREVIATIONS}

$\begin{array}{ll}\begin{array}{l}\text { ALARA } \\ \text { Argonne }\end{array} & \begin{array}{l}\text { as low as reasonably achievable } \\ \text { Argonne National Laboratory }\end{array} \\ \text { CDF } & \text { cumulative distribution function } \\ \text { DCF } & \text { dose conversion factor } \\ \text { DCGL } & \begin{array}{l}\text { derived concentration guideline level } \\ \text { DSR }\end{array} \\ \text { EBR } & \begin{array}{l}\text { Experimental Breeder Reactor } \\ \text { EPA }\end{array} \\ \text { Kd } & \text { distribution coefficient } \\ \text { NRC } & \text { U.S. Nuclear Regulatory Commission } \\ \text { TRU } & \text { transuranic } \\ \text { WD } & \text { water-dependent } \\ \text { WI } & \text { water-independent } \\ \text { W/o } & \text { without }\end{array}$

\section{UNITS OF MEASURE}

\begin{tabular}{|c|c|c|}
\hline cal calorie(s) & $\mathrm{L}$ & liter(s) \\
\hline & $\mathrm{m}$ & meter(s) \\
\hline $\operatorname{day}(\mathrm{s})$ & $\begin{array}{l}\mathrm{m}^{2} \\
\mathrm{~mol}\end{array}$ & $\begin{array}{l}\text { square meter(s) } \\
\text { mole }(\mathrm{s})\end{array}$ \\
\hline $\operatorname{gram}(\mathrm{s})$ & mrem & millirem \\
\hline hour(s) & $\mathrm{pCi}$ & picocurie(s) \\
\hline hectare(s) & & \\
\hline & $\mathrm{s}$ & second(s) \\
\hline kelvin & & \\
\hline kilogram(s) & $\mathrm{yr}$ & year(s) \\
\hline kilometer(s) & & \\
\hline
\end{tabular}




\section{INTRODUCTION}

The derived concentration guideline level (DCGL) is the allowable residual radionuclide concentration that can remain in soil after remediation of the site without radiological restrictions on the use of the site. It is sometimes called the single radionuclide soil guideline or the soil cleanup criteria. This report documents the methodology, scenarios, and parameters used in the analysis to support establishing radionuclide DCGLs for Argonne National Laboratory's Building 310 area.

\subsection{SITE DESCRIPTION}

Argonne National Laboratory (Argonne) is located $43 \mathrm{~km}$ southwest of downtown Chicago in DuPage County, Illinois. The site occupies 607 ha and is mostly surrounded by the Waterfall Glen Forest Preserve, which is used mainly as a recreational area. The Argonne site contains a number of small ponds and streams. The primary drainage on the site is through Sawmill Creek that runs through the site and enters the Des Plaines River. Some southernmost parts of the site drain directly into the Des Plaines River.

\subsection{BUILDING HISTORY}

Building 310 was constructed in 1950 and is made of concrete and structural steel with a brick facing. The building was initially known as the Experimental Waste Processing, Storage, and Shipping Building. It consists of two main floors, a partial basement service floor, and a fan loft on the third floor. Two tunnels extend south from the service floor in Building 310 to the adjacent Building 306; one is for personnel, and the other is for a system of liquid waste transfer pipes. Building 306 was used for decontamination activities and waste management operations. Any liquid or airborne releases from Building 306 (especially to the north) could be reflected in soil contamination south of Building 310.

The major radionuclides potentially present in the soils surrounding Building 310 are, in general, expected to be the same as those within the building. Soil contamination likely occurred as a result of liquid spills and airborne releases from various rooms and laboratories while the building was being used to support projects at Argonne. Hence, it is necessary to consider the previous uses of Building 310 to identify those radionuclides most likely to be present and of concern in nearby soils.

Building 310 was initially used for experimental waste processing activities and the storage and shipment of radioactive wastes offsite. The overall goal of the experimental waste processing program for liquid and solid wastes conducted in this building was to reduce the waste volume that had to be shipped offsite for disposal. The service floor retention tank facility in Building 310 provided capacity for the storage of liquid radioactive wastes prior to processing in Building 306. Some of the liquid radioactive wastes in these retention tanks were processed in Building 310 as part of developing treatment techniques for liquid wastes, generally involving 
solidification. The retention facility tanks also provided overflow capacity for contaminated liquid wastes that were initially received for processing in Building 306 through the liquid waste transfer pipes between the two buildings. On the basis of this history of operations, it is reasonable to expect that the same or a comparable mix of radionuclides would be present as residual contamination in both buildings.

Building 310 supported several additional projects, including acting as a staging area for dry waste that was being incinerated in a test facility at the site. The building was also used for a project involving the irradiation of food samples with spent nuclear fuel rods to study the effects of radiation on bacteria. It is expected that neither of these two projects would result in significant contamination of the building or nearby soils.

Building 310 was later expanded in the 1970s to provide support to the Experimental Breeder Reactor II (EBR-II) project, specifically the development and testing of prototypes for the EBR-II fuel cycle facility. The EBR-II facility was located at Argonne-West in Idaho, and Building 310 was generally used to house engineers and technicians supporting that project. The goal of the EBR-II project was demonstrating the recycle and reuse of the plutonium present in spent nuclear fuel.

Breeder reactors operate on the principal that more nuclear fuel (plutonium) is created than is used. Plutonium and uranium can only be recycled so many times in a nuclear reactor before the buildup of isotopes that absorb neutrons without causing fissions (neutron poisons) makes the fuel unusable. While detailed information on the specific activities conducted in Building 310 for the EBR-II project has not yet been found, it is possible that pilot-scale studies and experiments associated with spent nuclear fuel reprocessing and fuel assembly operations could have taken place in this building. Even if such activities were not conducted in Building 310, the radioactive contaminants associated with spent nuclear fuel were likely present in the radioactive wastes initially processed and staged in Buildings 310 and 306, as these activities were conducted at other facilities at Argonne at that time.

Reprocessing of spent fuel generally requires that the fuel rods be cut, dissolved in acids, and then processed to extract the useable materials from waste products. Such activities would result in the release of radionuclides with subsequent contamination of nearby areas with the fission products and actinides that are present in spent nuclear fuel. Neutron activation products in metal components would also likely be released by these activities, generally from the shearing of the fuel assemblies. The radioactive wastes generated by spent nuclear fuel reprocessing include fission and neutron activation products, as well as a number of transuranic (TRU) radionuclides such as plutonium and americium. As noted above, solid and liquid radioactive wastes associated with such operations were likely stored and possibly processed in Buildings 310 and 306.

Although a number of activities were conducted in Building 310 since 1950 (including using spent nuclear fuel as a source of gamma rays and providing support for the EBR-II project), it is expected that the radioactive waste processing activities previously conducted there would be the major source of contamination in the building. By inference, these same radionuclides would be expected to be present in nearby soils. The initial mission of Argonne 
was largely reactor development and the management of spent nuclear fuel. The wastes processed in Buildings 310 and 306 are expected to be very similar to the types of wastes associated with the West Valley Site, which is currently undergoing remediation. The major radionuclides of concern identified for that site, as given in the Phase I Decommissioning Plan developed for the U.S. Nuclear Regulatory Commission (NRC), were reviewed and used to develop the list of radionuclides expected to be of concern in soils in the vicinity of Building 310.

Characterization of the service floor retention tank facility in Building 310 in the 1990s identified the major radionuclides in this area as Sr-90, Cs-137, Pu-239, Am-241, and notable quantities of uranium isotopes. This information is consistent with the assumption that the radionuclides expected to be present in this building are similar to those at the West Valley Site. This list is not inclusive of all radionuclides that could be encountered during characterization and remediation of Building 310 soils. A screening evaluation was performed to identify those radionuclides that could reasonably be expected to be present in Building 310 soils and to present the greatest hazards to human health in the future.

The results of the screening analysis are given in Table 1 . The radionuclides in the first column of this table are the major radionuclides expected to be present in soils surrounding a spent nuclear fuel reprocessing plant (or a facility that handles comparable radioactive wastes) that could present a future hazard to human health and the environment. Subsequent screening was performed to reduce the list to a more tenable number for use with soils in the vicinity of Building 310.

Additional radionuclides are also likely to be present in Building 310 and the nearby soils, but these are expected to be present at lower activity concentrations or to present a lower hazard than those that were retained. These include hydrogen-3 (tritium), additional fission products such as various isotopes of europium, additional neutron activation products that may be present in activated metals (including the spent fuel cladding) such as Co-60 and several isotopes of nickel and iron, and additional TRU radionuclides.

Hydrogen-3 is not included here because of its relatively short half-life of 12.4 years. Fission and neutron activation products beyond those given in Table 1 (such as various isotopes of europium, nickel, and iron) were not included here because they generally have much smaller yields and shorter half-lives than those that were retained. These radionuclides would therefore have been produced in relatively low amounts, and much of the initial contamination would have since undergone significant radioactive decay. The exception is Co-60, which was detected as contamination within Building 310 and was retained as an indicator of possible activation product contamination. The prevalence of TRU radionuclides in spent nuclear fuel generally decreases as the atomic number increases, because they are largely produced by subsequent absorption of additional neutrons. The TRU radionuclides given here are those that are expected to be most prevalent and have the greatest potential for harm in the future. 


\section{TABLE 1 Screening Analysis for Radionuclides Present in Building 310 Soils}

\begin{tabular}{|c|c|c|}
\hline Radionuclide & $\begin{array}{l}\text { Screening } \\
\text { Result }\end{array}$ & Screening Rationale \\
\hline Americium-241 & Retained & Identified in previous characterization activities in Building 310 \\
\hline Carbon-14 & Retained & Neutron activation product present in stainless steel cladding \\
\hline Cobalt-60 & Retained & $\begin{array}{l}\text { An activation product observed as contamination within Building } 310 \text {, retained } \\
\text { as an indicator of possible activation product contamination }\end{array}$ \\
\hline Curium-243 & Eliminated & $\begin{array}{l}\text { Expected to be less prevalent than other TRU radionuclides that pose a } \\
\text { comparable or greater hazard to human health }\end{array}$ \\
\hline Curium-244 & Eliminated & $\begin{array}{l}\text { Expected to be less prevalent than other TRU radionuclides that pose a } \\
\text { comparable or greater hazard to human health }\end{array}$ \\
\hline Cesium-137 & Retained & Major fission product identified in characterization activities in Building 310 \\
\hline Iodine-129 & Retained & Long-lived fission product that is mobile in the environment \\
\hline Neptunium-237 & Retained & TRU radionuclide present in relatively high concentrations in spent nuclear fuel \\
\hline Plutonium-238 & Retained & $\begin{array}{l}\text { TRU radionuclide present in materials processed in Building } 310 \text { that would } \\
\text { accompany Pu- } 239\end{array}$ \\
\hline Plutonium-239 & Retained & $\begin{array}{l}\text { TRU radionuclide present in materials processed in Building } 310 \text { that was } \\
\text { identified in characterization activities in Building } 310\end{array}$ \\
\hline Plutonium-240 & Retained & $\begin{array}{l}\text { TRU radionuclide present in materials processed in Building } 310 \text { that would } \\
\text { accompany Pu- } 239\end{array}$ \\
\hline Plutonium-241 & Eliminated & $\begin{array}{l}\text { Less radiotoxic than the three plutonium isotopes that were retained (decays by } \\
\text { beta-particle emission with a half-life of } 14.4 \text { years) }\end{array}$ \\
\hline Strontium-90 & Retained & Major fission product identified in characterization activities in Building 310 \\
\hline Technetium-99 & Retained & Long-lived fission product that is mobile in the environment \\
\hline Uranium-232 & Eliminated & Expected to be less prevalent than the three uranium isotopes that were retained \\
\hline Uranium-233 & Eliminated & Expected to be less prevalent than the three uranium isotopes that were retained \\
\hline Uranium-234 & Retained & Component in the fuel elements initially charged to EBR-II \\
\hline Uranium-235 & Retained & Component in the fuel elements initially charged to EBR-II \\
\hline Uranium-238 & Retained & Component in the fuel elements initially charged to EBR-II \\
\hline
\end{tabular}


On the basis of this screening-level evaluation, the radionuclides addressed in this assessment are the following: Am-241, C-14, Co-60, Cs-137, I-129, Np-237, Pu-238, Pu-239, $\mathrm{Pu}-240$, Sr-90, Tc-99, U-234, U-235, and U-238. Remedial actions for contaminated soils in the vicinity of Building 310 would be conducted in accordance with Argonne's as low as reasonably achievable (ALARA) policy, which would be protective of human health and the environment.

\subsection{DERIVATION OF GUIDELINE LEVELS}

The contaminants of concern indentified for Building 310 are Am-241, C-14, Co-60, Cs-137, I-129, Np-237, Pu-238, Pu-239, Pu-240, Sr-90, Tc-99, U-234, U-235, and U-238 (Section 1.2). The soil guidelines for these radionuclides are calculated by using a dose constraint of $25 \mathrm{mrem} / \mathrm{yr}$ for all scenarios. It is assumed that the current industrial use of the site will continue, with an institutional control period of 100 years. All land use scenarios are described in Section 2. The soil guidelines are derived for all scenarios, and the results are presented in Section 4. The final DCGLs will be determined by the U.S. Department of Energy after taking other considerations into account, such as cost-benefit analysis, the ALARA policy, and their applicability to other portions of Argonne site. 


\section{SCENARIO DESCRIPTION}

\subsection{LAND USE CONSIDERATIONS}

The current land use for the site after it is remediated is industrial use with institutional control. However, after the institutional control period ends, the site could be rezoned for other purposes, such as recreational use or residential use, or it could continue being used as an industrial use site. In developing DCGLs for the Building 310 area's residual soil radioactivity, four potential exposure scenarios are considered to cover all likely future land uses within a 1,000-year time frame. However, if the peak dose were to occur at a later time, the time frame for the analysis would be increased to 10,000 years. The four scenarios vary with respect to the type of land use and food source consumed. Two scenarios (industrial worker onsite and surface water use offsite) capture the dose to different receptors for the current land use conditions. The other two scenarios (recreational use and subsistence farming) capture the potential dose to different receptors after the institutional control period has ended. The industrial use scenario after the institutional control has ended will result in lower doses than those associated with current industrial use because the peak dose in the time frame of 1-10,000 years is used and also because, as a result of the radioactive decay of some short-lived radionuclides that exist onsite, the dose would be less after the institutional control period of 100 years. Therefore, the industrial scenario after the institutional control period has ended is not considered in this analysis. During the institutional control period, it is possible that contaminants may migrate to offsite locations by either surface runoff or wind erosion. Therefore, offsite residents may be exposed to onsite contamination during and after the institutional control period. This scenario is included in the DCGL analysis.

\subsection{EXPOSURE SCENARIOS}

Four scenarios are considered in the derivation of soil guidelines (DCGLs) for the Building 310 area. Two scenarios (Scenarios A and B) are current use scenarios. The other two scenarios (Scenarios C and D) are future use scenarios. These four scenarios are described in detail here. The pathways and parameters considered in these four scenarios are discussed in Section 2.3.

Scenario A (the current use onsite worker scenario) assumes continued industrial use of the site. Under this scenario, it is assumed that a receptor would work onsite either indoors or outdoors (a probability distribution representative of an onsite worker will be assigned to the time fraction spent onsite). It is also assumed that the worker will not ingest contaminated water, plant, fish, meat, or milk obtained from the site. It is assumed that the dose to the worker comes only from the residual contamination in the soil in the vicinity of Building 310.

Scenario B (the current use offsite resident scenario) assumes the use of surface water by a hypothetical offsite resident. Under this scenario, it is assumed that the contamination from surface runoff mixes with the storm water drainage and discharges to Sawmill Creek. A hypothetical offsite resident uses this surface water for irrigation and feeding livestock. 
However, a public water supply is available in the area, and surface water is not used for drinking purposes. The exposure pathways considered are ingestion of plant food grown with the irrigation water from Sawmill Creek and ingestion of meat and milk from livestock that were fed with water from Sawmill Creek. The fish consumed by the resident are also caught from Sawmill Creek. It should be noted that currently very few people fish in Sawmill Creek or use the water for any purposes. Exposure to external radiation, inhalation of dust and radon, and incidental ingestion of soil are considered for this offsite resident scenario.

Scenario C (the likely future use scenario) assumes recreational use of the site. This scenario assumes that, after the institutional control period, the current forest preserve area bordering the site is expanded to include the Building 310 area. A hypothetical person (recreationist) camps on the remediated area for two weeks per year and is involved in recreational activities such as hiking, skiing, biking, and horseback riding. During this time period, it is assumed that all drinking water used by the hypothetical individual is drawn from the surface water (pond) that captures water from the surface runoff adjacent to the remediated area. Also the individual ingests fish taken from that pond. The site has clayey soil with low permeability and poor drainage; therefore, a pond that can capture surface water from runoff could easily be built. The recreationist does not ingest plant food growing in the area and also does not ingest milk from the livestock or wild animals raised in the area. However, part of the meat eaten is from the livestock or wild animals raised in the area.

Scenario D (possible but unlikely future use scenario) assumes residential use of the site after the institutional control period. For this scenario, it is assumed that after the institutional control of the site, the industrial activities end and the site is re-zoned for residential use. It is assumed that the site is used by a resident farmer. The resident farmer in this situation is exposed to direct external radiation; internal radiation from inhalation of contaminated dust; internal radiation from inhalation of radon and its progeny; and internal radiation from ingestion of water, plant foods, meat, milk, and fish and incidental ingestion of soil. All water used by the resident farmer for drinking, irrigation, and household usage is drawn from a deep well adjacent to the remediated area. The individual ingests fish caught from a nearby pond. A shallow well scenario is not considered because of the site's hydrogeological characteristics (Patton et al. 1990). Although a perched water table may exist onsite, the water yield of this groundwater system would be low and not capable of sustaining a resident farmer.

\subsection{PATHWAYS AND KEY PARAMETERS}

Potential radiation doses resulting from multiple exposure pathways are considered in this analysis for all exposure scenarios. These pathways include:

1. Direct exposure to external radiation from remediated soil material,

2. Internal radiation from inhalation of contaminated dust,

3. Internal radiation from inhalation of radon and its progeny, 
4. Internal radiation from ingestion of plant foods grown in the remediated area and irrigated with an onsite water source,

5. Internal radiation from ingestion of meat from livestock raised onsite and fed with fodder grown in the remediated area and irrigated with water drawn from an onsite well or pond (the water ingested by livestock is also drawn from an onsite water source),

6. Internal radiation from ingestion of milk from livestock raised onsite and fed with fodder grown in the remediated area and irrigated with onsite well water or pond water (the water ingested by milk cows is also drawn from an onsite water source),

7. Internal radiation from ingestion of fish from a pond located downgradient from the decontaminated area,

8. Internal radiation from incidental ingestion of onsite soil,

9. Internal radiation from drinking water from an onsite water source (groundwater or surface water),

10. Internal radiation from ingestion of plant foods grown offsite and irrigated with contaminated surface water,

11. Internal radiation from ingestion of meat from livestock raised offsite and fed with fodder grown offsite but irrigated with contaminated creek water (the water ingested by livestock is also drawn from the creek),

12. Internal radiation from ingestion of milk from livestock raised offsite and fed with fodder grown offsite but irrigated with contaminated creek water (the water ingested by milk cow is also drawn from the creek), and

13. Ingestion of fish from an offsite surface water source.

Table 2 summarizes the applicable exposure pathways for all scenarios, and Table 3 lists the key parameters for the applicable exposure pathways. 
TABLE 2 Summary of Applicable Exposure Pathways for Different Scenarios Considered for Argonne's Building 310 Area

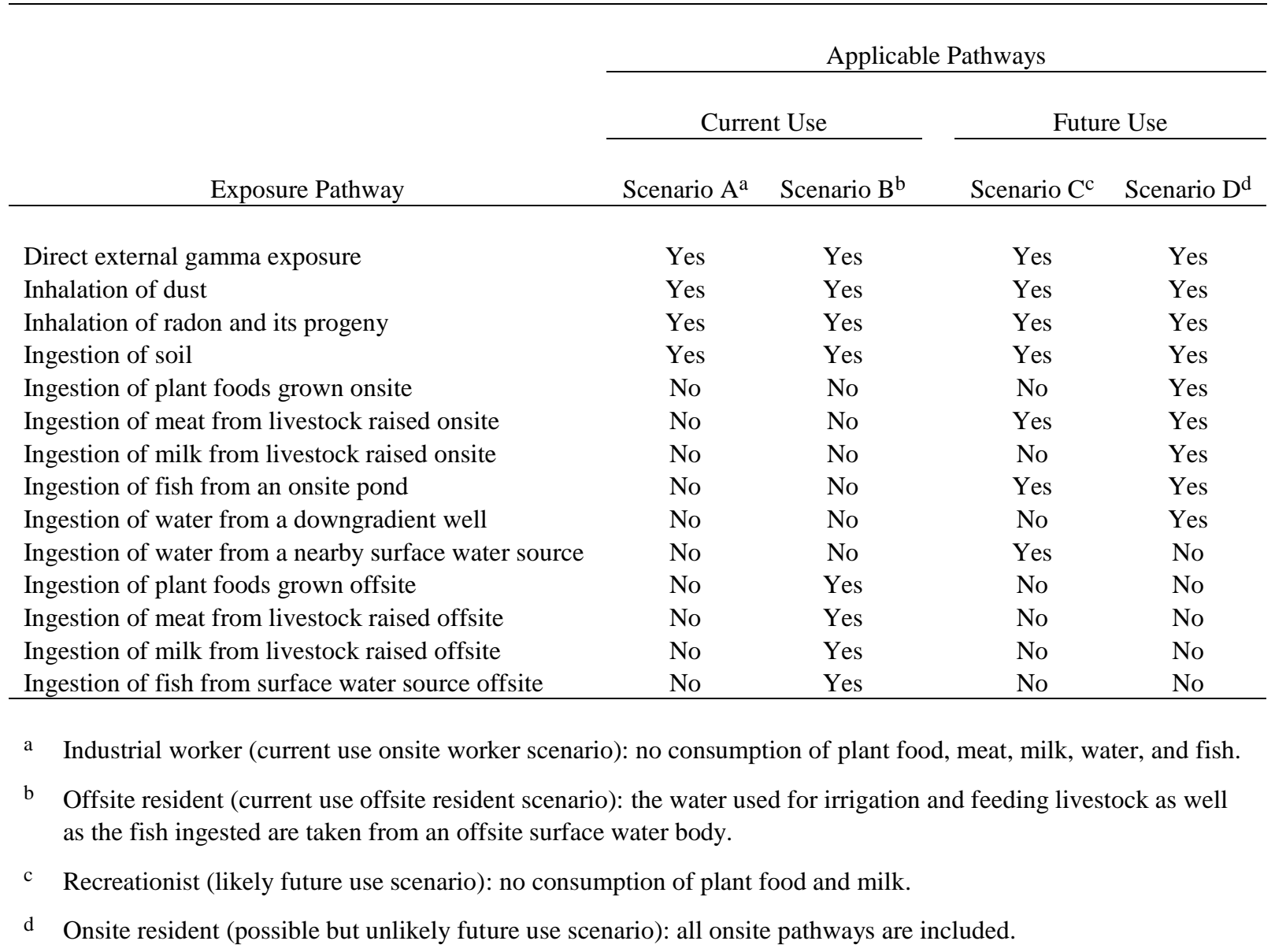




\section{TABLE 3 List of Key Parameters for Applicable Exposure Pathways}

Exposure Pathway

Direct external gamma exposure

Inhalation of dust

Inhalation of radon and its progeny

Ingestion of soil

Ingestion of plant foods grown onsite

Ingestion of meat from livestock raised onsite

Ingestion of milk from livestock raised onsite

Ingestion of fish from a nearby pond

Ingestion of water from a

downgradient well

Ingestion of water from a nearby surface water source

Ingestion of plant foods grown offsite

Ingestion of meat from livestock raised offsite

Ingestion of milk from livestock raised offsite

Ingestion of fish from surface water source offsite
Key Parameters

Time fraction spent onsite and external gamma shielding factor

Inhalation rate, time fraction spent onsite, mass loading for inhalation, and indoor dust filtration factor

Time fraction spent onsite, radon diffusion coefficient, and emanation coefficient

Soil ingestion rate and time spent onsite

Plant transfer factor, plant ingestion rate, water dilution factor, and release rate from the source

Plant transfer factor, meat transfer factor, meat ingestion rate, water dilution factor, and release rate from the source

Plant transfer factor, milk transfer factor, milk ingestion rate, water dilution factor, and release rate from the source

Fish bioaccumulation factor, aquatic food contaminated fraction, surface water dilution factor, and release rate from the source

Water ingestion rate, infiltration rate, distribution coefficient (i.e., Kd) values (or leach rate) for contaminants, and hydrogeological parameters for the site

Water ingestion rate, surface water dilution factor, and release rate from the source

Plant transfer factor, plant ingestion rate, surface water dilution factor, and release rate from the source

Plant transfer factor, meat transfer factor, meat ingestion rate, surface water dilution factor, and release rate from the source

Plant transfer factor, milk transfer factor, milk ingestion rate, surface water dilution factor, and release rate from the source

Fish bioaccumulation factor, aquatic food contaminated fraction, surface water dilution factor, and release rate from the source 


\section{DOSE-TO-SOURCE CONCENTRATION RATIOS}

Each scenario discussed in Section 2 was translated from its potential transport and environmental pathways into a specific set of parameter values. The RESRAD (onsite) and RESRAD-OFFSITE codes include many input parameters that can be classified as metabolic, behavioral, and physical parameters. The metabolic parameter represents a metabolic characteristic of the potential receptor, and its value is independent of the scenario but may be different for different population groups. Behavioral parameter values depend on the receptor's behavior in the scenario (e.g., parameter values for a recreationist scenario could be different from those for a subsistence farmer scenario). The physical parameters are source- and sitespecific, and their values do not change for a different group of receptors. The analysis was done

for an adult member of the population. For the behavioral parameters, scenario-specific, mean, or median values were used. For physical parameters, site-specific values (or distributions), whenever available, were used. If site-specific values were not available, RESRAD (onsite) default values were used. For probabilistic analysis, distributions from the NUREG/CR-6697 report (NRC 2000) were used.

Radionuclide dose-to-source (concentration) ratios (DSRs) were calculated from both probabilistic and deterministic analyses. Deterministic analysis uses a single value for each input parameter, resulting in a single dose output value. Probabilistic analysis uses parameter distributions to identify the variability and uncertainty in dose estimates resulting from variability and uncertainty in the input parameters. A probability distribution is specified for each input parameter with a distribution. A model is run repeatedly, using different values for each input parameter with a distribution for each run. Instead of producing a single model output (which results from a deterministic run), probabilistic analysis produces a set of outputs that are equal in number to the number of realizations selected (realizations are equal to the number of observations multiplied by the number of repetitions).

The RESRAD (onsite) computer code, Version 6.5 (Yu et al. 2001), was used for analyzing the industrial, recreational use, and onsite resident scenarios. The RESRAD-OFFSITE computer code, Version 2.6 (Yu et al. 2007), was used for analyzing the offsite resident scenario. The time frame considered in this analysis was 1,000 years. However, if the peak dose occurred at later time, the time frame for the analysis was increased to 10,000 years. Radioactive decay and ingrowth were considered in deriving dose/source concentration ratios. The various parameters used in the codes for probabilistic analysis are listed in the Appendix A. The various parameters used in the codes for deterministic analysis are listed in the Appendix B.

\subsection{PROBABILISTIC ANALYSIS}

The details of parameter selection are presented in Appendix A. Table A.1 lists all input parameter distributions/values used in the probabilistic dose analysis for different scenarios. Some of the parameters used in the analysis were radionuclide/element-specific (e.g., distribution coefficient or Kd values, transfer factors) and required multiple values because the analysis was 
conducted for multiple radionuclides; therefore, the radionuclide- and element-specific parameters used in the analysis are listed in separate tables.

Parameter distributions for Kd values are listed in Tables A.2 and A.3. Parameter distributions for plant transfer factors, meat transfer factors, milk transfer factors, and fish and crustacean transfer factors are listed in Tables A.3-A.7, respectively. The distribution function notations used in the RESRAD (onsite) input parameter table (Table A.1) are listed in Table A.8.

Some of the input parameters are correlated. In cases for which a clear relationship exists between parameters, strong correlations were used as input to ensure proper pairing. These include the pairs for bulk density and total porosity (correlation coefficient of -0.99), bulk density and effective porosity (correlation coefficient of -0.99), and total porosity and effective porosity (correlation coefficient of 0.99). Table A.9 lists the correlations used in the analysis. A few parameter values and distributions that are scenario-specific and distinguish the scenarios from one another (such as intake rates and time spent onsite) are specified for each scenario. For those parameters not specified with a single value, the default probabilistic distribution was used. This results in a dose probability distribution (presented as the cumulative distribution function, CDF). The RESRAD-OFFSITE code requires many more parameters than the RESRAD (onsite) code; the additional input parameters for the RESRAD-OFFSITE analysis are listed in Table A.10.

Since the peak dose depends linearly on radionuclide concentration, the analysis was done for the unit concentration of each radionuclide for four scenarios. For this analysis, 4,500 input sets (1,500 observations and 3 repetitions) were generated. For each set of sampled parameter values, the peak dose in the time interval 0-1,000 years was calculated. Cumulative probabilities were estimated from the resulting dose distribution for each scenario.

Tables 4-17 (at the end of Section 3.1) provide total peak DSR percentiles (mrem/yr per $\mathrm{pCi} / \mathrm{g}$ ) for different exposure scenarios. Also listed in Tables 4-17 are the mean of the peaks DSR and the peak of the means DSR. The mean of the peaks DSR is always greater than or equal to the peak of the means DSR. However, if the peak DSRs occurred at the same time for all input sets analyzed, then there would not be any difference between the mean of the peaks DSR and peak of the means DSR. The peak DSRs from 4,500 realizations were analyzed, and the percentiles for different exposure pathways are provided in Appendix C. Water-independent (WI) and water-dependent (WD) pathways are distinguished by the extensions WI and WD, respectively. Tables C.1-C.14 show the peak DSR percentiles (mrem/yr per $\mathrm{pCi} / \mathrm{g}$ ) for different exposure pathways for the industrial use scenario. RESRAD (onsite) Version 6.5 was used in the analysis. For Am-241, I-129, and Tc-99, external exposure and soil ingestion were the dominant exposure pathways. For C-14, only inhalation was the dominant exposure pathway. For Co-60, Cs-137, Np-237, Sr-90, U-235, and U-238, external exposure was the dominant exposure pathway. For $\mathrm{Pu}-238, \mathrm{Pu}-239$, and $\mathrm{Pu}-240$, soil ingestion and inhalation were two dominant exposure pathways. For U-234, radon inhalation was the dominant exposure pathway.

Tables C.15-C.28 show the peak DSR percentiles (mrem/yr per pCi/g) for different exposure pathways for individual radionuclides in the offsite resident scenario. RESRADOFFSITE Version 2.6 was used in the analysis. For all radionuclides except Co-60, WD 
pathways were dominant. For Am-241, C-14, Cs-137, and U-234, aquatic food ingestion resulted in the most dose. For Co-60, the external exposure pathway was dominant. For I-129, meat and milk resulted in the most dose. For $\mathrm{Np}-237, \mathrm{Pu}-238, \mathrm{Pu}-239$, and $\mathrm{Pu}-240$, fish and plant ingestion were dominant exposure pathways. For Sr-90 meat and fish ingestion resulted in the most dose. For Tc-99, plant, milk, and fish ingestion resulted in the most dose. For U-235 and U-238, external exposure and fish ingestion resulted in the most dose.

Tables C.29-C.42 show the peak DSR percentiles (mrem/yr per pCi/g) for different exposure pathways for individual radionuclides in the recreational use scenario. RESRAD (onsite) Version 6.5 was used in the analysis. For C-14, I-129, Np-237, and Tc-99, WD pathways were dominant, and for other radionuclides (Am-241, Co-60, Cs-137, Pu-238, Pu-239, Pu-240, Sr-90, U-234, U-235, and U-238), WI pathways were dominant. For Am-241, Co-60, U-235, and U-238, external exposure was dominant. For C-14, I-129, Np-237, and Tc-99, aquatic food ingestion resulted in the most dose. For Cs-137, external exposure and meat ingestion resulted in the most dose. For $\mathrm{Pu}-238, \mathrm{Pu}-239$, and $\mathrm{Pu}-240$, meat ingestion, soil ingestion, and inhalation were dominant exposure pathways. For Sr-90, meat ingestion resulted in the most dose. For U-234, meat ingestion and external exposure were dominant.

Tables C.43-C.56 show the peak DSR percentiles (mrem/yr per $\mathrm{pCi} / \mathrm{g}$ ) for different exposure pathways for individual radionuclides in the onsite resident scenario. RESRAD (onsite) Version 6.5 was used in the analysis. For C-14, I-129, Np-237, and Tc-99, WD pathways were dominant, and for other radionuclides (Am-241, Co-60, Cs-137, Pu-238, Pu-239, Pu-240, Sr-90, U-234, U-235, and U-238), WI pathways were dominant. For Am-241, Pu-238, Pu-239, Pu-240, and Sr-90, plant ingestion was dominant. For C-14, aquatic food ingestion resulted in the most dose. For Co-60, external exposure was dominant. For Cs-137, external exposure, meat and plant ingestion resulted in the most dose. For I-129, Np-237, and Tc-99 water ingestion resulted in the most dose. For U-234, radon inhalation resulted in the most dose. For U-235, external exposure and plant ingestion resulted in the most dose. For U-238, water ingestion, plant ingestion, and external exposure resulted in the most dose.

Table 4 shows the total peak DSR percentiles of Am-241 for different exposure scenarios considered in deriving DCGLs for the Building 310 area at Argonne. The future onsite resident (after the institutional control period of 100 years) would receive the highest dose.

Table 5 shows the total peak DSR percentiles of C-14 for different exposure scenarios considered in deriving DCGLs for the Building 310 area at Argonne. The current offsite resident (current use scenario) and recreational use (after the institutional control period of 100 years) would receive the highest dose.

Table 6 shows the total peak DSR percentiles of Co-60 for different exposure scenarios considered in deriving DCGLs for the Building 310 area at Argonne. The industrial worker (current use scenario) would receive the highest dose.

Table 7 shows the total peak DSR percentiles of Cs-137 for different exposure scenarios considered in deriving DCGLs for the Building 310 area at Argonne. The industrial worker (current use scenario) would receive the highest dose. 
Table 8 shows the total peak DSR percentiles of I-129 for different exposure scenarios considered in deriving DCGLs for the Building 310 area at Argonne. The future onsite resident (after the institutional control period of 100 years) would receive the highest dose.

Table 9 shows the total peak DSR percentiles of Np-237 for different exposure scenarios considered in deriving DCGLs for the Building 310 area at Argonne. The future onsite resident (after the institutional control period of 100 years) would receive the highest dose.

Table 10 shows the total peak DSR percentiles of Pu-238 for different exposure scenarios considered in deriving DCGLs for the Building 310 area at Argonne. The future onsite resident (after the institutional control period of 100 years) would receive the highest dose.

Table 11 shows the total peak DSR percentiles of $\mathrm{Pu}-239$ for different exposure scenarios considered in deriving DCGLs for the Building 310 area at Argonne. The future onsite resident (after the institutional control period of 100 years) would receive the highest dose.

Table 12 shows the total peak DSR percentiles of $\mathrm{Pu}-240$ for different exposure scenarios considered in deriving DCGLs for the Building 310 area at Argonne. The future onsite resident (after the institutional control period of 100 years) would receive the highest dose.

Table 13 shows the total peak DSR percentiles of Sr-90 for different exposure scenarios considered in deriving DCGLs for the Building 310 area at Argonne. The future onsite resident (after the institutional control period of 100 years) would receive the highest dose.

Table 14 shows the total peak DSR percentiles of Tc-99 for different exposure scenarios considered in deriving DCGLs for the Building 310 area at Argonne. The future onsite resident (after the institutional control period of 100 years) would receive the highest dose.

Table 15 shows the total peak DSR percentiles of U-234 for different exposure scenarios considered in deriving DCGLs for the Building 310 area at Argonne. The future onsite resident (after the institutional control period of 100 years) would receive the highest dose.

Table 16 shows the total peak DSR percentiles of U-235 for different exposure scenarios considered in deriving DCGLs for the Building 310 area at Argonne. The future onsite resident (after the institutional control period of 100 years) would receive the highest dose.

Table 17 shows the total peak DSR percentiles of U-238 for different exposure scenarios considered in deriving DCGLs for the Building 310 area at Argonne. The future onsite resident (after the institutional control period of 100 years) would receive the highest dose.

Table 18 shows the peak of the means DSR, mean of the peaks DSR, and 95th percentile of the peaks DSR of each radionuclide for different scenarios. These values were used in deriving DCGLs. The scenario that results in the most dose from an individual radionuclide is highlighted. 
TABLE 4 Total Peak DSR Percentiles (mrem/yr per pCi/g) of Am-241 for Different Exposure Scenarios for the Building 310 Area

\begin{tabular}{ccccc}
\hline & & & & \\
Percentile & Industrial Use & Offsite Resident & Recreational Use & Onsite Resident \\
\hline $5 \%$ & $1.47 \mathrm{E}-03$ & $2.16 \mathrm{E}-03$ & $1.81 \mathrm{E}-03$ & $2.96 \mathrm{E}-02$ \\
$10 \%$ & $4.06 \mathrm{E}-03$ & $2.69 \mathrm{E}-03$ & $1.96 \mathrm{E}-03$ & $3.61 \mathrm{E}-02$ \\
$15 \%$ & $6.76 \mathrm{E}-03$ & $3.32 \mathrm{E}-03$ & $2.05 \mathrm{E}-03$ & $4.05 \mathrm{E}-02$ \\
$20 \%$ & $9.42 \mathrm{E}-03$ & $4.17 \mathrm{E}-03$ & $2.11 \mathrm{E}-03$ & $4.48 \mathrm{E}-02$ \\
$25 \%$ & $1.11 \mathrm{E}-02$ & $4.91 \mathrm{E}-03$ & $2.16 \mathrm{E}-03$ & $4.91 \mathrm{E}-02$ \\
$30 \%$ & $1.21 \mathrm{E}-02$ & $5.62 \mathrm{E}-03$ & $2.22 \mathrm{E}-03$ & $5.33 \mathrm{E}-02$ \\
$35 \%$ & $1.27 \mathrm{E}-02$ & $6.21 \mathrm{E}-03$ & $2.26 \mathrm{E}-03$ & $5.75 \mathrm{E}-02$ \\
$40 \%$ & $1.33 \mathrm{E}-02$ & $6.66 \mathrm{E}-03$ & $2.31 \mathrm{E}-03$ & $6.29 \mathrm{E}-02$ \\
$45 \%$ & $1.37 \mathrm{E}-02$ & $6.92 \mathrm{E}-03$ & $2.36 \mathrm{E}-03$ & $6.81 \mathrm{E}-02$ \\
$50 \%$ & $1.43 \mathrm{E}-02$ & $7.12 \mathrm{E}-03$ & $2.40 \mathrm{E}-03$ & $7.46 \mathrm{E}-02$ \\
$55 \%$ & $1.47 \mathrm{E}-02$ & $7.31 \mathrm{E}-03$ & $2.44 \mathrm{E}-03$ & $8.03 \mathrm{E}-02$ \\
$60 \%$ & $1.52 \mathrm{E}-02$ & $7.49 \mathrm{E}-03$ & $2.49 \mathrm{E}-03$ & $8.78 \mathrm{E}-02$ \\
$65 \%$ & $1.57 \mathrm{E}-02$ & $7.69 \mathrm{E}-03$ & $2.54 \mathrm{E}-03$ & $9.62 \mathrm{E}-02$ \\
$70 \%$ & $1.62 \mathrm{E}-02$ & $7.95 \mathrm{E}-03$ & $2.58 \mathrm{E}-03$ & $1.07 \mathrm{E}-01$ \\
$75 \%$ & $1.68 \mathrm{E}-02$ & $8.26 \mathrm{E}-03$ & $2.64 \mathrm{E}-03$ & $1.20 \mathrm{E}-01$ \\
$80 \%$ & $1.75 \mathrm{E}-02$ & $8.66 \mathrm{E}-03$ & $2.71 \mathrm{E}-03$ & $1.37 \mathrm{E}-01$ \\
$85 \%$ & $1.84 \mathrm{E}-02$ & $9.24 \mathrm{E}-03$ & $2.79 \mathrm{E}-03$ & $1.61 \mathrm{E}-01$ \\
$90 \%$ & $1.97 \mathrm{E}-02$ & $1.00 \mathrm{E}-02$ & $2.89 \mathrm{E}-03$ & $1.96 \mathrm{E}-01$ \\
$95 \%$ & $2.19 \mathrm{E}-02$ & $1.19 \mathrm{E}-02$ & $3.05 \mathrm{E}-03$ & $2.68 \mathrm{E}-01$ \\
Mean of the peaks & $1.35 \mathrm{E}-02$ & $6.98 \mathrm{E}-03$ & $2.40 \mathrm{E}-03$ & $1.05 \mathrm{E}-01$ \\
Peak of the means & $1.35 \mathrm{E}-02$ & $6.87 \mathrm{E}-03$ & $2.40 \mathrm{E}-03$ & $1.05 \mathrm{E}-01$ \\
\hline
\end{tabular}


TABLE 5 Total Peak DSR Percentiles (mrem/yr per pCi/g) of C-14 for Different Exposure Scenarios for the Building 310 Area

\begin{tabular}{ccccc}
\hline Percentile & Industrial Use & Offsite Resident & Recreational Use & Onsite Resident \\
\hline $5 \%$ & $2.22 \mathrm{E}-06$ & $2.48 \mathrm{E}-04$ & $0.00 \mathrm{E}+00$ & $0.00 \mathrm{E}+00$ \\
$10 \%$ & $5.82 \mathrm{E}-06$ & $3.76 \mathrm{E}-04$ & $0.00 \mathrm{E}+00$ & $0.00 \mathrm{E}+00$ \\
$15 \%$ & $1.02 \mathrm{E}-05$ & $4.97 \mathrm{E}-04$ & $0.00 \mathrm{E}+00$ & $0.00 \mathrm{E}+00$ \\
$20 \%$ & $1.34 \mathrm{E}-05$ & $6.40 \mathrm{E}-04$ & $0.00 \mathrm{E}+00$ & $0.00 \mathrm{E}+00$ \\
$25 \%$ & $1.53 \mathrm{E}-05$ & $8.00 \mathrm{E}-04$ & $0.00 \mathrm{E}+00$ & $0.00 \mathrm{E}+00$ \\
$30 \%$ & $1.68 \mathrm{E}-05$ & $9.86 \mathrm{E}-04$ & $0.00 \mathrm{E}+00$ & $0.00 \mathrm{E}+00$ \\
$35 \%$ & $1.79 \mathrm{E}-05$ & $1.21 \mathrm{E}-03$ & $0.00 \mathrm{E}+00$ & $0.00 \mathrm{E}+00$ \\
$40 \%$ & $1.90 \mathrm{E}-05$ & $1.47 \mathrm{E}-03$ & $0.00 \mathrm{E}+00$ & $0.00 \mathrm{E}+00$ \\
$45 \%$ & $2.02 \mathrm{E}-05$ & $1.85 \mathrm{E}-03$ & $0.00 \mathrm{E}+00$ & $0.00 \mathrm{E}+00$ \\
$50 \%$ & $2.13 \mathrm{E}-05$ & $2.36 \mathrm{E}-03$ & $0.00 \mathrm{E}+00$ & $0.00 \mathrm{E}+00$ \\
$55 \%$ & $2.23 \mathrm{E}-05$ & $2.96 \mathrm{E}-03$ & $0.00 \mathrm{E}+00$ & $0.00 \mathrm{E}+00$ \\
$60 \%$ & $2.34 \mathrm{E}-05$ & $3.89 \mathrm{E}-03$ & $0.00 \mathrm{E}+00$ & $0.00 \mathrm{E}+00$ \\
$65 \%$ & $2.45 \mathrm{E}-05$ & $5.35 \mathrm{E}-03$ & $0.00 \mathrm{E}+00$ & $0.00 \mathrm{E}+00$ \\
$70 \%$ & $2.56 \mathrm{E}-05$ & $7.94 \mathrm{E}-03$ & $1.77 \mathrm{E}-17$ & $0.00 \mathrm{E}+00$ \\
$75 \%$ & $2.70 \mathrm{E}-05$ & $1.27 \mathrm{E}-02$ & $4.24 \mathrm{E}-03$ & $0.00 \mathrm{E}+00$ \\
$80 \%$ & $2.87 \mathrm{E}-05$ & $2.31 \mathrm{E}-02$ & $1.55 \mathrm{E}-02$ & $0.00 \mathrm{E}+00$ \\
$85 \%$ & $3.05 \mathrm{E}-05$ & $4.19 \mathrm{E}-02$ & $5.45 \mathrm{E}-02$ & $1.01 \mathrm{E}-03$ \\
$90 \%$ & $3.32 \mathrm{E}-05$ & $8.49 \mathrm{E}-02$ & $1.17 \mathrm{E}-01$ & $3.45 \mathrm{E}-03$ \\
$95 \%$ & $3.73 \mathrm{E}-05$ & $2.27 \mathrm{E}-01$ & $2.50 \mathrm{E}-01$ & $1.05 \mathrm{E}-02$ \\
Mean of the peaks & $2.10 \mathrm{E}-05$ & $5.95 \mathrm{E}-02$ & $4.61 \mathrm{E}-02$ & $3.36 \mathrm{E}-03$ \\
Peak of the means & $2.10 \mathrm{E}-05$ & $1.50 \mathrm{E}-02$ & $1.58 \mathrm{E}-02$ & $3.54 \mathrm{E}-03$ \\
\hline
\end{tabular}


TABLE 6 Total Peak DSR Percentiles (mrem/yr per pCi/g) of Co-60 for Different Exposure Scenarios for the Building 310 Area

\begin{tabular}{ccccc}
\hline Percentile & Industrial Use & Offsite Resident & Recreational Use & Onsite Resident \\
\hline $5 \%$ & $3.24 \mathrm{E}-01$ & $7.92 \mathrm{E}-04$ & $5.60 \mathrm{E}-07$ & $3.32 \mathrm{E}-06$ \\
$10 \%$ & $9.57 \mathrm{E}-01$ & $7.93 \mathrm{E}-04$ & $7.51 \mathrm{E}-07$ & $4.10 \mathrm{E}-06$ \\
$15 \%$ & $1.59 \mathrm{E}+00$ & $7.93 \mathrm{E}-04$ & $8.50 \mathrm{E}-07$ & $4.61 \mathrm{E}-06$ \\
$20 \%$ & $2.22 \mathrm{E}+00$ & $7.94 \mathrm{E}-04$ & $9.01 \mathrm{E}-07$ & $4.97 \mathrm{E}-06$ \\
$25 \%$ & $2.85 \mathrm{E}+00$ & $7.94 \mathrm{E}-04$ & $9.41 \mathrm{E}-07$ & $5.28 \mathrm{E}-06$ \\
$30 \%$ & $2.96 \mathrm{E}+00$ & $7.95 \mathrm{E}-04$ & $9.64 \mathrm{E}-07$ & $5.59 \mathrm{E}-06$ \\
$35 \%$ & $3.07 \mathrm{E}+00$ & $7.96 \mathrm{E}-04$ & $9.81 \mathrm{E}-07$ & $5.93 \mathrm{E}-06$ \\
$40 \%$ & $3.17 \mathrm{E}+00$ & $7.96 \mathrm{E}-04$ & $9.93 \mathrm{E}-07$ & $6.25 \mathrm{E}-06$ \\
$45 \%$ & $3.28 \mathrm{E}+00$ & $7.97 \mathrm{E}-04$ & $1.00 \mathrm{E}-06$ & $6.61 \mathrm{E}-06$ \\
$50 \%$ & $3.39 \mathrm{E}+00$ & $7.97 \mathrm{E}-04$ & $1.01 \mathrm{E}-06$ & $6.94 \mathrm{E}-06$ \\
$55 \%$ & $3.46 \mathrm{E}+00$ & $7.98 \mathrm{E}-04$ & $1.01 \mathrm{E}-06$ & $7.32 \mathrm{E}-06$ \\
$60 \%$ & $3.53 \mathrm{E}+00$ & $7.99 \mathrm{E}-04$ & $1.02 \mathrm{E}-06$ & $7.72 \mathrm{E}-06$ \\
$65 \%$ & $3.61 \mathrm{E}+00$ & $8.00 \mathrm{E}-04$ & $1.02 \mathrm{E}-06$ & $8.20 \mathrm{E}-06$ \\
$70 \%$ & $3.67 \mathrm{E}+00$ & $8.01 \mathrm{E}-04$ & $1.03 \mathrm{E}-06$ & $8.66 \mathrm{E}-06$ \\
$75 \%$ & $3.75 \mathrm{E}+00$ & $8.03 \mathrm{E}-04$ & $1.04 \mathrm{E}-06$ & $9.17 \mathrm{E}-06$ \\
$80 \%$ & $3.95 \mathrm{E}+00$ & $8.05 \mathrm{E}-04$ & $1.05 \mathrm{E}-06$ & $9.88 \mathrm{E}-06$ \\
$85 \%$ & $4.15 \mathrm{E}+00$ & $8.08 \mathrm{E}-04$ & $1.07 \mathrm{E}-06$ & $1.08 \mathrm{E}-05$ \\
$90 \%$ & $4.36 \mathrm{E}+00$ & $8.12 \mathrm{E}-04$ & $1.10 \mathrm{E}-06$ & $1.19 \mathrm{E}-05$ \\
$95 \%$ & $4.64 \mathrm{E}+00$ & $9.01 \mathrm{E}-04$ & $1.17 \mathrm{E}-06$ & $1.40 \mathrm{E}-05$ \\
Mean of the peaks & $3.11 \mathrm{E}+00$ & $8.20 \mathrm{E}-04$ & $9.65 \mathrm{E}-07$ & $7.54 \mathrm{E}-06$ \\
Peak of the means & $3.11 \mathrm{E}+00$ & $8.01 \mathrm{E}-04$ & $9.66 \mathrm{E}-07$ & $7.54 \mathrm{E}-06$ \\
\hline
\end{tabular}


TABLE 7 Total Peak DSR Percentiles (mrem/yr per pCi/g) of Cs-137 for Different Exposure Scenarios for the Building 310 Area

\begin{tabular}{ccccc}
\hline Percentile & Industrial Use & Offsite Resident & Recreational Use & Onsite Resident \\
\hline $5 \%$ & $7.12 \mathrm{E}-02$ & $2.69 \mathrm{E}-03$ & $1.27 \mathrm{E}-02$ & $7.02 \mathrm{E}-02$ \\
$10 \%$ & $2.09 \mathrm{E}-01$ & $3.10 \mathrm{E}-03$ & $1.33 \mathrm{E}-02$ & $7.93 \mathrm{E}-02$ \\
$15 \%$ & $3.47 \mathrm{E}-01$ & $3.45 \mathrm{E}-03$ & $1.37 \mathrm{E}-02$ & $8.78 \mathrm{E}-02$ \\
$20 \%$ & $4.85 \mathrm{E}-01$ & $3.76 \mathrm{E}-03$ & $1.42 \mathrm{E}-02$ & $9.41 \mathrm{E}-02$ \\
$25 \%$ & $6.22 \mathrm{E}-01$ & $4.08 \mathrm{E}-03$ & $1.46 \mathrm{E}-02$ & $1.00 \mathrm{E}-01$ \\
$30 \%$ & $6.46 \mathrm{E}-01$ & $4.39 \mathrm{E}-03$ & $1.51 \mathrm{E}-02$ & $1.07 \mathrm{E}-01$ \\
$35 \%$ & $6.70 \mathrm{E}-01$ & $4.71 \mathrm{E}-03$ & $1.55 \mathrm{E}-02$ & $1.14 \mathrm{E}-01$ \\
$40 \%$ & $6.93 \mathrm{E}-01$ & $5.05 \mathrm{E}-03$ & $1.60 \mathrm{E}-02$ & $1.21 \mathrm{E}-01$ \\
$45 \%$ & $7.17 \mathrm{E}-01$ & $5.41 \mathrm{E}-03$ & $1.65 \mathrm{E}-02$ & $1.28 \mathrm{E}-01$ \\
$50 \%$ & $7.41 \mathrm{E}-01$ & $5.76 \mathrm{E}-03$ & $1.71 \mathrm{E}-02$ & $1.36 \mathrm{E}-01$ \\
$55 \%$ & $7.56 \mathrm{E}-01$ & $6.13 \mathrm{E}-03$ & $1.77 \mathrm{E}-02$ & $1.43 \mathrm{E}-01$ \\
$60 \%$ & $7.71 \mathrm{E}-01$ & $6.54 \mathrm{E}-03$ & $1.83 \mathrm{E}-02$ & $1.52 \mathrm{E}-01$ \\
$65 \%$ & $7.87 \mathrm{E}-01$ & $7.07 \mathrm{E}-03$ & $1.92 \mathrm{E}-02$ & $1.61 \mathrm{E}-01$ \\
$70 \%$ & $8.02 \mathrm{E}-01$ & $7.62 \mathrm{E}-03$ & $2.00 \mathrm{E}-02$ & $1.71 \mathrm{E}-01$ \\
$75 \%$ & $8.18 \mathrm{E}-01$ & $8.38 \mathrm{E}-03$ & $2.11 \mathrm{E}-02$ & $1.83 \mathrm{E}-01$ \\
$80 \%$ & $8.62 \mathrm{E}-01$ & $9.14 \mathrm{E}-03$ & $2.25 \mathrm{E}-02$ & $1.99 \mathrm{E}-01$ \\
$85 \%$ & $9.06 \mathrm{E}-01$ & $1.03 \mathrm{E}-02$ & $2.45 \mathrm{E}-02$ & $2.23 \mathrm{E}-01$ \\
$90 \%$ & $9.51 \mathrm{E}-01$ & $1.18 \mathrm{E}-02$ & $2.76 \mathrm{E}-02$ & $2.56 \mathrm{E}-01$ \\
$95 \%$ & $1.01 \mathrm{E}+00$ & $1.49 \mathrm{E}-02$ & $3.49 \mathrm{E}-02$ & $3.17 \mathrm{E}-01$ \\
Mean of the peaks & $6.78 \mathrm{E}-01$ & $6.87 \mathrm{E}-03$ & $1.95 \mathrm{E}-02$ & $1.59 \mathrm{E}-01$ \\
Peak of the means & $6.78 \mathrm{E}-01$ & $6.88 \mathrm{E}-03$ & $1.95 \mathrm{E}-02$ & $1.59 \mathrm{E}-01$ \\
\hline
\end{tabular}


TABLE 8 Total Peak DSR Percentiles (mrem/yr per pCi/g) of I-129 for Different Exposure Scenarios for the Building 310 Area

\begin{tabular}{ccccc}
\hline Percentile & Industrial Use & Offsite Resident & Recreational Use & Onsite Resident \\
\hline $5 \%$ & $3.70 \mathrm{E}-04$ & $1.22 \mathrm{E}-02$ & $1.45 \mathrm{E}-17$ & $1.42 \mathrm{E}-06$ \\
$10 \%$ & $1.09 \mathrm{E}-03$ & $3.01 \mathrm{E}-02$ & $5.66 \mathrm{E}-12$ & $5.00 \mathrm{E}-03$ \\
$15 \%$ & $1.77 \mathrm{E}-03$ & $5.51 \mathrm{E}-02$ & $5.75 \mathrm{E}-09$ & $1.55 \mathrm{E}-01$ \\
$20 \%$ & $2.37 \mathrm{E}-03$ & $8.14 \mathrm{E}-02$ & $4.66 \mathrm{E}-07$ & $8.83 \mathrm{E}-01$ \\
$25 \%$ & $2.75 \mathrm{E}-03$ & $1.21 \mathrm{E}-01$ & $7.19 \mathrm{E}-06$ & $2.06 \mathrm{E}+00$ \\
$30 \%$ & $3.02 \mathrm{E}-03$ & $1.59 \mathrm{E}-01$ & $8.02 \mathrm{E}-05$ & $3.27 \mathrm{E}+00$ \\
$35 \%$ & $3.25 \mathrm{E}-03$ & $2.04 \mathrm{E}-01$ & $5.41 \mathrm{E}-04$ & $4.62 \mathrm{E}+00$ \\
$40 \%$ & $3.45 \mathrm{E}-03$ & $2.64 \mathrm{E}-01$ & $2.25 \mathrm{E}-03$ & $5.92 \mathrm{E}+00$ \\
$45 \%$ & $3.65 \mathrm{E}-03$ & $3.24 \mathrm{E}-01$ & $6.31 \mathrm{E}-03$ & $7.37 \mathrm{E}+00$ \\
$50 \%$ & $3.82 \mathrm{E}-03$ & $4.02 \mathrm{E}-01$ & $1.37 \mathrm{E}-02$ & $9.37 \mathrm{E}+00$ \\
$55 \%$ & $3.98 \mathrm{E}-03$ & $4.89 \mathrm{E}-01$ & $2.56 \mathrm{E}-02$ & $1.21 \mathrm{E}+01$ \\
$60 \%$ & $4.13 \mathrm{E}-03$ & $5.85 \mathrm{E}-01$ & $4.31 \mathrm{E}-02$ & $1.50 \mathrm{E}+01$ \\
$65 \%$ & $4.29 \mathrm{E}-03$ & $7.03 \mathrm{E}-01$ & $6.71 \mathrm{E}-02$ & $1.92 \mathrm{E}+01$ \\
$70 \%$ & $4.46 \mathrm{E}-03$ & $8.73 \mathrm{E}-01$ & $9.89 \mathrm{E}-02$ & $2.46 \mathrm{E}+01$ \\
$75 \%$ & $4.67 \mathrm{E}-03$ & $1.08 \mathrm{E}+00$ & $1.38 \mathrm{E}-01$ & $3.17 \mathrm{E}+01$ \\
$80 \%$ & $4.90 \mathrm{E}-03$ & $1.38 \mathrm{E}+00$ & $2.04 \mathrm{E}-01$ & $4.11 \mathrm{E}+01$ \\
$85 \%$ & $5.15 \mathrm{E}-03$ & $1.79 \mathrm{E}+00$ & $3.18 \mathrm{E}-01$ & $5.35 \mathrm{E}+01$ \\
$90 \%$ & $5.50 \mathrm{E}-03$ & $2.37 \mathrm{E}+00$ & $5.33 \mathrm{E}-01$ & $7.18 \mathrm{E}+01$ \\
$95 \%$ & $6.15 \mathrm{E}-03$ & $3.55 \mathrm{E}+00$ & $1.13 \mathrm{E}+00$ & $1.04 \mathrm{E}+02$ \\
Mean of the peaks & $3.64 \mathrm{E}-03$ & $9.30 \mathrm{E}-01$ & $2.25 \mathrm{E}-01$ & $2.51 \mathrm{E}+01$ \\
Peak of the means & $3.64 \mathrm{E}-03$ & $1.96 \mathrm{E}-01$ & $7.12 \mathrm{E}-02$ & $3.16 \mathrm{E}+00$ \\
\hline
\end{tabular}


TABLE 9 Total Peak DSR Percentiles (mrem/yr per pCi/g) of Np-237 for Different Exposure Scenarios for the Building 310 Area

\begin{tabular}{ccccc}
\hline Percentile & Industrial Use & Offsite Resident & Recreational Use & Onsite Resident \\
\hline $5 \%$ & $2.26 \mathrm{E}-02$ & $8.32 \mathrm{E}-04$ & $3.11 \mathrm{E}-08$ & $5.48 \mathrm{E}-07$ \\
$10 \%$ & $6.53 \mathrm{E}-02$ & $1.95 \mathrm{E}-03$ & $1.80 \mathrm{E}-07$ & $6.54 \mathrm{E}-06$ \\
$15 \%$ & $1.10 \mathrm{E}-01$ & $2.36 \mathrm{E}-03$ & $2.34 \mathrm{E}-04$ & $8.01 \mathrm{E}-03$ \\
$20 \%$ & $1.50 \mathrm{E}-01$ & $2.71 \mathrm{E}-03$ & $5.14 \mathrm{E}-03$ & $1.69 \mathrm{E}-01$ \\
$25 \%$ & $1.82 \mathrm{E}-01$ & $3.13 \mathrm{E}-03$ & $1.73 \mathrm{E}-02$ & $3.90 \mathrm{E}-01$ \\
$30 \%$ & $2.03 \mathrm{E}-01$ & $3.54 \mathrm{E}-03$ & $2.88 \mathrm{E}-02$ & $5.26 \mathrm{E}-01$ \\
$35 \%$ & $2.11 \mathrm{E}-01$ & $4.14 \mathrm{E}-03$ & $3.60 \mathrm{E}-02$ & $6.50 \mathrm{E}-01$ \\
$40 \%$ & $2.19 \mathrm{E}-01$ & $4.78 \mathrm{E}-03$ & $3.88 \mathrm{E}-02$ & $7.57 \mathrm{E}-01$ \\
$45 \%$ & $2.26 \mathrm{E}-01$ & $5.49 \mathrm{E}-03$ & $4.05 \mathrm{E}-02$ & $8.72 \mathrm{E}-01$ \\
$50 \%$ & $2.33 \mathrm{E}-01$ & $6.49 \mathrm{E}-03$ & $4.21 \mathrm{E}-02$ & $9.93 \mathrm{E}-01$ \\
$55 \%$ & $2.41 \mathrm{E}-01$ & $7.83 \mathrm{E}-03$ & $4.38 \mathrm{E}-02$ & $1.15 \mathrm{E}+00$ \\
$60 \%$ & $2.47 \mathrm{E}-01$ & $9.73 \mathrm{E}-03$ & $4.59 \mathrm{E}-02$ & $1.32 \mathrm{E}+00$ \\
$65 \%$ & $2.52 \mathrm{E}-01$ & $1.24 \mathrm{E}-02$ & $4.81 \mathrm{E}-02$ & $1.54 \mathrm{E}+00$ \\
$70 \%$ & $2.57 \mathrm{E}-01$ & $1.56 \mathrm{E}-02$ & $5.14 \mathrm{E}-02$ & $1.85 \mathrm{E}+00$ \\
$75 \%$ & $2.64 \mathrm{E}-01$ & $2.01 \mathrm{E}-02$ & $5.61 \mathrm{E}-02$ & $2.23 \mathrm{E}+00$ \\
$80 \%$ & $2.73 \mathrm{E}-01$ & $2.62 \mathrm{E}-02$ & $6.42 \mathrm{E}-02$ & $2.79 \mathrm{E}+00$ \\
$85 \%$ & $2.91 \mathrm{E}-01$ & $3.70 \mathrm{E}-02$ & $8.33 \mathrm{E}-02$ & $3.70 \mathrm{E}+00$ \\
$90 \%$ & $3.07 \mathrm{E}-01$ & $6.07 \mathrm{E}-02$ & $1.31 \mathrm{E}-01$ & $5.43 \mathrm{E}+00$ \\
$95 \%$ & $3.28 \mathrm{E}-01$ & $1.17 \mathrm{E}-01$ & $2.92 \mathrm{E}-01$ & $1.04 \mathrm{E}+01$ \\
Mean of the peaks & $2.15 \mathrm{E}-01$ & $3.48 \mathrm{E}-02$ & $9.41 \mathrm{E}-02$ & $2.64 \mathrm{E}+00$ \\
Peak of the means & $2.15 \mathrm{E}-01$ & $9.30 \mathrm{E}-03$ & $4.68 \mathrm{E}-02$ & $1.11 \mathrm{E}+00$ \\
\hline
\end{tabular}


TABLE 10 Total Peak DSR Percentiles (mrem/yr per pCi/g) of Pu-238 for Different Exposure Scenarios for the Building 310 Area

\begin{tabular}{ccccc}
\hline Percentile & Industrial Use & Offsite Resident & Recreational Use & Onsite Resident \\
\hline $5 \%$ & $6.12 \mathrm{E}-04$ & $8.84 \mathrm{E}-04$ & $6.07 \mathrm{E}-04$ & $1.42 \mathrm{E}-02$ \\
$10 \%$ & $1.65 \mathrm{E}-03$ & $1.18 \mathrm{E}-03$ & $6.87 \mathrm{E}-04$ & $1.74 \mathrm{E}-02$ \\
$15 \%$ & $2.51 \mathrm{E}-03$ & $1.37 \mathrm{E}-03$ & $7.47 \mathrm{E}-04$ & $2.00 \mathrm{E}-02$ \\
$20 \%$ & $3.24 \mathrm{E}-03$ & $1.51 \mathrm{E}-03$ & $8.00 \mathrm{E}-04$ & $2.22 \mathrm{E}-02$ \\
$25 \%$ & $3.85 \mathrm{E}-03$ & $1.65 \mathrm{E}-03$ & $8.37 \mathrm{E}-04$ & $2.48 \mathrm{E}-02$ \\
$30 \%$ & $4.36 \mathrm{E}-03$ & $1.75 \mathrm{E}-03$ & $8.72 \mathrm{E}-04$ & $2.75 \mathrm{E}-02$ \\
$35 \%$ & $4.81 \mathrm{E}-03$ & $1.88 \mathrm{E}-03$ & $9.09 \mathrm{E}-04$ & $3.03 \mathrm{E}-02$ \\
$40 \%$ & $5.21 \mathrm{E}-03$ & $2.02 \mathrm{E}-03$ & $9.42 \mathrm{E}-04$ & $3.33 \mathrm{E}-02$ \\
$45 \%$ & $5.56 \mathrm{E}-03$ & $2.15 \mathrm{E}-03$ & $9.83 \mathrm{E}-04$ & $3.65 \mathrm{E}-02$ \\
$50 \%$ & $5.92 \mathrm{E}-03$ & $2.29 \mathrm{E}-03$ & $1.02 \mathrm{E}-03$ & $4.05 \mathrm{E}-02$ \\
$55 \%$ & $6.27 \mathrm{E}-03$ & $2.47 \mathrm{E}-03$ & $1.06 \mathrm{E}-03$ & $4.43 \mathrm{E}-02$ \\
$60 \%$ & $6.65 \mathrm{E}-03$ & $2.65 \mathrm{E}-03$ & $1.11 \mathrm{E}-03$ & $4.90 \mathrm{E}-02$ \\
$65 \%$ & $7.07 \mathrm{E}-03$ & $2.89 \mathrm{E}-03$ & $1.15 \mathrm{E}-03$ & $5.36 \mathrm{E}-02$ \\
$70 \%$ & $7.54 \mathrm{E}-03$ & $3.17 \mathrm{E}-03$ & $1.20 \mathrm{E}-03$ & $5.97 \mathrm{E}-02$ \\
$75 \%$ & $7.97 \mathrm{E}-03$ & $3.49 \mathrm{E}-03$ & $1.25 \mathrm{E}-03$ & $6.82 \mathrm{E}-02$ \\
$80 \%$ & $8.46 \mathrm{E}-03$ & $3.91 \mathrm{E}-03$ & $1.32 \mathrm{E}-03$ & $7.85 \mathrm{E}-02$ \\
$85 \%$ & $9.12 \mathrm{E}-03$ & $4.62 \mathrm{E}-03$ & $1.40 \mathrm{E}-03$ & $9.26 \mathrm{E}-02$ \\
$90 \%$ & $1.00 \mathrm{E}-02$ & $5.69 \mathrm{E}-03$ & $1.49 \mathrm{E}-03$ & $1.13 \mathrm{E}-01$ \\
$95 \%$ & $1.13 \mathrm{E}-02$ & $8.18 \mathrm{E}-03$ & $1.61 \mathrm{E}-03$ & $1.56 \mathrm{E}-01$ \\
Mean of the peaks & $5.98 \mathrm{E}-03$ & $3.13 \mathrm{E}-03$ & $1.06 \mathrm{E}-03$ & $5.71 \mathrm{E}-02$ \\
Peak of the means & $5.98 \mathrm{E}-03$ & $3.12 \mathrm{E}-03$ & $1.06 \mathrm{E}-03$ & $5.71 \mathrm{E}-02$ \\
\hline
\end{tabular}


TABLE 11 Total Peak DSR Percentiles (mrem/yr per pCi/g) of Pu-239 for Different Exposure Scenarios for the Building 310 Area

\begin{tabular}{ccccc}
\hline Percentile & Industrial Use & Offsite Resident & Recreational Use & Onsite Resident \\
\hline $5 \%$ & $6.73 \mathrm{E}-04$ & $1.04 \mathrm{E}-03$ & $1.46 \mathrm{E}-03$ & $3.39 \mathrm{E}-02$ \\
$10 \%$ & $1.81 \mathrm{E}-03$ & $1.39 \mathrm{E}-03$ & $1.66 \mathrm{E}-03$ & $4.18 \mathrm{E}-02$ \\
$15 \%$ & $2.76 \mathrm{E}-03$ & $1.64 \mathrm{E}-03$ & $1.80 \mathrm{E}-03$ & $4.76 \mathrm{E}-02$ \\
$20 \%$ & $3.57 \mathrm{E}-03$ & $1.82 \mathrm{E}-03$ & $1.92 \mathrm{E}-03$ & $5.33 \mathrm{E}-02$ \\
$25 \%$ & $4.24 \mathrm{E}-03$ & $1.97 \mathrm{E}-03$ & $2.02 \mathrm{E}-03$ & $5.98 \mathrm{E}-02$ \\
$30 \%$ & $4.79 \mathrm{E}-03$ & $2.13 \mathrm{E}-03$ & $2.10 \mathrm{E}-03$ & $6.62 \mathrm{E}-02$ \\
$35 \%$ & $5.27 \mathrm{E}-03$ & $2.29 \mathrm{E}-03$ & $2.19 \mathrm{E}-03$ & $7.26 \mathrm{E}-02$ \\
$40 \%$ & $5.72 \mathrm{E}-03$ & $2.46 \mathrm{E}-03$ & $2.27 \mathrm{E}-03$ & $7.99 \mathrm{E}-02$ \\
$45 \%$ & $6.11 \mathrm{E}-03$ & $2.60 \mathrm{E}-03$ & $2.36 \mathrm{E}-03$ & $8.80 \mathrm{E}-02$ \\
$50 \%$ & $6.51 \mathrm{E}-03$ & $2.77 \mathrm{E}-03$ & $2.45 \mathrm{E}-03$ & $9.69 \mathrm{E}-02$ \\
$55 \%$ & $6.89 \mathrm{E}-03$ & $2.96 \mathrm{E}-03$ & $2.54 \mathrm{E}-03$ & $1.07 \mathrm{E}-01$ \\
$60 \%$ & $7.30 \mathrm{E}-03$ & $3.20 \mathrm{E}-03$ & $2.65 \mathrm{E}-03$ & $1.18 \mathrm{E}-01$ \\
$65 \%$ & $7.76 \mathrm{E}-03$ & $3.44 \mathrm{E}-03$ & $2.75 \mathrm{E}-03$ & $1.30 \mathrm{E}-01$ \\
$70 \%$ & $8.27 \mathrm{E}-03$ & $3.77 \mathrm{E}-03$ & $2.87 \mathrm{E}-03$ & $1.44 \mathrm{E}-01$ \\
$75 \%$ & $8.75 \mathrm{E}-03$ & $4.15 \mathrm{E}-03$ & $3.02 \mathrm{E}-03$ & $1.64 \mathrm{E}-01$ \\
$80 \%$ & $9.28 \mathrm{E}-03$ & $4.70 \mathrm{E}-03$ & $3.18 \mathrm{E}-03$ & $1.88 \mathrm{E}-01$ \\
$85 \%$ & $1.00 \mathrm{E}-02$ & $5.39 \mathrm{E}-03$ & $3.36 \mathrm{E}-03$ & $2.23 \mathrm{E}-01$ \\
$90 \%$ & $1.10 \mathrm{E}-02$ & $6.51 \mathrm{E}-03$ & $3.56 \mathrm{E}-03$ & $2.70 \mathrm{E}-01$ \\
$95 \%$ & $1.24 \mathrm{E}-02$ & $9.05 \mathrm{E}-03$ & $3.87 \mathrm{E}-03$ & $3.73 \mathrm{E}-01$ \\
Mean of the peaks & $6.56 \mathrm{E}-03$ & $3.67 \mathrm{E}-03$ & $2.54 \mathrm{E}-03$ & $1.37 \mathrm{E}-01$ \\
Peak of the means & $6.56 \mathrm{E}-03$ & $3.55 \mathrm{E}-03$ & $2.54 \mathrm{E}-03$ & $1.37 \mathrm{E}-01$ \\
\hline
\end{tabular}


TABLE 12 Total Peak DSR Percentiles (mrem/yr per pCi/g) of Pu-240 for Different Exposure Scenarios for the Building 310 Area

\begin{tabular}{ccccc}
\hline Percentile & Industrial Use & Offsite Resident & Recreational Use & Onsite Resident \\
\hline $5 \%$ & $6.44 \mathrm{E}-04$ & $1.90 \mathrm{E}-03$ & $1.38 \mathrm{E}-03$ & $3.33 \mathrm{E}-02$ \\
$10 \%$ & $1.61 \mathrm{E}-03$ & $2.14 \mathrm{E}-03$ & $1.55 \mathrm{E}-03$ & $3.97 \mathrm{E}-02$ \\
$15 \%$ & $2.49 \mathrm{E}-03$ & $2.33 \mathrm{E}-03$ & $1.69 \mathrm{E}-03$ & $4.62 \mathrm{E}-02$ \\
$20 \%$ & $3.28 \mathrm{E}-03$ & $2.53 \mathrm{E}-03$ & $1.79 \mathrm{E}-03$ & $5.26 \mathrm{E}-02$ \\
$25 \%$ & $3.80 \mathrm{E}-03$ & $2.75 \mathrm{E}-03$ & $1.90 \mathrm{E}-03$ & $5.88 \mathrm{E}-02$ \\
$30 \%$ & $4.33 \mathrm{E}-03$ & $2.95 \mathrm{E}-03$ & $1.98 \mathrm{E}-03$ & $6.50 \mathrm{E}-02$ \\
$35 \%$ & $4.80 \mathrm{E}-03$ & $3.16 \mathrm{E}-03$ & $2.06 \mathrm{E}-03$ & $7.12 \mathrm{E}-02$ \\
$40 \%$ & $5.22 \mathrm{E}-03$ & $3.39 \mathrm{E}-03$ & $2.14 \mathrm{E}-03$ & $7.86 \mathrm{E}-02$ \\
$45 \%$ & $5.60 \mathrm{E}-03$ & $3.64 \mathrm{E}-03$ & $2.23 \mathrm{E}-03$ & $8.66 \mathrm{E}-02$ \\
$50 \%$ & $5.97 \mathrm{E}-03$ & $3.94 \mathrm{E}-03$ & $2.32 \mathrm{E}-03$ & $9.51 \mathrm{E}-02$ \\
$55 \%$ & $6.29 \mathrm{E}-03$ & $4.25 \mathrm{E}-03$ & $2.42 \mathrm{E}-03$ & $1.05 \mathrm{E}-01$ \\
$60 \%$ & $6.67 \mathrm{E}-03$ & $4.61 \mathrm{E}-03$ & $2.51 \mathrm{E}-03$ & $1.16 \mathrm{E}-01$ \\
$65 \%$ & $7.05 \mathrm{E}-03$ & $5.00 \mathrm{E}-03$ & $2.61 \mathrm{E}-03$ & $1.28 \mathrm{E}-01$ \\
$70 \%$ & $7.48 \mathrm{E}-03$ & $5.47 \mathrm{E}-03$ & $2.73 \mathrm{E}-03$ & $1.44 \mathrm{E}-01$ \\
$75 \%$ & $7.90 \mathrm{E}-03$ & $6.04 \mathrm{E}-03$ & $2.84 \mathrm{E}-03$ & $1.62 \mathrm{E}-01$ \\
$80 \%$ & $8.37 \mathrm{E}-03$ & $6.90 \mathrm{E}-03$ & $2.98 \mathrm{E}-03$ & $1.85 \mathrm{E}-01$ \\
$85 \%$ & $8.94 \mathrm{E}-03$ & $8.07 \mathrm{E}-03$ & $3.15 \mathrm{E}-03$ & $2.18 \mathrm{E}-01$ \\
$90 \%$ & $9.68 \mathrm{E}-03$ & $9.76 \mathrm{E}-03$ & $3.38 \mathrm{E}-03$ & $2.69 \mathrm{E}-01$ \\
$95 \%$ & $1.09 \mathrm{E}-02$ & $1.42 \mathrm{E}-02$ & $3.71 \mathrm{E}-03$ & $3.71 \mathrm{E}-01$ \\
Mean of the peaks & $5.92 \mathrm{E}-03$ & $5.49 \mathrm{E}-03$ & $2.41 \mathrm{E}-03$ & $1.35 \mathrm{E}-01$ \\
Peak of the means & $5.93 \mathrm{E}-03$ & $5.49 \mathrm{E}-03$ & $2.40 \mathrm{E}-03$ & $1.35 \mathrm{E}-01$ \\
\hline
\end{tabular}


TABLE 13 Total Peak DSR Percentiles (mrem/yr per pCi/g) of Sr-90 for Different Exposure Scenarios for the Building 310 Area

\begin{tabular}{ccccc}
\hline Percentile & Industrial Use & Offsite Resident & Recreational Use & Onsite Resident \\
\hline $5 \%$ & $9.62 \mathrm{E}-04$ & $8.35 \mathrm{E}-04$ & $1.18 \mathrm{E}-04$ & $4.42 \mathrm{E}-03$ \\
$10 \%$ & $2.84 \mathrm{E}-03$ & $9.28 \mathrm{E}-04$ & $9.18 \mathrm{E}-04$ & $2.97 \mathrm{E}-02$ \\
$15 \%$ & $4.65 \mathrm{E}-03$ & $1.01 \mathrm{E}-03$ & $1.64 \mathrm{E}-03$ & $5.30 \mathrm{E}-02$ \\
$20 \%$ & $6.54 \mathrm{E}-03$ & $1.07 \mathrm{E}-03$ & $2.31 \mathrm{E}-03$ & $7.31 \mathrm{E}-02$ \\
$25 \%$ & $8.25 \mathrm{E}-03$ & $1.14 \mathrm{E}-03$ & $2.88 \mathrm{E}-03$ & $9.77 \mathrm{E}-02$ \\
$30 \%$ & $8.72 \mathrm{E}-03$ & $1.22 \mathrm{E}-03$ & $3.51 \mathrm{E}-03$ & $1.18 \mathrm{E}-01$ \\
$35 \%$ & $9.02 \mathrm{E}-03$ & $1.28 \mathrm{E}-03$ & $4.23 \mathrm{E}-03$ & $1.44 \mathrm{E}-01$ \\
$40 \%$ & $9.35 \mathrm{E}-03$ & $1.36 \mathrm{E}-03$ & $4.93 \mathrm{E}-03$ & $1.70 \mathrm{E}-01$ \\
$45 \%$ & $9.66 \mathrm{E}-03$ & $1.43 \mathrm{E}-03$ & $5.69 \mathrm{E}-03$ & $2.04 \mathrm{E}-01$ \\
$50 \%$ & $9.94 \mathrm{E}-03$ & $1.52 \mathrm{E}-03$ & $6.56 \mathrm{E}-03$ & $2.35 \mathrm{E}-01$ \\
$55 \%$ & $1.02 \mathrm{E}-02$ & $1.60 \mathrm{E}-03$ & $7.69 \mathrm{E}-03$ & $2.79 \mathrm{E}-01$ \\
$60 \%$ & $1.04 \mathrm{E}-02$ & $1.70 \mathrm{E}-03$ & $9.24 \mathrm{E}-03$ & $3.21 \mathrm{E}-01$ \\
$65 \%$ & $1.06 \mathrm{E}-02$ & $1.82 \mathrm{E}-03$ & $1.08 \mathrm{E}-02$ & $3.72 \mathrm{E}-01$ \\
$70 \%$ & $1.09 \mathrm{E}-02$ & $1.95 \mathrm{E}-03$ & $1.28 \mathrm{E}-02$ & $4.25 \mathrm{E}-01$ \\
$75 \%$ & $1.12 \mathrm{E}-02$ & $2.13 \mathrm{E}-03$ & $1.54 \mathrm{E}-02$ & $5.03 \mathrm{E}-01$ \\
$80 \%$ & $1.16 \mathrm{E}-02$ & $2.33 \mathrm{E}-03$ & $1.86 \mathrm{E}-02$ & $6.07 \mathrm{E}-01$ \\
$85 \%$ & $1.22 \mathrm{E}-02$ & $2.62 \mathrm{E}-03$ & $2.34 \mathrm{E}-02$ & $7.50 \mathrm{E}-01$ \\
$90 \%$ & $1.29 \mathrm{E}-02$ & $3.09 \mathrm{E}-03$ & $3.09 \mathrm{E}-02$ & $9.71 \mathrm{E}-01$ \\
$95 \%$ & $1.38 \mathrm{E}-02$ & $3.99 \mathrm{E}-03$ & $4.75 \mathrm{E}-02$ & $1.44 \mathrm{E}+00$ \\
Mean of the peaks & $9.15 \mathrm{E}-03$ & $1.86 \mathrm{E}-03$ & $1.35 \mathrm{E}-02$ & $4.17 \mathrm{E}-01$ \\
Peak of the means & $9.15 \mathrm{E}-03$ & $1.70 \mathrm{E}-03$ & $1.35 \mathrm{E}-02$ & $4.17 \mathrm{E}-01$ \\
\hline
\end{tabular}


TABLE 14 Total Peak DSR Percentiles (mrem/yr per pCi/g) of Tc-99 for Different Exposure Scenarios for the Building 310 Area

\begin{tabular}{ccccc}
\hline Percentile & Industrial Use & Offsite Resident & Recreational Use & Onsite Resident \\
\hline $5 \%$ & $3.44 \mathrm{E}-06$ & $1.03 \mathrm{E}-04$ & $5.78 \mathrm{E}-06$ & $1.60 \mathrm{E}-05$ \\
$10 \%$ & $9.83 \mathrm{E}-06$ & $2.50 \mathrm{E}-04$ & $1.69 \mathrm{E}-05$ & $4.02 \mathrm{E}-05$ \\
$15 \%$ & $1.62 \mathrm{E}-05$ & $3.90 \mathrm{E}-04$ & $3.34 \mathrm{E}-05$ & $8.08 \mathrm{E}-05$ \\
$20 \%$ & $2.27 \mathrm{E}-05$ & $5.63 \mathrm{E}-04$ & $5.84 \mathrm{E}-05$ & $1.58 \mathrm{E}-04$ \\
$25 \%$ & $2.65 \mathrm{E}-05$ & $7.84 \mathrm{E}-04$ & $9.76 \mathrm{E}-05$ & $5.40 \mathrm{E}-04$ \\
$30 \%$ & $2.87 \mathrm{E}-05$ & $1.02 \mathrm{E}-03$ & $1.50 \mathrm{E}-04$ & $1.68 \mathrm{E}-03$ \\
$35 \%$ & $3.04 \mathrm{E}-05$ & $1.29 \mathrm{E}-03$ & $2.16 \mathrm{E}-04$ & $3.40 \mathrm{E}-03$ \\
$40 \%$ & $3.18 \mathrm{E}-05$ & $1.53 \mathrm{E}-03$ & $3.41 \mathrm{E}-04$ & $5.71 \mathrm{E}-03$ \\
$45 \%$ & $3.30 \mathrm{E}-05$ & $1.79 \mathrm{E}-03$ & $4.72 \mathrm{E}-04$ & $8.61 \mathrm{E}-03$ \\
$50 \%$ & $3.41 \mathrm{E}-05$ & $2.03 \mathrm{E}-03$ & $6.48 \mathrm{E}-04$ & $1.29 \mathrm{E}-02$ \\
$55 \%$ & $3.54 \mathrm{E}-05$ & $2.31 \mathrm{E}-03$ & $8.73 \mathrm{E}-04$ & $1.95 \mathrm{E}-02$ \\
$60 \%$ & $3.65 \mathrm{E}-05$ & $2.59 \mathrm{E}-03$ & $1.17 \mathrm{E}-03$ & $2.79 \mathrm{E}-02$ \\
$65 \%$ & $3.77 \mathrm{E}-05$ & $2.86 \mathrm{E}-03$ & $1.50 \mathrm{E}-03$ & $3.98 \mathrm{E}-02$ \\
$70 \%$ & $3.89 \mathrm{E}-05$ & $3.18 \mathrm{E}-03$ & $1.93 \mathrm{E}-03$ & $5.62 \mathrm{E}-02$ \\
$75 \%$ & $4.02 \mathrm{E}-05$ & $3.54 \mathrm{E}-03$ & $2.49 \mathrm{E}-03$ & $7.78 \mathrm{E}-02$ \\
$80 \%$ & $4.18 \mathrm{E}-05$ & $4.02 \mathrm{E}-03$ & $3.31 \mathrm{E}-03$ & $1.03 \mathrm{E}-01$ \\
$85 \%$ & $4.38 \mathrm{E}-05$ & $4.62 \mathrm{E}-03$ & $4.36 \mathrm{E}-03$ & $1.36 \mathrm{E}-01$ \\
$90 \%$ & $4.70 \mathrm{E}-05$ & $5.50 \mathrm{E}-03$ & $6.31 \mathrm{E}-03$ & $1.83 \mathrm{E}-01$ \\
$95 \%$ & $5.18 \mathrm{E}-05$ & $7.06 \mathrm{E}-03$ & $1.07 \mathrm{E}-02$ & $2.54 \mathrm{E}-01$ \\
Mean of the peaks & $3.21 \mathrm{E}-05$ & $2.59 \mathrm{E}-03$ & $2.56 \mathrm{E}-03$ & $5.68 \mathrm{E}-02$ \\
Peak of the means & $3.21 \mathrm{E}-05$ & $1.06 \mathrm{E}-03$ & $9.62 \mathrm{E}-04$ & $5.41 \mathrm{E}-02$ \\
\hline
\end{tabular}


TABLE 15 Total Peak DSR Percentiles (mrem/yr per pCi/g) of U-234 for Different Exposure Scenarios for the Building 310 Area

\begin{tabular}{ccccc}
\hline Percentile & Industrial Use & Offsite Resident & Recreational Use & Onsite Resident \\
\hline $5 \%$ & $4.74 \mathrm{E}-03$ & $1.29 \mathrm{E}-03$ & $5.94 \mathrm{E}-04$ & $2.74 \mathrm{E}-02$ \\
$10 \%$ & $1.20 \mathrm{E}-02$ & $3.36 \mathrm{E}-03$ & $1.38 \mathrm{E}-03$ & $8.69 \mathrm{E}-02$ \\
$15 \%$ & $2.45 \mathrm{E}-02$ & $6.01 \mathrm{E}-03$ & $2.05 \mathrm{E}-03$ & $1.50 \mathrm{E}-01$ \\
$20 \%$ & $4.04 \mathrm{E}-02$ & $9.53 \mathrm{E}-03$ & $2.88 \mathrm{E}-03$ & $2.30 \mathrm{E}-01$ \\
$25 \%$ & $6.17 \mathrm{E}-02$ & $1.35 \mathrm{E}-02$ & $3.74 \mathrm{E}-03$ & $3.38 \mathrm{E}-01$ \\
$30 \%$ & $9.04 \mathrm{E}-02$ & $1.81 \mathrm{E}-02$ & $4.58 \mathrm{E}-03$ & $4.40 \mathrm{E}-01$ \\
$35 \%$ & $1.20 \mathrm{E}-01$ & $2.19 \mathrm{E}-02$ & $5.59 \mathrm{E}-03$ & $5.52 \mathrm{E}-01$ \\
$40 \%$ & $1.53 \mathrm{E}-01$ & $2.52 \mathrm{E}-02$ & $6.64 \mathrm{E}-03$ & $6.50 \mathrm{E}-01$ \\
$45 \%$ & $1.89 \mathrm{E}-01$ & $2.77 \mathrm{E}-02$ & $7.73 \mathrm{E}-03$ & $7.48 \mathrm{E}-01$ \\
$50 \%$ & $2.28 \mathrm{E}-01$ & $2.99 \mathrm{E}-02$ & $8.66 \mathrm{E}-03$ & $8.59 \mathrm{E}-01$ \\
$55 \%$ & $2.73 \mathrm{E}-01$ & $3.21 \mathrm{E}-02$ & $9.60 \mathrm{E}-03$ & $9.50 \mathrm{E}-01$ \\
$60 \%$ & $3.11 \mathrm{E}-01$ & $3.43 \mathrm{E}-02$ & $1.04 \mathrm{E}-02$ & $1.04 \mathrm{E}+00$ \\
$65 \%$ & $3.47 \mathrm{E}-01$ & $3.66 \mathrm{E}-02$ & $1.12 \mathrm{E}-02$ & $1.12 \mathrm{E}+00$ \\
$70 \%$ & $3.84 \mathrm{E}-01$ & $3.93 \mathrm{E}-02$ & $1.21 \mathrm{E}-02$ & $1.21 \mathrm{E}+00$ \\
$75 \%$ & $4.24 \mathrm{E}-01$ & $4.17 \mathrm{E}-02$ & $1.30 \mathrm{E}-02$ & $1.28 \mathrm{E}+00$ \\
$80 \%$ & $4.60 \mathrm{E}-01$ & $4.43 \mathrm{E}-02$ & $1.42 \mathrm{E}-02$ & $1.37 \mathrm{E}+00$ \\
$85 \%$ & $5.06 \mathrm{E}-01$ & $4.73 \mathrm{E}-02$ & $1.55 \mathrm{E}-02$ & $1.46 \mathrm{E}+00$ \\
$90 \%$ & $5.68 \mathrm{E}-01$ & $5.18 \mathrm{E}-02$ & $1.73 \mathrm{E}-02$ & $1.57 \mathrm{E}+00$ \\
$95 \%$ & $6.64 \mathrm{E}-01$ & $5.76 \mathrm{E}-02$ & $2.10 \mathrm{E}-02$ & $1.75 \mathrm{E}+00$ \\
Mean of the peaks & $2.65 \mathrm{E}-01$ & $2.89 \mathrm{E}-02$ & $1.00 \mathrm{E}-02$ & $8.57 \mathrm{E}-01$ \\
Peak of the means & $2.54 \mathrm{E}-01$ & $2.57 \mathrm{E}-02$ & $8.93 \mathrm{E}-03$ & $8.09 \mathrm{E}-01$ \\
\hline
\end{tabular}


TABLE 16 Total Peak DSR Percentiles (mrem/yr per pCi/g) of U-235 for Different Exposure Scenarios for the Building 310 Area

\begin{tabular}{ccccc}
\hline Percentile & Industrial Use & Offsite Resident & Recreational Use & Onsite Resident \\
\hline $5 \%$ & $1.69 \mathrm{E}-02$ & $6.30 \mathrm{E}-04$ & $6.68 \mathrm{E}-03$ & $6.84 \mathrm{E}-02$ \\
$10 \%$ & $4.98 \mathrm{E}-02$ & $1.06 \mathrm{E}-03$ & $1.68 \mathrm{E}-02$ & $1.35 \mathrm{E}-01$ \\
$15 \%$ & $8.11 \mathrm{E}-02$ & $1.76 \mathrm{E}-03$ & $2.18 \mathrm{E}-02$ & $1.61 \mathrm{E}-01$ \\
$20 \%$ & $1.14 \mathrm{E}-01$ & $2.71 \mathrm{E}-03$ & $2.41 \mathrm{E}-02$ & $1.81 \mathrm{E}-01$ \\
$25 \%$ & $1.41 \mathrm{E}-01$ & $3.83 \mathrm{E}-03$ & $2.52 \mathrm{E}-02$ & $1.97 \mathrm{E}-01$ \\
$30 \%$ & $1.47 \mathrm{E}-01$ & $5.02 \mathrm{E}-03$ & $2.58 \mathrm{E}-02$ & $2.13 \mathrm{E}-01$ \\
$35 \%$ & $1.54 \mathrm{E}-01$ & $6.17 \mathrm{E}-03$ & $2.62 \mathrm{E}-02$ & $2.27 \mathrm{E}-01$ \\
$40 \%$ & $1.61 \mathrm{E}-01$ & $7.04 \mathrm{E}-03$ & $2.66 \mathrm{E}-02$ & $2.41 \mathrm{E}-01$ \\
$45 \%$ & $1.67 \mathrm{E}-01$ & $7.70 \mathrm{E}-03$ & $2.69 \mathrm{E}-02$ & $2.56 \mathrm{E}-01$ \\
$50 \%$ & $1.71 \mathrm{E}-01$ & $8.45 \mathrm{E}-03$ & $2.74 \mathrm{E}-02$ & $2.72 \mathrm{E}-01$ \\
$55 \%$ & $1.75 \mathrm{E}-01$ & $9.07 \mathrm{E}-03$ & $2.79 \mathrm{E}-02$ & $2.90 \mathrm{E}-01$ \\
$60 \%$ & $1.80 \mathrm{E}-01$ & $9.68 \mathrm{E}-03$ & $2.85 \mathrm{E}-02$ & $3.08 \mathrm{E}-01$ \\
$65 \%$ & $1.84 \mathrm{E}-01$ & $1.05 \mathrm{E}-02$ & $2.92 \mathrm{E}-02$ & $3.29 \mathrm{E}-01$ \\
$70 \%$ & $1.92 \mathrm{E}-01$ & $1.15 \mathrm{E}-02$ & $3.00 \mathrm{E}-02$ & $3.52 \mathrm{E}-01$ \\
$75 \%$ & $2.00 \mathrm{E}-01$ & $1.26 \mathrm{E}-02$ & $3.08 \mathrm{E}-02$ & $3.79 \mathrm{E}-01$ \\
$80 \%$ & $2.08 \mathrm{E}-01$ & $1.37 \mathrm{E}-02$ & $3.20 \mathrm{E}-02$ & $4.14 \mathrm{E}-01$ \\
$85 \%$ & $2.18 \mathrm{E}-01$ & $1.51 \mathrm{E}-02$ & $3.31 \mathrm{E}-02$ & $4.59 \mathrm{E}-01$ \\
$90 \%$ & $2.29 \mathrm{E}-01$ & $1.67 \mathrm{E}-02$ & $3.41 \mathrm{E}-02$ & $5.21 \mathrm{E}-01$ \\
$95 \%$ & $2.65 \mathrm{E}-01$ & $1.88 \mathrm{E}-02$ & $3.58 \mathrm{E}-02$ & $6.49 \mathrm{E}-01$ \\
Mean of the peaks & $1.61 \mathrm{E}-01$ & $8.70 \mathrm{E}-03$ & $2.65 \mathrm{E}-02$ & $3.31 \mathrm{E}-01$ \\
Peak of the means & $1.52 \mathrm{E}-01$ & $7.81 \mathrm{E}-03$ & $2.51 \mathrm{E}-02$ & $2.44 \mathrm{E}-01$ \\
\hline
\end{tabular}


TABLE 17 Total Peak DSR Percentiles (mrem/yr per pCi/g) of U-238 for Different Exposure Scenarios for the Building 310 Area

\begin{tabular}{ccccc}
\hline Percentile & Industrial Use & Offsite Resident & Recreational Use & Onsite Resident \\
\hline $5 \%$ & $3.64 \mathrm{E}-03$ & $4.28 \mathrm{E}-04$ & $2.09 \mathrm{E}-03$ & $2.41 \mathrm{E}-02$ \\
$10 \%$ & $1.06 \mathrm{E}-02$ & $5.35 \mathrm{E}-04$ & $4.87 \mathrm{E}-03$ & $4.22 \mathrm{E}-02$ \\
$15 \%$ & $1.76 \mathrm{E}-02$ & $6.24 \mathrm{E}-04$ & $5.94 \mathrm{E}-03$ & $4.90 \mathrm{E}-02$ \\
$20 \%$ & $2.45 \mathrm{E}-02$ & $7.20 \mathrm{E}-04$ & $6.27 \mathrm{E}-03$ & $5.38 \mathrm{E}-02$ \\
$25 \%$ & $3.13 \mathrm{E}-02$ & $8.07 \mathrm{E}-04$ & $6.46 \mathrm{E}-03$ & $5.86 \mathrm{E}-02$ \\
$30 \%$ & $3.28 \mathrm{E}-02$ & $8.90 \mathrm{E}-04$ & $6.63 \mathrm{E}-03$ & $6.29 \mathrm{E}-02$ \\
$35 \%$ & $3.40 \mathrm{E}-02$ & $9.83 \mathrm{E}-04$ & $6.79 \mathrm{E}-03$ & $6.75 \mathrm{E}-02$ \\
$40 \%$ & $3.52 \mathrm{E}-02$ & $1.06 \mathrm{E}-03$ & $6.97 \mathrm{E}-03$ & $7.21 \mathrm{E}-02$ \\
$45 \%$ & $3.64 \mathrm{E}-02$ & $1.15 \mathrm{E}-03$ & $7.16 \mathrm{E}-03$ & $7.69 \mathrm{E}-02$ \\
$50 \%$ & $3.75 \mathrm{E}-02$ & $1.25 \mathrm{E}-03$ & $7.36 \mathrm{E}-03$ & $8.23 \mathrm{E}-02$ \\
$55 \%$ & $3.84 \mathrm{E}-02$ & $1.36 \mathrm{E}-03$ & $7.58 \mathrm{E}-03$ & $8.74 \mathrm{E}-02$ \\
$60 \%$ & $3.93 \mathrm{E}-02$ & $1.50 \mathrm{E}-03$ & $7.87 \mathrm{E}-03$ & $9.31 \mathrm{E}-02$ \\
$65 \%$ & $4.00 \mathrm{E}-02$ & $1.68 \mathrm{E}-03$ & $8.14 \mathrm{E}-03$ & $9.99 \mathrm{E}-02$ \\
$70 \%$ & $4.08 \mathrm{E}-02$ & $1.88 \mathrm{E}-03$ & $8.52 \mathrm{E}-03$ & $1.07 \mathrm{E}-01$ \\
$75 \%$ & $4.18 \mathrm{E}-02$ & $2.12 \mathrm{E}-03$ & $8.93 \mathrm{E}-03$ & $1.16 \mathrm{E}-01$ \\
$80 \%$ & $4.38 \mathrm{E}-02$ & $2.43 \mathrm{E}-03$ & $9.42 \mathrm{E}-03$ & $1.28 \mathrm{E}-01$ \\
$85 \%$ & $4.61 \mathrm{E}-02$ & $2.84 \mathrm{E}-03$ & $1.01 \mathrm{E}-02$ & $1.47 \mathrm{E}-01$ \\
$90 \%$ & $4.84 \mathrm{E}-02$ & $3.33 \mathrm{E}-03$ & $1.11 \mathrm{E}-02$ & $1.75 \mathrm{E}-01$ \\
$95 \%$ & $5.17 \mathrm{E}-02$ & $3.93 \mathrm{E}-03$ & $1.31 \mathrm{E}-02$ & $2.47 \mathrm{E}-01$ \\
Mean of the peaks & $3.45 \mathrm{E}-02$ & $1.64 \mathrm{E}-03$ & $7.90 \mathrm{E}-03$ & $1.28 \mathrm{E}-01$ \\
Peak of the means & $3.44 \mathrm{E}-02$ & $1.16 \mathrm{E}-03$ & $7.67 \mathrm{E}-03$ & $8.94 \mathrm{E}-02$ \\
\hline
\end{tabular}


TABLE 18 DSRs (mrem/yr per pCi/g) at Peak of the Means, Mean of the Peaks, and 95th Percentile of the Peaks for Different Scenarios

\begin{tabular}{|c|c|c|c|c|c|c|c|c|c|c|c|c|}
\hline \multirow[b]{2}{*}{ Nuclide } & \multicolumn{3}{|c|}{ Industrial Use } & \multicolumn{3}{|c|}{ Offsite Resident } & \multicolumn{3}{|c|}{ Recreational Use } & \multicolumn{3}{|c|}{ Onsite Resident } \\
\hline & $\begin{array}{c}\text { Peak of } \\
\text { the Means }\end{array}$ & $\begin{array}{l}\text { Mean of } \\
\text { the Peaks }\end{array}$ & $\begin{array}{l}95 \text { th } \% \text { of } \\
\text { the Peaks }\end{array}$ & $\begin{array}{c}\text { Peak of } \\
\text { the Means }\end{array}$ & $\begin{array}{c}\text { Mean of } \\
\text { the Peaks }\end{array}$ & $\begin{array}{l}95 \text { th } \% \text { of } \\
\text { the Peaks }\end{array}$ & $\begin{array}{c}\text { Peak of } \\
\text { the Means }\end{array}$ & $\begin{array}{l}\text { Mean of } \\
\text { the Peaks }\end{array}$ & $\begin{array}{l}95 \text { th } \% \text { of } \\
\text { the Peaks }\end{array}$ & $\begin{array}{c}\text { Peak of } \\
\text { the Means }\end{array}$ & $\begin{array}{c}\text { Mean of } \\
\text { the Peaks }\end{array}$ & $\begin{array}{l}95 \text { th } \% \text { of } \\
\text { the Peaks }\end{array}$ \\
\hline Am-241 & $1.35 \mathrm{E}-02$ & $1.35 \mathrm{E}-02$ & 2.19E-02 & $6.87 \mathrm{E}-03$ & $6.98 \mathrm{E}-03$ & $1.19 \mathrm{E}-02$ & $2.40 \mathrm{E}-03$ & $2.40 \mathrm{E}-03$ & $3.05 \mathrm{E}-03$ & $1.05 \mathrm{E}-01$ & $1.05 \mathrm{E}-01$ & $2.68 \mathrm{E}-01$ \\
\hline C-14 & $2.10 \mathrm{E}-05$ & $2.10 \mathrm{E}-05$ & $3.73 \mathrm{E}-05$ & $1.50 \mathrm{E}-02$ & $5.95 \mathrm{E}-02$ & 2.27E-01 & $1.58 \mathrm{E}-02$ & 4.61E-02 & $2.50 \mathrm{E}-01$ & $3.54 \mathrm{E}-03$ & $3.36 \mathrm{E}-03$ & $1.05 \mathrm{E}-02$ \\
\hline Co-60 & $3.11 \mathrm{E}+00$ & $3.11 \mathrm{E}+00$ & $4.64 \mathrm{E}+00$ & 8.01E-04 & $8.20 \mathrm{E}-04$ & $9.01 \mathrm{E}-04$ & $9.66 \mathrm{E}-07$ & $9.65 \mathrm{E}-07$ & $1.17 \mathrm{E}-06$ & 7.54E-06 & $7.54 \mathrm{E}-06$ & $1.40 \mathrm{E}-05$ \\
\hline Cs-137 & $6.78 \mathrm{E}-01$ & $6.78 \mathrm{E}-01$ & $1.01 \mathrm{E}+00$ & $6.88 \mathrm{E}-03$ & $6.87 \mathrm{E}-03$ & $1.49 \mathrm{E}-02$ & $1.95 \mathrm{E}-02$ & $1.95 \mathrm{E}-02$ & $3.49 \mathrm{E}-02$ & $1.59 \mathrm{E}-01$ & $1.59 \mathrm{E}-01$ & 3.17E-01 \\
\hline I-129 & $3.64 \mathrm{E}-03$ & $3.64 \mathrm{E}-03$ & $6.15 \mathrm{E}-03$ & $1.96 \mathrm{E}-01$ & $9.30 \mathrm{E}-01$ & $3.55 \mathrm{E}+00$ & $7.12 \mathrm{E}-02$ & $2.25 \mathrm{E}-01$ & $1.13 \mathrm{E}+00$ & $3.16 \mathrm{E}+00$ & $2.51 \mathrm{E}+01$ & $1.04 \mathrm{E}+02$ \\
\hline Np-237 & $2.15 \mathrm{E}-01$ & $2.15 \mathrm{E}-01$ & $3.28 \mathrm{E}-01$ & $9.30 \mathrm{E}-03$ & $3.48 \mathrm{E}-02$ & 1.17E-01 & $4.68 \mathrm{E}-02$ & $9.41 \mathrm{E}-02$ & $2.92 \mathrm{E}-01$ & $1.11 \mathrm{E}+00$ & $2.64 \mathrm{E}+00$ & $1.04 \mathrm{E}+01$ \\
\hline $\mathrm{Pu}-238$ & $5.98 \mathrm{E}-03$ & $5.98 \mathrm{E}-03$ & $1.13 \mathrm{E}-02$ & $3.12 \mathrm{E}-03$ & $3.13 \mathrm{E}-03$ & $8.18 \mathrm{E}-03$ & $1.06 \mathrm{E}-03$ & $1.06 \mathrm{E}-03$ & $1.61 \mathrm{E}-03$ & $5.71 \mathrm{E}-02$ & $5.71 \mathrm{E}-02$ & $1.56 \mathrm{E}-01$ \\
\hline $\mathrm{Pu}-239$ & $6.56 \mathrm{E}-03$ & $6.56 \mathrm{E}-03$ & $1.24 \mathrm{E}-02$ & $3.55 \mathrm{E}-03$ & 3.67E-03 & $9.05 \mathrm{E}-03$ & $2.54 \mathrm{E}-03$ & $2.54 \mathrm{E}-03$ & $3.87 \mathrm{E}-03$ & $1.37 \mathrm{E}-01$ & $1.37 \mathrm{E}-01$ & 3.73E-01 \\
\hline $\mathrm{Pu}-240$ & $5.93 \mathrm{E}-03$ & $5.92 \mathrm{E}-03$ & $1.09 \mathrm{E}-02$ & $5.49 \mathrm{E}-03$ & $5.49 \mathrm{E}-03$ & $1.42 \mathrm{E}-02$ & $2.40 \mathrm{E}-03$ & $2.41 \mathrm{E}-03$ & $3.71 \mathrm{E}-03$ & $1.35 \mathrm{E}-01$ & $1.35 \mathrm{E}-01$ & $3.71 \mathrm{E}-01$ \\
\hline Sr-90 & $9.15 \mathrm{E}-03$ & $9.15 \mathrm{E}-03$ & $1.38 \mathrm{E}-02$ & $1.70 \mathrm{E}-03$ & $1.86 \mathrm{E}-03$ & $3.99 \mathrm{E}-03$ & $1.35 \mathrm{E}-02$ & $1.35 \mathrm{E}-02$ & $4.75 \mathrm{E}-02$ & 4.17E-01 & 4.17E-01 & $1.44 \mathrm{E}+00$ \\
\hline Tc-99 & $3.21 \mathrm{E}-05$ & $3.21 \mathrm{E}-05$ & $5.18 \mathrm{E}-05$ & $1.06 \mathrm{E}-03$ & $2.59 \mathrm{E}-03$ & $7.06 \mathrm{E}-03$ & $9.62 \mathrm{E}-04$ & $2.56 \mathrm{E}-03$ & $1.07 \mathrm{E}-02$ & $5.41 \mathrm{E}-02$ & $5.68 \mathrm{E}-02$ & $2.54 \mathrm{E}-01$ \\
\hline U-234 & $2.54 \mathrm{E}-01$ & $2.65 \mathrm{E}-01$ & $6.64 \mathrm{E}-01$ & $2.57 \mathrm{E}-02$ & $2.89 \mathrm{E}-02$ & $5.76 \mathrm{E}-02$ & 8.93E-03 & $1.00 \mathrm{E}-02$ & $2.10 \mathrm{E}-02$ & 8.09E-01 & 8.57E-01 & $1.75 \mathrm{E}+00$ \\
\hline U-235 & $1.52 \mathrm{E}-01$ & $1.61 \mathrm{E}-01$ & $2.65 \mathrm{E}-01$ & $7.81 \mathrm{E}-03$ & $8.70 \mathrm{E}-03$ & $1.88 \mathrm{E}-02$ & $2.51 \mathrm{E}-02$ & $2.65 \mathrm{E}-02$ & $3.58 \mathrm{E}-02$ & $2.44 \mathrm{E}-01$ & 3.31E-01 & 6.49E-01 \\
\hline $\mathrm{U}-238$ & $3.44 \mathrm{E}-02$ & $3.45 \mathrm{E}-02$ & $5.17 \mathrm{E}-02$ & $1.16 \mathrm{E}-03$ & $1.64 \mathrm{E}-03$ & $3.93 \mathrm{E}-03$ & 7.67E-03 & $7.90 \mathrm{E}-03$ & $1.31 \mathrm{E}-02$ & 8.94E-02 & $1.28 \mathrm{E}-01$ & $2.47 \mathrm{E}-01$ \\
\hline
\end{tabular}




\subsection{DETERMINISTIC ANALYSIS}

Details on the parameters selected for the deterministic analysis are presented in Appendix B. Table B.1 lists all input parameter values used in the deterministic dose analysis for different scenarios. Some of the parameters used in the analysis were radionuclide/elementspecific (e.g., Kd values, transfer factors) and required multiple values because the analysis was conducted for multiple radionuclides. The parameter values used for these radionuclide- and element-specific analyses are listed in separate tables.

Parameter values for $\mathrm{Kd}$, plant transfer factors, meat transfer factors, and milk transfer factors are listed in Table B.2. Parameter values for fish and crustacean transfer factors are listed in Table B.3. The RESRAD-OFFSITE code requires many more parameters than the RESRAD (onsite) code; the additional input parameters for the RESRAD-OFFSITE analysis are listed in Table B.4.

Tables 19-23 appear at the end of this section (Section 3.2). Table 19 lists the calculated maximum DSRs (mrem/yr per $\mathrm{pCi} / \mathrm{g}$ ) for different exposure pathways for the industrial use scenario. RESRAD (onsite) Version 6.5 was used in the analysis. For Co-60, Cs-137, Np-237, Sr-90, U-235, and U-238, external exposure was the dominant exposure pathway. For Am-241, the external exposure, inhalation, and soil ingestion pathways contributed to the dose. For C-14, only inhalation was the dominant exposure pathway. For I-129 and Tc-99, soil ingestion and external exposure were the dominant exposure pathways. For $\mathrm{Pu}-238$, $\mathrm{Pu}-239$, and $\mathrm{Pu}-240$, inhalation and soil ingestion were the two dominant exposure pathways. For U-234, radon inhalation at later time contributed significantly to the dose. For most radionuclides, the maximum DSR would occur immediately following remedial action. For U-234, the maximum DSR would occur at a later time as a result of the buildup of progeny. To check if the peak dose would occur after 1,000 years, the time frame for the analysis was extended to 10,000 years. Table 19 also lists the time of maximum DSR for each radionuclide.

Table 20 lists the calculated maximum DSRs for different exposure pathways for the offsite resident scenario. RESRAD-OFFSITE Version 2.6 was used for this analysis. For most radionuclides, WD pathways were dominant. For Am-241, Np-237, $\mathrm{Pu}-238, \mathrm{Pu}-239$, and $\mathrm{Pu}-240$, fish and plant ingestion were dominant exposure pathways. For C-14, aquatic food ingestion resulted in the most dose. For Co-60, the external exposure pathway was dominant. For Cs-137, fish, meat, and milk ingestion resulted in the most dose. For I-129, milk ingestion resulted in the most dose. For Sr-90, U-234, U-235, and U-238, fish, meat, milk, and plant ingestion resulted in the most dose. For Tc-99, plant, fish, and milk ingestion resulted in the most dose. To check if the peak dose would occur after 1,000 years, the time frame for the analysis was extended to 10,000 years. Table 20 also lists the time of maximum DSR for each radionuclide.

Table 21 lists the maximum DSRs for different exposure pathways for the recreational use scenario. RESRAD (onsite) Version 6.5 was used in the analysis. The calculations were done for the time after the institutional control period of 100 years. Maximum DSRs would occur at 100 years (immediately after the institutional control period) for all radionuclides except U-234 and U-235. For U-234 and U-235, the maximum DSRs occur at later time as a result of the buildup of progeny. For C-14, I-129, and Tc-99, the maximum dose is from WD pathways 
(such as ingestion of food irrigated by contaminated water, water ingestion, and fish ingestion). To check if the peak dose would occur after 1,000 years, the time frame for the analysis was extended to 10,000 years. Table 21 also lists the time of maximum DSR for each radionuclide.

Table 22 lists the maximum DSRs for different exposure pathways for the onsite resident scenario. RESRAD (onsite) Version 6.5 was used in the analysis. The calculations were done for the time after the institutional control period of 100 years. Maximum DSRs occur at 100 years (immediately after the institutional control period) for all radionuclides except Np-237, U-234, and U-235. For C-14, I-129, Np-237, and Tc-99, the maximum dose is from WD pathways (such as ingestion of food irrigated by contaminated water, water ingestion, and fish ingestion). To check if the peak dose would occur after 1,000 years, the time frame for the analysis was extended to 10,000 years. Table 22 also lists the time of maximum DSR for each radionuclide.

Table 23 shows the peak DSR of each radionuclide in different scenarios. These values were used in deriving DCGL values. The scenario that results in the most dose for an individual radionuclide is highlighted. 
TABLE 19 Maximum DSRs for the Industrial (Current Use) Scenario for the Building 310 Area

\begin{tabular}{|c|c|c|c|c|c|}
\hline \multirow[b]{2}{*}{ Nuclide } & \multicolumn{4}{|c|}{$\begin{array}{l}\text { Maximum } \mathrm{DSR}^{\mathrm{a}}(\mathrm{mrem} / \mathrm{yr}) /(\mathrm{pCi} / \mathrm{g}) \\
\text { for the Industrial Use Scenario }\end{array}$} & \multirow{2}{*}{$\begin{array}{c}\text { Time of } \\
\text { Maximum } \\
\text { DSR (yr) }\end{array}$} \\
\hline & $\begin{array}{l}\text { External } \\
\text { Exposure }\end{array}$ & Inhalation & Radon & $\begin{array}{l}\text { Ingestion } \\
\text { of Soil } \\
\end{array}$ & \\
\hline Am-241 & $8.23 \mathrm{E}-03$ & $6.60 \mathrm{E}-03$ & $0.00 \mathrm{E}+00$ & $6.16 \mathrm{E}-03$ & $0.00 \mathrm{E}+00$ \\
\hline C-14 & 3.66E-07 & $1.78 \mathrm{E}-05$ & $0.00 \mathrm{E}+00$ & $2.65 \mathrm{E}-06$ & $0.00 \mathrm{E}+00$ \\
\hline Co-60 & $3.10 \mathrm{E}+00$ & $2.00 \mathrm{E}-06$ & $0.00 \mathrm{E}+00$ & $9.84 \mathrm{E}-05$ & $0.00 \mathrm{E}+00$ \\
\hline Cs-137 & $6.76 \mathrm{E}-01$ & $2.65 \mathrm{E}-06$ & $0.00 \mathrm{E}+00$ & $3.96 \mathrm{E}-04$ & $0.00 \mathrm{E}+00$ \\
\hline I-129 & $2.05 \mathrm{E}-03$ & $6.21 \mathrm{E}-06$ & $0.00 \mathrm{E}+00$ & $3.19 \mathrm{E}-03$ & $0.00 \mathrm{E}+00$ \\
\hline Np-237 & 2.19E-01 & 3.44E-03 & $0.00 \mathrm{E}+00$ & $3.41 \mathrm{E}-03$ & $0.00 \mathrm{E}+00$ \\
\hline Pu-238 & $2.62 \mathrm{E}-05$ & 7.54E-03 & $2.35 \mathrm{E}-13$ & $7.06 \mathrm{E}-03$ & $0.00 \mathrm{E}+00$ \\
\hline Pu-239 & $5.74 \mathrm{E}-05$ & $8.26 \mathrm{E}-03$ & $0.00 \mathrm{E}+00$ & 7.71E-03 & $0.00 \mathrm{E}+00$ \\
\hline $\mathrm{Pu}-240$ & $2.54 \mathrm{E}-05$ & $8.26 \mathrm{E}-03$ & $3.88 \mathrm{E}-24$ & 7.71E-03 & $0.00 \mathrm{E}+00$ \\
\hline Sr-90 & 8.69E-03 & $1.10 \mathrm{E}-05$ & $0.00 \mathrm{E}+00$ & $9.38 \mathrm{E}-04$ & $0.00 \mathrm{E}+00$ \\
\hline Тc-99 & 2.23E-05 & $8.41 \mathrm{E}-07$ & $0.00 \mathrm{E}+00$ & $1.86 \mathrm{E}-05$ & $0.00 \mathrm{E}+00$ \\
\hline $\mathrm{U}-234^{\mathrm{b}, \mathrm{c}}$ & 2.79E-02 & 2.63E-04 & $4.30 \mathrm{E}-01$ & $2.34 \mathrm{E}-03$ & $4.39 \mathrm{E}+03$ \\
\hline U-235 & $1.50 \mathrm{E}-01$ & $5.86 \mathrm{E}-04$ & $0.00 \mathrm{E}+00$ & $1.46 \mathrm{E}-03$ & $0.00 \mathrm{E}+00$ \\
\hline U-238 & $3.35 \mathrm{E}-02$ & $5.51 \mathrm{E}-04$ & $2.72 \mathrm{E}-19$ & $1.50 \mathrm{E}-03$ & $0.00 \mathrm{E}+00$ \\
\hline
\end{tabular}

a All values are reported to three significant figures. The maximum DSR occurs at time zero (immediately following remedial action) for all radionuclides except U-234.

b The maximum DSR occurs at a later time as a result of the buildup of progeny.

c For U-234, it is assumed that the worker spends all the time indoors in the contaminated area. 
TABLE 20 Maximum DSRs for the Offsite Resident (Current Use) Scenario for the Building 310 Area

\begin{tabular}{|c|c|c|c|c|c|c|c|c|c|}
\hline \multirow[b]{2}{*}{ Nuclide } & \multicolumn{8}{|c|}{ Maximum DSR ${ }^{\mathrm{a}}(\mathrm{mrem} / \mathrm{yr}) /(\mathrm{pCi} / \mathrm{g})$ for Offsite Resident Scenario } & \multirow{2}{*}{$\begin{array}{c}\text { Time of } \\
\text { Maximum } \\
\text { DSR (yr) }\end{array}$} \\
\hline & $\begin{array}{c}\text { External } \\
\text { Exposure }\end{array}$ & Inhalation & Radon & $\begin{array}{c}\text { Plant } \\
\text { Ingestion }\end{array}$ & $\begin{array}{c}\text { Meat } \\
\text { Ingestion }\end{array}$ & $\begin{array}{c}\text { Milk } \\
\text { Ingestion }\end{array}$ & $\begin{array}{c}\text { Soil } \\
\text { Ingestion }\end{array}$ & $\begin{array}{c}\text { Fish } \\
\text { Ingestion }\end{array}$ & \\
\hline Am-241 & $1.09 \mathrm{E}-06$ & $6.57 \mathrm{E}-06$ & $0.00 \mathrm{E}+00$ & $1.32 \mathrm{E}-03$ & $1.05 \mathrm{E}-05$ & $1.56 \mathrm{E}-06$ & $9.18 \mathrm{E}-07$ & 6.69E-03 & 5.86 \\
\hline C-14 & $3.98 \mathrm{E}-10$ & $0.00 \mathrm{E}+00$ & $0.00 \mathrm{E}+00$ & $8.02 \mathrm{E}-05$ & $4.52 \mathrm{E}-05$ & $4.57 \mathrm{E}-05$ & $3.54 \mathrm{E}-09$ & $3.38 \mathrm{E}-02$ & 152 \\
\hline Co- $60^{b}$ & 7.12E-04 & $1.76 \mathrm{E}-09$ & $0.00 \mathrm{E}+00$ & $1.55 \mathrm{E}-05$ & 4.73E-05 & $1.81 \mathrm{E}-05$ & $1.74 \mathrm{E}-09$ & $1.31 \mathrm{E}-04$ & 0.98 \\
\hline Cs-137 & $2.20 \mathrm{E}-04$ & 2.43E-09 & $0.00 \mathrm{E}+00$ & $8.76 \mathrm{E}-05$ & 4.07E-04 & $4.00 \mathrm{E}-04$ & 3.74E-08 & 4.39E-03 & 3.91 \\
\hline I-129 & $1.11 \mathrm{E}-05$ & $1.05 \mathrm{E}-14$ & $0.00 \mathrm{E}+00$ & $1.58 \mathrm{E}-01$ & $1.76 \mathrm{E}-01$ & $9.29 \mathrm{E}-01$ & $2.12 \mathrm{E}-05$ & $1.66 \mathrm{E}-01$ & 91.8 \\
\hline Np-237 & $1.45 \mathrm{E}-03$ & $6.02 \mathrm{E}-09$ & $0.00 \mathrm{E}+00$ & $4.88 \mathrm{E}-03$ & $6.78 \mathrm{E}-04$ & $1.21 \mathrm{E}-05$ & $2.40 \mathrm{E}-05$ & $9.56 \mathrm{E}-03$ & 3368 \\
\hline $\mathrm{Pu}-238$ & 2.79E-09 & 7.29E-06 & $1.99 \mathrm{E}-15$ & $1.53 \mathrm{E}-03$ & $2.44 \mathrm{E}-05$ & $9.05 \mathrm{E}-07$ & 8.70E-07 & $1.84 \mathrm{E}-03$ & 4.88 \\
\hline $\mathrm{Pu}-239$ & $1.10 \mathrm{E}-06$ & 7.75E-06 & $0.00 \mathrm{E}+00$ & $1.87 \mathrm{E}-03$ & $3.66 \mathrm{E}-05$ & $1.10 \mathrm{E}-06$ & $1.74 \mathrm{E}-04$ & $1.92 \mathrm{E}-03$ & 1118 \\
\hline $\mathrm{Pu}-240$ & $5.10 \mathrm{E}-09$ & $8.29 \mathrm{E}-06$ & $1.57 \mathrm{E}-20$ & $1.74 \mathrm{E}-03$ & $2.78 \mathrm{E}-05$ & $1.03 \mathrm{E}-06$ & $1.65 \mathrm{E}-06$ & $2.10 \mathrm{E}-03$ & 7.81 \\
\hline Sr-90 & 2.89E-06 & 9.73E-09 & $0.00 \mathrm{E}+00$ & $2.74 \mathrm{E}-04$ & $2.82 \mathrm{E}-04$ & 2.43E-04 & $1.11 \mathrm{E}-07$ & $3.94 \mathrm{E}-04$ & 4.88 \\
\hline Tc-99 & $1.44 \mathrm{E}-07$ & $2.02 \mathrm{E}-14$ & $0.00 \mathrm{E}+00$ & $2.70 \mathrm{E}-03$ & 2.49E-05 & 7.19E-04 & $1.43 \mathrm{E}-07$ & $5.74 \mathrm{E}-04$ & 75.2 \\
\hline U-234 & $2.78 \mathrm{E}-03$ & $4.75 \mathrm{E}-07$ & $5.42 \mathrm{E}-05$ & $2.28 \mathrm{E}-03$ & $5.43 \mathrm{E}-04$ & $3.56 \mathrm{E}-04$ & $1.39 \mathrm{E}-04$ & $3.68 \mathrm{E}-02$ & 5404 \\
\hline U-235 & $4.00 \mathrm{E}-03$ & $3.53 \mathrm{E}-06$ & $0.00 \mathrm{E}+00$ & $2.24 \mathrm{E}-03$ & $5.78 \mathrm{E}-04$ & $9.60 \mathrm{E}-05$ & 2.22E-04 & $3.36 \mathrm{E}-03$ & 5185 \\
\hline U-238 & 7.64E-04 & 4.29E-07 & $9.66 \mathrm{E}-08$ & $3.92 \mathrm{E}-04$ & $2.28 \mathrm{E}-05$ & $1.15 \mathrm{E}-04$ & $3.65 \mathrm{E}-05$ & $1.6 \mathrm{E}-04$ & 2553 \\
\hline
\end{tabular}

a All values are reported to three significant figures.

b For Co-60, the dose is from both WI and WD pathways. 
TABLE 21 Maximum DSRs for the Recreational (Likely Future Land Use) Scenario for the Building 310 Area

\begin{tabular}{|c|c|c|c|c|c|c|c|c|}
\hline \multirow[b]{2}{*}{ Nuclide } & \multicolumn{7}{|c|}{ Maximum DSR $^{\mathrm{a}}(\mathrm{mrem} / \mathrm{yr}) /(\mathrm{pCi} / \mathrm{g})$ for Recreational Use Scenario } & \multirow{2}{*}{$\begin{array}{l}\text { Time of } \\
\text { Maximum } \\
\text { DSR (yr) }\end{array}$} \\
\hline & $\begin{array}{l}\text { External } \\
\text { Exposure }\end{array}$ & Inhalation & Radon & $\begin{array}{c}\text { Water } \\
\text { Ingestion }\end{array}$ & $\begin{array}{c}\text { Meat } \\
\text { Ingestion }\end{array}$ & $\begin{array}{c}\text { Soil } \\
\text { Ingestion }\end{array}$ & $\begin{array}{c}\text { Fish } \\
\text { Ingestion }\end{array}$ & \\
\hline Am-241 & $1.18 \mathrm{E}-03$ & $1.16 \mathrm{E}-03$ & $0.00 \mathrm{E}+00$ & $0.00 \mathrm{E}+00$ & $3.05 \mathrm{E}-04$ & $8.81 \mathrm{E}-04$ & $0.00 \mathrm{E}+00$ & $1.00 \mathrm{E}+02$ \\
\hline $\mathrm{C}-14^{\mathrm{b}}$ & $0.00 \mathrm{E}+00$ & $0.00 \mathrm{E}+00$ & $0.00 \mathrm{E}+00$ & $4.24 \mathrm{E}-17$ & $7.52 \mathrm{E}-16$ & $0.00 \mathrm{E}+00$ & $6.58 \mathrm{E}-13$ & $1.00 \mathrm{E}+02$ \\
\hline Co-60 & $9.84 \mathrm{E}-07$ & $7.81 \mathrm{E}-13$ & $0.00 \mathrm{E}+00$ & $0.00 \mathrm{E}+00$ & $1.14 \mathrm{E}-08$ & $3.12 \mathrm{E}-11$ & $0.00 \mathrm{E}+00$ & $1.00 \mathrm{E}+02$ \\
\hline Cs-137 & $1.12 \mathrm{E}-02$ & $5.38 \mathrm{E}-08$ & $0.00 \mathrm{E}+00$ & $0.00 \mathrm{E}+00$ & $2.46 \mathrm{E}-03$ & $6.55 \mathrm{E}-06$ & $0.00 \mathrm{E}+00$ & $1.00 \mathrm{E}+02$ \\
\hline $\mathrm{I}-129^{\mathrm{b}}$ & $2.37 \mathrm{E}-10$ & $8.83 \mathrm{E}-13$ & $0.00 \mathrm{E}+00$ & $8.14 \mathrm{E}-04$ & $9.50 \mathrm{E}-05$ & $3.69 \mathrm{E}-10$ & $1.01 \mathrm{E}-02$ & $1.00 \mathrm{E}+02$ \\
\hline Np-237 & $2.72 \mathrm{E}-02$ & $5.26 \mathrm{E}-04$ & $0.00 \mathrm{E}+00$ & $0.00 \mathrm{E}+00$ & $4.11 \mathrm{E}-03$ & $4.25 \mathrm{E}-04$ & $0.00 \mathrm{E}+00$ & $1.00 \mathrm{E}+02$ \\
\hline $\mathrm{Pu}-238$ & $2.00 \mathrm{E}-06$ & $7.05 \mathrm{E}-04$ & $1.50 \mathrm{E}-13$ & $0.00 \mathrm{E}+00$ & $3.72 \mathrm{E}-04$ & $5.38 \mathrm{E}-04$ & $0.00 \mathrm{E}+00$ & $1.00 \mathrm{E}+02$ \\
\hline $\mathrm{Pu}-239$ & $9.59 \mathrm{E}-06$ & $1.70 \mathrm{E}-03$ & $0.00 \mathrm{E}+00$ & $0.00 \mathrm{E}+00$ & $8.92 \mathrm{E}-04$ & $1.29 \mathrm{E}-03$ & $0.00 \mathrm{E}+00$ & $1.00 \mathrm{E}+02$ \\
\hline $\mathrm{Pu}-240$ & $4.21 \mathrm{E}-06$ & $1.68 \mathrm{E}-03$ & $7.77 \mathrm{E}-18$ & $0.00 \mathrm{E}+00$ & $8.85 \mathrm{E}-04$ & $1.28 \mathrm{E}-03$ & $0.00 \mathrm{E}+00$ & $1.00 \mathrm{E}+02$ \\
\hline Sr-90 & $1.16 \mathrm{E}-04$ & $1.80 \mathrm{E}-07$ & $0.00 \mathrm{E}+00$ & $0.00 \mathrm{E}+00$ & $5.02 \mathrm{E}-03$ & $1.26 \mathrm{E}-05$ & $0.00 \mathrm{E}+00$ & $1.00 \mathrm{E}+02$ \\
\hline Tc-99b & $2.58 \mathrm{E}-12$ & $1.20 \mathrm{E}-13$ & $0.00 \mathrm{E}+00$ & $3.05 \mathrm{E}-06$ & $5.70 \mathrm{E}-09$ & $2.15 \mathrm{E}-12$ & $1.90 \mathrm{E}-05$ & $1.00 \mathrm{E}+02$ \\
\hline $\mathrm{U}-234$ & $7.28 \mathrm{E}-03$ & $1.29 \mathrm{E}-04$ & $4.04 \mathrm{E}-07$ & $0.00 \mathrm{E}+00$ & $3.14 \mathrm{E}-03$ & $4.10 \mathrm{E}-04$ & $0.00 \mathrm{E}+00$ & $5.15 \mathrm{E}+03$ \\
\hline U-235 & $2.09 \mathrm{E}-02$ & $6.97 \mathrm{E}-04$ & $0.00 \mathrm{E}+00$ & $0.00 \mathrm{E}+00$ & $8.85 \mathrm{E}-03$ & $7.68 \mathrm{E}-04$ & $0.00 \mathrm{E}+00$ & $4.39 \mathrm{E}+03$ \\
\hline U-238 & $5.58 \mathrm{E}-03$ & $1.13 \mathrm{E}-04$ & $1.81 \mathrm{E}-13$ & $0.00 \mathrm{E}+00$ & $6.05 \mathrm{E}-04$ & $2.49 \mathrm{E}-04$ & $0.00 \mathrm{E}+00$ & $1.00 \mathrm{E}+02$ \\
\hline
\end{tabular}

a All values are reported to three significant figures. The maximum DSR occurs at 100 years (immediately after the institutional control period) for all radionuclides except U-234 and U-235.

b For C-14, I-129, and Tc-99, the maximum dose is from WD pathways (such as ingestion of food irrigated by contaminated water, water ingestion, and fish ingestion). 


\section{TABLE 22 Maximum DSRs for the Onsite Resident (Unlikely Future Land Use) Scenario for}

the Building 310 Area

\begin{tabular}{|c|c|c|c|c|c|c|c|c|c|c|}
\hline \multirow[b]{2}{*}{ Nuclide } & \multicolumn{9}{|c|}{ Maximum DSR ${ }^{\mathrm{a}}(\mathrm{mrem} / \mathrm{yr}) /(\mathrm{pCi} / \mathrm{g})$ for Onsite Resident Scenario } & \multirow{2}{*}{$\begin{array}{c}\text { Time of } \\
\text { Maximum } \\
\text { DSR (yr) }\end{array}$} \\
\hline & $\begin{array}{c}\text { External } \\
\text { Exposure }\end{array}$ & Inhalation & Radon & $\begin{array}{c}\text { Water } \\
\text { Ingestion }\end{array}$ & $\begin{array}{c}\text { Plant } \\
\text { Ingestion }\end{array}$ & $\begin{array}{c}\text { Meat } \\
\text { Ingestion }\end{array}$ & $\begin{array}{c}\text { Milk } \\
\text { Ingestion }\end{array}$ & $\begin{array}{c}\text { Soil } \\
\text { Ingestion }\end{array}$ & $\begin{array}{c}\text { Fish } \\
\text { Ingestion }\end{array}$ & \\
\hline Am-241 & $1.72 \mathrm{E}-02$ & $6.46 \mathrm{E}-03$ & $0.00 \mathrm{E}+00$ & $0.00 \mathrm{E}+00$ & $5.47 \mathrm{E}-02$ & $5.63 \mathrm{E}-04$ & $3.21 \mathrm{E}-05$ & $1.76 \mathrm{E}-02$ & $0.00 \mathrm{E}+00$ & $1.00 \mathrm{E}+02$ \\
\hline $\mathrm{C}-14^{\mathrm{b}}$ & $0.00 \mathrm{E}+00$ & $0.00 \mathrm{E}+00$ & $0.00 \mathrm{E}+00$ & $4.63 \mathrm{E}-14$ & $6.49 \mathrm{E}-15$ & $3.98 \mathrm{E}-15$ & $6.24 \mathrm{E}-15$ & $0.00 \mathrm{E}+00$ & $3.45 \mathrm{E}-13$ & $1.00 \mathrm{E}+02$ \\
\hline Co-60 & $1.44 \mathrm{E}-05$ & $4.35 \mathrm{E}-12$ & $0.00 \mathrm{E}+00$ & $0.00 \mathrm{E}+00$ & $1.55 \mathrm{E}-07$ & 8.34E-08 & $1.00 \mathrm{E}-08$ & $6.23 \mathrm{E}-10$ & $0.00 \mathrm{E}+00$ & $1.00 \mathrm{E}+02$ \\
\hline Cs-137 & $1.64 \mathrm{E}-01$ & $3.00 \mathrm{E}-07$ & $0.00 \mathrm{E}+00$ & $0.00 \mathrm{E}+00$ & $1.63 \mathrm{E}-02$ & $1.42 \mathrm{E}-02$ & 4.65E-03 & $1.31 \mathrm{E}-04$ & $0.00 \mathrm{E}+00$ & $1.00 \mathrm{E}+02$ \\
\hline $\mathrm{I}-129^{\mathrm{b}}$ & 3.47E-09 & $4.92 \mathrm{E}-12$ & $0.00 \mathrm{E}+00$ & $8.71 \mathrm{E}-01$ & $3.38 \mathrm{E}-02$ & 4.28E-02 & $1.66 \mathrm{E}-01$ & 7.37E-09 & 4.59E-03 & $1.00 \mathrm{E}+02$ \\
\hline $\mathrm{Np}-237^{\mathrm{b}, \mathrm{c}}$ & $2.61 \mathrm{E}-04$ & $6.77 \mathrm{E}-06$ & $0.00 \mathrm{E}+00$ & $1.47 \mathrm{E}+00$ & $5.76 \mathrm{E}-02$ & $1.05 \mathrm{E}-02$ & $1.42 \mathrm{E}-04$ & $2.42 \mathrm{E}-03$ & $1.83 \mathrm{E}-02$ & $3.16 \mathrm{E}+03$ \\
\hline $\mathrm{Pu}-238$ & 2.92E-05 & $3.93 \mathrm{E}-03$ & $1.75 \mathrm{E}-07$ & $0.00 \mathrm{E}+00$ & $3.34 \mathrm{E}-02$ & $6.87 \mathrm{E}-04$ & $1.04 \mathrm{E}-05$ & $1.07 \mathrm{E}-02$ & $0.00 \mathrm{E}+00$ & $1.00 \mathrm{E}+02$ \\
\hline $\mathrm{Pu}-239$ & $1.40 \mathrm{E}-04$ & $9.45 \mathrm{E}-03$ & $0.00 \mathrm{E}+00$ & $0.00 \mathrm{E}+00$ & $8.00 \mathrm{E}-02$ & $1.65 \mathrm{E}-03$ & $2.35 \mathrm{E}-05$ & $2.57 \mathrm{E}-02$ & $0.00 \mathrm{E}+00$ & $1.00 \mathrm{E}+02$ \\
\hline $\mathrm{Pu}-240$ & $6.15 \mathrm{E}-05$ & $9.37 \mathrm{E}-03$ & $1.15 \mathrm{E}-15$ & $0.00 \mathrm{E}+00$ & 7.94E-02 & $1.63 \mathrm{E}-03$ & 2.33E-05 & $2.55 \mathrm{E}-02$ & $0.00 \mathrm{E}+00$ & $1.00 \mathrm{E}+02$ \\
\hline Sr-90 & $1.70 \mathrm{E}-03$ & $1.01 \mathrm{E}-06$ & $0.00 \mathrm{E}+00$ & $0.00 \mathrm{E}+00$ & 2.34E-01 & 4.72E-02 & $1.40 \mathrm{E}-02$ & $2.51 \mathrm{E}-04$ & $0.00 \mathrm{E}+00$ & $1.00 \mathrm{E}+02$ \\
\hline Tc- $99^{b}$ & $3.78 \mathrm{E}-11$ & $6.66 \mathrm{E}-13$ & $0.00 \mathrm{E}+00$ & $3.26 \mathrm{E}-03$ & $2.89 \mathrm{E}-04$ & $2.48 \mathrm{E}-06$ & $6.42 \mathrm{E}-05$ & $4.29 \mathrm{E}-11$ & 8.77E-06 & $1.00 \mathrm{E}+02$ \\
\hline $\mathrm{U}-234^{\mathrm{c}}$ & $9.42 \mathrm{E}-02$ & 7.22E-04 & $1.25 \mathrm{E}+00$ & $0.00 \mathrm{E}+00$ & $4.20 \mathrm{E}-02$ & $8.15 \mathrm{E}-03$ & $3.68 \mathrm{E}-03$ & $7.50 \mathrm{E}-03$ & $0.00 \mathrm{E}+00$ & $4.34 \mathrm{E}+03$ \\
\hline $\mathrm{U}-235^{\mathrm{c}}$ & $3.40 \mathrm{E}-01$ & $2.42 \mathrm{E}-03$ & $0.00 \mathrm{E}+00$ & $0.00 \mathrm{E}+00$ & $1.07 \mathrm{E}-01$ & $1.53 \mathrm{E}-02$ & $2.51 \mathrm{E}-03$ & $1.06 \mathrm{E}-02$ & $0.00 \mathrm{E}+00$ & $1.89 \mathrm{E}+03$ \\
\hline $\mathrm{U}-238$ & $8.16 \mathrm{E}-02$ & $6.28 \mathrm{E}-04$ & $2.11 \mathrm{E}-07$ & $0.00 \mathrm{E}+00$ & $3.87 \mathrm{E}-02$ & $1.28 \mathrm{E}-03$ & $3.13 \mathrm{E}-03$ & $4.98 \mathrm{E}-03$ & $0.00 \mathrm{E}+00$ & $1.00 \mathrm{E}+02$ \\
\hline
\end{tabular}

a All values are reported to three significant figures. The maximum DSR occurs at 100 years (immediately after the institutional control period) for all radionuclides.

b For C-14, I-129, Np-237, and Tc-99, the maximum dose is from WD pathways (such as food ingestion irrigated by contaminated water, water ingestion, and fish ingestion).

c The maximum dose occurs at later time as a result of the buildup of progeny. 
TABLE 23 Total Peak DSRs for Different Scenarios for the Building 310 Area

\begin{tabular}{|c|c|c|c|c|}
\hline \multirow[b]{2}{*}{ Nuclide } & \multicolumn{4}{|c|}{$\begin{array}{c}\text { Total Peak DSR }{ }^{\mathrm{a}}(\mathrm{mrem} / \mathrm{yr}) /(\mathrm{pCi} / \mathrm{g}) \text { for Different } \\
\text { Scenarios }\end{array}$} \\
\hline & $\begin{array}{l}\text { Industrial } \\
\text { Use }^{b}\end{array}$ & $\begin{array}{c}\text { Offsite } \\
\text { Resident }^{\mathrm{b}}\end{array}$ & $\begin{array}{c}\text { Onsite } \\
\text { Resident }^{\mathrm{c}}\end{array}$ & $\begin{array}{l}\text { Recreational } \\
\text { Use }^{\mathrm{d}}\end{array}$ \\
\hline Am-241 & $2.10 \mathrm{E}-02$ & 8.03E-03 & $9.66 \mathrm{E}-02$ & $3.52 \mathrm{E}-03$ \\
\hline C-14 & $2.08 \mathrm{E}-05$ & $3.40 \mathrm{E}-02$ & $4.08 \mathrm{E}-13$ & $6.59 \mathrm{E}-13$ \\
\hline Co-60 & $3.10 \mathrm{E}+00$ & $9.25 \mathrm{E}-04$ & $1.46 \mathrm{E}-05$ & $9.95 \mathrm{E}-07$ \\
\hline Cs-137 & $6.77 \mathrm{E}-01$ & $5.51 \mathrm{E}-03$ & $1.99 \mathrm{E}-01$ & $1.37 \mathrm{E}-02$ \\
\hline $\mathrm{I}-129$ & $5.24 \mathrm{E}-03$ & $1.43 \mathrm{E}+00$ & $1.12 \mathrm{E}+00$ & $1.10 \mathrm{E}-02$ \\
\hline Np-237 & $2.25 \mathrm{E}-01$ & $1.66 \mathrm{E}-02$ & $1.56 \mathrm{E}+00$ & $3.23 \mathrm{E}-02$ \\
\hline Pu-238 & $1.46 \mathrm{E}-02$ & $3.41 \mathrm{E}-03$ & $4.88 \mathrm{E}-02$ & $1.62 \mathrm{E}-03$ \\
\hline $\mathrm{Pu}-239$ & $1.60 \mathrm{E}-02$ & $4.02 \mathrm{E}-03$ & $1.17 \mathrm{E}-01$ & $3.89 \mathrm{E}-03$ \\
\hline $\mathrm{Pu}-240$ & $1.60 \mathrm{E}-02$ & $3.89 \mathrm{E}-03$ & $1.16 \mathrm{E}-01$ & $3.85 \mathrm{E}-03$ \\
\hline Sr-90 & $9.63 \mathrm{E}-03$ & $1.20 \mathrm{E}-03$ & $2.97 \mathrm{E}-01$ & $5.14 \mathrm{E}-03$ \\
\hline Tc-99 & $4.17 \mathrm{E}-05$ & $4.02 \mathrm{E}-03$ & $3.63 \mathrm{E}-03$ & $2.21 \mathrm{E}-05$ \\
\hline $\mathrm{U}-234$ & $4.60 \mathrm{E}-01$ & $4.29 \mathrm{E}-02$ & $1.41 \mathrm{E}+00$ & $1.10 \mathrm{E}-02$ \\
\hline U-235 & $1.53 \mathrm{E}-01$ & $1.04 \mathrm{E}-02$ & $4.78 \mathrm{E}-01$ & $3.12 \mathrm{E}-02$ \\
\hline U-238 & $3.56 \mathrm{E}-02$ & $1.50 \mathrm{E}-03$ & $1.30 \mathrm{E}-01$ & $6.54 \mathrm{E}-03$ \\
\hline
\end{tabular}

a All values are reported to three significant figures.

b Current use scenario.

c Future unlikely use scenario.

d Future likely use scenario. 


\section{DERIVED CONCENTRATION GUIDELINE LEVELS}

The DCGL is the concentration of residual radioactive material that can remain in the decontaminated area and still allow use of the area without radiological restrictions. Given a dose limit of $\mathrm{H}_{E L}$ for an individual, the DCGL for an individual radionuclide $(i)$ can be calculated as

$$
\mathrm{DCGL}_{i}(\mathrm{pCi} / \mathrm{g})=\mathrm{H}_{E L}(\mathrm{mrem} / \mathrm{yr}) / \mathrm{DSR}_{i}(\mathrm{mrem} / \mathrm{yr} \text { per pCi/g })
$$

where $\mathrm{DSR}_{i}$ is the total dose-to-source concentration ratio. The total DSRs for probabilistic analysis at the peak of the means, mean of the peaks, and the 95th percentile of the peaks are listed in Table 18; for deterministic analysis, they are listed in Table 23 for different scenarios. The dose limit, $\mathrm{H}_{E L}$, used in calculating DCGLs is $25 \mathrm{mrem} / \mathrm{yr}$.

\subsection{DCGLS BASED ON PROBABILISTIC ANALYSIS}

Table 24 shows the DCGLs derived from different scenarios at the mean and 95 th percentile of the peak DSRs based on a $25-\mathrm{mrem} / \mathrm{yr}$ dose. For some radionuclides, the current land use scenarios (industrial worker or offsite resident scenarios) resulted in the most restrictive DCGLs, and for others, the future land use (onsite resident) scenario resulted in the most restrictive DCGLs. The most restrictive DCGLs are highlighted in Table 24 and listed in the last column under the heading "Most Restrictive" for peak of the means, mean of the peaks, and 95th percentile of the peak DSRs.

\subsection{DCGLS BASED ON DETERMINISTIC ANALYSIS}

Table 25 shows the DCGLs derived from a deterministic analysis of different scenarios based on a $25-\mathrm{mrem} / \mathrm{yr}$ dose limit. For some radionuclides, the current land use scenarios (industrial worker or offsite resident scenarios) resulted in the most restrictive DCGLs, and for others, the future land use (onsite resident) scenario resulted in the most restrictive DCGLs. The most restrictive DCGL is highlighted in Table 25 and listed in the last column under the heading "Most Restrictive."

\subsection{SUMMARY DCGLS FOR BUILDING 310 AREA}

Table 26 compares the DCGLs derived from a probabilistic analysis at peak of the means, mean of the peaks, 95th percentile of the peak DSRs and the DCGLs derived from deterministic analysis for Building 310 area at Argonne. Also listed in the last column of Table 26 are the proposed DCGLs for the 14 radionuclides analyzed. These proposed DCGLs are selected by considering the results from probabilistic and deterministic analyses and the DCGLs used for other Argonne sites (Kamboj and Yu 2011). The proposed DCGLs are reported to two significant figures. 
TABLE 24 DCGLs (pCi/g) from Using Probabilistic Analysis at a 25-mrem/yr Dose Limit for the Building 310 Area

\begin{tabular}{|c|c|c|c|c|c|c|c|c|c|c|c|c|c|c|c|}
\hline \multirow[b]{2}{*}{ Nuclide } & \multicolumn{3}{|c|}{ Industrial Use } & \multicolumn{3}{|c|}{ Offsite Resident } & \multicolumn{3}{|c|}{ Recreational Use } & \multicolumn{3}{|c|}{ Onsite Resident } & \multicolumn{3}{|c|}{ Most Restrictive } \\
\hline & $\begin{array}{c}\text { Peak of } \\
\text { the Means }\end{array}$ & $\begin{array}{l}\text { Mean of } \\
\text { the Peaks }\end{array}$ & $\begin{array}{l}95 \text { th \% of } \\
\text { the Peaks }\end{array}$ & $\begin{array}{c}\text { Peak of } \\
\text { the Means }\end{array}$ & $\begin{array}{l}\text { Mean of } \\
\text { the Peaks }\end{array}$ & $\begin{array}{l}95 \text { th } \% \text { of } \\
\text { the Peaks }\end{array}$ & $\begin{array}{c}\text { Peak of } \\
\text { the Means }\end{array}$ & $\begin{array}{c}\text { Mean of } \\
\text { the Peaks }\end{array}$ & $\begin{array}{l}95 \text { th } \% \text { of } \\
\text { the Peaks }\end{array}$ & $\begin{array}{c}\text { Peak of } \\
\text { the Means }\end{array}$ & $\begin{array}{l}\text { Mean of } \\
\text { the Peaks }\end{array}$ & $\begin{array}{l}95 \text { th } \% \text { of } \\
\text { the Peaks }\end{array}$ & $\begin{array}{c}\text { Peak of } \\
\text { the Means }\end{array}$ & $\begin{array}{c}\text { Mean of } \\
\text { the Peaks }\end{array}$ & $\begin{array}{l}95 \text { th \% of } \\
\text { the Peaks }\end{array}$ \\
\hline Am-241 & $1.85 \mathrm{E}+03$ & $1.85 \mathrm{E}+03$ & $1.14 \mathrm{E}+03$ & $3.64 \mathrm{E}+04$ & $3.58 \mathrm{E}+03$ & $2.10 \mathrm{E}+03$ & $1.04 \mathrm{E}+04$ & $1.04 \mathrm{E}+04$ & $8.20 \mathrm{E}+03$ & $2.38 \mathrm{E}+02$ & $2.38 \mathrm{E}+02$ & $9.33 \mathrm{E}+01$ & $2.38 \mathrm{E}+02$ & $2.38 \mathrm{E}+02$ & $9.33 \mathrm{E}+01$ \\
\hline C-14 & $1.19 \mathrm{E}+06$ & $1.19 \mathrm{E}+06$ & $6.70 \mathrm{E}+05$ & $1.67 \mathrm{E}+03$ & $4.20 \mathrm{E}+02$ & $1.10 \mathrm{E}+02$ & $1.58 \mathrm{E}+03$ & $5.42 \mathrm{E}+02$ & $1.00 \mathrm{E}+02$ & $7.06 \mathrm{E}+03$ & $7.44 \mathrm{E}+03$ & $2.38 \mathrm{E}+03$ & $1.58 \mathrm{E}+03$ & $4.20 \mathrm{E}+02$ & $1.00 \mathrm{E}+02$ \\
\hline Co-60 & $8.04 \mathrm{E}+00$ & $8.04 \mathrm{E}+00$ & $5.39 \mathrm{E}+00$ & $3.12 \mathrm{E}+04$ & $3.05 \mathrm{E}+04$ & $2.77 \mathrm{E}+04$ & $2.59 \mathrm{E}+07$ & $2.59 \mathrm{E}+07$ & $2.14 \mathrm{E}+07$ & $3.32 \mathrm{E}+06$ & $3.32 \mathrm{E}+06$ & $1.79 \mathrm{E}+06$ & $8.04 \mathrm{E}+00$ & $8.04 \mathrm{E}+00$ & $5.39 \mathrm{E}+00$ \\
\hline Cs-137 & $3.69 \mathrm{E}+01$ & $3.69 \mathrm{E}+01$ & $2.48 \mathrm{E}+01$ & $3.63 \mathrm{E}+03$ & $3.64 \mathrm{E}+03$ & $1.68 \mathrm{E}+03$ & $1.28 \mathrm{E}+03$ & $1.28 \mathrm{E}+03$ & $7.16 \mathrm{E}+02$ & $1.57 \mathrm{E}+02$ & $1.57 \mathrm{E}+02$ & $7.89 \mathrm{E}+01$ & $3.69 \mathrm{E}+01$ & $3.69 \mathrm{E}+01$ & $2.48 \mathrm{E}+01$ \\
\hline I-129 & $6.87 \mathrm{E}+03$ & $6.87 \mathrm{E}+03$ & $4.07 \mathrm{E}+03$ & $1.28 \mathrm{E}+02$ & $2.69 \mathrm{E}+01$ & $7.04 \mathrm{E}+00$ & $3.51 \mathrm{E}+02$ & $1.11 \mathrm{E}+02$ & $2.21 \mathrm{E}+01$ & $7.91 \mathrm{E}+00$ & $9.96 \mathrm{E}-01$ & $2.40 \mathrm{E}-01$ & $7.91 \mathrm{E}+00$ & $9.96 \mathrm{E}-01$ & $2.40 \mathrm{E}-01$ \\
\hline Np-237 & $1.16 \mathrm{E}+02$ & $1.16 \mathrm{E}+02$ & $7.62 \mathrm{E}+01$ & $2.69 \mathrm{E}+03$ & $7.18 \mathrm{E}+02$ & $2.14 \mathrm{E}+02$ & $5.34 \mathrm{E}+02$ & $2.66 \mathrm{E}+02$ & $8.56 \mathrm{E}+01$ & $2.25 \mathrm{E}+01$ & $9.47 \mathrm{E}+00$ & $2.40 \mathrm{E}+00$ & $2.25 \mathrm{E}+01$ & $9.47 \mathrm{E}+00$ & $2.40 \mathrm{E}+00$ \\
\hline $\mathrm{Pu}-238$ & $4.18 \mathrm{E}+03$ & $4.18 \mathrm{E}+03$ & $2.21 \mathrm{E}+03$ & $8.01 \mathrm{E}+03$ & $7.99 \mathrm{E}+03$ & $3.06 \mathrm{E}+03$ & $2.36 \mathrm{E}+04$ & $2.36 \mathrm{E}+04$ & $1.55 \mathrm{E}+04$ & $4.38 \mathrm{E}+02$ & $4.38 \mathrm{E}+02$ & $1.60 \mathrm{E}+02$ & $4.38 \mathrm{E}+02$ & $4.38 \mathrm{E}+02$ & $1.60 \mathrm{E}+02$ \\
\hline $\mathrm{Pu}-239$ & $3.81 \mathrm{E}+03$ & $3.81 \mathrm{E}+03$ & $2.02 \mathrm{E}+03$ & $7.04 \mathrm{E}+03$ & $6.81 \mathrm{E}+03$ & $2.76 \mathrm{E}+03$ & $9.84 \mathrm{E}+03$ & $9.84 \mathrm{E}+03$ & $6.46 \mathrm{E}+03$ & $1.82 \mathrm{E}+02$ & $1.82 \mathrm{E}+02$ & $6.70 \mathrm{E}+01$ & $1.82 \mathrm{E}+02$ & $1.82 \mathrm{E}+02$ & $6.70 \mathrm{E}+01$ \\
\hline $\mathrm{Pu}-240$ & $4.22 \mathrm{E}+03$ & $4.22 \mathrm{E}+03$ & $2.29 \mathrm{E}+03$ & $4.55 \mathrm{E}+03$ & $4.55 \mathrm{E}+03$ & $1.76 \mathrm{E}+03$ & $1.04 \mathrm{E}+04$ & $1.04 \mathrm{E}+04$ & $6.74 \mathrm{E}+03$ & $1.85 \mathrm{E}+02$ & $1.85 \mathrm{E}+02$ & $6.74 \mathrm{E}+01$ & $1.85 \mathrm{E}+02$ & $1.85 \mathrm{E}+02$ & $6.74 \mathrm{E}+01$ \\
\hline Sr-90 & $2.73 \mathrm{E}+03$ & $2.73 \mathrm{E}+03$ & $1.81 \mathrm{E}+03$ & $1.47 \mathrm{E}+04$ & $1.34 \mathrm{E}+04$ & $6.27 \mathrm{E}+03$ & $1.85 \mathrm{E}+03$ & $1.85 \mathrm{E}+03$ & $5.26 \mathrm{E}+02$ & $6.00 \mathrm{E}+01$ & $6.00 \mathrm{E}+01$ & $1.74 \mathrm{E}+01$ & $6.00 \mathrm{E}+01$ & $6.00 \mathrm{E}+01$ & $1.74 \mathrm{E}+01$ \\
\hline Тc-99 & $7.79 \mathrm{E}+05$ & $7.79 \mathrm{E}+05$ & $4.83 \mathrm{E}+05$ & $2.36 \mathrm{E}+04$ & $9.65 \mathrm{E}+03$ & $3.54 \mathrm{E}+03$ & $2.60 \mathrm{E}+04$ & $9.77 \mathrm{E}+03$ & $2.34 \mathrm{E}+03$ & 4. $62 \mathrm{E}+02$ & $4.40 \mathrm{E}+02$ & $9.84 \mathrm{E}+01$ & $4.62 \mathrm{E}+02$ & $4.40 \mathrm{E}+02$ & $9.84 \mathrm{E}+01$ \\
\hline U-234 & $9.84 \mathrm{E}+01$ & $9.43 \mathrm{E}+01$ & $3.77 \mathrm{E}+01$ & $9.73 \mathrm{E}+02$ & $8.65 \mathrm{E}+02$ & $4.34 \mathrm{E}+02$ & $2.80 \mathrm{E}+03$ & $2.50 \mathrm{E}+03$ & $1.19 \mathrm{E}+03$ & $3.09 \mathrm{E}+01$ & $2.92 \mathrm{E}+01$ & $1.43 \mathrm{E}+01$ & $3.09 \mathrm{E}+01$ & $2.92 \mathrm{E}+01$ & $1.43 \mathrm{E}+01$ \\
\hline U-235 & $1.64 \mathrm{E}+02$ & $1.55 \mathrm{E}+02$ & $9.43 \mathrm{E}+01$ & $3.20 \mathrm{E}+03$ & $2.87 \mathrm{E}+03$ & $1.33 \mathrm{E}+03$ & $9.96 \mathrm{E}+02$ & $9.43 \mathrm{E}+02$ & $6.98 \mathrm{E}+02$ & $1.02 \mathrm{E}+02$ & $7.55 \mathrm{E}+01$ & $3.85 \mathrm{E}+01$ & $1.02 \mathrm{E}+02$ & $7.55 \mathrm{E}+01$ & $3.85 \mathrm{E}+01$ \\
\hline $\mathrm{U}-238$ & $7.27 \mathrm{E}+02$ & $7.25 \mathrm{E}+02$ & $4.84 \mathrm{E}+02$ & $2.16 \mathrm{E}+04$ & $1.52 \mathrm{E}+04$ & $6.36 \mathrm{E}+03$ & $3.26 \mathrm{E}+03$ & $3.16 \mathrm{E}+03$ & $1.91 \mathrm{E}+03$ & $2.80 \mathrm{E}+02$ & $1.95 \mathrm{E}+02$ & $1.01 \mathrm{E}+02$ & $2.80 \mathrm{E}+02$ & $1.95 \mathrm{E}+02$ & $1.01 \mathrm{E}+02$ \\
\hline
\end{tabular}


TABLE 25 DCGLs from Using Deterministic Analysis at a 25-mrem/yr Dose Limit for the Building 310 Area

\begin{tabular}{|c|c|c|c|c|c|}
\hline \multirow[b]{3}{*}{ Nuclide } & \multicolumn{5}{|c|}{$\begin{array}{l}\text { DCGLs (pCi/g) from Deterministic Analysis at the 25-mrem/yr } \\
\text { Dose Limit from Different Scenarios }\end{array}$} \\
\hline & \multicolumn{2}{|c|}{ Current Use } & \multicolumn{2}{|c|}{ Future Use } & \multirow[b]{2}{*}{ Most Restrictive } \\
\hline & Industrial & Offsite Resident & Onsite Resident & Recreational & \\
\hline Am-241 & $1.19 \mathrm{E}+03$ & $3.11 \mathrm{E}+03$ & $2.59 \mathrm{E}+02$ & $7.10 \mathrm{E}+03$ & $2.59 \mathrm{E}+02$ \\
\hline C-14 & $1.20 \mathrm{E}+06$ & $7.36 \mathrm{E}+02$ & $\mathrm{NA}^{\mathrm{a}}$ & $\mathrm{NA}^{\mathrm{a}}$ & $7.36 \mathrm{E}+02$ \\
\hline Co-60 & $8.07 \mathrm{E}+00$ & $2.70 \mathrm{E}+04$ & $1.71 \mathrm{E}+06$ & $2.51 \mathrm{E}+07$ & $8.07 \mathrm{E}+00$ \\
\hline Cs-137 & $3.70 \mathrm{E}+01$ & $4.54 \mathrm{E}+03$ & $1.26 \mathrm{E}+02$ & $1.83 \mathrm{E}+03$ & $3.70 \mathrm{E}+01$ \\
\hline I-129 & $4.77 \mathrm{E}+03$ & $1.75 E+01$ & $2.24 \mathrm{E}+01$ & $2.28 \mathrm{E}+03$ & $1.75 \mathrm{E}+01$ \\
\hline $\mathrm{Np}-237$ & $1.11 \mathrm{E}+02$ & $1.51 \mathrm{E}+03$ & $1.60 \mathrm{E}+01$ & $7.75 \mathrm{E}+02$ & $1.60 \mathrm{E}+01$ \\
\hline $\mathrm{Pu}-238$ & $1.71 \mathrm{E}+03$ & $7.33 \mathrm{E}+03$ & $5.13 \mathrm{E}+02$ & $1.55 \mathrm{E}+04$ & $5.13 \mathrm{E}+02$ \\
\hline $\mathrm{Pu}-239$ & $1.56 \mathrm{E}+03$ & $6.23 \mathrm{E}+03$ & $2.14 \mathrm{E}+02$ & $6.44 \mathrm{E}+03$ & $2.14 \mathrm{E}+02$ \\
\hline $\mathrm{Pu}-240$ & $1.56 \mathrm{E}+03$ & $6.43 \mathrm{E}+03$ & $2.16 \mathrm{E}+02$ & $6.49 \mathrm{E}+03$ & $2.16 \mathrm{E}+02$ \\
\hline Sr-90 & $2.60 \mathrm{E}+03$ & $2.09 \mathrm{E}+04$ & $8.42 \mathrm{E}+01$ & $4.86 \mathrm{E}+03$ & $8.42 \mathrm{E}+01$ \\
\hline Tc-99 & $5.99 \mathrm{E}+05$ & $6.22 \mathrm{E}+03$ & $6.89 \mathrm{E}+03$ & $1.13 \mathrm{E}+06$ & $6.22 \mathrm{E}+03$ \\
\hline U-234 & $5.43 \mathrm{E}+01$ & $5.83 \mathrm{E}+02$ & $1.78 \mathrm{E}+01$ & $2.28 \mathrm{E}+03$ & $1.78 \mathrm{E}+01$ \\
\hline U-235 & $1.64 \mathrm{E}+02$ & $2.40 \mathrm{E}+03$ & $5.23 \mathrm{E}+01$ & $8.01 \mathrm{E}+02$ & $5.23 \mathrm{E}+01$ \\
\hline $\mathrm{U}-238$ & $7.03 \mathrm{E}+02$ & $1.67 \mathrm{E}+04$ & $1.92 \mathrm{E}+02$ & $3.82 \mathrm{E}+03$ & $1.92 \mathrm{E}+02$ \\
\hline
\end{tabular}

a DCGLs at specific activity limit.

TABLE 26 Summary of DCGLs for the Building 310 Area

\begin{tabular}{|c|c|c|c|c|c|}
\hline \multirow[b]{2}{*}{ Nuclide } & \multicolumn{3}{|c|}{ Probabilistic DCGLs (pCi/g) } & \multirow[b]{2}{*}{$\begin{array}{c}\text { Deterministic } \\
\text { DCGLs (pCi/g) }\end{array}$} & \multirow[b]{2}{*}{ 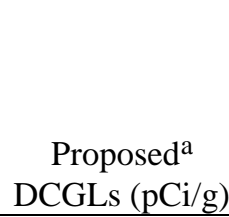 } \\
\hline & $\begin{array}{c}\text { At 95th } \\
\text { Percentile of the } \\
\text { Peak DSRs }\end{array}$ & $\begin{array}{c}\text { At Mean of the } \\
\text { Peak DSRs }\end{array}$ & $\begin{array}{l}\text { At Peak of the } \\
\text { Mean DSRs }\end{array}$ & & \\
\hline Am-241 & $9.33 \mathrm{E}+01$ & $2.38 \mathrm{E}+02$ & $2.38 \mathrm{E}+02$ & $2.59 \mathrm{E}+02$ & $2.4 \mathrm{E}+02$ \\
\hline C-14 & $1.00 \mathrm{E}+02$ & $4.20 \mathrm{E}+02$ & $1.58 \mathrm{E}+03$ & $7.36 \mathrm{E}+02$ & $5.4 \mathrm{E}+02$ \\
\hline Co-60 & $5.39 \mathrm{E}+00$ & $8.04 \mathrm{E}+00$ & $8.04 \mathrm{E}+00$ & $8.07 \mathrm{E}+00$ & $8.0 \mathrm{E}+00$ \\
\hline Cs-137 & $2.48 \mathrm{E}+01$ & $3.69 \mathrm{E}+01$ & $3.69 \mathrm{E}+01$ & $3.70 \mathrm{E}+01$ & $3.6 \mathrm{E}+01$ \\
\hline I-129 & $2.40 \mathrm{E}-01$ & $9.96 \mathrm{E}-01$ & $7.91 \mathrm{E}+00$ & $1.75 \mathrm{E}+01$ & $7.9 \mathrm{E}+00$ \\
\hline $\mathrm{Np}-237$ & $2.40 \mathrm{E}+00$ & $9.47 \mathrm{E}+00$ & $2.25 \mathrm{E}+01$ & $1.60 \mathrm{E}+01$ & $1.6 \mathrm{E}+01$ \\
\hline $\mathrm{Pu}-238$ & $1.60 \mathrm{E}+02$ & $4.38 \mathrm{E}+02$ & $4.38 \mathrm{E}+02$ & $5.13 \mathrm{E}+02$ & $4.3 \mathrm{E}+02$ \\
\hline $\mathrm{Pu}-239$ & $6.70 \mathrm{E}+01$ & $1.82 \mathrm{E}+02$ & $1.82 \mathrm{E}+02$ & $2.14 \mathrm{E}+02$ & $1.8 \mathrm{E}+02$ \\
\hline $\mathrm{Pu}-240$ & $6.74 \mathrm{E}+01$ & $1.85 \mathrm{E}+02$ & $1.85 \mathrm{E}+02$ & $2.16 \mathrm{E}+02$ & $1.8 \mathrm{E}+02$ \\
\hline Sr-90 & $1.74 \mathrm{E}+01$ & $6.00 \mathrm{E}+01$ & $6.00 \mathrm{E}+01$ & $8.42 \mathrm{E}+01$ & $6.0 \mathrm{E}+01$ \\
\hline Tc-99 & $9.84 \mathrm{E}+01$ & $4.40 \mathrm{E}+02$ & $4.62 \mathrm{E}+02$ & $6.22 \mathrm{E}+03$ & $4.6 \mathrm{E}+02$ \\
\hline U-234 & $1.43 \mathrm{E}+01$ & $2.92 \mathrm{E}+01$ & $3.09 \mathrm{E}+01$ & $1.78 \mathrm{E}+01$ & $1.7 \mathrm{E}+01$ \\
\hline U-235 & $3.85 \mathrm{E}+01$ & $7.55 \mathrm{E}+01$ & $1.02 \mathrm{E}+02$ & $5.23 \mathrm{E}+01$ & $5.2 \mathrm{E}+01$ \\
\hline U-238 & $1.01 \mathrm{E}+02$ & $1.95 \mathrm{E}+02$ & $2.80 \mathrm{E}+02$ & $1.92 \mathrm{E}+02$ & $1.9 \mathrm{E}+02$ \\
\hline
\end{tabular}

a The proposed DCGLs are rounded to two significant figures. 
The DCGLs listed in Table 26 are for a large, homogenously contaminated area. When implementing the DCGLs for decontamination of a site, the sum of fractions rule applies, as follows: The sum of the radionuclide concentrations $S_{i}$ remaining onsite for radionuclides $i$ divided by their DCGL $_{i}$ should not be greater than unity; that is,

$$
\sum_{\mathrm{i}} \mathrm{S}_{\mathrm{i}} / \mathrm{DCGL}_{\mathrm{i}} \leq 1 \text {. }
$$




\section{REFERENCES}

Kamboj, S., and C. Yu, 2011, Derived Concentration Guideline Levels for Argonne National Laboratory's Building 330 Area, ANL/EVS/TM/11-1, Argonne National Laboratory, Argonne, Ill.

NRC (U.S. Nuclear Regulatory Commission), 2000, Development of Probabilistic RESRAD 6.0 and RESRAD-BUILD 3.0 Computer Codes, NUREG/CR-6697 and ANL/EAD/TM-98, prepared by C. Yu et al., Argonne National Laboratory, Argonne, Ill., for NRC, Washington, D.C., Nov.

Patton, T.L., R.H. Pearl, and S.Y. Tsai, 1990, Hydrological Conditions at the 800 Area at Argonne National Laboratory, ANL/EAIS/TM-29, Argonne National Laboratory, Argonne, Ill., Aug.

Yu, C., et al., 2001, User's Manual for RESRAD Version 6, ANL/EAD-4, Argonne National Laboratory, Argonne, Ill.

Yu, C., et al., 2007, User's Manual for RESRAD-OFFSITE Version 2, ANL/EVS/TM/07-1, Argonne National Laboratory, Argonne, Ill. 


\section{APPENDIX A:}

\section{PARAMETERS USED FOR PROBABILISTIC ANALYSIS}

Tables A.1-A.10 list all input parameter distributions or parameter values used in the probabilistic dose analysis for different exposure scenarios. 


\section{TABLE A.1 Parameter Values and Distributions Used in the Probabilistic Analysis of Different Scenarios ${ }^{\mathrm{a}}$}

\begin{tabular}{|c|c|c|c|c|c|}
\hline \multirow[b]{2}{*}{ Input Parameters } & \multicolumn{2}{|c|}{ Current Use Scenarios } & \multicolumn{2}{|c|}{ Future Use Scenarios } & \multirow[b]{2}{*}{ Comments } \\
\hline & Industrial Worker & Offsite Resident ${ }^{\mathrm{b}}$ & Resident Farmer ${ }^{\mathrm{b}}$ & Recreationist $^{\mathrm{c}}$ & \\
\hline \multicolumn{6}{|l|}{ Title } \\
\hline Title & Scenario dependent & Scenario dependent & Scenario dependent & Scenario dependent & Scenario definition \\
\hline Dose factor library & ICRP-60 & ICRP-60 & ICRP-60 & ICRP-60 & $\begin{array}{l}\text { Dose conversion factors (DCFs) } \\
\text { based on ICRP-60 (ICRP 1991) } \\
\text { methodology (external from } \\
\text { FGR-12 [Eckerman and } \\
\text { Ryman 1993] and internal from } \\
\text { ICRP-72 [ICRP 1996]) }\end{array}$ \\
\hline Cut-off half-life (180 d or $30 \mathrm{~d})$ & $30 \mathrm{~d}$ & $30 \mathrm{~d}$ & $30 \mathrm{~d}$ & $30 \mathrm{~d}$ & RESRAD default \\
\hline Number of points $(32,64,128,256,512,1,024)$ & 32 & 32 & 32 & 32 & RESRAD default \\
\hline Linear spacing/log spacing & Log spacing & Log spacing & Log spacing & Log spacing & RESRAD default \\
\hline Maximum no. of points for dose & 17 & 17 & 17 & 17 & RESRAD default \\
\hline Maximum no. of points for risk & 1 & 1 & 1 & 1 & $\begin{array}{l}\text { Using a smaller integration point } \\
\text { will shorten the calculation time }\end{array}$ \\
\hline Use line draw character (yes/no) & Yes & Yes & Yes & Yes & RESRAD default \\
\hline Find peak pathway dose (yes/no) & Yes & Yes & Yes & Yes & \\
\hline Save all files after each run (yes/no) & Yes & Yes & Yes & Yes & \\
\hline Time integrated probabilistic risk (yes/no) & No & No & No & No & $\begin{array}{l}\text { Dose-to-source ratio (DSR) is } \\
\text { calculated }\end{array}$ \\
\hline \multicolumn{6}{|l|}{ Calculation Parameters } \\
\hline Basic radiation dose limit (mrem/yr) & 25 & 25 & 25 & 25 & Not used in DSR calculation \\
\hline Times for calculation $(\mathrm{yr})$ & $\begin{array}{c}1,3,10,30,100 \\
300,1,000\end{array}$ & $\begin{array}{c}1,3,10,30,100 \\
300,1,000\end{array}$ & $100,300,1,000$ & $100,300,1,000$ & $\begin{array}{l}\text { Up to the time horizon for dose } \\
\text { calculation }\end{array}$ \\
\hline \multicolumn{6}{|l|}{ Source } \\
\hline Nuclide concentration (pCi/g) & 1 & 1 & 1 & 1 & DSRs calculated \\
\hline \multicolumn{6}{|l|}{ Transport Factors } \\
\hline Distribution coefficient for all zones $\left(\mathrm{cm}^{3} / \mathrm{g}\right)$ & Tables A. 2 and A.3 & Tables A. 2 and A.3 & Tables A. 2 and A.3 & Tables A. 2 and A. 3 & $\begin{array}{l}\text { Distribution from Sheppard and } \\
\text { Thibault (1990) }\end{array}$ \\
\hline Number of unsaturated zones & 1 & 1 & 1 & 1 & RESRAD default \\
\hline Time since placement of material (yr) & 0 & 0 & 0 & 0 & RESRAD default \\
\hline Groundwater concentration (pCi/L) & 0 & 0 & 0 & 0 & RESRAD default \\
\hline Leach rate $(1 / \mathrm{yr})$ & 0 & 0 & 0 & 0 & RESRAD default \\
\hline
\end{tabular}


TABLE A.1 (Cont.)

\begin{tabular}{|c|c|c|c|c|c|}
\hline \multirow[b]{2}{*}{ Input Parameters } & \multicolumn{2}{|c|}{ Current Use Scenarios } & \multicolumn{2}{|c|}{ Future Use Scenarios } & \multirow[b]{2}{*}{ Comments } \\
\hline & Industrial Worker & Offsite Resident $\mathrm{t}^{\mathrm{b}}$ & Resident Farmer ${ }^{\mathrm{b}}$ & Recreationist $^{\mathrm{c}}$ & \\
\hline Solubility limit (mol/L) & 0 & 0 & 0 & 0 & RESRAD default \\
\hline Use plant/soil ration (check box) & No & No & No & No & RESRAD default \\
\hline \multicolumn{6}{|l|}{ Transfer Factors } \\
\hline $\begin{array}{l}\text { Plant transfer factor (wet-plant weight } \\
\text { concentration, } \mathrm{pCi} / \mathrm{g} / \mathrm{dry} \text { soil weight } \\
\text { concentration, } \mathrm{pCi} / \mathrm{g} \text { ) }\end{array}$ & $\mathrm{NR}^{\mathrm{d}}$ & Table A.4 & Table A.4 & NR & $\begin{array}{l}\text { Distribution from NUREG/CR-6697 } \\
\text { (NRC 2000) }\end{array}$ \\
\hline $\begin{array}{l}\text { Meat transfer factor (concentration in meat, } \\
\mathrm{pCi} / \mathrm{g} / \mathrm{rate} \text { of intake, } \mathrm{pCi} / \mathrm{d} \text { ) }\end{array}$ & NR & Table A.5 & Table A.5 & Table A.5 & $\begin{array}{l}\text { Distribution from NUREG/CR-6697 } \\
\text { (NRC 2000) }\end{array}$ \\
\hline $\begin{array}{l}\text { Milk transfer factor (concentration in milk, } \\
\mathrm{pCi} / \mathrm{L} / \mathrm{rate} \text { of intake, } \mathrm{pCi} / \mathrm{d} \text { ) }\end{array}$ & NR & Table A.6 & Table A.6 & NR & $\begin{array}{l}\text { Distribution from NUREG/CR-6697 } \\
\text { (NRC 2000) }\end{array}$ \\
\hline $\begin{array}{l}\text { Fish transfer factor (concentration in fish, } \\
\mathrm{pCi} / \mathrm{kg} / \text { concentration in water, } \mathrm{pCi} / \mathrm{L} \text { ) }\end{array}$ & NR & Table A.7 & Table A.7 & Table A.7 & $\begin{array}{l}\text { Distribution from NUREG/CR-6697 } \\
\text { (NRC 2000) }\end{array}$ \\
\hline $\begin{array}{l}\text { Crustacea transfer factor (concentration in } \\
\text { crustacea, } \mathrm{pCi} / \mathrm{kg} / \text { concentration in water, } \\
\mathrm{pCi} / \mathrm{L} \text { ) }\end{array}$ & NR & Table A.7 & Table A.7 & Table A.7 & RESRAD default \\
\hline \multicolumn{6}{|l|}{ Contaminated Zone Parameters } \\
\hline Area of contaminated zone $\left(\mathrm{m}^{2}\right)$ & 10,000 & 10,000 & 10,000 & 10,000 & Kamboj 2011; Matton 2011 \\
\hline Thickness of contaminated zone (m) & 1 & 1 & 1 & 1 & Kamboj 2011; Matton 2011 \\
\hline Length parallel to aquifer flow (m) & 100 & 100 & 100 & 100 & RESRAD default \\
\hline $\begin{array}{l}\text { Does the initial contamination penetrate the } \\
\text { water table? }\end{array}$ & No & No & No & No & RESRAD default \\
\hline \multicolumn{6}{|l|}{$\begin{array}{l}\text { Cover and Contaminated Zone Hydrological } \\
\text { Data }\end{array}$} \\
\hline Cover depth $(\mathrm{m})$ & 0 & 0 & 0 & 0 & No cover layer assumed \\
\hline Density of cover material $\left(\mathrm{g} / \mathrm{cm}^{3}\right)$ & NR & NR & NR & NR & $\begin{array}{l}\text { Not required when cover depth } \\
\text { equals zero }\end{array}$ \\
\hline 1.Cover erosion rate $(\mathrm{m} / \mathrm{yr})$ & NR & NR & NR & NR & $\begin{array}{l}\text { Not required when cover depth } \\
\text { equals zero }\end{array}$ \\
\hline
\end{tabular}


TABLE A.1 (Cont.)

\begin{tabular}{|c|c|c|c|c|c|}
\hline \multirow[b]{2}{*}{ Input Parameters } & \multicolumn{2}{|c|}{ Current Use Scenarios } & \multicolumn{2}{|c|}{ Future Use Scenarios } & \multirow[b]{2}{*}{ Comments } \\
\hline & Industrial Worker & Offsite Resident ${ }^{\mathrm{b}}$ & Resident Farmer ${ }^{b}$ & Recreationist $^{\mathrm{c}}$ & \\
\hline Density of primary contaminated zone $\left(\mathrm{g} / \mathrm{cm}^{3}\right)$ & $\begin{array}{l}\mathrm{TN}^{\mathrm{e}, \mathrm{f}}(1.696,0.1855 \\
\quad 0.001,0.999)\end{array}$ & $\begin{array}{c}\mathrm{TN}^{\mathrm{e}, \mathrm{f}}(1.696 \\
0.1855,0.001 \\
0.999)\end{array}$ & $\begin{array}{l}\mathrm{TN}^{\mathrm{e}, \mathrm{f}}(1.696,0.1855 \\
\quad 0.001,0.999)\end{array}$ & $\begin{array}{l}\mathrm{TN}^{\mathrm{e}, \mathrm{f}}(1.696 \\
0.1855,0.001 \\
0.999)\end{array}$ & $\begin{array}{l}\text { Site-specific soil type (silty clay - } \\
\text { Golchert et al. 2010) distribution }\end{array}$ \\
\hline Contaminated zone erosion rate $(\mathrm{m} / \mathrm{yr})$ & $1.65 \mathrm{E}-4$ & $1.65 \mathrm{E}-4^{\mathrm{g}}$ & $1.65 \mathrm{E}-4$ & $1.65 \mathrm{E}-4$ & $\begin{array}{l}\text { To match the soil erosion of } \\
1 \text { ton/acre/yr in Illinois } \\
\text { (NRCS 2010) }\end{array}$ \\
\hline Contaminated zone total porosity & $\begin{array}{c}\operatorname{TN}^{\mathrm{f}}(0.36,0.07 \\
0.001,0.999)\end{array}$ & $\begin{array}{c}\operatorname{TN}^{\mathrm{f}}(0.36,0.07 \\
0.001,0.999)\end{array}$ & $\begin{array}{c}\operatorname{TN}^{\mathrm{f}}(0.36,0.07 \\
0.001,0.999)\end{array}$ & $\begin{array}{c}\operatorname{TN}^{\mathrm{f}}(0.36,0.07 \\
0.001,0.999)\end{array}$ & $\begin{array}{l}\text { Site-specific soil type (silty clay - } \\
\text { Golchert et al. 2010) distribution }\end{array}$ \\
\hline Contaminated zone field capacity & 0.2 & 0.2 & 0.2 & 0.2 & RESRAD default \\
\hline $\begin{array}{l}\text { Contaminated zone hydraulic conductivity } \\
(\mathrm{m} / \mathrm{yr})\end{array}$ & 0.32 & 0.32 & 0.32 & 0.32 & Site-specific (Quinn 2010) \\
\hline Contaminated zone $\mathrm{b}$ parameter & $\begin{array}{c}\operatorname{TLN}^{\mathrm{f}}(2.29 \\
0.259,0.001,0.999)\end{array}$ & $\begin{array}{c}\operatorname{TLN}^{\mathrm{f}}(2.29 \\
0.259,0.001,0.999)\end{array}$ & $\begin{array}{c}\operatorname{TLN}^{\mathrm{f}}(2.29 \\
0.259,0.001,0.999)\end{array}$ & $\begin{array}{c}\operatorname{TLN}^{\mathrm{f}}(2.29 \\
0.259,0.001,0.999)\end{array}$ & $\begin{array}{l}\text { Site-specific soil type (silty clay - } \\
\text { Golchert et al. 2010) distribution }\end{array}$ \\
\hline Humidity in air $\left(\mathrm{g} / \mathrm{m}^{3}\right)$ & 8 & 8 & 8 & 8 & Used only for $\mathrm{H}-3$ \\
\hline Evapotranspiration coefficient & $\mathrm{U}(0.5,0.75)$ & $\mathrm{U}(0.5,0.75)$ & $\mathrm{U}(0.5,0.75)$ & $\mathrm{U}(0.5,0.75)$ & $\begin{array}{l}\text { Distribution from NUREG/CR-6697 } \\
\text { (NRC 2000) }\end{array}$ \\
\hline Wind speed $(\mathrm{m} / \mathrm{s})$ & 4.6 & NR & 4.6 & 4.6 & $\begin{array}{l}\text { Site-specific value for Chicago } \\
\text { (NCDC 2010) }\end{array}$ \\
\hline Precipitation rate $(\mathrm{m} / \mathrm{yr})$ & 0.9177 & 0.9177 & 0.9177 & 0.9177 & $\begin{array}{l}\text { Site-specific value from Golchert } \\
\text { et al. } 2010\end{array}$ \\
\hline Irrigation rate $(\mathrm{m} / \mathrm{yr})$ & 0.0 & 0.1 & 0.1 & 0.0 & Site-specific (Argonne 1996) \\
\hline Irrigation mode (overhead/ditch) & NR & NR & Overhead & NR & RESRAD default \\
\hline Runoff coefficient & 0.2 & 0.2 & 0.2 & 0.2 & $\begin{array}{l}\text { Site-specific for woodland (Yu et al. } \\
\text { 2001, page E-7) }\end{array}$ \\
\hline Watershed area for nearby stream or pond $\left(\mathrm{m}^{2}\right)$ & $1,000,000$ & NR & $1,000,000$ & $1,000,000$ & RESRAD default \\
\hline Accuracy for water/soil computation & 0.001 & NR & 0.001 & 0.001 & RESRAD default \\
\hline \multicolumn{6}{|l|}{ Saturated Zone Hydrological Data } \\
\hline Density of saturated zone $\left(\mathrm{g} / \mathrm{cm}^{3}\right)$ & NR & $\begin{array}{c}\mathrm{TN}(1.52,0.230 \\
0.001,0.999)\end{array}$ & $\begin{array}{c}\mathrm{TN}(1.52,0.230 \\
0.001,0.999)\end{array}$ & $\begin{array}{c}\mathrm{TN}(1.52,0.230 \\
0.001,0.999)\end{array}$ & Generic soil type distribution \\
\hline Saturated zone effective porosity & NR & $\begin{array}{c}\mathrm{TN}(0.355,0.0906 \\
0.001,0.999)\end{array}$ & $\begin{array}{l}\mathrm{TN}(0.355,0.0906 \\
0.001,0.999)\end{array}$ & $\begin{array}{c}\mathrm{TN}(0.355,0.0906 \\
0.001,0.999)\end{array}$ & Generic soil type distribution \\
\hline Saturated zone total porosity & NR & $\begin{array}{l}\mathrm{TN}(0.425,0.0867 \\
0.001,0.999)\end{array}$ & $\begin{array}{c}\mathrm{TN}(0.425,0.0867 \\
0.001,0.999)\end{array}$ & $\begin{array}{c}\mathrm{TN}(0.425,0.0867 \\
0.001,0.999)\end{array}$ & Generic soil type distribution \\
\hline
\end{tabular}


TABLE A.1 (Cont.)

\begin{tabular}{|c|c|c|c|c|c|}
\hline \multirow[b]{2}{*}{ Input Parameters } & \multicolumn{2}{|c|}{ Current Use Scenarios } & \multicolumn{2}{|c|}{ Future Use Scenarios } & \multirow[b]{2}{*}{ Comments } \\
\hline & Industrial Worker & Offsite Resident ${ }^{\mathrm{b}}$ & Resident Farmer ${ }^{\mathrm{b}}$ & Recreationist ${ }^{\mathrm{c}}$ & \\
\hline Saturated zone field capacity & NR & 0.2 & 0.2 & 0.2 & RESRAD default \\
\hline Saturated zone hydraulic conductivity (m/yr) & NR & 947 & 947 & 947 & Site-specific (Argonne 1996) \\
\hline Saturated zone $\mathrm{b}$ parameter & NR & NR & $\begin{array}{c}\operatorname{BLN}(1.06,0.66,0.5 \\
30)\end{array}$ & $\begin{array}{l}\operatorname{BLN}(1.06,0.66 \\
0.5,30)\end{array}$ & Generic soil type distribution \\
\hline Saturated zone hydraulic gradient & NR & $\mathrm{U}(0.007,0.023)$ & $\mathrm{U}(0.007,0.023)$ & $\mathrm{U}(0.007,0.023)$ & Site-specific (Patton et al. 1990) \\
\hline Water table drop rate $(\mathrm{m} / \mathrm{yr})$ & NR & NR & 0.001 & 0.001 & RESRAD default \\
\hline Well pump intake depth (m below water table) & NR & NR & 5 & 5 & Site-specific \\
\hline $\begin{array}{l}\text { Model for water transportation } \\
\text { (nondispersion/mass-balance) }\end{array}$ & NR & NR & Nondispersion & Nondispersion & RESRAD default \\
\hline Well pumping rate $\left(\mathrm{m}^{3} / \mathrm{yr}\right)$ & NR & NR & 250 & 250 & RESRAD default \\
\hline \multicolumn{6}{|l|}{ Unsaturated zone parameters } \\
\hline Thickness of unsaturated zone (m) & NR & 13.9 & 13.9 & 13.9 & $\begin{array}{l}\text { Site-specific }(48 \mathrm{ft}-1 \mathrm{~m}) \text { (Quinn } \\
\text { 2010) }\end{array}$ \\
\hline Density of unsaturated zone $\left(\mathrm{g} / \mathrm{cm}^{3}\right)$ & NR & $\begin{array}{l}\operatorname{TN}^{\mathrm{f}}(1.696,0.1855 \\
0.001,0.999)\end{array}$ & $\begin{array}{l}\mathrm{TN}^{\mathrm{f}}(1.696,0.1855 \\
0.001,0.999)\end{array}$ & $\begin{array}{l}\operatorname{TN}^{\mathrm{f}}(1.696,0.1855 \\
0.001,0.999)\end{array}$ & $\begin{array}{l}\text { Site-specific soil type (silty clay) } \\
\text { distribution }\end{array}$ \\
\hline Unsaturated zone effective porosity & NR & $\begin{array}{l}\mathrm{TN}^{\mathrm{f}}(0.289,0.0735 \\
0.001,0.999)\end{array}$ & $\begin{array}{l}\mathrm{TN}^{\mathrm{f}}(0.289,0.0735 \\
0.001,0.999)\end{array}$ & $\begin{array}{l}\mathrm{TN}^{\mathrm{f}}(0.289,0.0735 \\
0.001,0.999)\end{array}$ & $\begin{array}{l}\text { Site-specific soil type (silty clay) } \\
\text { distribution }\end{array}$ \\
\hline Unsaturated zone total porosity & NR & $\begin{array}{c}\mathrm{TN}^{\mathrm{f}}(0.36,0.07 \\
0.001,0.999)\end{array}$ & $\begin{array}{c}\mathrm{TN}^{\mathrm{f}}(0.36,0.07 \\
0.001,0.999)\end{array}$ & $\begin{array}{c}\mathrm{TN}^{\mathrm{f}}(0.36,0.07 \\
0.001,0.999)\end{array}$ & $\begin{array}{l}\text { Site-specific soil type (silty clay) } \\
\text { distribution }\end{array}$ \\
\hline Unsaturated zone field capacity & NR & 0.2 & 0.2 & 0.2 & RESRAD default \\
\hline Unsaturated zone hydraulic conductivity $(\mathrm{m} / \mathrm{yr})$ & NR & 0.32 & 0.32 & 0.32 & Site-specific (Quinn 2010) \\
\hline Unsaturated zone $\mathrm{b}$ parameter & NR & $\begin{array}{l}\operatorname{TLN}^{\mathrm{f}}(2.29,0.259 \\
0.001,0.999)\end{array}$ & $\begin{array}{l}\operatorname{TLN}^{\mathrm{f}}(2.29,0.259 \\
0.001,0.999)\end{array}$ & $\begin{array}{l}\operatorname{TLN}^{\mathrm{f}}(2.29,0.259 \\
0.001,0.999)\end{array}$ & $\begin{array}{l}\text { Site-specific soil type (silty clay) } \\
\text { distribution }\end{array}$ \\
\hline \multicolumn{6}{|l|}{$\begin{array}{l}\text { Occupancy, Inhalation, and External } \\
\text { Gamma Parameters }\end{array}$} \\
\hline Inhalation rate $\left(\mathrm{m}^{3} / \mathrm{yr}\right)$ & 11,400 & 8400 & 8400 & 14,000 & Scenario-specific \\
\hline Mass loading for inhalation $\left(\mathrm{g} / \mathrm{m}^{3}\right)$ & Empirical & Empirical & Empirical & Empirical & NRC 2000 \\
\hline Exposure duration (yr) & 25 & 30 & 30 & 30 & Not used in DSR calculations \\
\hline Indoor dust filtration factor & $\mathrm{U}(0.15,0.95)$ & $\mathrm{U}(0.15,0.95)$ & $\mathrm{U}(0.15,0.95)$ & $\mathrm{U}(0.15,0.95)$ & Distribution from NRC 2000 \\
\hline External gamma shielding factor & NR & $\begin{array}{l}\operatorname{BLN}(-1.3,0.59 \\
0.044,1)\end{array}$ & $\begin{array}{l}\operatorname{BLN}(-1.3,0.59 \\
0.044,1)\end{array}$ & NR & Distribution from NRC 2000 \\
\hline
\end{tabular}


TABLE A.1 (Cont.)

\begin{tabular}{|c|c|c|c|c|c|}
\hline \multirow[b]{2}{*}{ Input Parameters } & \multicolumn{2}{|c|}{ Current Use Scenarios } & \multicolumn{2}{|c|}{ Future Use Scenarios } & \multirow[b]{2}{*}{ Comments } \\
\hline & Industrial Worker & Offsite Resident ${ }^{\mathrm{b}}$ & Resident Farmer $^{\text {b }}$ & Recreationist $^{\mathrm{c}}$ & \\
\hline Indoor time fraction & 0/empirical & NR & 0.6833 & 0 & $\begin{array}{l}\text { Scenario-specific } \\
\text { (U.S. Environmental Protection } \\
\text { Agency [EPA] Exposure Factors } \\
\text { Handbook; for U-234 in the } \\
\text { industrial scenario, empirical data } \\
\text { for radon were used) }\end{array}$ \\
\hline Outdoor time fraction & Empirical/0 & NR & 0.0833 & 0.0384 & $\begin{array}{l}\text { Scenario-specific (EPA exposure } \\
\text { factor) }\end{array}$ \\
\hline $\begin{array}{l}\text { Shape of contaminated zone } \\
\text { (circular/noncircular) }\end{array}$ & Circular & Circular & Circular & Circular & RESRAD default \\
\hline \multicolumn{6}{|l|}{ Ingestion Pathway Dietary Data } \\
\hline Fruit, vegetable and grain consumption ( $\mathrm{kg} / \mathrm{yr})$ & NR & 160 & 160 & NR & Distribution from NRC 2000 \\
\hline Leafy vegetable consumption (kg/yr) & NR & 14 & 14 & NR & Distribution from NRC 2000 \\
\hline Milk consumption (L/yr) & NR & 92 & 92 & NR & Distribution from NRC 2000 \\
\hline Meat and poultry consumption (kg/yr) & NR & 63 & 63 & $\mathrm{~T}(7.0,19.0,61)$ & Scenario-specific \\
\hline Fish consumption $(\mathrm{kg} / \mathrm{yr})$ & NR & 5.4 & 5.4 & $\mathrm{~T}(4.7,12,57)$ & Scenario-specific \\
\hline Other sea food consumption ( $\mathrm{kg} / \mathrm{yr})$ & NR & 0.9 & 0.9 & NR & RESRAD default \\
\hline Soil ingestion $(\mathrm{g} / \mathrm{yr})$ & $\mathrm{T}(0,18.3,36.5)$ & $\mathrm{T}(0,18.3,36.5)$ & $\mathrm{T}(0,18.3,36.5)$ & $\mathrm{T}(0,18.3,36.5)$ & Distribution from NRC 2000 \\
\hline Drinking water intake (L/yr) & NR & 510 & 510 & $1.4 \mathrm{~L} / \mathrm{d}^{\mathrm{g}} 14=19.6 \mathrm{~L}$ & $\begin{array}{l}\text { Distribution from NRC } 2000 \text { and } \\
\text { scenario-specific for recreation use }\end{array}$ \\
\hline Drinking water contaminated fraction & NR & 0 & 1 & 1 & $\begin{array}{l}\text { RESRAD default and for offsite } \\
\text { receptor scenario, water is not } \\
\text { contaminated }\end{array}$ \\
\hline Household water contaminated fraction & 1 & 1 & 1 & NR & RESRAD default \\
\hline Livestock water contaminated fraction & NR & 1 & 1 & 1 & RESRAD default \\
\hline Irrigation water contaminated fraction & NR & 1 & 1 & 1 & RESRAD default \\
\hline Aquatic food contaminated fraction & NR & 0.5 & 0.5 & 0.5 & Distribution from NRC 2000 \\
\hline Plant food contaminated fraction & NR & -1 & -1 & NR & $\begin{array}{l}\text { Calculated by RESRAD from area } \\
\text { factor }\end{array}$ \\
\hline Meat contaminated fraction & NR & -1 & -1 & 1 & Scenario-specific \\
\hline Milk contaminated fraction & NR & -1 & -1 & NR & $\begin{array}{l}\text { Calculated by RESRAD from area } \\
\text { factor }\end{array}$ \\
\hline
\end{tabular}


TABLE A.1 (Cont.)

\begin{tabular}{|c|c|c|c|c|c|}
\hline \multirow[b]{2}{*}{ Input Parameters } & \multicolumn{2}{|c|}{ Current Use Scenarios } & \multicolumn{2}{|c|}{ Future Use Scenarios } & \multirow[b]{2}{*}{ Comments } \\
\hline & Industrial Worker & Offsite Resident ${ }^{\mathrm{b}}$ & Resident Farmer ${ }^{\mathrm{b}}$ & Recreationist $^{\mathrm{c}}$ & \\
\hline \multicolumn{6}{|l|}{ Ingestion Pathway, Nondietary Data } \\
\hline Livestock fodder intake for meat $(\mathrm{kg} / \mathrm{d})$ & NR & NR & 68 & 10.6 & $\begin{array}{l}\text { RESRAD default or scenario- } \\
\text { specific }\end{array}$ \\
\hline Livestock fodder intake for milk $(\mathrm{kg} / \mathrm{d})$ & NR & NR & 55 & NR & RESRAD default \\
\hline Livestock water intake for meat $(\mathrm{L} / \mathrm{d})$ & NR & 50 & 50 & 6.4 & $\begin{array}{l}\text { RESRAD default or scenario- } \\
\text { specific }\end{array}$ \\
\hline Livestock water intake for milk (L/d) & NR & 160 & 160 & NR & RESRAD default \\
\hline Livestock intake of soil for meat $(\mathrm{kg} / \mathrm{d})$ & NR & NR & 0.5 & 0.5 & RESRAD default \\
\hline Livestock intake of soil for milk (kg/d) & NR & NR & 0.5 & NR & RESRAD default \\
\hline Mass loading for foliar deposition $\left(\mathrm{g} / \mathrm{m}^{3}\right)$ & $\mathrm{NR}$ & NR & 0.0001 & 0.0001 & RESRAD default \\
\hline Depth of soil mixing layer $(\mathrm{m})$ & $\mathrm{T}(0.0,0.15,0.6)$ & $\mathrm{T}(0.0,0.15,0.6)$ & $\mathrm{T}(0.0,0.15,0.6)$ & $\mathrm{T}(0.0,0.15,0.6)$ & Distribution from NRC 2000 \\
\hline Depth of roots $(\mathrm{m})$ & NR & $\mathrm{U}(0.3,1.0)$ & $\mathrm{U}(0.3,1.0)$ & $\mathrm{U}(0.3,1.0)$ & $\begin{array}{l}\text { Upper bound equal to depth of } \\
\text { contamination }\end{array}$ \\
\hline $\begin{array}{l}\text { Drinking water fraction from groundwater } \\
\text { source }\end{array}$ & NR & 0 & 1 & 0 & Scenario-specific \\
\hline $\begin{array}{l}\text { Household water fraction from groundwater } \\
\text { source }\end{array}$ & NR & 0 & 1 & NR & Scenario-specific \\
\hline $\begin{array}{l}\text { Livestock water fraction from groundwater } \\
\text { source }\end{array}$ & NR & 0 & 1 & 0 & $\begin{array}{l}\text { RESRAD default for resident farmer } \\
\text { scenario and } 0 \text { for recreational use } \\
\text { scenario because surface water is } \\
\text { used by livestock }\end{array}$ \\
\hline $\begin{array}{l}\text { Irrigation water fraction from groundwater } \\
\text { source }\end{array}$ & NR & 0 & 1 & 0 & RESRAD default \\
\hline \multicolumn{6}{|l|}{ Plant Factors } \\
\hline $\begin{array}{l}\text { Wet weight crop yield for nonleafy vegetables } \\
\left(\mathrm{kg} / \mathrm{m}^{2}\right)\end{array}$ & NR & $\begin{array}{l}\operatorname{TLN}(0.56,0.48 \\
0.001,0.999)\end{array}$ & $\begin{array}{l}\operatorname{TLN}(0.56,0.48 \\
0.001,0.999)\end{array}$ & NR & Distribution from NRC 2000 \\
\hline $\begin{array}{l}\text { Length of growing season for nonleafy } \\
\text { vegetables }(\mathrm{yr})\end{array}$ & NR & 0.17 & 0.17 & NR & RESRAD default \\
\hline Translocation factor for nonleafy vegetables & NR & 0.1 & 0.1 & NR & RESRAD default \\
\hline Weathering removal constant $(1 / \mathrm{yr})$ & NR & $\mathrm{T}(5.1,18,84)$ & $\mathrm{T}(5.1,18,84)$ & $\mathrm{T}(5.1,18,84)$ & Distribution from NRC 2000 \\
\hline $\begin{array}{l}\text { Wet foliar interception fraction for nonleafy } \\
\text { vegetables }\end{array}$ & NR & 0.25 & 0.25 & NR & RESRAD default \\
\hline
\end{tabular}


TABLE A.1 (Cont.)

\begin{tabular}{|c|c|c|c|c|c|}
\hline \multirow[b]{2}{*}{ Input Parameters } & \multicolumn{2}{|c|}{ Current Use Scenarios } & \multicolumn{2}{|c|}{ Future Use Scenarios } & \multirow[b]{2}{*}{ Comments } \\
\hline & Industrial Worker & Offsite Resident ${ }^{b}$ & Resident Farmer ${ }^{\mathrm{b}}$ & Recreationist $^{\mathrm{c}}$ & \\
\hline $\begin{array}{l}\text { Dry foliar interception fraction for nonleafy } \\
\text { vegetables }\end{array}$ & NR & 0.25 & 0.25 & NR & RESRAD default \\
\hline $\begin{array}{l}\text { Wet weight crop yield for leafy vegetables } \\
\left(\mathrm{kg} / \mathrm{m}^{2}\right)\end{array}$ & NR & 1.5 & 1.5 & NR & RESRAD default \\
\hline $\begin{array}{l}\text { Length of growing season for leafy vegetables } \\
\text { (yr) }\end{array}$ & NR & 0.25 & 0.25 & NR & RESRAD default \\
\hline Translocation factor for leafy vegetables & NR & 1 & 1 & NR & RESRAD default \\
\hline $\begin{array}{l}\text { Wet foliar interception fraction for leafy } \\
\text { vegetables }\end{array}$ & NR & $\mathrm{T}(0.06,0.67,0.95)$ & $\mathrm{T}(0.06,0.67,0.95)$ & NR & Distribution from NRC 2000 \\
\hline $\begin{array}{l}\text { Dry foliar interception fraction for leafy } \\
\text { vegetables }\end{array}$ & NR & 0.25 & 0.25 & NR & RESRAD default \\
\hline Wet weight crop yield for fodder $\left(\mathrm{kg} / \mathrm{m}^{2}\right)$ & NR & NR & 1.1 & 1.1 & RESRAD default \\
\hline Length of growing season for fodder (yr) & NR & NR & 0.08 & 0.08 & RESRAD default \\
\hline Translocation factor for fodder & NR & NR & 1 & 1 & RESRAD default \\
\hline Wet foliar interception fraction for fodder & NR & NR & 0.25 & 0.25 & RESRAD default \\
\hline Dry foliar interception fraction for fodder & NR & NR & 0.25 & 0.25 & RESRAD default \\
\hline \multicolumn{6}{|l|}{ Storage-Times-Before-Use Data } \\
\hline $\begin{array}{l}\text { Storage time for fruits, nonleafy vegetables and } \\
\text { grain (d) }\end{array}$ & NR & 14 & 14 & NR & RESRAD default \\
\hline Storage time for leafy vegetables $(\mathrm{d})$ & NR & 1 & 1 & NR & RESRAD default \\
\hline Storage time for milk (d) & NR & 1 & 1 & NR & RESRAD default \\
\hline Storage time for meat (d) & NR & NR & 20 & 182.5 & $\begin{array}{l}\text { RESRAD default for resident farmer } \\
\text { and scenario-specific for } \\
\text { recreational use }\end{array}$ \\
\hline Storage time for fish (d) & NR & 7 & 7 & 0 & $\begin{array}{l}\text { RESRAD default and scenario- } \\
\text { specific for recreational use }\end{array}$ \\
\hline Storage time for crustacea and mollusks (d) & NR & 7 & 7 & 0 & $\begin{array}{l}\text { RESRAD default and scenario- } \\
\text { specific for recreational use }\end{array}$ \\
\hline Storage time for well water (d) & NR & 1 & 1 & NR & $\begin{array}{l}\text { RESRAD default for resident farmer } \\
\text { and not required for recreational } \\
\text { use }\end{array}$ \\
\hline Storage time for surface water $(\mathrm{d})$ & NR & 1 & 1 & 0 & $\begin{array}{l}\text { RESRAD default for resident farmer } \\
\text { and } 0 \text { for recreational use }\end{array}$ \\
\hline
\end{tabular}


TABLE A.1 (Cont.)

Current Use Scenarios Input Parameters
Industrial Worker

Offsite Res
Storage time for livestock fodder (d)

\section{Radon Data}

Cover total porosity

Cover volumetric water content

Cover radon diffusion coefficient

Total porosity of the house or building foundation

Volumetric water content of the foundation

Diffusion coefficient for radon gas in

foundation material $\left(\mathrm{m}^{2} / \mathrm{s}\right)$

A Diffusion coefficient for radon gas in

contaminated zone soil $\left(\mathrm{m}^{2} / \mathrm{s}\right)$

Emanating power of radon-222

Radon vertical dimension of mixing (m)

Average building air exchange rate $\left(\mathrm{h}^{-1}\right)$

Height of building (room) (m)

Building indoor area factor

Bulk density of house or building foundation $\left(\mathrm{g} / \mathrm{cm}^{3}\right)$

Thickness of house or building foundation (m)

Building depth below ground surface (m)

\section{Carbon-14 Data}

$\mathrm{C}-12$ concentration in local water $\left(\mathrm{g} / \mathrm{cm}^{3}\right)$

C-12 concentration in contaminated soil $(\mathrm{g} / \mathrm{g})$

Fraction of vegetation carbon absorbed from

soil

Fraction of vegetation carbon absorbed from air

Thickness of evasion layer of C-14 in soil (m)

C-14 evasion flux rate from soil $\left(\mathrm{s}^{-1}\right)$

Future Use Scenarios

Resident Farmer ${ }^{\mathrm{b}}$

Recreationist $^{\mathrm{c}}$

Comments

NR

45

NR

NR

NR

NR

0.1

0.03

3E-07

2E-06

0.28

2.0

0.5

2.5

0
2.4

0.15

0.15
-1

$2 \mathrm{E}-5$

0.03

0.02

0.98

0.3

7E-7
NR

NR
NR
NR
0.1

0.03
$3 \mathrm{E}-07$
$2 \mathrm{E}-06$
0.28
2.0
0.5
2.5
0
2.4
0.15
-1

2E-5

0.03

0.02

0.98

0.3

$\begin{array}{ccl}\text { NR } & \text { NR } & \text { No cover } \\ \text { NR } & \text { NR } & \begin{array}{l}\text { No cover } \\ \text { No cover } \\ \text { NR }\end{array} \\ 0.1 & \text { NR } & \text { RESRAD default } \\ & 0.1 & \\ 0.03 & 0.03 & \text { RESRAD default } \\ 3 E-07 & 3 E-07 & \text { RESRAD default } \\ 2 \text { E-06 } & 2 \mathrm{E}-06 & \text { For site-specific soil type } \\ 0.28 & 0.28 & \text { For site-specific soil type } \\ & & \text { (Yu et al. 1993) } \\ 2.0 & 2.0 & \text { RESRAD default } \\ 0.5 & 0.5 & \text { RESRAD default } \\ 2.5 & 2.5 & \text { RESRAD default } \\ 0 & 0 & \text { RESRAD default } \\ 2.4 & 2.4 & \text { RESRAD default } \\ 0.15 & 0.15 & \text { RESRAD default } \\ -1 & -1 & \text { RESRAD default }\end{array}$

2E-5 2E-5 RESRAD default

$0.03 \quad 0.03 \quad$ RESRAD default

$0.02 \quad 0.02 \quad$ RESRAD default

$0.98 \quad 0.98 \quad$ RESRAD default

$0.3 \quad 0.3 \quad$ RESRAD default and scenario-specific for

recreational use 
TABLE A.1 (Cont.)

\begin{tabular}{|c|c|c|c|c|c|}
\hline \multirow[b]{2}{*}{ Input Parameters } & \multicolumn{2}{|c|}{ Current Use Scenarios } & \multicolumn{2}{|c|}{ Future Use Scenarios } & \multirow[b]{2}{*}{ Comment } \\
\hline & Industrial Worker & Offsite Resident ${ }^{\mathrm{b}}$ & Resident Farmer ${ }^{\mathrm{b}}$ & Recreationist $\mathrm{c}^{\mathrm{c}}$ & \\
\hline C-12 evasion flux rate from soil $\left(\mathrm{s}^{-1}\right)$ & $1 \mathrm{E}-10$ & $1 \mathrm{E}-10$ & $1 \mathrm{E}-10$ & $1 \mathrm{E}-10$ & RESRAD default \\
\hline Grain fraction in beef cattle feed & 0.8 & 0.8 & 0.8 & 0.8 & RESRAD default \\
\hline Grain fraction in milk cow feed & 0.2 & 0.2 & 0.2 & 0.2 & RESRAD default \\
\hline
\end{tabular}

a RESRAD-OFFSITE code is used for analyzing offsite resident scenario, and RESRAD code is used for other scenarios. Extra parameters required for RESRAD-OFFSITE code are listed in Table A.10.

b For offsite resident and residential farmer scenarios, the parameter values for leafy vegetable consumption, meat and poultry consumption, and fish consumption are RESRAD defaults. Parameter values for inhalation rate, indoor and outdoor time fractions, livestock fodder intake for meat, livestock water intake for meat, livestock intake of soil, plant food contaminated fraction, and meat contaminated fractions are RESRAD defaults and are calculated on the basis of the contaminated area; storage times for meat, fish, surface water, and livestock fodder are RESRAD defaults.

c For the recreational use scenario, it is assumed that the individual would be involved in moderate activity onsite; therefore, an inhalation rate of moderate activity ( $\left.1.6 \mathrm{~m}^{3} / \mathrm{h}\right)$ is assumed (EPA 1997). The parameter value for meat and poultry consumption is based on the assumption that a recreationist kills a deer and brings the catch home and uses its meat over the course of a year; the distribution is based on the game consumption statistics among households that hunt. For fish consumption, it is assumed that a recreationist catches a fish and consumes it the same day; distribution is based on the home-caught fish consumption statistics among households that fish. The parameter values for indoor and outdoor time fractions are from the scenario description. All meat consumed is assumed to be contaminated. For the storage time of meat, since the meat is consumed over the course of a year, an average storage time of 182.5 days is assumed. For the storage time of fish, since the fish is consumed on the same day, a storage time of zero days is assumed. Since the game animal uses fresh forage and surface water, a storage time of zero days is assumed for both parameters.

d NR - value is not required for this scenario.

e See Table A.8 for the notation used for the type of distribution.

f It is assumed that the soil type is silty clay at the Building 310 area (Golchert et al. 2010), and distributions representative of silty clay soil are used.

$\mathrm{g}$ Cover and management are changed to 0.0506 to get this erosion rate in the RESRAD-OFFSITE code. 
TABLE A.2 Parameter Distributions Used in the Probabilistic Analysis for the Contaminated Zone and Unsaturated Zone Kd Values

\begin{tabular}{cccc}
\hline & \multicolumn{3}{c}{$\mathrm{Kd}\left(\mathrm{cm}^{3} / \mathrm{g}\right)$ Distribution ${ }^{\mathrm{a}}$} \\
\cline { 2 - 4 } & \multicolumn{3}{c}{ Lognormal Distribution } \\
\multicolumn{3}{c}{ Parameters } \\
\cline { 2 - 4 } Element & $\mu$ & $\sigma$ & $\exp (\mu)$ \\
\hline & & & \\
$\mathrm{Ac}$ & 7.8 & $3.22^{\mathrm{b}}$ & 2,400 \\
$\mathrm{Am}$ & 9.0 & 2.6 & 8,400 \\
$\mathrm{C}$ & 0.8 & $0.8^{\mathrm{c}}$ & 1 \\
$\mathrm{Co}$ & 6.3 & 1.8 & 550 \\
$\mathrm{Cs}$ & 7.5 & 1.6 & 1,900 \\
$\mathrm{I}$ & 0.5 & 1.5 & 1 \\
$\mathrm{~Np}$ & 4.0 & 3.8 & 55 \\
$\mathrm{~Pa}$ & 7.9 & $3.22^{\mathrm{b}}$ & 2,700 \\
$\mathrm{~Pb}$ & 6.3 & $2.76^{\mathrm{b}}$ & 550 \\
$\mathrm{Po}$ & 8.0 & $1.68^{\mathrm{b}}$ & 3,000 \\
$\mathrm{Pu}$ & 8.5 & 2.1 & 5,100 \\
$\mathrm{Ra}$ & 9.1 & 1.3 & 9,100 \\
$\mathrm{Sr}$ & 4.7 & 2.0 & 110 \\
$\mathrm{Tc}$ & 0.2 & 0.06 & 1 \\
$\mathrm{Th}$ & 8.6 & 2.6 & 5,800 \\
$\mathrm{U}$ & 7.3 & 2.9 & 1,600 \\
\hline
\end{tabular}

Source: Sheppard and Thibault (1990) unless noted otherwise

a The distributions are for clayey soil, which is the site-specific soil type for the contaminated zone and unsaturated zone.

b Value not available from Sheppard and Thibault (1990). The value used is default for generic soil type.

c Value not available from Sheppard and Thibault (1990). The value used is default for sandy soil type. 
TABLE A.3 Parameter Distributions Used in the Probabilistic Analysis for the Saturated Zone Kd Values

\begin{tabular}{cccc}
\hline & \multicolumn{3}{c}{$\mathrm{Kd}\left(\mathrm{cm}^{3} / \mathrm{g}\right)$ Distribution ${ }^{\mathrm{a}}$} \\
\cline { 2 - 4 } & \multicolumn{3}{c}{ Lognormal Distribution } \\
\multicolumn{3}{c}{ Parameters } \\
\cline { 2 - 4 } Element & $\mu$ & $\sigma$ & $\exp (\mu)$ \\
\hline & \multicolumn{3}{c}{} \\
$\mathrm{Ac}$ & 6.1 & $3.22^{\mathrm{b}}$ & 450 \\
$\mathrm{Am}$ & 7.6 & 2.6 & 1,900 \\
$\mathrm{C}$ & 1.1 & 0.8 & 5 \\
$\mathrm{Co}$ & 4.1 & 2.8 & 60 \\
$\mathrm{Cs}$ & 5.6 & 2.5 & 280 \\
$\mathrm{I}$ & 0.04 & 2.2 & 1 \\
$\mathrm{~Np}$ & 1.4 & 1.7 & 5 \\
$\mathrm{~Pa}$ & 6.3 & $3.22^{\mathrm{b}}$ & 550 \\
$\mathrm{~Pb}$ & 5.6 & $2.76^{\mathrm{b}}$ & 270 \\
$\mathrm{Po}$ & 5.0 & 1.6 & 150 \\
$\mathrm{Pu}$ & 6.3 & 1.7 & 550 \\
$\mathrm{Ra}$ & 6.2 & 3.2 & 500 \\
$\mathrm{Sr}$ & 2.6 & 1.6 & 15 \\
$\mathrm{Tc}$ & -2.0 & 1.8 & 0.1 \\
$\mathrm{Th}$ & 8.0 & 2.1 & 3,200 \\
$\mathrm{U}$ & 3.5 & 3.2 & 35 \\
\hline
\end{tabular}

Source: Sheppard and Thibault (1990) unless noted otherwise

a The distributions are for sandy soil, which is assumed to be the soil type for the saturated zone.

b Value not available from Sheppard and Thibault (1990). The value used is default for generic soil type. 
TABLE A.4 Parameter Distributions Used in the Probabilistic Analysis for the Plant Transfer Factor

\begin{tabular}{|c|c|c|c|}
\hline \multirow[b]{3}{*}{ Element } & \multicolumn{3}{|c|}{$\begin{array}{l}\text { Plant Transfer Factor (wet plant } \\
\text { concentration, } \mathrm{pCi} / \mathrm{g} / \text { dry soil } \\
\text { concentration, } \mathrm{pCi} / \mathrm{g} \text { ) Distribution }\end{array}$} \\
\hline & \multicolumn{3}{|c|}{$\begin{array}{c}\text { Lognormal Distribution } \\
\text { Parameters }\end{array}$} \\
\hline & $\mu$ & $\sigma$ & $\exp (\mu)$ \\
\hline $\mathrm{Ac}$ & -6.91 & 1.1 & $1.0 \times 10^{-3}$ \\
\hline $\mathrm{Am}$ & -6.91 & 0.9 & $1.0 \times 10^{-3}$ \\
\hline $\mathrm{C}$ & -0.36 & 0.9 & $7.0 \times 10^{-1}$ \\
\hline Co & -2.53 & 0.9 & $8.0 \times 10^{-2}$ \\
\hline Cs & -3.22 & 1.0 & $4.0 \times 10^{-2}$ \\
\hline I & -3.91 & 0.9 & $2.0 \times 10^{-2}$ \\
\hline $\mathrm{Np}$ & -3.91 & 0.9 & $2.0 \times 10^{-2}$ \\
\hline $\mathrm{Pa}$ & -4.61 & 1.1 & $1.0 \times 10^{-2}$ \\
\hline $\mathrm{Pb}$ & -5.52 & 0.9 & $4.0 \times 10^{-3}$ \\
\hline Po & -6.9 & 0.9 & $1.0 \times 10^{-3}$ \\
\hline $\mathrm{Pu}$ & -6.91 & 0.9 & $1.0 \times 10^{-3}$ \\
\hline $\mathrm{Ra}$ & -3.22 & 0.9 & $4.0 \times 10^{-2}$ \\
\hline $\mathrm{Sr}$ & -1.20 & 1.0 & $3.0 \times 10^{-1}$ \\
\hline $\mathrm{Tc}$ & 1.61 & 0.9 & $5.0 \times 10^{0}$ \\
\hline Th & -6.91 & 0.9 & $1.0 \times 10^{-3}$ \\
\hline $\mathrm{U}$ & -6.21 & 0.9 & $2.0 \times 10^{-3}$ \\
\hline
\end{tabular}

Source: NRC (2000) 
TABLE A.5 Parameter Distributions Used in the Probabilistic Analysis for the Meat Transfer Factor

\begin{tabular}{|c|c|c|c|}
\hline \multirow[b]{3}{*}{ Element } & \multicolumn{3}{|c|}{$\begin{array}{c}\text { Meat Transfer Factor } \\
\text { (concentration in meat, pCi/g/rate } \\
\text { of intake, } \mathrm{pCi} / \mathrm{d} \text { ) Distribution }\end{array}$} \\
\hline & \multicolumn{3}{|c|}{$\begin{array}{c}\text { Lognormal Distribution } \\
\text { Parameters }\end{array}$} \\
\hline & $\mu$ & $\sigma$ & $\exp (\mu)$ \\
\hline $\mathrm{Ac}$ & -10.82 & 1.0 & $1.0 \times 10^{-5}$ \\
\hline $\mathrm{Am}$ & -9.90 & 0.2 & $5.0 \times 10^{-5}$ \\
\hline $\mathrm{C}$ & -3.47 & 1 & $3.1 \times 10^{-2}$ \\
\hline Co & -3.51 & 1.0 & $3.0 \times 10^{-2}$ \\
\hline Cs & -3.00 & 0.4 & $5.0 \times 10^{-2}$ \\
\hline I & -3.22 & 0.4 & $1.2 \times 10^{-2}$ \\
\hline $\mathrm{Np}$ & -6.91 & 0.7 & $1.0 \times 10^{-3}$ \\
\hline $\mathrm{Pa}$ & -12.21 & 1.0 & $5.0 \times 10^{-6}$ \\
\hline $\mathrm{Pb}$ & -7.13 & 0.7 & $8.0 \times 10^{-4}$ \\
\hline Po & -5.30 & 0.7 & $5.0 \times 10^{-3}$ \\
\hline $\mathrm{Pu}$ & -9.21 & 0.2 & $1.0 \times 10^{-4}$ \\
\hline $\mathrm{Ra}$ & -6.91 & 0.7 & $1.0 \times 10^{-3}$ \\
\hline $\mathrm{Sr}$ & -4.61 & 0.4 & $1.0 \times 10^{-2}$ \\
\hline Tc & -9.21 & 0.7 & $1.0 \times 10^{-4}$ \\
\hline Th & -9.21 & 1.0 & $1.0 \times 10^{-4}$ \\
\hline $\mathrm{U}$ & -7.13 & 0.7 & $8.0 \times 10^{-4}$ \\
\hline
\end{tabular}

Source: NRC (2000) 
TABLE A.6 Parameter Distributions Used in the Probabilistic Analysis for the Milk Transfer Factor

\begin{tabular}{|c|c|c|c|}
\hline \multirow[b]{3}{*}{ Element } & \multicolumn{3}{|c|}{$\begin{array}{c}\text { Milk Transfer Factor } \\
\text { (concentration in milk, pCi/L/rate } \\
\text { of intake, } \mathrm{pCi} / \mathrm{d} \text { ) Distribution }\end{array}$} \\
\hline & \multicolumn{3}{|c|}{$\begin{array}{c}\text { Lognormal Distribution } \\
\text { Parameters }\end{array}$} \\
\hline & $\mu$ & $\sigma$ & $\exp (\mu)$ \\
\hline $\mathrm{Ac}$ & -13.12 & 0.9 & $2.0 \times 10^{-6}$ \\
\hline $\mathrm{Am}$ & -13.12 & 0.7 & $2.0 \times 10^{-6}$ \\
\hline $\mathrm{C}$ & -4.4 & 0.9 & $1.2 \times 10^{-2}$ \\
\hline $\mathrm{Co}$ & -6.21 & 0.7 & $2.0 \times 10^{-3}$ \\
\hline Cs & -4.61 & 0.5 & $1.0 \times 10^{-2}$ \\
\hline I & -4.61 & 0.5 & $1.0 \times 10^{-2}$ \\
\hline $\mathrm{Np}$ & -11.51 & 0.7 & $1.0 \times 10^{-5}$ \\
\hline $\mathrm{Pa}$ & -12.21 & 0.9 & $5.0 \times 10^{-6}$ \\
\hline $\mathrm{Pb}$ & -8.11 & 0.9 & $3.0 \times 10^{-4}$ \\
\hline Po & -7.82 & 0.7 & $4.0 \times 10^{-4}$ \\
\hline $\mathrm{Pu}$ & -13.82 & 0.5 & $1.0 \times 10^{-6}$ \\
\hline $\mathrm{Ra}$ & -6.91 & 0.5 & $1.0 \times 10^{-3}$ \\
\hline $\mathrm{Sr}$ & -6.21 & 0.5 & $2.0 \times 10^{-3}$ \\
\hline $\mathrm{Tc}$ & -6.91 & 0.7 & $1.0 \times 10^{-3}$ \\
\hline Th & -12.21 & 0.9 & $5.0 \times 10^{-6}$ \\
\hline $\mathrm{U}$ & -7.82 & 0.6 & $4.0 \times 10^{-4}$ \\
\hline
\end{tabular}

Source: NRC (2000) 
TABLE A.7 Parameter Values and Distributions Used in the Probabilistic Analysis for the Fish and Crustacea Transfer Factors

\begin{tabular}{|c|c|c|c|c|}
\hline \multirow[b]{3}{*}{ Element } & \multirow{3}{*}{$\begin{array}{c}\text { Crustacea Transfer } \\
\text { Factor (concentration } \\
\text { in crustacea, pCi } / \mathrm{kg} / \\
\text { concentration in water, } \\
\text { pCi } / \mathrm{L} \text { ) }\end{array}$} & \multirow{2}{*}{\multicolumn{3}{|c|}{$\begin{array}{c}\text { Fish Transfer Factor } \\
\text { (concentration in fish, } \\
\mathrm{pCi} / \mathrm{kg} / \text { concentration in water, } \\
\mathrm{pCi} / \mathrm{L} \text { ) Distributions } \\
\\
\text { Lognormal Distribution } \\
\text { Parameters } \\
\end{array}$}} \\
\hline & & & & \\
\hline & & $\mu$ & $\sigma$ & $\exp (\mu)$ \\
\hline $\mathrm{Ac}$ & 1,000 & 2.7 & 1.1 & 15 \\
\hline Am & 1,000 & 3.4 & 1.1 & 30 \\
\hline $\mathrm{C}$ & 9,100 & 10.8 & 1.1 & 50,000 \\
\hline $\mathrm{Co}$ & 200 & 5.7 & 1.1 & 300 \\
\hline Cs & 100 & 7.6 & 0.7 & 2,000 \\
\hline I & 5 & 3.7 & 1.1 & 40 \\
\hline $\mathrm{Np}$ & 400 & 3.4 & 1.1 & 30 \\
\hline $\mathrm{Pa}$ & 110 & 2.3 & 1.1 & 10 \\
\hline $\mathrm{Pb}$ & 100 & 5.7 & 1.1 & 300 \\
\hline Po & 20,000 & 4.6 & 1.1 & 100 \\
\hline $\mathrm{Pu}$ & 100 & 3.4 & 1.1 & 30 \\
\hline $\mathrm{Ra}$ & 250 & 3.9 & 1.1 & 50 \\
\hline $\mathrm{Sr}$ & 100 & 4.1 & 1.1 & 60 \\
\hline $\mathrm{Tc}$ & 5 & 3.0 & 1.1 & 20 \\
\hline Th & 500 & 4.6 & 1.1 & 100 \\
\hline $\mathrm{U}$ & 60 & 2.3 & 1.1 & 10 \\
\hline
\end{tabular}

Source: NRC (2000) 
TABLE A.8 Notations Used in Input Parameter Table A.1

\begin{tabular}{ll}
\hline \multicolumn{1}{c}{ Notation } & \multicolumn{1}{c}{ Type of Distribution } \\
\hline $\mathrm{TN}(\mu, \sigma, \mathrm{a}, \mathrm{b})^{\mathrm{a}}$ & Truncated normal \\
$\mathrm{TLN}(\mu, \sigma, \mathrm{a}, \mathrm{b})^{\mathrm{b}}$ & Truncated lognormal-N \\
$\mathrm{BLN}(\mu, \sigma, \mathrm{a}, \mathrm{b})^{\mathrm{c}}$ & Bounded lognormal-N \\
$\mathrm{U}(\mathrm{a}, \mathrm{b})^{\mathrm{d}}$ & Uniform \\
$\mathrm{LU}(\mathrm{a}, \mathrm{b})^{\mathrm{e}}$ & Log uniform \\
$\mathrm{T}(\mathrm{a}, \mathrm{c}, \mathrm{b})^{\mathrm{f}}$ & Triangular \\
Continuous linear $^{\mathrm{g}}$ & Empirical \\
${\text { Continuous } \log ^{\mathrm{h}}}^{\mathrm{C}}$ & Empirical \\
\hline
\end{tabular}

a For truncated normal distribution, $\mu$ is mean, $\sigma$ is standard deviation, $a$ is lower quantile, and $b$ is upper quantile of the distribution.

b For truncated lognormal-N distribution, $\mu$ is mean and $\sigma$ is standard deviation of underlying normal distribution; a is lower quantile and $b$ is upper quantile of the distribution.

c For bounded lognormal-N distribution, $\mu$ is mean and $\sigma$ is standard deviation of underlying normal distribution; a is minimum and $b$ is maximum of the distribution.

d For uniform distribution, $a$ and $b$ parameters represent the minimum and maximum values, respectively.

e For log uniform distribution, $a$ and $b$ parameters represent the minimum and maximum values, respectively.

f For triangular distribution, a is minimum, $\mathrm{c}$ is mode, and $b$ is maximum of the distribution.

g The continuous linear distribution is defined by the user for the $\mathrm{N}$ points, and the distribution is assumed to vary linearly between the cumulative distribution functions specified by the user for the $\mathrm{N}$ points.

$\mathrm{h}$ The continuous log distribution is defined by the user for the $\mathrm{N}$ points, and the distribution is assumed to vary logarithmically between the cumulative distribution functions specified by the user for the $\mathrm{N}$ points. 
TABLE A.9 Parameter Correlations for Probabilistic Analysis of Different Scenarios

\begin{tabular}{|c|c|c|c|}
\hline Parameter 1 & Parameter 2 & $\begin{array}{l}\text { Correlation } \\
\text { Coefficient } \\
\end{array}$ & Comments \\
\hline Contaminated zone soil density & $\begin{array}{l}\text { Contaminated zone } \\
\text { total porosity }\end{array}$ & -0.99 & $\begin{array}{l}\text { The two parameters are strongly } \\
\text { negatively correlated }\end{array}$ \\
\hline Unsaturated zone soil density & $\begin{array}{l}\text { Unsaturated zone } \\
\text { total porosity }\end{array}$ & -0.99 & $\begin{array}{l}\text { The two parameters are strongly } \\
\text { negatively correlated }\end{array}$ \\
\hline Unsaturated zone soil density & $\begin{array}{l}\text { Unsaturated zone } \\
\text { effective porosity }\end{array}$ & -0.99 & $\begin{array}{l}\text { The two parameters are strongly } \\
\text { negatively correlated }\end{array}$ \\
\hline Saturated zone soil density & $\begin{array}{l}\text { Saturated zone total } \\
\text { porosity }\end{array}$ & -0.99 & $\begin{array}{l}\text { The two parameters are strongly } \\
\text { negatively correlated }\end{array}$ \\
\hline Saturated zone soil density & $\begin{array}{l}\text { Saturated zone } \\
\text { effective porosity }\end{array}$ & -0.99 & $\begin{array}{l}\text { The two parameters are strongly } \\
\text { negatively correlated }\end{array}$ \\
\hline Unsaturated zone total porosity & $\begin{array}{l}\text { Unsaturated zone } \\
\text { effective porosity }\end{array}$ & 0.99 & $\begin{array}{l}\text { A correlation of } 0.99 \text { provides } \\
\text { satisfactory pairing of sampling data }\end{array}$ \\
\hline Saturated zone total porosity & $\begin{array}{l}\text { Saturated zone } \\
\text { effective porosity }\end{array}$ & 0.99 & $\begin{array}{l}\text { A correlation of } 0.99 \text { provides } \\
\text { satisfactory pairing of sampling data }\end{array}$ \\
\hline $\begin{array}{l}\text { Kd of U-234 in contaminated } \\
\text { zone }\end{array}$ & $\begin{array}{l}\mathrm{Kd} \text { of } \mathrm{U}-238 \text { in } \\
\text { contaminated zone }\end{array}$ & 0.99 & $\begin{array}{l}\text { The two parameters are strongly } \\
\text { correlated. }\end{array}$ \\
\hline $\mathrm{Kd}$ of $\mathrm{U}-234$ in unsaturated zone & $\begin{array}{l}\text { Kd of U-238 in } \\
\text { unsaturated zone }\end{array}$ & 0.99 & $\begin{array}{l}\text { The two parameters are strongly } \\
\text { correlated. }\end{array}$ \\
\hline $\mathrm{Kd}$ of U-234 in saturated zone & $\begin{array}{l}\mathrm{Kd} \text { of } \mathrm{U}-238 \text { in } \\
\text { saturated zone }\end{array}$ & 0.99 & $\begin{array}{l}\text { The two parameters are strongly } \\
\text { correlated. }\end{array}$ \\
\hline
\end{tabular}


TABLE A.10 Additional Input Parameters Used in the RESRAD-OFFSITE Probabilistic Analysis

Parameter

Site Layout ${ }^{\mathrm{a}}$

$\mathrm{X}$ dimension of primary contamination (m)

Y dimension of primary contamination (m)

Smaller X coordinate of the fruit, grain, nonleafy

vegetable plots $(\mathrm{m})$

Larger X coordinate of the fruit, grain, nonleafy vegetable plots $(\mathrm{m})$

Smaller Y coordinate of the fruit, grain, nonleafy vegetable plots $(\mathrm{m})$

Larger Y coordinate of the fruit, grain, nonleafy vegetable plots $(\mathrm{m})$

Smaller X coordinate of the leafy vegetable plots (m)

Larger X coordinate of the leafy vegetable plots (m)

Smaller Y coordinate of the leafy vegetable plots (m)

Larger Y coordinate of the leafy vegetable plots (m)

Smaller X coordinate of the pasture, silage growing area $(\mathrm{m})$

Larger X coordinate of the pasture, silage growing area $(\mathrm{m})$

Smaller Y coordinate of the pasture, silage growing area $(\mathrm{m})$

Larger Y coordinate of the pasture, silage growing area $(\mathrm{m})$

Smaller X coordinate of the grain fields (m)

Larger X coordinate of the grain fields (m)

Smaller Y coordinate of the grain fields (m)

Larger Y coordinate of the grain fields (m)

Smaller X coordinate of the dwelling site (m)

Larger X coordinate of the dwelling site (m)

Smaller Y coordinate of the dwelling site (m)

Larger Y coordinate of the dwelling site (m)

Smaller X coordinate of the surface water body

Larger X coordinate of the surface water body

Value

Comment

100

100

434.4

465.6

434

466

434.4

465.6

468

500

450

550

500

600

500

600

600

700

434.4

465.6

400

432

526.5

826.5
Total contaminated area $=10,000 \mathrm{~m}^{2}$

Closest distance to the site boundary

Assume the vegetable plot is $31.6 \mathrm{~m} \times$ $31.6 \mathrm{~m}$, with an area of $1,000 \mathrm{~m}^{2}$

Closest distance to the site boundary

Assume the vegetable plot is $31.6 \mathrm{~m} \times$ $31.6 \mathrm{~m}$, with an area of $1,000 \mathrm{~m}^{2}$

Closest distance to the site boundary

Assume the vegetable plot is $31.6 \mathrm{~m} \times$ $31.6 \mathrm{~m}$, with an area of $1,000 \mathrm{~m}^{2}$

Leafy vegetable plot is assumed to be next to the nonleafy vegetable plot

Assume the vegetable plot is $31.6 \mathrm{~m} \times$ $31.6 \mathrm{~m}$, with an area of $1,000 \mathrm{~m}^{2}$

Close to the vegetable plots

Assume the livestock feed area is $100 \mathrm{~m} \times 100 \mathrm{~m}$, with an area of $10,000 \mathrm{~m}^{2}$

Close to the vegetable plots

Assume the livestock feed area is $100 \mathrm{~m}$ $\times 100 \mathrm{~m}$, with an area of $10,000 \mathrm{~m}^{2}$

Close to the vegetable plots

Assume the livestock feed area is $100 \mathrm{~m}$ $\times 100 \mathrm{~m}$, with an area of $10,000 \mathrm{~m}^{2}$

Close to the vegetable plots

Assume the livestock feed area is $100 \mathrm{~m}$ $\times 100 \mathrm{~m}$, with an area of $10,000 \mathrm{~m}^{2}$

Closest distance to the site boundary

The dwelling site is assumed to be

$31.6 \mathrm{~m} \times 31.6 \mathrm{~m}$ and have an area of $1,000 \mathrm{~m}^{2}$

Closest distance to the site boundary

The dwelling site is assumed to be $31.6 \mathrm{~m} \times 31.6 \mathrm{~m}$ and have an area of $1,000 \mathrm{~m}^{2}$

The surface water body is assumed to be close to the dwelling site and the vegetable and livestock feed areas

The surface water body is assumed to be close to the dwelling site and the vegetable and livestock feed areas 
TABLE A.10 (Cont.)

\begin{tabular}{|c|c|c|}
\hline Parameter & Value & Comment \\
\hline Smaller Y coordinate of the surface water body & 356.8 & $\begin{array}{l}\text { The surface water body is assumed to } \\
\text { be close to the dwelling site and the } \\
\text { vegetable and livestock feed areas }\end{array}$ \\
\hline Larger Y coordinate of the surface water body & 656.8 & $\begin{array}{l}\text { The surface water body is assumed to } \\
\text { be close to the dwelling site and the } \\
\text { vegetable and livestock feed areas }\end{array}$ \\
\hline \multicolumn{3}{|l|}{ Source Release and Deposition Velocity } \\
\hline Deposition velocity $(\mathrm{m} / \mathrm{s})$ & 0.001 & $\begin{array}{l}\text { For all radionuclides except I-129 } \\
\quad(0.01)\end{array}$ \\
\hline \multicolumn{3}{|l|}{ Distribution Coefficients } \\
\hline Sediment in surface water body $\left(\mathrm{cm}^{3} / \mathrm{g}\right)$ & Table A.2 & \\
\hline Fruit, grain, nonleafy vegetable fields $\left(\mathrm{cm}^{3} / \mathrm{g}\right)$ & Table A. 2 & \\
\hline Leafy vegetable fields $\left(\mathrm{cm}^{3} / \mathrm{g}\right)$ & Table A. 2 & \\
\hline Pasture, silage growing areas $\left(\mathrm{cm}^{3} / \mathrm{g}\right)$ & Table A. 2 & \\
\hline Livestock feed grain fields $\left(\mathrm{cm}^{3} / \mathrm{g}\right)$ & Table A.2 & \\
\hline Offsite dwelling site $\left(\mathrm{cm}^{3} / \mathrm{g}\right)$ & Table A. 2 & \\
\hline \multicolumn{3}{|l|}{ Transfer Factors } \\
\hline Fruit, grain, nonleafy vegetable transfer factor & Table A.3 & \\
\hline Leafy vegetable transfer factor & Table A.3 & \\
\hline Pasture and silage transfer factor & Table A.3 & \\
\hline Livestock feed grain transfer factor & Table A.3 & \\
\hline \multicolumn{3}{|l|}{ Storage Time } \\
\hline Storage time for pasture and silage (d) & 1 & Default \\
\hline Storage time for livestock feed grain (d) & 45 & Default \\
\hline \multicolumn{3}{|l|}{ Primary Contamination } \\
\hline Deposition velocity of dust $(\mathrm{m} / \mathrm{s})$ & 0.001 & Default \\
\hline Rainfall and runoff factor & 160 & Default \\
\hline Slope-length-steepness factor & 0.4 & Default \\
\hline Cover and management factor & 0.0506 & $\begin{array}{l}\text { Adjusted to match site-specific erosion } \\
\text { rate }\end{array}$ \\
\hline Support practice factor & 1 & Default \\
\hline \multicolumn{3}{|l|}{ Contaminated Zone } \\
\hline Soil erodibility factor of contaminated zone & 0.4 & Default \\
\hline \multicolumn{3}{|l|}{ Clean Cover } \\
\hline Soil erodibility factor of clean cover & NR & No cover \\
\hline Volumetric water content of clean cover & NR & No cover \\
\hline \multicolumn{3}{|l|}{$\begin{array}{l}\text { Agriculture/Livestock Feed Growing/Offsite } \\
\text { Dwelling Area Parameters }\end{array}$} \\
\hline $\begin{array}{l}\text { Fraction of area directly over primary contamination } \\
\text { for all fields }\end{array}$ & 0 & Receptor offsite \\
\hline
\end{tabular}




\begin{tabular}{|c|c|c|}
\hline Parameter & Value & Comment \\
\hline Irrigation applied per year for all fields $(\mathrm{m} / \mathrm{yr})$ & 0.1 & Site specific \\
\hline Evapotranspiration coefficient for all fields & $\mathrm{U}(0.5,0.75)$ & Distribution \\
\hline Runoff coefficient for all fields & $\mathrm{U}(0.1,0.8)$ & Distribution \\
\hline $\begin{array}{l}\text { Depth of soil mixing layer or plow layer for all fields } \\
\text { (m) }\end{array}$ & See Table A.1 & \\
\hline Volumetric water content for all fields & 0.3 & Default \\
\hline Dry bulk density of soil for all fields $\left(\mathrm{g} / \mathrm{cm}^{3}\right)$ & $\begin{array}{l}\mathrm{TN}^{\mathrm{d}}(1.696 \\
0.1855,0.001 \\
0.999)\end{array}$ & $\begin{array}{l}\text { Site-specific soil type (silty clay - } \\
\text { Golchert et al. 2010) distribution from } \\
\text { NRC } 2000\end{array}$ \\
\hline Soil erodibility factor for all fields & 0.4 & Default \\
\hline Slope-length-steepness factor for all fields & 0.4 & Default \\
\hline Cover and management factor for all fields & 0.0506 & $\begin{array}{l}\text { Adjusted to match site-specific erosion } \\
\text { rate }\end{array}$ \\
\hline Support practice factor for all fields & 1 & Default \\
\hline \multicolumn{3}{|l|}{ Atmospheric Transport } \\
\hline Release height (m) & 1 & Default \\
\hline Release heat flux (cal/s) & 0 & Default \\
\hline Anemometer height (m) & 10 & Default \\
\hline Ambient temperature $(\mathrm{K})$ & 285 & Default \\
\hline AM atmospheric mixing height $(\mathrm{m})$ & 400 & Default \\
\hline PM atmospheric mixing height (m) & 1,600 & Default \\
\hline Dispersion model coefficients & $\begin{array}{l}\text { Pasquill- } \\
\text { Gifford }\end{array}$ & Default \\
\hline Wind speed terrain & Rural & Default \\
\hline $\begin{array}{l}\text { Elevation of offsite location, relative to ground level } \\
\text { at primary contamination, for all fields (m) }\end{array}$ & 0 & Default \\
\hline Grid spacing for areal integration (m) & 10 & Default \\
\hline $\begin{array}{l}\text { Joint frequency of wind speed and stability class for } \\
\text { a 16-sector wind rose }\end{array}$ & $\begin{array}{l}\text { Actual values } \\
\text { from Chicago } \\
\text { Midway }\end{array}$ & Site-specific \\
\hline \multicolumn{3}{|l|}{ Unsaturated Zone Parameters } \\
\hline Unsaturated zone longitudinal dispersivity (m) & 0.1 & Default \\
\hline \multicolumn{3}{|l|}{ Saturated Zone Hydrological Data } \\
\hline Thickness of saturated zone (m) & 18.3 & Default \\
\hline $\begin{array}{l}\text { Saturated zone longitudinal dispersivity to surface } \\
\text { water body }(\mathrm{m})\end{array}$ & 10 & Default \\
\hline $\begin{array}{l}\text { Saturated zone horizontal lateral dispersivity to } \\
\text { surface water body }(\mathrm{m})\end{array}$ & 1 & Default \\
\hline Disperse vertically & Check box & Yes \\
\hline $\begin{array}{l}\text { Saturated zone vertical lateral dispersivity to surface } \\
\text { water body }(\mathrm{m})\end{array}$ & 0.06 & Default \\
\hline $\begin{array}{l}\text { Depth of aquifer contributing to surface water body } \\
\text { (m) }\end{array}$ & 10 & Default \\
\hline
\end{tabular}




\section{TABLE A.10 (Cont.)}

\section{Parameter}

\section{Surface Water Body}

Sediment deliver ratio

Volume of surface water body $\left(\mathrm{m}^{3}\right)$

Mean residence time of water in surface water body (yr)

\section{Groundwater Transport Parameters}

Distance from downgradient edge of contamination to well in the direction parallel to aquifer flow (m)

Distance from downgradient edge of contamination to surface water body in the direction parallel to aquifer flow (m)

Distance from downgradient edge of contamination to well in the direction perpendicular to aquifer flow (m)

Distance from downgradient edge of contamination to the right edge of surface water body in the direction perpendicular to aquifer flow (m)

Distance from downgradient edge of contamination to the left edge of the surface water body in the direction perpendicular to aquifer flow (m)

Main subzones in saturated zone

Main subzones in each partially saturated zone

Nuclide-specific retardation in all subzones, longitudinal dispersion in all but the subzone of transformation?

\section{Water Use}

Quantity of water consumed by an individual (L/yr)

Number of individuals in household who consume and use water

Quantity of water for use indoors of dwelling per individual (L/d)

Quantity of water for beef cattle (L/d)

Number of beef cattle

Quantity of water for dairy cows (L/d)

Number of dairy cows

Well pumping rate $\left(\mathrm{m}^{3} / \mathrm{yr}\right)$

\section{Ingestion Rates}

Drinking water intake from affected area

Fish intake from affected area

Crustacea and mollusks intake from affected area

Fruit, grain, nonleafy vegetables fraction from affected area

Leafy vegetables fraction from affected area

Meat fraction from affected area
Value

Comment

$\begin{array}{cc}1 & \text { Default } \\ 150,000 & \text { Default } \\ 1 & \text { Default }\end{array}$

$-55 \quad$ Site-specific

$256.8 \quad$ Site-specific

$-424 \quad$ Site-specific

$-150 \quad$ Default

$150 \quad$ Default

$\begin{array}{cc}1 & \text { Default } \\ 1 & \text { Default } \\ \text { Yes } & \text { Default }\end{array}$

See Table A.1 Household water not contaminated 4 Default

$225 \quad$ Household water not contaminated

50 Default

2 Default

160 Default

2 Default

250 RESRAD default

0 Drinking water not contaminated

0.5 Default

0.5 Default

0.5 Default

0.5 Default

$0.5 \quad 50 \%$ of the meat consumed is from contaminated area 


\section{TABLE A.10 (Cont.)}

\begin{tabular}{|c|c|c|}
\hline Parameter & Value & Comment \\
\hline Milk fraction from affected area & 0.5 & $\begin{array}{l}50 \% \text { of the meat consumed is from } \\
\text { contaminated area }\end{array}$ \\
\hline \multicolumn{3}{|l|}{ Livestock intake } \\
\hline Pasture and silage intake for beef cattle $(\mathrm{kg} / \mathrm{d})$ & 14 & Default \\
\hline Grain intake for beef cattle $(\mathrm{kg} / \mathrm{d})$ & 54 & Default \\
\hline $\begin{array}{l}\text { Soil from pasture and silage intake for beef cattle } \\
(\mathrm{kg} / \mathrm{d})\end{array}$ & 0.1 & Default \\
\hline Soil from grain intake for beef cattle $(\mathrm{kg} / \mathrm{d})$ & 0.4 & Default \\
\hline Pasture and silage intake for dairy cows $(\mathrm{kg} / \mathrm{d})$ & 44 & Default \\
\hline Grain intake for dairy cows $(\mathrm{kg} / \mathrm{d})$ & 11 & Default \\
\hline $\begin{array}{l}\text { Soil from pasture and silage intake for dairy cows } \\
(\mathrm{kg} / \mathrm{d})\end{array}$ & 0.4 & Default \\
\hline Soil from grain intake for dairy cows $(\mathrm{kg} / \mathrm{d})$ & 0.1 & Default \\
\hline \multicolumn{3}{|l|}{$\begin{array}{l}\text { Livestock Feed Factors (for Pasture and } \\
\text { Silage, Grain) }\end{array}$} \\
\hline Wet weight crop yield $\left(\mathrm{kg} / \mathrm{m}^{2}\right)$ & $1.1,0.7$ & Default \\
\hline Duration of growing season (yr) & $0.08,0.17$ & Default \\
\hline Foliage to food transfer coefficient & $1,0.1$ & Default \\
\hline Weathering removal constant & 20,20 & Default \\
\hline Foliar interception factor for irrigation & $0.25,0.25$ & Default \\
\hline Foliar interception factor for dust deposition & $0.25,0.25$ & Default \\
\hline Root depth (m) & See Table A.1 & $\begin{array}{l}\text { Distribution from NUREG/CR-6697 } \\
\text { (NRC 2000) }\end{array}$ \\
\hline \multicolumn{3}{|l|}{ Occupancy Factors } \\
\hline Indoor time fraction on primary contamination & 0 & \\
\hline Outdoor time fraction on primary contamination & 0 & \\
\hline Indoor time fraction on offsite dwelling site & 0.5 & \\
\hline Outdoor time fraction on offsite dwelling site & 0.1 & \\
\hline $\begin{array}{l}\text { Time fraction in fruit, grain, and nonleafy vegetable } \\
\text { fields }\end{array}$ & 0.1 & \\
\hline Time fraction in leafy vegetable fields & 0.1 & \\
\hline Time fraction in pasture and silage fields & 0.1 & \\
\hline Time fraction in livestock grain fields & 0.1 & \\
\hline
\end{tabular}

a All the areas (dwelling, agricultural areas) are assumed to be less than $350 \mathrm{~m}$ away from the contaminated area. This is a conservative assumption because the distance to the site boundary from the Building 310 area is greater than $350 \mathrm{~m}$. 


\section{REFERENCES FOR APPENDIX A}

Argonne (Argonne National Laboratory), 1996, Interim Action Documentation Report: Decontamination and Demolition of the 317 Area South Vaults, Argonne, Ill., May 31.

Eckerman, K.F., and J.C. Ryman, 1993, External Exposure to Radionuclides in Air, Water, and Soil, Exposure to Dose Coefficients for General Application, Based on the 1987 Federal Radiation Protection Guidance, EPA 402-R-93-076, Federal Guidance Report No. 12, prepared by Oak Ridge National Laboratory, Oak Ridge, Tenn., for U.S. Environmental Protection Agency, Office of Radiation and Indoor Air, Washington, D.C.

EPA (U.S. Environmental Protection Agency), 1997, Exposure Factor Handbook, EPA/600/P-95/002Fa, Office of Research and Development, National Center for Environmental Assessment, Washington, D.C.

Golchert, N.W., T.M. Davis, and L.P. Moos, 2010, Argonne Site Environmental Report for Calendar Year 2009, ANL-10/02, Argonne National Laboratory, Argonne, Ill., Sept.

ICRP (International Commission on Radiological Protection), 1991, 1990 Recommendations of the International Commission on Radiological Protection, ICRP Publication 60, Ann. ICRP 21(1-3), Pergamon Press, Oxford, England.

ICRP, 1996, Age-Dependent Doses to Members of the Public from Intake of Radionuclides: Part 5, Compilation of Ingestion and Inhalation Coefficients, ICRP Publication 72, Ann. ICRP 26(1), Pergamon Press, Oxford, England.

Kamboj, S., 2011, "Bldg. 310 RESRAD Analysis Project Status," intraoffice personal communication between Kamboj and R.L. Johnson (Argonne National Laboratory, Argonne, Ill.), Jan. 7.

Matton, P.B., 2011, "Bldg. 330 D\&D Project-RESRAD, DCGLs, Clean-up Parameters," intraoffice personal communication between Matton and R.J. Johnson (Argonne National Laboratory, Argonne, Ill.), Jan. 14.

NCDC (National Climatic Data Center), 2010, “Average Wind Speed.” Available at http://lwf.ncdc.noaa.gov/oa/climate/online/ccd/avgwind.html.

NRC (U.S. Nuclear Regulatory Commission), 2000, Development of Probabilistic RESRAD 6.0 and RESRAD-BUILD 3.0 Computer Codes, NUREG/CR-6697 and ANL/EAD/TM-98, prepared by C. Yu et al., Argonne National Laboratory, Argonne, Ill., for U.S. Nuclear Regulatory Commission, Washington, D.C., Nov.

NRCS (Natural Resources Conservation Service), 2010, "Report Cards Are Out - Illinois NRI Gets Gold Star!," news release, May 25. 
Patton, T.L., R.H. Pearl, and S.Y. Tsai, 1990, Hydrological Conditions at the 800 Area at Argonne National Laboratory, ANL/EAIS/TM-29, Argonne National Laboratory, Argonne, Ill., August.

Quinn, J., 2010, intraoffice personal communication between Quinn and S. Kamboj (Argonne National Laboratory, Argonne, Ill.), Nov. 12.

Sheppard, M.I., and D.H. Thibault, 1990, "Default Soil Solid/Liquid Partition Coefficients, Kds, for Four Major Soil Types: A Compendium,” Health Physics 59(4):471-482.

Yu, C., et al., 1993, Data Collection Handbook to Support Modeling the Impacts of Radioactive Material in Soil, ANL/EAIS-8, Argonne National Laboratory, Argonne, Ill., April.

Yu, C., et al., 2001, User's Manual for RESRAD Version 6, ANL/EAD-4, Argonne National Laboratory, Argonne, Ill. 


\section{APPENDIX B:}

\section{PARAMETERS USED FOR DETERMINISTIC ANALYSIS}

Tables B.1-B.4 list all input parameter distributions or parameter values used in the deterministic dose analysis for different user scenarios. 


\section{TABLE B.1 Parameter Values Used in the Deterministic Analysis of Different Scenarios ${ }^{\mathrm{a}}$}

\begin{tabular}{|c|c|c|c|c|c|}
\hline \multirow[b]{2}{*}{ Input Parameters } & \multicolumn{2}{|c|}{ Current Use Scenarios } & \multicolumn{2}{|c|}{ Future Use Scenarios } & \multirow[b]{2}{*}{ Comments } \\
\hline & Industrial Worker & Offsite Resident ${ }^{\mathrm{b}}$ & Resident Farmer ${ }^{\mathrm{b}}$ & Recreationist $^{\mathrm{c}}$ & \\
\hline \multicolumn{6}{|l|}{ Title } \\
\hline Title & Scenario dependent & Scenario dependent & Scenario dependent & Scenario dependent & Scenario definition \\
\hline Dose factor library & ICRP-60 & ICRP-60 & ICRP-60 & ICRP-60 & $\begin{array}{l}\text { DCFs based on ICRP-60 } \\
\text { (ICRP 1991) methodology } \\
\text { (external from FGR-12 [Eckerman } \\
\text { and Ryman 1993] and internal } \\
\text { from ICRP-72 [ICRP 1996]) }\end{array}$ \\
\hline Cut-off half-life (180 d or $30 \mathrm{~d})$ & $30 \mathrm{~d}$ & $30 \mathrm{~d}$ & $30 \mathrm{~d}$ & $30 \mathrm{~d}$ & RESRAD default \\
\hline Number of points $(32,64,128,256,512,1024)$ & 32 & 32 & 32 & 32 & RESRAD default \\
\hline Linear spacing/log spacing & Log spacing & Log spacing & Log spacing & Log spacing & RESRAD default \\
\hline Maximum no. of points for dose & 17 & 17 & 17 & 17 & RESRAD default \\
\hline Maximum no. of points for risk & 1 & 1 & 1 & 1 & $\begin{array}{l}\text { Using a smaller integration point } \\
\text { will shorten the calculation time }\end{array}$ \\
\hline Use line draw character (yes/no) & Yes & Yes & Yes & Yes & RESRAD default \\
\hline Find peak pathway dose (yes/no) & Yes & Yes & Yes & Yes & \\
\hline Save all files after each run (yes/no) & Yes & Yes & Yes & Yes & \\
\hline Time integrated probabilistic risk (yes/no) & No & No & No & No & $\begin{array}{l}\text { Dose-to-source ratio (DSR) is } \\
\text { calculated }\end{array}$ \\
\hline \multicolumn{6}{|l|}{ Calculation Parameters } \\
\hline Basic radiation dose limit (mrem/yr) & 25 & 25 & 25 & 25 & Not used in DSR calculation \\
\hline Times for calculation (yr) & $\begin{array}{l}1,3,10,30,100 \\
300,1,000\end{array}$ & $\begin{array}{c}1,2,3,4,5,6,7,8 \\
970\end{array}$ & $100,300,1,000$ & $100,300,1,000$ & $\begin{array}{l}\text { Up to the time horizon for dose } \\
\text { calculation }\end{array}$ \\
\hline \multicolumn{6}{|l|}{ Source } \\
\hline Nuclide concentration (pCi/g) & 1 & 1 & 1 & 1 & DSRs calculated \\
\hline \multicolumn{6}{|l|}{ Transport Factors } \\
\hline Distribution coefficient for all zones $\left(\mathrm{cm}^{3} / \mathrm{g}\right)$ & Table B.2 & Table B.2 & Table B.2 & Table B.2 & Site-specific soil type \\
\hline Number of unsaturated zones & 1 & 1 & 1 & 1 & RESRAD default \\
\hline Time since placement of material (yr) & 0 & 0 & 0 & 0 & RESRAD default \\
\hline Groundwater concentration $(\mathrm{pCi} / \mathrm{L})$ & 0 & 0 & 0 & 0 & RESRAD default \\
\hline Leach rate $(1 / y r)$ & 0 & 0 & 0 & 0 & RESRAD default \\
\hline Solubility limit (mol/L) & 0 & 0 & 0 & 0 & RESRAD default \\
\hline Use plant/soil ration (check box) & No & No. & No & No & RESRAD default \\
\hline
\end{tabular}


TABLE B.1 (Cont.)

\begin{tabular}{|c|c|c|c|c|c|}
\hline \multirow[b]{2}{*}{ Input Parameters } & \multicolumn{2}{|c|}{ Current Use Scenarios } & \multicolumn{2}{|c|}{ Future Use Scenarios } & \multirow[b]{2}{*}{ Comments } \\
\hline & Industrial Worker & Offsite Resident ${ }^{\mathrm{b}}$ & Resident Farmer ${ }^{\mathrm{b}}$ & Recreationist $^{\mathrm{c}}$ & \\
\hline \multicolumn{6}{|l|}{ Transfer Factors } \\
\hline $\begin{array}{l}\text { Plant transfer factor (wet-plant weight } \\
\text { concentration, } \mathrm{pCi} / \mathrm{g} / \mathrm{dry} \text { soil weight } \\
\text { concentration, } \mathrm{pCi} / \mathrm{g} \text { ) }\end{array}$ & $\mathrm{NR}^{\mathrm{d}}$ & Table B.2 & Table B.2 & NR & RESRAD default \\
\hline $\begin{array}{l}\text { Meat transfer factor (concentration in meat, } \\
\mathrm{pCi} / \mathrm{g} / \mathrm{rate} \text { of intake, } \mathrm{pCi} / \mathrm{d})\end{array}$ & NR & Table B.2 & Table B.2 & Table A.2 & RESRAD default \\
\hline $\begin{array}{l}\text { Milk transfer factor (concentration in milk, } \\
\mathrm{pCi} / \mathrm{L} / \mathrm{rate} \text { of intake, } \mathrm{pCi} / \mathrm{d} \text { ) }\end{array}$ & NR & Table B.2 & Table B.2 & NR & RESRAD default \\
\hline $\begin{array}{l}\text { Fish transfer factor (concentration in fish, } \\
\mathrm{pCi} / \mathrm{Kg} / \text { concentration in water, } \mathrm{pCi} / \mathrm{L} \text { ) }\end{array}$ & NR & Table B.3 & Table B.3 & Table A.3 & RESRAD default \\
\hline $\begin{array}{l}\text { Crustacea transfer factor (concentration in } \\
\text { crustacea, } \mathrm{pCi} / \mathrm{kg} / \text { concentration in water, } \\
\mathrm{pCi} / \mathrm{L} \text { ) }\end{array}$ & NR & Table B.3 & Table B.3 & Table A.3 & RESRAD default \\
\hline \multicolumn{6}{|l|}{ Contaminated Zone Parameters } \\
\hline Area of contaminated zone $\left(\mathrm{m}^{2}\right)$ & 10,000 & 10,000 & 10,000 & 10,000 & Matton 2011; Kamboj 2011 \\
\hline Thickness of contaminated zone (m) & 1 & 1 & 1 & 1 & Matton 2011; Kamboj 2011 \\
\hline Length parallel to aquifer flow (m) & 100 & 100 & 100 & 100 & RESRAD default \\
\hline $\begin{array}{l}\text { Does the initial contamination penetrate the } \\
\text { water table? }\end{array}$ & No & No & No & No & RESRAD default \\
\hline \multirow{2}{*}{\multicolumn{6}{|c|}{$\begin{array}{l}\text { Cover and Contaminated Zone Hydrological } \\
\text { Data }\end{array}$}} \\
\hline & & & & & \\
\hline Cover depth (m) & 0 & 0 & 0 & 0 & No cover layer assumed \\
\hline Density of cover material $\left(\mathrm{g} / \mathrm{cm}^{3}\right)$ & NR & NR & NR & NR & $\begin{array}{l}\text { Not required when cover depth } \\
\text { equals zero }\end{array}$ \\
\hline 2. Cover erosion rate $(\mathrm{m} / \mathrm{yr})$ & NR & NR & NR & NR & $\begin{array}{l}\text { Not required when cover depth } \\
\text { equals zero }\end{array}$ \\
\hline Density of primary contaminated zone $\left(\mathrm{g} / \mathrm{cm}^{3}\right)$ & 1.76 & 1.76 & 1.76 & 1.76 & Site-specific (Argonne 1996) \\
\hline Contaminated zone erosion rate $(\mathrm{m} / \mathrm{y})$ & $1.65 \mathrm{E}-4$ & $1.65 \mathrm{E}-4^{\mathrm{e}}$ & $1.65 \mathrm{E}-4$ & $1.65 \mathrm{E}-4$ & $\begin{array}{l}\text { To match the soil erosion of } \\
1 \text { ton/acre/yr at Illinois } \\
\text { (NRCS 2010) }\end{array}$ \\
\hline Contaminated zone total porosity & 0.3 & 0.3 & 0.3 & 0.3 & Site-specific (Argonne 1996) \\
\hline Contaminated zone field capacity & 0.2 & 0.2 & 0.2 & 0.2 & RESRAD default \\
\hline
\end{tabular}


TABLE B.1 (Cont.)

\begin{tabular}{|c|c|c|c|c|c|}
\hline \multirow[b]{2}{*}{ Input Parameters } & \multicolumn{2}{|c|}{ Current Use Scenarios } & \multicolumn{2}{|c|}{ Future Use Scenarios } & \multirow[b]{2}{*}{ Comments } \\
\hline & Industrial Worker & Offsite Resident ${ }^{\mathrm{b}}$ & Resident Farmer $^{\mathrm{b}}$ & Recreationist $^{\mathrm{c}}$ & \\
\hline $\begin{array}{l}\text { Contaminated zone hydraulic conductivity } \\
(\mathrm{m} / \mathrm{yr})\end{array}$ & 0.32 & 0.32 & 0.32 & 0.32 & Site-specific (Quinn 2010) \\
\hline Contaminated zone b parameter & 10.4 & 10.4 & 10.4 & 10.4 & $\begin{array}{l}\text { Site-specific soil type (silty clay) } \\
\text { value from Yu et al. } 1993^{\mathrm{f}}\end{array}$ \\
\hline Humidity in air $\left(\mathrm{g} / \mathrm{m}^{3}\right)$ & 8 & 8 & 8 & 8 & Used only for $\mathrm{H}-3$ \\
\hline Evapotranspiration coefficient & 0.65 & 0.65 & 0.65 & 0.65 & Site-specific \\
\hline Wind speed $(\mathrm{m} / \mathrm{s})$ & 4.6 & NR & 4.6 & 4.6 & Site-specific value for Chicago \\
\hline Precipitation rate $(\mathrm{m} / \mathrm{yr})$ & 0.9177 & 0.9177 & 0.9177 & 0.9177 & $\begin{array}{l}\text { Site-specific value from Golchert } \\
\text { et al. } 1010\end{array}$ \\
\hline Irrigation rate $(\mathrm{m} / \mathrm{yr})$ & 0.0 & 0.1 & 0.1 & 0.0 & Site-specific (Argonne 1996) \\
\hline Irrigation mode (overhead/ditch) & NR & NR & Overhead & NR & RESRAD default \\
\hline Runoff coefficient & 0.2 & 0.2 & 0.2 & 0.2 & $\begin{array}{l}\text { Site-specific for woodland (Yu et } \\
\text { al. 2001, page E-7) }\end{array}$ \\
\hline Watershed area for nearby stream or pond $\left(\mathrm{m}^{2}\right)$ & $1,000,000$ & NR & $1,000,000$ & $1,000,000$ & RESRAD default \\
\hline Accuracy for water/soil computation & 0.001 & NR & 0.001 & 0.001 & RESRAD default \\
\hline \multicolumn{6}{|l|}{ Saturated Zone Hydrological Data } \\
\hline Density of saturated zone $\left(\mathrm{g} / \mathrm{cm}^{3}\right)$ & NR & 1.76 & 1.76 & 1.76 & Site-specific (Argonne 1996) \\
\hline Saturated zone effective porosity & NR & 0.14 & 0.14 & 0.14 & Site-specific \\
\hline Saturated zone total porosity & NR & 0.3 & 0.3 & 0.3 & Site-specific (Argonne 1996) \\
\hline Saturated zone field capacity & NR & 0.14 & 0.14 & 0.14 & Site-specific \\
\hline Saturated zone hydraulic conductivity (m/yr) & NR & 947 & 947 & 947 & Site-specific (Argonne 1996) \\
\hline Saturated zone $\mathrm{b}$ parameter & NR & 4.38 & 4.38 & 4.38 & Site-specific soil type \\
\hline Saturated zone hydraulic gradient & NR & 0.015 & 0.015 & 0.015 & Site-specific (Patton et al. 1990) \\
\hline Water table drop rate $(\mathrm{m} / \mathrm{yr})$ & NR & NR & 0.001 & 0.001 & RESRAD default \\
\hline Well pump intake depth (m below water table) & NR & NR & 5 & 5 & Site-specific \\
\hline $\begin{array}{l}\text { Model for water transportation } \\
\text { (nondispersion/mass-balance) }\end{array}$ & NR & NR & Nondispersion & Nondispersion & RESRAD default \\
\hline Well pumping rate $\left(\mathrm{m}^{3} / \mathrm{yr}\right)$ & NR & NR & 250 & 250 & RESRAD default \\
\hline \multicolumn{6}{|l|}{ Unsaturated Zone Parameters } \\
\hline Thickness of unsaturated zone (m) & NR & 13.9 & 13.9 & 13.9 & $\begin{array}{l}\text { Site-specific ( } 48 \mathrm{ft}-1 \mathrm{~m})(\mathrm{Quinn} \\
\text { 2010) or scenario-specific }\end{array}$ \\
\hline Density of unsaturated zone $\left(\mathrm{g} / \mathrm{cm}^{3}\right)$ & NR & 1.76 & 1.76 & 1.76 & Site-specific (Argonne 1996) \\
\hline
\end{tabular}


TABLE B.1 (Cont.)

\begin{tabular}{|c|c|c|c|c|c|}
\hline \multirow[b]{2}{*}{ Input Parameters } & \multicolumn{2}{|c|}{ Current Use Scenarios } & \multicolumn{2}{|c|}{ Future Use Scenarios } & \multirow[b]{2}{*}{ Comments } \\
\hline & Industrial Worker & Offsite Resident ${ }^{\mathrm{b}}$ & Resident Farmer ${ }^{b}$ & Recreationist $^{\mathrm{c}}$ & \\
\hline Unsaturated zone effective porosity & NR & 0.2 & 0.2 & 0.2 & Site-specific (Argonne 1996) \\
\hline Unsaturated zone total porosity & NR & 0.3 & 0.3 & 0.3 & Site-specific (Argonne 1996) \\
\hline Unsaturated zone field capacity & NR & 0.2 & 0.2 & 0.2 & RESRAD default \\
\hline Unsaturated zone hydraulic conductivity $(\mathrm{m} / \mathrm{yr})$ & NR & 0.32 & 0.32 & 0.32 & Site-specific (Quinn 2010) \\
\hline Unsaturated zone $b$ parameter & NR & 10.4 & 10.4 & 10.4 & $\begin{array}{l}\text { Site-specific soil type (silty clay) } \\
\text { value from Yu et al. } 1993\end{array}$ \\
\hline Occupancy, Inhalation, and External Gamma & & & & & \\
\hline Parameters & & & & & \\
\hline Inhalation rate $\left(\mathrm{m}^{3} / \mathrm{yr}\right)$ & 11,400 & 8,400 & 8,400 & 14,000 & Scenario-specific \\
\hline Mass loading for inhalation $\left(\mathrm{g} / \mathrm{m}^{3}\right)$ & $1 \mathrm{E}-4$ & $1 \mathrm{E}-4$ & $1 \mathrm{E}-4$ & $1 \mathrm{E}-4$ & RESRAD default \\
\hline Exposure duration $(\mathrm{yr})$ & 25 & 30 & 30 & 30 & Not used in DSR calculations \\
\hline Indoor dust filtration factor & 0.4 & 0.4 & 0.4 & 0.4 & RESRAD default \\
\hline External gamma shielding factor & NR & 0.7 & 0.7 & NR & RESRAD default \\
\hline Indoor time fraction & $0 / 0.2283$ & NR & 0.6833 & 0 & Scenario-specific \\
\hline Outdoor time fraction & $0.2283 / 0$ & NR & 0.0833 & 0.0384 & Scenario-specific \\
\hline $\begin{array}{l}\text { Shape of contaminated zone } \\
\text { (circular/noncircular) }\end{array}$ & Circular & Circular & Circular & Circular & RESRAD default \\
\hline \multicolumn{6}{|l|}{ Ingestion Pathway Dietary Data } \\
\hline Fruit, vegetable and grain consumption $(\mathrm{kg} / \mathrm{yr})$ & NR & 160 & 160 & NR & RESRAD default \\
\hline Leafy vegetable consumption $(\mathrm{kg} / \mathrm{yr})$ & NR & 14 & 14 & NR & RESRAD default \\
\hline Milk consumption $(\mathrm{L} / \mathrm{yr})$ & NR & 92 & 92 & NR & RESRAD default \\
\hline Meat and poultry consumption $(\mathrm{kg} / \mathrm{yr})$ & NR & 63 & 63 & 19 & $\begin{array}{l}\text { RESRAD default and scenario- } \\
\text { specific for recreational use }\end{array}$ \\
\hline Fish consumption $(\mathrm{kg} / \mathrm{yr})$ & NR & 5.4 & 5.4 & 12 & $\begin{array}{l}\text { RESRAD default and scenario- } \\
\text { specific for recreational use }\end{array}$ \\
\hline Other sea food consumption ( $\mathrm{kg} / \mathrm{yr})$ & NR & 0.9 & 0.9 & NR & RESRAD default \\
\hline Soil ingestion $(\mathrm{g} / \mathrm{yr})$ & 36.5 & 36.5 & 36.5 & 36.5 & RESRAD default \\
\hline Drinking water intake (L/yr) & NR & 510 & 510 & $1.4 \mathrm{~L} / \mathrm{d}^{\mathrm{g}} 14=19.6 \mathrm{~L}$ & $\begin{array}{l}\text { RESRAD default and scenario- } \\
\text { specific for recreational use }\end{array}$ \\
\hline Drinking water contaminated fraction & NR & 0 & 1 & 1 & $\begin{array}{l}\text { RESRAD default and for offsite } \\
\text { receptor scenario, water is not } \\
\text { contaminated }\end{array}$ \\
\hline
\end{tabular}


TABLE B.1 (Cont.)

\begin{tabular}{|c|c|c|c|c|c|}
\hline \multirow[b]{2}{*}{ Input Parameters } & \multicolumn{2}{|c|}{ Current Use Scenarios } & \multicolumn{2}{|c|}{ Future Use Scenarios } & \multirow[b]{2}{*}{ Comments } \\
\hline & Industrial Worker & Offsite Resident ${ }^{\mathrm{b}}$ & Resident Farmer ${ }^{\mathrm{b}}$ & Recreationist $^{\mathrm{c}}$ & \\
\hline Household water contaminated fraction & 1 & 1 & 1 & NR & RESRAD default \\
\hline Livestock water contaminated fraction & NR & 1 & 1 & 1 & RESRAD default \\
\hline Irrigation water contaminated fraction & NR & 1 & 1 & 1 & RESRAD default \\
\hline Aquatic food contaminated fraction & NR & 0.5 & 0.5 & 0.5 & Distribution from NRC 2000 \\
\hline Plant food contaminated fraction & NR & -1 & -1 & NR & $\begin{array}{l}\text { Calculated by RESRAD from area } \\
\text { factor }\end{array}$ \\
\hline Meat contaminated fraction & NR & -1 & -1 & 1 & Scenario specific \\
\hline Milk contaminated fraction & NR & -1 & -1 & NR & $\begin{array}{l}\text { Calculated by RESRAD from area } \\
\text { factor }\end{array}$ \\
\hline \multicolumn{6}{|l|}{ Ingestion Pathway, Nondietary Data } \\
\hline Livestock fodder intake for meat $(\mathrm{kg} / \mathrm{d})$ & NR & NR & 68 & 10.6 & $\begin{array}{l}\text { RESRAD default or scenario- } \\
\text { specific }\end{array}$ \\
\hline Livestock fodder intake for milk (kg/d) & NR & NR & 55 & NR & RESRAD default \\
\hline Livestock water intake for meat (L/d) & NR & 50 & 50 & 6.4 & $\begin{array}{l}\text { RESRAD default or scenario- } \\
\text { specific }\end{array}$ \\
\hline Livestock water intake for milk (L/d) & NR & 160 & 160 & NR & RESRAD default \\
\hline Livestock intake of soil for meat $(\mathrm{kg} / \mathrm{d})$ & NR & NR & 0.5 & 0.5 & RESRAD default \\
\hline Livestock intake of soil for milk (kg/d) & NR & NR & 0.5 & NR & RESRAD default \\
\hline Mass loading for foliar deposition $\left(\mathrm{g} / \mathrm{m}^{3}\right)$ & NR & NR & 0.0001 & 0.0001 & RESRAD default \\
\hline Depth of soil mixing layer $(\mathrm{m})$ & 0.15 & 0.15 & 0.15 & 0.15 & RESRAD default \\
\hline Depth of roots $(\mathrm{m})$ & NR & 0.9 & 0.9 & 0.9 & RESRAD default \\
\hline $\begin{array}{l}\text { Drinking water fraction from groundwater } \\
\text { source }\end{array}$ & NR & 0 & 1 & 0 & Scenario-specific \\
\hline $\begin{array}{l}\text { Household water fraction from groundwater } \\
\text { source }\end{array}$ & NR & 0 & 1 & NR & Scenario-specific \\
\hline $\begin{array}{l}\text { Livestock water fraction from groundwater } \\
\text { source }\end{array}$ & NR & 0 & 1 & 0 & $\begin{array}{l}\text { RESRAD default for resident farmer } \\
\text { scenario and } 0 \text { for recreational use } \\
\text { scenario because surface water is } \\
\text { used by livestock }\end{array}$ \\
\hline $\begin{array}{l}\text { Irrigation water fraction from groundwater } \\
\text { source }\end{array}$ & NR & 0 & 1 & 0 & RESRAD default \\
\hline
\end{tabular}


TABLE B.1 (Cont.)

\begin{tabular}{|c|c|c|c|c|c|}
\hline \multirow[b]{2}{*}{ Input Parameters } & \multicolumn{2}{|c|}{ Current Use Scenarios } & \multicolumn{2}{|c|}{ Future Use Scenarios } & \multirow[b]{2}{*}{ Comments } \\
\hline & Industrial Worker & Offsite Resident ${ }^{\mathrm{b}}$ & Resident Farmer ${ }^{b}$ & Recreationist $^{\mathrm{c}}$ & \\
\hline \multicolumn{6}{|l|}{ Plant Factors } \\
\hline $\begin{array}{l}\text { Wet weight crop yield for nonleafy vegetables } \\
\left(\mathrm{kg} / \mathrm{m}^{2}\right)\end{array}$ & NR & 0.7 & 0.7 & NR & RESRAD default \\
\hline $\begin{array}{l}\text { Length of growing season for nonleafy } \\
\text { vegetables }(\mathrm{yr})\end{array}$ & NR & 0.17 & 0.17 & NR & RESRAD default \\
\hline Translocation factor for nonleafy vegetables & NR & 0.1 & 0.1 & NR & RESRAD default \\
\hline Weathering removal constant $(1 / \mathrm{yr})$ & NR & 20 & 20 & 20 & Distribution from NRC 2000 \\
\hline $\begin{array}{l}\text { Wet foliar interception fraction for nonleafy } \\
\text { vegetables }\end{array}$ & NR & 0.25 & 0.25 & NR & RESRAD default \\
\hline $\begin{array}{l}\text { Dry foliar interception fraction or nonleafy } \\
\text { vegetables }\end{array}$ & NR & 0.25 & 0.25 & NR & RESRAD default \\
\hline $\begin{array}{l}\text { Wet weight crop yield for leafy vegetables } \\
\left(\mathrm{kg} / \mathrm{m}^{2}\right)\end{array}$ & NR & 1.5 & 1.5 & NR & RESRAD default \\
\hline $\begin{array}{l}\text { Length of growing season for leafy vegetables } \\
\text { (yr) }\end{array}$ & NR & 0.25 & 0.25 & NR & RESRAD default \\
\hline Translocation factor for leafy vegetables & NR & 1 & 1 & NR & RESRAD default \\
\hline $\begin{array}{l}\text { Wet foliar interception fraction for leafy } \\
\text { vegetables }\end{array}$ & NR & 0.25 & 0.25 & NR & RESRAD default \\
\hline $\begin{array}{l}\text { Dry foliar interception fraction for leafy } \\
\text { vegetables }\end{array}$ & NR & 0.25 & 0.25 & NR & RESRAD default \\
\hline Wet weight crop yield for fodder $\left(\mathrm{kg} / \mathrm{m}^{2}\right)$ & NR & NR & 1.1 & 1.1 & RESRAD default \\
\hline Length of growing season for fodder (yr) & NR & NR & 0.08 & 0.08 & RESRAD default \\
\hline Translocation factor for fodder & NR & NR & 1 & 1 & RESRAD default \\
\hline Wet foliar interception fraction for fodder & NR & NR & 0.25 & 0.25 & RESRAD default \\
\hline Dry foliar interception fraction for fodder & NR & NR & 0.25 & 0.25 & RESRAD default \\
\hline \multicolumn{6}{|l|}{ Storage-Times-Before-Use Data } \\
\hline $\begin{array}{l}\text { Storage time for fruits, nonleafy vegetables and } \\
\text { grain (d) }\end{array}$ & NR & 14 & 14 & NR & RESRAD default \\
\hline Storage time for leafy vegetables (d) & NR & 1 & 1 & NR & RESRAD default \\
\hline Storage time for milk (d) & NR & 1 & 1 & NR & RESRAD default \\
\hline Storage time for meat (d) & NR & NR & 20 & 182.5 & $\begin{array}{l}\text { RESRAD default for resident farmer } \\
\text { scenario and scenario-specific for } \\
\text { recreational use }\end{array}$ \\
\hline
\end{tabular}


TABLE B.1 (Cont.)

\begin{tabular}{|c|c|c|c|c|c|}
\hline \multirow[b]{2}{*}{ Input Parameters } & \multicolumn{2}{|c|}{ Current Use Scenarios } & \multicolumn{2}{|c|}{ Future Use Scenarios } & \multirow[b]{2}{*}{ Comments } \\
\hline & Industrial Worker & Offsite Resident ${ }^{b}$ & Resident Farmer ${ }^{\mathrm{b}}$ & Recreationist $^{\mathrm{c}}$ & \\
\hline Storage time for fish (d) & NR & 7 & 7 & 0 & $\begin{array}{l}\text { RESRAD default and scenario- } \\
\text { specific for recreational use }\end{array}$ \\
\hline Storage time for crustacea and mollusks (d) & NR & 7 & 7 & 0 & $\begin{array}{l}\text { RESRAD default and scenario- } \\
\text { specific for recreational use }\end{array}$ \\
\hline Storage time for well water (d) & NR & 1 & 1 & NR & $\begin{array}{l}\text { RESRAD default for resident farmer } \\
\text { scenario and not required for } \\
\text { recreational use scenario }\end{array}$ \\
\hline Storage time for surface water (d) & NR & 1 & 1 & 0 & $\begin{array}{l}\text { RESRAD default for resident farmer } \\
\text { scenario and } 0 \text { for recreational use } \\
\text { scenario }\end{array}$ \\
\hline Storage time for livestock fodder (d) & NR & NR & 45 & 0 & $\begin{array}{l}\text { RESRAD default for resident farmer } \\
\text { scenario and scenario-specific for } \\
\text { recreational use scenario }\end{array}$ \\
\hline \multicolumn{6}{|l|}{ Radon Datag } \\
\hline Cover total porosity & NR & NR & NR & NR & No cover \\
\hline Cover volumetric water content & NR & NR & NR & NR & No cover \\
\hline Cover radon diffusion coefficient & NR & NR & NR & NR & No cover \\
\hline $\begin{array}{l}\text { Total porosity of the house or building } \\
\text { foundation }\end{array}$ & 0.1 & 0.1 & 0.1 & 0.1 & RESRAD default \\
\hline Volumetric water content of the foundation & 0.03 & 0.03 & 0.03 & 0.03 & RESRAD default \\
\hline $\begin{array}{l}\text { Diffusion coefficient for radon gas in foundation } \\
\text { material }\left(\mathrm{m}^{2} / \mathrm{s}\right)\end{array}$ & $3 \mathrm{E}-07$ & $3 \mathrm{E}-07$ & $3 \mathrm{E}-07$ & $3 \mathrm{E}-07$ & RESRAD default \\
\hline $\begin{array}{l}\text { Diffusion coefficient for radon gas in } \\
\text { contaminated zone soil }\left(\mathrm{m}^{2} / \mathrm{s}\right)\end{array}$ & $2 \mathrm{E}-06$ & $2 \mathrm{E}-06$ & $2 \mathrm{E}-06$ & $2 \mathrm{E}-06$ & $\begin{array}{l}\text { RESRAD default (the value is } \\
\text { representative of silty clay soil } \\
\text { type) }\end{array}$ \\
\hline Emanating power of radon-222 & 0.28 & 0.28 & 0.28 & 0.28 & Clay soil type (Yu et al. 1993) \\
\hline Radon vertical dimension of mixing (m) & 2.0 & 2.0 & 2.0 & 2.0 & RESRAD default \\
\hline Average building air exchange rate $\left(\mathrm{h}^{-1}\right)$ & 0.5 & 0.5 & 0.5 & 0.5 & RESRAD default \\
\hline Height of building (room) (m) & 2.5 & 2.5 & 2.5 & 2.5 & RESRAD default \\
\hline Building indoor area factor & 0 & 0 & 0 & 0 & RESRAD default \\
\hline $\begin{array}{l}\text { Bulk density of house or building foundation } \\
\left(\mathrm{g} / \mathrm{cm}^{3}\right)\end{array}$ & 2.4 & 2.4 & 2.4 & 2.4 & RESRAD default \\
\hline Thickness of house or building foundation (m) & 0.15 & 0.15 & 0.15 & 0.15 & RESRAD default \\
\hline Building depth below ground surface $(\mathrm{m})$ & -1 & -1 & -1 & -1 & RESRAD default \\
\hline
\end{tabular}


TABLE B.1 (Cont.)

\begin{tabular}{|c|c|c|c|c|c|}
\hline \multirow[b]{2}{*}{ Input Parameters } & \multicolumn{2}{|c|}{ Current Use Scenarios } & \multicolumn{2}{|c|}{ Future Use Scenarios } & \multirow[b]{2}{*}{ Comments } \\
\hline & Industrial Worker & Offsite Resident ${ }^{\mathrm{b}}$ & Resident Farmer ${ }^{\mathrm{b}}$ & Recreationist $^{\mathrm{c}}$ & \\
\hline \multicolumn{6}{|l|}{ Carbon-14 Data ${ }^{g}$} \\
\hline C-12 concentration in local water $\left(\mathrm{g} / \mathrm{cm}^{3}\right)$ & $2 \mathrm{E}-5$ & $2 \mathrm{E}-5$ & $2 \mathrm{E}-5$ & $2 \mathrm{E}-5$ & RESRAD default \\
\hline C-12 concentration in contaminated soil $(\mathrm{g} / \mathrm{g})$ & 0.03 & 0.03 & 0.03 & 0.03 & RESRAD default \\
\hline $\begin{array}{l}\text { Fraction of vegetation carbon absorbed from } \\
\text { soil }\end{array}$ & 0.02 & 0.02 & 0.02 & 0.02 & RESRAD default \\
\hline Fraction of vegetation carbon absorbed from air & 0.98 & 0.98 & 0.98 & 0.98 & RESRAD default \\
\hline Thickness of evasion layer of C-14 in soil (m) & 0.3 & 0.3 & 0.3 & 0.3 & RESRAD default \\
\hline C-14 evasion flux rate from soil $\left(\mathrm{s}^{-1}\right)$ & $7 \mathrm{E}-7$ & $7 \mathrm{E}-7$ & $7 \mathrm{E}-7$ & $7 \mathrm{E}-7$ & RESRAD default \\
\hline C-12 evasion flux rate from soil $\left(\mathrm{s}^{-1}\right)$ & $1 \mathrm{E}-10$ & $1 \mathrm{E}-10$ & $1 \mathrm{E}-10$ & $1 \mathrm{E}-10$ & RESRAD default \\
\hline Grain fraction in beef cattle feed & 0.8 & 0.8 & 0.8 & 0.8 & RESRAD default \\
\hline Grain fraction in milk cow feed & 0.2 & 0.2 & 0.2 & 0.2 & RESRAD default \\
\hline
\end{tabular}

a RESRAD-OFFSITE code is used for analyzing offsite resident scenario, and RESRAD (onsite) code is used for other scenarios. Extra parameters required for RESRADOFFSITE code are listed in Table B.4.

b For offsite resident and residential farmer scenarios, the parameter values for food consumption are RESRAD defaults. The parameter values for inhalation rate and indoor and outdoor time fractions are scenario-specific. The parameter values for livestock fodder intake for meat, livestock water intake for meat, livestock intake of soil, plant food contaminated fraction, and meat contaminated fraction are code defaults and are calculated on the basis of the contaminated area. The parameter values for storage times for meat, fish, surface water, and livestock fodder are RESRAD defaults.

c For the recreational use scenario, it is assumed that the individual would be involved in moderate activity onsite; therefore, an inhalation rate of moderate activity $\left(1.6 \mathrm{~m}^{3} / \mathrm{h}\right)$ is assumed (EPA 1997). The parameter value for meat and poultry consumption is based on the assumption that a recreationist kills a deer and brings the catch home and uses its meat over the course of a year; the value is based on the game consumption statistics among households that hunt. For fish consumption, it is assumed that a recreationist catches a fish and consumes it the same day; the value is based on the home-caught fish consumption statistics among households that fish. The parameter values for indoor and outdoor time fractions are from the scenario description. All the meat consumed is assumed to be contaminated. For the storage time of meat, since the meat is consumed over the course of a year, an average storage time of 182.5 days is assumed. For the storage time of fish, since the fish is consumed on the same day, a storage time of zero days is assumed. Since the game animal uses fresh forage and surface water, a storage time of zero days is assumed for both parameters.

d NR - value is not required for this scenario.

e Cover and management are changed to 0.0506 to get site-specific erosion rate in RESRAD-OFFSITE areas.

f It is assumed that the soil type is silty clay at the Argonne site, and values representative of silty clay soil are used.

g All the parameters for radon (except radon emanation rate) and C-14 are kept at code default values. 
TABLE B.2 Parameter Values Used in the Deterministic Analysis for the Kd, Plant, Meat, and Milk Transfer Factors

\begin{tabular}{|c|c|c|c|c|c|}
\hline \multirow[b]{2}{*}{ Element } & \multicolumn{2}{|c|}{$\mathrm{Kd}\left(\mathrm{cm}^{3} / \mathrm{g}\right)$} & \multirow[b]{2}{*}{$\begin{array}{c}\text { Plant Transfer } \\
\text { Factor } \\
(\mathrm{pCi} / \mathrm{kg}) /(\mathrm{pCi} / \mathrm{kg})\end{array}$} & \multirow[b]{2}{*}{$\begin{array}{c}\text { Meat Transfer } \\
\text { Factor } \\
(\mathrm{pCi} / \mathrm{kg}) /(\mathrm{pCi} / \mathrm{d})\end{array}$} & \multirow[b]{2}{*}{$\begin{array}{c}\text { Milk Transfer } \\
\text { Factor } \\
(\mathrm{pCi} / \mathrm{L}) /(\mathrm{pCi} / \mathrm{d})\end{array}$} \\
\hline & $\begin{array}{c}\text { Saturated } \\
\text { Zone }^{\mathrm{a}} \\
\end{array}$ & $\begin{array}{l}\text { Contaminated and } \\
\text { Unsaturated Zone }\end{array}$ & & & \\
\hline $\mathrm{Ac}$ & 450 & 2,400 & $2.5 \times 10^{-3}$ & $2.0 \times 10^{-5}$ & $2.0 \times 10^{-5}$ \\
\hline $\mathrm{Am}$ & 1,900 & 8,400 & $1.0 \times 10^{-3}$ & $5.0 \times 10^{-5}$ & $2.0 \times 10^{-6}$ \\
\hline $\mathrm{C}$ & 5 & 1 & $5.5 \times 10^{0}$ & $3.1 \times 10^{-2}$ & $1.2 \times 10^{-2}$ \\
\hline $\mathrm{Co}$ & 60 & 550 & $8.0 \times 10^{-2}$ & $2.0 \times 10^{-2}$ & $2.0 \times 10^{-3}$ \\
\hline Cs & 280 & 1,900 & $4.0 \times 10^{-2}$ & $3.0 \times 10^{-2}$ & $8.0 \times 10^{-3}$ \\
\hline I & 1 & 1 & $2.0 \times 10^{-2}$ & $7.0 \times 10^{-3}$ & $1.0 \times 10^{-2}$ \\
\hline $\mathrm{Np}$ & 5 & 55 & $2.0 \times 10^{-2}$ & $1.0 \times 10^{-3}$ & $5.0 \times 10^{-6}$ \\
\hline $\mathrm{Pa}$ & 550 & 2,700 & $1.0 \times 10^{-2}$ & $5.0 \times 10^{-3}$ & $5.0 \times 10^{-6}$ \\
\hline $\mathrm{Pb}$ & 270 & 550 & $1.0 \times 10^{-2}$ & $8.0 \times 10^{-4}$ & $3.0 \times 10^{-4}$ \\
\hline Po & 150 & 3,000 & $1.0 \times 10^{-3}$ & $5.0 \times 10^{-3}$ & $3.4 \times 10^{-4}$ \\
\hline $\mathrm{Pu}$ & 550 & 5,100 & $1.0 \times 10^{-3}$ & $1.0 \times 10^{-4}$ & $1.0 \times 10^{-6}$ \\
\hline $\mathrm{Ra}$ & 500 & 9,100 & $4.0 \times 10^{-2}$ & $1.0 \times 10^{-3}$ & $1.0 \times 10^{-3}$ \\
\hline $\mathrm{Sr}$ & 15 & 110 & $3.0 \times 10^{-1}$ & $8.0 \times 10^{-3}$ & $2.0 \times 10^{-3}$ \\
\hline $\mathrm{Tc}$ & 0.1 & 1 & $5.0 \times 10^{0}$ & $1.0 \times 10^{-4}$ & $1.0 \times 10^{-3}$ \\
\hline Th & 3,200 & 5,800 & $1.0 \times 10^{-3}$ & $1.0 \times 10^{-4}$ & $5.0 \times 10^{-6}$ \\
\hline $\mathrm{U}$ & 35 & 1,600 & $2.5 \times 10^{-3}$ & $3.4 \times 10^{-4}$ & $6.0 \times 10^{-4}$ \\
\hline
\end{tabular}

a Based on sandy soil type from RESRAD data collection handbook or RESRAD default values for saturated zone.

b Based on clay soil type from RESRAD data collection handbook or RESRAD default values for contaminated and unsaturated zone. 
TABLE B.3 Parameter Values Used in the Deterministic Analysis for the Fish and Crustacea Transfer Factors

\begin{tabular}{ccc}
\hline Element & $\begin{array}{c}\text { Crustacea Transfer Factor } \\
\text { concentration in crustacea, } \mathrm{pCi} / \mathrm{kg} / \\
\text { concentration in water, } \mathrm{pCi} / \mathrm{L} \text { ) }\end{array}$ & $\begin{array}{c}\text { Fish Transfer Factor } \\
\text { (concentration in crustacea, } \mathrm{pCi} / \mathrm{kg} / \\
\text { concentration in water, } \mathrm{pCi} / \mathrm{L} \text { ) }\end{array}$ \\
\hline $\mathrm{Ac}$ & 1,000 & \\
$\mathrm{Am}$ & 1,000 & 15 \\
$\mathrm{C}$ & 9,100 & 30 \\
$\mathrm{Co}$ & 200 & 50,000 \\
$\mathrm{Cs}$ & 100 & 300 \\
$\mathrm{I}$ & 5 & 2,000 \\
$\mathrm{~Np}$ & 400 & 40 \\
$\mathrm{~Pa}$ & 110 & 30 \\
$\mathrm{~Pb}$ & 100 & 10 \\
$\mathrm{Po}$ & 20,000 & 300 \\
$\mathrm{Pu}$ & 100 & 100 \\
$\mathrm{Ra}$ & 250 & 30 \\
$\mathrm{Sr}$ & 100 & 50 \\
$\mathrm{Tc}$ & 5 & 60 \\
$\mathrm{Th}$ & 500 & 20 \\
$\mathrm{U}$ & 60 & 100 \\
\hline
\end{tabular}




\section{TABLE B.4 Additional Input Parameters Used in the RESRAD-OFFSITE Deterministic}

Analysis

$\begin{array}{lll}\text { Parameter } & \text { Value } & \text { Comment }\end{array}$

\section{Site Layout $^{\mathrm{a}}$}

$\mathrm{X}$ dimension of primary contamination (m)

Y dimension of primary contamination $(\mathrm{m})$

Smaller X coordinate of the fruit, grain, nonleafy vegetable plots $(\mathrm{m})$

Larger X coordinate of the fruit, grain, nonleafy vegetable plots $(\mathrm{m})$

Smaller Y coordinate of the fruit, grain, nonleafy vegetable plots $(\mathrm{m})$

Larger Y coordinate of the fruit, grain, nonleafy vegetable plots $(\mathrm{m})$

Smaller X coordinate of the leafy vegetable plots (m)

Larger X coordinate of the leafy vegetable plots (m)

Smaller Y coordinate of the leafy vegetable plots (m)

Larger Y coordinate of the leafy vegetable plots (m)

Smaller X coordinate of the pasture, silage growing area $(\mathrm{m})$

Larger X coordinate of the pasture, silage growing area $(\mathrm{m})$

Smaller Y coordinate of the pasture, silage growing area $(\mathrm{m})$

Larger Y coordinate of the pasture, silage growing area $(\mathrm{m})$

Smaller X coordinate of the grain fields (m)

Larger X coordinate of the grain fields (m)

Smaller Y coordinate of the grain fields (m)

Larger Y coordinate of the grain fields (m)

Smaller X coordinate of the dwelling site (m)

Larger X coordinate of the dwelling site (m)

Smaller Y coordinate of the dwelling site (m)

Larger Y coordinate of the dwelling site (m)

Smaller X coordinate of the surface water body

Larger X coordinate of the surface water body
100

100

434.4

466

434

466

434.4

465.6

468

500

450

550

500

600

500

600

600

700

434.4

465.6

400

432

526.5

826.5
Total contaminated area $=10,000 \mathrm{~m}^{2}$

Total contaminated area $=10,000 \mathrm{~m}^{2}$

Closest distance to the site boundary

Assume the vegetable plot is $31.6 \mathrm{~m} \times$ $31.6 \mathrm{~m}$, with an area of $1,000 \mathrm{~m}^{2}$

Closest distance to the site boundary

Assume the vegetable plot is $31.6 \mathrm{~m} \times$ $31.6 \mathrm{~m}$, with an area of $1,000 \mathrm{~m}^{2}$

Closest distance to the site boundary

Assume the vegetable plot is $31.6 \mathrm{~m} \times$ $31.6 \mathrm{~m}$, with an area of $1,000 \mathrm{~m}^{2}$

Leafy vegetable plot is assumed to be next to the nonleafy vegetable plot

Assume the vegetable plot is $31.6 \mathrm{~m} \times$ $31.6 \mathrm{~m}$, with an area of $1,000 \mathrm{~m}^{2}$

Close to the vegetable plots

Assume the livestock feed area is $100 \mathrm{~m}$ $\times 100 \mathrm{~m}$, with an area of $10,000 \mathrm{~m}^{2}$

Close to the vegetable plots

Assume the livestock feed area is $100 \mathrm{~m}$ $\times 100 \mathrm{~m}$, with an area of $10,000 \mathrm{~m}^{2}$

Close to the vegetable plots

Assume the livestock feed area is $100 \mathrm{~m}$ $\times 100 \mathrm{~m}$, with an area of $10,000 \mathrm{~m}^{2}$

Close to the vegetable plots

Assume the livestock feed area is $100 \mathrm{~m}$ $\times 100 \mathrm{~m}$, with an area of $10,000 \mathrm{~m}^{2}$

Closest distance to the site boundary

The dwelling site is assumed to be $31.6 \mathrm{~m} \times 31.6 \mathrm{~m}$ and have an area of $1,000 \mathrm{~m}^{2}$

Closest distance to the site boundary

The dwelling site is assumed to be

$31.6 \mathrm{~m} \times 31.6 \mathrm{~m}$ and have an area of $1,000 \mathrm{~m}^{2}$

The surface water body is assumed to be close to the dwelling site and the vegetable and livestock feed areas

The surface water body is assumed to be close to the dwelling site and the vegetable and livestock feed areas 
TABLE B.4 (Cont.)

Parameter

Smaller Y coordinate of the surface water body

Larger Y coordinate of the surface water body

\section{Source Release and Deposition Velocity}

Deposition velocity $(\mathrm{m} / \mathrm{s})$

\section{Distribution Coefficients}

Sediment in surface water body $\left(\mathrm{cm}^{3} / \mathrm{g}\right)$

Fruit, grain, nonleafy vegetable fields $\left(\mathrm{cm}^{3} / \mathrm{g}\right)$

Leafy vegetable fields $\left(\mathrm{cm}^{3} / \mathrm{g}\right)$

Pasture, silage growing areas $\left(\mathrm{cm}^{3} / \mathrm{g}\right)$

Livestock feed grain fields $\left(\mathrm{cm}^{3} / \mathrm{g}\right)$

Offsite dwelling site $\left(\mathrm{cm}^{3} / \mathrm{g}\right)$

\section{Transfer Factors}

Fruit, grain, nonleafy vegetable transfer factor

Leafy vegetable transfer factor

Pasture and silage transfer factor

Livestock feed grain transfer factor

\section{Storage Time}

Storage time for pasture and silage (d)

Storage time for livestock feed grain (d)

\section{Primary Contamination}

Deposition velocity of dust $(\mathrm{m} / \mathrm{s})$

Rainfall and runoff factor

Slope-length-steepness factor

Cover and management factor

Support practice factor

\section{Contaminated Zone}

Soil erodibility factor of contaminated zone

\section{Clean Cover}

Soil erodibility factor of clean cover

Volumetric water content of clean cover
Value

Comment

356.8

656.8

0.001

For all radionuclides except I-129 (0.01)
Table B.2

Table B.2

Table B.2

Table B.2

Table B.2

Table B.2

Table B.2

Table B. 2

Table B.2

Table B.2

$\begin{array}{cl}1 & \text { Default } \\ 45 & \text { Default }\end{array}$

$0.001 \quad$ Default

160 Default

0.4 Default

0.0506 Adjusted to match site-specific erosion rate

1 Default

$0.4 \quad$ Default

NR No cover

NR No cover

\section{Agriculture/Livestock Feed Growing/Offsite Dwelling Area Parameters}

Fraction of area directly over primary contamination for all fields

Irrigation applied per year for all fields (m/yr)

$0 \quad$ Receptor offsite

Site specific 
Parameter

Evapotranspiration coefficient for all fields

Runoff coefficient for all fields

Depth of soil mixing layer or plow layer for all fields (m)

Volumetric water content for all fields

Dry bulk density of soil for all fields $\left(\mathrm{g} / \mathrm{cm}^{3}\right)$

Soil erodibility factor for all fields

Slope-length-steepness factor for all fields

Cover and management factor for all fields

Support practice factor for all fields

\section{Atmospheric Transport}

Release height (m)

Release heat flux (cal/s)

Anemometer height (m)

Ambient temperature (K)

AM atmospheric mixing height (m)

PM atmospheric mixing height (m)

Dispersion model coefficients

Wind speed terrain

Elevation of offsite location relative to ground level at primary contamination for all fields (m)

Grid spacing for areal integration (m)

Joint frequency of wind speed and stability class for a 16-sector wind rose

\section{Unsaturated Zone Parameters}

Unsaturated zone longitudinal dispersivity (m)

\section{Saturated Zone Hydrological Data}

Thickness of saturated zone (m)

Saturated zone longitudinal dispersivity to surface water body (m)

Saturated zone horizontal lateral dispersivity to surface water body (m)

Disperse vertically

Saturated zone vertical lateral dispersivity to surface water body (m)

Depth of aquifer contributing to surface water body (m)

\section{Surface Water Body}

Sediment deliver ratio

Volume of surface water body $\left(\mathrm{m}^{3}\right)$

Value

Comment

Table B.1

Table B.1

Table B.1

\section{$0.3 \quad$ Default}

Table B.1

$\begin{array}{cl}0.4 & \text { Default } \\ 0.4 & \text { Default } \\ 0.0488 & \text { Adjusted to match site-specific erosion } \\ & \text { rate }\end{array}$

1 Default

$\begin{array}{cl}1 & \text { Default } \\ 0 & \text { Default } \\ 10 & \text { Default } \\ 285 & \text { Default } \\ 400 & \text { Default } \\ 1,600 & \text { Default } \\ \text { Pasquill- } & \text { Default } \\ \text { Gifford } & \\ \text { Rural } & \text { Default } \\ 0 & \text { Default } \\ 10 & \text { Default } \\ \text { Actual values } & \text { Site-specific } \\ \text { from Chicago } & \\ \text { Midway } & \end{array}$

$0.1 \quad$ Default

$\begin{array}{cl}18.3 & \text { Site-specific } \\ 10 & \text { Default } \\ 1 & \text { Default } \\ \text { Check box } & \text { Yes } \\ 0.06 & \text { Default } \\ 10 & \text { Default }\end{array}$




\begin{tabular}{|c|c|c|}
\hline Parameter & Value & Comment \\
\hline $\begin{array}{l}\text { Mean residence time of water in surface water body } \\
(\mathrm{yr})\end{array}$ & 1 & Default \\
\hline \multicolumn{3}{|l|}{ Groundwater Transport Parameters } \\
\hline $\begin{array}{l}\text { Distance from downgradient edge of contamination } \\
\text { to well in the direction parallel to aquifer flow (m) }\end{array}$ & -1 & Site-specific \\
\hline $\begin{array}{l}\text { Distance from downgradient edge of contamination } \\
\text { to surface water body in the direction parallel to } \\
\text { aquifer flow }(\mathrm{m})\end{array}$ & 256.8 & Site-specific \\
\hline $\begin{array}{l}\text { Distance from downgradient edge of contamination } \\
\text { to well in the direction perpendicular to aquifer } \\
\text { flow }(\mathrm{m})\end{array}$ & -424 & Site-specific \\
\hline $\begin{array}{l}\text { Distance from downgradient edge of contamination } \\
\text { to the right edge of surface water body in the } \\
\text { direction perpendicular to aquifer flow }(\mathrm{m})\end{array}$ & -150 & Default \\
\hline $\begin{array}{l}\text { Distance from downgradient edge of contamination } \\
\text { to the left edge of the surface water body in the } \\
\text { direction perpendicular to aquifer flow (m) }\end{array}$ & 150 & Default \\
\hline Main subzones in saturated zone & 1 & Default \\
\hline Main subzones in each partially saturated zone & 1 & Default \\
\hline $\begin{array}{l}\text { Nuclide-specific retardation in all subzones, } \\
\text { longitudinal dispersion in all but the subzone of } \\
\text { transformation? }\end{array}$ & Yes & Default \\
\hline \multicolumn{3}{|l|}{ Water Use } \\
\hline Quantity of water consumed by an individual (L/yr) & Table B.1 & Household water not contaminated \\
\hline $\begin{array}{l}\text { Number of household individuals consuming and } \\
\text { using water }\end{array}$ & 4 & Default \\
\hline $\begin{array}{l}\text { Quantity of water for use indoors of dwelling per } \\
\text { individual (L/d) }\end{array}$ & 225 & Household water not contaminated \\
\hline Quantity of water for beef cattle (L/d) & 50 & Default \\
\hline Number of beef cattle & 2 & Default \\
\hline Quantity of water for dairy cows (L/d) & 160 & Default \\
\hline Number of dairy cows & 2 & Default \\
\hline Well pumping rate $\left(\mathrm{m}^{3} / \mathrm{yr}\right)$ & NR & RESRAD default \\
\hline \multicolumn{3}{|l|}{ Ingestion Rates } \\
\hline Drinking water intake from affected area & 0 & Drinking water not contaminated \\
\hline Fish intake from affected area & 0.5 & Default \\
\hline Crustacea and mollusks intake from affected area & 0.5 & Default \\
\hline $\begin{array}{l}\text { Fruit, grain, nonleafy vegetables fraction from } \\
\text { affected area }\end{array}$ & 0.5 & Default \\
\hline Leafy vegetables fraction from affected area & 0.5 & Default \\
\hline Meat fraction from affected area & 0.5 & $\begin{array}{l}50 \% \text { of the meat consumed is from } \\
\text { contaminated area }\end{array}$ \\
\hline Milk fraction from affected area & 0.5 & $\begin{array}{l}50 \% \text { of the meat consumed is from } \\
\text { contaminated area }\end{array}$ \\
\hline
\end{tabular}




\section{Livestock Intake}

Pasture and silage intake for beef cattle $(\mathrm{kg} / \mathrm{d})$

Grain intake for beef cattle $(\mathrm{kg} / \mathrm{d})$

Soil from pasture and silage intake for beef cattle (kg/d)

Soil from grain intake for beef cattle $(\mathrm{kg} / \mathrm{d})$

Pasture and silage intake for dairy cows $(\mathrm{kg} / \mathrm{d})$

Grain intake for dairy cows $(\mathrm{kg} / \mathrm{d})$

Soil from pasture and silage intake for dairy cows (kg/d)

Soil from grain intake for dairy cows $(\mathrm{kg} / \mathrm{d})$

$\begin{array}{cc}14 & \text { Default } \\ 54 & \text { Default } \\ 0.1 & \text { Default } \\ 0.4 & \text { Default } \\ 44 & \text { Default } \\ 11 & \text { Default } \\ 0.4 & \text { Default } \\ 0.1 & \text { Default }\end{array}$

Livestock Feed Factors (for Pasture and Silage,

Grain)

Wet weight crop yield $\left(\mathrm{kg} / \mathrm{m}^{2}\right)$

Duration of growing season (yr)

Foliage to food transfer coefficient

Weathering removal constant

Foliar interception factor for irrigation

Foliar interception factor for dust deposition

Root depth (m)

$\begin{array}{cc}1.1,0.7 & \text { Default } \\ 0.08,0.17 & \text { Default } \\ 1,0.1 & \text { Default } \\ 20,20 & \text { Default } \\ 0.25,0.25 & \text { Default } \\ 0.25,0.25 & \text { Default } \\ \text { Table B.1 } & \end{array}$

\section{Occupancy Factors}

Indoor time fraction on primary contamination Outdoor time fraction on primary contamination Indoor time fraction on offsite dwelling site Outdoor time fraction on offsite dwelling site

Time fraction in fruit, grain, and nonleafy vegetable fields

Time fraction in leafy vegetable fields $\quad 0.1 \quad$ Default

Time fraction in pasture and silage fields $\quad 0.1 \quad$ Default

Time fraction in livestock grain fields

$0.1 \quad$ Default

a All the areas (dwelling, agricultural areas) are assumed to be less than $350 \mathrm{~m}$ away from the contaminated area. This is a conservative assumption because the distance to the site boundary from the Building 310 area is greater than $350 \mathrm{~m}$. 


\section{REFERENCES FOR APPENDIX B}

Argonne (Argonne National Laboratory), 1996, Interim Action Documentation Report: Decontamination and Demolition of the 317 Area South Vaults, Argonne, Ill., May 31.

Eckerman, K.F., and J.C. Ryman, 1993, External Exposure to Radionuclides in Air, Water, and Soil, Exposure to Dose Coefficients for General Application, Based on the 1987 Federal Radiation Protection Guidance, EPA 402-R-93-076, Federal Guidance Report No. 12, prepared by Oak Ridge National Laboratory, Oak Ridge, Tenn., for U.S. Environmental Protection Agency, Office of Radiation and Indoor Air, Washington, D.C.

EPA (U.S. Environmental Protection Agency), 1997, Exposure Factor Handbook, EPA/600/P-95/002Fa, Office of Research and Development, National Center for Environmental Assessment, Washington, D.C.

Golchert, N.W., T.M. Davis, and L.P. Moos, 2010, Argonne Site Environmental Report for Calendar Year 2009, ANL-10/02, Argonne National Laboratory, Argonne, Ill., September.

ICRP (International Commission on Radiological Protection), 1991, 1990 Recommendations of the International Commission on Radiological Protection, ICRP Publication 60, Ann. ICRP 21(1-3), Pergamon Press, Oxford, England.

ICRP, 1996, Age-Dependent Doses to Members of the Public from Intake of Radionuclides: Part 5, Compilation of Ingestion and Inhalation Coefficients, ICRP Publication 72, Ann. ICRP 26(1), Pergamon Press, Oxford, England.

Kamboj, S., 2011, "Bldg. 310 RESRAD Analysis Project Status," intraoffice personal communication between Kamboj and R.L. Johnson (Argonne National Laboratory, Argonne, Ill.), Jan. 7.

Matton, P.B., 2011, "Bldg. 330 D\&D Project-RESRAD, DCGLs, Clean-up Parameters," intraoffice personal communication between Matton and R.J. Johnson (Argonne National Laboratory, Argonne, Ill.), Jan. 14.

NRC (U.S. Nuclear Regulatory Commission), 2000, Development of Probabilistic RESRAD 6.0 and RESRAD-BUILD 3.0 Computer Codes, NUREG/CR-6697 and ANL/EAD/TM-98, prepared by C. Yu et al., Argonne National Laboratory, Argonne, Ill., for U.S. Nuclear Regulatory Commission, Washington, D.C., Nov.

NRCS (Natural Resources Conservation Service), 2010, "Report Cards Are Out—Illinois NRI Gets Gold Star!," news release, May 25.

Patton, T.L., R.H. Pearl, and S.Y. Tsai, 1990, Hydrological Conditions at the 800 Area at Argonne National Laboratory, ANL/EAIS/TM-29, Argonne National Laboratory, Argonne, Ill., August. 
Quinn, J., 2010, personal communication between Quinn and S. Kamboj (Argonne National Laboratory, Argonne, Ill.), Nov. 12.

Yu, C., et al., 1993, Data Collection Handbook to Support Modeling the Impacts of Radioactive Material in Soil, ANL/EAIS-8, Argonne National Laboratory, Argonne, Ill., April.

Yu, C., et al., 2001, User's Manual for RESRAD Version 6, ANL/EAD-4, Argonne National Laboratory, Argonne, Ill. 


\section{APPENDIX C:}

\section{DETAILED PROBABILISTIC ANALYSIS RESULTS}

Tables C.1-C.14 show the peak DSR percentiles (mrem/yr per pCi/g) for different exposure pathways for the industrial use scenario. RESRAD (onsite) Version 6.5 was used in the analysis. For Co-60, Cs-137, Np-237, Sr-90, U-235, and U-238, external exposure was the dominant exposure pathway. For Am-241, I-129, and Tc-99, soil ingestion and external exposure were the dominant exposure pathways. For C-14, only inhalation was the dominant exposure pathway. For $\mathrm{Pu}-238, \mathrm{Pu}-239$, and $\mathrm{Pu}-240$, soil ingestion and inhalation were two dominant exposure pathways. For U-234, radon inhalation was the dominant exposure pathway.

TABLE C.1 Peak DSR Percentiles (mrem/yr per pCi/g) for Different Exposure Pathways for Am-241 in Industrial Use Scenario

\begin{tabular}{ccccc}
\hline & & & & \\
Percentile & All Pathways & External & $\begin{array}{c}\text { w/o Radon } \\
\text { whal }\end{array}$ & Soil Ingestion \\
\hline & & & & \\
$5 \%$ & $1.47 \mathrm{E}-03$ & $8.66 \mathrm{E}-04$ & $1.86 \mathrm{E}-04$ & $2.76 \mathrm{E}-04$ \\
$10 \%$ & $4.06 \mathrm{E}-03$ & $2.54 \mathrm{E}-03$ & $4.46 \mathrm{E}-04$ & $6.60 \mathrm{E}-04$ \\
$15 \%$ & $6.76 \mathrm{E}-03$ & $4.23 \mathrm{E}-03$ & $6.94 \mathrm{E}-04$ & $1.00 \mathrm{E}-03$ \\
$20 \%$ & $9.42 \mathrm{E}-03$ & $5.89 \mathrm{E}-03$ & $9.35 \mathrm{E}-04$ & $1.33 \mathrm{E}-03$ \\
$25 \%$ & $1.11 \mathrm{E}-02$ & $7.57 \mathrm{E}-03$ & $1.14 \mathrm{E}-03$ & $1.65 \mathrm{E}-03$ \\
$30 \%$ & $1.21 \mathrm{E}-02$ & $7.86 \mathrm{E}-03$ & $1.30 \mathrm{E}-03$ & $1.95 \mathrm{E}-03$ \\
$35 \%$ & $1.27 \mathrm{E}-02$ & $8.15 \mathrm{E}-03$ & $1.44 \mathrm{E}-03$ & $2.27 \mathrm{E}-03$ \\
$40 \%$ & $1.33 \mathrm{E}-02$ & $8.43 \mathrm{E}-03$ & $1.57 \mathrm{E}-03$ & $2.53 \mathrm{E}-03$ \\
$45 \%$ & $1.37 \mathrm{E}-02$ & $8.72 \mathrm{E}-03$ & $1.70 \mathrm{E}-03$ & $2.78 \mathrm{E}-03$ \\
$50 \%$ & $1.43 \mathrm{E}-02$ & $9.01 \mathrm{E}-03$ & $1.82 \mathrm{E}-03$ & $3.02 \mathrm{E}-03$ \\
$55 \%$ & $1.47 \mathrm{E}-02$ & $9.20 \mathrm{E}-03$ & $1.97 \mathrm{E}-03$ & $3.26 \mathrm{E}-03$ \\
$60 \%$ & $1.52 \mathrm{E}-02$ & $9.39 \mathrm{E}-03$ & $2.14 \mathrm{E}-03$ & $3.50 \mathrm{E}-03$ \\
$65 \%$ & $1.57 \mathrm{E}-02$ & $9.58 \mathrm{E}-03$ & $2.34 \mathrm{E}-03$ & $3.73 \mathrm{E}-03$ \\
$70 \%$ & $1.62 \mathrm{E}-02$ & $9.76 \mathrm{E}-03$ & $2.53 \mathrm{E}-03$ & $4.00 \mathrm{E}-03$ \\
$75 \%$ & $1.68 \mathrm{E}-02$ & $9.95 \mathrm{E}-03$ & $2.78 \mathrm{E}-03$ & $4.31 \mathrm{E}-03$ \\
$80 \%$ & $1.75 \mathrm{E}-02$ & $1.05 \mathrm{E}-02$ & $3.07 \mathrm{E}-03$ & $4.64 \mathrm{E}-03$ \\
$85 \%$ & $1.84 \mathrm{E}-02$ & $1.10 \mathrm{E}-02$ & $3.46 \mathrm{E}-03$ & $5.04 \mathrm{E}-03$ \\
$90 \%$ & $1.97 \mathrm{E}-02$ & $1.16 \mathrm{E}-02$ & $3.99 \mathrm{E}-03$ & $5.53 \mathrm{E}-03$ \\
$95 \%$ & $2.19 \mathrm{E}-02$ & $1.23 \mathrm{E}-02$ & $4.87 \mathrm{E}-03$ & $6.27 \mathrm{E}-03$ \\
Mean & $1.35 \mathrm{E}-02$ & $8.26 \mathrm{E}-03$ & $2.11 \mathrm{E}-03$ & $3.09 \mathrm{E}-03$ \\
Min & $1.08 \mathrm{E}-04$ & $7.56 \mathrm{E}-05$ & $1.14 \mathrm{E}-06$ & $5.60 \mathrm{E}-06$ \\
Max & $4.08 \mathrm{E}-02$ & $1.71 \mathrm{E}-02$ & $1.65 \mathrm{E}-02$ & $1.15 \mathrm{E}-02$ \\
\hline & & & & \\
\hline
\end{tabular}


TABLE C.2 Peak DSR Percentiles (mrem/yr per pCi/g) for Different Exposure Pathways for C-14 in Industrial Use Scenario

\begin{tabular}{ccccc}
\hline & & & & \\
Percentile & All Pathways & External & $\begin{array}{c}\text { Inhalation } \\
\text { w/o Radon }\end{array}$ & Soil Ingestion \\
\hline & & & & \\
$5 \%$ & $2.22 \mathrm{E}-06$ & $3.91 \mathrm{E}-08$ & $1.94 \mathrm{E}-06$ & $1.19 \mathrm{E}-07$ \\
$10 \%$ & $5.82 \mathrm{E}-06$ & $1.14 \mathrm{E}-07$ & $5.28 \mathrm{E}-06$ & $2.85 \mathrm{E}-07$ \\
$15 \%$ & $1.02 \mathrm{E}-05$ & $1.89 \mathrm{E}-07$ & $9.27 \mathrm{E}-06$ & $4.35 \mathrm{E}-07$ \\
$20 \%$ & $1.34 \mathrm{E}-05$ & $2.64 \mathrm{E}-07$ & $1.20 \mathrm{E}-05$ & $5.79 \mathrm{E}-07$ \\
$25 \%$ & $1.53 \mathrm{E}-05$ & $3.37 \mathrm{E}-07$ & $1.38 \mathrm{E}-05$ & $7.13 \mathrm{E}-07$ \\
$30 \%$ & $1.68 \mathrm{E}-05$ & $3.51 \mathrm{E}-07$ & $1.50 \mathrm{E}-05$ & $8.45 \mathrm{E}-07$ \\
$35 \%$ & $1.79 \mathrm{E}-05$ & $3.65 \mathrm{E}-07$ & $1.62 \mathrm{E}-05$ & $9.74 \mathrm{E}-07$ \\
$40 \%$ & $1.90 \mathrm{E}-05$ & $3.78 \mathrm{E}-07$ & $1.72 \mathrm{E}-05$ & $1.10 \mathrm{E}-06$ \\
$45 \%$ & $2.02 \mathrm{E}-05$ & $3.90 \mathrm{E}-07$ & $1.84 \mathrm{E}-05$ & $1.20 \mathrm{E}-06$ \\
$50 \%$ & $2.13 \mathrm{E}-05$ & $4.02 \mathrm{E}-07$ & $1.93 \mathrm{E}-05$ & $1.31 \mathrm{E}-06$ \\
$55 \%$ & $2.23 \mathrm{E}-05$ & $4.12 \mathrm{E}-07$ & $2.03 \mathrm{E}-05$ & $1.41 \mathrm{E}-06$ \\
$60 \%$ & $2.34 \mathrm{E}-05$ & $4.21 \mathrm{E}-07$ & $2.14 \mathrm{E}-05$ & $1.52 \mathrm{E}-06$ \\
$65 \%$ & $2.45 \mathrm{E}-05$ & $4.28 \mathrm{E}-07$ & $2.24 \mathrm{E}-05$ & $1.61 \mathrm{E}-06$ \\
$70 \%$ & $2.56 \mathrm{E}-05$ & $4.37 \mathrm{E}-07$ & $2.36 \mathrm{E}-05$ & $1.73 \mathrm{E}-06$ \\
$75 \%$ & $2.70 \mathrm{E}-05$ & $4.47 \mathrm{E}-07$ & $2.50 \mathrm{E}-05$ & $1.86 \mathrm{E}-06$ \\
$80 \%$ & $2.87 \mathrm{E}-05$ & $4.69 \mathrm{E}-07$ & $2.64 \mathrm{E}-05$ & $2.00 \mathrm{E}-06$ \\
$85 \%$ & $3.05 \mathrm{E}-05$ & $4.93 \mathrm{E}-07$ & $2.83 \mathrm{E}-05$ & $2.18 \mathrm{E}-06$ \\
$90 \%$ & $3.32 \mathrm{E}-05$ & $5.18 \mathrm{E}-07$ & $3.11 \mathrm{E}-05$ & $2.39 \mathrm{E}-06$ \\
$95 \%$ & $3.73 \mathrm{E}-05$ & $5.54 \mathrm{E}-07$ & $3.50 \mathrm{E}-05$ & $2.71 \mathrm{E}-06$ \\
Mean & $2.10 \mathrm{E}-05$ & $3.69 \mathrm{E}-07$ & $1.93 \mathrm{E}-05$ & $1.34 \mathrm{E}-06$ \\
Min & $1.45 \mathrm{E}-07$ & $3.37 \mathrm{E}-09$ & $1.35 \mathrm{E}-07$ & $2.41 \mathrm{E}-09$ \\
Max & $7.84 \mathrm{E}-05$ & $7.68 \mathrm{E}-07$ & $7.40 \mathrm{E}-05$ & $5.03 \mathrm{E}-06$ \\
\hline & & & & \\
\hline
\end{tabular}


TABLE C.3 Peak DSR Percentiles (mrem/yr per pCi/g) for Different Exposure Pathways for Co-60 in Industrial Use Scenario

\begin{tabular}{ccccc}
\hline & & & & \\
Percentile & All Pathways & External & whation Radon & Soil Ingestion \\
\hline $5 \%$ & $3.24 \mathrm{E}-01$ & $3.24 \mathrm{E}-01$ & $4.40 \mathrm{E}-08$ & $4.25 \mathrm{E}-06$ \\
$10 \%$ & $9.57 \mathrm{E}-01$ & $9.57 \mathrm{E}-01$ & $1.13 \mathrm{E}-07$ & $1.00 \mathrm{E}-05$ \\
$15 \%$ & $1.59 \mathrm{E}+00$ & $1.59 \mathrm{E}+00$ & $1.92 \mathrm{E}-07$ & $1.62 \mathrm{E}-05$ \\
$20 \%$ & $2.22 \mathrm{E}+00$ & $2.22 \mathrm{E}+00$ & $2.49 \mathrm{E}-07$ & $2.16 \mathrm{E}-05$ \\
$25 \%$ & $2.85 \mathrm{E}+00$ & $2.85 \mathrm{E}+00$ & $3.05 \mathrm{E}-07$ & $2.65 \mathrm{E}-05$ \\
$30 \%$ & $2.96 \mathrm{E}+00$ & $2.96 \mathrm{E}+00$ & $3.48 \mathrm{E}-07$ & $3.12 \mathrm{E}-05$ \\
$35 \%$ & $3.07 \mathrm{E}+00$ & $3.07 \mathrm{E}+00$ & $3.82 \mathrm{E}-07$ & $3.59 \mathrm{E}-05$ \\
$40 \%$ & $3.17 \mathrm{E}+00$ & $3.17 \mathrm{E}+00$ & $4.14 \mathrm{E}-07$ & $4.04 \mathrm{E}-05$ \\
$45 \%$ & $3.28 \mathrm{E}+00$ & $3.28 \mathrm{E}+00$ & $4.44 \mathrm{E}-07$ & $4.48 \mathrm{E}-05$ \\
$50 \%$ & $3.39 \mathrm{E}+00$ & $3.39 \mathrm{E}+00$ & $4.77 \mathrm{E}-07$ & $4.85 \mathrm{E}-05$ \\
$55 \%$ & $3.46 \mathrm{E}+00$ & $3.46 \mathrm{E}+00$ & $5.08 \mathrm{E}-07$ & $5.23 \mathrm{E}-05$ \\
$60 \%$ & $3.53 \mathrm{E}+00$ & $3.53 \mathrm{E}+00$ & $5.39 \mathrm{E}-07$ & $5.60 \mathrm{E}-05$ \\
$65 \%$ & $3.61 \mathrm{E}+00$ & $3.61 \mathrm{E}+00$ & $5.71 \mathrm{E}-07$ & $6.01 \mathrm{E}-05$ \\
$70 \%$ & $3.67 \mathrm{E}+00$ & $3.67 \mathrm{E}+00$ & $6.05 \mathrm{E}-07$ & $6.41 \mathrm{E}-05$ \\
$75 \%$ & $3.75 \mathrm{E}+00$ & $3.75 \mathrm{E}+00$ & $6.40 \mathrm{E}-07$ & $6.90 \mathrm{E}-05$ \\
$80 \%$ & $3.95 \mathrm{E}+00$ & $3.95 \mathrm{E}+00$ & $6.80 \mathrm{E}-07$ & $7.39 \mathrm{E}-05$ \\
$85 \%$ & $4.15 \mathrm{E}+00$ & $4.15 \mathrm{E}+00$ & $7.40 \mathrm{E}-07$ & $8.01 \mathrm{E}-05$ \\
$90 \%$ & $4.36 \mathrm{E}+00$ & $4.36 \mathrm{E}+00$ & $8.31 \mathrm{E}-07$ & $8.74 \mathrm{E}-05$ \\
$95 \%$ & $4.64 \mathrm{E}+00$ & $4.64 \mathrm{E}+00$ & $1.01 \mathrm{E}-06$ & $9.96 \mathrm{E}-05$ \\
Mean & $3.11 \mathrm{E}+00$ & $3.11 \mathrm{E}+00$ & $4.93 \mathrm{E}-07$ & $4.94 \mathrm{E}-05$ \\
Min & $2.83 \mathrm{E}-02$ & $2.83 \mathrm{E}-02$ & $6.67 \mathrm{E}-10$ & $2.93 \mathrm{E}-08$ \\
Max & $6.43 \mathrm{E}+00$ & $6.43 \mathrm{E}+00$ & $2.64 \mathrm{E}-06$ & $1.81 \mathrm{E}-04$ \\
\hline
\end{tabular}


3. TABLE C.4 Peak DSR Percentiles (mrem/yr per pCi/g) for Different Exposure Pathways for Cs-137 in Industrial Use Scenario

\begin{tabular}{rcccc}
\hline & & & & \\
Percentile & All Pathways & External & $\begin{array}{c}\text { Inhation } \\
\text { w/o Radon }\end{array}$ & Soil Ingestion \\
\hline & & & & \\
$5 \%$ & $7.12 \mathrm{E}-02$ & $7.11 \mathrm{E}-02$ & $7.46 \mathrm{E}-08$ & $1.77 \mathrm{E}-05$ \\
$10 \%$ & $2.09 \mathrm{E}-01$ & $2.08 \mathrm{E}-01$ & $1.79 \mathrm{E}-07$ & $4.24 \mathrm{E}-05$ \\
$15 \%$ & $3.47 \mathrm{E}-01$ & $3.47 \mathrm{E}-01$ & $2.79 \mathrm{E}-07$ & $6.46 \mathrm{E}-05$ \\
$20 \%$ & $4.85 \mathrm{E}-01$ & $4.85 \mathrm{E}-01$ & $3.76 \mathrm{E}-07$ & $8.58 \mathrm{E}-05$ \\
$25 \%$ & $6.22 \mathrm{E}-01$ & $6.22 \mathrm{E}-01$ & $4.58 \mathrm{E}-07$ & $1.06 \mathrm{E}-04$ \\
$30 \%$ & $6.46 \mathrm{E}-01$ & $6.46 \mathrm{E}-01$ & $5.23 \mathrm{E}-07$ & $1.26 \mathrm{E}-04$ \\
$35 \%$ & $6.70 \mathrm{E}-01$ & $6.69 \mathrm{E}-01$ & $5.77 \mathrm{E}-07$ & $1.46 \mathrm{E}-04$ \\
$40 \%$ & $6.93 \mathrm{E}-01$ & $6.93 \mathrm{E}-01$ & $6.30 \mathrm{E}-07$ & $1.63 \mathrm{E}-04$ \\
$45 \%$ & $7.17 \mathrm{E}-01$ & $7.17 \mathrm{E}-01$ & $6.84 \mathrm{E}-07$ & $1.79 \mathrm{E}-04$ \\
$50 \%$ & $7.41 \mathrm{E}-01$ & $7.40 \mathrm{E}-01$ & $7.32 \mathrm{E}-07$ & $1.94 \mathrm{E}-04$ \\
$55 \%$ & $7.56 \mathrm{E}-01$ & $7.56 \mathrm{E}-01$ & $7.90 \mathrm{E}-07$ & $2.09 \mathrm{E}-04$ \\
$60 \%$ & $7.71 \mathrm{E}-01$ & $7.71 \mathrm{E}-01$ & $8.60 \mathrm{E}-07$ & $2.25 \mathrm{E}-04$ \\
$65 \%$ & $7.87 \mathrm{E}-01$ & $7.87 \mathrm{E}-01$ & $9.37 \mathrm{E}-07$ & $2.40 \mathrm{E}-04$ \\
$70 \%$ & $8.02 \mathrm{E}-01$ & $8.02 \mathrm{E}-01$ & $1.01 \mathrm{E}-06$ & $2.58 \mathrm{E}-04$ \\
$75 \%$ & $8.18 \mathrm{E}-01$ & $8.17 \mathrm{E}-01$ & $1.12 \mathrm{E}-06$ & $2.78 \mathrm{E}-04$ \\
$80 \%$ & $8.62 \mathrm{E}-01$ & $8.62 \mathrm{E}-01$ & $1.23 \mathrm{E}-06$ & $2.98 \mathrm{E}-04$ \\
$85 \%$ & $9.06 \mathrm{E}-01$ & $9.06 \mathrm{E}-01$ & $1.39 \mathrm{E}-06$ & $3.24 \mathrm{E}-04$ \\
$90 \%$ & $9.51 \mathrm{E}-01$ & $9.51 \mathrm{E}-01$ & $1.60 \mathrm{E}-06$ & $3.56 \mathrm{E}-04$ \\
$95 \%$ & $1.01 \mathrm{E}+00$ & $1.01 \mathrm{E}+00$ & $1.96 \mathrm{E}-06$ & $4.03 \mathrm{E}-04$ \\
Mean & $6.78 \mathrm{E}-01$ & $6.78 \mathrm{E}-01$ & $8.46 \mathrm{E}-07$ & $1.99 \mathrm{E}-04$ \\
Min & $6.21 \mathrm{E}-03$ & $6.20 \mathrm{E}-03$ & $4.58 \mathrm{E}-10$ & $3.60 \mathrm{E}-07$ \\
Max & $1.40 \mathrm{E}+00$ & $1.40 \mathrm{E}+00$ & $6.61 \mathrm{E}-06$ & $7.42 \mathrm{E}-04$ \\
\hline & & & & \\
\hline
\end{tabular}


TABLE C.5 Peak DSR Percentiles (mrem/yr per pCi/g) for Different Exposure Pathways for I-129 in Industrial Use Scenario

\begin{tabular}{ccccc}
\hline & & & & \\
Percentile & All Pathways & External & $\begin{array}{c}\text { Inhalation } \\
\text { w/o Radon }\end{array}$ & Soil Ingestion \\
\hline $5 \%$ & $3.70 \mathrm{E}-04$ & $2.14 \mathrm{E}-04$ & $1.36 \mathrm{E}-07$ & $1.32 \mathrm{E}-04$ \\
$10 \%$ & $1.09 \mathrm{E}-03$ & $6.26 \mathrm{E}-04$ & $3.48 \mathrm{E}-07$ & $3.24 \mathrm{E}-04$ \\
$15 \%$ & $1.77 \mathrm{E}-03$ & $1.04 \mathrm{E}-03$ & $5.84 \mathrm{E}-07$ & $5.25 \mathrm{E}-04$ \\
$20 \%$ & $2.37 \mathrm{E}-03$ & $1.43 \mathrm{E}-03$ & $7.66 \mathrm{E}-07$ & $6.95 \mathrm{E}-04$ \\
$25 \%$ & $2.75 \mathrm{E}-03$ & $1.75 \mathrm{E}-03$ & $9.34 \mathrm{E}-07$ & $8.48 \mathrm{E}-04$ \\
$30 \%$ & $3.02 \mathrm{E}-03$ & $1.91 \mathrm{E}-03$ & $1.06 \mathrm{E}-06$ & $1.00 \mathrm{E}-03$ \\
$35 \%$ & $3.25 \mathrm{E}-03$ & $2.01 \mathrm{E}-03$ & $1.17 \mathrm{E}-06$ & $1.14 \mathrm{E}-03$ \\
$40 \%$ & $3.45 \mathrm{E}-03$ & $2.08 \mathrm{E}-03$ & $1.28 \mathrm{E}-06$ & $1.29 \mathrm{E}-03$ \\
$45 \%$ & $3.65 \mathrm{E}-03$ & $2.14 \mathrm{E}-03$ & $1.36 \mathrm{E}-06$ & $1.44 \mathrm{E}-03$ \\
$50 \%$ & $3.82 \mathrm{E}-03$ & $2.20 \mathrm{E}-03$ & $1.46 \mathrm{E}-06$ & $1.56 \mathrm{E}-03$ \\
$55 \%$ & $3.98 \mathrm{E}-03$ & $2.27 \mathrm{E}-03$ & $1.56 \mathrm{E}-06$ & $1.69 \mathrm{E}-03$ \\
$60 \%$ & $4.13 \mathrm{E}-03$ & $2.33 \mathrm{E}-03$ & $1.65 \mathrm{E}-06$ & $1.81 \mathrm{E}-03$ \\
$65 \%$ & $4.29 \mathrm{E}-03$ & $2.39 \mathrm{E}-03$ & $1.76 \mathrm{E}-06$ & $1.92 \mathrm{E}-03$ \\
$70 \%$ & $4.46 \mathrm{E}-03$ & $2.45 \mathrm{E}-03$ & $1.86 \mathrm{E}-06$ & $2.07 \mathrm{E}-03$ \\
$75 \%$ & $4.67 \mathrm{E}-03$ & $2.51 \mathrm{E}-03$ & $1.97 \mathrm{E}-06$ & $2.22 \mathrm{E}-03$ \\
$80 \%$ & $4.90 \mathrm{E}-03$ & $2.61 \mathrm{E}-03$ & $2.11 \mathrm{E}-06$ & $2.39 \mathrm{E}-03$ \\
$85 \%$ & $5.15 \mathrm{E}-03$ & $2.74 \mathrm{E}-03$ & $2.30 \mathrm{E}-06$ & $2.59 \mathrm{E}-03$ \\
$90 \%$ & $5.50 \mathrm{E}-03$ & $2.91 \mathrm{E}-03$ & $2.57 \mathrm{E}-06$ & $2.83 \mathrm{E}-03$ \\
$95 \%$ & $6.15 \mathrm{E}-03$ & $3.16 \mathrm{E}-03$ & $3.15 \mathrm{E}-06$ & $3.24 \mathrm{E}-03$ \\
Mean & $3.64 \mathrm{E}-03$ & $2.04 \mathrm{E}-03$ & $1.52 \mathrm{E}-06$ & $1.59 \mathrm{E}-03$ \\
Min & $2.31 \mathrm{E}-05$ & $1.68 \mathrm{E}-05$ & $2.02 \mathrm{E}-09$ & $9.66 \mathrm{E}-07$ \\
Max & $1.01 \mathrm{E}-02$ & $4.47 \mathrm{E}-03$ & $8.27 \mathrm{E}-06$ & $5.91 \mathrm{E}-03$ \\
\hline & & & & \\
\hline
\end{tabular}


TABLE C.6 Peak DSR Percentiles (mrem/yr per pCi/g) for Different Exposure Pathways for Np-237 in Industrial Use Scenario

\begin{tabular}{ccccc}
\hline & & & & \\
Percentile & All Pathways & External & $\begin{array}{c}\text { Inhation } \\
\text { w/o Radon }\end{array}$ & Soil Ingestion \\
\hline $5 \%$ & $2.26 \mathrm{E}-02$ & $2.25 \mathrm{E}-02$ & $7.84 \mathrm{E}-05$ & $1.46 \mathrm{E}-04$ \\
$10 \%$ & $6.53 \mathrm{E}-02$ & $6.43 \mathrm{E}-02$ & $1.87 \mathrm{E}-04$ & $3.48 \mathrm{E}-04$ \\
$15 \%$ & $1.10 \mathrm{E}-01$ & $1.08 \mathrm{E}-01$ & $3.09 \mathrm{E}-04$ & $5.26 \mathrm{E}-04$ \\
$20 \%$ & $1.50 \mathrm{E}-01$ & $1.48 \mathrm{E}-01$ & $4.03 \mathrm{E}-04$ & $7.10 \mathrm{E}-04$ \\
$25 \%$ & $1.82 \mathrm{E}-01$ & $1.79 \mathrm{E}-01$ & $4.85 \mathrm{E}-04$ & $8.82 \mathrm{E}-04$ \\
$30 \%$ & $2.03 \mathrm{E}-01$ & $2.00 \mathrm{E}-01$ & $5.58 \mathrm{E}-04$ & $1.04 \mathrm{E}-03$ \\
$35 \%$ & $2.11 \mathrm{E}-01$ & $2.08 \mathrm{E}-01$ & $6.27 \mathrm{E}-04$ & $1.21 \mathrm{E}-03$ \\
$40 \%$ & $2.19 \mathrm{E}-01$ & $2.16 \mathrm{E}-01$ & $6.85 \mathrm{E}-04$ & $1.35 \mathrm{E}-03$ \\
$45 \%$ & $2.26 \mathrm{E}-01$ & $2.24 \mathrm{E}-01$ & $7.37 \mathrm{E}-04$ & $1.47 \mathrm{E}-03$ \\
$50 \%$ & $2.33 \mathrm{E}-01$ & $2.30 \mathrm{E}-01$ & $7.92 \mathrm{E}-04$ & $1.61 \mathrm{E}-03$ \\
$55 \%$ & $2.41 \mathrm{E}-01$ & $2.38 \mathrm{E}-01$ & $8.42 \mathrm{E}-04$ & $1.75 \mathrm{E}-03$ \\
$60 \%$ & $2.47 \mathrm{E}-01$ & $2.44 \mathrm{E}-01$ & $8.95 \mathrm{E}-04$ & $1.88 \mathrm{E}-03$ \\
$65 \%$ & $2.52 \mathrm{E}-01$ & $2.49 \mathrm{E}-01$ & $9.49 \mathrm{E}-04$ & $2.01 \mathrm{E}-03$ \\
$70 \%$ & $2.57 \mathrm{E}-01$ & $2.55 \mathrm{E}-01$ & $1.00 \mathrm{E}-03$ & $2.17 \mathrm{E}-03$ \\
$75 \%$ & $2.64 \mathrm{E}-01$ & $2.61 \mathrm{E}-01$ & $1.07 \mathrm{E}-03$ & $2.31 \mathrm{E}-03$ \\
$80 \%$ & $2.73 \mathrm{E}-01$ & $2.70 \mathrm{E}-01$ & $1.15 \mathrm{E}-03$ & $2.52 \mathrm{E}-03$ \\
$85 \%$ & $2.91 \mathrm{E}-01$ & $2.87 \mathrm{E}-01$ & $1.26 \mathrm{E}-03$ & $2.73 \mathrm{E}-03$ \\
$90 \%$ & $3.07 \mathrm{E}-01$ & $3.03 \mathrm{E}-01$ & $1.40 \mathrm{E}-03$ & $3.01 \mathrm{E}-03$ \\
$95 \%$ & $3.28 \mathrm{E}-01$ & $3.24 \mathrm{E}-01$ & $1.68 \mathrm{E}-03$ & $3.43 \mathrm{E}-03$ \\
Mean & $2.15 \mathrm{E}-01$ & $2.13 \mathrm{E}-01$ & $8.19 \mathrm{E}-04$ & $1.66 \mathrm{E}-03$ \\
Min & $1.84 \mathrm{E}-03$ & $1.83 \mathrm{E}-03$ & $2.39 \mathrm{E}-06$ & $6.32 \mathrm{E}-06$ \\
Max & $4.63 \mathrm{E}-01$ & $4.55 \mathrm{E}-01$ & $4.12 \mathrm{E}-03$ & $6.46 \mathrm{E}-03$ \\
\hline & & & &
\end{tabular}


TABLE C.7 Peak DSR Percentiles (mrem/yr per pCi/g) for Different Exposure Pathways for Pu-238 in Industrial Use Scenario

\begin{tabular}{cccccc}
\hline & & & & \\
Percentile & All Pathways & External & w/o Radon & Radon (WI) & Soil Ingestion \\
\hline & & & & & \\
$5 \%$ & $6.12 \mathrm{E}-04$ & $2.76 \mathrm{E}-06$ & $2.13 \mathrm{E}-04$ & $1.01 \mathrm{E}-11$ & $3.16 \mathrm{E}-04$ \\
$10 \%$ & $1.65 \mathrm{E}-03$ & $8.08 \mathrm{E}-06$ & $5.09 \mathrm{E}-04$ & $2.50 \mathrm{E}-11$ & $7.56 \mathrm{E}-04$ \\
$15 \%$ & $2.51 \mathrm{E}-03$ & $1.35 \mathrm{E}-05$ & $7.94 \mathrm{E}-04$ & $4.50 \mathrm{E}-11$ & $1.15 \mathrm{E}-03$ \\
$20 \%$ & $3.24 \mathrm{E}-03$ & $1.88 \mathrm{E}-05$ & $1.07 \mathrm{E}-03$ & $7.21 \mathrm{E}-11$ & $1.53 \mathrm{E}-03$ \\
$25 \%$ & $3.85 \mathrm{E}-03$ & $2.41 \mathrm{E}-05$ & $1.30 \mathrm{E}-03$ & $9.43 \mathrm{E}-11$ & $1.89 \mathrm{E}-03$ \\
$30 \%$ & $4.36 \mathrm{E}-03$ & $2.50 \mathrm{E}-05$ & $1.49 \mathrm{E}-03$ & $1.19 \mathrm{E}-10$ & $2.24 \mathrm{E}-03$ \\
$35 \%$ & $4.81 \mathrm{E}-03$ & $2.60 \mathrm{E}-05$ & $1.64 \mathrm{E}-03$ & $1.41 \mathrm{E}-10$ & $2.60 \mathrm{E}-03$ \\
$40 \%$ & $5.21 \mathrm{E}-03$ & $2.69 \mathrm{E}-05$ & $1.79 \mathrm{E}-03$ & $1.62 \mathrm{E}-10$ & $2.91 \mathrm{E}-03$ \\
$45 \%$ & $5.56 \mathrm{E}-03$ & $2.78 \mathrm{E}-05$ & $1.95 \mathrm{E}-03$ & $1.81 \mathrm{E}-10$ & $3.19 \mathrm{E}-03$ \\
$50 \%$ & $5.92 \mathrm{E}-03$ & $2.87 \mathrm{E}-05$ & $2.08 \mathrm{E}-03$ & $2.03 \mathrm{E}-10$ & $3.46 \mathrm{E}-03$ \\
$55 \%$ & $6.27 \mathrm{E}-03$ & $2.93 \mathrm{E}-05$ & $2.25 \mathrm{E}-03$ & $2.24 \mathrm{E}-10$ & $3.73 \mathrm{E}-03$ \\
$60 \%$ & $6.65 \mathrm{E}-03$ & $2.99 \mathrm{E}-05$ & $2.45 \mathrm{E}-03$ & $2.48 \mathrm{E}-10$ & $4.02 \mathrm{E}-03$ \\
$65 \%$ & $7.07 \mathrm{E}-03$ & $3.05 \mathrm{E}-05$ & $2.67 \mathrm{E}-03$ & $2.74 \mathrm{E}-10$ & $4.27 \mathrm{E}-03$ \\
$70 \%$ & $7.54 \mathrm{E}-03$ & $3.11 \mathrm{E}-05$ & $2.89 \mathrm{E}-03$ & $3.05 \mathrm{E}-10$ & $4.58 \mathrm{E}-03$ \\
$75 \%$ & $7.97 \mathrm{E}-03$ & $3.17 \mathrm{E}-05$ & $3.18 \mathrm{E}-03$ & $3.37 \mathrm{E}-10$ & $4.95 \mathrm{E}-03$ \\
$80 \%$ & $8.46 \mathrm{E}-03$ & $3.34 \mathrm{E}-05$ & $3.52 \mathrm{E}-03$ & $3.76 \mathrm{E}-10$ & $5.32 \mathrm{E}-03$ \\
$85 \%$ & $9.12 \mathrm{E}-03$ & $3.52 \mathrm{E}-05$ & $3.95 \mathrm{E}-03$ & $4.23 \mathrm{E}-10$ & $5.77 \mathrm{E}-03$ \\
$90 \%$ & $1.00 \mathrm{E}-02$ & $3.69 \mathrm{E}-05$ & $4.57 \mathrm{E}-03$ & $4.90 \mathrm{E}-10$ & $6.33 \mathrm{E}-03$ \\
$95 \%$ & $1.13 \mathrm{E}-02$ & $3.93 \mathrm{E}-05$ & $5.57 \mathrm{E}-03$ & $6.08 \mathrm{E}-10$ & $7.19 \mathrm{E}-03$ \\
Mean & $5.98 \mathrm{E}-03$ & $2.63 \mathrm{E}-05$ & $2.41 \mathrm{E}-03$ & $2.41 \mathrm{E}-10$ & $3.54 \mathrm{E}-03$ \\
Min & $2.74 \mathrm{E}-05$ & $2.41 \mathrm{E}-07$ & $1.30 \mathrm{E}-06$ & $9.48 \mathrm{E}-14$ & $6.42 \mathrm{E}-06$ \\
Max & $2.76 \mathrm{E}-02$ & $5.44 \mathrm{E}-05$ & $1.88 \mathrm{E}-02$ & $2.03 \mathrm{E}-09$ & $1.32 \mathrm{E}-02$ \\
\hline & & & & & \\
\hline & & & &
\end{tabular}


TABLE C.8 Peak DSR Percentiles (mrem/yr per pCi/g) for Different Exposure Pathways for Pu-239 in Industrial Use Scenario

\begin{tabular}{ccccc}
\hline & & & & \\
Percentile & All Pathways & External & $\begin{array}{c}\text { Inhation } \\
\text { w/o Radon }\end{array}$ & Soil Ingestion \\
\hline & & & & \\
$5 \%$ & $6.73 \mathrm{E}-04$ & $6.04 \mathrm{E}-06$ & $2.33 \mathrm{E}-04$ & $3.45 \mathrm{E}-04$ \\
$10 \%$ & $1.81 \mathrm{E}-03$ & $1.77 \mathrm{E}-05$ & $5.58 \mathrm{E}-04$ & $8.25 \mathrm{E}-04$ \\
$15 \%$ & $2.76 \mathrm{E}-03$ & $2.94 \mathrm{E}-05$ & $8.69 \mathrm{E}-04$ & $1.26 \mathrm{E}-03$ \\
$20 \%$ & $3.57 \mathrm{E}-03$ & $4.12 \mathrm{E}-05$ & $1.17 \mathrm{E}-03$ & $1.67 \mathrm{E}-03$ \\
$25 \%$ & $4.24 \mathrm{E}-03$ & $5.28 \mathrm{E}-05$ & $1.43 \mathrm{E}-03$ & $2.06 \mathrm{E}-03$ \\
$30 \%$ & $4.79 \mathrm{E}-03$ & $5.48 \mathrm{E}-05$ & $1.63 \mathrm{E}-03$ & $2.44 \mathrm{E}-03$ \\
$35 \%$ & $5.27 \mathrm{E}-03$ & $5.68 \mathrm{E}-05$ & $1.80 \mathrm{E}-03$ & $2.84 \mathrm{E}-03$ \\
$40 \%$ & $5.72 \mathrm{E}-03$ & $5.88 \mathrm{E}-05$ & $1.97 \mathrm{E}-03$ & $3.17 \mathrm{E}-03$ \\
$45 \%$ & $6.11 \mathrm{E}-03$ & $6.08 \mathrm{E}-05$ & $2.13 \mathrm{E}-03$ & $3.48 \mathrm{E}-03$ \\
$50 \%$ & $6.51 \mathrm{E}-03$ & $6.28 \mathrm{E}-05$ & $2.28 \mathrm{E}-03$ & $3.78 \mathrm{E}-03$ \\
$55 \%$ & $6.89 \mathrm{E}-03$ & $6.41 \mathrm{E}-05$ & $2.46 \mathrm{E}-03$ & $4.07 \mathrm{E}-03$ \\
$60 \%$ & $7.30 \mathrm{E}-03$ & $6.54 \mathrm{E}-05$ & $2.68 \mathrm{E}-03$ & $4.39 \mathrm{E}-03$ \\
$65 \%$ & $7.76 \mathrm{E}-03$ & $6.67 \mathrm{E}-05$ & $2.92 \mathrm{E}-03$ & $4.66 \mathrm{E}-03$ \\
$70 \%$ & $8.27 \mathrm{E}-03$ & $6.81 \mathrm{E}-05$ & $3.16 \mathrm{E}-03$ & $5.01 \mathrm{E}-03$ \\
$75 \%$ & $8.75 \mathrm{E}-03$ & $6.94 \mathrm{E}-05$ & $3.49 \mathrm{E}-03$ & $5.40 \mathrm{E}-03$ \\
$80 \%$ & $9.28 \mathrm{E}-03$ & $7.31 \mathrm{E}-05$ & $3.85 \mathrm{E}-03$ & $5.81 \mathrm{E}-03$ \\
$85 \%$ & $1.00 \mathrm{E}-02$ & $7.69 \mathrm{E}-05$ & $4.32 \mathrm{E}-03$ & $6.30 \mathrm{E}-03$ \\
$90 \%$ & $1.10 \mathrm{E}-02$ & $8.07 \mathrm{E}-05$ & $5.00 \mathrm{E}-03$ & $6.91 \mathrm{E}-03$ \\
$95 \%$ & $1.24 \mathrm{E}-02$ & $8.59 \mathrm{E}-05$ & $6.10 \mathrm{E}-03$ & $7.85 \mathrm{E}-03$ \\
Mean & $6.56 \mathrm{E}-03$ & $5.75 \mathrm{E}-05$ & $2.64 \mathrm{E}-03$ & $3.87 \mathrm{E}-03$ \\
Min & $3.02 \mathrm{E}-05$ & $5.27 \mathrm{E}-07$ & $1.43 \mathrm{E}-06$ & $7.00 \mathrm{E}-06$ \\
Max & $3.02 \mathrm{E}-02$ & $1.19 \mathrm{E}-04$ & $2.06 \mathrm{E}-02$ & $1.44 \mathrm{E}-02$ \\
\hline & & & & \\
\hline
\end{tabular}


TABLE C.9 Peak DSR Percentiles (mrem/yr per pCi/g) for Different Exposure Pathways for Pu-240 in Industrial Use Scenario

\begin{tabular}{rrrrrr}
\hline & & & & & \\
Percentile & All Pathways & External & Inhation & & \\
& & & & \\
& & & & & \\
$5 \%$ & $6.44 \mathrm{E}-04$ & $2.66 \mathrm{E}-06$ & $1.84 \mathrm{E}-04$ & $6.30 \mathrm{E}-16$ & $3.43 \mathrm{E}-04$ \\
$10 \%$ & $1.61 \mathrm{E}-03$ & $7.84 \mathrm{E}-06$ & $4.81 \mathrm{E}-04$ & $1.83 \mathrm{E}-15$ & $8.10 \mathrm{E}-04$ \\
$15 \%$ & $2.49 \mathrm{E}-03$ & $1.30 \mathrm{E}-05$ & $7.80 \mathrm{E}-04$ & $3.90 \mathrm{E}-15$ & $1.27 \mathrm{E}-03$ \\
$20 \%$ & $3.28 \mathrm{E}-03$ & $1.82 \mathrm{E}-05$ & $1.01 \mathrm{E}-03$ & $6.55 \mathrm{E}-15$ & $1.70 \mathrm{E}-03$ \\
$25 \%$ & $3.80 \mathrm{E}-03$ & $2.33 \mathrm{E}-05$ & $1.22 \mathrm{E}-03$ & $1.07 \mathrm{E}-14$ & $2.06 \mathrm{E}-03$ \\
$30 \%$ & $4.33 \mathrm{E}-03$ & $2.42 \mathrm{E}-05$ & $1.41 \mathrm{E}-03$ & $1.64 \mathrm{E}-14$ & $2.41 \mathrm{E}-03$ \\
$35 \%$ & $4.80 \mathrm{E}-03$ & $2.51 \mathrm{E}-05$ & $1.57 \mathrm{E}-03$ & $2.36 \mathrm{E}-14$ & $2.81 \mathrm{E}-03$ \\
$40 \%$ & $5.22 \mathrm{E}-03$ & $2.60 \mathrm{E}-05$ & $1.69 \mathrm{E}-03$ & $3.12 \mathrm{E}-14$ & $3.16 \mathrm{E}-03$ \\
$45 \%$ & $5.60 \mathrm{E}-03$ & $2.69 \mathrm{E}-05$ & $1.83 \mathrm{E}-03$ & $4.10 \mathrm{E}-14$ & $3.48 \mathrm{E}-03$ \\
$50 \%$ & $5.97 \mathrm{E}-03$ & $2.78 \mathrm{E}-05$ & $1.95 \mathrm{E}-03$ & $5.13 \mathrm{E}-14$ & $3.78 \mathrm{E}-03$ \\
$55 \%$ & $6.29 \mathrm{E}-03$ & $2.83 \mathrm{E}-05$ & $2.08 \mathrm{E}-03$ & $6.20 \mathrm{E}-14$ & $4.08 \mathrm{E}-03$ \\
$60 \%$ & $6.67 \mathrm{E}-03$ & $2.89 \mathrm{E}-05$ & $2.21 \mathrm{E}-03$ & $7.58 \mathrm{E}-14$ & $4.38 \mathrm{E}-03$ \\
$65 \%$ & $7.05 \mathrm{E}-03$ & $2.95 \mathrm{E}-05$ & $2.36 \mathrm{E}-03$ & $8.98 \mathrm{E}-14$ & $4.69 \mathrm{E}-03$ \\
$70 \%$ & $7.48 \mathrm{E}-03$ & $3.01 \mathrm{E}-05$ & $2.50 \mathrm{E}-03$ & $1.02 \mathrm{E}-13$ & $5.05 \mathrm{E}-03$ \\
$75 \%$ & $7.90 \mathrm{E}-03$ & $3.07 \mathrm{E}-05$ & $2.67 \mathrm{E}-03$ & $1.17 \mathrm{E}-13$ & $5.39 \mathrm{E}-03$ \\
$80 \%$ & $8.37 \mathrm{E}-03$ & $3.23 \mathrm{E}-05$ & $2.87 \mathrm{E}-03$ & $1.30 \mathrm{E}-13$ & $5.81 \mathrm{E}-03$ \\
$85 \%$ & $8.94 \mathrm{E}-03$ & $3.40 \mathrm{E}-05$ & $3.12 \mathrm{E}-03$ & $1.43 \mathrm{E}-13$ & $6.25 \mathrm{E}-03$ \\
$90 \%$ & $9.68 \mathrm{E}-03$ & $3.56 \mathrm{E}-05$ & $3.50 \mathrm{E}-03$ & $1.60 \mathrm{E}-13$ & $6.91 \mathrm{E}-03$ \\
$95 \%$ & $1.09 \mathrm{E}-02$ & $3.80 \mathrm{E}-05$ & $4.15 \mathrm{E}-03$ & $1.80 \mathrm{E}-13$ & $7.79 \mathrm{E}-03$ \\
Mean & $5.92 \mathrm{E}-03$ & $2.54 \mathrm{E}-05$ & $2.03 \mathrm{E}-03$ & $6.80 \mathrm{E}-14$ & $3.87 \mathrm{E}-03$ \\
Min & $3.55 \mathrm{E}-05$ & $2.36 \mathrm{E}-07$ & $2.01 \mathrm{E}-06$ & $6.14 \mathrm{E}-19$ & $1.51 \mathrm{E}-06$ \\
Max & $2.07 \mathrm{E}-02$ & $5.25 \mathrm{E}-05$ & $9.58 \mathrm{E}-03$ & $3.48 \mathrm{E}-13$ & $1.53 \mathrm{E}-02$ \\
\hline & & & & &
\end{tabular}


TABLE C.10 Peak DSR Percentiles (mrem/yr per pCi/g) for Different Exposure Pathways for Sr-90 in Industrial Use Scenario

\begin{tabular}{ccccc}
\hline & & & & \\
Percentile & All Pathways & External & $\begin{array}{c}\text { Inhation } \\
\text { w/o Radon }\end{array}$ & Soil Ingestion \\
\hline & & & & \\
$5 \%$ & $9.62 \mathrm{E}-04$ & $9.03 \mathrm{E}-04$ & $3.08 \mathrm{E}-07$ & $4.18 \mathrm{E}-05$ \\
$10 \%$ & $2.84 \mathrm{E}-03$ & $2.67 \mathrm{E}-03$ & $7.35 \mathrm{E}-07$ & $1.00 \mathrm{E}-04$ \\
$15 \%$ & $4.65 \mathrm{E}-03$ & $4.42 \mathrm{E}-03$ & $1.15 \mathrm{E}-06$ & $1.53 \mathrm{E}-04$ \\
$20 \%$ & $6.54 \mathrm{E}-03$ & $6.18 \mathrm{E}-03$ & $1.55 \mathrm{E}-06$ & $2.02 \mathrm{E}-04$ \\
$25 \%$ & $8.25 \mathrm{E}-03$ & $7.91 \mathrm{E}-03$ & $1.89 \mathrm{E}-06$ & $2.51 \mathrm{E}-04$ \\
$30 \%$ & $8.72 \mathrm{E}-03$ & $8.27 \mathrm{E}-03$ & $2.16 \mathrm{E}-06$ & $2.96 \mathrm{E}-04$ \\
$35 \%$ & $9.02 \mathrm{E}-03$ & $8.58 \mathrm{E}-03$ & $2.38 \mathrm{E}-06$ & $3.44 \mathrm{E}-04$ \\
$40 \%$ & $9.35 \mathrm{E}-03$ & $8.86 \mathrm{E}-03$ & $2.60 \mathrm{E}-06$ & $3.84 \mathrm{E}-04$ \\
$45 \%$ & $9.66 \mathrm{E}-03$ & $9.17 \mathrm{E}-03$ & $2.82 \mathrm{E}-06$ & $4.22 \mathrm{E}-04$ \\
$50 \%$ & $9.94 \mathrm{E}-03$ & $9.47 \mathrm{E}-03$ & $3.03 \mathrm{E}-06$ & $4.57 \mathrm{E}-04$ \\
$55 \%$ & $1.02 \mathrm{E}-02$ & $9.68 \mathrm{E}-03$ & $3.26 \mathrm{E}-06$ & $4.94 \mathrm{E}-04$ \\
$60 \%$ & $1.04 \mathrm{E}-02$ & $9.87 \mathrm{E}-03$ & $3.55 \mathrm{E}-06$ & $5.32 \mathrm{E}-04$ \\
$65 \%$ & $1.06 \mathrm{E}-02$ & $1.01 \mathrm{E}-02$ & $3.88 \mathrm{E}-06$ & $5.66 \mathrm{E}-04$ \\
$70 \%$ & $1.09 \mathrm{E}-02$ & $1.03 \mathrm{E}-02$ & $4.20 \mathrm{E}-06$ & $6.07 \mathrm{E}-04$ \\
$75 \%$ & $1.12 \mathrm{E}-02$ & $1.05 \mathrm{E}-02$ & $4.63 \mathrm{E}-06$ & $6.54 \mathrm{E}-04$ \\
$80 \%$ & $1.16 \mathrm{E}-02$ & $1.10 \mathrm{E}-02$ & $5.11 \mathrm{E}-06$ & $7.04 \mathrm{E}-04$ \\
$85 \%$ & $1.22 \mathrm{E}-02$ & $1.16 \mathrm{E}-02$ & $5.71 \mathrm{E}-06$ & $7.62 \mathrm{E}-04$ \\
$90 \%$ & $1.29 \mathrm{E}-02$ & $1.22 \mathrm{E}-02$ & $6.63 \mathrm{E}-06$ & $8.34 \mathrm{E}-04$ \\
$95 \%$ & $1.38 \mathrm{E}-02$ & $1.30 \mathrm{E}-02$ & $8.09 \mathrm{E}-06$ & $9.52 \mathrm{E}-04$ \\
Mean & $9.15 \mathrm{E}-03$ & $8.68 \mathrm{E}-03$ & $3.49 \mathrm{E}-06$ & $4.69 \mathrm{E}-04$ \\
Min & $7.80 \mathrm{E}-05$ & $7.57 \mathrm{E}-05$ & $1.90 \mathrm{E}-09$ & $8.52 \mathrm{E}-07$ \\
Max & $1.96 \mathrm{E}-02$ & $1.80 \mathrm{E}-02$ & $2.74 \mathrm{E}-05$ & $1.76 \mathrm{E}-03$ \\
\hline & & & & \\
\hline
\end{tabular}


TABLE C.11 Peak DSR Percentiles (mrem/yr per pCi/g) for Different Exposure Pathways for Tc-99 in Industrial Use Scenario

\begin{tabular}{rrrrr}
\hline & & & & \\
Percentile & All Pathways & External & $\begin{array}{c}\text { Inhalation } \\
\text { w/o Radon }\end{array}$ & Soil Ingestion \\
\hline & & & & \\
$5 \%$ & $3.44 \mathrm{E}-06$ & $2.35 \mathrm{E}-06$ & $2.37 \mathrm{E}-08$ & $8.40 \mathrm{E}-07$ \\
$10 \%$ & $9.83 \mathrm{E}-06$ & $6.91 \mathrm{E}-06$ & $5.70 \mathrm{E}-08$ & $2.00 \mathrm{E}-06$ \\
$15 \%$ & $1.62 \mathrm{E}-05$ & $1.15 \mathrm{E}-05$ & $8.88 \mathrm{E}-08$ & $3.05 \mathrm{E}-06$ \\
$20 \%$ & $2.27 \mathrm{E}-05$ & $1.61 \mathrm{E}-05$ & $1.19 \mathrm{E}-07$ & $4.06 \mathrm{E}-06$ \\
$25 \%$ & $2.65 \mathrm{E}-05$ & $2.05 \mathrm{E}-05$ & $1.46 \mathrm{E}-07$ & $5.00 \mathrm{E}-06$ \\
$30 \%$ & $2.87 \mathrm{E}-05$ & $2.14 \mathrm{E}-05$ & $1.67 \mathrm{E}-07$ & $5.90 \mathrm{E}-06$ \\
$35 \%$ & $3.04 \mathrm{E}-05$ & $2.22 \mathrm{E}-05$ & $1.84 \mathrm{E}-07$ & $6.85 \mathrm{E}-06$ \\
$40 \%$ & $3.18 \mathrm{E}-05$ & $2.30 \mathrm{E}-05$ & $2.02 \mathrm{E}-07$ & $7.71 \mathrm{E}-06$ \\
$45 \%$ & $3.30 \mathrm{E}-05$ & $2.38 \mathrm{E}-05$ & $2.17 \mathrm{E}-07$ & $8.44 \mathrm{E}-06$ \\
$50 \%$ & $3.41 \mathrm{E}-05$ & $2.45 \mathrm{E}-05$ & $2.34 \mathrm{E}-07$ & $9.17 \mathrm{E}-06$ \\
$55 \%$ & $3.54 \mathrm{E}-05$ & $2.51 \mathrm{E}-05$ & $2.52 \mathrm{E}-07$ & $9.88 \mathrm{E}-06$ \\
$60 \%$ & $3.65 \mathrm{E}-05$ & $2.56 \mathrm{E}-05$ & $2.75 \mathrm{E}-07$ & $1.06 \mathrm{E}-05$ \\
$65 \%$ & $3.77 \mathrm{E}-05$ & $2.61 \mathrm{E}-05$ & $2.99 \mathrm{E}-07$ & $1.13 \mathrm{E}-05$ \\
$70 \%$ & $3.89 \mathrm{E}-05$ & $2.66 \mathrm{E}-05$ & $3.25 \mathrm{E}-07$ & $1.21 \mathrm{E}-05$ \\
$75 \%$ & $4.02 \mathrm{E}-05$ & $2.73 \mathrm{E}-05$ & $3.58 \mathrm{E}-07$ & $1.31 \mathrm{E}-05$ \\
$80 \%$ & $4.18 \mathrm{E}-05$ & $2.85 \mathrm{E}-05$ & $3.94 \mathrm{E}-07$ & $1.41 \mathrm{E}-05$ \\
$85 \%$ & $4.38 \mathrm{E}-05$ & $3.00 \mathrm{E}-05$ & $4.42 \mathrm{E}-07$ & $1.53 \mathrm{E}-05$ \\
$90 \%$ & $4.70 \mathrm{E}-05$ & $3.16 \mathrm{E}-05$ & $5.10 \mathrm{E}-07$ & $1.67 \mathrm{E}-05$ \\
$95 \%$ & $5.18 \mathrm{E}-05$ & $3.39 \mathrm{E}-05$ & $6.23 \mathrm{E}-07$ & $1.90 \mathrm{E}-05$ \\
Mean & $3.21 \mathrm{E}-05$ & $2.25 \mathrm{E}-05$ & $2.70 \mathrm{E}-07$ & $9.37 \mathrm{E}-06$ \\
Min & $2.52 \mathrm{E}-07$ & $2.01 \mathrm{E}-07$ & $1.50 \mathrm{E}-10$ & $1.72 \mathrm{E}-08$ \\
Max & $8.21 \mathrm{E}-05$ & $4.69 \mathrm{E}-05$ & $2.13 \mathrm{E}-06$ & $3.57 \mathrm{E}-05$ \\
\hline & & & & \\
\hline
\end{tabular}




\section{TABLE C.12 Peak DSR Percentiles (mrem/yr per pCi/g) for Different Exposure Pathways for U-234 in Industrial Use Scenario}

\begin{tabular}{rcccccc}
\hline & & & & & \\
Inhalation & Radon & Radon & \\
Percentile & All Pathways & External & Wadon & (WI) & Soil Ingestion \\
\hline $5 \%$ & $4.74 \mathrm{E}-03$ & $9.68 \mathrm{E}-05$ & $7.53 \mathrm{E}-06$ & $4.49 \mathrm{E}-03$ & $0.00 \mathrm{E}+00$ & $8.54 \mathrm{E}-05$ \\
$10 \%$ & $1.20 \mathrm{E}-02$ & $2.81 \mathrm{E}-04$ & $1.60 \mathrm{E}-05$ & $1.15 \mathrm{E}-02$ & $0.00 \mathrm{E}+00$ & $1.85 \mathrm{E}-04$ \\
$15 \%$ & $2.45 \mathrm{E}-02$ & $5.40 \mathrm{E}-04$ & $2.61 \mathrm{E}-05$ & $2.38 \mathrm{E}-02$ & $0.00 \mathrm{E}+00$ & $2.97 \mathrm{E}-04$ \\
$20 \%$ & $4.04 \mathrm{E}-02$ & $9.45 \mathrm{E}-04$ & $3.40 \mathrm{E}-05$ & $3.90 \mathrm{E}-02$ & $0.00 \mathrm{E}+00$ & $4.08 \mathrm{E}-04$ \\
$25 \%$ & $6.17 \mathrm{E}-02$ & $1.46 \mathrm{E}-03$ & $4.13 \mathrm{E}-05$ & $5.97 \mathrm{E}-02$ & $0.00 \mathrm{E}+00$ & $5.02 \mathrm{E}-04$ \\
$30 \%$ & $9.04 \mathrm{E}-02$ & $2.10 \mathrm{E}-03$ & $4.90 \mathrm{E}-05$ & $8.75 \mathrm{E}-02$ & $0.00 \mathrm{E}+00$ & $5.91 \mathrm{E}-04$ \\
$35 \%$ & $1.20 \mathrm{E}-01$ & $2.83 \mathrm{E}-03$ & $5.67 \mathrm{E}-05$ & $1.17 \mathrm{E}-01$ & $0.00 \mathrm{E}+00$ & $6.78 \mathrm{E}-04$ \\
$40 \%$ & $1.53 \mathrm{E}-01$ & $3.72 \mathrm{E}-03$ & $6.53 \mathrm{E}-05$ & $1.48 \mathrm{E}-01$ & $0.00 \mathrm{E}+00$ & $7.61 \mathrm{E}-04$ \\
$45 \%$ & $1.89 \mathrm{E}-01$ & $4.69 \mathrm{E}-03$ & $7.33 \mathrm{E}-05$ & $1.84 \mathrm{E}-01$ & $0.00 \mathrm{E}+00$ & $8.43 \mathrm{E}-04$ \\
$50 \%$ & $2.28 \mathrm{E}-01$ & $5.86 \mathrm{E}-03$ & $8.17 \mathrm{E}-05$ & $2.21 \mathrm{E}-01$ & $0.00 \mathrm{E}+00$ & $9.23 \mathrm{E}-04$ \\
$55 \%$ & $2.73 \mathrm{E}-01$ & $6.96 \mathrm{E}-03$ & $9.02 \mathrm{E}-05$ & $2.63 \mathrm{E}-01$ & $0.00 \mathrm{E}+00$ & $1.01 \mathrm{E}-03$ \\
$60 \%$ & $3.11 \mathrm{E}-01$ & $8.33 \mathrm{E}-03$ & $9.95 \mathrm{E}-05$ & $3.00 \mathrm{E}-01$ & $0.00 \mathrm{E}+00$ & $1.10 \mathrm{E}-03$ \\
$65 \%$ & $3.47 \mathrm{E}-01$ & $9.66 \mathrm{E}-03$ & $1.09 \mathrm{E}-04$ & $3.35 \mathrm{E}-01$ & $0.00 \mathrm{E}+00$ & $1.18 \mathrm{E}-03$ \\
$70 \%$ & $3.84 \mathrm{E}-01$ & $1.10 \mathrm{E}-02$ & $1.20 \mathrm{E}-04$ & $3.72 \mathrm{E}-01$ & $0.00 \mathrm{E}+00$ & $1.28 \mathrm{E}-03$ \\
$75 \%$ & $4.24 \mathrm{E}-01$ & $1.29 \mathrm{E}-02$ & $1.34 \mathrm{E}-04$ & $4.09 \mathrm{E}-01$ & $0.00 \mathrm{E}+00$ & $1.39 \mathrm{E}-03$ \\
$80 \%$ & $4.60 \mathrm{E}-01$ & $1.53 \mathrm{E}-02$ & $1.52 \mathrm{E}-04$ & $4.46 \mathrm{E}-01$ & $0.00 \mathrm{E}+00$ & $1.55 \mathrm{E}-03$ \\
$85 \%$ & $5.06 \mathrm{E}-01$ & $1.76 \mathrm{E}-02$ & $1.70 \mathrm{E}-04$ & $4.89 \mathrm{E}-01$ & $0.00 \mathrm{E}+00$ & $1.75 \mathrm{E}-03$ \\
$90 \%$ & $5.68 \mathrm{E}-01$ & $2.16 \mathrm{E}-02$ & $1.99 \mathrm{E}-04$ & $5.51 \mathrm{E}-01$ & $9.60 \mathrm{E}-08$ & $2.03 \mathrm{E}-03$ \\
$95 \%$ & $6.64 \mathrm{E}-01$ & $2.83 \mathrm{E}-02$ & $2.46 \mathrm{E}-04$ & $6.42 \mathrm{E}-01$ & $1.34 \mathrm{E}-06$ & $2.47 \mathrm{E}-03$ \\
Mean & $2.65 \mathrm{E}-01$ & $8.92 \mathrm{E}-03$ & $9.82 \mathrm{E}-05$ & $2.56 \mathrm{E}-01$ & $5.68 \mathrm{E}-07$ & $1.04 \mathrm{E}-03$ \\
Min & $1.78 \mathrm{E}-05$ & $4.03 \mathrm{E}-07$ & $1.21 \mathrm{E}-07$ & $1.74 \mathrm{E}-05$ & $0.00 \mathrm{E}+00$ & $3.44 \mathrm{E}-06$ \\
Max & $1.33 \mathrm{E}+00$ & $8.87 \mathrm{E}-02$ & $7.08 \mathrm{E}-04$ & 1.277 & $1.27 \mathrm{E}-04$ & $5.27 \mathrm{E}-03$ \\
\hline & & & & & &
\end{tabular}


TABLE C.13 Peak DSR Percentiles (mrem/yr per pCi/g) for Different Exposure Pathways for U-235 in Industrial Use Scenario

\begin{tabular}{ccccc}
\hline & & & & \\
Percentile & All Pathways & External & $\begin{array}{c}\text { w/o Radon } \\
\text { Soil Ingestion }\end{array}$ \\
\hline & & & & \\
$5 \%$ & $1.69 \mathrm{E}-02$ & $1.68 \mathrm{E}-02$ & $3.14 \mathrm{E}-05$ & $1.18 \mathrm{E}-04$ \\
$10 \%$ & $4.98 \mathrm{E}-02$ & $4.92 \mathrm{E}-02$ & $7.06 \mathrm{E}-05$ & $2.59 \mathrm{E}-04$ \\
$15 \%$ & $8.11 \mathrm{E}-02$ & $8.05 \mathrm{E}-02$ & $1.03 \mathrm{E}-04$ & $3.85 \mathrm{E}-04$ \\
$20 \%$ & $1.14 \mathrm{E}-01$ & $1.13 \mathrm{E}-01$ & $1.28 \mathrm{E}-04$ & $5.13 \mathrm{E}-04$ \\
$25 \%$ & $1.41 \mathrm{E}-01$ & $1.40 \mathrm{E}-01$ & $1.51 \mathrm{E}-04$ & $6.14 \mathrm{E}-04$ \\
$30 \%$ & $1.47 \mathrm{E}-01$ & $1.46 \mathrm{E}-01$ & $1.78 \mathrm{E}-04$ & $7.31 \mathrm{E}-04$ \\
$35 \%$ & $1.54 \mathrm{E}-01$ & $1.53 \mathrm{E}-01$ & $2.04 \mathrm{E}-04$ & $8.30 \mathrm{E}-04$ \\
$40 \%$ & $1.61 \mathrm{E}-01$ & $1.59 \mathrm{E}-01$ & $2.37 \mathrm{E}-04$ & $9.27 \mathrm{E}-04$ \\
$45 \%$ & $1.67 \mathrm{E}-01$ & $1.66 \mathrm{E}-01$ & $2.79 \mathrm{E}-04$ & $1.03 \mathrm{E}-03$ \\
$50 \%$ & $1.71 \mathrm{E}-01$ & $1.70 \mathrm{E}-01$ & $3.40 \mathrm{E}-04$ & $1.16 \mathrm{E}-03$ \\
$55 \%$ & $1.75 \mathrm{E}-01$ & $1.74 \mathrm{E}-01$ & $4.06 \mathrm{E}-04$ & $1.31 \mathrm{E}-03$ \\
$60 \%$ & $1.80 \mathrm{E}-01$ & $1.78 \mathrm{E}-01$ & $4.84 \mathrm{E}-04$ & $1.47 \mathrm{E}-03$ \\
$65 \%$ & $1.84 \mathrm{E}-01$ & $1.82 \mathrm{E}-01$ & $5.79 \mathrm{E}-04$ & $1.68 \mathrm{E}-03$ \\
$70 \%$ & $1.92 \mathrm{E}-01$ & $1.90 \mathrm{E}-01$ & $6.93 \mathrm{E}-04$ & $1.97 \mathrm{E}-03$ \\
$75 \%$ & $2.00 \mathrm{E}-01$ & $1.98 \mathrm{E}-01$ & $8.17 \mathrm{E}-04$ & $2.35 \mathrm{E}-03$ \\
$80 \%$ & $2.08 \mathrm{E}-01$ & $2.06 \mathrm{E}-01$ & $9.55 \mathrm{E}-04$ & $2.76 \mathrm{E}-03$ \\
$85 \%$ & $2.18 \mathrm{E}-01$ & $2.15 \mathrm{E}-01$ & $1.13 \mathrm{E}-03$ & $3.28 \mathrm{E}-03$ \\
$90 \%$ & $2.29 \mathrm{E}-01$ & $2.26 \mathrm{E}-01$ & $1.34 \mathrm{E}-03$ & $3.92 \mathrm{E}-03$ \\
$95 \%$ & $2.65 \mathrm{E}-01$ & $2.60 \mathrm{E}-01$ & $1.73 \mathrm{E}-03$ & $5.01 \mathrm{E}-03$ \\
Mean & $1.61 \mathrm{E}-01$ & $1.59 \mathrm{E}-01$ & $5.59 \mathrm{E}-04$ & $1.69 \mathrm{E}-03$ \\
Min & $1.39 \mathrm{E}-03$ & $1.38 \mathrm{E}-03$ & $1.36 \mathrm{E}-07$ & $2.07 \mathrm{E}-06$ \\
Max & $3.97 \mathrm{E}-01$ & $3.90 \mathrm{E}-01$ & $5.56 \mathrm{E}-03$ & $1.13 \mathrm{E}-02$ \\
\hline & & & &
\end{tabular}


TABLE C.14 Peak DSR Percentiles (mrem/yr per pCi/g) for Different Exposure Pathways for U-238 in Industrial Use Scenario

\begin{tabular}{rrrrrr}
\hline & & & & & \\
Percentile & All Pathways & External & $\begin{array}{c}\text { Inhation } \\
\text { w/o Radon }\end{array}$ & Radon (WI) & Soil Ingestion \\
\hline $5 \%$ & $3.64 \mathrm{E}-03$ & $3.54 \mathrm{E}-03$ & $1.22 \mathrm{E}-05$ & $1.70 \mathrm{E}-11$ & $6.50 \mathrm{E}-05$ \\
$10 \%$ & $1.06 \mathrm{E}-02$ & $1.03 \mathrm{E}-02$ & $3.35 \mathrm{E}-05$ & $1.19 \mathrm{E}-10$ & $1.51 \mathrm{E}-04$ \\
$15 \%$ & $1.76 \mathrm{E}-02$ & $1.71 \mathrm{E}-02$ & $5.28 \mathrm{E}-05$ & $4.14 \mathrm{E}-10$ & $2.37 \mathrm{E}-04$ \\
$20 \%$ & $2.45 \mathrm{E}-02$ & $2.38 \mathrm{E}-02$ & $6.74 \mathrm{E}-05$ & $9.09 \mathrm{E}-10$ & $3.22 \mathrm{E}-04$ \\
$25 \%$ & $3.13 \mathrm{E}-02$ & $3.05 \mathrm{E}-02$ & $8.16 \mathrm{E}-05$ & $1.62 \mathrm{E}-09$ & $4.04 \mathrm{E}-04$ \\
$30 \%$ & $3.28 \mathrm{E}-02$ & $3.20 \mathrm{E}-02$ & $9.43 \mathrm{E}-05$ & $2.74 \mathrm{E}-09$ & $4.79 \mathrm{E}-04$ \\
$35 \%$ & $3.40 \mathrm{E}-02$ & $3.31 \mathrm{E}-02$ & $1.04 \mathrm{E}-04$ & $4.04 \mathrm{E}-09$ & $5.43 \mathrm{E}-04$ \\
$40 \%$ & $3.52 \mathrm{E}-02$ & $3.44 \mathrm{E}-02$ & $1.13 \mathrm{E}-04$ & $5.66 \mathrm{E}-09$ & $6.08 \mathrm{E}-04$ \\
$45 \%$ & $3.64 \mathrm{E}-02$ & $3.55 \mathrm{E}-02$ & $1.22 \mathrm{E}-04$ & $7.61 \mathrm{E}-09$ & $6.70 \mathrm{E}-04$ \\
$50 \%$ & $3.75 \mathrm{E}-02$ & $3.67 \mathrm{E}-02$ & $1.30 \mathrm{E}-04$ & $9.27 \mathrm{E}-09$ & $7.34 \mathrm{E}-04$ \\
$55 \%$ & $3.84 \mathrm{E}-02$ & $3.74 \mathrm{E}-02$ & $1.39 \mathrm{E}-04$ & $1.11 \mathrm{E}-08$ & $7.92 \mathrm{E}-04$ \\
$60 \%$ & $3.93 \mathrm{E}-02$ & $3.82 \mathrm{E}-02$ & $1.48 \mathrm{E}-04$ & $1.29 \mathrm{E}-08$ & $8.52 \mathrm{E}-04$ \\
$65 \%$ & $4.00 \mathrm{E}-02$ & $3.90 \mathrm{E}-02$ & $1.58 \mathrm{E}-04$ & $1.47 \mathrm{E}-08$ & $9.17 \mathrm{E}-04$ \\
$70 \%$ & $4.08 \mathrm{E}-02$ & $3.98 \mathrm{E}-02$ & $1.68 \mathrm{E}-04$ & $1.64 \mathrm{E}-08$ & $9.77 \mathrm{E}-04$ \\
$75 \%$ & $4.18 \mathrm{E}-02$ & $4.06 \mathrm{E}-02$ & $1.78 \mathrm{E}-04$ & $1.79 \mathrm{E}-08$ & $1.05 \mathrm{E}-03$ \\
$80 \%$ & $4.38 \mathrm{E}-02$ & $4.27 \mathrm{E}-02$ & $1.89 \mathrm{E}-04$ & $1.95 \mathrm{E}-08$ & $1.13 \mathrm{E}-03$ \\
$85 \%$ & $4.61 \mathrm{E}-02$ & $4.49 \mathrm{E}-02$ & $2.06 \mathrm{E}-04$ & $2.11 \mathrm{E}-08$ & $1.23 \mathrm{E}-03$ \\
$90 \%$ & $4.84 \mathrm{E}-02$ & $4.71 \mathrm{E}-02$ & $2.29 \mathrm{E}-04$ & $2.30 \mathrm{E}-08$ & $1.35 \mathrm{E}-03$ \\
$95 \%$ & $5.17 \mathrm{E}-02$ & $5.03 \mathrm{E}-02$ & $2.68 \mathrm{E}-04$ & $2.59 \mathrm{E}-08$ & $1.53 \mathrm{E}-03$ \\
Mean & $3.45 \mathrm{E}-02$ & $3.36 \mathrm{E}-02$ & $1.35 \mathrm{E}-04$ & $1.06 \mathrm{E}-08$ & $7.51 \mathrm{E}-04$ \\
Min & $3.32 \mathrm{E}-04$ & $3.18 \mathrm{E}-04$ & $7.36 \mathrm{E}-09$ & $2.49 \mathrm{E}-15$ & $3.00 \mathrm{E}-06$ \\
Max & $7.18 \mathrm{E}-02$ & $7.00 \mathrm{E}-02$ & $6.35 \mathrm{E}-04$ & $4.52 \mathrm{E}-08$ & $2.50 \mathrm{E}-03$ \\
\hline
\end{tabular}

Tables C.15-C.28 show the peak DSR percentiles (mrem/yr per pCi/g) for different exposure pathways for individual radionuclides in the offsite resident scenario. RESRADOFFSITE Version 2.6 was used in the analysis. For all radionuclides except Co-60, WD pathways were dominant, and for Co-60, the WI pathway was dominant. For Am-241, C-14, Cs-137, and U-234, aquatic food ingestion resulted in the most dose. For Np-237, Pu-238, $\mathrm{Pu}-239$, and $\mathrm{Pu}-240$, fish and plant ingestion were dominant exposure pathways. For Co-60, the external exposure pathway was dominant. For I-129, meat and milk resulted in the most dose. For Sr-90, fish, meat, and plant ingestion resulted in most dose. For Tc-99, plant and fish ingestion resulted in the most dose. For U-235 and U-238, external exposure and fish ingestion resulted in the most dose. 
TABLE C.15 Peak DSR Percentiles (mrem/yr per pCi/g) for Different Exposure Pathways for Am-241 in Offsite Resident Scenario

\begin{tabular}{|c|c|c|c|c|c|c|c|c|c|c|c|c|c|}
\hline Percentile & Total & External-WD & Fish & Plant-WD & Meat-WD & Milk-WD & Soil-WD & External-WI & Inhalation & Plant-WI & Meat-WI & Milk-WI & Soil-WI \\
\hline $5 \%$ & $2.16 \mathrm{E}-03$ & $2.72 \mathrm{E}-06$ & $1.99 \mathrm{E}-03$ & $1.21 \mathrm{E}-04$ & $2.90 \mathrm{E}-06$ & 3.07E-07 & $9.24 \mathrm{E}-07$ & 9.69E-08 & $6.63 \mathrm{E}-06$ & $5.17 \mathrm{E}-09$ & $3.51 \mathrm{E}-11$ & $1.72 \mathrm{E}-12$ & $2.15 \mathrm{E}-10$ \\
\hline $10 \%$ & $2.69 \mathrm{E}-03$ & $5.72 \mathrm{E}-06$ & $2.47 \mathrm{E}-03$ & $1.62 \mathrm{E}-04$ & $3.68 \mathrm{E}-06$ & $4.41 \mathrm{E}-07$ & $1.95 \mathrm{E}-06$ & $9.70 \mathrm{E}-08$ & 6.64E-06 & $6.08 \mathrm{E}-09$ & 3.86E-11 & 2.24E-12 & $3.08 \mathrm{E}-10$ \\
\hline $15 \%$ & $3.32 \mathrm{E}-03$ & $8.77 \mathrm{E}-06$ & $3.07 \mathrm{E}-03$ & $2.00 \mathrm{E}-04$ & $4.50 \mathrm{E}-06$ & $5.44 \mathrm{E}-07$ & $2.96 \mathrm{E}-06$ & $9.70 \mathrm{E}-08$ & 6.64E-06 & 6.76E-09 & $4.15 \mathrm{E}-11$ & $2.66 \mathrm{E}-12$ & $3.89 \mathrm{E}-10$ \\
\hline $20 \%$ & $4.17 \mathrm{E}-03$ & $1.18 \mathrm{E}-05$ & $3.85 \mathrm{E}-03$ & 2.35E-04 & $5.50 \mathrm{E}-06$ & $6.53 \mathrm{E}-07$ & 4.01E-06 & $9.70 \mathrm{E}-08$ & 6.64E-06 & 7.40E-09 & 4.41E-11 & 3.02E-12 & $4.80 \mathrm{E}-10$ \\
\hline $25 \%$ & $4.91 \mathrm{E}-03$ & $1.46 \mathrm{E}-05$ & $4.56 \mathrm{E}-03$ & $2.68 \mathrm{E}-04$ & $6.61 \mathrm{E}-06$ & $7.47 \mathrm{E}-07$ & $4.96 \mathrm{E}-06$ & $9.70 \mathrm{E}-08$ & $6.64 \mathrm{E}-06$ & 8.02E-09 & $4.63 \mathrm{E}-11$ & $3.43 \mathrm{E}-12$ & $5.47 \mathrm{E}-10$ \\
\hline $30 \%$ & $5.62 \mathrm{E}-03$ & 1.69E-05 & $5.21 \mathrm{E}-03$ & $2.97 \mathrm{E}-04$ & 7.37E-06 & $8.52 \mathrm{E}-07$ & 5.92E-06 & $9.70 \mathrm{E}-08$ & $6.64 \mathrm{E}-06$ & 8.68E-09 & $4.88 \mathrm{E}-11$ & $3.80 \mathrm{E}-12$ & $6.16 \mathrm{E}-10$ \\
\hline $35 \%$ & $6.21 \mathrm{E}-03$ & $1.94 \mathrm{E}-05$ & $5.75 \mathrm{E}-03$ & 3.30E-04 & 8.04E-06 & $9.51 \mathrm{E}-07$ & $6.97 \mathrm{E}-06$ & $9.70 \mathrm{E}-08$ & $6.64 \mathrm{E}-06$ & 9.27E-09 & $5.07 \mathrm{E}-11$ & $4.21 \mathrm{E}-12$ & $6.85 \mathrm{E}-10$ \\
\hline $40 \%$ & $6.66 \mathrm{E}-03$ & 2.19E-05 & $6.14 \mathrm{E}-03$ & $3.58 \mathrm{E}-04$ & $8.62 \mathrm{E}-06$ & $1.05 \mathrm{E}-06$ & 8.13E-06 & $9.70 \mathrm{E}-08$ & $6.64 \mathrm{E}-06$ & 9.83E-09 & $5.30 \mathrm{E}-11$ & $4.63 \mathrm{E}-12$ & $7.48 \mathrm{E}-10$ \\
\hline $45 \%$ & $6.92 \mathrm{E}-03$ & $2.41 \mathrm{E}-05$ & $6.40 \mathrm{E}-03$ & $3.86 \mathrm{E}-04$ & $9.11 \mathrm{E}-06$ & $1.17 \mathrm{E}-06$ & $9.27 \mathrm{E}-06$ & $9.70 \mathrm{E}-08$ & 6.64E-06 & $1.05 \mathrm{E}-08$ & $5.53 \mathrm{E}-11$ & $5.07 \mathrm{E}-12$ & $8.09 \mathrm{E}-10$ \\
\hline $50 \%$ & 7.12E-03 & 2.62E-05 & $6.57 \mathrm{E}-03$ & 4.15E-04 & $9.62 \mathrm{E}-06$ & $1.29 \mathrm{E}-06$ & $1.03 \mathrm{E}-05$ & $9.70 \mathrm{E}-08$ & $6.64 \mathrm{E}-06$ & $1.12 \mathrm{E}-08$ & $5.78 \mathrm{E}-11$ & $5.59 \mathrm{E}-12$ & $8.71 \mathrm{E}-10$ \\
\hline $55 \%$ & 7.31E-03 & $2.78 \mathrm{E}-05$ & $6.72 \mathrm{E}-03$ & $4.50 \mathrm{E}-04$ & $1.01 \mathrm{E}-05$ & $1.45 \mathrm{E}-06$ & $1.14 \mathrm{E}-05$ & $9.70 \mathrm{E}-08$ & $6.64 \mathrm{E}-06$ & $1.19 \mathrm{E}-08$ & $6.03 \mathrm{E}-11$ & $6.10 \mathrm{E}-12$ & $9.31 \mathrm{E}-10$ \\
\hline $60 \%$ & 7.49E-03 & $2.93 \mathrm{E}-05$ & $6.88 \mathrm{E}-03$ & $4.88 \mathrm{E}-04$ & $1.06 \mathrm{E}-05$ & $1.61 \mathrm{E}-06$ & $1.27 \mathrm{E}-05$ & $9.70 \mathrm{E}-08$ & $6.64 \mathrm{E}-06$ & $1.27 \mathrm{E}-08$ & $6.32 \mathrm{E}-11$ & $6.76 \mathrm{E}-12$ & $9.95 \mathrm{E}-10$ \\
\hline $65 \%$ & 7.69E-03 & 3.07E-05 & 7.09E-03 & $5.27 \mathrm{E}-04$ & $1.10 \mathrm{E}-05$ & $1.77 \mathrm{E}-06$ & $1.40 \mathrm{E}-05$ & $9.70 \mathrm{E}-08$ & $6.64 \mathrm{E}-06$ & $1.35 \mathrm{E}-08$ & $6.62 \mathrm{E}-11$ & $7.40 \mathrm{E}-12$ & $1.06 \mathrm{E}-09$ \\
\hline $70 \%$ & $7.95 \mathrm{E}-03$ & 3.22E-05 & 7.30E-03 & 5.64E-04 & $1.14 \mathrm{E}-05$ & $1.95 \mathrm{E}-06$ & $1.52 \mathrm{E}-05$ & $9.70 \mathrm{E}-08$ & $6.64 \mathrm{E}-06$ & $1.45 \mathrm{E}-08$ & $7.00 \mathrm{E}-11$ & $8.06 \mathrm{E}-12$ & $1.13 \mathrm{E}-09$ \\
\hline $75 \%$ & $8.26 \mathrm{E}-03$ & 3.33E-05 & $7.58 \mathrm{E}-03$ & $6.18 \mathrm{E}-04$ & $1.19 \mathrm{E}-05$ & 2.22E-06 & $1.66 \mathrm{E}-05$ & $9.70 \mathrm{E}-08$ & 6.64E-06 & $1.54 \mathrm{E}-08$ & $7.43 \mathrm{E}-11$ & $9.02 \mathrm{E}-12$ & $1.20 \mathrm{E}-09$ \\
\hline $80 \%$ & 8.66E-03 & $3.47 \mathrm{E}-05$ & 8.01E-03 & $6.75 \mathrm{E}-04$ & $1.24 \mathrm{E}-05$ & $2.50 \mathrm{E}-06$ & $1.81 \mathrm{E}-05$ & $9.70 \mathrm{E}-08$ & 6.64E-06 & $1.67 \mathrm{E}-08$ & $7.91 \mathrm{E}-11$ & $1.01 \mathrm{E}-11$ & $1.28 \mathrm{E}-09$ \\
\hline $85 \%$ & $9.24 \mathrm{E}-03$ & $3.61 \mathrm{E}-05$ & 8.59E-03 & $7.46 \mathrm{E}-04$ & $1.30 \mathrm{E}-05$ & 2.89E-06 & $1.99 \mathrm{E}-05$ & $9.70 \mathrm{E}-08$ & $6.64 \mathrm{E}-06$ & $1.86 \mathrm{E}-08$ & $8.56 \mathrm{E}-11$ & $1.16 \mathrm{E}-11$ & $1.39 \mathrm{E}-09$ \\
\hline $90 \%$ & $1.00 \mathrm{E}-02$ & $3.78 \mathrm{E}-05$ & $9.36 \mathrm{E}-03$ & $8.68 \mathrm{E}-04$ & $1.38 \mathrm{E}-05$ & $3.42 \mathrm{E}-06$ & $2.22 \mathrm{E}-05$ & $9.70 \mathrm{E}-08$ & $6.64 \mathrm{E}-06$ & 2.12E-08 & $9.50 \mathrm{E}-11$ & $1.37 \mathrm{E}-11$ & $1.50 \mathrm{E}-09$ \\
\hline $95 \%$ & 1.19E-02 & 4.02E-05 & $1.13 \mathrm{E}-02$ & $1.06 \mathrm{E}-03$ & $1.49 \mathrm{E}-05$ & $4.56 \mathrm{E}-06$ & $2.57 \mathrm{E}-05$ & $9.70 \mathrm{E}-08$ & $6.64 \mathrm{E}-06$ & $2.56 \mathrm{E}-08$ & $1.12 \mathrm{E}-10$ & $1.79 \mathrm{E}-11$ & 1.69E-09 \\
\hline Mean & $6.98 \mathrm{E}-03$ & $2.38 \mathrm{E}-05$ & $6.49 \mathrm{E}-03$ & 4.80E-04 & $9.24 \mathrm{E}-06$ & $1.72 \mathrm{E}-06$ & $1.14 \mathrm{E}-05$ & $9.70 \mathrm{E}-08$ & $6.64 \mathrm{E}-06$ & $1.28 \mathrm{E}-08$ & $6.37 \mathrm{E}-11$ & 7.17E-12 & $8.95 \mathrm{E}-10$ \\
\hline Min & $1.49 \mathrm{E}-03$ & $1.90 \mathrm{E}-07$ & $1.42 \mathrm{E}-03$ & 3.37E-05 & $1.64 \mathrm{E}-06$ & 5.33E-08 & 4.81E-08 & $9.46 \mathrm{E}-08$ & $6.47 \mathrm{E}-06$ & $1.97 \mathrm{E}-09$ & $2.27 \mathrm{E}-11$ & $6.44 \mathrm{E}-13$ & $1.13 \mathrm{E}-11$ \\
\hline $\operatorname{Max}$ & $1.08 \mathrm{E}-01$ & $4.98 \mathrm{E}-05$ & $1.08 \mathrm{E}-01$ & $3.70 \mathrm{E}-03$ & 2.14E-05 & $1.26 \mathrm{E}-05$ & $3.97 \mathrm{E}-05$ & $9.70 \mathrm{E}-08$ & 6.64E-06 & 2.13E-07 & $3.07 \mathrm{E}-10$ & 7.49E-11 & 2.43E-09 \\
\hline
\end{tabular}


TABLE C.16 Peak DSR Percentiles (mrem/yr per pCi/g) for Different Exposure Pathways for C-14 in Offsite Resident Scenario

\begin{tabular}{|c|c|c|c|c|c|c|c|c|c|c|c|c|c|}
\hline Percentile & Total & External-WD & Fish & Plant-WD & Meat-WD & Milk-WD & Soil-WD & External-WI & Inhalation & Plant-WI & Meat-WI & Milk-WI & Soil-WI \\
\hline $5 \%$ & $2.48 \mathrm{E}-04$ & 4.57E-12 & $2.43 \mathrm{E}-04$ & $4.02 \mathrm{E}-07$ & 4.44E-07 & $1.03 \mathrm{E}-06$ & $1.40 \mathrm{E}-11$ & $1.27 \mathrm{E}-15$ & $9.49 \mathrm{E}-07$ & $2.40 \mathrm{E}-11$ & $1.54 \mathrm{E}-11$ & $2.95 \mathrm{E}-11$ & $6.03 \mathrm{E}-16$ \\
\hline $10 \%$ & $3.76 \mathrm{E}-04$ & $6.03 \mathrm{E}-12$ & $3.66 \mathrm{E}-04$ & $5.31 \mathrm{E}-07$ & $5.01 \mathrm{E}-07$ & $1.06 \mathrm{E}-06$ & $2.03 \mathrm{E}-11$ & $1.31 \mathrm{E}-15$ & $9.95 \mathrm{E}-07$ & $3.04 \mathrm{E}-11$ & $1.68 \mathrm{E}-11$ & $3.07 \mathrm{E}-11$ & $8.64 \mathrm{E}-16$ \\
\hline $15 \%$ & 4.97E-04 & $7.71 \mathrm{E}-12$ & $4.86 \mathrm{E}-04$ & $7.40 \mathrm{E}-07$ & $5.82 \mathrm{E}-07$ & $1.09 \mathrm{E}-06$ & $2.71 \mathrm{E}-11$ & $1.34 \mathrm{E}-15$ & $1.03 \mathrm{E}-06$ & $3.72 \mathrm{E}-11$ & $1.82 \mathrm{E}-11$ & $3.15 \mathrm{E}-11$ & $1.14 \mathrm{E}-15$ \\
\hline $20 \%$ & $6.40 \mathrm{E}-04$ & $9.77 \mathrm{E}-12$ & $6.27 \mathrm{E}-04$ & $1.04 \mathrm{E}-06$ & $6.71 \mathrm{E}-07$ & $1.12 \mathrm{E}-06$ & $3.53 \mathrm{E}-11$ & $1.38 \mathrm{E}-15$ & $1.05 \mathrm{E}-06$ & $4.49 \mathrm{E}-11$ & $1.97 \mathrm{E}-11$ & $3.23 \mathrm{E}-11$ & $1.38 \mathrm{E}-15$ \\
\hline $25 \%$ & $8.00 \mathrm{E}-04$ & $1.19 \mathrm{E}-11$ & 7.79E-04 & $1.36 \mathrm{E}-06$ & 7.97E-07 & $1.15 \mathrm{E}-06$ & $4.42 \mathrm{E}-11$ & $1.42 \mathrm{E}-15$ & $1.07 \mathrm{E}-06$ & $5.40 \mathrm{E}-11$ & $2.14 \mathrm{E}-11$ & $3.29 \mathrm{E}-11$ & $1.61 \mathrm{E}-15$ \\
\hline $30 \%$ & $9.86 \mathrm{E}-04$ & $1.38 \mathrm{E}-11$ & $9.68 \mathrm{E}-04$ & $1.71 \mathrm{E}-06$ & $9.40 \mathrm{E}-07$ & $1.18 \mathrm{E}-06$ & $5.63 \mathrm{E}-11$ & $1.47 \mathrm{E}-15$ & $1.09 \mathrm{E}-06$ & $6.51 \mathrm{E}-11$ & $2.33 \mathrm{E}-11$ & $3.36 \mathrm{E}-11$ & $1.91 \mathrm{E}-15$ \\
\hline $35 \%$ & $1.21 \mathrm{E}-03$ & $1.57 \mathrm{E}-11$ & $1.19 \mathrm{E}-03$ & 2.07E-06 & $1.15 \mathrm{E}-06$ & $1.23 \mathrm{E}-06$ & $6.97 \mathrm{E}-11$ & $1.52 \mathrm{E}-15$ & $1.11 \mathrm{E}-06$ & $8.00 \mathrm{E}-11$ & $2.53 \mathrm{E}-11$ & $3.43 \mathrm{E}-11$ & $2.25 \mathrm{E}-15$ \\
\hline $40 \%$ & $1.47 \mathrm{E}-03$ & $1.75 \mathrm{E}-11$ & $1.44 \mathrm{E}-03$ & $2.55 \mathrm{E}-06$ & $1.40 \mathrm{E}-06$ & $1.29 \mathrm{E}-06$ & $8.51 \mathrm{E}-11$ & $1.57 \mathrm{E}-15$ & $1.12 \mathrm{E}-06$ & $9.45 \mathrm{E}-11$ & $2.76 \mathrm{E}-11$ & $3.50 \mathrm{E}-11$ & $2.61 \mathrm{E}-15$ \\
\hline $45 \%$ & $1.85 \mathrm{E}-03$ & $2.04 \mathrm{E}-11$ & $1.80 \mathrm{E}-03$ & $3.21 \mathrm{E}-06$ & $1.70 \mathrm{E}-06$ & $1.39 \mathrm{E}-06$ & $1.06 \mathrm{E}-10$ & $1.63 \mathrm{E}-15$ & $1.14 \mathrm{E}-06$ & $1.08 \mathrm{E}-10$ & $3.03 \mathrm{E}-11$ & $3.56 \mathrm{E}-11$ & $2.97 \mathrm{E}-15$ \\
\hline $50 \%$ & $2.36 \mathrm{E}-03$ & $3.00 \mathrm{E}-11$ & $2.32 \mathrm{E}-03$ & $4.26 \mathrm{E}-06$ & $2.32 \mathrm{E}-06$ & $1.69 \mathrm{E}-06$ & $1.40 \mathrm{E}-10$ & $1.66 \mathrm{E}-15$ & $1.16 \mathrm{E}-06$ & $1.19 \mathrm{E}-10$ & $3.32 \mathrm{E}-11$ & $3.63 \mathrm{E}-11$ & $3.39 \mathrm{E}-15$ \\
\hline $55 \%$ & $2.96 \mathrm{E}-03$ & $6.93 \mathrm{E}-11$ & $2.89 \mathrm{E}-03$ & $6.15 \mathrm{E}-06$ & $3.42 \mathrm{E}-06$ & $2.99 \mathrm{E}-06$ & $2.55 \mathrm{E}-10$ & $1.69 \mathrm{E}-15$ & $1.17 \mathrm{E}-06$ & $1.34 \mathrm{E}-10$ & 3.63E-11 & $3.71 \mathrm{E}-11$ & $3.86 \mathrm{E}-15$ \\
\hline $60 \%$ & $3.89 \mathrm{E}-03$ & $1.46 \mathrm{E}-10$ & $3.79 \mathrm{E}-03$ & $1.07 \mathrm{E}-05$ & $6.52 \mathrm{E}-06$ & $5.74 \mathrm{E}-06$ & $5.35 \mathrm{E}-10$ & $1.72 \mathrm{E}-15$ & $1.19 \mathrm{E}-06$ & $1.49 \mathrm{E}-10$ & $3.99 \mathrm{E}-11$ & $3.79 \mathrm{E}-11$ & $4.37 \mathrm{E}-15$ \\
\hline $65 \%$ & $5.35 \mathrm{E}-03$ & $2.68 \mathrm{E}-10$ & $5.19 \mathrm{E}-03$ & $1.94 \mathrm{E}-05$ & $1.21 \mathrm{E}-05$ & $1.16 \mathrm{E}-05$ & $1.07 \mathrm{E}-09$ & $1.74 \mathrm{E}-15$ & $1.20 \mathrm{E}-06$ & $1.65 \mathrm{E}-10$ & 4.42E-11 & $3.87 \mathrm{E}-11$ & $4.88 \mathrm{E}-15$ \\
\hline $70 \%$ & $7.94 \mathrm{E}-03$ & $5.22 \mathrm{E}-10$ & $7.56 \mathrm{E}-03$ & $3.54 \mathrm{E}-05$ & $2.12 \mathrm{E}-05$ & $2.25 \mathrm{E}-05$ & $2.06 \mathrm{E}-09$ & $1.77 \mathrm{E}-15$ & $1.22 \mathrm{E}-06$ & $1.84 \mathrm{E}-10$ & $4.92 \mathrm{E}-11$ & $3.97 \mathrm{E}-11$ & $5.42 \mathrm{E}-15$ \\
\hline $75 \%$ & $1.27 \mathrm{E}-02$ & $9.98 \mathrm{E}-10$ & $1.23 \mathrm{E}-02$ & 7.13E-05 & $3.86 \mathrm{E}-05$ & $4.26 \mathrm{E}-05$ & $3.76 \mathrm{E}-09$ & $1.79 \mathrm{E}-15$ & $1.24 \mathrm{E}-06$ & $2.11 \mathrm{E}-10$ & $5.44 \mathrm{E}-11$ & $4.09 \mathrm{E}-11$ & $5.97 \mathrm{E}-15$ \\
\hline $80 \%$ & $2.31 \mathrm{E}-02$ & $1.74 \mathrm{E}-09$ & $2.24 \mathrm{E}-02$ & $1.37 \mathrm{E}-04$ & $7.48 \mathrm{E}-05$ & $7.08 \mathrm{E}-05$ & $6.97 \mathrm{E}-09$ & $1.82 \mathrm{E}-15$ & $1.26 \mathrm{E}-06$ & $2.39 \mathrm{E}-10$ & $6.16 \mathrm{E}-11$ & $4.26 \mathrm{E}-11$ & $6.60 \mathrm{E}-15$ \\
\hline $85 \%$ & $4.19 \mathrm{E}-02$ & $3.28 \mathrm{E}-09$ & $4.00 \mathrm{E}-02$ & 3.03E-04 & $1.62 \mathrm{E}-04$ & $1.33 \mathrm{E}-04$ & $1.30 \mathrm{E}-08$ & $1.85 \mathrm{E}-15$ & $1.29 \mathrm{E}-06$ & $2.76 \mathrm{E}-10$ & $7.10 \mathrm{E}-11$ & $4.45 \mathrm{E}-11$ & $7.32 \mathrm{E}-15$ \\
\hline $90 \%$ & $8.49 \mathrm{E}-02$ & $6.40 \mathrm{E}-09$ & $8.30 \mathrm{E}-02$ & $6.75 \mathrm{E}-04$ & $3.80 \mathrm{E}-04$ & 2.92E-04 & $2.53 \mathrm{E}-08$ & $1.88 \mathrm{E}-15$ & $1.32 \mathrm{E}-06$ & $3.42 \mathrm{E}-10$ & $8.41 \mathrm{E}-11$ & 4.76E-11 & $8.23 \mathrm{E}-15$ \\
\hline $95 \%$ & $2.27 \mathrm{E}-01$ & $1.40 \mathrm{E}-08$ & $2.26 \mathrm{E}-01$ & $2.26 \mathrm{E}-03$ & $1.20 \mathrm{E}-03$ & $6.93 \mathrm{E}-04$ & $5.96 \mathrm{E}-08$ & $1.94 \mathrm{E}-15$ & $1.36 \mathrm{E}-06$ & 4.79E-10 & $1.11 \mathrm{E}-10$ & $5.36 \mathrm{E}-11$ & $9.64 \mathrm{E}-15$ \\
\hline Mean & $5.95 \mathrm{E}-02$ & $2.68 \mathrm{E}-09$ & $5.89 \mathrm{E}-02$ & $4.88 \mathrm{E}-04$ & $2.43 \mathrm{E}-04$ & $1.21 \mathrm{E}-04$ & $1.16 \mathrm{E}-08$ & $1.62 \mathrm{E}-15$ & $1.16 \mathrm{E}-06$ & $1.68 \mathrm{E}-10$ & $4.59 \mathrm{E}-11$ & $3.93 \mathrm{E}-11$ & $4.09 \mathrm{E}-15$ \\
\hline Min & $4.91 \mathrm{E}-05$ & $2.14 \mathrm{E}-12$ & $4.66 \mathrm{E}-05$ & $1.92 \mathrm{E}-07$ & $3.40 \mathrm{E}-07$ & $9.64 \mathrm{E}-07$ & $4.29 \mathrm{E}-13$ & $1.16 \mathrm{E}-15$ & 7.82E-07 & $9.76 \mathrm{E}-12$ & $1.22 \mathrm{E}-11$ & $2.74 \mathrm{E}-11$ & $1.81 \mathrm{E}-17$ \\
\hline Max & $9.51 \mathrm{E}+00$ & $1.51 \mathrm{E}-07$ & $9.51 \mathrm{E}+00$ & $5.68 \mathrm{E}-02$ & $3.86 \mathrm{E}-02$ & $9.22 \mathrm{E}-03$ & $6.30 \mathrm{E}-07$ & $4.26 \mathrm{E}-15$ & $1.55 \mathrm{E}-06$ & $5.22 \mathrm{E}-09$ & $1.39 \mathrm{E}-09$ & $1.76 \mathrm{E}-09$ & $8.85 \mathrm{E}-14$ \\
\hline
\end{tabular}


TABLE C.17 Peak DSR Percentiles (mrem/yr per pCi/g) for Different Exposure Pathways for Co-60 in Offsite Resident Scenario

\begin{tabular}{|c|c|c|c|c|c|c|c|c|c|c|c|c|c|}
\hline Percentile & Total & External-WD & Fish & Plant-WD & Meat-WD & Milk-WD & Soil-WD & External-WI & Inhalation & Plant-WI & Meat-WI & Milk-WI & Soil-WI \\
\hline $5 \%$ & 7.92E-04 & $6.63 \mathrm{E}-05$ & $1.67 \mathrm{E}-05$ & $1.79 \mathrm{E}-06$ & 7.98E-06 & $3.10 \mathrm{E}-06$ & $5.87 \mathrm{E}-10$ & 7.85E-04 & $2.06 \mathrm{E}-09$ & $8.75 \mathrm{E}-11$ & $9.86 \mathrm{E}-11$ & $2.68 \mathrm{E}-11$ & 8.11E-14 \\
\hline $10 \%$ & 7.93E-04 & $7.02 \mathrm{E}-05$ & $2.15 \mathrm{E}-05$ & $2.07 \mathrm{E}-06$ & $1.15 \mathrm{E}-05$ & 4.01E-06 & $8.24 \mathrm{E}-10$ & $7.85 \mathrm{E}-04$ & 2.07E-09 & $1.02 \mathrm{E}-10$ & $1.49 \mathrm{E}-10$ & $3.50 \mathrm{E}-11$ & $1.15 \mathrm{E}-13$ \\
\hline $15 \%$ & 7.93E-04 & $7.26 \mathrm{E}-05$ & 2.63E-05 & $2.34 \mathrm{E}-06$ & $1.50 \mathrm{E}-05$ & 4.75E-06 & $1.01 \mathrm{E}-09$ & $7.85 \mathrm{E}-04$ & 2.07E-09 & $1.14 \mathrm{E}-10$ & $2.00 \mathrm{E}-10$ & 4.17E-11 & $1.40 \mathrm{E}-13$ \\
\hline $20 \%$ & 7.94E-04 & $7.45 \mathrm{E}-05$ & $3.09 \mathrm{E}-05$ & $2.53 \mathrm{E}-06$ & $1.84 \mathrm{E}-05$ & $5.47 \mathrm{E}-06$ & $1.15 \mathrm{E}-09$ & $7.85 \mathrm{E}-04$ & 2.07E-09 & $1.25 \mathrm{E}-10$ & $2.47 \mathrm{E}-10$ & $4.83 \mathrm{E}-11$ & $1.61 \mathrm{E}-13$ \\
\hline $25 \%$ & 7.94E-04 & $7.63 \mathrm{E}-05$ & $3.59 \mathrm{E}-05$ & $2.72 \mathrm{E}-06$ & $2.21 \mathrm{E}-05$ & 6.17E-06 & $1.28 \mathrm{E}-09$ & $7.85 \mathrm{E}-04$ & 2.07E-09 & $1.36 \mathrm{E}-10$ & $2.96 \mathrm{E}-10$ & $5.49 \mathrm{E}-11$ & $1.79 \mathrm{E}-13$ \\
\hline $30 \%$ & 7.95E-04 & $7.80 \mathrm{E}-05$ & 4.09E-05 & $2.92 \mathrm{E}-06$ & 2.57E-05 & $6.95 \mathrm{E}-06$ & $1.41 \mathrm{E}-09$ & $7.85 \mathrm{E}-04$ & 2.07E-09 & $1.47 \mathrm{E}-10$ & $3.52 \mathrm{E}-10$ & $6.15 \mathrm{E}-11$ & $1.97 \mathrm{E}-13$ \\
\hline $35 \%$ & 7.96E-04 & 7.94E-05 & 4.68E-05 & $3.10 \mathrm{E}-06$ & $2.96 \mathrm{E}-05$ & $7.56 \mathrm{E}-06$ & $1.53 \mathrm{E}-09$ & $7.85 \mathrm{E}-04$ & 2.07E-09 & $1.58 \mathrm{E}-10$ & $4.17 \mathrm{E}-10$ & $6.77 \mathrm{E}-11$ & $2.12 \mathrm{E}-13$ \\
\hline $40 \%$ & 7.96E-04 & 8.08E-05 & $5.34 \mathrm{E}-05$ & $3.27 \mathrm{E}-06$ & 3.40E-05 & 8.34E-06 & $1.64 \mathrm{E}-09$ & 7.85E-04 & 2.07E-09 & $1.68 \mathrm{E}-10$ & $4.86 \mathrm{E}-10$ & 7.47E-11 & $2.28 \mathrm{E}-13$ \\
\hline $45 \%$ & 7.97E-04 & $8.20 \mathrm{E}-05$ & $6.05 \mathrm{E}-05$ & 3.46E-06 & 3.89E-05 & $9.06 \mathrm{E}-06$ & $1.76 \mathrm{E}-09$ & $7.85 \mathrm{E}-04$ & 2.07E-09 & $1.81 \mathrm{E}-10$ & $5.70 \mathrm{E}-10$ & $8.22 \mathrm{E}-11$ & $2.43 \mathrm{E}-13$ \\
\hline $50 \%$ & 7.97E-04 & 8.35E-05 & 6.84E-05 & 3.65E-06 & 4.49E-05 & $1.00 \mathrm{E}-05$ & $1.89 \mathrm{E}-09$ & 7.85E-04 & 2.07E-09 & $1.94 \mathrm{E}-10$ & $6.67 \mathrm{E}-10$ & $9.16 \mathrm{E}-11$ & $2.59 \mathrm{E}-13$ \\
\hline $55 \%$ & 7.98E-04 & 8.49E-05 & 7.81E-05 & 3.87E-06 & $5.15 \mathrm{E}-05$ & $1.11 \mathrm{E}-05$ & 2.02E-09 & 7.85E-04 & 2.07E-09 & $2.07 \mathrm{E}-10$ & 7.69E-10 & 1.00E-10 & 2.75E-13 \\
\hline $60 \%$ & 7.99E-04 & 8.66E-05 & 8.84E-05 & 4.12E-06 & $5.83 \mathrm{E}-05$ & $1.21 \mathrm{E}-05$ & 2.13E-09 & 7.85E-04 & 2.07E-09 & $2.21 \mathrm{E}-10$ & $9.08 \mathrm{E}-10$ & $1.11 \mathrm{E}-10$ & 2.91E-13 \\
\hline $65 \%$ & $8.00 \mathrm{E}-04$ & $8.82 \mathrm{E}-05$ & $1.01 \mathrm{E}-04$ & 4.41E-06 & $6.78 \mathrm{E}-05$ & $1.33 \mathrm{E}-05$ & $2.28 \mathrm{E}-09$ & $7.85 \mathrm{E}-04$ & 2.07E-09 & $2.36 \mathrm{E}-10$ & 1.06E-09 & $1.23 \mathrm{E}-10$ & $3.08 \mathrm{E}-13$ \\
\hline $70 \%$ & $8.01 \mathrm{E}-04$ & $9.02 \mathrm{E}-05$ & $1.17 \mathrm{E}-04$ & 4.72E-06 & 7.85E-05 & $1.47 \mathrm{E}-05$ & $2.40 \mathrm{E}-09$ & $7.85 \mathrm{E}-04$ & 2.07E-09 & $2.57 \mathrm{E}-10$ & 1.26E-09 & $1.36 \mathrm{E}-10$ & $3.26 \mathrm{E}-13$ \\
\hline $75 \%$ & 8.03E-04 & 9.23E-05 & $1.38 \mathrm{E}-04$ & $5.10 \mathrm{E}-06$ & $9.15 \mathrm{E}-05$ & $1.64 \mathrm{E}-05$ & 2.57E-09 & $7.85 \mathrm{E}-04$ & 2.07E-09 & $2.79 \mathrm{E}-10$ & $1.52 \mathrm{E}-09$ & $1.55 \mathrm{E}-10$ & $3.44 \mathrm{E}-13$ \\
\hline $80 \%$ & 8.05E-04 & $9.54 \mathrm{E}-05$ & $1.65 \mathrm{E}-04$ & $5.56 \mathrm{E}-06$ & $1.08 \mathrm{E}-04$ & $1.85 \mathrm{E}-05$ & 2.73E-09 & $7.85 \mathrm{E}-04$ & 2.07E-09 & $3.07 \mathrm{E}-10$ & $1.88 \mathrm{E}-09$ & $1.80 \mathrm{E}-10$ & $3.67 \mathrm{E}-13$ \\
\hline $85 \%$ & 8.08E-04 & 9.92E-05 & $2.00 \mathrm{E}-04$ & $6.16 \mathrm{E}-06$ & $1.33 \mathrm{E}-04$ & $2.13 \mathrm{E}-05$ & 2.92E-09 & $7.85 \mathrm{E}-04$ & 2.07E-09 & $3.41 \mathrm{E}-10$ & 2.35E-09 & $2.13 \mathrm{E}-10$ & $3.90 \mathrm{E}-13$ \\
\hline $90 \%$ & 8.12E-04 & $1.06 \mathrm{E}-04$ & 2.67E-04 & $7.05 \mathrm{E}-06$ & $1.74 \mathrm{E}-04$ & $2.54 \mathrm{E}-05$ & 3.19E-09 & $7.85 \mathrm{E}-04$ & 2.07E-09 & $4.01 \mathrm{E}-10$ & 3.38E-09 & $2.67 \mathrm{E}-10$ & $4.22 \mathrm{E}-13$ \\
\hline $95 \%$ & 9.01E-04 & $1.23 \mathrm{E}-04$ & $3.95 \mathrm{E}-04$ & 8.64E-06 & $2.55 \mathrm{E}-04$ & $3.29 \mathrm{E}-05$ & $3.72 \mathrm{E}-09$ & $7.85 \mathrm{E}-04$ & 2.07E-09 & $5.01 \mathrm{E}-10$ & $5.10 \mathrm{E}-09$ & $3.88 \mathrm{E}-10$ & $4.70 \mathrm{E}-13$ \\
\hline Mean & $8.20 \mathrm{E}-04$ & 8.76E-05 & $1.22 \mathrm{E}-04$ & 4.27E-06 & $7.80 \mathrm{E}-05$ & $1.31 \mathrm{E}-05$ & 2.01E-09 & 7.85E-04 & 2.07E-09 & $2.38 \mathrm{E}-10$ & $1.52 \mathrm{E}-09$ & $1.39 \mathrm{E}-10$ & 2.67E-13 \\
\hline Min & 7.87E-04 & 3.99E-05 & 7.87E-06 & $6.78 \mathrm{E}-07$ & $1.71 \mathrm{E}-06$ & $1.08 \mathrm{E}-06$ & $1.87 \mathrm{E}-11$ & 7.78E-04 & 2.05E-09 & $2.46 \mathrm{E}-11$ & $2.19 \mathrm{E}-11$ & 8.92E-12 & $2.69 \mathrm{E}-15$ \\
\hline Max & $5.17 \mathrm{E}-03$ & $3.10 \mathrm{E}-04$ & $4.78 \mathrm{E}-03$ & 4.30E-05 & $2.73 \mathrm{E}-03$ & $1.62 \mathrm{E}-04$ & $2.43 \mathrm{E}-08$ & $7.85 \mathrm{E}-04$ & 2.07E-09 & 3.94E-09 & $1.13 \mathrm{E}-07$ & 5.97E-09 & $2.60 \mathrm{E}-12$ \\
\hline
\end{tabular}




\section{TABLE C.18 Peak DSR Percentiles (mrem/yr per pCi/g) for Different Exposure Pathways for Cs-137 in Offsite Resident Scenario}

\begin{tabular}{|c|c|c|c|c|c|c|c|c|c|c|c|c|c|}
\hline Percentile & Total & External-WD & Fish & Plant-WD & Meat-WD & Milk-WD & Soil-WD & External-WI & Inhalation & Plant-WI & Meat-WI & Milk-WI & Soil-WI \\
\hline $5 \%$ & $2.69 \mathrm{E}-03$ & $1.81 \mathrm{E}-04$ & $1.31 \mathrm{E}-03$ & $1.72 \mathrm{E}-05$ & 3.22E-04 & 2.16E-04 & 2.45E-08 & $1.76 \mathrm{E}-04$ & 2.67E-09 & $4.75 \mathrm{E}-10$ & $1.47 \mathrm{E}-09$ & $7.21 \mathrm{E}-10$ & $1.61 \mathrm{E}-12$ \\
\hline $10 \%$ & $3.10 \mathrm{E}-03$ & $2.10 \mathrm{E}-04$ & $1.71 \mathrm{E}-03$ & $2.08 \mathrm{E}-05$ & $3.76 \mathrm{E}-04$ & $2.56 \mathrm{E}-04$ & $3.48 \mathrm{E}-08$ & $1.76 \mathrm{E}-04$ & 2.67E-09 & $5.72 \mathrm{E}-10$ & $1.72 \mathrm{E}-09$ & $8.52 \mathrm{E}-10$ & $2.27 \mathrm{E}-12$ \\
\hline $15 \%$ & $3.45 \mathrm{E}-03$ & 2.22E-04 & $2.03 \mathrm{E}-03$ & 2.34E-05 & $4.18 \mathrm{E}-04$ & $2.88 \mathrm{E}-04$ & 4.39E-08 & $1.76 \mathrm{E}-04$ & 2.67E-09 & $6.43 \mathrm{E}-10$ & 1.94E-09 & $9.60 \mathrm{E}-10$ & $2.84 \mathrm{E}-12$ \\
\hline $20 \%$ & $3.76 \mathrm{E}-03$ & 2.32E-04 & $2.31 \mathrm{E}-03$ & 2.57E-05 & 4.56E-04 & $3.17 \mathrm{E}-04$ & 5.04E-08 & $1.76 \mathrm{E}-04$ & 2.67E-09 & $7.22 \mathrm{E}-10$ & 2.14E-09 & $1.06 \mathrm{E}-09$ & $3.27 \mathrm{E}-12$ \\
\hline $25 \%$ & $4.08 \mathrm{E}-03$ & 2.38E-04 & $2.62 \mathrm{E}-03$ & 2.77E-05 & 4.91E-04 & 3.44E-04 & $5.70 \mathrm{E}-08$ & $1.76 \mathrm{E}-04$ & 2.67E-09 & $7.98 \mathrm{E}-10$ & 2.32E-09 & $1.16 \mathrm{E}-09$ & $3.66 \mathrm{E}-12$ \\
\hline $30 \%$ & $4.39 \mathrm{E}-03$ & 2.44E-04 & $2.90 \mathrm{E}-03$ & 2.97E-05 & $5.25 \mathrm{E}-04$ & $3.70 \mathrm{E}-04$ & $6.26 \mathrm{E}-08$ & $1.76 \mathrm{E}-04$ & 2.67E-09 & $8.74 \mathrm{E}-10$ & $2.50 \mathrm{E}-09$ & $1.24 \mathrm{E}-09$ & $3.99 \mathrm{E}-12$ \\
\hline $35 \%$ & 4.71E-03 & 2.49E-04 & 3.19E-03 & 3.19E-05 & 5.54E-04 & $3.97 \mathrm{E}-04$ & $6.81 \mathrm{E}-08$ & $1.76 \mathrm{E}-04$ & 2.67E-09 & $9.42 \mathrm{E}-10$ & 2.70E-09 & $1.33 \mathrm{E}-09$ & $4.31 \mathrm{E}-12$ \\
\hline $40 \%$ & $5.05 \mathrm{E}-03$ & $2.54 \mathrm{E}-04$ & $3.50 \mathrm{E}-03$ & 3.38E-05 & $5.87 \mathrm{E}-04$ & 4.22E-04 & 7.31E-08 & $1.76 \mathrm{E}-04$ & 2.67E-09 & 1.03E-09 & 2.92E-09 & $1.42 \mathrm{E}-09$ & $4.59 \mathrm{E}-12$ \\
\hline $45 \%$ & $5.41 \mathrm{E}-03$ & $2.58 \mathrm{E}-04$ & $3.87 \mathrm{E}-03$ & 3.61E-05 & $6.22 \mathrm{E}-04$ & 4.51E-04 & 7.80E-08 & $1.76 \mathrm{E}-04$ & 2.67E-09 & $1.10 \mathrm{E}-09$ & 3.14E-09 & $1.52 \mathrm{E}-09$ & $4.88 \mathrm{E}-12$ \\
\hline $50 \%$ & $5.76 \mathrm{E}-03$ & 2.62E-04 & 4.21E-03 & $3.86 \mathrm{E}-05$ & $6.55 \mathrm{E}-04$ & 4.75E-04 & 8.29E-08 & $1.76 \mathrm{E}-04$ & 2.67E-09 & 1.19E-09 & 3.37E-09 & $1.62 \mathrm{E}-09$ & $5.16 \mathrm{E}-12$ \\
\hline $55 \%$ & $6.13 \mathrm{E}-03$ & $2.66 \mathrm{E}-04$ & $4.60 \mathrm{E}-03$ & 4.11E-05 & $6.92 \mathrm{E}-04$ & 5.07E-04 & 8.75E-08 & $1.76 \mathrm{E}-04$ & 2.67E-09 & $1.31 \mathrm{E}-09$ & 3.63E-09 & $1.74 \mathrm{E}-09$ & $5.48 \mathrm{E}-12$ \\
\hline $60 \%$ & $6.54 \mathrm{E}-03$ & 2.70E-04 & $5.04 \mathrm{E}-03$ & 4.41E-05 & $7.28 \mathrm{E}-04$ & $5.40 \mathrm{E}-04$ & $9.25 \mathrm{E}-08$ & $1.76 \mathrm{E}-04$ & 2.67E-09 & $1.42 \mathrm{E}-09$ & 3.94E-09 & $1.86 \mathrm{E}-09$ & $5.75 \mathrm{E}-12$ \\
\hline $65 \%$ & 7.07E-03 & $2.75 \mathrm{E}-04$ & $5.50 \mathrm{E}-03$ & 4.70E-05 & 7.73E-04 & $5.73 \mathrm{E}-04$ & $9.84 \mathrm{E}-08$ & $1.76 \mathrm{E}-04$ & 2.67E-09 & $1.57 \mathrm{E}-09$ & 4.25E-09 & $1.99 \mathrm{E}-09$ & $6.04 \mathrm{E}-12$ \\
\hline $70 \%$ & 7.62E-03 & 2.79E-04 & $6.08 \mathrm{E}-03$ & 5.09E-05 & 8.19E-04 & $6.15 \mathrm{E}-04$ & 1.04E-07 & $1.76 \mathrm{E}-04$ & 2.67E-09 & $1.72 \mathrm{E}-09$ & 4.63E-09 & $2.15 \mathrm{E}-09$ & $6.42 \mathrm{E}-12$ \\
\hline $75 \%$ & $8.38 \mathrm{E}-03$ & $2.84 \mathrm{E}-04$ & $6.75 \mathrm{E}-03$ & $5.56 \mathrm{E}-05$ & $8.73 \mathrm{E}-04$ & $6.61 \mathrm{E}-04$ & $1.11 \mathrm{E}-07$ & $1.76 \mathrm{E}-04$ & 2.67E-09 & $1.94 \mathrm{E}-09$ & $5.08 \mathrm{E}-09$ & 2.33E-09 & $6.81 \mathrm{E}-12$ \\
\hline $80 \%$ & $9.14 \mathrm{E}-03$ & $2.90 \mathrm{E}-04$ & 7.60E-03 & $6.12 \mathrm{E}-05$ & 9.39E-04 & $7.20 \mathrm{E}-04$ & $1.18 \mathrm{E}-07$ & $1.76 \mathrm{E}-04$ & $2.67 \mathrm{E}-09$ & 2.26E-09 & $5.67 \mathrm{E}-09$ & $2.58 \mathrm{E}-09$ & $7.21 \mathrm{E}-12$ \\
\hline $85 \%$ & $1.03 \mathrm{E}-02$ & 2.97E-04 & 8.70E-03 & $6.83 \mathrm{E}-05$ & $1.02 \mathrm{E}-03$ & 7.90E-04 & $1.27 \mathrm{E}-07$ & $1.76 \mathrm{E}-04$ & 2.67E-09 & $2.75 \mathrm{E}-09$ & $6.57 \mathrm{E}-09$ & 2.87E-09 & 7.73E-12 \\
\hline $90 \%$ & $1.18 \mathrm{E}-02$ & $3.06 \mathrm{E}-04$ & $1.03 \mathrm{E}-02$ & $7.85 \mathrm{E}-05$ & $1.14 \mathrm{E}-03$ & 8.84E-04 & $1.38 \mathrm{E}-07$ & $1.76 \mathrm{E}-04$ & 2.67E-09 & 3.54E-09 & 8.06E-09 & 3.36E-09 & $8.42 \mathrm{E}-12$ \\
\hline $95 \%$ & $1.49 \mathrm{E}-02$ & $3.17 \mathrm{E}-04$ & $1.34 \mathrm{E}-02$ & $1.01 \mathrm{E}-04$ & $1.31 \mathrm{E}-03$ & $1.06 \mathrm{E}-03$ & $1.53 \mathrm{E}-07$ & $1.76 \mathrm{E}-04$ & 2.67E-09 & $5.16 \mathrm{E}-09$ & $1.14 \mathrm{E}-08$ & 4.31E-09 & $9.31 \mathrm{E}-12$ \\
\hline Mean & $6.87 \mathrm{E}-03$ & $2.58 \mathrm{E}-04$ & $5.38 \mathrm{E}-03$ & 4.74E-05 & $7.16 \mathrm{E}-04$ & $5.35 \mathrm{E}-04$ & $8.56 \mathrm{E}-08$ & $1.76 \mathrm{E}-04$ & 2.67E-09 & $1.84 \mathrm{E}-09$ & 4.55E-09 & $1.99 \mathrm{E}-09$ & $5.30 \mathrm{E}-12$ \\
\hline Min & $9.86 \mathrm{E}-04$ & 4.90E-05 & 3.63E-04 & $6.18 \mathrm{E}-06$ & $1.06 \mathrm{E}-04$ & 6.61E-05 & $1.36 \mathrm{E}-09$ & $1.75 \mathrm{E}-04$ & 2.65E-09 & $2.00 \mathrm{E}-10$ & $7.65 \mathrm{E}-10$ & 3.55E-10 & 8.30E-14 \\
\hline Max & $5.79 \mathrm{E}-02$ & $3.91 \mathrm{E}-04$ & $5.56 \mathrm{E}-02$ & 8.40E-04 & $2.60 \mathrm{E}-03$ & $2.20 \mathrm{E}-03$ & $3.60 \mathrm{E}-07$ & $1.76 \mathrm{E}-04$ & 2.67E-09 & 5.23E-08 & $7.55 \mathrm{E}-08$ & $3.10 \mathrm{E}-08$ & $2.08 \mathrm{E}-11$ \\
\hline
\end{tabular}




\section{TABLE C.19 Peak DSR Percentiles (mrem/yr per pCi/g) for Different Exposure Pathways for I-129 in Offsite Resident Scenario}

\begin{tabular}{|c|c|c|c|c|c|c|c|c|c|c|c|c|c|}
\hline Percentile & Total & External-WD & Fish & Plant-WD & Meat-WD & Milk-WD & Soil-WD & External-WI & Inhalation & Plant-WI & Meat-WI & Milk-WI & Soil-WI \\
\hline $5 \%$ & $1.22 \mathrm{E}-02$ & $1.28 \mathrm{E}-07$ & 6.49E-04 & 2.92E-04 & $5.17 \mathrm{E}-03$ & 4.66E-03 & $9.22 \mathrm{E}-08$ & 5.37E-12 & 4.91E-09 & $1.83 \mathrm{E}-08$ & $6.16 \mathrm{E}-08$ & $4.25 \mathrm{E}-08$ & $3.21 \mathrm{E}-12$ \\
\hline $10 \%$ & $3.01 \mathrm{E}-02$ & $3.29 \mathrm{E}-07$ & $1.64 \mathrm{E}-03$ & $7.94 \mathrm{E}-04$ & $1.23 \mathrm{E}-02$ & $1.10 \mathrm{E}-02$ & $2.53 \mathrm{E}-07$ & $7.68 \mathrm{E}-12$ & $4.92 \mathrm{E}-09$ & $2.15 \mathrm{E}-08$ & $7.20 \mathrm{E}-08$ & $5.03 \mathrm{E}-08$ & $4.79 \mathrm{E}-12$ \\
\hline $15 \%$ & $5.51 \mathrm{E}-02$ & $5.92 \mathrm{E}-07$ & $2.96 \mathrm{E}-03$ & $1.36 \mathrm{E}-03$ & $2.25 \mathrm{E}-02$ & $2.05 \mathrm{E}-02$ & 4.67E-07 & $9.97 \mathrm{E}-12$ & $4.92 \mathrm{E}-09$ & $2.40 \mathrm{E}-08$ & 7.93E-08 & $5.66 \mathrm{E}-08$ & $6.69 \mathrm{E}-12$ \\
\hline $20 \%$ & $8.14 \mathrm{E}-02$ & $9.22 \mathrm{E}-07$ & $4.76 \mathrm{E}-03$ & $2.06 \mathrm{E}-03$ & $3.39 \mathrm{E}-02$ & $3.24 \mathrm{E}-02$ & $6.84 \mathrm{E}-07$ & $1.20 \mathrm{E}-11$ & $4.93 \mathrm{E}-09$ & $2.65 \mathrm{E}-08$ & $8.58 \mathrm{E}-08$ & $6.18 \mathrm{E}-08$ & $8.28 \mathrm{E}-12$ \\
\hline $25 \%$ & $1.21 \mathrm{E}-01$ & $1.35 \mathrm{E}-06$ & $6.62 \mathrm{E}-03$ & $3.05 \mathrm{E}-03$ & $4.73 \mathrm{E}-02$ & $4.53 \mathrm{E}-02$ & $9.88 \mathrm{E}-07$ & $1.42 \mathrm{E}-11$ & $4.94 \mathrm{E}-09$ & $2.87 \mathrm{E}-08$ & $9.19 \mathrm{E}-08$ & $6.72 \mathrm{E}-08$ & $1.01 \mathrm{E}-11$ \\
\hline $30 \%$ & $1.59 \mathrm{E}-01$ & $1.79 \mathrm{E}-06$ & $9.21 \mathrm{E}-03$ & 4.03E-03 & $6.64 \mathrm{E}-02$ & 6.07E-02 & $1.37 \mathrm{E}-06$ & $1.62 \mathrm{E}-11$ & 4.95E-09 & $3.10 \mathrm{E}-08$ & $9.81 \mathrm{E}-08$ & 7.19E-08 & $1.20 \mathrm{E}-11$ \\
\hline $35 \%$ & 2.04E-01 & $2.40 \mathrm{E}-06$ & $1.22 \mathrm{E}-02$ & $5.24 \mathrm{E}-03$ & $8.68 \mathrm{E}-02$ & 7.92E-02 & $1.85 \mathrm{E}-06$ & $1.84 \mathrm{E}-11$ & 4.97E-09 & $3.35 \mathrm{E}-08$ & $1.03 \mathrm{E}-07$ & 7.69E-08 & $1.41 \mathrm{E}-11$ \\
\hline $40 \%$ & 2.64E-01 & $3.08 \mathrm{E}-06$ & $1.58 \mathrm{E}-02$ & $6.83 \mathrm{E}-03$ & $1.08 \mathrm{E}-01$ & $9.91 \mathrm{E}-02$ & $2.41 \mathrm{E}-06$ & $2.08 \mathrm{E}-11$ & 4.98E-09 & $3.56 \mathrm{E}-08$ & $1.09 \mathrm{E}-07$ & 8.17E-08 & $1.60 \mathrm{E}-11$ \\
\hline $45 \%$ & $3.24 \mathrm{E}-01$ & $3.74 \mathrm{E}-06$ & $2.05 \mathrm{E}-02$ & $8.65 \mathrm{E}-03$ & $1.37 \mathrm{E}-01$ & $1.24 \mathrm{E}-01$ & $3.04 \mathrm{E}-06$ & $2.31 \mathrm{E}-11$ & $5.00 \mathrm{E}-09$ & $3.81 \mathrm{E}-08$ & $1.15 \mathrm{E}-07$ & 8.69E-08 & $1.88 \mathrm{E}-11$ \\
\hline $50 \%$ & 4.02E-01 & $4.71 \mathrm{E}-06$ & $2.60 \mathrm{E}-02$ & 1.07E-02 & $1.70 \mathrm{E}-01$ & $1.51 \mathrm{E}-01$ & $3.80 \mathrm{E}-06$ & $2.59 \mathrm{E}-11$ & $5.02 \mathrm{E}-09$ & $4.08 \mathrm{E}-08$ & $1.21 \mathrm{E}-07$ & $9.19 \mathrm{E}-08$ & $2.15 \mathrm{E}-11$ \\
\hline $55 \%$ & 4.89E-01 & $5.68 \mathrm{E}-06$ & $3.36 \mathrm{E}-02$ & $1.30 \mathrm{E}-02$ & $2.07 \mathrm{E}-01$ & $1.83 \mathrm{E}-01$ & $4.70 \mathrm{E}-06$ & $2.88 \mathrm{E}-11$ & $5.03 \mathrm{E}-09$ & 4.37E-08 & $1.28 \mathrm{E}-07$ & $9.75 \mathrm{E}-08$ & $2.43 \mathrm{E}-11$ \\
\hline $60 \%$ & $5.85 \mathrm{E}-01$ & $6.99 \mathrm{E}-06$ & $4.28 \mathrm{E}-02$ & $1.58 \mathrm{E}-02$ & $2.53 \mathrm{E}-01$ & $2.25 \mathrm{E}-01$ & $5.75 \mathrm{E}-06$ & $3.25 \mathrm{E}-11$ & $5.05 \mathrm{E}-09$ & 4.64E-08 & $1.35 \mathrm{E}-07$ & $1.04 \mathrm{E}-07$ & $2.81 \mathrm{E}-11$ \\
\hline $65 \%$ & 7.03E-01 & $8.41 \mathrm{E}-06$ & $5.43 \mathrm{E}-02$ & $1.93 \mathrm{E}-02$ & $3.02 \mathrm{E}-01$ & $2.82 \mathrm{E}-01$ & 7.29E-06 & $3.66 \mathrm{E}-11$ & $5.06 \mathrm{E}-09$ & 4.93E-08 & $1.42 \mathrm{E}-07$ & $1.10 \mathrm{E}-07$ & 3.24E-11 \\
\hline $70 \%$ & $8.73 \mathrm{E}-01$ & $1.07 \mathrm{E}-05$ & $7.02 \mathrm{E}-02$ & $2.35 \mathrm{E}-02$ & $3.73 \mathrm{E}-01$ & $3.45 \mathrm{E}-01$ & $9.31 \mathrm{E}-06$ & $4.16 \mathrm{E}-11$ & $5.08 \mathrm{E}-09$ & $5.26 \mathrm{E}-08$ & $1.51 \mathrm{E}-07$ & $1.17 \mathrm{E}-07$ & $3.75 \mathrm{E}-11$ \\
\hline $75 \%$ & $1.08 \mathrm{E}+00$ & $1.34 \mathrm{E}-05$ & $9.32 \mathrm{E}-02$ & $2.89 \mathrm{E}-02$ & $4.70 \mathrm{E}-01$ & 4.23E-01 & $1.16 \mathrm{E}-05$ & $4.75 \mathrm{E}-11$ & $5.09 \mathrm{E}-09$ & $5.68 \mathrm{E}-08$ & $1.61 \mathrm{E}-07$ & $1.26 \mathrm{E}-07$ & $4.51 \mathrm{E}-11$ \\
\hline $80 \%$ & $1.38 \mathrm{E}+00$ & $1.72 \mathrm{E}-05$ & $1.23 \mathrm{E}-01$ & $3.69 \mathrm{E}-02$ & $5.98 \mathrm{E}-01$ & $5.40 \mathrm{E}-01$ & $1.54 \mathrm{E}-05$ & $5.43 \mathrm{E}-11$ & $5.11 \mathrm{E}-09$ & $6.15 \mathrm{E}-08$ & $1.73 \mathrm{E}-07$ & $1.36 \mathrm{E}-07$ & $5.35 \mathrm{E}-11$ \\
\hline $85 \%$ & $1.79 \mathrm{E}+00$ & $2.24 \mathrm{E}-05$ & $1.71 \mathrm{E}-01$ & $4.81 \mathrm{E}-02$ & 7.66E-01 & 7.18E-01 & $2.09 \mathrm{E}-05$ & $6.36 \mathrm{E}-11$ & 5.13E-09 & $6.77 \mathrm{E}-08$ & $1.86 \mathrm{E}-07$ & $1.50 \mathrm{E}-07$ & $6.42 \mathrm{E}-11$ \\
\hline $90 \%$ & $2.37 \mathrm{E}+00$ & $3.02 \mathrm{E}-05$ & $2.53 \mathrm{E}-01$ & $6.35 \mathrm{E}-02$ & $1.07 \mathrm{E}+00$ & $9.90 \mathrm{E}-01$ & $3.00 \mathrm{E}-05$ & 7.67E-11 & $5.14 \mathrm{E}-09$ & $7.68 \mathrm{E}-08$ & $2.06 \mathrm{E}-07$ & $1.68 \mathrm{E}-07$ & $8.14 \mathrm{E}-11$ \\
\hline $95 \%$ & $3.55 \mathrm{E}+00$ & 4.83E-05 & $4.45 \mathrm{E}-01$ & $1.00 \mathrm{E}-01$ & $1.58 \mathrm{E}+00$ & $1.47 \mathrm{E}+00$ & 4.83E-05 & $1.02 \mathrm{E}-10$ & $5.16 \mathrm{E}-09$ & $9.25 \mathrm{E}-08$ & $2.38 \mathrm{E}-07$ & $2.00 \mathrm{E}-07$ & $1.16 \mathrm{E}-10$ \\
\hline Mean & $9.30 \mathrm{E}-01$ & $1.23 \mathrm{E}-05$ & $1.14 \mathrm{E}-01$ & $2.53 \mathrm{E}-02$ & $4.06 \mathrm{E}-01$ & $3.85 \mathrm{E}-01$ & $1.19 \mathrm{E}-05$ & $3.71 \mathrm{E}-11$ & $5.02 \mathrm{E}-09$ & 4.59E-08 & $1.32 \mathrm{E}-07$ & $1.03 \mathrm{E}-07$ & 3.63E-11 \\
\hline Min & $8.40 \mathrm{E}-04$ & $2.98 \mathrm{E}-09$ & $1.36 \mathrm{E}-05$ & $2.00 \mathrm{E}-05$ & $3.20 \mathrm{E}-04$ & $2.00 \mathrm{E}-04$ & $1.20 \mathrm{E}-09$ & $1.91 \mathrm{E}-12$ & 4.91E-09 & $6.57 \mathrm{E}-09$ & $3.51 \mathrm{E}-08$ & $2.15 \mathrm{E}-08$ & $8.38 \mathrm{E}-14$ \\
\hline Max & $3.56 \mathrm{E}+01$ & 4.49E-04 & $9.49 \mathrm{E}+00$ & $5.66 \mathrm{E}-01$ & $1.39 \mathrm{E}+01$ & $1.42 \mathrm{E}+01$ & $5.01 \mathrm{E}-04$ & $5.91 \mathrm{E}-10$ & $5.17 \mathrm{E}-09$ & $5.11 \mathrm{E}-07$ & 4.57E-07 & $3.88 \mathrm{E}-07$ & $8.08 \mathrm{E}-10$ \\
\hline
\end{tabular}




\section{TABLE C.20 Peak DSR Percentiles (mrem/yr per pCi/g) for Different Exposure Pathways for Np-237 in Offsite Resident Scenario}

\begin{tabular}{|c|c|c|c|c|c|c|c|c|c|c|c|c|c|}
\hline Percentile & Total & External-WD & Fish & Plant-WD & Meat-WD & Milk-WD & Soil-WD & External-WI & Inhalation & Plant-WI & Meat-WI & Milk-WI & Soil-WI \\
\hline $5 \%$ & 8.32E-04 & $1.05 \mathrm{E}-05$ & 6.41E-04 & 8.62E-05 & $3.14 \mathrm{E}-05$ & $1.21 \mathrm{E}-06$ & $7.58 \mathrm{E}-08$ & $3.14 \mathrm{E}-05$ & $3.28 \mathrm{E}-06$ & 3.07E-09 & $1.71 \mathrm{E}-10$ & $5.35 \mathrm{E}-12$ & $3.95 \mathrm{E}-12$ \\
\hline $10 \%$ & $1.95 \mathrm{E}-03$ & $4.38 \mathrm{E}-05$ & $1.44 \mathrm{E}-03$ & $1.93 \mathrm{E}-04$ & $5.41 \mathrm{E}-05$ & $2.27 \mathrm{E}-06$ & $3.14 \mathrm{E}-07$ & $3.15 \mathrm{E}-05$ & $3.30 \mathrm{E}-06$ & $3.80 \mathrm{E}-09$ & $2.26 \mathrm{E}-10$ & $6.98 \mathrm{E}-12$ & 7.34E-12 \\
\hline $15 \%$ & $2.36 \mathrm{E}-03$ & 8.73E-05 & $1.60 \mathrm{E}-03$ & $2.54 \mathrm{E}-04$ & 7.43E-05 & $3.13 \mathrm{E}-06$ & 7.27E-07 & $3.18 \mathrm{E}-05$ & $3.33 \mathrm{E}-06$ & 4.44E-09 & $2.77 \mathrm{E}-10$ & $8.52 \mathrm{E}-12$ & $1.11 \mathrm{E}-11$ \\
\hline $20 \%$ & $2.71 \mathrm{E}-03$ & $1.54 \mathrm{E}-04$ & $1.73 \mathrm{E}-03$ & $3.20 \mathrm{E}-04$ & $9.40 \mathrm{E}-05$ & $3.84 \mathrm{E}-06$ & $1.28 \mathrm{E}-06$ & $3.22 \mathrm{E}-05$ & $3.37 \mathrm{E}-06$ & $5.08 \mathrm{E}-09$ & $3.23 \mathrm{E}-10$ & $1.01 \mathrm{E}-11$ & $1.47 \mathrm{E}-11$ \\
\hline $25 \%$ & $3.13 \mathrm{E}-03$ & $2.36 \mathrm{E}-04$ & $1.85 \mathrm{E}-03$ & $3.88 \mathrm{E}-04$ & $1.16 \mathrm{E}-04$ & 4.61E-06 & $1.97 \mathrm{E}-06$ & $3.25 \mathrm{E}-05$ & $3.40 \mathrm{E}-06$ & $5.66 \mathrm{E}-09$ & $3.74 \mathrm{E}-10$ & $1.16 \mathrm{E}-11$ & $1.97 \mathrm{E}-11$ \\
\hline $30 \%$ & $3.54 \mathrm{E}-03$ & $3.29 \mathrm{E}-04$ & $2.02 \mathrm{E}-03$ & $4.59 \mathrm{E}-04$ & $1.39 \mathrm{E}-04$ & $5.46 \mathrm{E}-06$ & 2.69E-06 & $3.27 \mathrm{E}-05$ & $3.42 \mathrm{E}-06$ & $6.23 \mathrm{E}-09$ & $4.31 \mathrm{E}-10$ & $1.33 \mathrm{E}-11$ & $2.66 \mathrm{E}-11$ \\
\hline $35 \%$ & 4.14E-03 & $4.36 \mathrm{E}-04$ & $2.22 \mathrm{E}-03$ & $5.36 \mathrm{E}-04$ & $1.63 \mathrm{E}-04$ & $6.26 \mathrm{E}-06$ & $3.59 \mathrm{E}-06$ & $3.29 \mathrm{E}-05$ & $3.44 \mathrm{E}-06$ & $6.95 \mathrm{E}-09$ & $4.86 \mathrm{E}-10$ & $1.50 \mathrm{E}-11$ & $3.70 \mathrm{E}-11$ \\
\hline $40 \%$ & $4.78 \mathrm{E}-03$ & 5.67E-04 & $2.45 \mathrm{E}-03$ & $6.48 \mathrm{E}-04$ & $1.92 \mathrm{E}-04$ & 7.17E-06 & 4.67E-06 & $3.29 \mathrm{E}-05$ & $3.45 \mathrm{E}-06$ & 7.68E-09 & $5.51 \mathrm{E}-10$ & $1.72 \mathrm{E}-11$ & $5.05 \mathrm{E}-11$ \\
\hline $45 \%$ & $5.49 \mathrm{E}-03$ & 7.12E-04 & 2.73E-03 & 8.02E-04 & $2.25 \mathrm{E}-04$ & 8.12E-06 & $5.92 \mathrm{E}-06$ & $3.30 \mathrm{E}-05$ & $3.45 \mathrm{E}-06$ & 8.58E-09 & $6.28 \mathrm{E}-10$ & $1.95 \mathrm{E}-11$ & $6.91 \mathrm{E}-11$ \\
\hline $50 \%$ & $6.49 \mathrm{E}-03$ & $9.20 \mathrm{E}-04$ & 3.17E-03 & $9.88 \mathrm{E}-04$ & $2.64 \mathrm{E}-04$ & $9.51 \mathrm{E}-06$ & $7.32 \mathrm{E}-06$ & $3.30 \mathrm{E}-05$ & $3.45 \mathrm{E}-06$ & $9.66 \mathrm{E}-09$ & $7.19 \mathrm{E}-10$ & $2.26 \mathrm{E}-11$ & $9.12 \mathrm{E}-11$ \\
\hline $55 \%$ & $7.83 \mathrm{E}-03$ & $1.19 \mathrm{E}-03$ & $3.68 \mathrm{E}-03$ & $1.25 \mathrm{E}-03$ & $3.20 \mathrm{E}-04$ & $1.11 \mathrm{E}-05$ & $9.35 \mathrm{E}-06$ & $3.30 \mathrm{E}-05$ & $3.46 \mathrm{E}-06$ & $1.11 \mathrm{E}-08$ & $8.46 \mathrm{E}-10$ & $2.62 \mathrm{E}-11$ & $1.17 \mathrm{E}-10$ \\
\hline $60 \%$ & $9.73 \mathrm{E}-03$ & $1.57 \mathrm{E}-03$ & $4.32 \mathrm{E}-03$ & $1.67 \mathrm{E}-03$ & $3.84 \mathrm{E}-04$ & $1.30 \mathrm{E}-05$ & $1.24 \mathrm{E}-05$ & $3.31 \mathrm{E}-05$ & $3.46 \mathrm{E}-06$ & $1.30 \mathrm{E}-08$ & $9.76 \mathrm{E}-10$ & $3.06 \mathrm{E}-11$ & $1.50 \mathrm{E}-10$ \\
\hline $65 \%$ & $1.24 \mathrm{E}-02$ & $2.10 \mathrm{E}-03$ & $5.33 \mathrm{E}-03$ & $2.18 \mathrm{E}-03$ & $4.68 \mathrm{E}-04$ & $1.56 \mathrm{E}-05$ & $1.67 \mathrm{E}-05$ & $3.31 \mathrm{E}-05$ & $3.46 \mathrm{E}-06$ & $1.55 \mathrm{E}-08$ & $1.16 \mathrm{E}-09$ & $3.57 \mathrm{E}-11$ & $1.93 \mathrm{E}-10$ \\
\hline $70 \%$ & $1.56 \mathrm{E}-02$ & $2.80 \mathrm{E}-03$ & $6.73 \mathrm{E}-03$ & $2.91 \mathrm{E}-03$ & $5.90 \mathrm{E}-04$ & $1.92 \mathrm{E}-05$ & $2.29 \mathrm{E}-05$ & $3.31 \mathrm{E}-05$ & $3.46 \mathrm{E}-06$ & $1.93 \mathrm{E}-08$ & $1.41 \mathrm{E}-09$ & $4.24 \mathrm{E}-11$ & $2.43 \mathrm{E}-10$ \\
\hline $75 \%$ & $2.01 \mathrm{E}-02$ & $4.06 \mathrm{E}-03$ & $9.38 \mathrm{E}-03$ & $3.90 \mathrm{E}-03$ & $7.84 \mathrm{E}-04$ & $2.50 \mathrm{E}-05$ & $3.18 \mathrm{E}-05$ & $3.31 \mathrm{E}-05$ & $3.46 \mathrm{E}-06$ & $2.55 \mathrm{E}-08$ & $1.77 \mathrm{E}-09$ & $5.12 \mathrm{E}-11$ & 3.13E-10 \\
\hline $80 \%$ & $2.62 \mathrm{E}-02$ & $6.28 \mathrm{E}-03$ & $1.35 \mathrm{E}-02$ & $5.49 \mathrm{E}-03$ & $1.08 \mathrm{E}-03$ & $3.45 \mathrm{E}-05$ & $4.68 \mathrm{E}-05$ & $3.31 \mathrm{E}-05$ & $3.46 \mathrm{E}-06$ & $3.63 \mathrm{E}-08$ & $2.38 \mathrm{E}-09$ & $6.26 \mathrm{E}-11$ & $4.30 \mathrm{E}-10$ \\
\hline $85 \%$ & $3.70 \mathrm{E}-02$ & $1.01 \mathrm{E}-02$ & $2.12 \mathrm{E}-02$ & 7.83E-03 & $1.60 \mathrm{E}-03$ & $5.27 \mathrm{E}-05$ & 7.30E-05 & $3.31 \mathrm{E}-05$ & $3.46 \mathrm{E}-06$ & 5.63E-08 & $3.34 \mathrm{E}-09$ & $8.46 \mathrm{E}-11$ & $6.32 \mathrm{E}-10$ \\
\hline $90 \%$ & $6.07 \mathrm{E}-02$ & $1.48 \mathrm{E}-02$ & $3.88 \mathrm{E}-02$ & $1.26 \mathrm{E}-02$ & $2.73 \mathrm{E}-03$ & $8.95 \mathrm{E}-05$ & $1.16 \mathrm{E}-04$ & $3.31 \mathrm{E}-05$ & $3.46 \mathrm{E}-06$ & $9.33 \mathrm{E}-08$ & $4.98 \mathrm{E}-09$ & $1.15 \mathrm{E}-10$ & $1.01 \mathrm{E}-09$ \\
\hline $95 \%$ & $1.17 \mathrm{E}-01$ & $2.05 \mathrm{E}-02$ & $8.35 \mathrm{E}-02$ & $2.41 \mathrm{E}-02$ & $6.04 \mathrm{E}-03$ & $2.09 \mathrm{E}-04$ & $1.84 \mathrm{E}-04$ & $3.31 \mathrm{E}-05$ & $3.46 \mathrm{E}-06$ & $1.71 \mathrm{E}-07$ & $9.62 \mathrm{E}-09$ & $1.98 \mathrm{E}-10$ & 2.35E-09 \\
\hline Mean & $3.48 \mathrm{E}-02$ & 4.47E-03 & $2.41 \mathrm{E}-02$ & $6.21 \mathrm{E}-03$ & $1.70 \mathrm{E}-03$ & $6.03 \mathrm{E}-05$ & $3.79 \mathrm{E}-05$ & $3.27 \mathrm{E}-05$ & $3.42 \mathrm{E}-06$ & 3.67E-08 & $2.26 \mathrm{E}-09$ & $5.09 \mathrm{E}-11$ & $5.37 \mathrm{E}-10$ \\
\hline Min & $1.87 \mathrm{E}-04$ & $5.10 \mathrm{E}-07$ & $1.44 \mathrm{E}-04$ & $1.26 \mathrm{E}-05$ & $2.07 \mathrm{E}-06$ & $8.76 \mathrm{E}-08$ & $1.23 \mathrm{E}-09$ & $3.14 \mathrm{E}-05$ & $3.28 \mathrm{E}-06$ & $8.75 \mathrm{E}-10$ & $4.98 \mathrm{E}-11$ & $1.71 \mathrm{E}-12$ & $1.90 \mathrm{E}-13$ \\
\hline Max & $9.72 \mathrm{E}+00$ & $1.61 \mathrm{E}-01$ & $8.95 \mathrm{E}+00$ & 7.43E-01 & $3.88 \mathrm{E}-01$ & 7.11E-03 & $1.74 \mathrm{E}-03$ & $3.39 \mathrm{E}-05$ & $3.55 \mathrm{E}-06$ & $1.39 \mathrm{E}-06$ & $1.47 \mathrm{E}-07$ & 1.32E-09 & $2.52 \mathrm{E}-08$ \\
\hline
\end{tabular}


TABLE C.21 Peak DSR Percentiles (mrem/yr per pCi/g) for Different Exposure Pathways for Pu-238 in Offsite Resident Scenario

\begin{tabular}{|c|c|c|c|c|c|c|c|c|c|c|c|c|c|c|c|}
\hline Percentile & Total & External-WD & Fish & Radon-WD & Plant-WD & Meat-WD & Milk-WD & Soil-WD & External-WI & Inhalation & Radon & Plant-WI & Meat-WI & Milk-WI & Soil-WI \\
\hline $5 \%$ & $8.84 \mathrm{E}-04$ & $1.40 \mathrm{E}-08$ & $6.03 \mathrm{E}-04$ & $1.33 \mathrm{E}-10$ & $1.82 \mathrm{E}-04$ & $9.05 \mathrm{E}-06$ & $2.99 \mathrm{E}-07$ & $7.24 \mathrm{E}-07$ & $1.11 \mathrm{E}-10$ & $7.58 \mathrm{E}-06$ & $2.25 \mathrm{E}-10$ & $4.95 \mathrm{E}-09$ & $6.17 \mathrm{E}-11$ & $1.26 \mathrm{E}-12$ & 7.45E-11 \\
\hline $10 \%$ & $1.18 \mathrm{E}-03$ & $1.64 \mathrm{E}-08$ & 7.77E-04 & $3.25 \mathrm{E}-10$ & $2.39 \mathrm{E}-04$ & $1.24 \mathrm{E}-05$ & $3.75 \mathrm{E}-07$ & $1.11 \mathrm{E}-06$ & $1.11 \mathrm{E}-10$ & $7.58 \mathrm{E}-06$ & $5.65 \mathrm{E}-10$ & $5.85 \mathrm{E}-09$ & $6.64 \mathrm{E}-11$ & $1.47 \mathrm{E}-12$ & $1.09 \mathrm{E}-10$ \\
\hline $15 \%$ & $1.37 \mathrm{E}-03$ & $1.77 \mathrm{E}-08$ & 8.74E-04 & $4.88 \mathrm{E}-10$ & $2.80 \mathrm{E}-04$ & 1.49E-05 & 4.45E-07 & $1.46 \mathrm{E}-06$ & $1.26 \mathrm{E}-10$ & $7.58 \mathrm{E}-06$ & $8.38 \mathrm{E}-10$ & $6.61 \mathrm{E}-09$ & $6.98 \mathrm{E}-11$ & $1.65 \mathrm{E}-12$ & $1.35 \mathrm{E}-10$ \\
\hline $20 \%$ & $1.51 \mathrm{E}-03$ & $1.86 \mathrm{E}-08$ & $9.73 \mathrm{E}-04$ & $6.19 \mathrm{E}-10$ & $3.16 \mathrm{E}-04$ & $1.68 \mathrm{E}-05$ & $5.05 \mathrm{E}-07$ & $1.85 \mathrm{E}-06$ & $1.62 \mathrm{E}-10$ & $7.58 \mathrm{E}-06$ & $1.07 \mathrm{E}-09$ & 7.28E-09 & $7.27 \mathrm{E}-11$ & $1.81 \mathrm{E}-12$ & $1.55 \mathrm{E}-10$ \\
\hline $25 \%$ & $1.65 \mathrm{E}-03$ & $1.95 \mathrm{E}-08$ & $1.06 \mathrm{E}-03$ & $7.08 \mathrm{E}-10$ & $3.46 \mathrm{E}-04$ & $1.81 \mathrm{E}-05$ & $5.59 \mathrm{E}-07$ & 2.17E-06 & $1.88 \mathrm{E}-10$ & $7.58 \mathrm{E}-06$ & $1.21 \mathrm{E}-09$ & 7.85E-09 & $7.52 \mathrm{E}-11$ & $1.95 \mathrm{E}-12$ & $1.76 \mathrm{E}-10$ \\
\hline $30 \%$ & $1.75 \mathrm{E}-03$ & 2.04E-08 & $1.17 \mathrm{E}-03$ & 7.74E-10 & $3.72 \mathrm{E}-04$ & $1.92 \mathrm{E}-05$ & $6.05 \mathrm{E}-07$ & $2.46 \mathrm{E}-06$ & $2.06 \mathrm{E}-10$ & $7.58 \mathrm{E}-06$ & $1.32 \mathrm{E}-09$ & 8.45E-09 & 7.74E-11 & 2.09E-12 & $1.94 \mathrm{E}-10$ \\
\hline $35 \%$ & $1.88 \mathrm{E}-03$ & 2.12E-08 & $1.27 \mathrm{E}-03$ & $8.17 \mathrm{E}-10$ & $4.05 \mathrm{E}-04$ & 2.01E-05 & $6.56 \mathrm{E}-07$ & $2.72 \mathrm{E}-06$ & $2.18 \mathrm{E}-10$ & $7.58 \mathrm{E}-06$ & $1.39 \mathrm{E}-09$ & $9.01 \mathrm{E}-09$ & $7.97 \mathrm{E}-11$ & 2.23E-12 & $2.09 \mathrm{E}-10$ \\
\hline $40 \%$ & $2.02 \mathrm{E}-03$ & 2.21E-08 & $1.38 \mathrm{E}-03$ & $8.45 \mathrm{E}-10$ & $4.36 \mathrm{E}-04$ & $2.10 \mathrm{E}-05$ & 7.09E-07 & 3.04E-06 & $2.27 \mathrm{E}-10$ & 7.58E-06 & $1.44 \mathrm{E}-09$ & $9.70 \mathrm{E}-09$ & $8.19 \mathrm{E}-11$ & $2.37 \mathrm{E}-12$ & $2.26 \mathrm{E}-10$ \\
\hline $45 \%$ & $2.15 \mathrm{E}-03$ & 2.29E-08 & $1.50 \mathrm{E}-03$ & $8.68 \mathrm{E}-10$ & $4.67 \mathrm{E}-04$ & $2.20 \mathrm{E}-05$ & 7.61E-07 & 3.32E-06 & 2.33E-10 & $7.58 \mathrm{E}-06$ & $1.49 \mathrm{E}-09$ & 1.03E- 08 & $8.42 \mathrm{E}-11$ & $2.52 \mathrm{E}-12$ & $2.40 \mathrm{E}-10$ \\
\hline $50 \%$ & $2.29 \mathrm{E}-03$ & $2.37 \mathrm{E}-08$ & $1.66 \mathrm{E}-03$ & $8.84 \mathrm{E}-10$ & $5.01 \mathrm{E}-04$ & $2.27 \mathrm{E}-05$ & $8.16 \mathrm{E}-07$ & $3.58 \mathrm{E}-06$ & $2.38 \mathrm{E}-10$ & $7.58 \mathrm{E}-06$ & $1.54 \mathrm{E}-09$ & $1.09 \mathrm{E}-08$ & $8.65 \mathrm{E}-11$ & $2.67 \mathrm{E}-12$ & $2.57 \mathrm{E}-10$ \\
\hline $55 \%$ & $2.47 \mathrm{E}-03$ & 2.44E-08 & $1.80 \mathrm{E}-03$ & 8.96E-10 & 5.34E-04 & 2.35E-05 & 8.65E-07 & $3.87 \mathrm{E}-06$ & $2.42 \mathrm{E}-10$ & $7.58 \mathrm{E}-06$ & $1.59 \mathrm{E}-09$ & $1.17 \mathrm{E}-08$ & $8.87 \mathrm{E}-11$ & $2.83 \mathrm{E}-12$ & $2.72 \mathrm{E}-10$ \\
\hline $60 \%$ & $2.65 \mathrm{E}-03$ & $2.50 \mathrm{E}-08$ & $1.99 \mathrm{E}-03$ & $9.05 \mathrm{E}-10$ & $5.77 \mathrm{E}-04$ & $2.42 \mathrm{E}-05$ & $9.35 \mathrm{E}-07$ & 4.14E-06 & $2.45 \mathrm{E}-10$ & $7.58 \mathrm{E}-06$ & $1.62 \mathrm{E}-09$ & $1.25 \mathrm{E}-08$ & $9.13 \mathrm{E}-11$ & $3.01 \mathrm{E}-12$ & $2.86 \mathrm{E}-10$ \\
\hline $65 \%$ & $2.89 \mathrm{E}-03$ & $2.57 \mathrm{E}-08$ & $2.21 \mathrm{E}-03$ & $9.11 \mathrm{E}-10$ & $6.26 \mathrm{E}-04$ & $2.51 \mathrm{E}-05$ & $1.01 \mathrm{E}-06$ & 4.40E-06 & $2.47 \mathrm{E}-10$ & 7.58E-06 & $1.66 \mathrm{E}-09$ & $1.33 \mathrm{E}-08$ & $9.40 \mathrm{E}-11$ & $3.20 \mathrm{E}-12$ & $3.00 \mathrm{E}-10$ \\
\hline $70 \%$ & $3.17 \mathrm{E}-03$ & 2.64E-08 & $2.46 \mathrm{E}-03$ & $9.16 \mathrm{E}-10$ & $6.76 \mathrm{E}-04$ & $2.60 \mathrm{E}-05$ & $1.08 \mathrm{E}-06$ & 4.74E-06 & 2.49E-10 & $7.58 \mathrm{E}-06$ & $1.70 \mathrm{E}-09$ & 1.43E- 08 & $9.68 \mathrm{E}-11$ & 3.42E-12 & $3.18 \mathrm{E}-10$ \\
\hline $75 \%$ & $3.49 \mathrm{E}-03$ & 2.73E-08 & $2.80 \mathrm{E}-03$ & $9.20 \mathrm{E}-10$ & 7.30E-04 & 2.69E-05 & $1.17 \mathrm{E}-06$ & $5.07 \mathrm{E}-06$ & $2.50 \mathrm{E}-10$ & 7.58E-06 & $1.74 \mathrm{E}-09$ & $1.55 \mathrm{E}-08$ & $1.00 \mathrm{E}-10$ & $3.66 \mathrm{E}-12$ & $3.37 \mathrm{E}-10$ \\
\hline $80 \%$ & $3.91 \mathrm{E}-03$ & 2.83E-08 & $3.22 \mathrm{E}-03$ & $9.25 \mathrm{E}-10$ & 7.94E-04 & $2.80 \mathrm{E}-05$ & 1.27E-06 & 5.41E-06 & $2.51 \mathrm{E}-10$ & $7.58 \mathrm{E}-06$ & $1.79 \mathrm{E}-09$ & $1.69 \mathrm{E}-08$ & $1.04 \mathrm{E}-10$ & $3.97 \mathrm{E}-12$ & $3.57 \mathrm{E}-10$ \\
\hline $85 \%$ & $4.62 \mathrm{E}-03$ & 2.93E-08 & 3.92E-03 & $9.36 \mathrm{E}-10$ & $8.76 \mathrm{E}-04$ & $2.93 \mathrm{E}-05$ & $1.40 \mathrm{E}-06$ & $5.88 \mathrm{E}-06$ & $2.52 \mathrm{E}-10$ & $7.58 \mathrm{E}-06$ & $1.84 \mathrm{E}-09$ & $1.87 \mathrm{E}-08$ & $1.08 \mathrm{E}-10$ & $4.35 \mathrm{E}-12$ & $3.84 \mathrm{E}-10$ \\
\hline $90 \%$ & $5.69 \mathrm{E}-03$ & $3.08 \mathrm{E}-08$ & $5.02 \mathrm{E}-03$ & $9.61 \mathrm{E}-10$ & $9.87 \mathrm{E}-04$ & 3.09E-05 & $1.58 \mathrm{E}-06$ & 6.45E-06 & 2.53E-10 & 7.58E-06 & $1.90 \mathrm{E}-09$ & $2.09 \mathrm{E}-08$ & $1.14 \mathrm{E}-10$ & $4.89 \mathrm{E}-12$ & $4.15 \mathrm{E}-10$ \\
\hline $95 \%$ & $8.18 \mathrm{E}-03$ & $3.31 \mathrm{E}-08$ & $7.38 \mathrm{E}-03$ & $1.01 \mathrm{E}-09$ & $1.18 \mathrm{E}-03$ & $3.34 \mathrm{E}-05$ & $1.92 \mathrm{E}-06$ & 7.34E-06 & $2.54 \mathrm{E}-10$ & $7.58 \mathrm{E}-06$ & $1.99 \mathrm{E}-09$ & $2.52 \mathrm{E}-08$ & $1.24 \mathrm{E}-10$ & $5.80 \mathrm{E}-12$ & $4.59 \mathrm{E}-10$ \\
\hline Mean & $3.13 \mathrm{E}-03$ & $2.36 \mathrm{E}-08$ & $2.53 \mathrm{E}-03$ & 7.69E-10 & $5.72 \mathrm{E}-04$ & $2.23 \mathrm{E}-05$ & $9.19 \mathrm{E}-07$ & $3.72 \mathrm{E}-06$ & $2.12 \mathrm{E}-10$ & $7.58 \mathrm{E}-06$ & $1.40 \mathrm{E}-09$ & $1.24 \mathrm{E}-08$ & $8.92 \mathrm{E}-11$ & $2.98 \mathrm{E}-12$ & $2.60 \mathrm{E}-10$ \\
\hline Min & 3.37E-04 & 2.72E-09 & $2.13 \mathrm{E}-04$ & $4.46 \mathrm{E}-12$ & $6.07 \mathrm{E}-05$ & $4.24 \mathrm{E}-06$ & $8.48 \mathrm{E}-08$ & $3.08 \mathrm{E}-08$ & $1.10 \mathrm{E}-10$ & $7.51 \mathrm{E}-06$ & $6.34 \mathrm{E}-12$ & 2.05E-09 & 4.61E-11 & $6.27 \mathrm{E}-13$ & $6.05 \mathrm{E}-12$ \\
\hline Max & 4.32E-02 & $6.72 \mathrm{E}-08$ & $4.25 \mathrm{E}-02$ & $1.25 \mathrm{E}-09$ & $3.09 \mathrm{E}-03$ & 4.83E-05 & 3.72E-06 & $1.21 \mathrm{E}-05$ & $2.56 \mathrm{E}-10$ & $7.58 \mathrm{E}-06$ & 2.37E-09 & $5.38 \mathrm{E}-08$ & $3.31 \mathrm{E}-10$ & $1.24 \mathrm{E}-11$ & $8.37 \mathrm{E}-10$ \\
\hline
\end{tabular}




\section{TABLE C.22 Peak DSR Percentiles (mrem/yr per pCi/g) for Different Exposure Pathways for Pu-239 in Offsite Resident Scenario}

\begin{tabular}{|c|c|c|c|c|c|c|c|c|c|c|c|c|c|}
\hline Percentile & Total & External-WD & Fish & Plant-WD & Meat-WD & Milk-WD & Soil-WD & External-WI & Inhalation & Plant-WI & Meat-WI & Milk-WI & Soil-WI \\
\hline $5 \%$ & $1.04 \mathrm{E}-03$ & $1.50 \mathrm{E}-07$ & $6.68 \mathrm{E}-04$ & 2.32E-04 & 1.03E-05 & $3.22 \mathrm{E}-07$ & 7.83E-06 & $6.93 \mathrm{E}-09$ & 8.30E-06 & 9.77E-09 & $1.49 \mathrm{E}-10$ & $2.60 \mathrm{E}-12$ & 7.67E-10 \\
\hline $10 \%$ & $1.39 \mathrm{E}-03$ & $2.33 \mathrm{E}-07$ & $8.83 \mathrm{E}-04$ & $3.29 \mathrm{E}-04$ & $1.44 \mathrm{E}-05$ & $4.31 \mathrm{E}-07$ & $1.30 \mathrm{E}-05$ & $6.93 \mathrm{E}-09$ & $8.30 \mathrm{E}-06$ & $1.18 \mathrm{E}-08$ & $1.89 \mathrm{E}-10$ & $3.26 \mathrm{E}-12$ & $1.16 \mathrm{E}-09$ \\
\hline $15 \%$ & $1.64 \mathrm{E}-03$ & $2.97 \mathrm{E}-07$ & $9.99 \mathrm{E}-04$ & $3.93 \mathrm{E}-04$ & $1.80 \mathrm{E}-05$ & $5.08 \mathrm{E}-07$ & $1.83 \mathrm{E}-05$ & $6.93 \mathrm{E}-09$ & $8.30 \mathrm{E}-06$ & $1.35 \mathrm{E}-08$ & $2.18 \mathrm{E}-10$ & $3.77 \mathrm{E}-12$ & $1.53 \mathrm{E}-09$ \\
\hline $20 \%$ & $1.82 \mathrm{E}-03$ & $3.55 \mathrm{E}-07$ & $1.10 \mathrm{E}-03$ & $4.48 \mathrm{E}-04$ & $2.09 \mathrm{E}-05$ & $5.77 \mathrm{E}-07$ & $2.26 \mathrm{E}-05$ & $6.93 \mathrm{E}-09$ & $8.30 \mathrm{E}-06$ & $1.50 \mathrm{E}-08$ & $2.39 \mathrm{E}-10$ & $4.22 \mathrm{E}-12$ & $1.84 \mathrm{E}-09$ \\
\hline $25 \%$ & $1.97 \mathrm{E}-03$ & $4.16 \mathrm{E}-07$ & $1.20 \mathrm{E}-03$ & $5.02 \mathrm{E}-04$ & $2.26 \mathrm{E}-05$ & $6.45 \mathrm{E}-07$ & $2.69 \mathrm{E}-05$ & $6.93 \mathrm{E}-09$ & $8.30 \mathrm{E}-06$ & $1.65 \mathrm{E}-08$ & $2.58 \mathrm{E}-10$ & $4.65 \mathrm{E}-12$ & $2.19 \mathrm{E}-09$ \\
\hline $30 \%$ & $2.13 \mathrm{E}-03$ & $4.71 \mathrm{E}-07$ & $1.30 \mathrm{E}-03$ & $5.49 \mathrm{E}-04$ & $2.42 \mathrm{E}-05$ & $7.14 \mathrm{E}-07$ & $3.18 \mathrm{E}-05$ & $6.93 \mathrm{E}-09$ & $8.30 \mathrm{E}-06$ & $1.80 \mathrm{E}-08$ & $2.75 \mathrm{E}-10$ & $5.04 \mathrm{E}-12$ & $2.54 \mathrm{E}-09$ \\
\hline $35 \%$ & $2.29 \mathrm{E}-03$ & 5.31E-07 & $1.43 \mathrm{E}-03$ & $5.91 \mathrm{E}-04$ & $2.56 \mathrm{E}-05$ & 7.71E-07 & 3.61E-05 & 6.93E-09 & $8.30 \mathrm{E}-06$ & $1.95 \mathrm{E}-08$ & $2.90 \mathrm{E}-10$ & $5.45 \mathrm{E}-12$ & $2.85 \mathrm{E}-09$ \\
\hline $40 \%$ & $2.46 \mathrm{E}-03$ & $5.83 \mathrm{E}-07$ & $1.55 \mathrm{E}-03$ & $6.37 \mathrm{E}-04$ & $2.68 \mathrm{E}-05$ & $8.25 \mathrm{E}-07$ & $4.12 \mathrm{E}-05$ & 6.93E-09 & $8.30 \mathrm{E}-06$ & $2.09 \mathrm{E}-08$ & $3.06 \mathrm{E}-10$ & $5.85 \mathrm{E}-12$ & $3.14 \mathrm{E}-09$ \\
\hline $45 \%$ & $2.60 \mathrm{E}-03$ & $6.41 \mathrm{E}-07$ & $1.70 \mathrm{E}-03$ & $6.85 \mathrm{E}-04$ & $2.81 \mathrm{E}-05$ & $8.92 \mathrm{E}-07$ & 4.61E-05 & $6.93 \mathrm{E}-09$ & $8.30 \mathrm{E}-06$ & $2.21 \mathrm{E}-08$ & $3.22 \mathrm{E}-10$ & $6.30 \mathrm{E}-12$ & $3.49 \mathrm{E}-09$ \\
\hline $50 \%$ & $2.77 \mathrm{E}-03$ & $6.93 \mathrm{E}-07$ & $1.87 \mathrm{E}-03$ & 7.38E-04 & $2.92 \mathrm{E}-05$ & $9.60 \mathrm{E}-07$ & $5.11 \mathrm{E}-05$ & $6.93 \mathrm{E}-09$ & $8.30 \mathrm{E}-06$ & $2.37 \mathrm{E}-08$ & $3.39 \mathrm{E}-10$ & $6.80 \mathrm{E}-12$ & $3.80 \mathrm{E}-09$ \\
\hline $55 \%$ & $2.96 \mathrm{E}-03$ & 7.39E-07 & $2.03 \mathrm{E}-03$ & $7.86 \mathrm{E}-04$ & $3.02 \mathrm{E}-05$ & $1.02 \mathrm{E}-06$ & $5.63 \mathrm{E}-05$ & $6.93 \mathrm{E}-09$ & $8.30 \mathrm{E}-06$ & $2.54 \mathrm{E}-08$ & $3.54 \mathrm{E}-10$ & 7.33E-12 & $4.12 \mathrm{E}-09$ \\
\hline $60 \%$ & $3.20 \mathrm{E}-03$ & 7.77E-07 & $2.27 \mathrm{E}-03$ & $8.44 \mathrm{E}-04$ & $3.14 \mathrm{E}-05$ & $1.09 \mathrm{E}-06$ & $6.13 \mathrm{E}-05$ & $6.93 \mathrm{E}-09$ & $8.30 \mathrm{E}-06$ & $2.76 \mathrm{E}-08$ & $3.71 \mathrm{E}-10$ & $7.93 \mathrm{E}-12$ & $4.43 \mathrm{E}-09$ \\
\hline $65 \%$ & $3.44 \mathrm{E}-03$ & 8.17E-07 & $2.51 \mathrm{E}-03$ & $9.06 \mathrm{E}-04$ & $3.26 \mathrm{E}-05$ & $1.17 \mathrm{E}-06$ & $6.74 \mathrm{E}-05$ & $6.93 \mathrm{E}-09$ & $8.30 \mathrm{E}-06$ & $2.98 \mathrm{E}-08$ & $3.89 \mathrm{E}-10$ & $8.53 \mathrm{E}-12$ & 4.76E-09 \\
\hline $70 \%$ & $3.77 \mathrm{E}-03$ & $8.54 \mathrm{E}-07$ & $2.82 \mathrm{E}-03$ & $9.71 \mathrm{E}-04$ & $3.40 \mathrm{E}-05$ & $1.26 \mathrm{E}-06$ & $7.31 \mathrm{E}-05$ & $6.93 \mathrm{E}-09$ & $8.30 \mathrm{E}-06$ & $3.22 \mathrm{E}-08$ & $4.09 \mathrm{E}-10$ & $9.21 \mathrm{E}-12$ & $5.12 \mathrm{E}-09$ \\
\hline $75 \%$ & $4.15 \mathrm{E}-03$ & $8.89 \mathrm{E}-07$ & $3.24 \mathrm{E}-03$ & $1.05 \mathrm{E}-03$ & $3.55 \mathrm{E}-05$ & $1.35 \mathrm{E}-06$ & $7.94 \mathrm{E}-05$ & $6.93 \mathrm{E}-09$ & $8.30 \mathrm{E}-06$ & $3.50 \mathrm{E}-08$ & $4.33 \mathrm{E}-10$ & $1.01 \mathrm{E}-11$ & $5.53 \mathrm{E}-09$ \\
\hline $80 \%$ & $4.70 \mathrm{E}-03$ & $9.24 \mathrm{E}-07$ & $3.80 \mathrm{E}-03$ & $1.14 \mathrm{E}-03$ & $3.71 \mathrm{E}-05$ & $1.47 \mathrm{E}-06$ & $8.66 \mathrm{E}-05$ & $6.93 \mathrm{E}-09$ & $8.30 \mathrm{E}-06$ & $3.86 \mathrm{E}-08$ & $4.63 \mathrm{E}-10$ & $1.11 \mathrm{E}-11$ & $5.96 \mathrm{E}-09$ \\
\hline $85 \%$ & $5.39 \mathrm{E}-03$ & $9.59 \mathrm{E}-07$ & $4.51 \mathrm{E}-03$ & $1.25 \mathrm{E}-03$ & $3.91 \mathrm{E}-05$ & $1.63 \mathrm{E}-06$ & $9.55 \mathrm{E}-05$ & $6.93 \mathrm{E}-09$ & $8.30 \mathrm{E}-06$ & $4.34 \mathrm{E}-08$ & $4.95 \mathrm{E}-10$ & $1.21 \mathrm{E}-11$ & $6.44 \mathrm{E}-09$ \\
\hline $90 \%$ & $6.51 \mathrm{E}-03$ & $1.00 \mathrm{E}-06$ & $5.64 \mathrm{E}-03$ & $1.42 \mathrm{E}-03$ & 4.17E-05 & $1.86 \mathrm{E}-06$ & $1.07 \mathrm{E}-04$ & $6.93 \mathrm{E}-09$ & $8.30 \mathrm{E}-06$ & $5.15 \mathrm{E}-08$ & $5.34 \mathrm{E}-10$ & $1.39 \mathrm{E}-11$ & 7.07E-09 \\
\hline $95 \%$ & $9.05 \mathrm{E}-03$ & $1.06 \mathrm{E}-06$ & 8.27E-03 & $1.75 \mathrm{E}-03$ & $4.55 \mathrm{E}-05$ & $2.21 \mathrm{E}-06$ & $1.23 \mathrm{E}-04$ & $6.93 \mathrm{E}-09$ & 8.30E-06 & $6.64 \mathrm{E}-08$ & $6.03 \mathrm{E}-10$ & $1.65 \mathrm{E}-11$ & 8.01E-09 \\
\hline Mean & 3.67E-03 & $6.50 \mathrm{E}-07$ & $2.87 \mathrm{E}-03$ & $8.28 \mathrm{E}-04$ & 2.89E-05 & $1.07 \mathrm{E}-06$ & $5.61 \mathrm{E}-05$ & $6.93 \mathrm{E}-09$ & $8.30 \mathrm{E}-06$ & $2.91 \mathrm{E}-08$ & $3.53 \mathrm{E}-10$ & $7.88 \mathrm{E}-12$ & $3.99 \mathrm{E}-09$ \\
\hline Min & $3.32 \mathrm{E}-04$ & $9.91 \mathrm{E}-09$ & $1.95 \mathrm{E}-04$ & 5.53E-05 & 4.34E-06 & $1.00 \mathrm{E}-07$ & $3.31 \mathrm{E}-07$ & $6.84 \mathrm{E}-09$ & 8.19E-06 & $3.50 \mathrm{E}-09$ & $5.98 \mathrm{E}-11$ & $9.87 \mathrm{E}-13$ & $8.48 \mathrm{E}-11$ \\
\hline Max & 7.18E-02 & $1.30 \mathrm{E}-06$ & 7.04E-02 & $3.56 \mathrm{E}-03$ & $6.86 \mathrm{E}-05$ & $4.76 \mathrm{E}-06$ & $1.94 \mathrm{E}-04$ & $6.93 \mathrm{E}-09$ & $8.30 \mathrm{E}-06$ & $2.35 \mathrm{E}-07$ & $1.00 \mathrm{E}-09$ & $3.56 \mathrm{E}-11$ & $1.25 \mathrm{E}-08$ \\
\hline
\end{tabular}




\section{TABLE C.23 Peak DSR Percentiles (mrem/yr per pCi/g) for Different Exposure Pathways for Pu-240 in Offsite Resident Scenario}

\begin{tabular}{|c|c|c|c|c|c|c|c|c|c|c|c|c|c|c|c|}
\hline Percentile & Total & External-WD & Fish & Radon-WD & Plant-WD & Meat-WD & Milk-WD & Soil-WD & External-WI & Inhalation & Radon & Plant-WI & Meat-WI & Milk-WI & Soil-WI \\
\hline $5 \%$ & $1.90 \mathrm{E}-03$ & $8.78 \mathrm{E}-08$ & $1.13 \mathrm{E}-03$ & $3.17 \mathrm{E}-17$ & 4.41E-04 & $2.38 \mathrm{E}-05$ & $5.88 \mathrm{E}-07$ & $1.10 \mathrm{E}-05$ & $5.52 \mathrm{E}-10$ & $8.30 \mathrm{E}-06$ & $7.78 \mathrm{E}-15$ & $9.50 \mathrm{E}-09$ & $1.45 \mathrm{E}-10$ & $2.55 \mathrm{E}-12$ & $8.30 \mathrm{E}-10$ \\
\hline $10 \%$ & $2.14 \mathrm{E}-03$ & $1.21 \mathrm{E}-07$ & $1.30 \mathrm{E}-03$ & $4.68 \mathrm{E}-17$ & $5.15 \mathrm{E}-04$ & $2.60 \mathrm{E}-05$ & $6.99 \mathrm{E}-07$ & $1.66 \mathrm{E}-05$ & $5.52 \mathrm{E}-10$ & $8.30 \mathrm{E}-06$ & $1.22 \mathrm{E}-14$ & $1.15 \mathrm{E}-08$ & $1.85 \mathrm{E}-10$ & $3.21 \mathrm{E}-12$ & $1.20 \mathrm{E}-09$ \\
\hline $15 \%$ & $2.33 \mathrm{E}-03$ & $1.50 \mathrm{E}-07$ & 1.44E-03 & $5.59 \mathrm{E}-17$ & $5.80 \mathrm{E}-04$ & $2.75 \mathrm{E}-05$ & $7.88 \mathrm{E}-07$ & $2.13 \mathrm{E}-05$ & $5.52 \mathrm{E}-10$ & $8.30 \mathrm{E}-06$ & $1.53 \mathrm{E}-14$ & $1.30 \mathrm{E}-08$ & 2.13E-10 & $3.81 \mathrm{E}-12$ & $1.61 \mathrm{E}-09$ \\
\hline $20 \%$ & $2.53 \mathrm{E}-03$ & $1.78 \mathrm{E}-07$ & $1.60 \mathrm{E}-03$ & $6.33 \mathrm{E}-17$ & $6.36 \mathrm{E}-04$ & $2.87 \mathrm{E}-05$ & $8.74 \mathrm{E}-07$ & $2.72 \mathrm{E}-05$ & $5.52 \mathrm{E}-10$ & $8.30 \mathrm{E}-06$ & $1.74 \mathrm{E}-14$ & $1.46 \mathrm{E}-08$ & $2.39 \mathrm{E}-10$ & $4.29 \mathrm{E}-12$ & 2.05E-09 \\
\hline $25 \%$ & $2.75 \mathrm{E}-03$ & $2.12 \mathrm{E}-07$ & $1.78 \mathrm{E}-03$ & $6.87 \mathrm{E}-17$ & $6.86 \mathrm{E}-04$ & 3.00E-05 & $9.50 \mathrm{E}-07$ & $3.31 \mathrm{E}-05$ & $5.52 \mathrm{E}-10$ & $8.30 \mathrm{E}-06$ & $1.93 \mathrm{E}-14$ & $1.59 \mathrm{E}-08$ & 2.63E-10 & $4.77 \mathrm{E}-12$ & 2.57E- 09 \\
\hline $30 \%$ & $2.95 \mathrm{E}-03$ & $2.46 \mathrm{E}-07$ & $1.94 \mathrm{E}-03$ & $7.31 \mathrm{E}-17$ & 7.35E-04 & 3.13E-05 & $1.03 \mathrm{E}-06$ & $3.95 \mathrm{E}-05$ & $5.52 \mathrm{E}-10$ & $8.30 \mathrm{E}-06$ & $2.09 \mathrm{E}-14$ & $1.73 \mathrm{E}-08$ & $2.82 \mathrm{E}-10$ & $5.15 \mathrm{E}-12$ & 3.02E-09 \\
\hline $35 \%$ & $3.16 \mathrm{E}-03$ & $2.83 \mathrm{E}-07$ & $2.15 \mathrm{E}-03$ & $7.75 \mathrm{E}-17$ & 7.87E-04 & 3.23E-05 & $1.11 \mathrm{E}-06$ & $4.59 \mathrm{E}-05$ & $5.52 \mathrm{E}-10$ & $8.30 \mathrm{E}-06$ & $2.24 \mathrm{E}-14$ & $1.87 \mathrm{E}-08$ & $3.02 \mathrm{E}-10$ & $5.54 \mathrm{E}-12$ & 3.60E-09 \\
\hline $40 \%$ & $3.39 \mathrm{E}-03$ & $3.29 \mathrm{E}-07$ & $2.35 \mathrm{E}-03$ & $8.19 \mathrm{E}-17$ & $8.36 \mathrm{E}-04$ & 3.35E-05 & $1.18 \mathrm{E}-06$ & $5.44 \mathrm{E}-05$ & $5.52 \mathrm{E}-10$ & 8.30E-06 & $2.37 \mathrm{E}-14$ & 2.03E- 08 & $3.19 \mathrm{E}-10$ & $5.95 \mathrm{E}-12$ & 4.15E-09 \\
\hline $45 \%$ & 3.64E-03 & $3.75 \mathrm{E}-07$ & $2.58 \mathrm{E}-03$ & $8.62 \mathrm{E}-17$ & $8.91 \mathrm{E}-04$ & 3.46E-05 & $1.28 \mathrm{E}-06$ & $6.31 \mathrm{E}-05$ & $5.52 \mathrm{E}-10$ & $8.30 \mathrm{E}-06$ & $2.51 \mathrm{E}-14$ & $2.15 \mathrm{E}-08$ & $3.35 \mathrm{E}-10$ & $6.38 \mathrm{E}-12$ & 4.71E- 09 \\
\hline $50 \%$ & $3.94 \mathrm{E}-03$ & 4.16E-07 & $2.82 \mathrm{E}-03$ & $9.05 \mathrm{E}-17$ & $9.54 \mathrm{E}-04$ & 3.60E-05 & $1.36 \mathrm{E}-06$ & 7.13E-05 & $5.52 \mathrm{E}-10$ & $8.30 \mathrm{E}-06$ & $2.64 \mathrm{E}-14$ & $2.30 \mathrm{E}-08$ & 3.52E-10 & $6.85 \mathrm{E}-12$ & $5.28 \mathrm{E}-09$ \\
\hline $55 \%$ & $4.25 \mathrm{E}-03$ & 4.66E-07 & $3.10 \mathrm{E}-03$ & $9.53 \mathrm{E}-17$ & $1.02 \mathrm{E}-03$ & $3.74 \mathrm{E}-05$ & $1.45 \mathrm{E}-06$ & $8.06 \mathrm{E}-05$ & $5.52 \mathrm{E}-10$ & 8.30E-06 & $2.78 \mathrm{E}-14$ & 2.49E-08 & $3.71 \mathrm{E}-10$ & 7.38E-12 & 6.03E-09 \\
\hline $60 \%$ & $4.61 \mathrm{E}-03$ & $5.18 \mathrm{E}-07$ & 3.42E- 03 & $1.00 \mathrm{E}-16$ & $1.08 \mathrm{E}-03$ & 3.91E-05 & $1.56 \mathrm{E}-06$ & $9.14 \mathrm{E}-05$ & $5.52 \mathrm{E}-10$ & $8.30 \mathrm{E}-06$ & $2.92 \mathrm{E}-14$ & $2.69 \mathrm{E}-08$ & 3.87E-10 & 7.96E-12 & $6.85 \mathrm{E}-09$ \\
\hline $65 \%$ & $5.00 \mathrm{E}-03$ & $5.78 \mathrm{E}-07$ & 3.82E- 03 & $1.04 \mathrm{E}-16$ & $1.15 \mathrm{E}-03$ & 4.09E-05 & $1.67 \mathrm{E}-06$ & $1.05 \mathrm{E}-04$ & $5.52 \mathrm{E}-10$ & $8.30 \mathrm{E}-06$ & $3.04 \mathrm{E}-14$ & $2.90 \mathrm{E}-08$ & 4.07E-10 & $8.60 \mathrm{E}-12$ & 7.74E- 09 \\
\hline $70 \%$ & $5.47 \mathrm{E}-03$ & 6.44E-07 & $4.28 \mathrm{E}-03$ & $1.09 \mathrm{E}-16$ & $1.24 \mathrm{E}-03$ & $4.28 \mathrm{E}-05$ & $1.79 \mathrm{E}-06$ & $1.19 \mathrm{E}-04$ & $5.52 \mathrm{E}-10$ & $8.30 \mathrm{E}-06$ & $3.20 \mathrm{E}-14$ & $3.14 \mathrm{E}-08$ & $4.26 \mathrm{E}-10$ & $9.28 \mathrm{E}-12$ & $8.65 \mathrm{E}-09$ \\
\hline $75 \%$ & $6.04 \mathrm{E}-03$ & $7.11 \mathrm{E}-07$ & $4.84 \mathrm{E}-03$ & $1.15 \mathrm{E}-16$ & $1.33 \mathrm{E}-03$ & 4.53E-05 & $1.95 \mathrm{E}-06$ & $1.36 \mathrm{E}-04$ & $5.52 \mathrm{E}-10$ & $8.30 \mathrm{E}-06$ & $3.36 \mathrm{E}-14$ & $3.46 \mathrm{E}-08$ & $4.50 \mathrm{E}-10$ & $1.00 \mathrm{E}-11$ & $9.71 \mathrm{E}-09$ \\
\hline $80 \%$ & $6.90 \mathrm{E}-03$ & 7.85E-07 & $5.61 \mathrm{E}-03$ & $1.20 \mathrm{E}-16$ & $1.46 \mathrm{E}-03$ & 4.87E- 05 & 2.16E-06 & $1.55 \mathrm{E}-04$ & $5.52 \mathrm{E}-10$ & $8.30 \mathrm{E}-06$ & $3.56 \mathrm{E}-14$ & $3.88 \mathrm{E}-08$ & $4.75 \mathrm{E}-10$ & $1.11 \mathrm{E}-11$ & $1.09 \mathrm{E}-08$ \\
\hline $85 \%$ & 8.07E-03 & $8.60 \mathrm{E}-07$ & $6.72 \mathrm{E}-03$ & $1.28 \mathrm{E}-16$ & $1.61 \mathrm{E}-03$ & $5.39 \mathrm{E}-05$ & $2.41 \mathrm{E}-06$ & $1.78 \mathrm{E}-04$ & $5.52 \mathrm{E}-10$ & $8.30 \mathrm{E}-06$ & $3.80 \mathrm{E}-14$ & 4.39E-08 & $5.02 \mathrm{E}-10$ & $1.24 \mathrm{E}-11$ & $1.22 \mathrm{E}-08$ \\
\hline $90 \%$ & $9.76 \mathrm{E}-03$ & 9.37E-07 & $8.49 \mathrm{E}-03$ & $1.38 \mathrm{E}-16$ & $1.85 \mathrm{E}-03$ & 6.06E-05 & 2.78E-06 & $2.07 \mathrm{E}-04$ & $5.52 \mathrm{E}-10$ & $8.30 \mathrm{E}-06$ & $4.10 \mathrm{E}-14$ & $5.19 \mathrm{E}-08$ & $5.41 \mathrm{E}-10$ & $1.42 \mathrm{E}-11$ & $1.41 \mathrm{E}-08$ \\
\hline $95 \%$ & $1.42 \mathrm{E}-02$ & $1.03 \mathrm{E}-06$ & $1.28 \mathrm{E}-02$ & $1.52 \mathrm{E}-16$ & $2.27 \mathrm{E}-03$ & 7.06E-05 & $3.40 \mathrm{E}-06$ & $2.53 \mathrm{E}-04$ & $5.52 \mathrm{E}-10$ & $8.30 \mathrm{E}-06$ & $4.54 \mathrm{E}-14$ & $6.83 \mathrm{E}-08$ & $5.96 \mathrm{E}-10$ & $1.71 \mathrm{E}-11$ & $1.71 \mathrm{E}-08$ \\
\hline Mean & $5.49 \mathrm{E}-03$ & 4.78E-07 & $4.41 \mathrm{E}-03$ & $9.16 \mathrm{E}-17$ & $1.10 \mathrm{E}-03$ & 3.99E-05 & $1.59 \mathrm{E}-06$ & $9.45 \mathrm{E}-05$ & $5.52 \mathrm{E}-10$ & $8.30 \mathrm{E}-06$ & $2.66 \mathrm{E}-14$ & $2.91 \mathrm{E}-08$ & 3.60E-10 & 7.98E-12 & $6.70 \mathrm{E}-09$ \\
\hline Min & $1.16 \mathrm{E}-03$ & $2.26 \mathrm{E}-08$ & 8.37E-04 & $1.69 \mathrm{E}-18$ & $1.77 \mathrm{E}-04$ & $1.62 \mathrm{E}-05$ & $2.87 \mathrm{E}-07$ & $5.24 \mathrm{E}-07$ & $5.45 \mathrm{E}-10$ & $8.20 \mathrm{E}-06$ & 3.89E-16 & 4.29E-09 & 5.67E-11 & $8.38 \mathrm{E}-13$ & $3.11 \mathrm{E}-11$ \\
\hline Max & $1.76 \mathrm{E}-01$ & $1.48 \mathrm{E}-06$ & $1.73 \mathrm{E}-01$ & $2.17 \mathrm{E}-16$ & $5.47 \mathrm{E}-03$ & $1.40 \mathrm{E}-04$ & $1.08 \mathrm{E}-05$ & $5.46 \mathrm{E}-04$ & $5.53 \mathrm{E}-10$ & $8.30 \mathrm{E}-06$ & 7.12E-14 & 2.83E-07 & $1.01 \mathrm{E}-09$ & 3.69E-11 & $3.28 \mathrm{E}-08$ \\
\hline
\end{tabular}


TABLE C.24 Peak DSR Percentiles (mrem/yr per pCi/g) for Different Exposure Pathways for Sr-90 in Offsite Resident Scenario

\begin{tabular}{|c|c|c|c|c|c|c|c|c|c|c|c|c|c|}
\hline Percentile & Total & External-WD & Fish & Plant-WD & Meat-WD & Milk-WD & Soil-WD & External-WI & Inhalation & Plant-WI & Meat-WI & Milk-WI & Soil-WI \\
\hline $5 \%$ & $8.35 \mathrm{E}-04$ & $9.80 \mathrm{E}-07$ & $1.34 \mathrm{E}-04$ & 7.27E- 05 & 2.05E-04 & $1.20 \mathrm{E}-04$ & 3.24E- 08 & $2.09 \mathrm{E}-06$ & $1.08 \mathrm{E}-08$ & 2.46E-09 & 2.34E-09 & 7.32E-10 & $1.83 \mathrm{E}-12$ \\
\hline $10 \%$ & $9.28 \mathrm{E}-04$ & $1.27 \mathrm{E}-06$ & $1.59 \mathrm{E}-04$ & $8.92 \mathrm{E}-05$ & $2.48 \mathrm{E}-04$ & $1.43 \mathrm{E}-04$ & $4.71 \mathrm{E}-08$ & $2.11 \mathrm{E}-06$ & $1.09 \mathrm{E}-08$ & $3.20 \mathrm{E}-09$ & 3.13E-09 & $9.52 \mathrm{E}-10$ & $2.71 \mathrm{E}-12$ \\
\hline $15 \%$ & $1.01 \mathrm{E}-03$ & $1.50 \mathrm{E}-06$ & $1.84 \mathrm{E}-04$ & $1.02 \mathrm{E}-04$ & $2.80 \mathrm{E}-04$ & $1.62 \mathrm{E}-04$ & $5.90 \mathrm{E}-08$ & $2.12 \mathrm{E}-06$ & $1.10 \mathrm{E}-08$ & 3.87E-09 & 3.97E-09 & $1.15 \mathrm{E}-09$ & 3.36E-12 \\
\hline $20 \%$ & $1.07 \mathrm{E}-03$ & $1.71 \mathrm{E}-06$ & $2.06 \mathrm{E}-04$ & $1.14 \mathrm{E}-04$ & $3.10 \mathrm{E}-04$ & $1.78 \mathrm{E}-04$ & 7.00E- 08 & $2.12 \mathrm{E}-06$ & $1.10 \mathrm{E}-08$ & 4.46E- 09 & 4.77E-09 & $1.37 \mathrm{E}-09$ & 4.02E-12 \\
\hline $25 \%$ & $1.14 \mathrm{E}-03$ & $1.91 \mathrm{E}-06$ & 2.33E-04 & $1.26 \mathrm{E}-04$ & $3.38 \mathrm{E}-04$ & $1.93 \mathrm{E}-04$ & $8.01 \mathrm{E}-08$ & $2.12 \mathrm{E}-06$ & $1.10 \mathrm{E}-08$ & $5.15 \mathrm{E}-09$ & $5.58 \mathrm{E}-09$ & $1.59 \mathrm{E}-09$ & 4.64E-12 \\
\hline $30 \%$ & $1.22 \mathrm{E}-03$ & 2.08E-06 & $2.59 \mathrm{E}-04$ & $1.39 \mathrm{E}-04$ & 3.65E-04 & 2.07E-04 & 9.03E- 08 & 2.13E-06 & $1.10 \mathrm{E}-08$ & $5.87 \mathrm{E}-09$ & 6.35E-09 & $1.85 \mathrm{E}-09$ & $5.21 \mathrm{E}-12$ \\
\hline $35 \%$ & $1.28 \mathrm{E}-03$ & 2.23E-06 & $2.86 \mathrm{E}-04$ & $1.52 \mathrm{E}-04$ & 3.92E-04 & $2.22 \mathrm{E}-04$ & $1.01 \mathrm{E}-07$ & 2.13E- 06 & $1.10 \mathrm{E}-08$ & $6.71 \mathrm{E}-09$ & 7.26E-09 & 2.08E-09 & $5.84 \mathrm{E}-12$ \\
\hline $40 \%$ & $1.36 \mathrm{E}-03$ & $2.38 \mathrm{E}-06$ & $3.18 \mathrm{E}-04$ & $1.69 \mathrm{E}-04$ & 4.23E-04 & $2.37 \mathrm{E}-04$ & $1.10 \mathrm{E}-07$ & 2.13E-06 & $1.10 \mathrm{E}-08$ & $7.58 \mathrm{E}-09$ & $8.23 \mathrm{E}-09$ & $2.38 \mathrm{E}-09$ & $6.42 \mathrm{E}-12$ \\
\hline $45 \%$ & $1.43 \mathrm{E}-03$ & $2.51 \mathrm{E}-06$ & $3.54 \mathrm{E}-04$ & $1.86 \mathrm{E}-04$ & 4.53E-04 & $2.52 \mathrm{E}-04$ & $1.20 \mathrm{E}-07$ & 2.13E-06 & $1.10 \mathrm{E}-08$ & $8.72 \mathrm{E}-09$ & $9.24 \mathrm{E}-09$ & $2.70 \mathrm{E}-09$ & $6.99 \mathrm{E}-12$ \\
\hline $50 \%$ & $1.52 \mathrm{E}-03$ & 2.61E-06 & $3.92 \mathrm{E}-04$ & 2.02E-04 & 4.87E-04 & $2.69 \mathrm{E}-04$ & $1.30 \mathrm{E}-07$ & 2.13E-06 & $1.10 \mathrm{E}-08$ & $9.82 \mathrm{E}-09$ & $1.05 \mathrm{E}-08$ & 3.04E-09 & 7.62E-12 \\
\hline $55 \%$ & $1.60 \mathrm{E}-03$ & 2.71E-06 & $4.40 \mathrm{E}-04$ & $2.24 \mathrm{E}-04$ & $5.24 \mathrm{E}-04$ & $2.88 \mathrm{E}-04$ & $1.41 \mathrm{E}-07$ & 2.13E-06 & $1.10 \mathrm{E}-08$ & 1.12E-08 & $1.18 \mathrm{E}-08$ & $3.52 \mathrm{E}-09$ & $8.29 \mathrm{E}-12$ \\
\hline $60 \%$ & $1.70 \mathrm{E}-03$ & 2.81E-06 & $4.92 \mathrm{E}-04$ & $2.48 \mathrm{E}-04$ & $5.68 \mathrm{E}-04$ & 3.06E-04 & $1.52 \mathrm{E}-07$ & 2.13E-06 & $1.10 \mathrm{E}-08$ & $1.28 \mathrm{E}-08$ & $1.35 \mathrm{E}-08$ & 3.99E-09 & $8.90 \mathrm{E}-12$ \\
\hline $65 \%$ & $1.82 \mathrm{E}-03$ & 2.90E-06 & $5.56 \mathrm{E}-04$ & 2.76E-04 & $6.23 \mathrm{E}-04$ & $3.30 \mathrm{E}-04$ & $1.63 \mathrm{E}-07$ & 2.13E-06 & $1.10 \mathrm{E}-08$ & $1.44 \mathrm{E}-08$ & $1.54 \mathrm{E}-08$ & 4.53E- 09 & $9.57 \mathrm{E}-12$ \\
\hline $70 \%$ & $1.95 \mathrm{E}-03$ & 3.00E-06 & 6.34E-04 & 3.12E-04 & $6.80 \mathrm{E}-04$ & 3.55E-04 & $1.74 \mathrm{E}-07$ & 2.13E-06 & $1.10 \mathrm{E}-08$ & $1.66 \mathrm{E}-08$ & $1.78 \mathrm{E}-08$ & 5.27E- 09 & $1.02 \mathrm{E}-11$ \\
\hline $75 \%$ & $2.13 \mathrm{E}-03$ & 3.11E-06 & 7.31E-04 & 3.57E-04 & 7.65E-04 & $3.84 \mathrm{E}-04$ & $1.87 \mathrm{E}-07$ & 2.13E-06 & $1.10 \mathrm{E}-08$ & $1.96 \mathrm{E}-08$ & $2.08 \mathrm{E}-08$ & $6.18 \mathrm{E}-09$ & $1.10 \mathrm{E}-11$ \\
\hline $80 \%$ & $2.33 \mathrm{E}-03$ & $3.21 \mathrm{E}-06$ & $8.67 \mathrm{E}-04$ & $4.30 \mathrm{E}-04$ & $8.66 \mathrm{E}-04$ & $4.20 \mathrm{E}-04$ & $2.02 \mathrm{E}-07$ & 2.13E-06 & $1.10 \mathrm{E}-08$ & $2.37 \mathrm{E}-08$ & 2.44E- 08 & 7.40E- 09 & $1.19 \mathrm{E}-11$ \\
\hline $85 \%$ & $2.62 \mathrm{E}-03$ & $3.34 \mathrm{E}-06$ & $1.06 \mathrm{E}-03$ & $5.19 \mathrm{E}-04$ & $1.02 \mathrm{E}-03$ & 4.67E-04 & $2.21 \mathrm{E}-07$ & 2.13E-06 & $1.10 \mathrm{E}-08$ & $2.95 \mathrm{E}-08$ & $3.08 \mathrm{E}-08$ & 9.06E-09 & $1.30 \mathrm{E}-11$ \\
\hline $90 \%$ & $3.09 \mathrm{E}-03$ & 3.49E-06 & $1.35 \mathrm{E}-03$ & $6.71 \mathrm{E}-04$ & $1.28 \mathrm{E}-03$ & $5.46 \mathrm{E}-04$ & $2.45 \mathrm{E}-07$ & $2.13 \mathrm{E}-06$ & $1.10 \mathrm{E}-08$ & 3.86E- 08 & $4.00 \mathrm{E}-08$ & $1.22 \mathrm{E}-08$ & $1.44 \mathrm{E}-11$ \\
\hline $95 \%$ & $3.99 \mathrm{E}-03$ & $3.70 \mathrm{E}-06$ & $1.95 \mathrm{E}-03$ & $1.01 \mathrm{E}-03$ & $1.89 \mathrm{E}-03$ & $6.77 \mathrm{E}-04$ & $2.82 \mathrm{E}-07$ & $2.13 \mathrm{E}-06$ & $1.10 \mathrm{E}-08$ & $5.93 \mathrm{E}-08$ & $6.28 \mathrm{E}-08$ & $1.87 \mathrm{E}-08$ & $1.65 \mathrm{E}-11$ \\
\hline Mean & $1.86 \mathrm{E}-03$ & $2.50 \mathrm{E}-06$ & $6.49 \mathrm{E}-04$ & $3.34 \mathrm{E}-04$ & $7.18 \mathrm{E}-04$ & 3.23E-04 & $1.40 \mathrm{E}-07$ & $2.12 \mathrm{E}-06$ & $1.10 \mathrm{E}-08$ & $1.80 \mathrm{E}-08$ & $1.93 \mathrm{E}-08$ & $5.70 \mathrm{E}-09$ & 8.17E-12 \\
\hline Min & $3.60 \mathrm{E}-04$ & $3.22 \mathrm{E}-07$ & 7.43E-05 & $2.32 \mathrm{E}-05$ & $8.90 \mathrm{E}-05$ & $4.80 \mathrm{E}-05$ & $2.25 \mathrm{E}-09$ & $1.69 \mathrm{E}-06$ & $8.78 \mathrm{E}-09$ & $6.76 \mathrm{E}-10$ & $5.37 \mathrm{E}-10$ & 2.42E-10 & $1.31 \mathrm{E}-13$ \\
\hline Max & 3.53E- 02 & $1.19 \mathrm{E}-05$ & $1.21 \mathrm{E}-02$ & $7.99 \mathrm{E}-03$ & $3.15 \mathrm{E}-02$ & $8.94 \mathrm{E}-03$ & $1.01 \mathrm{E}-06$ & 2.13E-06 & $1.10 \mathrm{E}-08$ & 4.79E-07 & $1.08 \mathrm{E}-06$ & 4.14E- 07 & $4.31 \mathrm{E}-11$ \\
\hline
\end{tabular}




\section{TABLE C.25 Peak DSR Percentiles (mrem/yr per pCi/g) for Different Exposure Pathways for Tc-99 in Offsite Resident Scenario}

\begin{tabular}{|c|c|c|c|c|c|c|c|c|c|c|c|c|c|}
\hline Percentile & Total & External-WD & Fish & Plant-WD & Meat-WD & Milk-WD & Soil-WD & External-WI & Inhalation & Plant-WI & Meat-WI & Milk-WI & Soil-WI \\
\hline $5 \%$ & $1.03 \mathrm{E}-04$ & 4.68E-09 & $9.77 \mathrm{E}-06$ & $4.88 \mathrm{E}-05$ & $6.78 \mathrm{E}-07$ & $1.78 \mathrm{E}-05$ & $1.65 \mathrm{E}-09$ & $1.59 \mathrm{E}-09$ & $8.28 \mathrm{E}-10$ & $1.12 \mathrm{E}-10$ & $4.95 \mathrm{E}-13$ & 7.63E-12 & 4.35E-15 \\
\hline $10 \%$ & $2.50 \mathrm{E}-04$ & $9.03 \mathrm{E}-09$ & $2.18 \mathrm{E}-05$ & $1.21 \mathrm{E}-04$ & $1.76 \mathrm{E}-06$ & $3.97 \mathrm{E}-05$ & 3.65E-09 & $1.59 \mathrm{E}-09$ & $8.31 \mathrm{E}-10$ & $1.39 \mathrm{E}-10$ & $6.94 \mathrm{E}-13$ & $1.07 \mathrm{E}-11$ & $6.23 \mathrm{E}-15$ \\
\hline $15 \%$ & $3.90 \mathrm{E}-04$ & $1.39 \mathrm{E}-08$ & $3.59 \mathrm{E}-05$ & $1.99 \mathrm{E}-04$ & $2.87 \mathrm{E}-06$ & $6.69 \mathrm{E}-05$ & $5.68 \mathrm{E}-09$ & $1.60 \mathrm{E}-09$ & 8.33E-10 & $1.65 \mathrm{E}-10$ & $8.80 \mathrm{E}-13$ & $1.30 \mathrm{E}-11$ & $7.58 \mathrm{E}-15$ \\
\hline $20 \%$ & $5.63 \mathrm{E}-04$ & 2.02E-08 & $5.29 \mathrm{E}-05$ & $2.85 \mathrm{E}-04$ & 4.16E-06 & $9.71 \mathrm{E}-05$ & 8.34E-09 & $1.60 \mathrm{E}-09$ & $8.35 \mathrm{E}-10$ & $1.89 \mathrm{E}-10$ & $1.04 \mathrm{E}-12$ & $1.53 \mathrm{E}-11$ & $8.68 \mathrm{E}-15$ \\
\hline $25 \%$ & $7.84 \mathrm{E}-04$ & 2.74E-08 & 7.27E-05 & $4.02 \mathrm{E}-04$ & $5.66 \mathrm{E}-06$ & $1.34 \mathrm{E}-04$ & 1.12E-08 & $1.61 \mathrm{E}-09$ & $8.37 \mathrm{E}-10$ & $2.10 \mathrm{E}-10$ & $1.21 \mathrm{E}-12$ & $1.79 \mathrm{E}-11$ & $9.70 \mathrm{E}-15$ \\
\hline $30 \%$ & $1.02 \mathrm{E}-03$ & 3.77E-08 & 9.33E-05 & $5.15 \mathrm{E}-04$ & 7.54E-06 & $1.73 \mathrm{E}-04$ & $1.45 \mathrm{E}-08$ & $1.61 \mathrm{E}-09$ & $8.38 \mathrm{E}-10$ & $2.39 \mathrm{E}-10$ & $1.39 \mathrm{E}-12$ & $2.07 \mathrm{E}-11$ & $1.05 \mathrm{E}-14$ \\
\hline $35 \%$ & $1.29 \mathrm{E}-03$ & 4.93E-08 & $1.20 \mathrm{E}-04$ & $6.41 \mathrm{E}-04$ & $9.16 \mathrm{E}-06$ & $2.18 \mathrm{E}-04$ & $1.84 \mathrm{E}-08$ & $1.61 \mathrm{E}-09$ & $8.40 \mathrm{E}-10$ & $2.65 \mathrm{E}-10$ & $1.59 \mathrm{E}-12$ & $2.36 \mathrm{E}-11$ & $1.15 \mathrm{E}-14$ \\
\hline $40 \%$ & $1.53 \mathrm{E}-03$ & $6.12 \mathrm{E}-08$ & $1.47 \mathrm{E}-04$ & $7.81 \mathrm{E}-04$ & $1.10 \mathrm{E}-05$ & $2.59 \mathrm{E}-04$ & $2.28 \mathrm{E}-08$ & $1.61 \mathrm{E}-09$ & $8.42 \mathrm{E}-10$ & $2.92 \mathrm{E}-10$ & $1.81 \mathrm{E}-12$ & $2.66 \mathrm{E}-11$ & $1.23 \mathrm{E}-14$ \\
\hline $45 \%$ & $1.79 \mathrm{E}-03$ & 7.24E-08 & $1.74 \mathrm{E}-04$ & 8.92E-04 & 1.30E-05 & 3.06E-04 & $2.77 \mathrm{E}-08$ & 1.62E-09 & 8.44E-10 & $3.20 \mathrm{E}-10$ & $2.06 \mathrm{E}-12$ & $3.02 \mathrm{E}-11$ & $1.31 \mathrm{E}-14$ \\
\hline $50 \%$ & 2.03E-03 & $8.20 \mathrm{E}-08$ & 2.07E-04 & $1.02 \mathrm{E}-03$ & 1.53E-05 & 3.58E-04 & $3.21 \mathrm{E}-08$ & 1.62E-09 & $8.45 \mathrm{E}-10$ & $3.51 \mathrm{E}-10$ & $2.28 \mathrm{E}-12$ & $3.43 \mathrm{E}-11$ & $1.38 \mathrm{E}-14$ \\
\hline $55 \%$ & 2.31E-03 & 8.91E-08 & 2.44E-04 & $1.16 \mathrm{E}-03$ & $1.76 \mathrm{E}-05$ & 4.14E-04 & 3.71E-08 & 1.62E-09 & 8.47E-10 & $3.86 \mathrm{E}-10$ & $2.57 \mathrm{E}-12$ & $3.89 \mathrm{E}-11$ & $1.46 \mathrm{E}-14$ \\
\hline $60 \%$ & $2.59 \mathrm{E}-03$ & $9.60 \mathrm{E}-08$ & 2.97E-04 & $1.33 \mathrm{E}-03$ & 2.01E-05 & 4.77E-04 & 4.22E-08 & 1.63E-09 & 8.49E-10 & $4.31 \mathrm{E}-10$ & 2.92E-12 & 4.32E-11 & $1.54 \mathrm{E}-14$ \\
\hline $65 \%$ & $2.86 \mathrm{E}-03$ & $1.03 \mathrm{E}-07$ & $3.59 \mathrm{E}-04$ & $1.51 \mathrm{E}-03$ & 2.32E-05 & $5.43 \mathrm{E}-04$ & 4.78E-08 & $1.63 \mathrm{E}-09$ & $8.51 \mathrm{E}-10$ & $4.80 \mathrm{E}-10$ & $3.36 \mathrm{E}-12$ & $4.92 \mathrm{E}-11$ & $1.63 \mathrm{E}-14$ \\
\hline $70 \%$ & $3.18 \mathrm{E}-03$ & $1.10 \mathrm{E}-07$ & 4.24E-04 & $1.72 \mathrm{E}-03$ & $2.72 \mathrm{E}-05$ & $6.25 \mathrm{E}-04$ & 5.34E-08 & $1.63 \mathrm{E}-09$ & $8.52 \mathrm{E}-10$ & $5.39 \mathrm{E}-10$ & $3.86 \mathrm{E}-12$ & $5.64 \mathrm{E}-11$ & $1.73 \mathrm{E}-14$ \\
\hline $75 \%$ & $3.54 \mathrm{E}-03$ & $1.17 \mathrm{E}-07$ & $5.18 \mathrm{E}-04$ & $1.98 \mathrm{E}-03$ & 3.19E-05 & 7.27E-04 & $5.96 \mathrm{E}-08$ & $1.64 \mathrm{E}-09$ & $8.54 \mathrm{E}-10$ & $6.05 \mathrm{E}-10$ & 4.47E-12 & $6.46 \mathrm{E}-11$ & $1.82 \mathrm{E}-14$ \\
\hline $80 \%$ & $4.02 \mathrm{E}-03$ & $1.25 \mathrm{E}-07$ & $6.50 \mathrm{E}-04$ & $2.30 \mathrm{E}-03$ & $3.81 \mathrm{E}-05$ & $8.45 \mathrm{E}-04$ & 6.67E-08 & $1.64 \mathrm{E}-09$ & $8.56 \mathrm{E}-10$ & $6.92 \mathrm{E}-10$ & $5.31 \mathrm{E}-12$ & $7.54 \mathrm{E}-11$ & $1.95 \mathrm{E}-14$ \\
\hline $85 \%$ & $4.62 \mathrm{E}-03$ & $1.33 \mathrm{E}-07$ & $8.48 \mathrm{E}-04$ & $2.67 \mathrm{E}-03$ & 4.60E-05 & $1.02 \mathrm{E}-03$ & 7.49E-08 & $1.64 \mathrm{E}-09$ & $8.57 \mathrm{E}-10$ & 8.23E-10 & $6.37 \mathrm{E}-12$ & $9.32 \mathrm{E}-11$ & $2.07 \mathrm{E}-14$ \\
\hline $90 \%$ & $5.50 \mathrm{E}-03$ & $1.46 \mathrm{E}-07$ & 1.19E-03 & $3.35 \mathrm{E}-03$ & 5.97E-05 & $1.24 \mathrm{E}-03$ & 8.47E-08 & $1.65 \mathrm{E}-09$ & $8.59 \mathrm{E}-10$ & 1.03E-09 & 8.02E-12 & $1.19 \mathrm{E}-10$ & $2.22 \mathrm{E}-14$ \\
\hline $95 \%$ & 7.06E-03 & $1.63 \mathrm{E}-07$ & $1.82 \mathrm{E}-03$ & $4.70 \mathrm{E}-03$ & 8.63E-05 & $1.75 \mathrm{E}-03$ & $1.00 \mathrm{E}-07$ & $1.65 \mathrm{E}-09$ & $8.61 \mathrm{E}-10$ & 1.39E-09 & $1.18 \mathrm{E}-11$ & $1.71 \mathrm{E}-10$ & $2.49 \mathrm{E}-14$ \\
\hline Mean & $2.59 \mathrm{E}-03$ & $7.80 \mathrm{E}-08$ & 4.92E-04 & $1.53 \mathrm{E}-03$ & 2.57E-05 & $5.48 \mathrm{E}-04$ & 3.91E-08 & $1.62 \mathrm{E}-09$ & $8.45 \mathrm{E}-10$ & $5.01 \mathrm{E}-10$ & $3.70 \mathrm{E}-12$ & $5.54 \mathrm{E}-11$ & $1.41 \mathrm{E}-14$ \\
\hline Min & 3.93E-06 & $3.55 \mathrm{E}-10$ & $1.76 \mathrm{E}-07$ & $1.74 \mathrm{E}-06$ & $1.17 \mathrm{E}-08$ & $5.68 \mathrm{E}-07$ & $1.99 \mathrm{E}-11$ & $1.56 \mathrm{E}-09$ & $8.12 \mathrm{E}-10$ & $3.54 \mathrm{E}-11$ & 8.99E-14 & $1.49 \mathrm{E}-12$ & $1.35 \mathrm{E}-16$ \\
\hline $\operatorname{Max}$ & 4.25E-02 & $2.36 \mathrm{E}-07$ & 4.07E-02 & $2.62 \mathrm{E}-02$ & $6.81 \mathrm{E}-04$ & $1.36 \mathrm{E}-02$ & $1.93 \mathrm{E}-07$ & $1.67 \mathrm{E}-09$ & $8.69 \mathrm{E}-10$ & 5.23E-09 & 7.73E-11 & $2.54 \mathrm{E}-09$ & $3.76 \mathrm{E}-14$ \\
\hline
\end{tabular}


TABLE C.26 Peak DSR Percentiles (mrem/yr per pCi/g) for Different Exposure Pathways for U-234 in Offsite Resident Scenario

\begin{tabular}{|c|c|c|c|c|c|c|c|c|c|c|c|c|c|c|c|}
\hline Percentile & Total & External-WD & Fish & Radon-WD & Plant-WD & Meat-WD & Milk-WD & Soil-WD & External-WI & Inhalation & Radon & Plant-WI & Meat-WI & Milk-WI & Soil-WI \\
\hline $5 \%$ & $1.29 \mathrm{E}-03$ & $1.17 \mathrm{E}-04$ & $1.05 \mathrm{E}-03$ & $1.20 \mathrm{E}-06$ & $1.19 \mathrm{E}-04$ & $4.49 \mathrm{E}-05$ & $6.01 \mathrm{E}-05$ & $1.99 \mathrm{E}-06$ & $2.32 \mathrm{E}-07$ & $6.46 \mathrm{E}-07$ & $1.01 \mathrm{E}-06$ & $2.60 \mathrm{E}-09$ & $2.66 \mathrm{E}-10$ & $2.28 \mathrm{E}-10$ & $8.46 \mathrm{E}-11$ \\
\hline $10 \%$ & $3.36 \mathrm{E}-03$ & $3.09 \mathrm{E}-04$ & $2.76 \mathrm{E}-03$ & $3.17 \mathrm{E}-06$ & $1.75 \mathrm{E}-04$ & 7.53E-05 & $8.51 \mathrm{E}-05$ & $5.30 \mathrm{E}-06$ & $6.24 \mathrm{E}-07$ & $6.49 \mathrm{E}-07$ & $2.78 \mathrm{E}-06$ & $4.09 \mathrm{E}-09$ & $5.55 \mathrm{E}-10$ & $3.86 \mathrm{E}-10$ & $2.30 \mathrm{E}-10$ \\
\hline $15 \%$ & $6.01 \mathrm{E}-03$ & $6.15 \mathrm{E}-04$ & $4.91 \mathrm{E}-03$ & $5.72 \mathrm{E}-06$ & $2.56 \mathrm{E}-04$ & $1.11 \mathrm{E}-04$ & $1.14 \mathrm{E}-04$ & $1.07 \mathrm{E}-05$ & $1.15 \mathrm{E}-06$ & $6.50 \mathrm{E}-07$ & $5.37 \mathrm{E}-06$ & $6.22 \mathrm{E}-09$ & $9.66 \mathrm{E}-10$ & $5.84 \mathrm{E}-10$ & 4.75E-10 \\
\hline $20 \%$ & $9.53 \mathrm{E}-03$ & $9.18 \mathrm{E}-04$ & $8.11 \mathrm{E}-03$ & $9.66 \mathrm{E}-06$ & 3.67E-04 & $1.60 \mathrm{E}-04$ & $1.40 \mathrm{E}-04$ & $1.60 \mathrm{E}-05$ & $1.87 \mathrm{E}-06$ & $6.50 \mathrm{E}-07$ & $8.44 \mathrm{E}-06$ & $9.98 \mathrm{E}-09$ & $1.53 \mathrm{E}-09$ & $8.01 \mathrm{E}-10$ & 7.52E-10 \\
\hline $25 \%$ & $1.35 \mathrm{E}-02$ & $1.32 \mathrm{E}-03$ & $1.16 \mathrm{E}-02$ & $1.40 \mathrm{E}-05$ & 4.94E-04 & $2.19 \mathrm{E}-04$ & $1.68 \mathrm{E}-04$ & 2.13E-05 & $2.59 \mathrm{E}-06$ & $6.50 \mathrm{E}-07$ & $1.14 \mathrm{E}-05$ & $1.49 \mathrm{E}-08$ & $2.18 \mathrm{E}-09$ & $1.13 \mathrm{E}-09$ & 1.05E-09 \\
\hline $30 \%$ & $1.81 \mathrm{E}-02$ & $1.76 \mathrm{E}-03$ & $1.56 \mathrm{E}-02$ & $1.84 \mathrm{E}-05$ & $6.31 \mathrm{E}-04$ & $2.79 \mathrm{E}-04$ & $1.93 \mathrm{E}-04$ & $2.71 \mathrm{E}-05$ & $3.30 \mathrm{E}-06$ & $6.50 \mathrm{E}-07$ & $1.42 \mathrm{E}-05$ & 2.05E-08 & $2.91 \mathrm{E}-09$ & $1.41 \mathrm{E}-09$ & $1.38 \mathrm{E}-09$ \\
\hline $35 \%$ & $2.19 \mathrm{E}-02$ & $2.10 \mathrm{E}-03$ & $1.88 \mathrm{E}-02$ & $2.24 \mathrm{E}-05$ & 7.55E-04 & 3.37E-04 & $2.16 \mathrm{E}-04$ & 3.37E-05 & $3.85 \mathrm{E}-06$ & $6.50 \mathrm{E}-07$ & $1.64 \mathrm{E}-05$ & $2.58 \mathrm{E}-08$ & 3.46E-09 & $1.66 \mathrm{E}-09$ & $1.72 \mathrm{E}-09$ \\
\hline $40 \%$ & $2.52 \mathrm{E}-02$ & $2.35 \mathrm{E}-03$ & $2.18 \mathrm{E}-02$ & $2.57 \mathrm{E}-05$ & $8.62 \mathrm{E}-04$ & $3.80 \mathrm{E}-04$ & $2.40 \mathrm{E}-04$ & 4.04E-05 & 4.30E-06 & $6.50 \mathrm{E}-07$ & $1.85 \mathrm{E}-05$ & 2.99E-08 & 3.99E-09 & $1.92 \mathrm{E}-09$ & 2.07E-09 \\
\hline $45 \%$ & $2.77 \mathrm{E}-02$ & $2.61 \mathrm{E}-03$ & $2.39 \mathrm{E}-02$ & $2.80 \mathrm{E}-05$ & $9.43 \mathrm{E}-04$ & $4.17 \mathrm{E}-04$ & $2.61 \mathrm{E}-04$ & $4.56 \mathrm{E}-05$ & 4.68E-06 & $6.51 \mathrm{E}-07$ & $2.02 \mathrm{E}-05$ & 3.33E-08 & 4.46E-09 & 2.16E-09 & 2.42E-09 \\
\hline $50 \%$ & $2.99 \mathrm{E}-02$ & $2.84 \mathrm{E}-03$ & $2.58 \mathrm{E}-02$ & 3.03E-05 & $1.02 \mathrm{E}-03$ & $4.52 \mathrm{E}-04$ & $2.81 \mathrm{E}-04$ & $5.27 \mathrm{E}-05$ & $4.98 \mathrm{E}-06$ & $6.51 \mathrm{E}-07$ & $2.17 \mathrm{E}-05$ & $3.65 \mathrm{E}-08$ & $4.92 \mathrm{E}-09$ & $2.39 \mathrm{E}-09$ & $2.74 \mathrm{E}-09$ \\
\hline $55 \%$ & $3.21 \mathrm{E}-02$ & $3.06 \mathrm{E}-03$ & $2.77 \mathrm{E}-02$ & $3.24 \mathrm{E}-05$ & $1.10 \mathrm{E}-03$ & $4.84 \mathrm{E}-04$ & $3.02 \mathrm{E}-04$ & 5.93E-05 & $5.27 \mathrm{E}-06$ & 6.67E-07 & 2.33E-05 & 3.93E-08 & $5.39 \mathrm{E}-09$ & 2.61E-09 & 3.13E-09 \\
\hline $60 \%$ & $3.43 \mathrm{E}-02$ & $3.30 \mathrm{E}-03$ & $2.96 \mathrm{E}-02$ & $3.44 \mathrm{E}-05$ & $1.17 \mathrm{E}-03$ & $5.21 \mathrm{E}-04$ & $3.21 \mathrm{E}-04$ & $6.71 \mathrm{E}-05$ & $5.53 \mathrm{E}-06$ & 7.33E-07 & $2.48 \mathrm{E}-05$ & $4.22 \mathrm{E}-08$ & $5.84 \mathrm{E}-09$ & $2.89 \mathrm{E}-09$ & $3.54 \mathrm{E}-09$ \\
\hline $65 \%$ & 3.66E-02 & $3.59 \mathrm{E}-03$ & 3.17E-02 & $3.65 \mathrm{E}-05$ & $1.25 \mathrm{E}-03$ & $5.54 \mathrm{E}-04$ & $3.41 \mathrm{E}-04$ & 7.57E-05 & $5.77 \mathrm{E}-06$ & 7.81E-07 & 2.62E-05 & $4.52 \mathrm{E}-08$ & $6.31 \mathrm{E}-09$ & 3.19E-09 & 4.03E-09 \\
\hline $70 \%$ & 3.93E-02 & $3.89 \mathrm{E}-03$ & $3.42 \mathrm{E}-02$ & $3.88 \mathrm{E}-05$ & $1.33 \mathrm{E}-03$ & $5.90 \mathrm{E}-04$ & 3.67E-04 & 8.66E-05 & $6.00 \mathrm{E}-06$ & $8.12 \mathrm{E}-07$ & $2.77 \mathrm{E}-05$ & $4.80 \mathrm{E}-08$ & $6.82 \mathrm{E}-09$ & $3.50 \mathrm{E}-09$ & $4.59 \mathrm{E}-09$ \\
\hline $75 \%$ & $4.17 \mathrm{E}-02$ & $4.27 \mathrm{E}-03$ & $3.62 \mathrm{E}-02$ & $4.10 \mathrm{E}-05$ & $1.43 \mathrm{E}-03$ & $6.29 \mathrm{E}-04$ & $3.89 \mathrm{E}-04$ & $9.85 \mathrm{E}-05$ & $6.25 \mathrm{E}-06$ & $8.43 \mathrm{E}-07$ & $2.93 \mathrm{E}-05$ & $5.07 \mathrm{E}-08$ & 7.42E-09 & $3.90 \mathrm{E}-09$ & $5.29 \mathrm{E}-09$ \\
\hline $80 \%$ & 4.43E-02 & $4.71 \mathrm{E}-03$ & $3.86 \mathrm{E}-02$ & 4.33E-05 & $1.54 \mathrm{E}-03$ & $6.73 \mathrm{E}-04$ & $4.16 \mathrm{E}-04$ & $1.13 \mathrm{E}-04$ & $6.48 \mathrm{E}-06$ & $8.66 \mathrm{E}-07$ & $3.10 \mathrm{E}-05$ & $5.40 \mathrm{E}-08$ & $8.12 \mathrm{E}-09$ & 4.40E-09 & $6.15 \mathrm{E}-09$ \\
\hline $85 \%$ & 4.73E-02 & $5.28 \mathrm{E}-03$ & $4.14 \mathrm{E}-02$ & 4.63E-05 & $1.65 \mathrm{E}-03$ & $7.19 \mathrm{E}-04$ & $4.47 \mathrm{E}-04$ & $1.33 \mathrm{E}-04$ & $6.77 \mathrm{E}-06$ & $8.89 \mathrm{E}-07$ & 3.33E-05 & $5.83 \mathrm{E}-08$ & $9.22 \mathrm{E}-09$ & $5.11 \mathrm{E}-09$ & 7.19E-09 \\
\hline $90 \%$ & $5.18 \mathrm{E}-02$ & $5.97 \mathrm{E}-03$ & $4.52 \mathrm{E}-02$ & 4.98E-05 & $1.83 \mathrm{E}-03$ & $7.84 \mathrm{E}-04$ & $4.88 \mathrm{E}-04$ & $1.63 \mathrm{E}-04$ & 7.15E-06 & $9.15 \mathrm{E}-07$ & $3.60 \mathrm{E}-05$ & $6.35 \mathrm{E}-08$ & $1.07 \mathrm{E}-08$ & $6.02 \mathrm{E}-09$ & 8.92E-09 \\
\hline $95 \%$ & $5.76 \mathrm{E}-02$ & $6.89 \mathrm{E}-03$ & 4.99E-02 & $5.46 \mathrm{E}-05$ & $2.12 \mathrm{E}-03$ & $8.95 \mathrm{E}-04$ & $5.75 \mathrm{E}-04$ & $2.10 \mathrm{E}-04$ & 7.69E-06 & $9.43 \mathrm{E}-07$ & $4.01 \mathrm{E}-05$ & 7.17E-08 & $1.40 \mathrm{E}-08$ & $8.20 \mathrm{E}-09$ & $1.22 \mathrm{E}-08$ \\
\hline Mean & $2.89 \mathrm{E}-02$ & $3.02 \mathrm{E}-03$ & $2.51 \mathrm{E}-02$ & $2.85 \mathrm{E}-05$ & $1.03 \mathrm{E}-03$ & 4.53E-04 & $2.98 \mathrm{E}-04$ & $7.10 \mathrm{E}-05$ & $4.44 \mathrm{E}-06$ & 7.38E-07 & $2.08 \mathrm{E}-05$ & $3.52 \mathrm{E}-08$ & $5.70 \mathrm{E}-09$ & $3.06 \mathrm{E}-09$ & 3.87E- 09 \\
\hline Min & 7.07E-05 & $2.11 \mathrm{E}-06$ & $3.51 \mathrm{E}-05$ & $2.26 \mathrm{E}-08$ & 7.45E-06 & $3.54 \mathrm{E}-06$ & 7.83E-06 & $2.41 \mathrm{E}-08$ & 5.36E-09 & $6.17 \mathrm{E}-07$ & $0.00 \mathrm{E}+00$ & $7.56 \mathrm{E}-10$ & $3.24 \mathrm{E}-11$ & $4.12 \mathrm{E}-11$ & $2.15 \mathrm{E}-12$ \\
\hline Max & $2.31 \mathrm{E}-01$ & $1.68 \mathrm{E}-02$ & $2.12 \mathrm{E}-01$ & $2.22 \mathrm{E}-04$ & $7.81 \mathrm{E}-03$ & $4.91 \mathrm{E}-03$ & $7.01 \mathrm{E}-03$ & $5.01 \mathrm{E}-04$ & $1.02 \mathrm{E}-05$ & $1.05 \mathrm{E}-06$ & $6.22 \mathrm{E}-05$ & $1.89 \mathrm{E}-07$ & $1.49 \mathrm{E}-07$ & $7.86 \mathrm{E}-08$ & $3.02 \mathrm{E}-08$ \\
\hline
\end{tabular}


TABLE C.27 Peak DSR Percentiles (mrem/yr per pCi/g) for Different Exposure Pathways for U-235 in Offsite Resident Scenario

\begin{tabular}{|c|c|c|c|c|c|c|c|c|c|c|c|c|c|}
\hline Percentile & Total & External-WD & Fish & Plant-WD & Meat-WD & Milk-WD & Soil-WD & External-WI & Inhalation & Plant-WI & Meat-WI & Milk-WI & Soil-WI \\
\hline $5 \%$ & $6.30 \mathrm{E}-04$ & $1.85 \mathrm{E}-04$ & 2.19E-04 & $1.17 \mathrm{E}-04$ & 4.84E-05 & $3.88 \mathrm{E}-05$ & $2.68 \mathrm{E}-06$ & $1.78 \mathrm{E}-05$ & $5.85 \mathrm{E}-07$ & 2.59E-09 & $3.72 \mathrm{E}-10$ & $1.69 \mathrm{E}-10$ & $1.10 \mathrm{E}-10$ \\
\hline $10 \%$ & $1.06 \mathrm{E}-03$ & 3.81E-04 & $3.76 \mathrm{E}-04$ & $1.86 \mathrm{E}-04$ & $8.20 \mathrm{E}-05$ & $4.96 \mathrm{E}-05$ & 7.63E-06 & 1.79E-05 & 5.87E-07 & 4.43E-09 & $9.28 \mathrm{E}-10$ & $2.42 \mathrm{E}-10$ & $3.30 \mathrm{E}-10$ \\
\hline $15 \%$ & $1.76 \mathrm{E}-03$ & $6.49 \mathrm{E}-04$ & 5.99E-04 & 2.72E-04 & $1.24 \mathrm{E}-04$ & 5.79E-05 & $1.39 \mathrm{E}-05$ & 1.79E-05 & $5.88 \mathrm{E}-07$ & 7.48E-09 & $1.74 \mathrm{E}-09$ & $3.08 \mathrm{E}-10$ & $6.03 \mathrm{E}-10$ \\
\hline $20 \%$ & $2.71 \mathrm{E}-03$ & $9.54 \mathrm{E}-04$ & $8.87 \mathrm{E}-04$ & $3.99 \mathrm{E}-04$ & $1.87 \mathrm{E}-04$ & $6.57 \mathrm{E}-05$ & $2.12 \mathrm{E}-05$ & $1.79 \mathrm{E}-05$ & $6.49 \mathrm{E}-07$ & $1.25 \mathrm{E}-08$ & 2.77E-09 & $3.91 \mathrm{E}-10$ & $9.97 \mathrm{E}-10$ \\
\hline $25 \%$ & $3.83 \mathrm{E}-03$ & $1.25 \mathrm{E}-03$ & $1.30 \mathrm{E}-03$ & $5.39 \mathrm{E}-04$ & $2.59 \mathrm{E}-04$ & 7.32E-05 & $3.02 \mathrm{E}-05$ & $1.79 \mathrm{E}-05$ & $9.80 \mathrm{E}-07$ & $1.86 \mathrm{E}-08$ & 4.14E-09 & $4.73 \mathrm{E}-10$ & $1.44 \mathrm{E}-09$ \\
\hline $30 \%$ & $5.02 \mathrm{E}-03$ & $1.52 \mathrm{E}-03$ & $1.71 \mathrm{E}-03$ & 7.03E-04 & $3.32 \mathrm{E}-04$ & $8.00 \mathrm{E}-05$ & $4.03 \mathrm{E}-05$ & $1.79 \mathrm{E}-05$ & $1.39 \mathrm{E}-06$ & $2.51 \mathrm{E}-08$ & 5.54E-09 & $5.76 \mathrm{E}-10$ & $1.92 \mathrm{E}-09$ \\
\hline $35 \%$ & $6.17 \mathrm{E}-03$ & $1.74 \mathrm{E}-03$ & $2.12 \mathrm{E}-03$ & $8.62 \mathrm{E}-04$ & $4.05 \mathrm{E}-04$ & $8.95 \mathrm{E}-05$ & $4.89 \mathrm{E}-05$ & $1.79 \mathrm{E}-05$ & $1.80 \mathrm{E}-06$ & $3.23 \mathrm{E}-08$ & 7.02E-09 & $6.72 \mathrm{E}-10$ & 2.47E-09 \\
\hline $40 \%$ & $7.04 \mathrm{E}-03$ & $1.95 \mathrm{E}-03$ & $2.47 \mathrm{E}-03$ & $9.78 \mathrm{E}-04$ & 4.62E-04 & $9.88 \mathrm{E}-05$ & 5.89E-05 & $1.79 \mathrm{E}-05$ & $2.29 \mathrm{E}-06$ & $3.88 \mathrm{E}-08$ & 8.32E-09 & $7.89 \mathrm{E}-10$ & 2.97E-09 \\
\hline $45 \%$ & $7.70 \mathrm{E}-03$ & $2.19 \mathrm{E}-03$ & $2.78 \mathrm{E}-03$ & $1.08 \mathrm{E}-03$ & $5.10 \mathrm{E}-04$ & $1.07 \mathrm{E}-04$ & $6.87 \mathrm{E}-05$ & $1.79 \mathrm{E}-05$ & $2.69 \mathrm{E}-06$ & 4.39E-08 & $9.36 \mathrm{E}-09$ & $9.12 \mathrm{E}-10$ & $3.54 \mathrm{E}-09$ \\
\hline $50 \%$ & $8.45 \mathrm{E}-03$ & $2.43 \mathrm{E}-03$ & $3.04 \mathrm{E}-03$ & $1.18 \mathrm{E}-03$ & $5.57 \mathrm{E}-04$ & $1.16 \mathrm{E}-04$ & 7.89E-05 & 1.79E-05 & 2.99E-06 & 4.84E-08 & $1.03 \mathrm{E}-08$ & $1.06 \mathrm{E}-09$ & $4.08 \mathrm{E}-09$ \\
\hline $55 \%$ & 9.07E-03 & $2.74 \mathrm{E}-03$ & $3.25 \mathrm{E}-03$ & $1.28 \mathrm{E}-03$ & 5.99E-04 & $1.27 \mathrm{E}-04$ & 8.87E-05 & 1.79E-05 & $3.23 \mathrm{E}-06$ & 5.24E-08 & $1.12 \mathrm{E}-08$ & $1.24 \mathrm{E}-09$ & $4.68 \mathrm{E}-09$ \\
\hline $60 \%$ & $9.68 \mathrm{E}-03$ & $3.22 \mathrm{E}-03$ & $3.47 \mathrm{E}-03$ & $1.37 \mathrm{E}-03$ & $6.40 \mathrm{E}-04$ & $1.38 \mathrm{E}-04$ & $1.00 \mathrm{E}-04$ & $1.88 \mathrm{E}-05$ & $3.45 \mathrm{E}-06$ & 5.69E-08 & $1.21 \mathrm{E}-08$ & $1.42 \mathrm{E}-09$ & $5.31 \mathrm{E}-09$ \\
\hline $65 \%$ & $1.05 \mathrm{E}-02$ & $3.83 \mathrm{E}-03$ & $3.67 \mathrm{E}-03$ & $1.48 \mathrm{E}-03$ & $6.78 \mathrm{E}-04$ & $1.51 \mathrm{E}-04$ & $1.13 \mathrm{E}-04$ & $1.97 \mathrm{E}-05$ & 3.63E-06 & 6.07E-08 & $1.30 \mathrm{E}-08$ & $1.68 \mathrm{E}-09$ & $6.01 \mathrm{E}-09$ \\
\hline $70 \%$ & $1.15 \mathrm{E}-02$ & $4.72 \mathrm{E}-03$ & $3.88 \mathrm{E}-03$ & $1.58 \mathrm{E}-03$ & 7.17E-04 & $1.67 \mathrm{E}-04$ & $1.28 \mathrm{E}-04$ & 2.04E-05 & $3.79 \mathrm{E}-06$ & $6.54 \mathrm{E}-08$ & $1.38 \mathrm{E}-08$ & $1.98 \mathrm{E}-09$ & $6.84 \mathrm{E}-09$ \\
\hline $75 \%$ & $1.26 \mathrm{E}-02$ & $5.87 \mathrm{E}-03$ & $4.11 \mathrm{E}-03$ & $1.69 \mathrm{E}-03$ & 7.64E-04 & $1.85 \mathrm{E}-04$ & $1.46 \mathrm{E}-04$ & $2.09 \mathrm{E}-05$ & $3.97 \mathrm{E}-06$ & $6.99 \mathrm{E}-08$ & $1.47 \mathrm{E}-08$ & $2.34 \mathrm{E}-09$ & $7.86 \mathrm{E}-09$ \\
\hline $80 \%$ & $1.37 \mathrm{E}-02$ & 7.34E-03 & $4.38 \mathrm{E}-03$ & $1.80 \mathrm{E}-03$ & 8.14E-04 & $2.08 \mathrm{E}-04$ & $1.65 \mathrm{E}-04$ & $2.12 \mathrm{E}-05$ & 4.14E-06 & 7.47E-08 & $1.58 \mathrm{E}-08$ & 2.83E-09 & $9.01 \mathrm{E}-09$ \\
\hline $85 \%$ & $1.51 \mathrm{E}-02$ & 8.94E-03 & $4.76 \mathrm{E}-03$ & $1.96 \mathrm{E}-03$ & $8.81 \mathrm{E}-04$ & 2.37E-04 & $1.94 \mathrm{E}-04$ & $2.15 \mathrm{E}-05$ & $4.36 \mathrm{E}-06$ & 8.08E-08 & $1.71 \mathrm{E}-08$ & $3.51 \mathrm{E}-09$ & $1.06 \mathrm{E}-08$ \\
\hline $90 \%$ & $1.67 \mathrm{E}-02$ & $1.07 \mathrm{E}-02$ & 5.33E-03 & $2.19 \mathrm{E}-03$ & $9.77 \mathrm{E}-04$ & $2.78 \mathrm{E}-04$ & $2.28 \mathrm{E}-04$ & $2.18 \mathrm{E}-05$ & $4.60 \mathrm{E}-06$ & 8.80E-08 & $1.87 \mathrm{E}-08$ & 4.53E-09 & $1.29 \mathrm{E}-08$ \\
\hline $95 \%$ & $1.88 \mathrm{E}-02$ & $1.28 \mathrm{E}-02$ & $6.42 \mathrm{E}-03$ & $2.57 \mathrm{E}-03$ & $1.13 \mathrm{E}-03$ & 3.69E-04 & $2.87 \mathrm{E}-04$ & $2.21 \mathrm{E}-05$ & 4.89E-06 & $1.02 \mathrm{E}-07$ & $2.17 \mathrm{E}-08$ & $6.38 \mathrm{E}-09$ & $1.65 \mathrm{E}-08$ \\
\hline Mean & $8.70 \mathrm{E}-03$ & $4.09 \mathrm{E}-03$ & $2.98 \mathrm{E}-03$ & $1.21 \mathrm{E}-03$ & $5.51 \mathrm{E}-04$ & $1.54 \mathrm{E}-04$ & $1.01 \mathrm{E}-04$ & $1.91 \mathrm{E}-05$ & $2.68 \mathrm{E}-06$ & $4.81 \mathrm{E}-08$ & $1.03 \mathrm{E}-08$ & $1.94 \mathrm{E}-09$ & $5.51 \mathrm{E}-09$ \\
\hline Min & 4.77E-05 & $3.95 \mathrm{E}-06$ & $1.65 \mathrm{E}-05$ & $5.69 \mathrm{E}-06$ & $3.73 \mathrm{E}-06$ & $5.76 \mathrm{E}-06$ & $8.27 \mathrm{E}-09$ & $1.70 \mathrm{E}-05$ & $5.58 \mathrm{E}-07$ & $5.53 \mathrm{E}-10$ & $4.04 \mathrm{E}-11$ & $4.87 \mathrm{E}-11$ & $6.56 \mathrm{E}-13$ \\
\hline Max & $4.16 \mathrm{E}-02$ & $2.15 \mathrm{E}-02$ & 1.94E-02 & $8.81 \mathrm{E}-03$ & $6.37 \mathrm{E}-03$ & $8.45 \mathrm{E}-03$ & $6.20 \mathrm{E}-04$ & $2.33 \mathrm{E}-05$ & $6.03 \mathrm{E}-06$ & 3.39E-07 & $1.52 \mathrm{E}-07$ & $3.48 \mathrm{E}-08$ & $3.70 \mathrm{E}-08$ \\
\hline
\end{tabular}




\section{TABLE C.28 Peak DSR Percentiles (mrem/yr per pCi/g) for Different Exposure Pathways for U-238 in Offsite Resident Scenario}

\begin{tabular}{|c|c|c|c|c|c|c|c|c|c|c|c|c|c|c|c|}
\hline Percentile & Total & External-WD & Fish & Radon-WD & Plant-WD & Meat-WD & Milk-WD & Soil-WD & External-WI & Inhalation & Radon & Plant-WI & Meat-WI & Milk-WI & Soil-WI \\
\hline $5 \%$ & $4.28 \mathrm{E}-04$ & $4.16 \mathrm{E}-05$ & $1.22 \mathrm{E}-04$ & $3.30 \mathrm{E}-10$ & $8.23 \mathrm{E}-05$ & $2.19 \mathrm{E}-05$ & 3.92E-05 & 7.15E-07 & 7.14E-06 & $5.51 \mathrm{E}-07$ & $1.84 \mathrm{E}-10$ & $1.69 \mathrm{E}-09$ & $1.56 \mathrm{E}-10$ & $1.67 \mathrm{E}-10$ & $3.55 \mathrm{E}-11$ \\
\hline $10 \%$ & $5.35 \mathrm{E}-04$ & $6.61 \mathrm{E}-05$ & $1.46 \mathrm{E}-04$ & $2.76 \mathrm{E}-09$ & $1.02 \mathrm{E}-04$ & $2.89 \mathrm{E}-05$ & 4.94E-05 & $1.27 \mathrm{E}-06$ & 7.17E-06 & $5.53 \mathrm{E}-07$ & $1.57 \mathrm{E}-09$ & 2.09E-09 & $2.48 \mathrm{E}-10$ & 2.43E-10 & $6.63 \mathrm{E}-11$ \\
\hline $15 \%$ & $6.24 \mathrm{E}-04$ & $9.23 \mathrm{E}-05$ & $1.70 \mathrm{E}-04$ & $8.39 \mathrm{E}-09$ & $1.17 \mathrm{E}-04$ & $3.56 \mathrm{E}-05$ & $5.90 \mathrm{E}-05$ & $1.84 \mathrm{E}-06$ & 7.18E-06 & $5.53 \mathrm{E}-07$ & $5.20 \mathrm{E}-09$ & $2.44 \mathrm{E}-09$ & 3.43E-10 & $3.26 \mathrm{E}-10$ & $9.92 \mathrm{E}-11$ \\
\hline $20 \%$ & $7.20 \mathrm{E}-04$ & $1.19 \mathrm{E}-04$ & $1.89 \mathrm{E}-04$ & $2.24 \mathrm{E}-08$ & $1.31 \mathrm{E}-04$ & $4.18 \mathrm{E}-05$ & $6.73 \mathrm{E}-05$ & 2.43E-06 & 7.18E-06 & $5.54 \mathrm{E}-07$ & $1.30 \mathrm{E}-08$ & $2.75 \mathrm{E}-09$ & 4.55E-10 & $4.12 \mathrm{E}-10$ & $1.35 \mathrm{E}-10$ \\
\hline $25 \%$ & 8.07E-04 & $1.46 \mathrm{E}-04$ & $2.10 \mathrm{E}-04$ & $4.14 \mathrm{E}-08$ & $1.44 \mathrm{E}-04$ & $4.82 \mathrm{E}-05$ & 7.49E-05 & $3.00 \mathrm{E}-06$ & 7.18E-06 & $5.54 \mathrm{E}-07$ & $2.50 \mathrm{E}-08$ & $3.12 \mathrm{E}-09$ & $5.69 \mathrm{E}-10$ & $5.01 \mathrm{E}-10$ & $1.71 \mathrm{E}-10$ \\
\hline $30 \%$ & $8.90 \mathrm{E}-04$ & $1.76 \mathrm{E}-04$ & $2.30 \mathrm{E}-04$ & $6.68 \mathrm{E}-08$ & $1.58 \mathrm{E}-04$ & $5.48 \mathrm{E}-05$ & $8.32 \mathrm{E}-05$ & $3.87 \mathrm{E}-06$ & 7.18E-06 & $5.54 \mathrm{E}-07$ & $3.80 \mathrm{E}-08$ & 3.49E-09 & 7.07E-10 & $5.94 \mathrm{E}-10$ & $2.21 \mathrm{E}-10$ \\
\hline $35 \%$ & $9.83 \mathrm{E}-04$ & $2.12 \mathrm{E}-04$ & $2.48 \mathrm{E}-04$ & $9.14 \mathrm{E}-08$ & $1.71 \mathrm{E}-04$ & $6.20 \mathrm{E}-05$ & $9.10 \mathrm{E}-05$ & 4.77E-06 & 7.18E-06 & $5.54 \mathrm{E}-07$ & $4.92 \mathrm{E}-08$ & $3.87 \mathrm{E}-09$ & $8.53 \mathrm{E}-10$ & $6.99 \mathrm{E}-10$ & $2.72 \mathrm{E}-10$ \\
\hline $40 \%$ & $1.06 \mathrm{E}-03$ & $2.53 \mathrm{E}-04$ & $2.66 \mathrm{E}-04$ & $1.15 \mathrm{E}-07$ & $1.84 \mathrm{E}-04$ & $6.90 \mathrm{E}-05$ & $9.97 \mathrm{E}-05$ & $5.73 \mathrm{E}-06$ & 7.18E-06 & $5.54 \mathrm{E}-07$ & $6.00 \mathrm{E}-08$ & 4.32E-09 & $1.02 \mathrm{E}-09$ & $8.22 \mathrm{E}-10$ & $3.25 \mathrm{E}-10$ \\
\hline $45 \%$ & $1.15 \mathrm{E}-03$ & $3.01 \mathrm{E}-04$ & $2.86 \mathrm{E}-04$ & $1.34 \mathrm{E}-07$ & $1.98 \mathrm{E}-04$ & 7.66E-05 & $1.08 \mathrm{E}-04$ & $6.85 \mathrm{E}-06$ & 7.19E-06 & $5.54 \mathrm{E}-07$ & 7.02E-08 & 4.82E-09 & $1.22 \mathrm{E}-09$ & $9.56 \mathrm{E}-10$ & $3.90 \mathrm{E}-10$ \\
\hline $50 \%$ & $1.25 \mathrm{E}-03$ & $3.66 \mathrm{E}-04$ & 3.06E-04 & $1.53 \mathrm{E}-07$ & 2.12E-04 & 8.47E-05 & $1.18 \mathrm{E}-04$ & 8.33E-06 & 7.19E-06 & $5.54 \mathrm{E}-07$ & $7.86 \mathrm{E}-08$ & $5.35 \mathrm{E}-09$ & $1.43 \mathrm{E}-09$ & $1.13 \mathrm{E}-09$ & 4.74E-10 \\
\hline $55 \%$ & $1.36 \mathrm{E}-03$ & 4.60E-04 & 3.30E-04 & $1.68 \mathrm{E}-07$ & $2.26 \mathrm{E}-04$ & $9.46 \mathrm{E}-05$ & $1.28 \mathrm{E}-04$ & $1.02 \mathrm{E}-05$ & 7.19E-06 & $5.54 \mathrm{E}-07$ & 8.73E-08 & $5.97 \mathrm{E}-09$ & $1.67 \mathrm{E}-09$ & $1.30 \mathrm{E}-09$ & $5.94 \mathrm{E}-10$ \\
\hline $60 \%$ & $1.50 \mathrm{E}-03$ & $5.85 \mathrm{E}-04$ & 3.53E-04 & $1.85 \mathrm{E}-07$ & 2.44E-04 & $1.06 \mathrm{E}-04$ & $1.39 \mathrm{E}-04$ & $1.26 \mathrm{E}-05$ & 7.19E-06 & $5.54 \mathrm{E}-07$ & $9.55 \mathrm{E}-08$ & $6.56 \mathrm{E}-09$ & $1.94 \mathrm{E}-09$ & 1.49E-09 & 7.56E-10 \\
\hline $65 \%$ & $1.68 \mathrm{E}-03$ & $7.28 \mathrm{E}-04$ & $3.81 \mathrm{E}-04$ & $2.00 \mathrm{E}-07$ & $2.65 \mathrm{E}-04$ & $1.20 \mathrm{E}-04$ & $1.52 \mathrm{E}-04$ & $1.59 \mathrm{E}-05$ & 7.19E-06 & $5.54 \mathrm{E}-07$ & $1.05 \mathrm{E}-07$ & 7.34E-09 & 2.32E-09 & $1.71 \mathrm{E}-09$ & $9.56 \mathrm{E}-10$ \\
\hline $70 \%$ & $1.88 \mathrm{E}-03$ & $9.53 \mathrm{E}-04$ & $4.14 \mathrm{E}-04$ & $2.17 \mathrm{E}-07$ & $2.84 \mathrm{E}-04$ & $1.35 \mathrm{E}-04$ & $1.69 \mathrm{E}-04$ & 2.07E-05 & 7.19E-06 & $5.54 \mathrm{E}-07$ & $1.14 \mathrm{E}-07$ & $8.36 \mathrm{E}-09$ & $2.75 \mathrm{E}-09$ & $1.99 \mathrm{E}-09$ & $1.24 \mathrm{E}-09$ \\
\hline $75 \%$ & $2.12 \mathrm{E}-03$ & $1.25 \mathrm{E}-03$ & $4.51 \mathrm{E}-04$ & 2.37E-07 & $3.14 \mathrm{E}-04$ & $1.56 \mathrm{E}-04$ & $1.86 \mathrm{E}-04$ & $2.66 \mathrm{E}-05$ & 7.19E-06 & $5.54 \mathrm{E}-07$ & $1.24 \mathrm{E}-07$ & $9.47 \mathrm{E}-09$ & $3.26 \mathrm{E}-09$ & $2.31 \mathrm{E}-09$ & $1.65 \mathrm{E}-09$ \\
\hline $80 \%$ & 2.43E-03 & $1.58 \mathrm{E}-03$ & 4.93E-04 & $2.56 \mathrm{E}-07$ & 3.48E-04 & $1.79 \mathrm{E}-04$ & $2.12 \mathrm{E}-04$ & $3.42 \mathrm{E}-05$ & 7.19E-06 & $5.54 \mathrm{E}-07$ & $1.35 \mathrm{E}-07$ & $1.11 \mathrm{E}-08$ & 3.91E-09 & 2.77E-09 & $2.09 \mathrm{E}-09$ \\
\hline $85 \%$ & $2.84 \mathrm{E}-03$ & $1.96 \mathrm{E}-03$ & $5.61 \mathrm{E}-04$ & $2.80 \mathrm{E}-07$ & $3.89 \mathrm{E}-04$ & $2.12 \mathrm{E}-04$ & $2.45 \mathrm{E}-04$ & $4.55 \mathrm{E}-05$ & 7.19E-06 & $5.54 \mathrm{E}-07$ & $1.49 \mathrm{E}-07$ & $1.32 \mathrm{E}-08$ & 4.95E-09 & 3.45E-09 & $2.71 \mathrm{E}-09$ \\
\hline $90 \%$ & $3.33 \mathrm{E}-03$ & $2.48 \mathrm{E}-03$ & $6.87 \mathrm{E}-04$ & $3.13 \mathrm{E}-07$ & 4.62E-04 & $2.64 \mathrm{E}-04$ & $2.86 \mathrm{E}-04$ & $6.12 \mathrm{E}-05$ & 7.19E-06 & $5.57 \mathrm{E}-07$ & $1.68 \mathrm{E}-07$ & $1.63 \mathrm{E}-08$ & $6.42 \mathrm{E}-09$ & 4.37E-09 & $3.74 \mathrm{E}-09$ \\
\hline $95 \%$ & $3.93 \mathrm{E}-03$ & $3.04 \mathrm{E}-03$ & $9.59 \mathrm{E}-04$ & 3.68E-07 & $6.20 \mathrm{E}-04$ & $3.76 \mathrm{E}-04$ & $3.80 \mathrm{E}-04$ & $8.23 \mathrm{E}-05$ & 7.23E-06 & $5.61 \mathrm{E}-07$ & $1.95 \mathrm{E}-07$ & $2.31 \mathrm{E}-08$ & $9.77 \mathrm{E}-09$ & $6.46 \mathrm{E}-09$ & 4.97E- 09 \\
\hline Mean & $1.64 \mathrm{E}-03$ & 8.33E-04 & 4.09E-04 & $1.56 \mathrm{E}-07$ & $2.70 \mathrm{E}-04$ & $1.34 \mathrm{E}-04$ & $1.56 \mathrm{E}-04$ & $2.05 \mathrm{E}-05$ & 7.18E-06 & $5.54 \mathrm{E}-07$ & $8.22 \mathrm{E}-08$ & $8.02 \mathrm{E}-09$ & $2.80 \mathrm{E}-09$ & $1.96 \mathrm{E}-09$ & $1.23 \mathrm{E}-09$ \\
\hline Min & $1.44 \mathrm{E}-05$ & $1.12 \mathrm{E}-06$ & $1.76 \mathrm{E}-05$ & $1.94 \mathrm{E}-13$ & $1.18 \mathrm{E}-05$ & $2.42 \mathrm{E}-06$ & $3.41 \mathrm{E}-06$ & $2.85 \mathrm{E}-08$ & $6.82 \mathrm{E}-06$ & $5.26 \mathrm{E}-07$ & $0.00 \mathrm{E}+00$ & $5.73 \mathrm{E}-10$ & 2.79E-11 & 3.94E-11 & $1.28 \mathrm{E}-12$ \\
\hline Max & $2.82 \mathrm{E}-02$ & $4.81 \mathrm{E}-03$ & $1.61 \mathrm{E}-02$ & $1.15 \mathrm{E}-06$ & $8.12 \mathrm{E}-03$ & $6.03 \mathrm{E}-03$ & $3.56 \mathrm{E}-03$ & $2.14 \mathrm{E}-04$ & $7.35 \mathrm{E}-06$ & $5.70 \mathrm{E}-07$ & 3.46E-07 & $1.13 \mathrm{E}-07$ & $1.65 \mathrm{E}-07$ & $2.81 \mathrm{E}-08$ & $1.28 \mathrm{E}-08$ \\
\hline
\end{tabular}


Tables C.29-C.42 show the peak DSR percentiles (mrem/yr per pCi/g) for different exposure pathways for individual radionuclides in the recreational use scenario. RESRAD (onsite) Version 6.5 was used in the analysis. For C-14, I-129, Np-237, and Tc-99, WD pathways were dominant; for the other radionuclides (Am-241, Co-60, Cs-137, $\mathrm{Pu}-238, \mathrm{Pu}-239, \mathrm{Pu}-240$, Sr-90, U-234, U-235, and U-238), WI pathways were dominant. For Am-241, Co-60, U-235, and U-238, external exposure was dominant. For Pu-238, Pu-239, and Pu-240, meat ingestion, soil ingestion, and inhalation were dominant exposure pathways. For U-234, meat ingestion and external exposure were dominant. For C-14, I-129, Np-237, and Tc-99, aquatic food ingestion resulted in the most dose. For Cs-137, meat ingestion and external exposure resulted in the most dose. For Sr-90, meat ingestion resulted in the most dose.

TABLE C.29 Peak DSR Percentiles (mrem/yr per pCi/g) for Different Exposure Pathways for Am-241 in Recreational Use Scenario

\begin{tabular}{|c|c|c|c|c|c|c|c|c|}
\hline Percentile & External & $\begin{array}{l}\text { Inhalation } \\
\text { w/o Radon }\end{array}$ & $\begin{array}{c}\text { Meat } \\
\text { Ingestion } \\
\text { (WI) }\end{array}$ & $\begin{array}{c}\text { Soil } \\
\text { Ingestion }\end{array}$ & $\begin{array}{c}\text { Water } \\
\text { Ingestion }\end{array}$ & $\begin{array}{l}\text { Aquatic } \\
\text { Foods }\end{array}$ & $\begin{array}{c}\text { Meat } \\
\text { Ingestion } \\
\text { (WD) }\end{array}$ & $\begin{array}{c}\text { All } \\
\text { Pathways }\end{array}$ \\
\hline $5 \%$ & $1.00 \mathrm{E}-03$ & $1.18 \mathrm{E}-04$ & $1.78 \mathrm{E}-04$ & $1.27 \mathrm{E}-04$ & $0.00 \mathrm{E}+00$ & $0.00 \mathrm{E}+00$ & $0.00 \mathrm{E}+00$ & $1.81 \mathrm{E}-03$ \\
\hline $10 \%$ & $1.11 \mathrm{E}-03$ & $1.63 \mathrm{E}-04$ & $2.18 \mathrm{E}-04$ & $1.84 \mathrm{E}-04$ & $0.00 \mathrm{E}+00$ & $0.00 \mathrm{E}+00$ & $0.00 \mathrm{E}+00$ & $1.96 \mathrm{E}-03$ \\
\hline $15 \%$ & $1.14 \mathrm{E}-03$ & $1.92 \mathrm{E}-04$ & $2.51 \mathrm{E}-04$ & $2.27 \mathrm{E}-04$ & $0.00 \mathrm{E}+00$ & $0.00 \mathrm{E}+00$ & $0.00 \mathrm{E}+00$ & $2.05 \mathrm{E}-03$ \\
\hline $20 \%$ & $1.16 \mathrm{E}-03$ & $2.12 \mathrm{E}-04$ & $2.78 \mathrm{E}-04$ & $2.64 \mathrm{E}-04$ & $0.00 \mathrm{E}+00$ & $0.00 \mathrm{E}+00$ & $0.00 \mathrm{E}+00$ & $2.11 \mathrm{E}-03$ \\
\hline $25 \%$ & $1.17 \mathrm{E}-03$ & $2.28 \mathrm{E}-04$ & $3.01 \mathrm{E}-04$ & $2.95 \mathrm{E}-04$ & $0.00 \mathrm{E}+00$ & $0.00 \mathrm{E}+00$ & $0.00 \mathrm{E}+00$ & $2.16 \mathrm{E}-03$ \\
\hline $30 \%$ & $1.17 \mathrm{E}-03$ & $2.47 \mathrm{E}-04$ & $3.24 \mathrm{E}-04$ & $3.25 \mathrm{E}-04$ & $0.00 \mathrm{E}+00$ & $0.00 \mathrm{E}+00$ & $0.00 \mathrm{E}+00$ & $2.22 \mathrm{E}-03$ \\
\hline $35 \%$ & $1.17 \mathrm{E}-03$ & $2.63 \mathrm{E}-04$ & $3.49 \mathrm{E}-04$ & $3.54 \mathrm{E}-04$ & $0.00 \mathrm{E}+00$ & $0.00 \mathrm{E}+00$ & $0.00 \mathrm{E}+00$ & $2.26 \mathrm{E}-03$ \\
\hline $40 \%$ & $1.17 \mathrm{E}-03$ & $2.80 \mathrm{E}-04$ & $3.73 \mathrm{E}-04$ & $3.81 \mathrm{E}-04$ & $0.00 \mathrm{E}+00$ & $0.00 \mathrm{E}+00$ & $0.00 \mathrm{E}+00$ & $2.31 \mathrm{E}-03$ \\
\hline $45 \%$ & $1.18 \mathrm{E}-03$ & $2.96 \mathrm{E}-04$ & $3.99 \mathrm{E}-04$ & $4.06 \mathrm{E}-04$ & $0.00 \mathrm{E}+00$ & $0.00 \mathrm{E}+00$ & $0.00 \mathrm{E}+00$ & $2.36 \mathrm{E}-03$ \\
\hline $50 \%$ & $1.18 \mathrm{E}-03$ & $3.13 \mathrm{E}-04$ & 4.31E-04 & $4.29 \mathrm{E}-04$ & $0.00 \mathrm{E}+00$ & $0.00 \mathrm{E}+00$ & $0.00 \mathrm{E}+00$ & $2.40 \mathrm{E}-03$ \\
\hline $55 \%$ & $1.18 \mathrm{E}-03$ & $3.34 \mathrm{E}-04$ & $4.60 \mathrm{E}-04$ & $4.50 \mathrm{E}-04$ & $0.00 \mathrm{E}+00$ & $0.00 \mathrm{E}+00$ & $0.00 \mathrm{E}+00$ & $2.44 \mathrm{E}-03$ \\
\hline $60 \%$ & $1.18 \mathrm{E}-03$ & $3.59 \mathrm{E}-04$ & $4.93 \mathrm{E}-04$ & $4.73 \mathrm{E}-04$ & $0.00 \mathrm{E}+00$ & $0.00 \mathrm{E}+00$ & $0.00 \mathrm{E}+00$ & $2.49 \mathrm{E}-03$ \\
\hline $65 \%$ & $1.18 \mathrm{E}-03$ & $3.86 \mathrm{E}-04$ & $5.27 \mathrm{E}-04$ & $4.99 \mathrm{E}-04$ & $0.00 \mathrm{E}+00$ & $0.00 \mathrm{E}+00$ & $0.00 \mathrm{E}+00$ & $2.54 \mathrm{E}-03$ \\
\hline $70 \%$ & $1.18 \mathrm{E}-03$ & 4.12E-04 & $5.63 \mathrm{E}-04$ & $5.27 \mathrm{E}-04$ & $0.00 \mathrm{E}+00$ & $0.00 \mathrm{E}+00$ & $0.00 \mathrm{E}+00$ & $2.58 \mathrm{E}-03$ \\
\hline $75 \%$ & $1.18 \mathrm{E}-03$ & $4.45 \mathrm{E}-04$ & $6.03 \mathrm{E}-04$ & 5.59E-04 & $0.00 \mathrm{E}+00$ & $0.00 \mathrm{E}+00$ & $0.00 \mathrm{E}+00$ & $2.64 \mathrm{E}-03$ \\
\hline $80 \%$ & $1.18 \mathrm{E}-03$ & $4.88 \mathrm{E}-04$ & $6.47 \mathrm{E}-04$ & $5.92 \mathrm{E}-04$ & $0.00 \mathrm{E}+00$ & $0.00 \mathrm{E}+00$ & $0.00 \mathrm{E}+00$ & $2.71 \mathrm{E}-03$ \\
\hline $85 \%$ & $1.18 \mathrm{E}-03$ & $5.47 \mathrm{E}-04$ & $6.98 \mathrm{E}-04$ & $6.27 \mathrm{E}-04$ & $5.86 \mathrm{E}-11$ & $1.68 \mathrm{E}-09$ & $1.23 \mathrm{E}-12$ & $2.79 \mathrm{E}-03$ \\
\hline $90 \%$ & $1.18 \mathrm{E}-03$ & $6.13 \mathrm{E}-04$ & $7.66 \mathrm{E}-04$ & $6.72 \mathrm{E}-04$ & $3.34 \mathrm{E}-09$ & $9.40 \mathrm{E}-08$ & $6.80 \mathrm{E}-11$ & $2.89 \mathrm{E}-03$ \\
\hline $95 \%$ & $1.18 \mathrm{E}-03$ & $7.26 \mathrm{E}-04$ & 8.80E-04 & 7.33E-04 & $4.30 \mathrm{E}-08$ & $1.23 \mathrm{E}-06$ & $9.07 \mathrm{E}-10$ & $3.05 \mathrm{E}-03$ \\
\hline Mean & $1.14 \mathrm{E}-03$ & $3.59 \mathrm{E}-04$ & 4.67E-04 & $4.28 \mathrm{E}-04$ & $6.01 \mathrm{E}-09$ & $2.76 \mathrm{E}-07$ & $1.81 \mathrm{E}-10$ & $2.40 \mathrm{E}-03$ \\
\hline Min & $2.58 \mathrm{E}-08$ & $2.78 \mathrm{E}-10$ & $5.15 \mathrm{E}-09$ & $5.46 \mathrm{E}-10$ & $0.00 \mathrm{E}+00$ & $0.00 \mathrm{E}+00$ & $0.00 \mathrm{E}+00$ & $5.24 \mathrm{E}-08$ \\
\hline Max & $1.18 \mathrm{E}-03$ & $2.55 \mathrm{E}-03$ & $1.46 \mathrm{E}-03$ & 8.80E-04 & $2.24 \mathrm{E}-07$ & $4.41 \mathrm{E}-05$ & $1.58 \mathrm{E}-08$ & $4.99 \mathrm{E}-03$ \\
\hline
\end{tabular}


TABLE C.30 Peak DSR Percentiles (mrem/yr per pCi/g) for Different Exposure Pathways for C-14 in Recreational Use Scenario

\begin{tabular}{|c|c|c|c|c|c|c|c|c|}
\hline Percentile & External & $\begin{array}{l}\text { Inhalation } \\
\text { w/o Radon }\end{array}$ & $\begin{array}{c}\text { Meat } \\
\text { Ingestion } \\
(\mathrm{WI})\end{array}$ & $\begin{array}{c}\text { Soil } \\
\text { Ingestion }\end{array}$ & $\begin{array}{c}\text { Water } \\
\text { Ingestion }\end{array}$ & $\begin{array}{l}\text { Aquatic } \\
\text { Foods }\end{array}$ & $\begin{array}{c}\text { Meat } \\
\text { Ingestion } \\
\text { (WD) }\end{array}$ & $\begin{array}{c}\text { All } \\
\text { Pathways }\end{array}$ \\
\hline $5 \%$ & $0.00 \mathrm{E}+00$ & $0.00 \mathrm{E}+00$ & $0.00 \mathrm{E}+00$ & $0.00 \mathrm{E}+00$ & $0.00 \mathrm{E}+00$ & $0.00 \mathrm{E}+00$ & $0.00 \mathrm{E}+00$ & $0.00 \mathrm{E}+00$ \\
\hline $10 \%$ & $0.00 \mathrm{E}+00$ & $0.00 \mathrm{E}+00$ & $0.00 \mathrm{E}+00$ & $0.00 \mathrm{E}+00$ & $0.00 \mathrm{E}+00$ & $0.00 \mathrm{E}+00$ & $0.00 \mathrm{E}+00$ & $0.00 \mathrm{E}+00$ \\
\hline $15 \%$ & $0.00 \mathrm{E}+00$ & $0.00 \mathrm{E}+00$ & $0.00 \mathrm{E}+00$ & $0.00 \mathrm{E}+00$ & $0.00 \mathrm{E}+00$ & $0.00 \mathrm{E}+00$ & $0.00 \mathrm{E}+00$ & $0.00 \mathrm{E}+00$ \\
\hline $20 \%$ & $0.00 \mathrm{E}+00$ & $0.00 \mathrm{E}+00$ & $0.00 \mathrm{E}+00$ & $0.00 \mathrm{E}+00$ & $0.00 \mathrm{E}+00$ & $0.00 \mathrm{E}+00$ & $0.00 \mathrm{E}+00$ & $0.00 \mathrm{E}+00$ \\
\hline $25 \%$ & $0.00 \mathrm{E}+00$ & $0.00 \mathrm{E}+00$ & $0.00 \mathrm{E}+00$ & $0.00 \mathrm{E}+00$ & $0.00 \mathrm{E}+00$ & $0.00 \mathrm{E}+00$ & $0.00 \mathrm{E}+00$ & $0.00 \mathrm{E}+00$ \\
\hline $30 \%$ & $0.00 \mathrm{E}+00$ & $0.00 \mathrm{E}+00$ & $0.00 \mathrm{E}+00$ & $0.00 \mathrm{E}+00$ & $0.00 \mathrm{E}+00$ & $0.00 \mathrm{E}+00$ & $0.00 \mathrm{E}+00$ & $0.00 \mathrm{E}+00$ \\
\hline $35 \%$ & $0.00 \mathrm{E}+00$ & $0.00 \mathrm{E}+00$ & $0.00 \mathrm{E}+00$ & $0.00 \mathrm{E}+00$ & $0.00 \mathrm{E}+00$ & $0.00 \mathrm{E}+00$ & $0.00 \mathrm{E}+00$ & $0.00 \mathrm{E}+00$ \\
\hline $40 \%$ & $0.00 \mathrm{E}+00$ & $0.00 \mathrm{E}+00$ & $0.00 \mathrm{E}+00$ & $0.00 \mathrm{E}+00$ & $0.00 \mathrm{E}+00$ & $0.00 \mathrm{E}+00$ & $0.00 \mathrm{E}+00$ & $0.00 \mathrm{E}+00$ \\
\hline $45 \%$ & $0.00 \mathrm{E}+00$ & $0.00 \mathrm{E}+00$ & $0.00 \mathrm{E}+00$ & $0.00 \mathrm{E}+00$ & $0.00 \mathrm{E}+00$ & $0.00 \mathrm{E}+00$ & $0.00 \mathrm{E}+00$ & $0.00 \mathrm{E}+00$ \\
\hline $50 \%$ & $0.00 \mathrm{E}+00$ & $0.00 \mathrm{E}+00$ & $0.00 \mathrm{E}+00$ & $0.00 \mathrm{E}+00$ & $0.00 \mathrm{E}+00$ & $0.00 \mathrm{E}+00$ & $0.00 \mathrm{E}+00$ & $0.00 \mathrm{E}+00$ \\
\hline $55 \%$ & $0.00 \mathrm{E}+00$ & $0.00 \mathrm{E}+00$ & $0.00 \mathrm{E}+00$ & $0.00 \mathrm{E}+00$ & $0.00 \mathrm{E}+00$ & $0.00 \mathrm{E}+00$ & $0.00 \mathrm{E}+00$ & $0.00 \mathrm{E}+00$ \\
\hline $60 \%$ & $0.00 \mathrm{E}+00$ & $0.00 \mathrm{E}+00$ & $0.00 \mathrm{E}+00$ & $0.00 \mathrm{E}+00$ & $0.00 \mathrm{E}+00$ & $0.00 \mathrm{E}+00$ & $0.00 \mathrm{E}+00$ & $0.00 \mathrm{E}+00$ \\
\hline $65 \%$ & $0.00 \mathrm{E}+00$ & $0.00 \mathrm{E}+00$ & $0.00 \mathrm{E}+00$ & $0.00 \mathrm{E}+00$ & $0.00 \mathrm{E}+00$ & $0.00 \mathrm{E}+00$ & $0.00 \mathrm{E}+00$ & $0.00 \mathrm{E}+00$ \\
\hline $70 \%$ & $0.00 \mathrm{E}+00$ & $0.00 \mathrm{E}+00$ & $0.00 \mathrm{E}+00$ & $0.00 \mathrm{E}+00$ & $1.24 \mathrm{E}-21$ & $1.71 \mathrm{E}-17$ & $9.54 \mathrm{E}-22$ & $1.77 \mathrm{E}-17$ \\
\hline $75 \%$ & $0.00 \mathrm{E}+00$ & $0.00 \mathrm{E}+00$ & $0.00 \mathrm{E}+00$ & $0.00 \mathrm{E}+00$ & $2.58 \mathrm{E}-07$ & 4.23E-03 & $1.88 \mathrm{E}-07$ & $4.24 \mathrm{E}-03$ \\
\hline $80 \%$ & $0.00 \mathrm{E}+00$ & $0.00 \mathrm{E}+00$ & $0.00 \mathrm{E}+00$ & $0.00 \mathrm{E}+00$ & $5.02 \mathrm{E}-07$ & $1.55 \mathrm{E}-02$ & $3.84 \mathrm{E}-07$ & $1.55 \mathrm{E}-02$ \\
\hline $85 \%$ & $0.00 \mathrm{E}+00$ & $0.00 \mathrm{E}+00$ & $0.00 \mathrm{E}+00$ & $0.00 \mathrm{E}+00$ & $9.02 \mathrm{E}-07$ & $5.45 \mathrm{E}-02$ & $6.66 \mathrm{E}-07$ & $5.45 \mathrm{E}-02$ \\
\hline $90 \%$ & $6.11 \mathrm{E}-08$ & $3.03 \mathrm{E}-06$ & $6.00 \mathrm{E}-02$ & $1.62 \mathrm{E}-07$ & $1.48 \mathrm{E}-06$ & $1.17 \mathrm{E}-01$ & $1.17 \mathrm{E}-06$ & $1.17 \mathrm{E}-01$ \\
\hline $95 \%$ & 6.19E-08 & $3.90 \mathrm{E}-06$ & $9.22 \mathrm{E}-02$ & $2.56 \mathrm{E}-07$ & $2.81 \mathrm{E}-06$ & $2.50 \mathrm{E}-01$ & $2.26 \mathrm{E}-06$ & $2.50 \mathrm{E}-01$ \\
\hline Mean & $8.40 \mathrm{E}-09$ & $5.08 \mathrm{E}-07$ & $1.20 \mathrm{E}-02$ & $3.04 \mathrm{E}-08$ & $4.66 \mathrm{E}-07$ & 4.61E-02 & $3.97 \mathrm{E}-07$ & 4.61E-02 \\
\hline Min & $0.00 \mathrm{E}+00$ & $0.00 \mathrm{E}+00$ & $0.00 \mathrm{E}+00$ & $0.00 \mathrm{E}+00$ & $0.00 \mathrm{E}+00$ & $0.00 \mathrm{E}+00$ & $0.00 \mathrm{E}+00$ & $0.00 \mathrm{E}+00$ \\
\hline Max & $6.26 \mathrm{E}-08$ & $9.13 \mathrm{E}-06$ & $3.04 \mathrm{E}-01$ & $4.30 \mathrm{E}-07$ & $1.53 \mathrm{E}-05$ & $1.14 \mathrm{E}+01$ & 2.02E-05 & $1.14 \mathrm{E}+01$ \\
\hline
\end{tabular}


TABLE C.31 Peak DSR Percentiles (mrem/yr per pCi/g) for Different Exposure Pathways for Co-60 in Recreational Use Scenario

\begin{tabular}{|c|c|c|c|c|c|c|c|c|}
\hline Percentile & External & $\begin{array}{c}\text { Inhalation } \\
\text { w/o Radon }\end{array}$ & $\begin{array}{c}\text { Meat } \\
\text { Ingestion } \\
(\mathrm{WI})\end{array}$ & $\begin{array}{c}\text { Soil } \\
\text { Ingestion } \\
\end{array}$ & $\begin{array}{c}\text { Water } \\
\text { Ingestion }\end{array}$ & $\begin{array}{l}\text { Aquatic } \\
\text { Foods }\end{array}$ & $\begin{array}{c}\text { Meat } \\
\text { Ingestion } \\
\text { (WD) }\end{array}$ & $\begin{array}{c}\text { All } \\
\text { Pathways }\end{array}$ \\
\hline $5 \%$ & 5.33E-07 & $6.21 \mathrm{E}-14$ & 2.74E-09 & $3.53 \mathrm{E}-12$ & $0.00 \mathrm{E}+00$ & $0.00 \mathrm{E}+00$ & $0.00 \mathrm{E}+00$ & $5.60 \mathrm{E}-07$ \\
\hline $10 \%$ & 7.17E-07 & $8.22 \mathrm{E}-14$ & 4.50E-09 & $5.60 \mathrm{E}-12$ & $0.00 \mathrm{E}+00$ & $0.00 \mathrm{E}+00$ & $0.00 \mathrm{E}+00$ & $7.51 \mathrm{E}-07$ \\
\hline $15 \%$ & $8.17 \mathrm{E}-07$ & $1.02 \mathrm{E}-13$ & $6.19 \mathrm{E}-09$ & $7.09 \mathrm{E}-12$ & $0.00 \mathrm{E}+00$ & $0.00 \mathrm{E}+00$ & $0.00 \mathrm{E}+00$ & $8.50 \mathrm{E}-07$ \\
\hline $20 \%$ & 8.69E-07 & $1.20 \mathrm{E}-13$ & 8.08E-09 & $8.41 \mathrm{E}-12$ & $0.00 \mathrm{E}+00$ & $0.00 \mathrm{E}+00$ & $0.00 \mathrm{E}+00$ & $9.01 \mathrm{E}-07$ \\
\hline $25 \%$ & $9.05 \mathrm{E}-07$ & $1.30 \mathrm{E}-13$ & $1.00 \mathrm{E}-08$ & $9.70 \mathrm{E}-12$ & $0.00 \mathrm{E}+00$ & $0.00 \mathrm{E}+00$ & $0.00 \mathrm{E}+00$ & $9.41 \mathrm{E}-07$ \\
\hline $30 \%$ & $9.30 \mathrm{E}-07$ & $1.38 \mathrm{E}-13$ & $1.20 \mathrm{E}-08$ & $1.08 \mathrm{E}-11$ & $0.00 \mathrm{E}+00$ & $0.00 \mathrm{E}+00$ & $0.00 \mathrm{E}+00$ & $9.64 \mathrm{E}-07$ \\
\hline $35 \%$ & $9.48 \mathrm{E}-07$ & $1.46 \mathrm{E}-13$ & $1.42 \mathrm{E}-08$ & $1.17 \mathrm{E}-11$ & $0.00 \mathrm{E}+00$ & $0.00 \mathrm{E}+00$ & $0.00 \mathrm{E}+00$ & $9.81 \mathrm{E}-07$ \\
\hline $40 \%$ & $9.62 \mathrm{E}-07$ & $1.54 \mathrm{E}-13$ & $1.67 \mathrm{E}-08$ & $1.26 \mathrm{E}-11$ & $0.00 \mathrm{E}+00$ & $0.00 \mathrm{E}+00$ & $0.00 \mathrm{E}+00$ & $9.93 \mathrm{E}-07$ \\
\hline $45 \%$ & $9.72 \mathrm{E}-07$ & $1.63 \mathrm{E}-13$ & $1.98 \mathrm{E}-08$ & $1.35 \mathrm{E}-11$ & $0.00 \mathrm{E}+00$ & $0.00 \mathrm{E}+00$ & $0.00 \mathrm{E}+00$ & $1.00 \mathrm{E}-06$ \\
\hline $50 \%$ & $9.81 \mathrm{E}-07$ & $1.71 \mathrm{E}-13$ & $2.36 \mathrm{E}-08$ & $1.45 \mathrm{E}-11$ & $0.00 \mathrm{E}+00$ & $0.00 \mathrm{E}+00$ & $0.00 \mathrm{E}+00$ & $1.01 \mathrm{E}-06$ \\
\hline $55 \%$ & $9.88 \mathrm{E}-07$ & $1.80 \mathrm{E}-13$ & $2.77 \mathrm{E}-08$ & $1.53 \mathrm{E}-11$ & $0.00 \mathrm{E}+00$ & $0.00 \mathrm{E}+00$ & $0.00 \mathrm{E}+00$ & $1.01 \mathrm{E}-06$ \\
\hline $60 \%$ & $9.93 \mathrm{E}-07$ & $1.88 \mathrm{E}-13$ & $3.23 \mathrm{E}-08$ & $1.63 \mathrm{E}-11$ & $0.00 \mathrm{E}+00$ & $0.00 \mathrm{E}+00$ & $0.00 \mathrm{E}+00$ & $1.02 \mathrm{E}-06$ \\
\hline $65 \%$ & $9.97 \mathrm{E}-07$ & $1.97 \mathrm{E}-13$ & $3.80 \mathrm{E}-08$ & $1.72 \mathrm{E}-11$ & $0.00 \mathrm{E}+00$ & $0.00 \mathrm{E}+00$ & $0.00 \mathrm{E}+00$ & $1.02 \mathrm{E}-06$ \\
\hline $70 \%$ & $1.00 \mathrm{E}-06$ & $2.07 \mathrm{E}-13$ & $4.57 \mathrm{E}-08$ & $1.82 \mathrm{E}-11$ & $0.00 \mathrm{E}+00$ & $0.00 \mathrm{E}+00$ & $0.00 \mathrm{E}+00$ & $1.03 \mathrm{E}-06$ \\
\hline $75 \%$ & $1.00 \mathrm{E}-06$ & $2.16 \mathrm{E}-13$ & $5.47 \mathrm{E}-08$ & $1.92 \mathrm{E}-11$ & $0.00 \mathrm{E}+00$ & $0.00 \mathrm{E}+00$ & $0.00 \mathrm{E}+00$ & $1.04 \mathrm{E}-06$ \\
\hline $80 \%$ & $1.01 \mathrm{E}-06$ & $2.26 \mathrm{E}-13$ & $6.87 \mathrm{E}-08$ & $2.04 \mathrm{E}-11$ & $0.00 \mathrm{E}+00$ & $0.00 \mathrm{E}+00$ & $0.00 \mathrm{E}+00$ & $1.05 \mathrm{E}-06$ \\
\hline $85 \%$ & $1.01 \mathrm{E}-06$ & $2.40 \mathrm{E}-13$ & 8.77E-08 & $2.18 \mathrm{E}-11$ & $0.00 \mathrm{E}+00$ & $0.00 \mathrm{E}+00$ & $0.00 \mathrm{E}+00$ & $1.07 \mathrm{E}-06$ \\
\hline $90 \%$ & $1.01 \mathrm{E}-06$ & $2.71 \mathrm{E}-13$ & $1.21 \mathrm{E}-07$ & $2.32 \mathrm{E}-11$ & $0.00 \mathrm{E}+00$ & $0.00 \mathrm{E}+00$ & $0.00 \mathrm{E}+00$ & $1.10 \mathrm{E}-06$ \\
\hline $95 \%$ & $1.01 \mathrm{E}-06$ & $3.08 \mathrm{E}-13$ & $1.95 \mathrm{E}-07$ & $2.56 \mathrm{E}-11$ & $0.00 \mathrm{E}+00$ & $0.00 \mathrm{E}+00$ & $0.00 \mathrm{E}+00$ & $1.17 \mathrm{E}-06$ \\
\hline Mean & $9.11 \mathrm{E}-07$ & $1.78 \mathrm{E}-13$ & $5.45 \mathrm{E}-08$ & $1.45 \mathrm{E}-11$ & $6.86 \mathrm{E}-14$ & $3.11 \mathrm{E}-12$ & $1.19 \mathrm{E}-13$ & $9.65 \mathrm{E}-07$ \\
\hline Min & $7.27 \mathrm{E}-13$ & $1.26 \mathrm{E}-19$ & $6.30 \mathrm{E}-14$ & 7.77E-18 & $0.00 \mathrm{E}+00$ & $0.00 \mathrm{E}+00$ & $0.00 \mathrm{E}+00$ & $7.90 \mathrm{E}-13$ \\
\hline Max & $1.01 \mathrm{E}-06$ & $7.69 \mathrm{E}-13$ & 4.36E-06 & $3.19 \mathrm{E}-11$ & $3.08 \mathrm{E}-10$ & $1.40 \mathrm{E}-08$ & $5.35 \mathrm{E}-10$ & $5.31 \mathrm{E}-06$ \\
\hline
\end{tabular}


TABLE C.32 Peak DSR Percentiles (mrem/yr per pCi/g) for Different Exposure Pathways for Cs-137 in Recreational Use Scenario

\begin{tabular}{|c|c|c|c|c|c|c|c|c|}
\hline Percentile & External & $\begin{array}{c}\text { Inhalation } \\
\text { w/o Radon }\end{array}$ & $\begin{array}{c}\text { Meat } \\
\text { Ingestion } \\
(\mathrm{WI})\end{array}$ & $\begin{array}{c}\text { Soil } \\
\text { Ingestion } \\
\end{array}$ & $\begin{array}{c}\text { Water } \\
\text { Ingestion }\end{array}$ & $\begin{array}{l}\text { Aquatic } \\
\text { Foods }\end{array}$ & $\begin{array}{c}\text { Meat } \\
\text { Ingestion } \\
\text { (WD) }\end{array}$ & $\begin{array}{c}\text { All } \\
\text { Pathways }\end{array}$ \\
\hline $5 \%$ & 9.79E-03 & $5.80 \mathrm{E}-09$ & $1.90 \mathrm{E}-03$ & $9.80 \mathrm{E}-07$ & $0.00 \mathrm{E}+00$ & $0.00 \mathrm{E}+00$ & $0.00 \mathrm{E}+00$ & $1.27 \mathrm{E}-02$ \\
\hline $10 \%$ & $1.04 \mathrm{E}-02$ & 7.82E-09 & 2.38E-03 & $1.42 \mathrm{E}-06$ & $0.00 \mathrm{E}+00$ & $0.00 \mathrm{E}+00$ & $0.00 \mathrm{E}+00$ & $1.33 \mathrm{E}-02$ \\
\hline $15 \%$ & $1.07 \mathrm{E}-02$ & 8.99E-09 & $2.84 \mathrm{E}-03$ & $1.73 \mathrm{E}-06$ & $0.00 \mathrm{E}+00$ & $0.00 \mathrm{E}+00$ & $0.00 \mathrm{E}+00$ & $1.37 \mathrm{E}-02$ \\
\hline $20 \%$ & 1.09E-02 & $9.87 \mathrm{E}-09$ & $3.23 \mathrm{E}-03$ & $2.01 \mathrm{E}-06$ & $0.00 \mathrm{E}+00$ & $0.00 \mathrm{E}+00$ & $0.00 \mathrm{E}+00$ & $1.42 \mathrm{E}-02$ \\
\hline $25 \%$ & $1.10 \mathrm{E}-02$ & $1.07 \mathrm{E}-08$ & $3.67 \mathrm{E}-03$ & $2.23 \mathrm{E}-06$ & $0.00 \mathrm{E}+00$ & $0.00 \mathrm{E}+00$ & $0.00 \mathrm{E}+00$ & $1.46 \mathrm{E}-02$ \\
\hline $30 \%$ & $1.10 \mathrm{E}-02$ & $1.15 \mathrm{E}-08$ & $4.11 \mathrm{E}-03$ & $2.46 \mathrm{E}-06$ & $0.00 \mathrm{E}+00$ & $0.00 \mathrm{E}+00$ & $0.00 \mathrm{E}+00$ & $1.51 \mathrm{E}-02$ \\
\hline $35 \%$ & $1.11 \mathrm{E}-02$ & $1.23 \mathrm{E}-08$ & $4.51 \mathrm{E}-03$ & $2.65 \mathrm{E}-06$ & $0.00 \mathrm{E}+00$ & $0.00 \mathrm{E}+00$ & $0.00 \mathrm{E}+00$ & $1.55 \mathrm{E}-02$ \\
\hline $40 \%$ & $1.11 \mathrm{E}-02$ & $1.31 \mathrm{E}-08$ & $5.03 \mathrm{E}-03$ & $2.85 \mathrm{E}-06$ & $0.00 \mathrm{E}+00$ & $0.00 \mathrm{E}+00$ & $0.00 \mathrm{E}+00$ & $1.60 \mathrm{E}-02$ \\
\hline $45 \%$ & $1.11 \mathrm{E}-02$ & $1.38 \mathrm{E}-08$ & $5.52 \mathrm{E}-03$ & $3.03 \mathrm{E}-06$ & $0.00 \mathrm{E}+00$ & $0.00 \mathrm{E}+00$ & $0.00 \mathrm{E}+00$ & $1.65 \mathrm{E}-02$ \\
\hline $50 \%$ & $1.12 \mathrm{E}-02$ & $1.46 \mathrm{E}-08$ & $6.09 \mathrm{E}-03$ & $3.21 \mathrm{E}-06$ & $0.00 \mathrm{E}+00$ & $0.00 \mathrm{E}+00$ & $0.00 \mathrm{E}+00$ & $1.71 \mathrm{E}-02$ \\
\hline $55 \%$ & $1.12 \mathrm{E}-02$ & $1.56 \mathrm{E}-08$ & $6.61 \mathrm{E}-03$ & $3.37 \mathrm{E}-06$ & $0.00 \mathrm{E}+00$ & $0.00 \mathrm{E}+00$ & $0.00 \mathrm{E}+00$ & $1.77 \mathrm{E}-02$ \\
\hline $60 \%$ & $1.12 \mathrm{E}-02$ & $1.67 \mathrm{E}-08$ & 7.32E-03 & $3.55 \mathrm{E}-06$ & $0.00 \mathrm{E}+00$ & $0.00 \mathrm{E}+00$ & $0.00 \mathrm{E}+00$ & $1.83 \mathrm{E}-02$ \\
\hline $65 \%$ & $1.12 \mathrm{E}-02$ & $1.79 \mathrm{E}-08$ & $8.15 \mathrm{E}-03$ & $3.75 \mathrm{E}-06$ & $0.00 \mathrm{E}+00$ & $0.00 \mathrm{E}+00$ & $0.00 \mathrm{E}+00$ & $1.92 \mathrm{E}-02$ \\
\hline $70 \%$ & $1.12 \mathrm{E}-02$ & $1.92 \mathrm{E}-08$ & $9.00 \mathrm{E}-03$ & $3.94 \mathrm{E}-06$ & $0.00 \mathrm{E}+00$ & $0.00 \mathrm{E}+00$ & $0.00 \mathrm{E}+00$ & $2.00 \mathrm{E}-02$ \\
\hline $75 \%$ & $1.13 \mathrm{E}-02$ & $2.07 \mathrm{E}-08$ & $1.01 \mathrm{E}-02$ & 4.17E-06 & $0.00 \mathrm{E}+00$ & $0.00 \mathrm{E}+00$ & $0.00 \mathrm{E}+00$ & $2.11 \mathrm{E}-02$ \\
\hline $80 \%$ & $1.13 \mathrm{E}-02$ & $2.26 \mathrm{E}-08$ & $1.16 \mathrm{E}-02$ & $4.40 \mathrm{E}-06$ & $0.00 \mathrm{E}+00$ & $0.00 \mathrm{E}+00$ & $0.00 \mathrm{E}+00$ & $2.25 \mathrm{E}-02$ \\
\hline $85 \%$ & $1.13 \mathrm{E}-02$ & $2.55 \mathrm{E}-08$ & $1.35 \mathrm{E}-02$ & $4.67 \mathrm{E}-06$ & $0.00 \mathrm{E}+00$ & $0.00 \mathrm{E}+00$ & $0.00 \mathrm{E}+00$ & $2.45 \mathrm{E}-02$ \\
\hline $90 \%$ & $1.13 \mathrm{E}-02$ & $2.87 \mathrm{E}-08$ & $1.65 \mathrm{E}-02$ & $5.00 \mathrm{E}-06$ & $0.00 \mathrm{E}+00$ & $0.00 \mathrm{E}+00$ & $0.00 \mathrm{E}+00$ & $2.76 \mathrm{E}-02$ \\
\hline $95 \%$ & $1.13 \mathrm{E}-02$ & $3.35 \mathrm{E}-08$ & $2.38 \mathrm{E}-02$ & $5.43 \mathrm{E}-06$ & $0.00 \mathrm{E}+00$ & $0.00 \mathrm{E}+00$ & $0.00 \mathrm{E}+00$ & $3.49 \mathrm{E}-02$ \\
\hline Mean & $1.09 \mathrm{E}-02$ & $1.68 \mathrm{E}-08$ & $8.62 \mathrm{E}-03$ & $3.20 \mathrm{E}-06$ & $0.00 \mathrm{E}+00$ & $0.00 \mathrm{E}+00$ & $0.00 \mathrm{E}+00$ & $1.95 \mathrm{E}-02$ \\
\hline Min & 1.69E-04 & $3.90 \mathrm{E}-11$ & $4.22 \mathrm{E}-05$ & 4.19E-08 & $0.00 \mathrm{E}+00$ & $0.00 \mathrm{E}+00$ & $0.00 \mathrm{E}+00$ & $2.11 \mathrm{E}-04$ \\
\hline Max & $1.13 \mathrm{E}-02$ & $1.19 \mathrm{E}-07$ & $1.80 \mathrm{E}-01$ & $6.50 \mathrm{E}-06$ & $0.00 \mathrm{E}+00$ & $0.00 \mathrm{E}+00$ & $0.00 \mathrm{E}+00$ & $1.91 \mathrm{E}-01$ \\
\hline
\end{tabular}


TABLE C.33 Peak DSR Percentiles (mrem/yr per pCi/g) for Different Exposure Pathways for I-129 in Recreational Use Scenario

\begin{tabular}{|c|c|c|c|c|c|c|c|c|}
\hline Percentile & External & $\begin{array}{c}\text { Inhalation } \\
\text { w/o Radon }\end{array}$ & $\begin{array}{c}\text { Meat } \\
\text { Ingestion } \\
(\mathrm{WI})\end{array}$ & $\begin{array}{c}\text { Soil } \\
\text { Ingestion } \\
\end{array}$ & $\begin{array}{c}\text { Water } \\
\text { Ingestion }\end{array}$ & $\begin{array}{c}\text { Aquatic } \\
\text { Foods }\end{array}$ & $\begin{array}{c}\text { Meat } \\
\text { Ingestion } \\
\text { (WD) }\end{array}$ & $\begin{array}{c}\text { All } \\
\text { Pathways }\end{array}$ \\
\hline $5 \%$ & $2.74 \mathrm{E}-25$ & $1.78 \mathrm{E}-28$ & $2.60 \mathrm{E}-22$ & $1.96 \mathrm{E}-25$ & $0.00 \mathrm{E}+00$ & $0.00 \mathrm{E}+00$ & $0.00 \mathrm{E}+00$ & $1.45 \mathrm{E}-17$ \\
\hline $10 \%$ & $2.60 \mathrm{E}-20$ & $1.81 \mathrm{E}-23$ & 2.89E-17 & $1.71 \mathrm{E}-20$ & $0.00 \mathrm{E}+00$ & $0.00 \mathrm{E}+00$ & $0.00 \mathrm{E}+00$ & $5.66 \mathrm{E}-12$ \\
\hline $15 \%$ & $1.51 \mathrm{E}-16$ & $1.22 \mathrm{E}-19$ & $1.75 \mathrm{E}-13$ & $9.90 \mathrm{E}-17$ & $0.00 \mathrm{E}+00$ & $0.00 \mathrm{E}+00$ & $0.00 \mathrm{E}+00$ & 5.75E-09 \\
\hline $20 \%$ & $3.42 \mathrm{E}-14$ & $3.32 \mathrm{E}-17$ & $3.84 \mathrm{E}-11$ & $2.71 \mathrm{E}-14$ & $0.00 \mathrm{E}+00$ & $0.00 \mathrm{E}+00$ & $0.00 \mathrm{E}+00$ & $4.66 \mathrm{E}-07$ \\
\hline $25 \%$ & $3.11 \mathrm{E}-12$ & $2.47 \mathrm{E}-15$ & $3.18 \mathrm{E}-09$ & $2.22 \mathrm{E}-12$ & $0.00 \mathrm{E}+00$ & $0.00 \mathrm{E}+00$ & $0.00 \mathrm{E}+00$ & 7.19E-06 \\
\hline $30 \%$ & $9.61 \mathrm{E}-11$ & 7.91E-14 & 1.09E-07 & $6.10 \mathrm{E}-11$ & $0.00 \mathrm{E}+00$ & $0.00 \mathrm{E}+00$ & $0.00 \mathrm{E}+00$ & $8.02 \mathrm{E}-05$ \\
\hline $35 \%$ & $1.71 \mathrm{E}-09$ & $1.32 \mathrm{E}-12$ & $1.65 \mathrm{E}-06$ & $1.18 \mathrm{E}-09$ & $0.00 \mathrm{E}+00$ & $0.00 \mathrm{E}+00$ & $0.00 \mathrm{E}+00$ & $5.41 \mathrm{E}-04$ \\
\hline $40 \%$ & $1.58 \mathrm{E}-08$ & $1.26 \mathrm{E}-11$ & $1.54 \mathrm{E}-05$ & $1.04 \mathrm{E}-08$ & $0.00 \mathrm{E}+00$ & $0.00 \mathrm{E}+00$ & $0.00 \mathrm{E}+00$ & $2.25 \mathrm{E}-03$ \\
\hline $45 \%$ & $1.03 \mathrm{E}-07$ & $7.94 \mathrm{E}-11$ & $9.15 \mathrm{E}-05$ & 7.14E-08 & $3.67 \mathrm{E}-10$ & 4.43E-09 & $3.23 \mathrm{E}-10$ & $6.31 \mathrm{E}-03$ \\
\hline $50 \%$ & $4.53 \mathrm{E}-07$ & $3.69 \mathrm{E}-10$ & 4.43E-04 & $3.06 \mathrm{E}-07$ & $2.23 \mathrm{E}-06$ & $2.67 \mathrm{E}-05$ & $2.04 \mathrm{E}-06$ & $1.37 \mathrm{E}-02$ \\
\hline $55 \%$ & $1.81 \mathrm{E}-06$ & $1.51 \mathrm{E}-09$ & $1.65 \mathrm{E}-03$ & $1.27 \mathrm{E}-06$ & $1.28 \mathrm{E}-04$ & $1.16 \mathrm{E}-03$ & 1.19E-04 & $2.56 \mathrm{E}-02$ \\
\hline $60 \%$ & $4.84 \mathrm{E}-06$ & $4.07 \mathrm{E}-09$ & $4.66 \mathrm{E}-03$ & $3.28 \mathrm{E}-06$ & $6.52 \mathrm{E}-04$ & $5.69 \mathrm{E}-03$ & $5.30 \mathrm{E}-04$ & $4.31 \mathrm{E}-02$ \\
\hline $65 \%$ & $1.36 \mathrm{E}-05$ & $1.03 \mathrm{E}-08$ & $1.24 \mathrm{E}-02$ & $9.30 \mathrm{E}-06$ & $1.50 \mathrm{E}-03$ & $1.40 \mathrm{E}-02$ & $1.13 \mathrm{E}-03$ & $6.71 \mathrm{E}-02$ \\
\hline $70 \%$ & $3.08 \mathrm{E}-05$ & $2.55 \mathrm{E}-08$ & $3.07 \mathrm{E}-02$ & $2.17 \mathrm{E}-05$ & $2.36 \mathrm{E}-03$ & $2.81 \mathrm{E}-02$ & $1.85 \mathrm{E}-03$ & $9.89 \mathrm{E}-02$ \\
\hline $75 \%$ & $6.94 \mathrm{E}-05$ & $5.74 \mathrm{E}-08$ & $6.55 \mathrm{E}-02$ & $4.55 \mathrm{E}-05$ & $3.50 \mathrm{E}-03$ & $5.25 \mathrm{E}-02$ & $3.05 \mathrm{E}-03$ & $1.38 \mathrm{E}-01$ \\
\hline $80 \%$ & $1.37 \mathrm{E}-04$ & $1.12 \mathrm{E}-07$ & $1.06 \mathrm{E}-01$ & 8.83E-05 & $5.24 \mathrm{E}-03$ & $1.01 \mathrm{E}-01$ & 4.72E-03 & $2.04 \mathrm{E}-01$ \\
\hline $85 \%$ & 2.69E-04 & $1.84 \mathrm{E}-07$ & $1.56 \mathrm{E}-01$ & $1.49 \mathrm{E}-04$ & $9.35 \mathrm{E}-03$ & $2.28 \mathrm{E}-01$ & 7.79E-03 & $3.18 \mathrm{E}-01$ \\
\hline $90 \%$ & $3.28 \mathrm{E}-04$ & $2.60 \mathrm{E}-07$ & $2.20 \mathrm{E}-01$ & $2.27 \mathrm{E}-04$ & $1.79 \mathrm{E}-02$ & 4.63E-01 & $1.35 \mathrm{E}-02$ & 5.33E-01 \\
\hline $95 \%$ & $3.50 \mathrm{E}-04$ & $3.33 \mathrm{E}-07$ & $3.23 \mathrm{E}-01$ & $3.06 \mathrm{E}-04$ & $3.82 \mathrm{E}-02$ & $1.04 \mathrm{E}+00$ & 2.99E-02 & $1.13 \mathrm{E}+00$ \\
\hline Mean & $6.90 \mathrm{E}-05$ & $6.29 \mathrm{E}-08$ & $6.22 \mathrm{E}-02$ & $5.35 \mathrm{E}-05$ & $6.00 \mathrm{E}-03$ & $1.90 \mathrm{E}-01$ & $5.13 \mathrm{E}-03$ & $2.25 \mathrm{E}-01$ \\
\hline Min & $0.00 \mathrm{E}+00$ & $0.00 \mathrm{E}+00$ & $0.00 \mathrm{E}+00$ & $0.00 \mathrm{E}+00$ & $0.00 \mathrm{E}+00$ & $0.00 \mathrm{E}+00$ & $0.00 \mathrm{E}+00$ & $0.00 \mathrm{E}+00$ \\
\hline Max & $3.66 \mathrm{E}-04$ & $1.15 \mathrm{E}-06$ & $1.77 \mathrm{E}+00$ & $5.27 \mathrm{E}-04$ & $1.48 \mathrm{E}-01$ & $1.49 \mathrm{E}+01$ & $2.86 \mathrm{E}-01$ & $1.51 \mathrm{E}+01$ \\
\hline
\end{tabular}


TABLE C.34 Peak DSR Percentiles (mrem/yr per pCi/g) for Different Exposure Pathways for Np-237 in Recreational Use Scenario

\begin{tabular}{|c|c|c|c|c|c|c|c|c|}
\hline Percentile & External & $\begin{array}{l}\text { Inhalation } \\
\text { w/o Radon }\end{array}$ & $\begin{array}{c}\text { Meat } \\
\text { Ingestion } \\
(\mathrm{WI})\end{array}$ & $\begin{array}{c}\text { Soil } \\
\text { Ingestion }\end{array}$ & $\begin{array}{c}\text { Water } \\
\text { Ingestion }\end{array}$ & $\begin{array}{l}\text { Aquatic } \\
\text { Foods }\end{array}$ & $\begin{array}{c}\text { Meat } \\
\text { Ingestion } \\
\text { (WD) }\end{array}$ & $\begin{array}{c}\text { All } \\
\text { Pathways }\end{array}$ \\
\hline $5 \%$ & 4.73E-09 & $3.41 \mathrm{E}-10$ & $1.92 \mathrm{E}-08$ & $1.12 \mathrm{E}-09$ & $0.00 \mathrm{E}+00$ & $0.00 \mathrm{E}+00$ & $0.00 \mathrm{E}+00$ & $3.11 \mathrm{E}-08$ \\
\hline $10 \%$ & $1.42 \mathrm{E}-08$ & $1.01 \mathrm{E}-09$ & 7.19E-08 & 3.33E-09 & $0.00 \mathrm{E}+00$ & $0.00 \mathrm{E}+00$ & $0.00 \mathrm{E}+00$ & $1.80 \mathrm{E}-07$ \\
\hline $15 \%$ & $3.96 \mathrm{E}-05$ & 2.09E-07 & 9.99E-06 & $2.93 \mathrm{E}-07$ & $0.00 \mathrm{E}+00$ & $0.00 \mathrm{E}+00$ & $0.00 \mathrm{E}+00$ & $2.34 \mathrm{E}-04$ \\
\hline $20 \%$ & $2.02 \mathrm{E}-03$ & 7.76E-06 & $4.21 \mathrm{E}-04$ & $1.31 \mathrm{E}-05$ & $0.00 \mathrm{E}+00$ & $0.00 \mathrm{E}+00$ & $0.00 \mathrm{E}+00$ & $5.14 \mathrm{E}-03$ \\
\hline $25 \%$ & 8.87E-03 & $3.17 \mathrm{E}-05$ & $1.39 \mathrm{E}-03$ & 4.63E-05 & $0.00 \mathrm{E}+00$ & $0.00 \mathrm{E}+00$ & $0.00 \mathrm{E}+00$ & $1.73 \mathrm{E}-02$ \\
\hline $30 \%$ & $1.81 \mathrm{E}-02$ & $6.02 \mathrm{E}-05$ & $2.08 \mathrm{E}-03$ & $8.10 \mathrm{E}-05$ & $0.00 \mathrm{E}+00$ & $0.00 \mathrm{E}+00$ & $0.00 \mathrm{E}+00$ & $2.88 \mathrm{E}-02$ \\
\hline $35 \%$ & $2.52 \mathrm{E}-02$ & 7.87E-05 & $2.80 \mathrm{E}-03$ & $1.14 \mathrm{E}-04$ & $0.00 \mathrm{E}+00$ & $0.00 \mathrm{E}+00$ & $0.00 \mathrm{E}+00$ & $3.60 \mathrm{E}-02$ \\
\hline $40 \%$ & 2.92E-02 & 9.77E-05 & $3.46 \mathrm{E}-03$ & $1.43 \mathrm{E}-04$ & $0.00 \mathrm{E}+00$ & $0.00 \mathrm{E}+00$ & $0.00 \mathrm{E}+00$ & $3.88 \mathrm{E}-02$ \\
\hline $45 \%$ & $3.15 \mathrm{E}-02$ & $1.12 \mathrm{E}-04$ & $4.23 \mathrm{E}-03$ & $1.71 \mathrm{E}-04$ & $0.00 \mathrm{E}+00$ & $0.00 \mathrm{E}+00$ & $0.00 \mathrm{E}+00$ & $4.05 \mathrm{E}-02$ \\
\hline $50 \%$ & $3.35 \mathrm{E}-02$ & $1.22 \mathrm{E}-04$ & $5.00 \mathrm{E}-03$ & $1.97 \mathrm{E}-04$ & $0.00 \mathrm{E}+00$ & $0.00 \mathrm{E}+00$ & $0.00 \mathrm{E}+00$ & $4.21 \mathrm{E}-02$ \\
\hline $55 \%$ & $3.46 \mathrm{E}-02$ & $1.33 \mathrm{E}-04$ & 5.92E-03 & $2.17 \mathrm{E}-04$ & $0.00 \mathrm{E}+00$ & $0.00 \mathrm{E}+00$ & $0.00 \mathrm{E}+00$ & 4.38E-02 \\
\hline $60 \%$ & $3.55 \mathrm{E}-02$ & $1.42 \mathrm{E}-04$ & $6.84 \mathrm{E}-03$ & $2.40 \mathrm{E}-04$ & $0.00 \mathrm{E}+00$ & $0.00 \mathrm{E}+00$ & $0.00 \mathrm{E}+00$ & $4.59 \mathrm{E}-02$ \\
\hline $65 \%$ & $3.60 \mathrm{E}-02$ & $1.52 \mathrm{E}-04$ & $7.78 \mathrm{E}-03$ & $2.62 \mathrm{E}-04$ & $0.00 \mathrm{E}+00$ & $0.00 \mathrm{E}+00$ & $0.00 \mathrm{E}+00$ & $4.81 \mathrm{E}-02$ \\
\hline $70 \%$ & $3.63 \mathrm{E}-02$ & $1.63 \mathrm{E}-04$ & $9.12 \mathrm{E}-03$ & $2.86 \mathrm{E}-04$ & $1.20 \mathrm{E}-06$ & $3.45 \mathrm{E}-05$ & $1.87 \mathrm{E}-08$ & $5.14 \mathrm{E}-02$ \\
\hline $75 \%$ & $3.65 \mathrm{E}-02$ & $1.74 \mathrm{E}-04$ & $1.05 \mathrm{E}-02$ & $3.07 \mathrm{E}-04$ & $7.06 \mathrm{E}-05$ & $2.46 \mathrm{E}-03$ & $1.45 \mathrm{E}-06$ & $5.61 \mathrm{E}-02$ \\
\hline $80 \%$ & $3.66 \mathrm{E}-02$ & $1.85 \mathrm{E}-04$ & $1.24 \mathrm{E}-02$ & $3.34 \mathrm{E}-04$ & $5.89 \mathrm{E}-04$ & $1.93 \mathrm{E}-02$ & $1.08 \mathrm{E}-05$ & $6.42 \mathrm{E}-02$ \\
\hline $85 \%$ & 3.67E-02 & $1.98 \mathrm{E}-04$ & $1.53 \mathrm{E}-02$ & $3.61 \mathrm{E}-04$ & $1.63 \mathrm{E}-03$ & $6.27 \mathrm{E}-02$ & $2.84 \mathrm{E}-05$ & 8.33E-02 \\
\hline $90 \%$ & $3.68 \mathrm{E}-02$ & 2.17E-04 & $1.87 \mathrm{E}-02$ & $3.95 \mathrm{E}-04$ & $3.33 \mathrm{E}-03$ & $1.17 \mathrm{E}-01$ & 7.13E-05 & $1.31 \mathrm{E}-01$ \\
\hline $95 \%$ & $3.68 \mathrm{E}-02$ & $2.59 \mathrm{E}-04$ & $2.69 \mathrm{E}-02$ & $4.41 \mathrm{E}-04$ & $6.92 \mathrm{E}-03$ & $2.82 \mathrm{E}-01$ & $1.86 \mathrm{E}-04$ & $2.92 \mathrm{E}-01$ \\
\hline Mean & $2.46 \mathrm{E}-02$ & $1.16 \mathrm{E}-04$ & $8.11 \mathrm{E}-03$ & $1.93 \mathrm{E}-04$ & $1.40 \mathrm{E}-03$ & 6.64E-02 & $4.08 \mathrm{E}-05$ & $9.41 \mathrm{E}-02$ \\
\hline Min & $1.62 \mathrm{E}-10$ & $1.41 \mathrm{E}-12$ & $2.24 \mathrm{E}-12$ & 4.64E-12 & $0.00 \mathrm{E}+00$ & $0.00 \mathrm{E}+00$ & $0.00 \mathrm{E}+00$ & $2.07 \mathrm{E}-10$ \\
\hline Max & $3.76 \mathrm{E}-02$ & $6.89 \mathrm{E}-04$ & $1.90 \mathrm{E}-01$ & $5.54 \mathrm{E}-04$ & $1.26 \mathrm{E}-01$ & $1.42 \mathrm{E}+01$ & 5.13E-03 & $1.42 \mathrm{E}+01$ \\
\hline
\end{tabular}


TABLE C.35 Peak DSR Percentiles (mrem/yr per pCi/g) for Different Exposure Pathways for $\mathrm{Pu}-238$ in Recreational Use Scenario

\begin{tabular}{|c|c|c|c|c|c|c|c|c|c|}
\hline Percentile & External & $\begin{array}{l}\text { Inhalation } \\
\text { w/o Radon } \\
\end{array}$ & $\begin{array}{c}\text { Radon } \\
\text { (WI) }\end{array}$ & $\begin{array}{c}\text { Meat } \\
\text { Ingestion } \\
(\mathrm{WI})\end{array}$ & $\begin{array}{c}\text { Soil } \\
\text { Ingestion } \\
\end{array}$ & $\begin{array}{c}\text { Water } \\
\text { Ingestion }\end{array}$ & $\begin{array}{c}\text { Aquatic } \\
\text { Foods }\end{array}$ & $\begin{array}{c}\text { Meat } \\
\text { Ingestion } \\
\text { (WD) }\end{array}$ & $\begin{array}{c}\text { All } \\
\text { Pathways } \\
\end{array}$ \\
\hline $5 \%$ & $1.78 \mathrm{E}-06$ & $7.65 \mathrm{E}-05$ & $6.21 \mathrm{E}-14$ & $2.25 \mathrm{E}-04$ & $7.97 \mathrm{E}-05$ & $0.00 \mathrm{E}+00$ & $0.00 \mathrm{E}+00$ & $0.00 \mathrm{E}+00$ & $6.07 \mathrm{E}-04$ \\
\hline $10 \%$ & $1.90 \mathrm{E}-06$ & $1.02 \mathrm{E}-04$ & $7.85 \mathrm{E}-14$ & $2.74 \mathrm{E}-04$ & $1.15 \mathrm{E}-04$ & $0.00 \mathrm{E}+00$ & $0.00 \mathrm{E}+00$ & $0.00 \mathrm{E}+00$ & $6.87 \mathrm{E}-04$ \\
\hline $15 \%$ & $1.94 \mathrm{E}-06$ & $1.18 \mathrm{E}-04$ & $9.13 \mathrm{E}-14$ & 3.07E-04 & $1.43 \mathrm{E}-04$ & $0.00 \mathrm{E}+00$ & $0.00 \mathrm{E}+00$ & $0.00 \mathrm{E}+00$ & 7.47E-04 \\
\hline $20 \%$ & $1.96 \mathrm{E}-06$ & $1.30 \mathrm{E}-04$ & $1.01 \mathrm{E}-13$ & $3.42 \mathrm{E}-04$ & $1.65 \mathrm{E}-04$ & $0.00 \mathrm{E}+00$ & $0.00 \mathrm{E}+00$ & $0.00 \mathrm{E}+00$ & $8.00 \mathrm{E}-04$ \\
\hline $25 \%$ & $1.97 \mathrm{E}-06$ & $1.40 \mathrm{E}-04$ & $1.10 \mathrm{E}-13$ & $3.73 \mathrm{E}-04$ & $1.84 \mathrm{E}-04$ & $0.00 \mathrm{E}+00$ & $0.00 \mathrm{E}+00$ & $0.00 \mathrm{E}+00$ & 8.37E-04 \\
\hline $30 \%$ & $1.98 \mathrm{E}-06$ & $1.51 \mathrm{E}-04$ & $1.19 \mathrm{E}-13$ & 4.03E-04 & $2.02 \mathrm{E}-04$ & $0.00 \mathrm{E}+00$ & $0.00 \mathrm{E}+00$ & $0.00 \mathrm{E}+00$ & $8.72 \mathrm{E}-04$ \\
\hline $35 \%$ & $1.99 \mathrm{E}-06$ & $1.61 \mathrm{E}-04$ & $1.29 \mathrm{E}-13$ & 4.33E-04 & 2.19E-04 & $0.00 \mathrm{E}+00$ & $0.00 \mathrm{E}+00$ & $0.00 \mathrm{E}+00$ & $9.09 \mathrm{E}-04$ \\
\hline $40 \%$ & $1.99 \mathrm{E}-06$ & $1.72 \mathrm{E}-04$ & $1.39 \mathrm{E}-13$ & 4.64E-04 & $2.35 \mathrm{E}-04$ & $0.00 \mathrm{E}+00$ & $0.00 \mathrm{E}+00$ & $0.00 \mathrm{E}+00$ & $9.42 \mathrm{E}-04$ \\
\hline $45 \%$ & $2.00 \mathrm{E}-06$ & $1.81 \mathrm{E}-04$ & $1.49 \mathrm{E}-13$ & $4.95 \mathrm{E}-04$ & $2.51 \mathrm{E}-04$ & $0.00 \mathrm{E}+00$ & $0.00 \mathrm{E}+00$ & $0.00 \mathrm{E}+00$ & $9.83 \mathrm{E}-04$ \\
\hline $50 \%$ & $2.00 \mathrm{E}-06$ & $1.91 \mathrm{E}-04$ & $1.59 \mathrm{E}-13$ & 5.23E-04 & 2.64E-04 & $0.00 \mathrm{E}+00$ & $0.00 \mathrm{E}+00$ & $0.00 \mathrm{E}+00$ & $1.02 \mathrm{E}-03$ \\
\hline $55 \%$ & $2.00 \mathrm{E}-06$ & 2.03E-04 & $1.69 \mathrm{E}-13$ & $5.61 \mathrm{E}-04$ & 2.77E-04 & $0.00 \mathrm{E}+00$ & $0.00 \mathrm{E}+00$ & $0.00 \mathrm{E}+00$ & $1.06 \mathrm{E}-03$ \\
\hline $60 \%$ & $2.00 \mathrm{E}-06$ & 2.19E-04 & $1.81 \mathrm{E}-13$ & $5.98 \mathrm{E}-04$ & 2.92E-04 & $0.00 \mathrm{E}+00$ & $0.00 \mathrm{E}+00$ & $0.00 \mathrm{E}+00$ & $1.11 \mathrm{E}-03$ \\
\hline $65 \%$ & $2.00 \mathrm{E}-06$ & $2.35 \mathrm{E}-04$ & $1.91 \mathrm{E}-13$ & $6.40 \mathrm{E}-04$ & $3.06 \mathrm{E}-04$ & $0.00 \mathrm{E}+00$ & $0.00 \mathrm{E}+00$ & $0.00 \mathrm{E}+00$ & $1.15 \mathrm{E}-03$ \\
\hline $70 \%$ & $2.00 \mathrm{E}-06$ & $2.51 \mathrm{E}-04$ & $2.06 \mathrm{E}-13$ & $6.86 \mathrm{E}-04$ & $3.23 \mathrm{E}-04$ & $0.00 \mathrm{E}+00$ & $0.00 \mathrm{E}+00$ & $0.00 \mathrm{E}+00$ & $1.20 \mathrm{E}-03$ \\
\hline $75 \%$ & $2.00 \mathrm{E}-06$ & $2.71 \mathrm{E}-04$ & $2.22 \mathrm{E}-13$ & 7.29E-04 & $3.41 \mathrm{E}-04$ & $0.00 \mathrm{E}+00$ & $0.00 \mathrm{E}+00$ & $0.00 \mathrm{E}+00$ & $1.25 \mathrm{E}-03$ \\
\hline $80 \%$ & $2.00 \mathrm{E}-06$ & $2.97 \mathrm{E}-04$ & $2.42 \mathrm{E}-13$ & 7.91E-04 & $3.61 \mathrm{E}-04$ & $0.00 \mathrm{E}+00$ & $0.00 \mathrm{E}+00$ & $0.00 \mathrm{E}+00$ & $1.32 \mathrm{E}-03$ \\
\hline $85 \%$ & $2.00 \mathrm{E}-06$ & $3.34 \mathrm{E}-04$ & $2.66 \mathrm{E}-13$ & 8.60E-04 & $3.83 \mathrm{E}-04$ & $0.00 \mathrm{E}+00$ & $0.00 \mathrm{E}+00$ & $0.00 \mathrm{E}+00$ & $1.40 \mathrm{E}-03$ \\
\hline $90 \%$ & $2.00 \mathrm{E}-06$ & $3.78 \mathrm{E}-04$ & $3.06 \mathrm{E}-13$ & $9.42 \mathrm{E}-04$ & 4.11E-04 & $0.00 \mathrm{E}+00$ & $0.00 \mathrm{E}+00$ & $0.00 \mathrm{E}+00$ & $1.49 \mathrm{E}-03$ \\
\hline $95 \%$ & $2.00 \mathrm{E}-06$ & 4.44E-04 & $3.63 \mathrm{E}-13$ & $1.07 \mathrm{E}-03$ & 4.47E-04 & $0.00 \mathrm{E}+00$ & $0.00 \mathrm{E}+00$ & $0.00 \mathrm{E}+00$ & $1.61 \mathrm{E}-03$ \\
\hline Mean & $1.95 \mathrm{E}-06$ & $2.20 \mathrm{E}-04$ & $1.78 \mathrm{E}-13$ & $5.73 \mathrm{E}-04$ & $2.63 \mathrm{E}-04$ & $1.47 \mathrm{E}-10$ & 1.02E-09 & $1.56 \mathrm{E}-12$ & $1.06 \mathrm{E}-03$ \\
\hline Min & $3.28 \mathrm{E}-10$ & $1.23 \mathrm{E}-09$ & 2.67E-15 & $9.70 \mathrm{E}-09$ & $1.89 \mathrm{E}-09$ & $0.00 \mathrm{E}+00$ & $0.00 \mathrm{E}+00$ & $0.00 \mathrm{E}+00$ & $1.32 \mathrm{E}-08$ \\
\hline Max & $2.00 \mathrm{E}-06$ & $1.55 \mathrm{E}-03$ & $1.03 \mathrm{E}-12$ & $1.95 \mathrm{E}-03$ & 5.32E-04 & $2.86 \mathrm{E}-07$ & $2.84 \mathrm{E}-06$ & $2.77 \mathrm{E}-09$ & $2.49 \mathrm{E}-03$ \\
\hline
\end{tabular}




\section{TABLE C.36 Peak DSR Percentiles (mrem/yr per pCi/g) for Different Exposure Pathways for}

Pu-239 in Recreational Use Scenario

\begin{tabular}{|c|c|c|c|c|c|c|c|c|}
\hline Percentile & External & $\begin{array}{c}\text { Inhalation } \\
\text { w/o Radon } \\
\end{array}$ & $\begin{array}{c}\text { Meat } \\
\text { Ingestion } \\
(\mathrm{WI})\end{array}$ & $\begin{array}{c}\text { Soil } \\
\text { Ingestion } \\
\end{array}$ & $\begin{array}{c}\text { Water } \\
\text { Ingestion }\end{array}$ & $\begin{array}{c}\text { Aquatic } \\
\text { Foods }\end{array}$ & $\begin{array}{c}\text { Meat } \\
\text { Ingestion } \\
\text { (WD) }\end{array}$ & $\begin{array}{c}\text { All } \\
\text { Pathways }\end{array}$ \\
\hline $5 \%$ & $8.56 \mathrm{E}-06$ & $1.81 \mathrm{E}-04$ & $5.41 \mathrm{E}-04$ & $1.91 \mathrm{E}-04$ & $0.00 \mathrm{E}+00$ & $0.00 \mathrm{E}+00$ & $0.00 \mathrm{E}+00$ & $1.46 \mathrm{E}-03$ \\
\hline $10 \%$ & $9.10 \mathrm{E}-06$ & $2.48 \mathrm{E}-04$ & $6.58 \mathrm{E}-04$ & $2.77 \mathrm{E}-04$ & $0.00 \mathrm{E}+00$ & $0.00 \mathrm{E}+00$ & $0.00 \mathrm{E}+00$ & $1.66 \mathrm{E}-03$ \\
\hline $15 \%$ & 9.32E-06 & 2.84E-04 & 7.39E-04 & $3.41 \mathrm{E}-04$ & $0.00 \mathrm{E}+00$ & $0.00 \mathrm{E}+00$ & $0.00 \mathrm{E}+00$ & $1.80 \mathrm{E}-03$ \\
\hline $20 \%$ & $9.41 \mathrm{E}-06$ & $3.14 \mathrm{E}-04$ & $8.17 \mathrm{E}-04$ & 3.94E-04 & $0.00 \mathrm{E}+00$ & $0.00 \mathrm{E}+00$ & $0.00 \mathrm{E}+00$ & $1.92 \mathrm{E}-03$ \\
\hline $25 \%$ & $9.48 \mathrm{E}-06$ & 3.39E-04 & 8.91E-04 & 4.42E-04 & $0.00 \mathrm{E}+00$ & $0.00 \mathrm{E}+00$ & $0.00 \mathrm{E}+00$ & $2.02 \mathrm{E}-03$ \\
\hline $30 \%$ & $9.51 \mathrm{E}-06$ & 3.66E-04 & $9.61 \mathrm{E}-04$ & 4.83E-04 & $0.00 \mathrm{E}+00$ & $0.00 \mathrm{E}+00$ & $0.00 \mathrm{E}+00$ & $2.10 \mathrm{E}-03$ \\
\hline $35 \%$ & $9.54 \mathrm{E}-06$ & $3.89 \mathrm{E}-04$ & $1.03 \mathrm{E}-03$ & $5.24 \mathrm{E}-04$ & $0.00 \mathrm{E}+00$ & $0.00 \mathrm{E}+00$ & $0.00 \mathrm{E}+00$ & $2.19 \mathrm{E}-03$ \\
\hline $40 \%$ & $9.56 \mathrm{E}-06$ & 4.13E-04 & $1.11 \mathrm{E}-03$ & $5.63 \mathrm{E}-04$ & $0.00 \mathrm{E}+00$ & $0.00 \mathrm{E}+00$ & $0.00 \mathrm{E}+00$ & $2.27 \mathrm{E}-03$ \\
\hline $45 \%$ & $9.58 \mathrm{E}-06$ & 4.36E-04 & $1.18 \mathrm{E}-03$ & $5.96 \mathrm{E}-04$ & $0.00 \mathrm{E}+00$ & $0.00 \mathrm{E}+00$ & $0.00 \mathrm{E}+00$ & $2.36 \mathrm{E}-03$ \\
\hline $50 \%$ & $9.59 \mathrm{E}-06$ & $4.61 \mathrm{E}-04$ & $1.26 \mathrm{E}-03$ & $6.31 \mathrm{E}-04$ & $0.00 \mathrm{E}+00$ & $0.00 \mathrm{E}+00$ & $0.00 \mathrm{E}+00$ & $2.45 \mathrm{E}-03$ \\
\hline $55 \%$ & $9.60 \mathrm{E}-06$ & 4.94E-04 & $1.35 \mathrm{E}-03$ & 6.64E-04 & $0.00 \mathrm{E}+00$ & $0.00 \mathrm{E}+00$ & $0.00 \mathrm{E}+00$ & $2.54 \mathrm{E}-03$ \\
\hline $60 \%$ & $9.60 \mathrm{E}-06$ & $5.28 \mathrm{E}-04$ & $1.43 \mathrm{E}-03$ & $7.00 \mathrm{E}-04$ & $0.00 \mathrm{E}+00$ & $0.00 \mathrm{E}+00$ & $0.00 \mathrm{E}+00$ & $2.65 \mathrm{E}-03$ \\
\hline $65 \%$ & $9.61 \mathrm{E}-06$ & $5.66 \mathrm{E}-04$ & $1.53 \mathrm{E}-03$ & 7.36E-04 & $0.00 \mathrm{E}+00$ & $0.00 \mathrm{E}+00$ & $0.00 \mathrm{E}+00$ & $2.75 \mathrm{E}-03$ \\
\hline $70 \%$ & $9.61 \mathrm{E}-06$ & $6.06 \mathrm{E}-04$ & $1.64 \mathrm{E}-03$ & 7.72E-04 & $0.00 \mathrm{E}+00$ & $0.00 \mathrm{E}+00$ & $0.00 \mathrm{E}+00$ & $2.87 \mathrm{E}-03$ \\
\hline $75 \%$ & $9.62 \mathrm{E}-06$ & $6.52 \mathrm{E}-04$ & $1.75 \mathrm{E}-03$ & $8.16 \mathrm{E}-04$ & $0.00 \mathrm{E}+00$ & $0.00 \mathrm{E}+00$ & $0.00 \mathrm{E}+00$ & $3.02 \mathrm{E}-03$ \\
\hline $80 \%$ & $9.62 \mathrm{E}-06$ & 7.13E-04 & $1.90 \mathrm{E}-03$ & $8.65 \mathrm{E}-04$ & $0.00 \mathrm{E}+00$ & $0.00 \mathrm{E}+00$ & $0.00 \mathrm{E}+00$ & $3.18 \mathrm{E}-03$ \\
\hline $85 \%$ & $9.62 \mathrm{E}-06$ & $8.00 \mathrm{E}-04$ & $2.06 \mathrm{E}-03$ & $9.22 \mathrm{E}-04$ & $0.00 \mathrm{E}+00$ & $0.00 \mathrm{E}+00$ & $0.00 \mathrm{E}+00$ & $3.36 \mathrm{E}-03$ \\
\hline $90 \%$ & $9.62 \mathrm{E}-06$ & $9.02 \mathrm{E}-04$ & $2.26 \mathrm{E}-03$ & 9.87E-04 & $0.00 \mathrm{E}+00$ & $0.00 \mathrm{E}+00$ & $0.00 \mathrm{E}+00$ & $3.56 \mathrm{E}-03$ \\
\hline $95 \%$ & $9.62 \mathrm{E}-06$ & $1.06 \mathrm{E}-03$ & $2.56 \mathrm{E}-03$ & $1.08 \mathrm{E}-03$ & $0.00 \mathrm{E}+00$ & $0.00 \mathrm{E}+00$ & $0.00 \mathrm{E}+00$ & $3.87 \mathrm{E}-03$ \\
\hline Mean & $9.38 \mathrm{E}-06$ & $5.30 \mathrm{E}-04$ & $1.37 \mathrm{E}-03$ & $6.31 \mathrm{E}-04$ & $1.82 \mathrm{E}-14$ & $2.24 \mathrm{E}-13$ & $3.38 \mathrm{E}-16$ & $2.54 \mathrm{E}-03$ \\
\hline Min & $6.72 \mathrm{E}-08$ & $1.24 \mathrm{E}-06$ & $1.23 \mathrm{E}-05$ & $2.42 \mathrm{E}-06$ & $0.00 \mathrm{E}+00$ & $0.00 \mathrm{E}+00$ & $0.00 \mathrm{E}+00$ & $1.82 \mathrm{E}-05$ \\
\hline Max & $9.62 \mathrm{E}-06$ & $3.74 \mathrm{E}-03$ & 4.63E- 03 & $1.29 \mathrm{E}-03$ & $2.78 \mathrm{E}-11$ & $4.04 \mathrm{E}-10$ & $7.19 \mathrm{E}-13$ & $6.03 \mathrm{E}-03$ \\
\hline
\end{tabular}


TABLE C.37 Peak DSR Percentiles (mrem/yr per pCi/g) for Different Exposure Pathways for Pu-240 in Recreational Use Scenario

\begin{tabular}{|c|c|c|c|c|c|c|c|c|c|}
\hline Percentile & External & $\begin{array}{l}\text { Inhalation } \\
\text { w/o Radon }\end{array}$ & $\begin{array}{c}\text { Radon } \\
\text { (WI) }\end{array}$ & $\begin{array}{c}\text { Meat } \\
\text { Ingestion } \\
\text { (WI) }\end{array}$ & $\begin{array}{c}\text { Soil } \\
\text { Ingestion }\end{array}$ & $\begin{array}{c}\text { Water } \\
\text { Ingestion }\end{array}$ & $\begin{array}{l}\text { Aquatic } \\
\text { Foods }\end{array}$ & $\begin{array}{c}\text { Meat } \\
\text { Ingestion } \\
\text { (WD) }\end{array}$ & $\begin{array}{c}\text { All } \\
\text { Pathways }\end{array}$ \\
\hline $5 \%$ & $3.75 \mathrm{E}-06$ & $1.64 \mathrm{E}-04$ & $4.42 \mathrm{E}-18$ & 5.23E-04 & $1.92 \mathrm{E}-04$ & $0.00 \mathrm{E}+00$ & $0.00 \mathrm{E}+00$ & $0.00 \mathrm{E}+00$ & $1.38 \mathrm{E}-03$ \\
\hline $10 \%$ & $4.00 \mathrm{E}-06$ & $2.17 \mathrm{E}-04$ & $5.51 \mathrm{E}-18$ & $6.43 \mathrm{E}-04$ & $2.74 \mathrm{E}-04$ & $0.00 \mathrm{E}+00$ & $0.00 \mathrm{E}+00$ & $0.00 \mathrm{E}+00$ & $1.55 \mathrm{E}-03$ \\
\hline $15 \%$ & 4.08E-06 & 2.64E-04 & $5.95 \mathrm{E}-18$ & 7.33E-04 & 3.37E-04 & $0.00 \mathrm{E}+00$ & $0.00 \mathrm{E}+00$ & $0.00 \mathrm{E}+00$ & $1.69 \mathrm{E}-03$ \\
\hline $20 \%$ & 4.13E-06 & 2.84E-04 & $6.22 \mathrm{E}-18$ & 8.04E-04 & 3.95E-04 & $0.00 \mathrm{E}+00$ & $0.00 \mathrm{E}+00$ & $0.00 \mathrm{E}+00$ & $1.79 \mathrm{E}-03$ \\
\hline $25 \%$ & 4.16E-06 & $3.00 \mathrm{E}-04$ & $6.42 \mathrm{E}-18$ & 8.80E-04 & 4.39E-04 & $0.00 \mathrm{E}+00$ & $0.00 \mathrm{E}+00$ & $0.00 \mathrm{E}+00$ & $1.90 \mathrm{E}-03$ \\
\hline $30 \%$ & 4.17E-06 & $3.18 \mathrm{E}-04$ & $6.58 \mathrm{E}-18$ & $9.56 \mathrm{E}-04$ & 4.82E-04 & $0.00 \mathrm{E}+00$ & $0.00 \mathrm{E}+00$ & $0.00 \mathrm{E}+00$ & $1.98 \mathrm{E}-03$ \\
\hline $35 \%$ & 4.18E-06 & $3.35 \mathrm{E}-04$ & $6.76 \mathrm{E}-18$ & $1.02 \mathrm{E}-03$ & $5.21 \mathrm{E}-04$ & $0.00 \mathrm{E}+00$ & $0.00 \mathrm{E}+00$ & $0.00 \mathrm{E}+00$ & $2.06 \mathrm{E}-03$ \\
\hline $40 \%$ & 4.19E-06 & $3.54 \mathrm{E}-04$ & $6.89 \mathrm{E}-18$ & $1.10 \mathrm{E}-03$ & $5.56 \mathrm{E}-04$ & $0.00 \mathrm{E}+00$ & $0.00 \mathrm{E}+00$ & $0.00 \mathrm{E}+00$ & $2.14 \mathrm{E}-03$ \\
\hline $45 \%$ & 4.20E-06 & $3.71 \mathrm{E}-04$ & 7.02E-18 & $1.18 \mathrm{E}-03$ & 5.91E-04 & $0.00 \mathrm{E}+00$ & $0.00 \mathrm{E}+00$ & $0.00 \mathrm{E}+00$ & $2.23 \mathrm{E}-03$ \\
\hline $50 \%$ & 4.20E-06 & $3.88 \mathrm{E}-04$ & $7.16 \mathrm{E}-18$ & $1.26 \mathrm{E}-03$ & $6.27 \mathrm{E}-04$ & $0.00 \mathrm{E}+00$ & $0.00 \mathrm{E}+00$ & $0.00 \mathrm{E}+00$ & $2.32 \mathrm{E}-03$ \\
\hline $55 \%$ & 4.21E-06 & $4.05 \mathrm{E}-04$ & 7.27E-18 & $1.34 \mathrm{E}-03$ & $6.58 \mathrm{E}-04$ & $0.00 \mathrm{E}+00$ & $0.00 \mathrm{E}+00$ & $0.00 \mathrm{E}+00$ & $2.42 \mathrm{E}-03$ \\
\hline $60 \%$ & $4.21 \mathrm{E}-06$ & $4.22 \mathrm{E}-04$ & $7.40 \mathrm{E}-18$ & $1.43 \mathrm{E}-03$ & $6.94 \mathrm{E}-04$ & $0.00 \mathrm{E}+00$ & $0.00 \mathrm{E}+00$ & $0.00 \mathrm{E}+00$ & $2.51 \mathrm{E}-03$ \\
\hline $65 \%$ & 4.21E-06 & $4.41 \mathrm{E}-04$ & $7.53 \mathrm{E}-18$ & $1.53 \mathrm{E}-03$ & $7.30 \mathrm{E}-04$ & $0.00 \mathrm{E}+00$ & $0.00 \mathrm{E}+00$ & $0.00 \mathrm{E}+00$ & $2.61 \mathrm{E}-03$ \\
\hline $70 \%$ & $4.21 \mathrm{E}-06$ & $4.58 \mathrm{E}-04$ & $7.65 \mathrm{E}-18$ & $1.63 \mathrm{E}-03$ & 7.69E-04 & $0.00 \mathrm{E}+00$ & $0.00 \mathrm{E}+00$ & $0.00 \mathrm{E}+00$ & $2.73 \mathrm{E}-03$ \\
\hline $75 \%$ & 4.22E-06 & 4.77E-04 & $7.78 \mathrm{E}-18$ & $1.75 \mathrm{E}-03$ & $8.15 \mathrm{E}-04$ & $0.00 \mathrm{E}+00$ & $0.00 \mathrm{E}+00$ & $0.00 \mathrm{E}+00$ & $2.84 \mathrm{E}-03$ \\
\hline $80 \%$ & 4.22E-06 & 4.96E-04 & 7.94E-18 & $1.88 \mathrm{E}-03$ & 8.63E-04 & $0.00 \mathrm{E}+00$ & $0.00 \mathrm{E}+00$ & $0.00 \mathrm{E}+00$ & $2.98 \mathrm{E}-03$ \\
\hline $85 \%$ & 4.22E-06 & 5.37E-04 & $8.11 \mathrm{E}-18$ & $2.04 \mathrm{E}-03$ & $9.15 \mathrm{E}-04$ & $0.00 \mathrm{E}+00$ & $0.00 \mathrm{E}+00$ & $0.00 \mathrm{E}+00$ & $3.15 \mathrm{E}-03$ \\
\hline $90 \%$ & 4.22E-06 & $6.04 \mathrm{E}-04$ & 8.34E-18 & $2.24 \mathrm{E}-03$ & $9.78 \mathrm{E}-04$ & $0.00 \mathrm{E}+00$ & $0.00 \mathrm{E}+00$ & $0.00 \mathrm{E}+00$ & $3.38 \mathrm{E}-03$ \\
\hline $95 \%$ & 4.22E-06 & $6.71 \mathrm{E}-04$ & 8.69E-18 & $2.53 \mathrm{E}-03$ & $1.06 \mathrm{E}-03$ & $0.00 \mathrm{E}+00$ & $0.00 \mathrm{E}+00$ & $0.00 \mathrm{E}+00$ & $3.71 \mathrm{E}-03$ \\
\hline Mean & 4.12E-06 & 4.04E-04 & $2.48 \mathrm{E}-17$ & $1.36 \mathrm{E}-03$ & $6.26 \mathrm{E}-04$ & $5.98 \mathrm{E}-07$ & $1.78 \mathrm{E}-05$ & $1.18 \mathrm{E}-09$ & $2.41 \mathrm{E}-03$ \\
\hline Min & 3.69E-09 & $3.19 \mathrm{E}-07$ & $1.45 \mathrm{E}-19$ & $5.48 \mathrm{E}-07$ & $6.48 \mathrm{E}-07$ & $0.00 \mathrm{E}+00$ & $0.00 \mathrm{E}+00$ & $0.00 \mathrm{E}+00$ & $1.52 \mathrm{E}-06$ \\
\hline Max & 4.26E-06 & $1.58 \mathrm{E}-03$ & $1.76 \mathrm{E}-14$ & $4.71 \mathrm{E}-03$ & $1.26 \mathrm{E}-03$ & $4.53 \mathrm{E}-04$ & $2.60 \mathrm{E}-02$ & $9.22 \mathrm{E}-07$ & $2.65 \mathrm{E}-02$ \\
\hline
\end{tabular}




\section{TABLE C.38 Peak DSR Percentiles (mrem/yr per pCi/g) for Different Exposure Pathways for}

Sr-90 in Recreational Use Scenario

\begin{tabular}{|c|c|c|c|c|c|c|c|c|}
\hline Percentile & External & $\begin{array}{c}\text { Inhalation } \\
\text { w/o Radon }\end{array}$ & $\begin{array}{c}\text { Meat } \\
\text { Ingestion } \\
(\mathrm{WI})\end{array}$ & $\begin{array}{c}\text { Soil } \\
\text { Ingestion } \\
\end{array}$ & $\begin{array}{c}\text { Water } \\
\text { Ingestion }\end{array}$ & $\begin{array}{c}\text { Aquatic } \\
\text { Foods }\end{array}$ & $\begin{array}{c}\text { Meat } \\
\text { Ingestion } \\
\text { (WD) }\end{array}$ & $\begin{array}{c}\text { All } \\
\text { Pathways }\end{array}$ \\
\hline $5 \%$ & $1.57 \mathrm{E}-06$ & $6.82 \mathrm{E}-10$ & $1.07 \mathrm{E}-04$ & 8.55E-08 & $0.00 \mathrm{E}+00$ & $0.00 \mathrm{E}+00$ & $0.00 \mathrm{E}+00$ & $1.18 \mathrm{E}-04$ \\
\hline $10 \%$ & $1.49 \mathrm{E}-05$ & $5.84 \mathrm{E}-09$ & $8.34 \mathrm{E}-04$ & $5.86 \mathrm{E}-07$ & $0.00 \mathrm{E}+00$ & $0.00 \mathrm{E}+00$ & $0.00 \mathrm{E}+00$ & $9.18 \mathrm{E}-04$ \\
\hline $15 \%$ & $3.59 \mathrm{E}-05$ & $1.19 \mathrm{E}-08$ & $1.57 \mathrm{E}-03$ & $1.24 \mathrm{E}-06$ & $0.00 \mathrm{E}+00$ & $0.00 \mathrm{E}+00$ & $0.00 \mathrm{E}+00$ & $1.64 \mathrm{E}-03$ \\
\hline $20 \%$ & $5.40 \mathrm{E}-05$ & $1.75 \mathrm{E}-08$ & $2.21 \mathrm{E}-03$ & $1.87 \mathrm{E}-06$ & $0.00 \mathrm{E}+00$ & $0.00 \mathrm{E}+00$ & $0.00 \mathrm{E}+00$ & $2.31 \mathrm{E}-03$ \\
\hline $25 \%$ & 7.09E-05 & $2.30 \mathrm{E}-08$ & $2.78 \mathrm{E}-03$ & $2.45 \mathrm{E}-06$ & $0.00 \mathrm{E}+00$ & $0.00 \mathrm{E}+00$ & $0.00 \mathrm{E}+00$ & $2.88 \mathrm{E}-03$ \\
\hline $30 \%$ & $8.41 \mathrm{E}-05$ & $2.77 \mathrm{E}-08$ & $3.40 \mathrm{E}-03$ & $3.04 \mathrm{E}-06$ & $0.00 \mathrm{E}+00$ & $0.00 \mathrm{E}+00$ & $0.00 \mathrm{E}+00$ & $3.51 \mathrm{E}-03$ \\
\hline $35 \%$ & $9.46 \mathrm{E}-05$ & $3.21 \mathrm{E}-08$ & $4.11 \mathrm{E}-03$ & $3.60 \mathrm{E}-06$ & $0.00 \mathrm{E}+00$ & $0.00 \mathrm{E}+00$ & $0.00 \mathrm{E}+00$ & $4.23 \mathrm{E}-03$ \\
\hline $40 \%$ & $1.03 \mathrm{E}-04$ & $3.53 \mathrm{E}-08$ & $4.78 \mathrm{E}-03$ & $4.14 \mathrm{E}-06$ & $0.00 \mathrm{E}+00$ & $0.00 \mathrm{E}+00$ & $0.00 \mathrm{E}+00$ & $4.93 \mathrm{E}-03$ \\
\hline $45 \%$ & $1.09 \mathrm{E}-04$ & $3.88 \mathrm{E}-08$ & $5.55 \mathrm{E}-03$ & $4.62 \mathrm{E}-06$ & $0.00 \mathrm{E}+00$ & $0.00 \mathrm{E}+00$ & $0.00 \mathrm{E}+00$ & $5.69 \mathrm{E}-03$ \\
\hline $50 \%$ & $1.14 \mathrm{E}-04$ & 4.22E-08 & $6.44 \mathrm{E}-03$ & $5.14 \mathrm{E}-06$ & $0.00 \mathrm{E}+00$ & $0.00 \mathrm{E}+00$ & $0.00 \mathrm{E}+00$ & $6.56 \mathrm{E}-03$ \\
\hline $55 \%$ & $1.19 \mathrm{E}-04$ & $4.57 \mathrm{E}-08$ & $7.55 \mathrm{E}-03$ & $5.55 \mathrm{E}-06$ & $0.00 \mathrm{E}+00$ & $0.00 \mathrm{E}+00$ & $0.00 \mathrm{E}+00$ & 7.69E-03 \\
\hline $60 \%$ & $1.22 \mathrm{E}-04$ & $4.94 \mathrm{E}-08$ & $9.10 \mathrm{E}-03$ & $6.03 \mathrm{E}-06$ & $0.00 \mathrm{E}+00$ & $0.00 \mathrm{E}+00$ & $0.00 \mathrm{E}+00$ & $9.24 \mathrm{E}-03$ \\
\hline $65 \%$ & $1.25 \mathrm{E}-04$ & $5.36 \mathrm{E}-08$ & $1.07 \mathrm{E}-02$ & $6.52 \mathrm{E}-06$ & $0.00 \mathrm{E}+00$ & $0.00 \mathrm{E}+00$ & $0.00 \mathrm{E}+00$ & $1.08 \mathrm{E}-02$ \\
\hline $70 \%$ & $1.28 \mathrm{E}-04$ & $5.82 \mathrm{E}-08$ & $1.27 \mathrm{E}-02$ & $7.00 \mathrm{E}-06$ & $0.00 \mathrm{E}+00$ & $0.00 \mathrm{E}+00$ & $0.00 \mathrm{E}+00$ & $1.28 \mathrm{E}-02$ \\
\hline $75 \%$ & $1.30 \mathrm{E}-04$ & $6.41 \mathrm{E}-08$ & $1.52 \mathrm{E}-02$ & $7.50 \mathrm{E}-06$ & $0.00 \mathrm{E}+00$ & $0.00 \mathrm{E}+00$ & $0.00 \mathrm{E}+00$ & $1.54 \mathrm{E}-02$ \\
\hline $80 \%$ & $1.31 \mathrm{E}-04$ & 7.04E-08 & $1.85 \mathrm{E}-02$ & $8.06 \mathrm{E}-06$ & $0.00 \mathrm{E}+00$ & $0.00 \mathrm{E}+00$ & $0.00 \mathrm{E}+00$ & $1.86 \mathrm{E}-02$ \\
\hline $85 \%$ & $1.33 \mathrm{E}-04$ & 7.87E-08 & 2.33E-02 & $8.76 \mathrm{E}-06$ & $0.00 \mathrm{E}+00$ & $0.00 \mathrm{E}+00$ & $0.00 \mathrm{E}+00$ & $2.34 \mathrm{E}-02$ \\
\hline $90 \%$ & $1.33 \mathrm{E}-04$ & 8.97E-08 & $3.08 \mathrm{E}-02$ & $9.72 \mathrm{E}-06$ & $0.00 \mathrm{E}+00$ & $0.00 \mathrm{E}+00$ & $0.00 \mathrm{E}+00$ & $3.09 \mathrm{E}-02$ \\
\hline $95 \%$ & $1.34 \mathrm{E}-04$ & $1.13 \mathrm{E}-07$ & $4.74 \mathrm{E}-02$ & $1.07 \mathrm{E}-05$ & $0.00 \mathrm{E}+00$ & $0.00 \mathrm{E}+00$ & $0.00 \mathrm{E}+00$ & $4.75 \mathrm{E}-02$ \\
\hline Mean & $9.53 \mathrm{E}-05$ & $4.72 \mathrm{E}-08$ & $1.34 \mathrm{E}-02$ & $5.15 \mathrm{E}-06$ & $2.21 \mathrm{E}-07$ & $1.35 \mathrm{E}-05$ & $5.66 \mathrm{E}-08$ & $1.35 \mathrm{E}-02$ \\
\hline Min & $0.00 \mathrm{E}+00$ & $0.00 \mathrm{E}+00$ & 4.49E-29 & $0.00 \mathrm{E}+00$ & $0.00 \mathrm{E}+00$ & $0.00 \mathrm{E}+00$ & $0.00 \mathrm{E}+00$ & 4.49E-29 \\
\hline Max & $1.35 \mathrm{E}-04$ & $4.56 \mathrm{E}-07$ & $5.62 \mathrm{E}-01$ & $1.43 \mathrm{E}-05$ & $1.01 \mathrm{E}-04$ & $2.47 \mathrm{E}-02$ & $2.84 \mathrm{E}-05$ & $5.62 \mathrm{E}-01$ \\
\hline
\end{tabular}


TABLE C.39 Peak DSR Percentiles (mrem/yr per pCi/g) for Different Exposure Pathways for Tc-99 in Recreational Use Scenario

\begin{tabular}{|c|c|c|c|c|c|c|c|c|}
\hline Percentile & External & $\begin{array}{c}\text { Inhalation } \\
\text { w/o Radon }\end{array}$ & $\begin{array}{c}\text { Meat } \\
\text { Ingestion } \\
(\mathrm{WI})\end{array}$ & $\begin{array}{c}\text { Soil } \\
\text { Ingestion } \\
\end{array}$ & $\begin{array}{c}\text { Water } \\
\text { Ingestion }\end{array}$ & $\begin{array}{c}\text { Aquatic } \\
\text { Foods }\end{array}$ & $\begin{array}{c}\text { Meat } \\
\text { Ingestion } \\
\text { (WD) }\end{array}$ & $\begin{array}{c}\text { All } \\
\text { Pathways }\end{array}$ \\
\hline $5 \%$ & $1.05 \mathrm{E}-13$ & $1.14 \mathrm{E}-15$ & $6.12 \mathrm{E}-12$ & $3.41 \mathrm{E}-14$ & 7.79E-07 & $4.17 \mathrm{E}-06$ & 1.41E-09 & $5.78 \mathrm{E}-06$ \\
\hline $10 \%$ & $2.34 \mathrm{E}-13$ & $2.81 \mathrm{E}-15$ & $1.57 \mathrm{E}-11$ & $8.11 \mathrm{E}-14$ & $1.76 \mathrm{E}-06$ & $1.30 \mathrm{E}-05$ & 4.07E-09 & $1.69 \mathrm{E}-05$ \\
\hline $15 \%$ & $4.25 \mathrm{E}-13$ & $5.20 \mathrm{E}-15$ & $3.34 \mathrm{E}-11$ & $1.58 \mathrm{E}-13$ & $3.16 \mathrm{E}-06$ & $2.78 \mathrm{E}-05$ & 7.40E-09 & 3.34E-05 \\
\hline $20 \%$ & $7.26 \mathrm{E}-13$ & $9.21 \mathrm{E}-15$ & $6.30 \mathrm{E}-11$ & $2.67 \mathrm{E}-13$ & $4.95 \mathrm{E}-06$ & $4.95 \mathrm{E}-05$ & $1.23 \mathrm{E}-08$ & $5.84 \mathrm{E}-05$ \\
\hline $25 \%$ & $1.24 \mathrm{E}-12$ & $1.62 \mathrm{E}-14$ & $1.12 \mathrm{E}-10$ & $4.43 \mathrm{E}-13$ & $7.74 \mathrm{E}-06$ & 8.31E-05 & $1.93 \mathrm{E}-08$ & $9.76 \mathrm{E}-05$ \\
\hline $30 \%$ & $1.97 \mathrm{E}-12$ & $2.61 \mathrm{E}-14$ & $1.88 \mathrm{E}-10$ & 7.09E-13 & $1.17 \mathrm{E}-05$ & $1.25 \mathrm{E}-04$ & $3.05 \mathrm{E}-08$ & $1.50 \mathrm{E}-04$ \\
\hline $35 \%$ & $3.16 \mathrm{E}-12$ & $4.17 \mathrm{E}-14$ & $3.04 \mathrm{E}-10$ & $1.17 \mathrm{E}-12$ & $1.78 \mathrm{E}-05$ & $1.80 \mathrm{E}-04$ & $4.51 \mathrm{E}-08$ & $2.16 \mathrm{E}-04$ \\
\hline $40 \%$ & $5.01 \mathrm{E}-12$ & $6.51 \mathrm{E}-14$ & $5.03 \mathrm{E}-10$ & $1.87 \mathrm{E}-12$ & $2.68 \mathrm{E}-05$ & $2.79 \mathrm{E}-04$ & $6.95 \mathrm{E}-08$ & $3.41 \mathrm{E}-04$ \\
\hline $45 \%$ & $8.06 \mathrm{E}-12$ & $1.05 \mathrm{E}-13$ & $9.03 \mathrm{E}-10$ & $3.06 \mathrm{E}-12$ & $3.89 \mathrm{E}-05$ & $3.97 \mathrm{E}-04$ & $9.58 \mathrm{E}-08$ & 4.72E-04 \\
\hline $50 \%$ & $1.36 \mathrm{E}-11$ & $1.80 \mathrm{E}-13$ & $1.63 \mathrm{E}-09$ & $5.11 \mathrm{E}-12$ & $5.52 \mathrm{E}-05$ & $5.40 \mathrm{E}-04$ & $1.30 \mathrm{E}-07$ & $6.48 \mathrm{E}-04$ \\
\hline $55 \%$ & $2.45 \mathrm{E}-11$ & $3.38 \mathrm{E}-13$ & $3.26 \mathrm{E}-09$ & $9.62 \mathrm{E}-12$ & $7.81 \mathrm{E}-05$ & $7.46 \mathrm{E}-04$ & $1.71 \mathrm{E}-07$ & $8.73 \mathrm{E}-04$ \\
\hline $60 \%$ & $5.72 \mathrm{E}-11$ & $8.07 \mathrm{E}-13$ & $1.14 \mathrm{E}-08$ & $2.25 \mathrm{E}-11$ & $1.06 \mathrm{E}-04$ & $1.02 \mathrm{E}-03$ & $2.28 \mathrm{E}-07$ & $1.17 \mathrm{E}-03$ \\
\hline $65 \%$ & $3.76 \mathrm{E}-10$ & $6.00 \mathrm{E}-12$ & $1.67 \mathrm{E}-07$ & $1.50 \mathrm{E}-10$ & $1.41 \mathrm{E}-04$ & $1.33 \mathrm{E}-03$ & $2.88 \mathrm{E}-07$ & $1.50 \mathrm{E}-03$ \\
\hline $70 \%$ & $3.75 \mathrm{E}-06$ & $2.93 \mathrm{E}-08$ & $7.50 \mathrm{E}-05$ & 8.08E-07 & $1.79 \mathrm{E}-04$ & $1.76 \mathrm{E}-03$ & $3.52 \mathrm{E}-07$ & $1.93 \mathrm{E}-03$ \\
\hline $75 \%$ & $3.77 \mathrm{E}-06$ & $3.75 \mathrm{E}-08$ & $1.44 \mathrm{E}-04$ & $1.18 \mathrm{E}-06$ & $2.11 \mathrm{E}-04$ & $2.30 \mathrm{E}-03$ & 4.30E-07 & $2.49 \mathrm{E}-03$ \\
\hline $80 \%$ & $3.79 \mathrm{E}-06$ & $4.50 \mathrm{E}-08$ & $2.21 \mathrm{E}-04$ & $1.47 \mathrm{E}-06$ & 2.33E-04 & $3.10 \mathrm{E}-03$ & 5.34E-07 & $3.31 \mathrm{E}-03$ \\
\hline $85 \%$ & $3.80 \mathrm{E}-06$ & 5.33E-08 & $3.27 \mathrm{E}-04$ & $1.69 \mathrm{E}-06$ & $2.50 \mathrm{E}-04$ & $4.16 \mathrm{E}-03$ & $6.64 \mathrm{E}-07$ & 4.36E-03 \\
\hline $90 \%$ & $3.81 \mathrm{E}-06$ & $6.56 \mathrm{E}-08$ & $4.93 \mathrm{E}-04$ & $1.96 \mathrm{E}-06$ & $2.65 \mathrm{E}-04$ & $6.14 \mathrm{E}-03$ & 8.70E-07 & $6.31 \mathrm{E}-03$ \\
\hline $95 \%$ & $3.82 \mathrm{E}-06$ & $8.47 \mathrm{E}-08$ & $8.44 \mathrm{E}-04$ & $2.32 \mathrm{E}-06$ & $2.81 \mathrm{E}-04$ & $1.05 \mathrm{E}-02$ & $1.25 \mathrm{E}-06$ & $1.07 \mathrm{E}-02$ \\
\hline Mean & $1.30 \mathrm{E}-06$ & $1.94 \mathrm{E}-08$ & $1.62 \mathrm{E}-04$ & $5.45 \mathrm{E}-07$ & $1.03 \mathrm{E}-04$ & $2.46 \mathrm{E}-03$ & $3.29 \mathrm{E}-07$ & $2.56 \mathrm{E}-03$ \\
\hline Min & $1.71 \mathrm{E}-15$ & $1.79 \mathrm{E}-17$ & $5.18 \mathrm{E}-14$ & $8.70 \mathrm{E}-16$ & $0.00 \mathrm{E}+00$ & $0.00 \mathrm{E}+00$ & $0.00 \mathrm{E}+00$ & $1.18 \mathrm{E}-09$ \\
\hline Max & $3.86 \mathrm{E}-06$ & 2.43E-07 & $1.15 \mathrm{E}-02$ & $3.12 \mathrm{E}-06$ & $3.60 \mathrm{E}-04$ & $1.28 \mathrm{E}-01$ & 7.23E-06 & $1.29 \mathrm{E}-01$ \\
\hline
\end{tabular}


TABLE C.40 Peak DSR Percentiles (mrem/yr per pCi/g) for Different Exposure Pathways for U-234 in Recreational Use Scenario

\begin{tabular}{|c|c|c|c|c|c|c|c|c|c|}
\hline Percentile & External & $\begin{array}{l}\text { Inhalation } \\
\text { w/o Radon }\end{array}$ & $\begin{array}{c}\text { Radon } \\
\text { (WI) }\end{array}$ & $\begin{array}{c}\text { Meat } \\
\text { Ingestion } \\
(\mathrm{WI})\end{array}$ & $\begin{array}{c}\text { Soil } \\
\text { Ingestion } \\
\end{array}$ & $\begin{array}{c}\text { Water } \\
\text { Ingestion }\end{array}$ & $\begin{array}{l}\text { Aquatic } \\
\text { Foods }\end{array}$ & $\begin{array}{c}\text { Meat } \\
\text { Ingestion } \\
\text { (WD) }\end{array}$ & $\begin{array}{c}\text { All } \\
\text { Pathways }\end{array}$ \\
\hline $5 \%$ & $8.96 \mathrm{E}-06$ & $6.25 \mathrm{E}-06$ & $1.12 \mathrm{E}-09$ & 4.43E-04 & $2.08 \mathrm{E}-05$ & $0.00 \mathrm{E}+00$ & $0.00 \mathrm{E}+00$ & $0.00 \mathrm{E}+00$ & $5.94 \mathrm{E}-04$ \\
\hline $10 \%$ & $1.47 \mathrm{E}-05$ & $1.35 \mathrm{E}-05$ & $1.46 \mathrm{E}-09$ & $8.51 \mathrm{E}-04$ & 4.45E-05 & $0.00 \mathrm{E}+00$ & $0.00 \mathrm{E}+00$ & $0.00 \mathrm{E}+00$ & $1.38 \mathrm{E}-03$ \\
\hline $15 \%$ & $1.74 \mathrm{E}-05$ & $1.73 \mathrm{E}-05$ & $1.66 \mathrm{E}-09$ & $1.16 \mathrm{E}-03$ & $6.26 \mathrm{E}-05$ & $0.00 \mathrm{E}+00$ & $0.00 \mathrm{E}+00$ & $0.00 \mathrm{E}+00$ & $2.05 \mathrm{E}-03$ \\
\hline $20 \%$ & $1.93 \mathrm{E}-05$ & $2.10 \mathrm{E}-05$ & $1.94 \mathrm{E}-09$ & $1.52 \mathrm{E}-03$ & 7.69E-05 & $0.00 \mathrm{E}+00$ & $0.00 \mathrm{E}+00$ & $0.00 \mathrm{E}+00$ & $2.88 \mathrm{E}-03$ \\
\hline $25 \%$ & 7.42E-04 & 2.32E-05 & $1.11 \mathrm{E}-07$ & $1.85 \mathrm{E}-03$ & $9.06 \mathrm{E}-05$ & $0.00 \mathrm{E}+00$ & $0.00 \mathrm{E}+00$ & $0.00 \mathrm{E}+00$ & $3.74 \mathrm{E}-03$ \\
\hline $30 \%$ & $1.59 \mathrm{E}-03$ & $2.51 \mathrm{E}-05$ & $2.04 \mathrm{E}-07$ & $2.14 \mathrm{E}-03$ & $1.03 \mathrm{E}-04$ & $0.00 \mathrm{E}+00$ & $0.00 \mathrm{E}+00$ & $0.00 \mathrm{E}+00$ & $4.58 \mathrm{E}-03$ \\
\hline $35 \%$ & $2.42 \mathrm{E}-03$ & 2.70E-05 & $2.86 \mathrm{E}-07$ & $2.49 \mathrm{E}-03$ & $1.15 \mathrm{E}-04$ & $0.00 \mathrm{E}+00$ & $0.00 \mathrm{E}+00$ & $0.00 \mathrm{E}+00$ & $5.59 \mathrm{E}-03$ \\
\hline $40 \%$ & $3.20 \mathrm{E}-03$ & $2.88 \mathrm{E}-05$ & $3.51 \mathrm{E}-07$ & $2.85 \mathrm{E}-03$ & $1.26 \mathrm{E}-04$ & $0.00 \mathrm{E}+00$ & $0.00 \mathrm{E}+00$ & $0.00 \mathrm{E}+00$ & $6.64 \mathrm{E}-03$ \\
\hline $45 \%$ & $4.01 \mathrm{E}-03$ & $3.06 \mathrm{E}-05$ & 4.04E-07 & $3.23 \mathrm{E}-03$ & $1.36 \mathrm{E}-04$ & $0.00 \mathrm{E}+00$ & $0.00 \mathrm{E}+00$ & $0.00 \mathrm{E}+00$ & 7.73E-03 \\
\hline $50 \%$ & $4.78 \mathrm{E}-03$ & $3.24 \mathrm{E}-05$ & $4.59 \mathrm{E}-07$ & $3.61 \mathrm{E}-03$ & $1.46 \mathrm{E}-04$ & $0.00 \mathrm{E}+00$ & $0.00 \mathrm{E}+00$ & $0.00 \mathrm{E}+00$ & $8.66 \mathrm{E}-03$ \\
\hline $55 \%$ & $5.51 \mathrm{E}-03$ & $3.43 \mathrm{E}-05$ & $5.08 \mathrm{E}-07$ & $4.05 \mathrm{E}-03$ & $1.58 \mathrm{E}-04$ & $0.00 \mathrm{E}+00$ & $0.00 \mathrm{E}+00$ & $0.00 \mathrm{E}+00$ & $9.60 \mathrm{E}-03$ \\
\hline $60 \%$ & $6.12 \mathrm{E}-03$ & 3.60E-05 & $5.50 \mathrm{E}-07$ & 4.53E-03 & $1.72 \mathrm{E}-04$ & $0.00 \mathrm{E}+00$ & $0.00 \mathrm{E}+00$ & $0.00 \mathrm{E}+00$ & $1.04 \mathrm{E}-02$ \\
\hline $65 \%$ & $6.70 \mathrm{E}-03$ & $3.78 \mathrm{E}-05$ & $5.86 \mathrm{E}-07$ & $5.03 \mathrm{E}-03$ & $1.84 \mathrm{E}-04$ & $0.00 \mathrm{E}+00$ & $0.00 \mathrm{E}+00$ & $0.00 \mathrm{E}+00$ & $1.12 \mathrm{E}-02$ \\
\hline $70 \%$ & 7.18E-03 & $3.96 \mathrm{E}-05$ & 6.19E-07 & $5.61 \mathrm{E}-03$ & $1.99 \mathrm{E}-04$ & $0.00 \mathrm{E}+00$ & $0.00 \mathrm{E}+00$ & $0.00 \mathrm{E}+00$ & $1.21 \mathrm{E}-02$ \\
\hline $75 \%$ & $7.66 \mathrm{E}-03$ & $4.21 \mathrm{E}-05$ & $6.52 \mathrm{E}-07$ & $6.36 \mathrm{E}-03$ & $2.17 \mathrm{E}-04$ & $0.00 \mathrm{E}+00$ & $0.00 \mathrm{E}+00$ & $0.00 \mathrm{E}+00$ & $1.30 \mathrm{E}-02$ \\
\hline $80 \%$ & $8.08 \mathrm{E}-03$ & $4.55 \mathrm{E}-05$ & $6.81 \mathrm{E}-07$ & $7.20 \mathrm{E}-03$ & $2.37 \mathrm{E}-04$ & $0.00 \mathrm{E}+00$ & $0.00 \mathrm{E}+00$ & $0.00 \mathrm{E}+00$ & $1.42 \mathrm{E}-02$ \\
\hline $85 \%$ & $8.42 \mathrm{E}-03$ & $4.90 \mathrm{E}-05$ & 7.13E-07 & 8.38E-03 & $2.62 \mathrm{E}-04$ & $3.27 \mathrm{E}-11$ & 8.01E-09 & $1.32 \mathrm{E}-12$ & $1.55 \mathrm{E}-02$ \\
\hline $90 \%$ & 8.79E-03 & $5.38 \mathrm{E}-05$ & 7.49E-07 & $1.00 \mathrm{E}-02$ & $2.99 \mathrm{E}-04$ & $3.96 \mathrm{E}-06$ & 7.73E-05 & $6.50 \mathrm{E}-08$ & $1.73 \mathrm{E}-02$ \\
\hline $95 \%$ & $9.13 \mathrm{E}-03$ & $6.66 \mathrm{E}-05$ & 8.01E-07 & $1.29 \mathrm{E}-02$ & $3.46 \mathrm{E}-04$ & 4.74E-05 & $7.79 \mathrm{E}-04$ & $8.24 \mathrm{E}-07$ & $2.10 \mathrm{E}-02$ \\
\hline Mean & $4.46 \mathrm{E}-03$ & $3.38 \mathrm{E}-05$ & $4.06 \mathrm{E}-07$ & $4.75 \mathrm{E}-03$ & $1.61 \mathrm{E}-04$ & $2.99 \mathrm{E}-05$ & $1.22 \mathrm{E}-03$ & $5.83 \mathrm{E}-07$ & $1.00 \mathrm{E}-02$ \\
\hline Min & $1.34 \mathrm{E}-07$ & $2.21 \mathrm{E}-09$ & 3.64E-11 & $2.94 \mathrm{E}-08$ & $5.26 \mathrm{E}-09$ & $0.00 \mathrm{E}+00$ & $0.00 \mathrm{E}+00$ & $0.00 \mathrm{E}+00$ & $1.73 \mathrm{E}-07$ \\
\hline Max & $1.01 \mathrm{E}-02$ & $1.71 \mathrm{E}-04$ & $1.01 \mathrm{E}-06$ & $4.28 \mathrm{E}-02$ & $5.78 \mathrm{E}-04$ & $1.83 \mathrm{E}-02$ & $2.46 \mathrm{E}+00$ & $3.60 \mathrm{E}-04$ & $2.46 \mathrm{E}+00$ \\
\hline
\end{tabular}


TABLE C.41 Peak DSR Percentiles (mrem/yr per pCi/g) for Different Exposure Pathways for U-235 in Recreational Use Scenario

\begin{tabular}{|c|c|c|c|c|c|c|c|c|}
\hline Percentile & External & $\begin{array}{l}\text { Inhalation } \\
\text { w/o Radon }\end{array}$ & $\begin{array}{c}\text { Meat } \\
\text { Ingestion } \\
(\mathrm{WI})\end{array}$ & $\begin{array}{c}\text { Soil } \\
\text { Ingestion } \\
\end{array}$ & $\begin{array}{c}\text { Water } \\
\text { Ingestion }\end{array}$ & $\begin{array}{c}\text { Aquatic } \\
\text { Foods }\end{array}$ & $\begin{array}{c}\text { Meat } \\
\text { Ingestion } \\
\text { (WD) }\end{array}$ & $\begin{array}{c}\text { All } \\
\text { Pathways }\end{array}$ \\
\hline $5 \%$ & $5.93 \mathrm{E}-03$ & $6.05 \mathrm{E}-06$ & 2.73E-04 & $1.85 \mathrm{E}-05$ & $0.00 \mathrm{E}+00$ & $0.00 \mathrm{E}+00$ & $0.00 \mathrm{E}+00$ & $6.68 \mathrm{E}-03$ \\
\hline $10 \%$ & $1.51 \mathrm{E}-02$ & $1.31 \mathrm{E}-05$ & $4.68 \mathrm{E}-04$ & $4.30 \mathrm{E}-05$ & $0.00 \mathrm{E}+00$ & $0.00 \mathrm{E}+00$ & $0.00 \mathrm{E}+00$ & $1.68 \mathrm{E}-02$ \\
\hline $15 \%$ & $1.97 \mathrm{E}-02$ & $1.78 \mathrm{E}-05$ & $6.58 \mathrm{E}-04$ & $6.13 \mathrm{E}-05$ & $0.00 \mathrm{E}+00$ & $0.00 \mathrm{E}+00$ & $0.00 \mathrm{E}+00$ & $2.18 \mathrm{E}-02$ \\
\hline $20 \%$ & $2.21 \mathrm{E}-02$ & $2.15 \mathrm{E}-05$ & $8.18 \mathrm{E}-04$ & 7.53E-05 & $0.00 \mathrm{E}+00$ & $0.00 \mathrm{E}+00$ & $0.00 \mathrm{E}+00$ & $2.41 \mathrm{E}-02$ \\
\hline $25 \%$ & 2.33E-02 & $2.38 \mathrm{E}-05$ & $9.63 \mathrm{E}-04$ & 8.92E-05 & $0.00 \mathrm{E}+00$ & $0.00 \mathrm{E}+00$ & $0.00 \mathrm{E}+00$ & $2.52 \mathrm{E}-02$ \\
\hline $30 \%$ & $2.40 \mathrm{E}-02$ & 2.62E-05 & $1.10 \mathrm{E}-03$ & $1.01 \mathrm{E}-04$ & $0.00 \mathrm{E}+00$ & $0.00 \mathrm{E}+00$ & $0.00 \mathrm{E}+00$ & $2.58 \mathrm{E}-02$ \\
\hline $35 \%$ & $2.45 \mathrm{E}-02$ & $2.86 \mathrm{E}-05$ & $1.26 \mathrm{E}-03$ & $1.12 \mathrm{E}-04$ & $0.00 \mathrm{E}+00$ & $0.00 \mathrm{E}+00$ & $0.00 \mathrm{E}+00$ & $2.62 \mathrm{E}-02$ \\
\hline $40 \%$ & $2.48 \mathrm{E}-02$ & $3.10 \mathrm{E}-05$ & $1.44 \mathrm{E}-03$ & $1.23 \mathrm{E}-04$ & $0.00 \mathrm{E}+00$ & $0.00 \mathrm{E}+00$ & $0.00 \mathrm{E}+00$ & $2.66 \mathrm{E}-02$ \\
\hline $45 \%$ & $2.50 \mathrm{E}-02$ & $3.34 \mathrm{E}-05$ & $1.60 \mathrm{E}-03$ & $1.33 \mathrm{E}-04$ & $0.00 \mathrm{E}+00$ & $0.00 \mathrm{E}+00$ & $0.00 \mathrm{E}+00$ & $2.69 \mathrm{E}-02$ \\
\hline $50 \%$ & $2.51 \mathrm{E}-02$ & $3.58 \mathrm{E}-05$ & $1.78 \mathrm{E}-03$ & $1.44 \mathrm{E}-04$ & $0.00 \mathrm{E}+00$ & $0.00 \mathrm{E}+00$ & $0.00 \mathrm{E}+00$ & $2.74 \mathrm{E}-02$ \\
\hline $55 \%$ & $2.52 \mathrm{E}-02$ & $3.88 \mathrm{E}-05$ & $1.98 \mathrm{E}-03$ & $1.56 \mathrm{E}-04$ & $0.00 \mathrm{E}+00$ & $0.00 \mathrm{E}+00$ & $0.00 \mathrm{E}+00$ & 2.79E-02 \\
\hline $60 \%$ & $2.53 \mathrm{E}-02$ & 4.38E-05 & $2.21 \mathrm{E}-03$ & $1.71 \mathrm{E}-04$ & $0.00 \mathrm{E}+00$ & $0.00 \mathrm{E}+00$ & $0.00 \mathrm{E}+00$ & $2.85 \mathrm{E}-02$ \\
\hline $65 \%$ & $2.54 \mathrm{E}-02$ & $5.32 \mathrm{E}-05$ & $2.44 \mathrm{E}-03$ & $1.89 \mathrm{E}-04$ & $0.00 \mathrm{E}+00$ & $0.00 \mathrm{E}+00$ & $0.00 \mathrm{E}+00$ & $2.92 \mathrm{E}-02$ \\
\hline $70 \%$ & $2.59 \mathrm{E}-02$ & 7.89E-05 & $2.76 \mathrm{E}-03$ & $2.14 \mathrm{E}-04$ & $0.00 \mathrm{E}+00$ & $0.00 \mathrm{E}+00$ & $0.00 \mathrm{E}+00$ & $3.00 \mathrm{E}-02$ \\
\hline $75 \%$ & $2.71 \mathrm{E}-02$ & $1.18 \mathrm{E}-04$ & $3.16 \mathrm{E}-03$ & $2.58 \mathrm{E}-04$ & $0.00 \mathrm{E}+00$ & $0.00 \mathrm{E}+00$ & $0.00 \mathrm{E}+00$ & $3.08 \mathrm{E}-02$ \\
\hline $80 \%$ & $2.85 \mathrm{E}-02$ & $1.58 \mathrm{E}-04$ & $3.61 \mathrm{E}-03$ & $3.65 \mathrm{E}-04$ & $0.00 \mathrm{E}+00$ & $0.00 \mathrm{E}+00$ & $0.00 \mathrm{E}+00$ & $3.20 \mathrm{E}-02$ \\
\hline $85 \%$ & $2.97 \mathrm{E}-02$ & $1.92 \mathrm{E}-04$ & 4.22E-03 & 4.61E-04 & $0.00 \mathrm{E}+00$ & $0.00 \mathrm{E}+00$ & $0.00 \mathrm{E}+00$ & $3.31 \mathrm{E}-02$ \\
\hline $90 \%$ & $3.10 \mathrm{E}-02$ & $2.28 \mathrm{E}-04$ & $5.11 \mathrm{E}-03$ & $5.67 \mathrm{E}-04$ & $0.00 \mathrm{E}+00$ & $0.00 \mathrm{E}+00$ & $0.00 \mathrm{E}+00$ & $3.41 \mathrm{E}-02$ \\
\hline $95 \%$ & $3.20 \mathrm{E}-02$ & $2.90 \mathrm{E}-04$ & $6.87 \mathrm{E}-03$ & 7.00E-04 & $2.08 \mathrm{E}-06$ & $1.89 \mathrm{E}-05$ & $6.08 \mathrm{E}-09$ & $3.58 \mathrm{E}-02$ \\
\hline mean & $2.38 \mathrm{E}-02$ & 8.33E-05 & $2.48 \mathrm{E}-03$ & 2.23E-04 & $2.68 \mathrm{E}-06$ & $7.82 \mathrm{E}-05$ & $3.19 \mathrm{E}-08$ & $2.65 \mathrm{E}-02$ \\
\hline $\min$ & $4.36 \mathrm{E}-11$ & $1.55 \mathrm{E}-12$ & $3.47 \mathrm{E}-13$ & $1.03 \mathrm{E}-12$ & $0.00 \mathrm{E}+00$ & $0.00 \mathrm{E}+00$ & $0.00 \mathrm{E}+00$ & $4.66 \mathrm{E}-11$ \\
\hline $\max$ & $3.32 \mathrm{E}-02$ & $7.65 \mathrm{E}-04$ & $3.92 \mathrm{E}-02$ & $1.25 \mathrm{E}-03$ & $2.11 \mathrm{E}-03$ & 7.96E-02 & 3.33E-05 & $8.25 \mathrm{E}-02$ \\
\hline
\end{tabular}


TABLE C.42 Peak DSR Percentiles (mrem/yr per pCi/g) for Different Exposure Pathways for U-238 in Recreational Use Scenario

\begin{tabular}{|c|c|c|c|c|c|c|c|c|c|}
\hline Percentile & External & $\begin{array}{l}\text { Inhalation } \\
\text { w/o Radon }\end{array}$ & $\begin{array}{c}\text { Radon } \\
\text { (WI) }\end{array}$ & $\begin{array}{c}\text { Meat } \\
\text { Ingestion } \\
(\mathrm{WI})\end{array}$ & $\begin{array}{c}\text { Soil } \\
\text { Ingestion } \\
\end{array}$ & $\begin{array}{c}\text { Water } \\
\text { Ingestion }\end{array}$ & $\begin{array}{c}\text { Aquatic } \\
\text { Foods }\end{array}$ & $\begin{array}{c}\text { Meat } \\
\text { Ingestion } \\
\text { (WD) }\end{array}$ & $\begin{array}{c}\text { All } \\
\text { Pathways }\end{array}$ \\
\hline $5 \%$ & $1.46 \mathrm{E}-03$ & $3.96 \mathrm{E}-06$ & $8.20 \mathrm{E}-14$ & 2.77E-04 & $1.63 \mathrm{E}-05$ & $0.00 \mathrm{E}+00$ & $0.00 \mathrm{E}+00$ & $0.00 \mathrm{E}+00$ & $2.09 \mathrm{E}-03$ \\
\hline $10 \%$ & $3.53 \mathrm{E}-03$ & $1.01 \mathrm{E}-05$ & $1.23 \mathrm{E}-13$ & $5.05 \mathrm{E}-04$ & $3.67 \mathrm{E}-05$ & $0.00 \mathrm{E}+00$ & $0.00 \mathrm{E}+00$ & $0.00 \mathrm{E}+00$ & $4.87 \mathrm{E}-03$ \\
\hline $15 \%$ & 4.47E-03 & $1.30 \mathrm{E}-05$ & $1.38 \mathrm{E}-13$ & $6.72 \mathrm{E}-04$ & $5.02 \mathrm{E}-05$ & $0.00 \mathrm{E}+00$ & $0.00 \mathrm{E}+00$ & $0.00 \mathrm{E}+00$ & $5.94 \mathrm{E}-03$ \\
\hline $20 \%$ & 4.95E-03 & $1.59 \mathrm{E}-05$ & $1.45 \mathrm{E}-13$ & 8.13E-04 & $6.13 \mathrm{E}-05$ & $0.00 \mathrm{E}+00$ & $0.00 \mathrm{E}+00$ & $0.00 \mathrm{E}+00$ & $6.27 \mathrm{E}-03$ \\
\hline $25 \%$ & $5.19 \mathrm{E}-03$ & $1.82 \mathrm{E}-05$ & $1.51 \mathrm{E}-13$ & $9.46 \mathrm{E}-04$ & 7.19E-05 & $0.00 \mathrm{E}+00$ & $0.00 \mathrm{E}+00$ & $0.00 \mathrm{E}+00$ & $6.46 \mathrm{E}-03$ \\
\hline $30 \%$ & $5.34 \mathrm{E}-03$ & $1.94 \mathrm{E}-05$ & $1.55 \mathrm{E}-13$ & $1.09 \mathrm{E}-03$ & 8.07E-05 & $0.00 \mathrm{E}+00$ & $0.00 \mathrm{E}+00$ & $0.00 \mathrm{E}+00$ & $6.63 \mathrm{E}-03$ \\
\hline $35 \%$ & $5.44 \mathrm{E}-03$ & $2.06 \mathrm{E}-05$ & $1.59 \mathrm{E}-13$ & $1.27 \mathrm{E}-03$ & $8.96 \mathrm{E}-05$ & $0.00 \mathrm{E}+00$ & $0.00 \mathrm{E}+00$ & $0.00 \mathrm{E}+00$ & $6.79 \mathrm{E}-03$ \\
\hline $40 \%$ & $5.50 \mathrm{E}-03$ & 2.19E-05 & $1.62 \mathrm{E}-13$ & $1.41 \mathrm{E}-03$ & $9.83 \mathrm{E}-05$ & $0.00 \mathrm{E}+00$ & $0.00 \mathrm{E}+00$ & $0.00 \mathrm{E}+00$ & $6.97 \mathrm{E}-03$ \\
\hline $45 \%$ & $5.55 \mathrm{E}-03$ & $2.31 \mathrm{E}-05$ & $1.66 \mathrm{E}-13$ & $1.59 \mathrm{E}-03$ & $1.06 \mathrm{E}-04$ & $0.00 \mathrm{E}+00$ & $0.00 \mathrm{E}+00$ & $0.00 \mathrm{E}+00$ & $7.16 \mathrm{E}-03$ \\
\hline $50 \%$ & $5.57 \mathrm{E}-03$ & $2.44 \mathrm{E}-05$ & $1.69 \mathrm{E}-13$ & $1.77 \mathrm{E}-03$ & $1.14 \mathrm{E}-04$ & $0.00 \mathrm{E}+00$ & $0.00 \mathrm{E}+00$ & $0.00 \mathrm{E}+00$ & $7.36 \mathrm{E}-03$ \\
\hline $55 \%$ & $5.59 \mathrm{E}-03$ & $2.57 \mathrm{E}-05$ & $1.72 \mathrm{E}-13$ & $1.98 \mathrm{E}-03$ & $1.21 \mathrm{E}-04$ & $0.00 \mathrm{E}+00$ & $0.00 \mathrm{E}+00$ & $0.00 \mathrm{E}+00$ & $7.58 \mathrm{E}-03$ \\
\hline $60 \%$ & $5.60 \mathrm{E}-03$ & 2.69E-05 & $1.75 \mathrm{E}-13$ & $2.24 \mathrm{E}-03$ & $1.28 \mathrm{E}-04$ & $0.00 \mathrm{E}+00$ & $0.00 \mathrm{E}+00$ & $0.00 \mathrm{E}+00$ & $7.87 \mathrm{E}-03$ \\
\hline $65 \%$ & $5.61 \mathrm{E}-03$ & $2.83 \mathrm{E}-05$ & $1.79 \mathrm{E}-13$ & $2.50 \mathrm{E}-03$ & $1.35 \mathrm{E}-04$ & $0.00 \mathrm{E}+00$ & $0.00 \mathrm{E}+00$ & $0.00 \mathrm{E}+00$ & $8.14 \mathrm{E}-03$ \\
\hline $70 \%$ & $5.62 \mathrm{E}-03$ & $2.97 \mathrm{E}-05$ & $1.83 \mathrm{E}-13$ & $2.87 \mathrm{E}-03$ & $1.43 \mathrm{E}-04$ & $0.00 \mathrm{E}+00$ & $0.00 \mathrm{E}+00$ & $0.00 \mathrm{E}+00$ & $8.52 \mathrm{E}-03$ \\
\hline $75 \%$ & $5.63 \mathrm{E}-03$ & $3.11 \mathrm{E}-05$ & $1.87 \mathrm{E}-13$ & $3.26 \mathrm{E}-03$ & $1.52 \mathrm{E}-04$ & $0.00 \mathrm{E}+00$ & $0.00 \mathrm{E}+00$ & $0.00 \mathrm{E}+00$ & $8.93 \mathrm{E}-03$ \\
\hline $80 \%$ & $5.63 \mathrm{E}-03$ & $3.27 \mathrm{E}-05$ & $1.92 \mathrm{E}-13$ & $3.74 \mathrm{E}-03$ & $1.61 \mathrm{E}-04$ & $0.00 \mathrm{E}+00$ & $0.00 \mathrm{E}+00$ & $0.00 \mathrm{E}+00$ & $9.42 \mathrm{E}-03$ \\
\hline $85 \%$ & $5.63 \mathrm{E}-03$ & $3.48 \mathrm{E}-05$ & $1.99 \mathrm{E}-13$ & 4.40E-03 & $1.73 \mathrm{E}-04$ & $0.00 \mathrm{E}+00$ & $0.00 \mathrm{E}+00$ & $0.00 \mathrm{E}+00$ & $1.01 \mathrm{E}-02$ \\
\hline $90 \%$ & $5.63 \mathrm{E}-03$ & $3.90 \mathrm{E}-05$ & $2.17 \mathrm{E}-13$ & $5.35 \mathrm{E}-03$ & $1.86 \mathrm{E}-04$ & $0.00 \mathrm{E}+00$ & $0.00 \mathrm{E}+00$ & $0.00 \mathrm{E}+00$ & $1.11 \mathrm{E}-02$ \\
\hline $95 \%$ & $5.70 \mathrm{E}-03$ & $4.42 \mathrm{E}-05$ & $2.94 \mathrm{E}-09$ & $7.25 \mathrm{E}-03$ & $2.04 \mathrm{E}-04$ & $0.00 \mathrm{E}+00$ & $0.00 \mathrm{E}+00$ & $0.00 \mathrm{E}+00$ & $1.31 \mathrm{E}-02$ \\
\hline Mean & $5.02 \mathrm{E}-03$ & $2.49 \mathrm{E}-05$ & $2.44 \mathrm{E}-10$ & $2.52 \mathrm{E}-03$ & $1.12 \mathrm{E}-04$ & $1.63 \mathrm{E}-05$ & $2.79 \mathrm{E}-04$ & $2.70 \mathrm{E}-07$ & $7.90 \mathrm{E}-03$ \\
\hline Min & 8.35E-13 & $9.50 \mathrm{E}-15$ & $2.27 \mathrm{E}-16$ & $3.40 \mathrm{E}-13$ & $3.68 \mathrm{E}-14$ & $0.00 \mathrm{E}+00$ & $0.00 \mathrm{E}+00$ & $0.00 \mathrm{E}+00$ & $1.23 \mathrm{E}-12$ \\
\hline Max & $5.78 \mathrm{E}-03$ & $1.12 \mathrm{E}-04$ & $4.26 \mathrm{E}-09$ & $2.47 \mathrm{E}-02$ & $2.50 \mathrm{E}-04$ & $1.33 \mathrm{E}-02$ & $1.79 \mathrm{E}-01$ & $1.68 \mathrm{E}-04$ & $1.78 \mathrm{E}-01$ \\
\hline
\end{tabular}

Tables C.43-C.56 show the peak DSR percentiles (mrem/yr per pCi/g) for different exposure pathways for individual radionuclides in the onsite resident scenario. RESRAD (onsite) Version 6.5 was used in the analysis. For C-14, I-129, Np-237, and Tc-99, WD pathways were dominant; for other radionuclides (Am-241, Co-60, Cs-137, Pu-238, Pu-239, Pu-240, Sr-90, U-234, U-235, and U-238), WI pathways were dominant. For Am-241, Pu-238, Pu-239, Pu-240, and Sr-90, plant ingestion was dominant. For C-14, aquatic food ingestion resulted in the most dose. For Co-60, external exposure was dominant. For Cs-137, external exposure, meat and plant ingestion resulted in the most dose. For I-129, Np-237, and Tc-99 water ingestion resulted in the most dose. For U-234, radon inhalation resulted in the most dose. For U-235, external exposure and plant ingestion resulted in the most dose. For U-238, water ingestion, plant ingestion, and external exposure resulted in the most dose. 
TABLE C.43 Peak DSR Percentiles (mrem/yr per pCi/g) for Different Exposure Pathways for Am-241 in Onsite Resident Scenario

\begin{tabular}{|c|c|c|c|c|c|c|c|c|c|c|c|c|}
\hline Percentile & External & $\begin{array}{l}\text { Inhalation } \\
\text { w/o Radon }\end{array}$ & $\begin{array}{c}\text { Plant } \\
\text { Ingestion } \\
(\mathrm{WI})\end{array}$ & $\begin{array}{c}\text { Meat } \\
\text { Ingestion } \\
(\mathrm{WI})\end{array}$ & $\begin{array}{c}\text { Milk } \\
\text { Ingestion } \\
(\mathrm{WI})\end{array}$ & $\begin{array}{c}\text { Soil } \\
\text { Ingestion }\end{array}$ & $\begin{array}{c}\text { Water } \\
\text { Ingestion }\end{array}$ & $\begin{array}{c}\text { Aquatic } \\
\text { Foods }\end{array}$ & $\begin{array}{c}\text { Plant } \\
\text { Ingestion } \\
\text { (WD) }\end{array}$ & $\begin{array}{c}\text { Meat } \\
\text { Ingestion } \\
\text { (WD) }\end{array}$ & $\begin{array}{c}\text { Milk } \\
\text { Ingestion } \\
\text { (WD) }\end{array}$ & $\begin{array}{c}\text { All } \\
\text { Pathways }\end{array}$ \\
\hline $5 \%$ & $4.45 \mathrm{E}-03$ & $6.67 \mathrm{E}-04$ & $1.13 \mathrm{E}-02$ & $3.75 \mathrm{E}-04$ & $9.85 \mathrm{E}-06$ & $2.50 \mathrm{E}-03$ & $0.00 \mathrm{E}+00$ & $0.00 \mathrm{E}+00$ & $0.00 \mathrm{E}+00$ & $0.00 \mathrm{E}+00$ & $0.00 \mathrm{E}+00$ & $2.96 \mathrm{E}-02$ \\
\hline $10 \%$ & $5.00 \mathrm{E}-03$ & $9.09 \mathrm{E}-04$ & $1.59 \mathrm{E}-02$ & 4.14E-04 & $1.28 \mathrm{E}-05$ & $3.66 \mathrm{E}-03$ & $0.00 \mathrm{E}+00$ & $0.00 \mathrm{E}+00$ & $0.00 \mathrm{E}+00$ & $0.00 \mathrm{E}+00$ & $0.00 \mathrm{E}+00$ & $3.61 \mathrm{E}-02$ \\
\hline $15 \%$ & $5.44 \mathrm{E}-03$ & $1.06 \mathrm{E}-03$ & $2.00 \mathrm{E}-02$ & $4.42 \mathrm{E}-04$ & $1.54 \mathrm{E}-05$ & $4.54 \mathrm{E}-03$ & $0.00 \mathrm{E}+00$ & $0.00 \mathrm{E}+00$ & $0.00 \mathrm{E}+00$ & $0.00 \mathrm{E}+00$ & $0.00 \mathrm{E}+00$ & $4.05 \mathrm{E}-02$ \\
\hline $20 \%$ & $5.84 \mathrm{E}-03$ & $1.20 \mathrm{E}-03$ & $2.40 \mathrm{E}-02$ & 4.64E-04 & $1.78 \mathrm{E}-05$ & $5.29 \mathrm{E}-03$ & $0.00 \mathrm{E}+00$ & $0.00 \mathrm{E}+00$ & $0.00 \mathrm{E}+00$ & $0.00 \mathrm{E}+00$ & $0.00 \mathrm{E}+00$ & $4.48 \mathrm{E}-02$ \\
\hline $30 \%$ & $6.53 \mathrm{E}-03$ & $1.53 \mathrm{E}-03$ & $3.24 \mathrm{E}-02$ & $5.04 \mathrm{E}-04$ & $2.24 \mathrm{E}-05$ & $6.55 \mathrm{E}-03$ & $0.00 \mathrm{E}+00$ & $0.00 \mathrm{E}+00$ & $0.00 \mathrm{E}+00$ & $0.00 \mathrm{E}+00$ & $0.00 \mathrm{E}+00$ & 5.33E-02 \\
\hline $35 \%$ & $6.87 \mathrm{E}-03$ & $1.70 \mathrm{E}-03$ & $3.67 \mathrm{E}-02$ & $5.23 \mathrm{E}-04$ & $2.46 \mathrm{E}-05$ & $7.10 \mathrm{E}-03$ & $0.00 \mathrm{E}+00$ & $0.00 \mathrm{E}+00$ & $0.00 \mathrm{E}+00$ & $0.00 \mathrm{E}+00$ & $0.00 \mathrm{E}+00$ & $5.75 \mathrm{E}-02$ \\
\hline $40 \%$ & 7.24E-03 & $1.85 \mathrm{E}-03$ & $4.16 \mathrm{E}-02$ & $5.40 \mathrm{E}-04$ & $2.70 \mathrm{E}-05$ & 7.61E-03 & $0.00 \mathrm{E}+00$ & $0.00 \mathrm{E}+00$ & $0.00 \mathrm{E}+00$ & $0.00 \mathrm{E}+00$ & $0.00 \mathrm{E}+00$ & $6.29 \mathrm{E}-02$ \\
\hline $45 \%$ & $7.61 \mathrm{E}-03$ & $2.01 \mathrm{E}-03$ & $4.68 \mathrm{E}-02$ & $5.55 \mathrm{E}-04$ & $2.96 \mathrm{E}-05$ & $8.10 \mathrm{E}-03$ & $0.00 \mathrm{E}+00$ & $0.00 \mathrm{E}+00$ & $0.00 \mathrm{E}+00$ & $0.00 \mathrm{E}+00$ & $0.00 \mathrm{E}+00$ & $6.81 \mathrm{E}-02$ \\
\hline $50 \%$ & $8.00 \mathrm{E}-03$ & $2.18 \mathrm{E}-03$ & $5.24 \mathrm{E}-02$ & 5.72E-04 & $3.22 \mathrm{E}-05$ & 8.57E-03 & $0.00 \mathrm{E}+00$ & $0.00 \mathrm{E}+00$ & $0.00 \mathrm{E}+00$ & $0.00 \mathrm{E}+00$ & $0.00 \mathrm{E}+00$ & $7.46 \mathrm{E}-02$ \\
\hline $55 \%$ & $8.41 \mathrm{E}-03$ & $2.35 \mathrm{E}-03$ & $5.92 \mathrm{E}-02$ & $5.88 \mathrm{E}-04$ & $3.51 \mathrm{E}-05$ & $9.00 \mathrm{E}-03$ & $0.00 \mathrm{E}+00$ & $0.00 \mathrm{E}+00$ & $0.00 \mathrm{E}+00$ & $0.00 \mathrm{E}+00$ & $0.00 \mathrm{E}+00$ & 8.03E-02 \\
\hline $60 \%$ & $8.86 \mathrm{E}-03$ & $2.55 \mathrm{E}-03$ & $6.64 \mathrm{E}-02$ & $6.06 \mathrm{E}-04$ & $3.88 \mathrm{E}-05$ & $9.47 \mathrm{E}-03$ & $0.00 \mathrm{E}+00$ & $0.00 \mathrm{E}+00$ & $0.00 \mathrm{E}+00$ & $0.00 \mathrm{E}+00$ & $0.00 \mathrm{E}+00$ & $8.78 \mathrm{E}-02$ \\
\hline $65 \%$ & $9.40 \mathrm{E}-03$ & $2.76 \mathrm{E}-03$ & $7.46 \mathrm{E}-02$ & $6.28 \mathrm{E}-04$ & 4.24E-05 & $9.98 \mathrm{E}-03$ & $0.00 \mathrm{E}+00$ & $0.00 \mathrm{E}+00$ & $0.00 \mathrm{E}+00$ & $0.00 \mathrm{E}+00$ & $0.00 \mathrm{E}+00$ & $9.62 \mathrm{E}-02$ \\
\hline $80 \%$ & $1.14 \mathrm{E}-02$ & $3.59 \mathrm{E}-03$ & $1.14 \mathrm{E}-01$ & 7.03E-04 & $5.92 \mathrm{E}-05$ & $1.18 \mathrm{E}-02$ & $0.00 \mathrm{E}+00$ & $0.00 \mathrm{E}+00$ & $0.00 \mathrm{E}+00$ & $0.00 \mathrm{E}+00$ & $0.00 \mathrm{E}+00$ & $1.37 \mathrm{E}-01$ \\
\hline $85 \%$ & $1.25 \mathrm{E}-02$ & $4.08 \mathrm{E}-03$ & $1.38 \mathrm{E}-01$ & 7.44E-04 & $6.79 \mathrm{E}-05$ & $1.25 \mathrm{E}-02$ & $1.95 \mathrm{E}-07$ & 2.20E-09 & 7.47E-09 & $1.23 \mathrm{E}-09$ & $3.58 \mathrm{E}-11$ & $1.61 \mathrm{E}-01$ \\
\hline $90 \%$ & $1.40 \mathrm{E}-02$ & $4.73 \mathrm{E}-03$ & $1.72 \mathrm{E}-01$ & 8.04E-04 & $8.06 \mathrm{E}-05$ & $1.34 \mathrm{E}-02$ & $5.21 \mathrm{E}-06$ & $6.92 \mathrm{E}-08$ & $2.01 \mathrm{E}-07$ & $3.60 \mathrm{E}-08$ & $9.94 \mathrm{E}-10$ & $1.96 \mathrm{E}-01$ \\
\hline $95 \%$ & $1.64 \mathrm{E}-02$ & $5.87 \mathrm{E}-03$ & $2.37 \mathrm{E}-01$ & $8.98 \mathrm{E}-04$ & $1.05 \mathrm{E}-04$ & $1.46 \mathrm{E}-02$ & $6.35 \mathrm{E}-05$ & $7.90 \mathrm{E}-07$ & $2.46 \mathrm{E}-06$ & $4.25 \mathrm{E}-07$ & $1.04 \mathrm{E}-08$ & $2.68 \mathrm{E}-01$ \\
\hline Mean & 8.83E-03 & $2.57 \mathrm{E}-03$ & 8.04E-02 & $5.95 \mathrm{E}-04$ & $4.16 \mathrm{E}-05$ & 8.54E-03 & $4.51 \mathrm{E}-03$ & $1.13 \mathrm{E}-04$ & $1.25 \mathrm{E}-04$ & $1.48 \mathrm{E}-06$ & $1.55 \mathrm{E}-07$ & $1.05 \mathrm{E}-01$ \\
\hline Min & $1.40 \mathrm{E}-11$ & $5.49 \mathrm{E}-12$ & $2.30 \mathrm{E}-10$ & $2.22 \mathrm{E}-12$ & $1.35 \mathrm{E}-13$ & $2.85 \mathrm{E}-11$ & $0.00 \mathrm{E}+00$ & $0.00 \mathrm{E}+00$ & $0.00 \mathrm{E}+00$ & $0.00 \mathrm{E}+00$ & $0.00 \mathrm{E}+00$ & $2.81 \mathrm{E}-10$ \\
\hline Max & $2.37 \mathrm{E}-02$ & $1.96 \mathrm{E}-02$ & $2.19 \mathrm{E}+00$ & $2.34 \mathrm{E}-03$ & 4.19E-04 & $1.97 \mathrm{E}-02$ & $4.68 \mathrm{E}+00$ & $1.18 \mathrm{E}-01$ & $9.48 \mathrm{E}-02$ & $1.08 \mathrm{E}-03$ & $1.04 \mathrm{E}-04$ & $4.89 \mathrm{E}+00$ \\
\hline
\end{tabular}


TABLE C.44 Peak DSR Percentiles (mrem/yr per pCi/g) for Different Exposure Pathways for C-14 in Onsite Resident Scenario

\begin{tabular}{|c|c|c|c|c|c|c|c|c|c|c|c|c|}
\hline Percentile & External & $\begin{array}{l}\text { Inhalation } \\
\text { w/o Radon }\end{array}$ & $\begin{array}{c}\text { Plant } \\
\text { Ingestion } \\
(\mathrm{WI})\end{array}$ & $\begin{array}{c}\text { Meat } \\
\text { Ingestion } \\
(\mathrm{WI})\end{array}$ & $\begin{array}{c}\text { Milk } \\
\text { Ingestion } \\
(\mathrm{WI})\end{array}$ & $\begin{array}{c}\text { Soil } \\
\text { Ingestion }\end{array}$ & $\begin{array}{c}\text { Water } \\
\text { Ingestion }\end{array}$ & $\begin{array}{c}\text { Aquatic } \\
\text { Foods }\end{array}$ & $\begin{array}{c}\text { Plant } \\
\text { Ingestion } \\
\text { (WD) }\end{array}$ & $\begin{array}{c}\text { Meat } \\
\text { Ingestion } \\
\text { (WD) }\end{array}$ & $\begin{array}{c}\text { Milk } \\
\text { Ingestion } \\
\text { (WD) }\end{array}$ & $\begin{array}{c}\text { All } \\
\text { Pathways }\end{array}$ \\
\hline $5 \%$ & $0.00 \mathrm{E}+00$ & $0.00 \mathrm{E}+00$ & $0.00 \mathrm{E}+00$ & $0.00 \mathrm{E}+00$ & $0.00 \mathrm{E}+00$ & $0.00 \mathrm{E}+00$ & $0.00 \mathrm{E}+00$ & $0.00 \mathrm{E}+00$ & $0.00 \mathrm{E}+00$ & $0.00 \mathrm{E}+00$ & $0.00 \mathrm{E}+00$ & $0.00 \mathrm{E}+00$ \\
\hline $10 \%$ & $0.00 \mathrm{E}+00$ & $0.00 \mathrm{E}+00$ & $0.00 \mathrm{E}+00$ & $0.00 \mathrm{E}+00$ & $0.00 \mathrm{E}+00$ & $0.00 \mathrm{E}+00$ & $0.00 \mathrm{E}+00$ & $0.00 \mathrm{E}+00$ & $0.00 \mathrm{E}+00$ & $0.00 \mathrm{E}+00$ & $0.00 \mathrm{E}+00$ & $0.00 \mathrm{E}+00$ \\
\hline $15 \%$ & $0.00 \mathrm{E}+00$ & $0.00 \mathrm{E}+00$ & $0.00 \mathrm{E}+00$ & $0.00 \mathrm{E}+00$ & $0.00 \mathrm{E}+00$ & $0.00 \mathrm{E}+00$ & $0.00 \mathrm{E}+00$ & $0.00 \mathrm{E}+00$ & $0.00 \mathrm{E}+00$ & $0.00 \mathrm{E}+00$ & $0.00 \mathrm{E}+00$ & $0.00 \mathrm{E}+00$ \\
\hline $20 \%$ & $0.00 \mathrm{E}+00$ & $0.00 \mathrm{E}+00$ & $0.00 \mathrm{E}+00$ & $0.00 \mathrm{E}+00$ & $0.00 \mathrm{E}+00$ & $0.00 \mathrm{E}+00$ & $0.00 \mathrm{E}+00$ & $0.00 \mathrm{E}+00$ & $0.00 \mathrm{E}+00$ & $0.00 \mathrm{E}+00$ & $0.00 \mathrm{E}+00$ & $0.00 \mathrm{E}+00$ \\
\hline $25 \%$ & $0.00 \mathrm{E}+00$ & $0.00 \mathrm{E}+00$ & $0.00 \mathrm{E}+00$ & $0.00 \mathrm{E}+00$ & $0.00 \mathrm{E}+00$ & $0.00 \mathrm{E}+00$ & $0.00 \mathrm{E}+00$ & $0.00 \mathrm{E}+00$ & $0.00 \mathrm{E}+00$ & $0.00 \mathrm{E}+00$ & $0.00 \mathrm{E}+00$ & $0.00 \mathrm{E}+00$ \\
\hline $30 \%$ & $0.00 \mathrm{E}+00$ & $0.00 \mathrm{E}+00$ & $0.00 \mathrm{E}+00$ & $0.00 \mathrm{E}+00$ & $0.00 \mathrm{E}+00$ & $0.00 \mathrm{E}+00$ & $0.00 \mathrm{E}+00$ & $0.00 \mathrm{E}+00$ & $0.00 \mathrm{E}+00$ & $0.00 \mathrm{E}+00$ & $0.00 \mathrm{E}+00$ & $0.00 \mathrm{E}+00$ \\
\hline $35 \%$ & $0.00 \mathrm{E}+00$ & $0.00 \mathrm{E}+00$ & $0.00 \mathrm{E}+00$ & $0.00 \mathrm{E}+00$ & $0.00 \mathrm{E}+00$ & $0.00 \mathrm{E}+00$ & $0.00 \mathrm{E}+00$ & $0.00 \mathrm{E}+00$ & $0.00 \mathrm{E}+00$ & $0.00 \mathrm{E}+00$ & $0.00 \mathrm{E}+00$ & $0.00 \mathrm{E}+00$ \\
\hline $40 \%$ & $0.00 \mathrm{E}+00$ & $0.00 \mathrm{E}+00$ & $0.00 \mathrm{E}+00$ & $0.00 \mathrm{E}+00$ & $0.00 \mathrm{E}+00$ & $0.00 \mathrm{E}+00$ & $0.00 \mathrm{E}+00$ & $0.00 \mathrm{E}+00$ & $0.00 \mathrm{E}+00$ & $0.00 \mathrm{E}+00$ & $0.00 \mathrm{E}+00$ & $0.00 \mathrm{E}+00$ \\
\hline $45 \%$ & $0.00 \mathrm{E}+00$ & $0.00 \mathrm{E}+00$ & $0.00 \mathrm{E}+00$ & $0.00 \mathrm{E}+00$ & $0.00 \mathrm{E}+00$ & $0.00 \mathrm{E}+00$ & $0.00 \mathrm{E}+00$ & $0.00 \mathrm{E}+00$ & $0.00 \mathrm{E}+00$ & $0.00 \mathrm{E}+00$ & $0.00 \mathrm{E}+00$ & $0.00 \mathrm{E}+00$ \\
\hline $50 \%$ & $0.00 \mathrm{E}+00$ & $0.00 \mathrm{E}+00$ & $0.00 \mathrm{E}+00$ & $0.00 \mathrm{E}+00$ & $0.00 \mathrm{E}+00$ & $0.00 \mathrm{E}+00$ & $0.00 \mathrm{E}+00$ & $0.00 \mathrm{E}+00$ & $0.00 \mathrm{E}+00$ & $0.00 \mathrm{E}+00$ & $0.00 \mathrm{E}+00$ & $0.00 \mathrm{E}+00$ \\
\hline $55 \%$ & $0.00 \mathrm{E}+00$ & $0.00 \mathrm{E}+00$ & $0.00 \mathrm{E}+00$ & $0.00 \mathrm{E}+00$ & $0.00 \mathrm{E}+00$ & $0.00 \mathrm{E}+00$ & $0.00 \mathrm{E}+00$ & $0.00 \mathrm{E}+00$ & $0.00 \mathrm{E}+00$ & $0.00 \mathrm{E}+00$ & $0.00 \mathrm{E}+00$ & $0.00 \mathrm{E}+00$ \\
\hline $60 \%$ & $0.00 \mathrm{E}+00$ & $0.00 \mathrm{E}+00$ & $0.00 \mathrm{E}+00$ & $0.00 \mathrm{E}+00$ & $0.00 \mathrm{E}+00$ & $0.00 \mathrm{E}+00$ & $0.00 \mathrm{E}+00$ & $0.00 \mathrm{E}+00$ & $0.00 \mathrm{E}+00$ & $0.00 \mathrm{E}+00$ & $0.00 \mathrm{E}+00$ & $0.00 \mathrm{E}+00$ \\
\hline $65 \%$ & $0.00 \mathrm{E}+00$ & $0.00 \mathrm{E}+00$ & $0.00 \mathrm{E}+00$ & $0.00 \mathrm{E}+00$ & $0.00 \mathrm{E}+00$ & $0.00 \mathrm{E}+00$ & $0.00 \mathrm{E}+00$ & $0.00 \mathrm{E}+00$ & $0.00 \mathrm{E}+00$ & $0.00 \mathrm{E}+00$ & $0.00 \mathrm{E}+00$ & $0.00 \mathrm{E}+00$ \\
\hline $70 \%$ & $0.00 \mathrm{E}+00$ & $0.00 \mathrm{E}+00$ & $0.00 \mathrm{E}+00$ & $0.00 \mathrm{E}+00$ & $0.00 \mathrm{E}+00$ & $0.00 \mathrm{E}+00$ & $0.00 \mathrm{E}+00$ & $0.00 \mathrm{E}+00$ & $0.00 \mathrm{E}+00$ & $0.00 \mathrm{E}+00$ & $0.00 \mathrm{E}+00$ & $0.00 \mathrm{E}+00$ \\
\hline $75 \%$ & $0.00 \mathrm{E}+00$ & $0.00 \mathrm{E}+00$ & $0.00 \mathrm{E}+00$ & $0.00 \mathrm{E}+00$ & $0.00 \mathrm{E}+00$ & $0.00 \mathrm{E}+00$ & $0.00 \mathrm{E}+00$ & $0.00 \mathrm{E}+00$ & $0.00 \mathrm{E}+00$ & $0.00 \mathrm{E}+00$ & $0.00 \mathrm{E}+00$ & $0.00 \mathrm{E}+00$ \\
\hline $80 \%$ & $0.00 \mathrm{E}+00$ & $0.00 \mathrm{E}+00$ & $0.00 \mathrm{E}+00$ & $0.00 \mathrm{E}+00$ & $0.00 \mathrm{E}+00$ & $0.00 \mathrm{E}+00$ & $0.00 \mathrm{E}+00$ & $0.00 \mathrm{E}+00$ & $0.00 \mathrm{E}+00$ & $0.00 \mathrm{E}+00$ & $0.00 \mathrm{E}+00$ & $0.00 \mathrm{E}+00$ \\
\hline $85 \%$ & $0.00 \mathrm{E}+00$ & $0.00 \mathrm{E}+00$ & $0.00 \mathrm{E}+00$ & $0.00 \mathrm{E}+00$ & $0.00 \mathrm{E}+00$ & $0.00 \mathrm{E}+00$ & $1.77 \mathrm{E}-04$ & $5.95 \mathrm{E}-04$ & $1.94 \mathrm{E}-05$ & $8.11 \mathrm{E}-06$ & $2.26 \mathrm{E}-05$ & $1.01 \mathrm{E}-03$ \\
\hline $90 \%$ & $0.00 \mathrm{E}+00$ & $0.00 \mathrm{E}+00$ & $0.00 \mathrm{E}+00$ & $0.00 \mathrm{E}+00$ & $0.00 \mathrm{E}+00$ & $0.00 \mathrm{E}+00$ & $5.70 \mathrm{E}-04$ & $2.46 \mathrm{E}-03$ & $6.22 \mathrm{E}-05$ & $2.52 \mathrm{E}-05$ & $7.21 \mathrm{E}-05$ & $3.45 \mathrm{E}-03$ \\
\hline $95 \%$ & $0.00 \mathrm{E}+00$ & $0.00 \mathrm{E}+00$ & $0.00 \mathrm{E}+00$ & $0.00 \mathrm{E}+00$ & $0.00 \mathrm{E}+00$ & $0.00 \mathrm{E}+00$ & $1.21 \mathrm{E}-03$ & $7.98 \mathrm{E}-03$ & $1.35 \mathrm{E}-04$ & $5.33 \mathrm{E}-05$ & $1.51 \mathrm{E}-04$ & $1.05 \mathrm{E}-02$ \\
\hline Mean & $7.48 \mathrm{E}-10$ & $5.07 \mathrm{E}-08$ & $3.01 \mathrm{E}-04$ & $1.17 \mathrm{E}-04$ & $4.00 \mathrm{E}-05$ & $5.17 \mathrm{E}-09$ & $1.91 \mathrm{E}-04$ & $3.11 \mathrm{E}-03$ & $2.19 \mathrm{E}-05$ & $8.52 \mathrm{E}-06$ & $2.40 \mathrm{E}-05$ & $3.36 \mathrm{E}-03$ \\
\hline Min & $0.00 \mathrm{E}+00$ & $0.00 \mathrm{E}+00$ & $0.00 \mathrm{E}+00$ & $0.00 \mathrm{E}+00$ & $0.00 \mathrm{E}+00$ & $0.00 \mathrm{E}+00$ & $0.00 \mathrm{E}+00$ & $0.00 \mathrm{E}+00$ & $0.00 \mathrm{E}+00$ & $0.00 \mathrm{E}+00$ & $0.00 \mathrm{E}+00$ & $0.00 \mathrm{E}+00$ \\
\hline $\operatorname{Max}$ & $1.02 \mathrm{E}-06$ & 5.13E-05 & $3.02 \mathrm{E}-01$ & $1.17 \mathrm{E}-01$ & 4.01E-02 & 7.72E-06 & $1.88 \mathrm{E}-02$ & $3.00 \mathrm{E}+00$ & $1.78 \mathrm{E}-03$ & 7.99E-04 & $2.35 \mathrm{E}-03$ & $3.00 \mathrm{E}+00$ \\
\hline
\end{tabular}


TABLE C.45 Peak DSR Percentiles (mrem/yr per pCi/g) for Different Exposure Pathways for Co-60 in Onsite Resident Scenario

\begin{tabular}{|c|c|c|c|c|c|c|c|c|c|c|c|c|}
\hline Percentile & External & $\begin{array}{c}\text { Inhalation } \\
\text { w/o Radon }\end{array}$ & $\begin{array}{c}\text { Plant } \\
\text { Ingestion } \\
(\mathrm{WI})\end{array}$ & $\begin{array}{c}\text { Meat } \\
\text { Ingestion } \\
(\mathrm{WI})\end{array}$ & $\begin{array}{c}\text { Milk } \\
\text { Ingestion } \\
(\mathrm{WI})\end{array}$ & $\begin{array}{c}\text { Soil } \\
\text { Ingestion } \\
\end{array}$ & $\begin{array}{c}\text { Water } \\
\text { Ingestion }\end{array}$ & $\begin{array}{c}\text { Aquatic } \\
\text { Foods }\end{array}$ & $\begin{array}{c}\text { Plant } \\
\text { Ingestion } \\
\text { (WD) }\end{array}$ & $\begin{array}{c}\text { Meat } \\
\text { Ingestion } \\
\text { (WD) }\end{array}$ & $\begin{array}{c}\text { Milk } \\
\text { Ingestion } \\
\text { (WD) }\end{array}$ & $\begin{array}{c}\text { All } \\
\text { Pathways }\end{array}$ \\
\hline $5 \%$ & 2.99E-06 & $3.47 \mathrm{E}-13$ & $2.60 \mathrm{E}-08$ & $1.20 \mathrm{E}-08$ & $1.45 \mathrm{E}-09$ & $6.98 \mathrm{E}-11$ & $0.00 \mathrm{E}+00$ & $0.00 \mathrm{E}+00$ & $0.00 \mathrm{E}+00$ & $0.00 \mathrm{E}+00$ & $0.00 \mathrm{E}+00$ & $3.32 \mathrm{E}-06$ \\
\hline $10 \%$ & $3.78 \mathrm{E}-06$ & $4.79 \mathrm{E}-13$ & $3.94 \mathrm{E}-08$ & $2.08 \mathrm{E}-08$ & $2.27 \mathrm{E}-09$ & $1.11 \mathrm{E}-10$ & $0.00 \mathrm{E}+00$ & $0.00 \mathrm{E}+00$ & $0.00 \mathrm{E}+00$ & $0.00 \mathrm{E}+00$ & $0.00 \mathrm{E}+00$ & $4.10 \mathrm{E}-06$ \\
\hline $15 \%$ & $4.20 \mathrm{E}-06$ & $5.68 \mathrm{E}-13$ & $5.14 \mathrm{E}-08$ & $2.85 \mathrm{E}-08$ & 2.97E-09 & $1.42 \mathrm{E}-10$ & $0.00 \mathrm{E}+00$ & $0.00 \mathrm{E}+00$ & $0.00 \mathrm{E}+00$ & $0.00 \mathrm{E}+00$ & $0.00 \mathrm{E}+00$ & 4.61E-06 \\
\hline $20 \%$ & $4.53 \mathrm{E}-06$ & $6.53 \mathrm{E}-13$ & $6.27 \mathrm{E}-08$ & $3.60 \mathrm{E}-08$ & $3.67 \mathrm{E}-09$ & $1.69 \mathrm{E}-10$ & $0.00 \mathrm{E}+00$ & $0.00 \mathrm{E}+00$ & $0.00 \mathrm{E}+00$ & $0.00 \mathrm{E}+00$ & $0.00 \mathrm{E}+00$ & $4.97 \mathrm{E}-06$ \\
\hline $25 \%$ & $4.85 \mathrm{E}-06$ & $7.24 \mathrm{E}-13$ & 7.44E-08 & 4.49E-08 & 4.42E-09 & $1.94 \mathrm{E}-10$ & $0.00 \mathrm{E}+00$ & $0.00 \mathrm{E}+00$ & $0.00 \mathrm{E}+00$ & $0.00 \mathrm{E}+00$ & $0.00 \mathrm{E}+00$ & $5.28 \mathrm{E}-06$ \\
\hline $30 \%$ & $5.16 \mathrm{E}-06$ & $8.07 \mathrm{E}-13$ & $8.55 \mathrm{E}-08$ & $5.52 \mathrm{E}-08$ & $5.28 \mathrm{E}-09$ & $2.15 \mathrm{E}-10$ & $0.00 \mathrm{E}+00$ & $0.00 \mathrm{E}+00$ & $0.00 \mathrm{E}+00$ & $0.00 \mathrm{E}+00$ & $0.00 \mathrm{E}+00$ & $5.59 \mathrm{E}-06$ \\
\hline $35 \%$ & $5.44 \mathrm{E}-06$ & $8.86 \mathrm{E}-13$ & $9.74 \mathrm{E}-08$ & $6.67 \mathrm{E}-08$ & $6.18 \mathrm{E}-09$ & $2.34 \mathrm{E}-10$ & $0.00 \mathrm{E}+00$ & $0.00 \mathrm{E}+00$ & $0.00 \mathrm{E}+00$ & $0.00 \mathrm{E}+00$ & $0.00 \mathrm{E}+00$ & $5.93 \mathrm{E}-06$ \\
\hline $40 \%$ & $5.74 \mathrm{E}-06$ & $9.57 \mathrm{E}-13$ & $1.11 \mathrm{E}-07$ & $7.95 \mathrm{E}-08$ & $7.20 \mathrm{E}-09$ & $2.52 \mathrm{E}-10$ & $0.00 \mathrm{E}+00$ & $0.00 \mathrm{E}+00$ & $0.00 \mathrm{E}+00$ & $0.00 \mathrm{E}+00$ & $0.00 \mathrm{E}+00$ & $6.25 \mathrm{E}-06$ \\
\hline $45 \%$ & $6.04 \mathrm{E}-06$ & $1.04 \mathrm{E}-12$ & $1.26 \mathrm{E}-07$ & $9.49 \mathrm{E}-08$ & $8.24 \mathrm{E}-09$ & $2.69 \mathrm{E}-10$ & $0.00 \mathrm{E}+00$ & $0.00 \mathrm{E}+00$ & $0.00 \mathrm{E}+00$ & $0.00 \mathrm{E}+00$ & $0.00 \mathrm{E}+00$ & 6.61E-06 \\
\hline $50 \%$ & $6.39 \mathrm{E}-06$ & $1.13 \mathrm{E}-12$ & $1.41 \mathrm{E}-07$ & $1.13 \mathrm{E}-07$ & $9.44 \mathrm{E}-09$ & $2.88 \mathrm{E}-10$ & $0.00 \mathrm{E}+00$ & $0.00 \mathrm{E}+00$ & $0.00 \mathrm{E}+00$ & $0.00 \mathrm{E}+00$ & $0.00 \mathrm{E}+00$ & $6.94 \mathrm{E}-06$ \\
\hline $55 \%$ & $6.75 \mathrm{E}-06$ & $1.23 \mathrm{E}-12$ & $1.59 \mathrm{E}-07$ & $1.34 \mathrm{E}-07$ & $1.08 \mathrm{E}-08$ & $3.05 \mathrm{E}-10$ & $0.00 \mathrm{E}+00$ & $0.00 \mathrm{E}+00$ & $0.00 \mathrm{E}+00$ & $0.00 \mathrm{E}+00$ & $0.00 \mathrm{E}+00$ & $7.32 \mathrm{E}-06$ \\
\hline $60 \%$ & $7.15 \mathrm{E}-06$ & $1.32 \mathrm{E}-12$ & $1.79 \mathrm{E}-07$ & $1.61 \mathrm{E}-07$ & $1.23 \mathrm{E}-08$ & $3.21 \mathrm{E}-10$ & $0.00 \mathrm{E}+00$ & $0.00 \mathrm{E}+00$ & $0.00 \mathrm{E}+00$ & $0.00 \mathrm{E}+00$ & $0.00 \mathrm{E}+00$ & $7.72 \mathrm{E}-06$ \\
\hline $65 \%$ & $7.56 \mathrm{E}-06$ & $1.43 \mathrm{E}-12$ & $2.03 \mathrm{E}-07$ & $1.94 \mathrm{E}-07$ & $1.44 \mathrm{E}-08$ & $3.41 \mathrm{E}-10$ & $0.00 \mathrm{E}+00$ & $0.00 \mathrm{E}+00$ & $0.00 \mathrm{E}+00$ & $0.00 \mathrm{E}+00$ & $0.00 \mathrm{E}+00$ & $8.20 \mathrm{E}-06$ \\
\hline $70 \%$ & 8.08E-06 & $1.54 \mathrm{E}-12$ & $2.33 \mathrm{E}-07$ & $2.34 \mathrm{E}-07$ & $1.67 \mathrm{E}-08$ & $3.60 \mathrm{E}-10$ & $0.00 \mathrm{E}+00$ & $0.00 \mathrm{E}+00$ & $0.00 \mathrm{E}+00$ & $0.00 \mathrm{E}+00$ & $0.00 \mathrm{E}+00$ & $8.66 \mathrm{E}-06$ \\
\hline $75 \%$ & $8.63 \mathrm{E}-06$ & $1.67 \mathrm{E}-12$ & $2.69 \mathrm{E}-07$ & $2.94 \mathrm{E}-07$ & $1.96 \mathrm{E}-08$ & $3.83 \mathrm{E}-10$ & $0.00 \mathrm{E}+00$ & $0.00 \mathrm{E}+00$ & $0.00 \mathrm{E}+00$ & $0.00 \mathrm{E}+00$ & $0.00 \mathrm{E}+00$ & $9.17 \mathrm{E}-06$ \\
\hline $80 \%$ & $9.30 \mathrm{E}-06$ & $1.82 \mathrm{E}-12$ & $3.09 \mathrm{E}-07$ & $3.69 \mathrm{E}-07$ & $2.39 \mathrm{E}-08$ & $4.08 \mathrm{E}-10$ & $0.00 \mathrm{E}+00$ & $0.00 \mathrm{E}+00$ & $0.00 \mathrm{E}+00$ & $0.00 \mathrm{E}+00$ & $0.00 \mathrm{E}+00$ & $9.88 \mathrm{E}-06$ \\
\hline $85 \%$ & $1.01 \mathrm{E}-05$ & $1.98 \mathrm{E}-12$ & $3.75 \mathrm{E}-07$ & $4.84 \mathrm{E}-07$ & $2.91 \mathrm{E}-08$ & $4.36 \mathrm{E}-10$ & $0.00 \mathrm{E}+00$ & $0.00 \mathrm{E}+00$ & $0.00 \mathrm{E}+00$ & $0.00 \mathrm{E}+00$ & $0.00 \mathrm{E}+00$ & $1.08 \mathrm{E}-05$ \\
\hline $90 \%$ & $1.13 \mathrm{E}-05$ & $2.21 \mathrm{E}-12$ & 4.69E-07 & $6.67 \mathrm{E}-07$ & $3.79 \mathrm{E}-08$ & $4.66 \mathrm{E}-10$ & $0.00 \mathrm{E}+00$ & $0.00 \mathrm{E}+00$ & $0.00 \mathrm{E}+00$ & $0.00 \mathrm{E}+00$ & $0.00 \mathrm{E}+00$ & $1.19 \mathrm{E}-05$ \\
\hline $95 \%$ & $1.33 \mathrm{E}-05$ & $2.63 \mathrm{E}-12$ & $6.49 \mathrm{E}-07$ & $1.09 \mathrm{E}-06$ & $5.87 \mathrm{E}-08$ & $5.11 \mathrm{E}-10$ & $0.00 \mathrm{E}+00$ & $0.00 \mathrm{E}+00$ & $0.00 \mathrm{E}+00$ & $0.00 \mathrm{E}+00$ & $0.00 \mathrm{E}+00$ & $1.40 \mathrm{E}-05$ \\
\hline Mean & 7.02E-06 & $1.27 \mathrm{E}-12$ & $2.17 \mathrm{E}-07$ & $2.83 \mathrm{E}-07$ & $1.75 \mathrm{E}-08$ & $2.89 \mathrm{E}-10$ & $2.78 \mathrm{E}-13$ & $7.84 \mathrm{E}-15$ & $1.07 \mathrm{E}-14$ & 4.07E-13 & $5.55 \mathrm{E}-15$ & $7.54 \mathrm{E}-06$ \\
\hline Min & $3.95 \mathrm{E}-12$ & $1.25 \mathrm{E}-18$ & $7.19 \mathrm{E}-14$ & $3.91 \mathrm{E}-14$ & $1.02 \mathrm{E}-14$ & $1.76 \mathrm{E}-16$ & $0.00 \mathrm{E}+00$ & $0.00 \mathrm{E}+00$ & $0.00 \mathrm{E}+00$ & $0.00 \mathrm{E}+00$ & $0.00 \mathrm{E}+00$ & $4.07 \mathrm{E}-12$ \\
\hline Max & $2.01 \mathrm{E}-05$ & $6.50 \mathrm{E}-12$ & $3.12 \mathrm{E}-06$ & $1.07 \mathrm{E}-05$ & $8.51 \mathrm{E}-07$ & $6.32 \mathrm{E}-10$ & $1.25 \mathrm{E}-09$ & $3.53 \mathrm{E}-11$ & $4.82 \mathrm{E}-11$ & $1.83 \mathrm{E}-09$ & $2.50 \mathrm{E}-11$ & $2.20 \mathrm{E}-05$ \\
\hline
\end{tabular}


TABLE C.46 Peak DSR Percentiles (mrem/yr per pCi/g) for Different Exposure Pathways for Cs-137 in Onsite Resident Scenario

\begin{tabular}{|c|c|c|c|c|c|c|c|c|c|c|c|c|}
\hline Percentile & External & $\begin{array}{l}\text { Inhalation } \\
\text { w/o Radon }\end{array}$ & $\begin{array}{c}\text { Plant } \\
\text { Ingestion } \\
\text { (WI) }\end{array}$ & $\begin{array}{c}\text { Meat } \\
\text { Ingestion } \\
(\mathrm{WI})\end{array}$ & $\begin{array}{c}\text { Milk } \\
\text { Ingestion } \\
(\mathrm{WI})\end{array}$ & $\begin{array}{c}\text { Soil } \\
\text { Ingestion } \\
\end{array}$ & $\begin{array}{c}\text { Water } \\
\text { Ingestion }\end{array}$ & $\begin{array}{c}\text { Aquatic } \\
\text { Foods }\end{array}$ & $\begin{array}{c}\text { Plant } \\
\text { Ingestion } \\
\text { (WD) }\end{array}$ & $\begin{array}{c}\text { Meat } \\
\text { Ingestion } \\
\text { (WD) }\end{array}$ & $\begin{array}{c}\text { Milk } \\
\text { Ingestion } \\
\text { (WD) }\end{array}$ & $\begin{array}{c}\text { All } \\
\text { Pathways }\end{array}$ \\
\hline $5 \%$ & $4.31 \mathrm{E}-02$ & $3.22 \mathrm{E}-08$ & $3.03 \mathrm{E}-03$ & $6.08 \mathrm{E}-03$ & $1.47 \mathrm{E}-03$ & $2.00 \mathrm{E}-05$ & $0.00 \mathrm{E}+00$ & $0.00 \mathrm{E}+00$ & $0.00 \mathrm{E}+00$ & $0.00 \mathrm{E}+00$ & $0.00 \mathrm{E}+00$ & 7.02E-02 \\
\hline $10 \%$ & $4.80 \mathrm{E}-02$ & $4.36 \mathrm{E}-08$ & $4.37 \mathrm{E}-03$ & $7.97 \mathrm{E}-03$ & $1.93 \mathrm{E}-03$ & $2.81 \mathrm{E}-05$ & $0.00 \mathrm{E}+00$ & $0.00 \mathrm{E}+00$ & $0.00 \mathrm{E}+00$ & $0.00 \mathrm{E}+00$ & $0.00 \mathrm{E}+00$ & $7.93 \mathrm{E}-02$ \\
\hline $15 \%$ & $5.20 \mathrm{E}-02$ & $5.04 \mathrm{E}-08$ & $5.60 \mathrm{E}-03$ & $9.57 \mathrm{E}-03$ & $2.37 \mathrm{E}-03$ & $3.44 \mathrm{E}-05$ & $0.00 \mathrm{E}+00$ & $0.00 \mathrm{E}+00$ & $0.00 \mathrm{E}+00$ & $0.00 \mathrm{E}+00$ & $0.00 \mathrm{E}+00$ & $8.78 \mathrm{E}-02$ \\
\hline $20 \%$ & $5.55 \mathrm{E}-02$ & $5.70 \mathrm{E}-08$ & $6.83 \mathrm{E}-03$ & $1.12 \mathrm{E}-02$ & $2.76 \mathrm{E}-03$ & $3.98 \mathrm{E}-05$ & $0.00 \mathrm{E}+00$ & $0.00 \mathrm{E}+00$ & $0.00 \mathrm{E}+00$ & $0.00 \mathrm{E}+00$ & $0.00 \mathrm{E}+00$ & $9.41 \mathrm{E}-02$ \\
\hline $25 \%$ & $5.90 \mathrm{E}-02$ & $6.43 \mathrm{E}-08$ & 8.07E-03 & $1.29 \mathrm{E}-02$ & $3.15 \mathrm{E}-03$ & $4.46 \mathrm{E}-05$ & $0.00 \mathrm{E}+00$ & $0.00 \mathrm{E}+00$ & $0.00 \mathrm{E}+00$ & $0.00 \mathrm{E}+00$ & $0.00 \mathrm{E}+00$ & $1.00 \mathrm{E}-01$ \\
\hline $30 \%$ & $6.23 \mathrm{E}-02$ & $7.29 \mathrm{E}-08$ & $9.35 \mathrm{E}-03$ & $1.45 \mathrm{E}-02$ & $3.61 \mathrm{E}-03$ & $4.89 \mathrm{E}-05$ & $0.00 \mathrm{E}+00$ & $0.00 \mathrm{E}+00$ & $0.00 \mathrm{E}+00$ & $0.00 \mathrm{E}+00$ & $0.00 \mathrm{E}+00$ & $1.07 \mathrm{E}-01$ \\
\hline $35 \%$ & $6.55 \mathrm{E}-02$ & 8.04E-08 & $1.07 \mathrm{E}-02$ & $1.65 \mathrm{E}-02$ & 4.07E-03 & 5.31E-05 & $0.00 \mathrm{E}+00$ & $0.00 \mathrm{E}+00$ & $0.00 \mathrm{E}+00$ & $0.00 \mathrm{E}+00$ & $0.00 \mathrm{E}+00$ & $1.14 \mathrm{E}-01$ \\
\hline $40 \%$ & $6.89 \mathrm{E}-02$ & $8.63 \mathrm{E}-08$ & $1.22 \mathrm{E}-02$ & $1.84 \mathrm{E}-02$ & $4.60 \mathrm{E}-03$ & $5.67 \mathrm{E}-05$ & $0.00 \mathrm{E}+00$ & $0.00 \mathrm{E}+00$ & $0.00 \mathrm{E}+00$ & $0.00 \mathrm{E}+00$ & $0.00 \mathrm{E}+00$ & $1.21 \mathrm{E}-01$ \\
\hline $45 \%$ & $7.22 \mathrm{E}-02$ & $9.40 \mathrm{E}-08$ & $1.40 \mathrm{E}-02$ & $2.07 \mathrm{E}-02$ & $5.12 \mathrm{E}-03$ & $6.05 \mathrm{E}-05$ & $0.00 \mathrm{E}+00$ & $0.00 \mathrm{E}+00$ & $0.00 \mathrm{E}+00$ & $0.00 \mathrm{E}+00$ & $0.00 \mathrm{E}+00$ & $1.28 \mathrm{E}-01$ \\
\hline $50 \%$ & 7.64E-02 & $1.02 \mathrm{E}-07$ & $1.58 \mathrm{E}-02$ & $2.33 \mathrm{E}-02$ & $5.79 \mathrm{E}-03$ & $6.37 \mathrm{E}-05$ & $0.00 \mathrm{E}+00$ & $0.00 \mathrm{E}+00$ & $0.00 \mathrm{E}+00$ & $0.00 \mathrm{E}+00$ & $0.00 \mathrm{E}+00$ & $1.36 \mathrm{E}-01$ \\
\hline $55 \%$ & $8.02 \mathrm{E}-02$ & $1.10 \mathrm{E}-07$ & $1.79 \mathrm{E}-02$ & $2.62 \mathrm{E}-02$ & $6.49 \mathrm{E}-03$ & $6.73 \mathrm{E}-05$ & $0.00 \mathrm{E}+00$ & $0.00 \mathrm{E}+00$ & $0.00 \mathrm{E}+00$ & $0.00 \mathrm{E}+00$ & $0.00 \mathrm{E}+00$ & $1.43 \mathrm{E}-01$ \\
\hline $60 \%$ & $8.46 \mathrm{E}-02$ & $1.19 \mathrm{E}-07$ & $2.03 \mathrm{E}-02$ & $2.94 \mathrm{E}-02$ & 7.34E-03 & $7.06 \mathrm{E}-05$ & $0.00 \mathrm{E}+00$ & $0.00 \mathrm{E}+00$ & $0.00 \mathrm{E}+00$ & $0.00 \mathrm{E}+00$ & $0.00 \mathrm{E}+00$ & $1.52 \mathrm{E}-01$ \\
\hline $65 \%$ & $8.95 \mathrm{E}-02$ & $1.29 \mathrm{E}-07$ & $2.32 \mathrm{E}-02$ & $3.39 \mathrm{E}-02$ & 8.31E-03 & 7.47E-05 & $0.00 \mathrm{E}+00$ & $0.00 \mathrm{E}+00$ & $0.00 \mathrm{E}+00$ & $0.00 \mathrm{E}+00$ & $0.00 \mathrm{E}+00$ & $1.61 \mathrm{E}-01$ \\
\hline $70 \%$ & $9.48 \mathrm{E}-02$ & $1.39 \mathrm{E}-07$ & $2.68 \mathrm{E}-02$ & $3.83 \mathrm{E}-02$ & $9.43 \mathrm{E}-03$ & 7.84E-05 & $0.00 \mathrm{E}+00$ & $0.00 \mathrm{E}+00$ & $0.00 \mathrm{E}+00$ & $0.00 \mathrm{E}+00$ & $0.00 \mathrm{E}+00$ & $1.71 \mathrm{E}-01$ \\
\hline $75 \%$ & $1.01 \mathrm{E}-01$ & $1.51 \mathrm{E}-07$ & $3.11 \mathrm{E}-02$ & $4.40 \mathrm{E}-02$ & $1.09 \mathrm{E}-02$ & $8.29 \mathrm{E}-05$ & $0.00 \mathrm{E}+00$ & $0.00 \mathrm{E}+00$ & $0.00 \mathrm{E}+00$ & $0.00 \mathrm{E}+00$ & $0.00 \mathrm{E}+00$ & $1.83 \mathrm{E}-01$ \\
\hline $80 \%$ & $1.09 \mathrm{E}-01$ & $1.66 \mathrm{E}-07$ & $3.66 \mathrm{E}-02$ & $5.17 \mathrm{E}-02$ & $1.31 \mathrm{E}-02$ & $8.79 \mathrm{E}-05$ & $0.00 \mathrm{E}+00$ & $0.00 \mathrm{E}+00$ & $0.00 \mathrm{E}+00$ & $0.00 \mathrm{E}+00$ & $0.00 \mathrm{E}+00$ & $1.99 \mathrm{E}-01$ \\
\hline $85 \%$ & $1.19 \mathrm{E}-01$ & $1.89 \mathrm{E}-07$ & $4.45 \mathrm{E}-02$ & $6.23 \mathrm{E}-02$ & $1.58 \mathrm{E}-02$ & $9.33 \mathrm{E}-05$ & $0.00 \mathrm{E}+00$ & $0.00 \mathrm{E}+00$ & $0.00 \mathrm{E}+00$ & $0.00 \mathrm{E}+00$ & $0.00 \mathrm{E}+00$ & $2.23 \mathrm{E}-01$ \\
\hline $90 \%$ & $1.33 \mathrm{E}-01$ & $2.21 \mathrm{E}-07$ & $5.65 \mathrm{E}-02$ & $8.16 \mathrm{E}-02$ & $2.01 \mathrm{E}-02$ & $9.98 \mathrm{E}-05$ & $0.00 \mathrm{E}+00$ & $0.00 \mathrm{E}+00$ & $0.00 \mathrm{E}+00$ & $0.00 \mathrm{E}+00$ & $0.00 \mathrm{E}+00$ & $2.56 \mathrm{E}-01$ \\
\hline $95 \%$ & $1.55 \mathrm{E}-01$ & $2.73 \mathrm{E}-07$ & $8.11 \mathrm{E}-02$ & $1.19 \mathrm{E}-01$ & $2.90 \mathrm{E}-02$ & $1.09 \mathrm{E}-04$ & $0.00 \mathrm{E}+00$ & $0.00 \mathrm{E}+00$ & $0.00 \mathrm{E}+00$ & $0.00 \mathrm{E}+00$ & $0.00 \mathrm{E}+00$ & $3.17 \mathrm{E}-01$ \\
\hline Mean & $8.42 \mathrm{E}-02$ & $1.20 \mathrm{E}-07$ & $2.60 \mathrm{E}-02$ & $3.87 \mathrm{E}-02$ & $9.63 \mathrm{E}-03$ & $6.39 \mathrm{E}-05$ & $0.00 \mathrm{E}+00$ & $0.00 \mathrm{E}+00$ & $0.00 \mathrm{E}+00$ & $0.00 \mathrm{E}+00$ & $0.00 \mathrm{E}+00$ & $1.59 \mathrm{E}-01$ \\
\hline Min & $2.59 \mathrm{E}-03$ & $2.95 \mathrm{E}-10$ & $5.07 \mathrm{E}-04$ & $1.41 \mathrm{E}-03$ & $2.54 \mathrm{E}-04$ & $2.00 \mathrm{E}-06$ & $0.00 \mathrm{E}+00$ & $0.00 \mathrm{E}+00$ & $0.00 \mathrm{E}+00$ & $0.00 \mathrm{E}+00$ & $0.00 \mathrm{E}+00$ & $5.56 \mathrm{E}-03$ \\
\hline Max & $2.23 \mathrm{E}-01$ & $9.13 \mathrm{E}-07$ & $7.17 \mathrm{E}-01$ & $1.21 \mathrm{E}+00$ & $4.52 \mathrm{E}-01$ & $1.29 \mathrm{E}-04$ & $0.00 \mathrm{E}+00$ & $0.00 \mathrm{E}+00$ & $0.00 \mathrm{E}+00$ & $0.00 \mathrm{E}+00$ & $0.00 \mathrm{E}+00$ & $2.45 \mathrm{E}+00$ \\
\hline
\end{tabular}


TABLE C.47 Peak DSR Percentiles (mrem/yr per pCi/g) for Different Exposure Pathways for I-129 in Onsite Resident Scenario

\begin{tabular}{|c|c|c|c|c|c|c|c|c|c|c|c|c|}
\hline Percentile & External & $\begin{array}{l}\text { Inhalation } \\
\text { w/o Radon }\end{array}$ & $\begin{array}{c}\text { Plant } \\
\text { Ingestion } \\
(\mathrm{WI})\end{array}$ & $\begin{array}{c}\text { Meat } \\
\text { Ingestion } \\
(\mathrm{WI})\end{array}$ & $\begin{array}{c}\text { Milk } \\
\text { Ingestion } \\
(\mathrm{WI})\end{array}$ & $\begin{array}{c}\text { Soil } \\
\text { Ingestion }\end{array}$ & $\begin{array}{c}\text { Water } \\
\text { Ingestion }\end{array}$ & $\begin{array}{c}\text { Aquatic } \\
\text { Foods }\end{array}$ & $\begin{array}{c}\text { Plant } \\
\text { Ingestion } \\
\text { (WD) }\end{array}$ & $\begin{array}{c}\text { Meat } \\
\text { Ingestion } \\
\text { (WD) }\end{array}$ & $\begin{array}{c}\text { Milk } \\
\text { Ingestion } \\
\text { (WD) }\end{array}$ & $\begin{array}{c}\text { All } \\
\text { Pathways }\end{array}$ \\
\hline $5 \%$ & $2.51 \mathrm{E}-19$ & $2.54 \mathrm{E}-22$ & $7.91 \mathrm{E}-17$ & $1.01 \mathrm{E}-16$ & $3.41 \mathrm{E}-17$ & $5.18 \mathrm{E}-19$ & $3.06 \mathrm{E}-29$ & $0.00 \mathrm{E}+00$ & $1.21 \mathrm{E}-30$ & $1.12 \mathrm{E}-29$ & $7.49 \mathrm{E}-30$ & $1.42 \mathrm{E}-06$ \\
\hline $10 \%$ & $7.11 \mathrm{E}-13$ & $4.14 \mathrm{E}-16$ & $1.42 \mathrm{E}-10$ & $3.17 \mathrm{E}-10$ & $6.84 \mathrm{E}-11$ & $1.30 \mathrm{E}-12$ & $2.54 \mathrm{E}-05$ & $1.09 \mathrm{E}-07$ & $9.86 \mathrm{E}-07$ & $6.87 \mathrm{E}-06$ & $4.40 \mathrm{E}-06$ & $5.00 \mathrm{E}-03$ \\
\hline $15 \%$ & $1.41 \mathrm{E}-09$ & $1.17 \mathrm{E}-12$ & $4.45 \mathrm{E}-07$ & $6.66 \mathrm{E}-07$ & $1.94 \mathrm{E}-07$ & $2.72 \mathrm{E}-09$ & $2.89 \mathrm{E}-02$ & $1.18 \mathrm{E}-04$ & $1.12 \mathrm{E}-03$ & $8.29 \mathrm{E}-03$ & 5.37E-03 & $1.55 \mathrm{E}-01$ \\
\hline $20 \%$ & $2.65 \mathrm{E}-07$ & $1.79 \mathrm{E}-10$ & $7.59 \mathrm{E}-05$ & $1.05 \mathrm{E}-04$ & $2.97 \mathrm{E}-05$ & $5.15 \mathrm{E}-07$ & $3.97 \mathrm{E}-01$ & $1.31 \mathrm{E}-03$ & $1.54 \mathrm{E}-02$ & $9.83 \mathrm{E}-02$ & $6.75 \mathrm{E}-02$ & $8.83 \mathrm{E}-01$ \\
\hline $25 \%$ & 7.09E-06 & $5.31 \mathrm{E}-09$ & $1.83 \mathrm{E}-03$ & $3.00 \mathrm{E}-03$ & $8.64 \mathrm{E}-04$ & $1.34 \mathrm{E}-05$ & $1.10 \mathrm{E}+00$ & $3.54 \mathrm{E}-03$ & $4.24 \mathrm{E}-02$ & $2.92 \mathrm{E}-01$ & $1.81 \mathrm{E}-01$ & $2.06 \mathrm{E}+00$ \\
\hline $30 \%$ & 7.91E-05 & $5.12 \mathrm{E}-08$ & $2.59 \mathrm{E}-02$ & $3.51 \mathrm{E}-02$ & $1.10 \mathrm{E}-02$ & $1.37 \mathrm{E}-04$ & $1.93 \mathrm{E}+00$ & $6.41 \mathrm{E}-03$ & $7.46 \mathrm{E}-02$ & $4.76 \mathrm{E}-01$ & $3.36 \mathrm{E}-01$ & $3.27 \mathrm{E}+00$ \\
\hline $35 \%$ & $5.01 \mathrm{E}-04$ & $3.19 \mathrm{E}-07$ & $1.04 \mathrm{E}-01$ & $1.92 \mathrm{E}-01$ & $6.06 \mathrm{E}-02$ & $7.79 \mathrm{E}-04$ & $2.77 \mathrm{E}+00$ & $9.71 \mathrm{E}-03$ & $1.08 \mathrm{E}-01$ & $6.95 \mathrm{E}-01$ & $4.91 \mathrm{E}-01$ & $4.62 \mathrm{E}+00$ \\
\hline $40 \%$ & $1.27 \mathrm{E}-03$ & $7.18 \mathrm{E}-07$ & $1.75 \mathrm{E}-01$ & $3.20 \mathrm{E}-01$ & $9.76 \mathrm{E}-02$ & $1.75 \mathrm{E}-03$ & $3.69 \mathrm{E}+00$ & $1.37 \mathrm{E}-02$ & $1.43 \mathrm{E}-01$ & $9.60 \mathrm{E}-01$ & $6.50 \mathrm{E}-01$ & $5.92 \mathrm{E}+00$ \\
\hline $45 \%$ & $1.52 \mathrm{E}-03$ & $9.81 \mathrm{E}-07$ & $2.41 \mathrm{E}-01$ & $4.11 \mathrm{E}-01$ & $1.28 \mathrm{E}-01$ & $2.56 \mathrm{E}-03$ & $4.68 \mathrm{E}+00$ & $1.94 \mathrm{E}-02$ & $1.81 \mathrm{E}-01$ & $1.25 \mathrm{E}+00$ & $8.43 \mathrm{E}-01$ & $7.37 \mathrm{E}+00$ \\
\hline $50 \%$ & $1.70 \mathrm{E}-03$ & $1.20 \mathrm{E}-06$ & $3.01 \mathrm{E}-01$ & 4.87E-01 & $1.55 \mathrm{E}-01$ & $3.19 \mathrm{E}-03$ & $6.03 \mathrm{E}+00$ & $2.66 \mathrm{E}-02$ & $2.33 \mathrm{E}-01$ & $1.63 \mathrm{E}+00$ & $1.10 \mathrm{E}+00$ & $9.37 \mathrm{E}+00$ \\
\hline $55 \%$ & $1.88 \mathrm{E}-03$ & $1.40 \mathrm{E}-06$ & $3.75 \mathrm{E}-01$ & $5.77 \mathrm{E}-01$ & $1.80 \mathrm{E}-01$ & $3.83 \mathrm{E}-03$ & $7.69 \mathrm{E}+00$ & $3.61 \mathrm{E}-02$ & $2.99 \mathrm{E}-01$ & $2.03 \mathrm{E}+00$ & $1.43 \mathrm{E}+00$ & $1.21 \mathrm{E}+01$ \\
\hline $60 \%$ & $2.03 \mathrm{E}-03$ & $1.62 \mathrm{E}-06$ & $4.49 \mathrm{E}-01$ & $6.74 \mathrm{E}-01$ & $2.11 \mathrm{E}-01$ & $4.36 \mathrm{E}-03$ & $9.72 \mathrm{E}+00$ & $4.80 \mathrm{E}-02$ & $3.75 \mathrm{E}-01$ & $2.69 \mathrm{E}+00$ & $1.85 \mathrm{E}+00$ & $1.50 \mathrm{E}+01$ \\
\hline $65 \%$ & $2.20 \mathrm{E}-03$ & $1.84 \mathrm{E}-06$ & $5.39 \mathrm{E}-01$ & $7.64 \mathrm{E}-01$ & $2.45 \mathrm{E}-01$ & $4.84 \mathrm{E}-03$ & $1.22 \mathrm{E}+01$ & $6.17 \mathrm{E}-02$ & $4.74 \mathrm{E}-01$ & $3.48 \mathrm{E}+00$ & $2.38 \mathrm{E}+00$ & $1.92 \mathrm{E}+01$ \\
\hline $70 \%$ & $2.37 \mathrm{E}-03$ & 2.07E-06 & $6.52 \mathrm{E}-01$ & $8.96 \mathrm{E}-01$ & $2.84 \mathrm{E}-01$ & $5.29 \mathrm{E}-03$ & $1.59 \mathrm{E}+01$ & $8.38 \mathrm{E}-02$ & $6.14 \mathrm{E}-01$ & $4.44 \mathrm{E}+00$ & $2.99 \mathrm{E}+00$ & $2.46 \mathrm{E}+01$ \\
\hline $75 \%$ & $2.57 \mathrm{E}-03$ & $2.28 \mathrm{E}-06$ & $7.75 \mathrm{E}-01$ & $1.04 \mathrm{E}+00$ & $3.29 \mathrm{E}-01$ & $5.77 \mathrm{E}-03$ & $2.03 \mathrm{E}+01$ & $1.12 \mathrm{E}-01$ & $7.85 \mathrm{E}-01$ & $5.83 \mathrm{E}+00$ & $3.98 \mathrm{E}+00$ & $3.17 \mathrm{E}+01$ \\
\hline $80 \%$ & $2.81 \mathrm{E}-03$ & $2.54 \mathrm{E}-06$ & $9.22 \mathrm{E}-01$ & $1.24 \mathrm{E}+00$ & $3.92 \mathrm{E}-01$ & $6.33 \mathrm{E}-03$ & $2.65 \mathrm{E}+01$ & $1.55 \mathrm{E}-01$ & $1.03 \mathrm{E}+00$ & $7.43 \mathrm{E}+00$ & $5.10 \mathrm{E}+00$ & $4.11 \mathrm{E}+01$ \\
\hline $85 \%$ & $3.12 \mathrm{E}-03$ & $2.86 \mathrm{E}-06$ & $1.14 \mathrm{E}+00$ & $1.51 \mathrm{E}+00$ & $4.70 \mathrm{E}-01$ & $6.86 \mathrm{E}-03$ & $3.42 \mathrm{E}+01$ & $2.21 \mathrm{E}-01$ & $1.32 \mathrm{E}+00$ & $9.88 \mathrm{E}+00$ & $6.65 \mathrm{E}+00$ & $5.35 \mathrm{E}+01$ \\
\hline $90 \%$ & $3.53 \mathrm{E}-03$ & $3.25 \mathrm{E}-06$ & $1.45 \mathrm{E}+00$ & $1.90 \mathrm{E}+00$ & $5.85 \mathrm{E}-01$ & $7.59 \mathrm{E}-03$ & $4.64 \mathrm{E}+01$ & $3.56 \mathrm{E}-01$ & $1.79 \mathrm{E}+00$ & $1.35 \mathrm{E}+01$ & $9.32 \mathrm{E}+00$ & $7.18 \mathrm{E}+01$ \\
\hline $95 \%$ & $4.29 \mathrm{E}-03$ & $3.92 \mathrm{E}-06$ & $2.01 \mathrm{E}+00$ & $2.63 \mathrm{E}+00$ & $8.30 \mathrm{E}-01$ & $8.47 \mathrm{E}-03$ & $6.86 \mathrm{E}+01$ & $6.46 \mathrm{E}-01$ & $2.65 \mathrm{E}+00$ & $2.06 \mathrm{E}+01$ & $1.46 \mathrm{E}+01$ & $1.04 \mathrm{E}+02$ \\
\hline Mean & $1.64 \mathrm{E}-03$ & $1.41 \mathrm{E}-06$ & $5.60 \mathrm{E}-01$ & 7.62E-01 & $2.44 \mathrm{E}-01$ & $3.34 \mathrm{E}-03$ & $1.61 \mathrm{E}+01$ & $1.47 \mathrm{E}-01$ & $6.22 \mathrm{E}-01$ & $4.78 \mathrm{E}+00$ & $3.41 \mathrm{E}+00$ & $2.51 \mathrm{E}+01$ \\
\hline Min & $0.00 \mathrm{E}+00$ & $0.00 \mathrm{E}+00$ & $0.00 \mathrm{E}+00$ & $0.00 \mathrm{E}+00$ & $0.00 \mathrm{E}+00$ & $0.00 \mathrm{E}+00$ & $0.00 \mathrm{E}+00$ & $0.00 \mathrm{E}+00$ & $0.00 \mathrm{E}+00$ & $0.00 \mathrm{E}+00$ & $0.00 \mathrm{E}+00$ & $0.00 \mathrm{E}+00$ \\
\hline $\operatorname{Max}$ & $7.21 \mathrm{E}-03$ & $1.17 \mathrm{E}-05$ & $1.01 \mathrm{E}+01$ & $1.25 \mathrm{E}+01$ & $5.69 \mathrm{E}+00$ & $1.10 \mathrm{E}-02$ & $2.10 \mathrm{E}+02$ & $1.52 \mathrm{E}+01$ & $8.19 \mathrm{E}+00$ & $1.12 \mathrm{E}+02$ & $1.01 \mathrm{E}+02$ & $3.34 \mathrm{E}+02$ \\
\hline
\end{tabular}


TABLE C.48 Peak DSR Percentiles (mrem/yr per pCi/g) for Different Exposure Pathways for Np-237 in Onsite Resident Scenario

\begin{tabular}{|c|c|c|c|c|c|c|c|c|c|c|c|c|}
\hline Percentile & External & $\begin{array}{l}\text { Inhalation } \\
\text { w/o Radon }\end{array}$ & $\begin{array}{c}\text { Plant } \\
\text { Ingestion } \\
\text { (WI) }\end{array}$ & $\begin{array}{c}\text { Meat } \\
\text { Ingestion } \\
(\mathrm{WI})\end{array}$ & $\begin{array}{c}\text { Milk } \\
\text { Ingestion } \\
(\mathrm{WI})\end{array}$ & $\begin{array}{c}\text { Soil } \\
\text { Ingestion }\end{array}$ & $\begin{array}{c}\text { Water } \\
\text { Ingestion }\end{array}$ & $\begin{array}{c}\text { Aquatic } \\
\text { Foods }\end{array}$ & $\begin{array}{c}\text { Plant } \\
\text { Ingestion } \\
\text { (WD) }\end{array}$ & $\begin{array}{c}\text { Meat } \\
\text { Ingestion } \\
(\mathrm{WD})\end{array}$ & $\begin{array}{c}\text { Milk } \\
\text { Ingestion } \\
\text { (WD) }\end{array}$ & $\begin{array}{c}\text { All } \\
\text { Pathways }\end{array}$ \\
\hline $5 \%$ & 3.74E-08 & $2.52 \mathrm{E}-09$ & $3.23 \mathrm{E}-07$ & $3.34 \mathrm{E}-08$ & 2.04E-08 & 2.64E-08 & $0.00 \mathrm{E}+00$ & $0.00 \mathrm{E}+00$ & $0.00 \mathrm{E}+00$ & $0.00 \mathrm{E}+00$ & $0.00 \mathrm{E}+00$ & $5.48 \mathrm{E}-07$ \\
\hline $10 \%$ & $1.97 \mathrm{E}-07$ & 8.22E-09 & $1.95 \mathrm{E}-06$ & $1.41 \mathrm{E}-07$ & $6.84 \mathrm{E}-08$ & 7.93E-08 & $0.00 \mathrm{E}+00$ & $0.00 \mathrm{E}+00$ & $0.00 \mathrm{E}+00$ & $0.00 \mathrm{E}+00$ & $0.00 \mathrm{E}+00$ & $6.54 \mathrm{E}-06$ \\
\hline $15 \%$ & $4.71 \mathrm{E}-04$ & $2.22 \mathrm{E}-06$ & $1.50 \mathrm{E}-03$ & $5.98 \mathrm{E}-05$ & 8.94E-07 & $9.75 \mathrm{E}-06$ & $0.00 \mathrm{E}+00$ & $0.00 \mathrm{E}+00$ & $0.00 \mathrm{E}+00$ & $0.00 \mathrm{E}+00$ & $0.00 \mathrm{E}+00$ & $8.01 \mathrm{E}-03$ \\
\hline $20 \%$ & $2.33 \mathrm{E}-02$ & 8.09E-05 & $4.87 \mathrm{E}-02$ & $1.92 \mathrm{E}-03$ & $2.49 \mathrm{E}-05$ & 3.99E-04 & $0.00 \mathrm{E}+00$ & $0.00 \mathrm{E}+00$ & $0.00 \mathrm{E}+00$ & $0.00 \mathrm{E}+00$ & $0.00 \mathrm{E}+00$ & $1.69 \mathrm{E}-01$ \\
\hline $25 \%$ & $8.83 \mathrm{E}-02$ & $2.66 \mathrm{E}-04$ & $1.28 \mathrm{E}-01$ & $4.64 \mathrm{E}-03$ & $5.80 \mathrm{E}-05$ & $1.15 \mathrm{E}-03$ & $0.00 \mathrm{E}+00$ & $0.00 \mathrm{E}+00$ & $0.00 \mathrm{E}+00$ & $0.00 \mathrm{E}+00$ & $0.00 \mathrm{E}+00$ & $3.90 \mathrm{E}-01$ \\
\hline $30 \%$ & $1.32 \mathrm{E}-01$ & 4.05E-04 & $1.89 \mathrm{E}-01$ & $6.53 \mathrm{E}-03$ & 8.92E-05 & $1.91 \mathrm{E}-03$ & $0.00 \mathrm{E}+00$ & $0.00 \mathrm{E}+00$ & $0.00 \mathrm{E}+00$ & $0.00 \mathrm{E}+00$ & $0.00 \mathrm{E}+00$ & $5.26 \mathrm{E}-01$ \\
\hline $35 \%$ & $1.52 \mathrm{E}-01$ & $5.11 \mathrm{E}-04$ & $2.48 \mathrm{E}-01$ & $8.57 \mathrm{E}-03$ & $1.18 \mathrm{E}-04$ & $2.54 \mathrm{E}-03$ & $0.00 \mathrm{E}+00$ & $0.00 \mathrm{E}+00$ & $0.00 \mathrm{E}+00$ & $0.00 \mathrm{E}+00$ & $0.00 \mathrm{E}+00$ & $6.50 \mathrm{E}-01$ \\
\hline $40 \%$ & $1.68 \mathrm{E}-01$ & $5.96 \mathrm{E}-04$ & $3.06 \mathrm{E}-01$ & $1.07 \mathrm{E}-02$ & $1.44 \mathrm{E}-04$ & $3.09 \mathrm{E}-03$ & $0.00 \mathrm{E}+00$ & $0.00 \mathrm{E}+00$ & $0.00 \mathrm{E}+00$ & $0.00 \mathrm{E}+00$ & $0.00 \mathrm{E}+00$ & 7.57E-01 \\
\hline $45 \%$ & $1.82 \mathrm{E}-01$ & $6.87 \mathrm{E}-04$ & 3.67E-01 & $1.32 \mathrm{E}-02$ & $1.70 \mathrm{E}-04$ & $3.63 \mathrm{E}-03$ & $0.00 \mathrm{E}+00$ & $0.00 \mathrm{E}+00$ & $0.00 \mathrm{E}+00$ & $0.00 \mathrm{E}+00$ & $0.00 \mathrm{E}+00$ & $8.72 \mathrm{E}-01$ \\
\hline $50 \%$ & $1.96 \mathrm{E}-01$ & 7.79E-04 & $4.37 \mathrm{E}-01$ & $1.56 \mathrm{E}-02$ & $1.97 \mathrm{E}-04$ & $4.14 \mathrm{E}-03$ & $0.00 \mathrm{E}+00$ & $0.00 \mathrm{E}+00$ & $0.00 \mathrm{E}+00$ & $0.00 \mathrm{E}+00$ & $0.00 \mathrm{E}+00$ & $9.93 \mathrm{E}-01$ \\
\hline $55 \%$ & $2.11 \mathrm{E}-01$ & $8.75 \mathrm{E}-04$ & $5.18 \mathrm{E}-01$ & $1.83 \mathrm{E}-02$ & $2.29 \mathrm{E}-04$ & $4.54 \mathrm{E}-03$ & $0.00 \mathrm{E}+00$ & $0.00 \mathrm{E}+00$ & $0.00 \mathrm{E}+00$ & $0.00 \mathrm{E}+00$ & $0.00 \mathrm{E}+00$ & $1.15 \mathrm{E}+00$ \\
\hline $60 \%$ & $2.27 \mathrm{E}-01$ & $9.74 \mathrm{E}-04$ & $6.04 \mathrm{E}-01$ & $2.13 \mathrm{E}-02$ & $2.61 \mathrm{E}-04$ & 4.97E-03 & $0.00 \mathrm{E}+00$ & $0.00 \mathrm{E}+00$ & $0.00 \mathrm{E}+00$ & $0.00 \mathrm{E}+00$ & $0.00 \mathrm{E}+00$ & $1.32 \mathrm{E}+00$ \\
\hline $65 \%$ & $2.44 \mathrm{E}-01$ & $1.08 \mathrm{E}-03$ & $6.98 \mathrm{E}-01$ & $2.47 \mathrm{E}-02$ & $3.05 \mathrm{E}-04$ & $5.39 \mathrm{E}-03$ & $3.94 \mathrm{E}-03$ & $4.65 \mathrm{E}-05$ & $1.52 \mathrm{E}-04$ & $2.40 \mathrm{E}-05$ & $8.56 \mathrm{E}-07$ & $1.54 \mathrm{E}+00$ \\
\hline $70 \%$ & $2.62 \mathrm{E}-01$ & $1.19 \mathrm{E}-03$ & 8.07E-01 & $2.87 \mathrm{E}-02$ & 3.64E-04 & $5.81 \mathrm{E}-03$ & $1.05 \mathrm{E}-01$ & $1.25 \mathrm{E}-03$ & $4.05 \mathrm{E}-03$ & $6.30 \mathrm{E}-04$ & $1.79 \mathrm{E}-05$ & $1.85 \mathrm{E}+00$ \\
\hline $75 \%$ & $2.83 \mathrm{E}-01$ & $1.32 \mathrm{E}-03$ & $9.45 \mathrm{E}-01$ & $3.35 \mathrm{E}-02$ & 4.37E-04 & $6.27 \mathrm{E}-03$ & $5.66 \mathrm{E}-01$ & $6.66 \mathrm{E}-03$ & $2.18 \mathrm{E}-02$ & $3.23 \mathrm{E}-03$ & $9.24 \mathrm{E}-05$ & $2.23 \mathrm{E}+00$ \\
\hline $80 \%$ & $3.08 \mathrm{E}-01$ & $1.46 \mathrm{E}-03$ & $1.13 \mathrm{E}+00$ & $4.06 \mathrm{E}-02$ & $5.24 \mathrm{E}-04$ & $6.77 \mathrm{E}-03$ & $1.39 \mathrm{E}+00$ & $1.62 \mathrm{E}-02$ & $5.34 \mathrm{E}-02$ & $9.21 \mathrm{E}-03$ & $2.26 \mathrm{E}-04$ & $2.79 \mathrm{E}+00$ \\
\hline $85 \%$ & $3.38 \mathrm{E}-01$ & $1.62 \mathrm{E}-03$ & $1.38 \mathrm{E}+00$ & $5.16 \mathrm{E}-02$ & $6.46 \mathrm{E}-04$ & 7.36E-03 & $2.56 \mathrm{E}+00$ & $3.15 \mathrm{E}-02$ & $9.87 \mathrm{E}-02$ & $1.74 \mathrm{E}-02$ & 4.74E-04 & $3.70 \mathrm{E}+00$ \\
\hline $90 \%$ & $3.82 \mathrm{E}-01$ & $1.84 \mathrm{E}-03$ & $1.75 \mathrm{E}+00$ & $6.57 \mathrm{E}-02$ & $8.56 \mathrm{E}-04$ & $8.03 \mathrm{E}-03$ & $4.63 \mathrm{E}+00$ & $5.54 \mathrm{E}-02$ & $1.79 \mathrm{E}-01$ & $3.38 \mathrm{E}-02$ & $9.26 \mathrm{E}-04$ & $5.43 \mathrm{E}+00$ \\
\hline $95 \%$ & $4.58 \mathrm{E}-01$ & $2.14 \mathrm{E}-03$ & $2.48 \mathrm{E}+00$ & $9.89 \mathrm{E}-02$ & $1.22 \mathrm{E}-03$ & $8.99 \mathrm{E}-03$ & $9.52 \mathrm{E}+00$ & $1.26 \mathrm{E}-01$ & 3.67E-01 & $7.60 \mathrm{E}-02$ & $2.11 \mathrm{E}-03$ & $1.04 \mathrm{E}+01$ \\
\hline Mean & $1.98 \mathrm{E}-01$ & $8.74 \mathrm{E}-04$ & 7.36E-01 & $2.87 \mathrm{E}-02$ & $3.58 \mathrm{E}-04$ & $4.01 \mathrm{E}-03$ & $1.79 \mathrm{E}+00$ & $2.55 \mathrm{E}-02$ & $6.91 \mathrm{E}-02$ & $1.50 \mathrm{E}-02$ & $4.11 \mathrm{E}-04$ & $2.64 \mathrm{E}+00$ \\
\hline Min & $3.92 \mathrm{E}-10$ & $4.21 \mathrm{E}-12$ & $1.18 \mathrm{E}-10$ & $3.18 \mathrm{E}-12$ & $4.04 \mathrm{E}-13$ & $1.12 \mathrm{E}-11$ & $0.00 \mathrm{E}+00$ & $0.00 \mathrm{E}+00$ & $0.00 \mathrm{E}+00$ & $0.00 \mathrm{E}+00$ & $0.00 \mathrm{E}+00$ & $1.72 \mathrm{E}-09$ \\
\hline Max & $7.28 \mathrm{E}-01$ & $5.40 \mathrm{E}-03$ & $1.79 \mathrm{E}+01$ & $6.83 \mathrm{E}-01$ & $2.50 \mathrm{E}-02$ & $1.13 \mathrm{E}-02$ & $1.29 \mathrm{E}+02$ & $5.03 \mathrm{E}+00$ & $4.99 \mathrm{E}+00$ & $2.36 \mathrm{E}+00$ & $3.84 \mathrm{E}-02$ & $1.37 \mathrm{E}+02$ \\
\hline
\end{tabular}


TABLE C.49 Peak DSR Percentiles (mrem/yr per pCi/g) for Different Exposure Pathways for Pu-238 in Onsite Resident Scenario

\begin{tabular}{|c|c|c|c|c|c|c|c|c|c|c|c|c|c|c|}
\hline Percentile & External & $\begin{array}{c}\text { Inhalation } \\
\text { w/o Radon }\end{array}$ & $\begin{array}{l}\text { Radon } \\
\text { (WI) }\end{array}$ & $\begin{array}{c}\text { Plant } \\
\text { Ingestion } \\
\text { (WI) }\end{array}$ & $\begin{array}{c}\text { Meat } \\
\text { Ingestion } \\
(\mathrm{WI})\end{array}$ & $\begin{array}{c}\text { Milk } \\
\text { Ingestion } \\
\text { (WI) }\end{array}$ & $\begin{array}{c}\text { Soil } \\
\text { Ingestion }\end{array}$ & $\begin{array}{c}\text { Water } \\
\text { Ingestion }\end{array}$ & $\begin{array}{c}\text { Aquatic } \\
\text { Foods }\end{array}$ & $\begin{array}{l}\text { Radon } \\
\text { (WD) }\end{array}$ & $\begin{array}{c}\text { Plant } \\
\text { Ingestion } \\
\text { (WD) }\end{array}$ & $\begin{array}{c}\text { Meat } \\
\text { Ingestion } \\
\text { (WD) }\end{array}$ & $\begin{array}{c}\text { Milk } \\
\text { Ingestion } \\
\text { (WD) }\end{array}$ & $\begin{array}{c}\text { All } \\
\text { Pathways } \\
\end{array}$ \\
\hline $5 \%$ & 7.67E-06 & 4.19E-04 & $8.08 \mathrm{E}-08$ & $7.18 \mathrm{E}-03$ & 4.69E-04 & $4.78 \mathrm{E}-06$ & $1.61 \mathrm{E}-03$ & $0.00 \mathrm{E}+00$ & $0.00 \mathrm{E}+00$ & $0.00 \mathrm{E}+00$ & $0.00 \mathrm{E}+00$ & $0.00 \mathrm{E}+00$ & $0.00 \mathrm{E}+00$ & $1.42 \mathrm{E}-02$ \\
\hline $10 \%$ & $8.58 \mathrm{E}-06$ & $5.69 \mathrm{E}-04$ & $9.58 \mathrm{E}-08$ & $9.90 \mathrm{E}-03$ & $5.13 \mathrm{E}-04$ & $5.71 \mathrm{E}-06$ & $2.28 \mathrm{E}-03$ & $0.00 \mathrm{E}+00$ & $0.00 \mathrm{E}+00$ & $0.00 \mathrm{E}+00$ & $0.00 \mathrm{E}+00$ & $0.00 \mathrm{E}+00$ & $0.00 \mathrm{E}+00$ & $1.74 \mathrm{E}-02$ \\
\hline $15 \%$ & 9.34E-06 & $6.59 \mathrm{E}-04$ & $1.05 \mathrm{E}-07$ & $1.25 \mathrm{E}-02$ & $5.41 \mathrm{E}-04$ & $6.46 \mathrm{E}-06$ & $2.82 \mathrm{E}-03$ & $0.00 \mathrm{E}+00$ & $0.00 \mathrm{E}+00$ & $0.00 \mathrm{E}+00$ & $0.00 \mathrm{E}+00$ & $0.00 \mathrm{E}+00$ & $0.00 \mathrm{E}+00$ & $2.00 \mathrm{E}-02$ \\
\hline $20 \%$ & 9.94E-06 & 7.47E-04 & $1.11 \mathrm{E}-07$ & $1.49 \mathrm{E}-02$ & $5.68 \mathrm{E}-04$ & 7.05E-06 & $3.29 \mathrm{E}-03$ & $0.00 \mathrm{E}+00$ & $0.00 \mathrm{E}+00$ & $0.00 \mathrm{E}+00$ & $0.00 \mathrm{E}+00$ & $0.00 \mathrm{E}+00$ & $0.00 \mathrm{E}+00$ & $2.22 \mathrm{E}-02$ \\
\hline $25 \%$ & $1.05 \mathrm{E}-05$ & $8.42 \mathrm{E}-04$ & $1.16 \mathrm{E}-07$ & $1.74 \mathrm{E}-02$ & $5.93 \mathrm{E}-04$ & 7.63E-06 & $3.67 \mathrm{E}-03$ & $0.00 \mathrm{E}+00$ & $0.00 \mathrm{E}+00$ & $0.00 \mathrm{E}+00$ & $0.00 \mathrm{E}+00$ & $0.00 \mathrm{E}+00$ & $0.00 \mathrm{E}+00$ & $2.48 \mathrm{E}-02$ \\
\hline $30 \%$ & $1.11 \mathrm{E}-05$ & $9.55 \mathrm{E}-04$ & $1.21 \mathrm{E}-07$ & $2.01 \mathrm{E}-02$ & $6.13 \mathrm{E}-04$ & $8.10 \mathrm{E}-06$ & $4.03 \mathrm{E}-03$ & $0.00 \mathrm{E}+00$ & $0.00 \mathrm{E}+00$ & $0.00 \mathrm{E}+00$ & $0.00 \mathrm{E}+00$ & $0.00 \mathrm{E}+00$ & $0.00 \mathrm{E}+00$ & $2.75 \mathrm{E}-02$ \\
\hline $35 \%$ & $1.18 \mathrm{E}-05$ & $1.04 \mathrm{E}-03$ & $1.27 \mathrm{E}-07$ & $2.28 \mathrm{E}-02$ & $6.33 \mathrm{E}-04$ & $8.62 \mathrm{E}-06$ & $4.37 \mathrm{E}-03$ & $0.00 \mathrm{E}+00$ & $0.00 \mathrm{E}+00$ & $0.00 \mathrm{E}+00$ & $0.00 \mathrm{E}+00$ & $0.00 \mathrm{E}+00$ & $0.00 \mathrm{E}+00$ & $3.03 \mathrm{E}-02$ \\
\hline $40 \%$ & $1.24 \mathrm{E}-05$ & $1.13 \mathrm{E}-03$ & $1.31 \mathrm{E}-07$ & $2.57 \mathrm{E}-02$ & $6.54 \mathrm{E}-04$ & 9.17E-06 & $4.68 \mathrm{E}-03$ & $0.00 \mathrm{E}+00$ & $0.00 \mathrm{E}+00$ & $0.00 \mathrm{E}+00$ & $0.00 \mathrm{E}+00$ & $0.00 \mathrm{E}+00$ & $0.00 \mathrm{E}+00$ & $3.33 \mathrm{E}-02$ \\
\hline $45 \%$ & $1.30 \mathrm{E}-05$ & $1.23 \mathrm{E}-03$ & $1.37 \mathrm{E}-07$ & $2.89 \mathrm{E}-02$ & $6.77 \mathrm{E}-04$ & $9.76 \mathrm{E}-06$ & $4.98 \mathrm{E}-03$ & $0.00 \mathrm{E}+00$ & $0.00 \mathrm{E}+00$ & $0.00 \mathrm{E}+00$ & $0.00 \mathrm{E}+00$ & $0.00 \mathrm{E}+00$ & $0.00 \mathrm{E}+00$ & $3.65 \mathrm{E}-02$ \\
\hline $50 \%$ & $1.36 \mathrm{E}-05$ & $1.33 \mathrm{E}-03$ & $1.41 \mathrm{E}-07$ & $3.25 \mathrm{E}-02$ & $6.97 \mathrm{E}-04$ & $1.03 \mathrm{E}-05$ & $5.26 \mathrm{E}-03$ & $0.00 \mathrm{E}+00$ & $0.00 \mathrm{E}+00$ & $0.00 \mathrm{E}+00$ & $0.00 \mathrm{E}+00$ & $0.00 \mathrm{E}+00$ & $0.00 \mathrm{E}+00$ & $4.05 \mathrm{E}-02$ \\
\hline $55 \%$ & $1.44 \mathrm{E}-05$ & $1.44 \mathrm{E}-03$ & $1.45 \mathrm{E}-07$ & $3.65 \mathrm{E}-02$ & 7.18E-04 & $1.10 \mathrm{E}-05$ & $5.52 \mathrm{E}-03$ & $0.00 \mathrm{E}+00$ & $0.00 \mathrm{E}+00$ & $0.00 \mathrm{E}+00$ & $0.00 \mathrm{E}+00$ & $0.00 \mathrm{E}+00$ & $0.00 \mathrm{E}+00$ & $4.43 \mathrm{E}-02$ \\
\hline $60 \%$ & $1.52 \mathrm{E}-05$ & $1.56 \mathrm{E}-03$ & $1.50 \mathrm{E}-07$ & $4.09 \mathrm{E}-02$ & 7.39E-04 & $1.16 \mathrm{E}-05$ & $5.82 \mathrm{E}-03$ & $0.00 \mathrm{E}+00$ & $0.00 \mathrm{E}+00$ & $0.00 \mathrm{E}+00$ & $0.00 \mathrm{E}+00$ & $0.00 \mathrm{E}+00$ & $0.00 \mathrm{E}+00$ & $4.90 \mathrm{E}-02$ \\
\hline $65 \%$ & $1.60 \mathrm{E}-05$ & $1.69 \mathrm{E}-03$ & $1.56 \mathrm{E}-07$ & $4.60 \mathrm{E}-02$ & $7.65 \mathrm{E}-04$ & $1.24 \mathrm{E}-05$ & $6.12 \mathrm{E}-03$ & $0.00 \mathrm{E}+00$ & $0.00 \mathrm{E}+00$ & $0.00 \mathrm{E}+00$ & $0.00 \mathrm{E}+00$ & $0.00 \mathrm{E}+00$ & $0.00 \mathrm{E}+00$ & $5.36 \mathrm{E}-02$ \\
\hline $70 \%$ & $1.70 \mathrm{E}-05$ & $1.83 \mathrm{E}-03$ & $1.62 \mathrm{E}-07$ & $5.23 \mathrm{E}-02$ & 7.93E-04 & $1.32 \mathrm{E}-05$ & $6.47 \mathrm{E}-03$ & $0.00 \mathrm{E}+00$ & $0.00 \mathrm{E}+00$ & $0.00 \mathrm{E}+00$ & $0.00 \mathrm{E}+00$ & $0.00 \mathrm{E}+00$ & $0.00 \mathrm{E}+00$ & $5.97 \mathrm{E}-02$ \\
\hline $75 \%$ & $1.82 \mathrm{E}-05$ & $1.99 \mathrm{E}-03$ & $1.70 \mathrm{E}-07$ & $6.03 \mathrm{E}-02$ & $8.22 \mathrm{E}-04$ & $1.42 \mathrm{E}-05$ & $6.81 \mathrm{E}-03$ & $0.00 \mathrm{E}+00$ & $0.00 \mathrm{E}+00$ & $0.00 \mathrm{E}+00$ & $0.00 \mathrm{E}+00$ & $0.00 \mathrm{E}+00$ & $0.00 \mathrm{E}+00$ & $6.82 \mathrm{E}-02$ \\
\hline $80 \%$ & $1.96 \mathrm{E}-05$ & $2.19 \mathrm{E}-03$ & $1.77 \mathrm{E}-07$ & 7.03E-02 & 8.63E-04 & $1.55 \mathrm{E}-05$ & $7.21 \mathrm{E}-03$ & $0.00 \mathrm{E}+00$ & $0.00 \mathrm{E}+00$ & $0.00 \mathrm{E}+00$ & $0.00 \mathrm{E}+00$ & $0.00 \mathrm{E}+00$ & $0.00 \mathrm{E}+00$ & $7.85 \mathrm{E}-02$ \\
\hline $85 \%$ & $2.14 \mathrm{E}-05$ & $2.49 \mathrm{E}-03$ & $1.87 \mathrm{E}-07$ & 8.47E-02 & $9.10 \mathrm{E}-04$ & $1.70 \mathrm{E}-05$ & $7.66 \mathrm{E}-03$ & $0.00 \mathrm{E}+00$ & $0.00 \mathrm{E}+00$ & $0.00 \mathrm{E}+00$ & $0.00 \mathrm{E}+00$ & $0.00 \mathrm{E}+00$ & $0.00 \mathrm{E}+00$ & $9.26 \mathrm{E}-02$ \\
\hline $90 \%$ & 2.37E-05 & 2.90E-03 & 1.99E-07 & $1.05 \mathrm{E}-01$ & $9.80 \mathrm{E}-04$ & $1.94 \mathrm{E}-05$ & $8.21 \mathrm{E}-03$ & $0.00 \mathrm{E}+00$ & $0.00 \mathrm{E}+00$ & $0.00 \mathrm{E}+00$ & $0.00 \mathrm{E}+00$ & $0.00 \mathrm{E}+00$ & $0.00 \mathrm{E}+00$ & $1.13 \mathrm{E}-01$ \\
\hline $95 \%$ & $2.76 \mathrm{E}-05$ & $3.60 \mathrm{E}-03$ & $2.21 \mathrm{E}-07$ & $1.48 \mathrm{E}-01$ & $1.08 \mathrm{E}-03$ & $2.31 \mathrm{E}-05$ & 8.92E-03 & $0.00 \mathrm{E}+00$ & $0.00 \mathrm{E}+00$ & $0.00 \mathrm{E}+00$ & $0.00 \mathrm{E}+00$ & $0.00 \mathrm{E}+00$ & $0.00 \mathrm{E}+00$ & $1.56 \mathrm{E}-01$ \\
\hline Mean & $1.51 \mathrm{E}-05$ & $1.58 \mathrm{E}-03$ & $1.44 \mathrm{E}-07$ & 4.95E-02 & $7.28 \mathrm{E}-04$ & 1.17E-05 & $5.25 \mathrm{E}-03$ & $1.36 \mathrm{E}-07$ & $9.78 \mathrm{E}-10$ & $9.88 \mathrm{E}-17$ & $5.22 \mathrm{E}-09$ & $1.12 \mathrm{E}-09$ & $1.41 \mathrm{E}-09$ & $5.71 \mathrm{E}-02$ \\
\hline Min & $1.73 \mathrm{E}-09$ & 4.82E-09 & 4.67E-09 & $1.46 \mathrm{E}-06$ & $8.59 \mathrm{E}-08$ & $3.59 \mathrm{E}-08$ & $3.21 \mathrm{E}-08$ & $0.00 \mathrm{E}+00$ & $0.00 \mathrm{E}+00$ & $0.00 \mathrm{E}+00$ & $0.00 \mathrm{E}+00$ & $0.00 \mathrm{E}+00$ & $0.00 \mathrm{E}+00$ & $1.65 \mathrm{E}-06$ \\
\hline Max & $3.97 \mathrm{E}-05$ & $1.19 \mathrm{E}-02$ & $3.51 \mathrm{E}-07$ & 8.99E-01 & 3.63E-03 & $8.14 \mathrm{E}-05$ & $1.07 \mathrm{E}-02$ & 2.67E-04 & $3.52 \mathrm{E}-06$ & $2.86 \mathrm{E}-13$ & $1.03 \mathrm{E}-05$ & $3.01 \mathrm{E}-06$ & $3.26 \mathrm{E}-06$ & $9.12 \mathrm{E}-01$ \\
\hline
\end{tabular}




\section{TABLE C.50 Peak DSR Percentiles (mrem/yr per pCi/g) for Different Exposure Pathways for Pu-239 in Onsite Resident Scenario}

\begin{tabular}{|c|c|c|c|c|c|c|c|c|c|c|c|c|}
\hline Percentile & External & $\begin{array}{c}\text { Inhalation } \\
\text { w/o Radon }\end{array}$ & $\begin{array}{c}\text { Plant } \\
\text { Ingestion } \\
(\mathrm{WI})\end{array}$ & $\begin{array}{c}\text { Meat } \\
\text { Ingestion } \\
(\mathrm{WI})\end{array}$ & $\begin{array}{c}\text { Milk } \\
\text { Ingestion } \\
(\mathrm{WI})\end{array}$ & $\begin{array}{c}\text { Soil } \\
\text { Ingestion } \\
\end{array}$ & $\begin{array}{c}\text { Water } \\
\text { Ingestion }\end{array}$ & $\begin{array}{c}\text { Aquatic } \\
\text { Foods }\end{array}$ & $\begin{array}{c}\text { Plant } \\
\text { Ingestion } \\
\text { (WD) }\end{array}$ & $\begin{array}{c}\text { Meat } \\
\text { Ingestion } \\
\text { (WD) }\end{array}$ & $\begin{array}{c}\text { Milk } \\
\text { Ingestion } \\
\text { (WD) }\end{array}$ & $\begin{array}{c}\text { All } \\
\text { Pathways }\end{array}$ \\
\hline $5 \%$ & $3.69 \mathrm{E}-05$ & $1.00 \mathrm{E}-03$ & $1.68 \mathrm{E}-02$ & $1.12 \mathrm{E}-03$ & $1.05 \mathrm{E}-05$ & $3.86 \mathrm{E}-03$ & $0.00 \mathrm{E}+00$ & $0.00 \mathrm{E}+00$ & $0.00 \mathrm{E}+00$ & $0.00 \mathrm{E}+00$ & $0.00 \mathrm{E}+00$ & 3.39E-02 \\
\hline $10 \%$ & 4.13E-05 & $1.37 \mathrm{E}-03$ & $2.38 \mathrm{E}-02$ & $1.23 \mathrm{E}-03$ & $1.27 \mathrm{E}-05$ & $5.50 \mathrm{E}-03$ & $0.00 \mathrm{E}+00$ & $0.00 \mathrm{E}+00$ & $0.00 \mathrm{E}+00$ & $0.00 \mathrm{E}+00$ & $0.00 \mathrm{E}+00$ & 4.18E-02 \\
\hline $15 \%$ & 4.47E-05 & $1.59 \mathrm{E}-03$ & $3.00 \mathrm{E}-02$ & $1.30 \mathrm{E}-03$ & $1.44 \mathrm{E}-05$ & $6.80 \mathrm{E}-03$ & $0.00 \mathrm{E}+00$ & $0.00 \mathrm{E}+00$ & $0.00 \mathrm{E}+00$ & $0.00 \mathrm{E}+00$ & $0.00 \mathrm{E}+00$ & 4.76E-02 \\
\hline $20 \%$ & $4.78 \mathrm{E}-05$ & $1.80 \mathrm{E}-03$ & $3.57 \mathrm{E}-02$ & $1.36 \mathrm{E}-03$ & $1.58 \mathrm{E}-05$ & $7.91 \mathrm{E}-03$ & $0.00 \mathrm{E}+00$ & $0.00 \mathrm{E}+00$ & $0.00 \mathrm{E}+00$ & $0.00 \mathrm{E}+00$ & $0.00 \mathrm{E}+00$ & 5.33E-02 \\
\hline $25 \%$ & $5.07 \mathrm{E}-05$ & 2.03E-03 & 4.18E-02 & $1.42 \mathrm{E}-03$ & $1.71 \mathrm{E}-05$ & $8.80 \mathrm{E}-03$ & $0.00 \mathrm{E}+00$ & $0.00 \mathrm{E}+00$ & $0.00 \mathrm{E}+00$ & $0.00 \mathrm{E}+00$ & $0.00 \mathrm{E}+00$ & $5.98 \mathrm{E}-02$ \\
\hline $30 \%$ & 5.34E-05 & $2.30 \mathrm{E}-03$ & $4.80 \mathrm{E}-02$ & $1.47 \mathrm{E}-03$ & $1.83 \mathrm{E}-05$ & $9.74 \mathrm{E}-03$ & $0.00 \mathrm{E}+00$ & $0.00 \mathrm{E}+00$ & $0.00 \mathrm{E}+00$ & $0.00 \mathrm{E}+00$ & $0.00 \mathrm{E}+00$ & $6.62 \mathrm{E}-02$ \\
\hline $35 \%$ & $5.65 \mathrm{E}-05$ & $2.51 \mathrm{E}-03$ & $5.44 \mathrm{E}-02$ & $1.52 \mathrm{E}-03$ & $1.96 \mathrm{E}-05$ & $1.05 \mathrm{E}-02$ & $0.00 \mathrm{E}+00$ & $0.00 \mathrm{E}+00$ & $0.00 \mathrm{E}+00$ & $0.00 \mathrm{E}+00$ & $0.00 \mathrm{E}+00$ & $7.26 \mathrm{E}-02$ \\
\hline $40 \%$ & $5.93 \mathrm{E}-05$ & $2.74 \mathrm{E}-03$ & $6.15 \mathrm{E}-02$ & $1.57 \mathrm{E}-03$ & $2.08 \mathrm{E}-05$ & $1.12 \mathrm{E}-02$ & $0.00 \mathrm{E}+00$ & $0.00 \mathrm{E}+00$ & $0.00 \mathrm{E}+00$ & $0.00 \mathrm{E}+00$ & $0.00 \mathrm{E}+00$ & $7.99 \mathrm{E}-02$ \\
\hline $45 \%$ & $6.24 \mathrm{E}-05$ & 2.97E-03 & $7.00 \mathrm{E}-02$ & $1.62 \mathrm{E}-03$ & $2.23 \mathrm{E}-05$ & $1.19 \mathrm{E}-02$ & $0.00 \mathrm{E}+00$ & $0.00 \mathrm{E}+00$ & $0.00 \mathrm{E}+00$ & $0.00 \mathrm{E}+00$ & $0.00 \mathrm{E}+00$ & $8.80 \mathrm{E}-02$ \\
\hline $50 \%$ & $6.55 \mathrm{E}-05$ & $3.20 \mathrm{E}-03$ & $7.83 \mathrm{E}-02$ & $1.67 \mathrm{E}-03$ & $2.37 \mathrm{E}-05$ & $1.26 \mathrm{E}-02$ & $0.00 \mathrm{E}+00$ & $0.00 \mathrm{E}+00$ & $0.00 \mathrm{E}+00$ & $0.00 \mathrm{E}+00$ & $0.00 \mathrm{E}+00$ & $9.69 \mathrm{E}-02$ \\
\hline $55 \%$ & $6.89 \mathrm{E}-05$ & $3.46 \mathrm{E}-03$ & $8.76 \mathrm{E}-02$ & $1.72 \mathrm{E}-03$ & $2.51 \mathrm{E}-05$ & $1.32 \mathrm{E}-02$ & $0.00 \mathrm{E}+00$ & $0.00 \mathrm{E}+00$ & $0.00 \mathrm{E}+00$ & $0.00 \mathrm{E}+00$ & $0.00 \mathrm{E}+00$ & $1.07 \mathrm{E}-01$ \\
\hline $60 \%$ & $7.26 \mathrm{E}-05$ & $3.75 \mathrm{E}-03$ & $9.92 \mathrm{E}-02$ & $1.77 \mathrm{E}-03$ & $2.66 \mathrm{E}-05$ & $1.39 \mathrm{E}-02$ & $0.00 \mathrm{E}+00$ & $0.00 \mathrm{E}+00$ & $0.00 \mathrm{E}+00$ & $0.00 \mathrm{E}+00$ & $0.00 \mathrm{E}+00$ & $1.18 \mathrm{E}-01$ \\
\hline $65 \%$ & 7.67E-05 & $4.06 \mathrm{E}-03$ & $1.11 \mathrm{E}-01$ & $1.84 \mathrm{E}-03$ & $2.85 \mathrm{E}-05$ & $1.47 \mathrm{E}-02$ & $0.00 \mathrm{E}+00$ & $0.00 \mathrm{E}+00$ & $0.00 \mathrm{E}+00$ & $0.00 \mathrm{E}+00$ & $0.00 \mathrm{E}+00$ & $1.30 \mathrm{E}-01$ \\
\hline $70 \%$ & $8.16 \mathrm{E}-05$ & $4.39 \mathrm{E}-03$ & $1.26 \mathrm{E}-01$ & $1.91 \mathrm{E}-03$ & $3.05 \mathrm{E}-05$ & $1.55 \mathrm{E}-02$ & $0.00 \mathrm{E}+00$ & $0.00 \mathrm{E}+00$ & $0.00 \mathrm{E}+00$ & $0.00 \mathrm{E}+00$ & $0.00 \mathrm{E}+00$ & $1.44 \mathrm{E}-01$ \\
\hline $75 \%$ & $8.70 \mathrm{E}-05$ & $4.78 \mathrm{E}-03$ & $1.45 \mathrm{E}-01$ & $1.98 \mathrm{E}-03$ & $3.28 \mathrm{E}-05$ & $1.63 \mathrm{E}-02$ & $0.00 \mathrm{E}+00$ & $0.00 \mathrm{E}+00$ & $0.00 \mathrm{E}+00$ & $0.00 \mathrm{E}+00$ & $0.00 \mathrm{E}+00$ & $1.64 \mathrm{E}-01$ \\
\hline $80 \%$ & $9.35 \mathrm{E}-05$ & $5.26 \mathrm{E}-03$ & $1.69 \mathrm{E}-01$ & $2.06 \mathrm{E}-03$ & $3.57 \mathrm{E}-05$ & $1.73 \mathrm{E}-02$ & $0.00 \mathrm{E}+00$ & $0.00 \mathrm{E}+00$ & $0.00 \mathrm{E}+00$ & $0.00 \mathrm{E}+00$ & $0.00 \mathrm{E}+00$ & $1.88 \mathrm{E}-01$ \\
\hline $85 \%$ & $1.02 \mathrm{E}-04$ & $6.00 \mathrm{E}-03$ & $2.03 \mathrm{E}-01$ & $2.18 \mathrm{E}-03$ & $3.96 \mathrm{E}-05$ & $1.84 \mathrm{E}-02$ & $0.00 \mathrm{E}+00$ & $0.00 \mathrm{E}+00$ & $0.00 \mathrm{E}+00$ & $0.00 \mathrm{E}+00$ & $0.00 \mathrm{E}+00$ & $2.23 \mathrm{E}-01$ \\
\hline $90 \%$ & $1.14 \mathrm{E}-04$ & $6.97 \mathrm{E}-03$ & $2.51 \mathrm{E}-01$ & $2.35 \mathrm{E}-03$ & $4.52 \mathrm{E}-05$ & $1.97 \mathrm{E}-02$ & $0.00 \mathrm{E}+00$ & $0.00 \mathrm{E}+00$ & $0.00 \mathrm{E}+00$ & $0.00 \mathrm{E}+00$ & $0.00 \mathrm{E}+00$ & $2.70 \mathrm{E}-01$ \\
\hline $95 \%$ & $1.33 \mathrm{E}-04$ & $8.61 \mathrm{E}-03$ & $3.52 \mathrm{E}-01$ & $2.59 \mathrm{E}-03$ & $5.44 \mathrm{E}-05$ & $2.13 \mathrm{E}-02$ & $0.00 \mathrm{E}+00$ & $0.00 \mathrm{E}+00$ & $0.00 \mathrm{E}+00$ & $0.00 \mathrm{E}+00$ & $0.00 \mathrm{E}+00$ & $3.73 \mathrm{E}-01$ \\
\hline Mean & $7.23 \mathrm{E}-05$ & 3.79E-03 & $1.19 \mathrm{E}-01$ & $1.74 \mathrm{E}-03$ & 2.69E-05 & $1.26 \mathrm{E}-02$ & $6.47 \mathrm{E}-11$ & $1.72 \mathrm{E}-13$ & $2.49 \mathrm{E}-12$ & $2.39 \mathrm{E}-13$ & $3.84 \mathrm{E}-13$ & $1.37 \mathrm{E}-01$ \\
\hline Min & $7.59 \mathrm{E}-07$ & $9.27 \mathrm{E}-06$ & $2.85 \mathrm{E}-04$ & $1.19 \mathrm{E}-05$ & $4.10 \mathrm{E}-07$ & $1.46 \mathrm{E}-04$ & $0.00 \mathrm{E}+00$ & $0.00 \mathrm{E}+00$ & $0.00 \mathrm{E}+00$ & $0.00 \mathrm{E}+00$ & $0.00 \mathrm{E}+00$ & 4.63E-04 \\
\hline $\operatorname{Max}$ & $1.91 \mathrm{E}-04$ & $2.87 \mathrm{E}-02$ & $2.15 \mathrm{E}+00$ & $8.69 \mathrm{E}-03$ & $1.91 \mathrm{E}-04$ & $2.57 \mathrm{E}-02$ & $1.61 \mathrm{E}-07$ & $3.34 \mathrm{E}-10$ & $6.19 \mathrm{E}-09$ & $5.22 \mathrm{E}-10$ & $5.42 \mathrm{E}-10$ & $2.18 \mathrm{E}+00$ \\
\hline
\end{tabular}


TABLE C.51 Peak DSR Percentiles (mrem/yr per pCi/g) for Different Exposure Pathways for Pu-240 in Onsite Resident Scenario

\begin{tabular}{|c|c|c|c|c|c|c|c|c|c|c|c|c|c|c|}
\hline Percentile & External & $\begin{array}{c}\text { Inhalation } \\
\text { w/o Radon }\end{array}$ & $\begin{array}{l}\text { Radon } \\
\text { (WI) }\end{array}$ & $\begin{array}{c}\text { Plant } \\
\text { Ingestion } \\
\text { (WI) }\end{array}$ & $\begin{array}{c}\text { Meat } \\
\text { Ingestion } \\
\text { (WI) }\end{array}$ & $\begin{array}{c}\text { Milk } \\
\text { Ingestion } \\
\text { (WI) }\end{array}$ & $\begin{array}{c}\text { Soil } \\
\text { Ingestion } \\
\end{array}$ & $\begin{array}{c}\text { Water } \\
\text { Ingestion }\end{array}$ & $\begin{array}{l}\text { Aquatic } \\
\text { Foods }\end{array}$ & $\begin{array}{l}\text { Radon } \\
\text { (WD) }\end{array}$ & $\begin{array}{c}\text { Plant } \\
\text { Ingestion } \\
\text { (WD) }\end{array}$ & $\begin{array}{c}\text { Meat } \\
\text { Ingestion } \\
\text { (WD) } \\
\end{array}$ & $\begin{array}{c}\text { Milk } \\
\text { Ingestion } \\
\text { (WD) }\end{array}$ & $\begin{array}{c}\text { All } \\
\text { Pathways } \\
\end{array}$ \\
\hline $5 \%$ & $1.61 \mathrm{E}-05$ & $9.19 \mathrm{E}-04$ & $6.31 \mathrm{E}-16$ & $1.70 \mathrm{E}-02$ & $1.11 \mathrm{E}-03$ & $1.04 \mathrm{E}-05$ & $3.81 \mathrm{E}-03$ & $0.00 \mathrm{E}+00$ & $0.00 \mathrm{E}+00$ & $0.00 \mathrm{E}+00$ & $0.00 \mathrm{E}+00$ & $0.00 \mathrm{E}+00$ & $0.00 \mathrm{E}+00$ & 3.33E-02 \\
\hline $10 \%$ & $1.80 \mathrm{E}-05$ & $1.20 \mathrm{E}-03$ & $8.11 \mathrm{E}-16$ & $2.37 \mathrm{E}-02$ & $1.22 \mathrm{E}-03$ & $1.25 \mathrm{E}-05$ & $5.46 \mathrm{E}-03$ & $0.00 \mathrm{E}+00$ & $0.00 \mathrm{E}+00$ & $0.00 \mathrm{E}+00$ & $0.00 \mathrm{E}+00$ & $0.00 \mathrm{E}+00$ & $0.00 \mathrm{E}+00$ & 3.97E-02 \\
\hline $15 \%$ & $1.95 \mathrm{E}-05$ & $1.40 \mathrm{E}-03$ & $8.82 \mathrm{E}-16$ & $2.97 \mathrm{E}-02$ & $1.29 \mathrm{E}-03$ & $1.42 \mathrm{E}-05$ & $6.77 \mathrm{E}-03$ & $0.00 \mathrm{E}+00$ & $0.00 \mathrm{E}+00$ & $0.00 \mathrm{E}+00$ & $0.00 \mathrm{E}+00$ & $0.00 \mathrm{E}+00$ & $0.00 \mathrm{E}+00$ & 4.62E-02 \\
\hline $20 \%$ & $2.09 \mathrm{E}-05$ & $1.57 \mathrm{E}-03$ & $9.26 \mathrm{E}-16$ & $3.57 \mathrm{E}-02$ & $1.36 \mathrm{E}-03$ & $1.56 \mathrm{E}-05$ & $7.80 \mathrm{E}-03$ & $0.00 \mathrm{E}+00$ & $0.00 \mathrm{E}+00$ & $0.00 \mathrm{E}+00$ & $0.00 \mathrm{E}+00$ & $0.00 \mathrm{E}+00$ & $0.00 \mathrm{E}+00$ & $5.26 \mathrm{E}-02$ \\
\hline $25 \%$ & 2.23E-05 & $1.73 \mathrm{E}-03$ & $9.56 \mathrm{E}-16$ & 4.15E-02 & $1.41 \mathrm{E}-03$ & $1.69 \mathrm{E}-05$ & $8.74 \mathrm{E}-03$ & $0.00 \mathrm{E}+00$ & $0.00 \mathrm{E}+00$ & $0.00 \mathrm{E}+00$ & $0.00 \mathrm{E}+00$ & $0.00 \mathrm{E}+00$ & $0.00 \mathrm{E}+00$ & $5.88 \mathrm{E}-02$ \\
\hline $30 \%$ & $2.34 \mathrm{E}-05$ & $1.88 \mathrm{E}-03$ & $9.82 \mathrm{E}-16$ & $4.75 \mathrm{E}-02$ & $1.46 \mathrm{E}-03$ & $1.83 \mathrm{E}-05$ & $9.59 \mathrm{E}-03$ & $0.00 \mathrm{E}+00$ & $0.00 \mathrm{E}+00$ & $0.00 \mathrm{E}+00$ & $0.00 \mathrm{E}+00$ & $0.00 \mathrm{E}+00$ & $0.00 \mathrm{E}+00$ & $6.50 \mathrm{E}-02$ \\
\hline $35 \%$ & $2.48 \mathrm{E}-05$ & $2.08 \mathrm{E}-03$ & $1.01 \mathrm{E}-15$ & $5.41 \mathrm{E}-02$ & $1.51 \mathrm{E}-03$ & $1.96 \mathrm{E}-05$ & $1.04 \mathrm{E}-02$ & $0.00 \mathrm{E}+00$ & $0.00 \mathrm{E}+00$ & $0.00 \mathrm{E}+00$ & $0.00 \mathrm{E}+00$ & $0.00 \mathrm{E}+00$ & $0.00 \mathrm{E}+00$ & 7.12E-02 \\
\hline $40 \%$ & $2.60 \mathrm{E}-05$ & $2.26 \mathrm{E}-03$ & $1.03 \mathrm{E}-15$ & $6.10 \mathrm{E}-02$ & $1.56 \mathrm{E}-03$ & $2.08 \mathrm{E}-05$ & $1.11 \mathrm{E}-02$ & $0.00 \mathrm{E}+00$ & $0.00 \mathrm{E}+00$ & $0.00 \mathrm{E}+00$ & $0.00 \mathrm{E}+00$ & $0.00 \mathrm{E}+00$ & $0.00 \mathrm{E}+00$ & 7.86E-02 \\
\hline $45 \%$ & $2.74 \mathrm{E}-05$ & $2.42 \mathrm{E}-03$ & $1.04 \mathrm{E}-15$ & $6.91 \mathrm{E}-02$ & $1.61 \mathrm{E}-03$ & $2.21 \mathrm{E}-05$ & $1.18 \mathrm{E}-02$ & $0.00 \mathrm{E}+00$ & $0.00 \mathrm{E}+00$ & $0.00 \mathrm{E}+00$ & $0.00 \mathrm{E}+00$ & $0.00 \mathrm{E}+00$ & $0.00 \mathrm{E}+00$ & $8.66 \mathrm{E}-02$ \\
\hline $50 \%$ & $2.88 \mathrm{E}-05$ & $2.63 \mathrm{E}-03$ & $1.06 \mathrm{E}-15$ & 7.78E-02 & $1.66 \mathrm{E}-03$ & $2.35 \mathrm{E}-05$ & $1.25 \mathrm{E}-02$ & $0.00 \mathrm{E}+00$ & $0.00 \mathrm{E}+00$ & $0.00 \mathrm{E}+00$ & $0.00 \mathrm{E}+00$ & $0.00 \mathrm{E}+00$ & $0.00 \mathrm{E}+00$ & $9.51 \mathrm{E}-02$ \\
\hline $55 \%$ & 3.03E-05 & $2.80 \mathrm{E}-03$ & $1.08 \mathrm{E}-15$ & $8.70 \mathrm{E}-02$ & $1.71 \mathrm{E}-03$ & $2.50 \mathrm{E}-05$ & $1.32 \mathrm{E}-02$ & $0.00 \mathrm{E}+00$ & $0.00 \mathrm{E}+00$ & $0.00 \mathrm{E}+00$ & $0.00 \mathrm{E}+00$ & $0.00 \mathrm{E}+00$ & $0.00 \mathrm{E}+00$ & $1.05 \mathrm{E}-01$ \\
\hline $60 \%$ & $3.20 \mathrm{E}-05$ & $3.01 \mathrm{E}-03$ & $1.10 \mathrm{E}-15$ & $9.78 \mathrm{E}-02$ & $1.77 \mathrm{E}-03$ & $2.66 \mathrm{E}-05$ & $1.39 \mathrm{E}-02$ & $0.00 \mathrm{E}+00$ & $0.00 \mathrm{E}+00$ & $0.00 \mathrm{E}+00$ & $0.00 \mathrm{E}+00$ & $0.00 \mathrm{E}+00$ & $0.00 \mathrm{E}+00$ & $1.16 \mathrm{E}-01$ \\
\hline $65 \%$ & $3.37 \mathrm{E}-05$ & $3.20 \mathrm{E}-03$ & $1.12 \mathrm{E}-15$ & $1.10 \mathrm{E}-01$ & $1.82 \mathrm{E}-03$ & $2.83 \mathrm{E}-05$ & $1.46 \mathrm{E}-02$ & $0.00 \mathrm{E}+00$ & $0.00 \mathrm{E}+00$ & $0.00 \mathrm{E}+00$ & $0.00 \mathrm{E}+00$ & $0.00 \mathrm{E}+00$ & $0.00 \mathrm{E}+00$ & $1.28 \mathrm{E}-01$ \\
\hline $70 \%$ & 3.57E-05 & $3.42 \mathrm{E}-03$ & $1.14 \mathrm{E}-15$ & $1.25 \mathrm{E}-01$ & $1.88 \mathrm{E}-03$ & $3.04 \mathrm{E}-05$ & $1.54 \mathrm{E}-02$ & $0.00 \mathrm{E}+00$ & $0.00 \mathrm{E}+00$ & $0.00 \mathrm{E}+00$ & $0.00 \mathrm{E}+00$ & $0.00 \mathrm{E}+00$ & $0.00 \mathrm{E}+00$ & $1.44 \mathrm{E}-01$ \\
\hline $75 \%$ & $3.82 \mathrm{E}-05$ & $3.70 \mathrm{E}-03$ & $1.16 \mathrm{E}-15$ & $1.44 \mathrm{E}-01$ & $1.96 \mathrm{E}-03$ & $3.28 \mathrm{E}-05$ & $1.62 \mathrm{E}-02$ & $0.00 \mathrm{E}+00$ & $0.00 \mathrm{E}+00$ & $0.00 \mathrm{E}+00$ & $0.00 \mathrm{E}+00$ & $0.00 \mathrm{E}+00$ & $0.00 \mathrm{E}+00$ & $1.62 \mathrm{E}-01$ \\
\hline $80 \%$ & 4.12E-05 & $4.02 \mathrm{E}-03$ & $1.18 \mathrm{E}-15$ & $1.67 \mathrm{E}-01$ & $2.04 \mathrm{E}-03$ & $3.56 \mathrm{E}-05$ & $1.72 \mathrm{E}-02$ & $0.00 \mathrm{E}+00$ & $0.00 \mathrm{E}+00$ & $0.00 \mathrm{E}+00$ & $0.00 \mathrm{E}+00$ & $0.00 \mathrm{E}+00$ & $0.00 \mathrm{E}+00$ & $1.85 \mathrm{E}-01$ \\
\hline $85 \%$ & 4.47E-05 & $4.40 \mathrm{E}-03$ & $1.21 \mathrm{E}-15$ & $1.99 \mathrm{E}-01$ & $2.15 \mathrm{E}-03$ & 3.92E-05 & $1.83 \mathrm{E}-02$ & $0.00 \mathrm{E}+00$ & $0.00 \mathrm{E}+00$ & $0.00 \mathrm{E}+00$ & $0.00 \mathrm{E}+00$ & $0.00 \mathrm{E}+00$ & $0.00 \mathrm{E}+00$ & $2.18 \mathrm{E}-01$ \\
\hline $90 \%$ & $4.99 \mathrm{E}-05$ & $4.94 \mathrm{E}-03$ & $1.24 \mathrm{E}-15$ & $2.49 \mathrm{E}-01$ & $2.31 \mathrm{E}-03$ & $4.45 \mathrm{E}-05$ & $1.95 \mathrm{E}-02$ & $0.00 \mathrm{E}+00$ & $0.00 \mathrm{E}+00$ & $0.00 \mathrm{E}+00$ & $0.00 \mathrm{E}+00$ & $0.00 \mathrm{E}+00$ & $0.00 \mathrm{E}+00$ & $2.69 \mathrm{E}-01$ \\
\hline $95 \%$ & $5.85 \mathrm{E}-05$ & $5.83 \mathrm{E}-03$ & $1.29 \mathrm{E}-15$ & $3.51 \mathrm{E}-01$ & $2.58 \mathrm{E}-03$ & $5.34 \mathrm{E}-05$ & $2.12 \mathrm{E}-02$ & $0.00 \mathrm{E}+00$ & $0.00 \mathrm{E}+00$ & $0.00 \mathrm{E}+00$ & $0.00 \mathrm{E}+00$ & $0.00 \mathrm{E}+00$ & $0.00 \mathrm{E}+00$ & 3.71E-01 \\
\hline Mean & $3.17 \mathrm{E}-05$ & $2.89 \mathrm{E}-03$ & $4.11 \mathrm{E}-15$ & $1.18 \mathrm{E}-01$ & $1.73 \mathrm{E}-03$ & $2.66 \mathrm{E}-05$ & $1.25 \mathrm{E}-02$ & 7.34E-04 & $6.91 \mathrm{E}-06$ & $9.15 \mathrm{E}-20$ & 2.82E-05 & $5.08 \mathrm{E}-07$ & $1.25 \mathrm{E}-08$ & $1.35 \mathrm{E}-01$ \\
\hline Min & $4.92 \mathrm{E}-12$ & $3.16 \mathrm{E}-11$ & 3.07E-17 & $1.82 \mathrm{E}-09$ & $2.96 \mathrm{E}-10$ & $2.76 \mathrm{E}-10$ & $2.11 \mathrm{E}-10$ & $0.00 \mathrm{E}+00$ & $0.00 \mathrm{E}+00$ & $0.00 \mathrm{E}+00$ & $0.00 \mathrm{E}+00$ & $0.00 \mathrm{E}+00$ & $0.00 \mathrm{E}+00$ & 2.64E-09 \\
\hline Max & $8.41 \mathrm{E}-05$ & $1.38 \mathrm{E}-02$ & $3.74 \mathrm{E}-12$ & $2.07 \mathrm{E}+00$ & $6.32 \mathrm{E}-03$ & $1.42 \mathrm{E}-04$ & $2.55 \mathrm{E}-02$ & $1.25 \mathrm{E}+00$ & $1.25 \mathrm{E}-02$ & $1.45 \mathrm{E}-16$ & 4.79E-02 & 7.74E-04 & $1.73 \mathrm{E}-05$ & $2.10 \mathrm{E}+00$ \\
\hline
\end{tabular}




\section{TABLE C.52 Peak DSR Percentiles (mrem/yr per pCi/g) for Different Exposure Pathways for Sr-90 in Onsite Resident Scenario}

\begin{tabular}{|c|c|c|c|c|c|c|c|c|c|c|c|c|}
\hline Percentile & External & $\begin{array}{l}\text { Inhalation } \\
\text { w/o Radon }\end{array}$ & $\begin{array}{c}\text { Plant } \\
\text { Ingestion } \\
(\mathrm{WI})\end{array}$ & $\begin{array}{c}\text { Meat } \\
\text { Ingestion } \\
(\mathrm{WI})\end{array}$ & $\begin{array}{c}\text { Milk } \\
\text { Ingestion } \\
(\mathrm{WI})\end{array}$ & $\begin{array}{c}\text { Soil } \\
\text { Ingestion }\end{array}$ & $\begin{array}{c}\text { Water } \\
\text { Ingestion }\end{array}$ & $\begin{array}{c}\text { Aquatic } \\
\text { Foods }\end{array}$ & $\begin{array}{c}\text { Plant } \\
\text { Ingestion } \\
\text { (WD) }\end{array}$ & $\begin{array}{c}\text { Meat } \\
\text { Ingestion } \\
\text { (WD) }\end{array}$ & $\begin{array}{c}\text { Milk } \\
\text { Ingestion } \\
\text { (WD) }\end{array}$ & $\begin{array}{c}\text { All } \\
\text { Pathways }\end{array}$ \\
\hline $5 \%$ & $1.35 \mathrm{E}-05$ & $5.27 \mathrm{E}-09$ & $3.13 \mathrm{E}-03$ & 8.29E-04 & $1.96 \mathrm{E}-04$ & $1.79 \mathrm{E}-06$ & $0.00 \mathrm{E}+00$ & $0.00 \mathrm{E}+00$ & $0.00 \mathrm{E}+00$ & $0.00 \mathrm{E}+00$ & $0.00 \mathrm{E}+00$ & $4.42 \mathrm{E}-03$ \\
\hline $10 \%$ & $1.05 \mathrm{E}-04$ & 3.67E-08 & $2.06 \mathrm{E}-02$ & 5.19E-03 & $1.17 \mathrm{E}-03$ & $1.27 \mathrm{E}-05$ & $0.00 \mathrm{E}+00$ & $0.00 \mathrm{E}+00$ & $0.00 \mathrm{E}+00$ & $0.00 \mathrm{E}+00$ & $0.00 \mathrm{E}+00$ & $2.97 \mathrm{E}-02$ \\
\hline $15 \%$ & 2.37E-04 & $7.46 \mathrm{E}-08$ & $3.82 \mathrm{E}-02$ & $9.20 \mathrm{E}-03$ & $2.26 \mathrm{E}-03$ & $2.57 \mathrm{E}-05$ & $0.00 \mathrm{E}+00$ & $0.00 \mathrm{E}+00$ & $0.00 \mathrm{E}+00$ & $0.00 \mathrm{E}+00$ & $0.00 \mathrm{E}+00$ & $5.30 \mathrm{E}-02$ \\
\hline $20 \%$ & $3.58 \mathrm{E}-04$ & $1.06 \mathrm{E}-07$ & 5.33E-02 & $1.34 \mathrm{E}-02$ & $3.15 \mathrm{E}-03$ & $3.85 \mathrm{E}-05$ & $0.00 \mathrm{E}+00$ & $0.00 \mathrm{E}+00$ & $0.00 \mathrm{E}+00$ & $0.00 \mathrm{E}+00$ & $0.00 \mathrm{E}+00$ & $7.31 \mathrm{E}-02$ \\
\hline $25 \%$ & $4.44 \mathrm{E}-04$ & $1.37 \mathrm{E}-07$ & $7.15 \mathrm{E}-02$ & $1.75 \mathrm{E}-02$ & $4.14 \mathrm{E}-03$ & $5.10 \mathrm{E}-05$ & $0.00 \mathrm{E}+00$ & $0.00 \mathrm{E}+00$ & $0.00 \mathrm{E}+00$ & $0.00 \mathrm{E}+00$ & $0.00 \mathrm{E}+00$ & $9.77 \mathrm{E}-02$ \\
\hline $30 \%$ & $5.01 \mathrm{E}-04$ & $1.69 \mathrm{E}-07$ & 8.81E-02 & 2.17E-02 & $5.06 \mathrm{E}-03$ & $6.22 \mathrm{E}-05$ & $0.00 \mathrm{E}+00$ & $0.00 \mathrm{E}+00$ & $0.00 \mathrm{E}+00$ & $0.00 \mathrm{E}+00$ & $0.00 \mathrm{E}+00$ & $1.18 \mathrm{E}-01$ \\
\hline $35 \%$ & $5.53 \mathrm{E}-04$ & $1.91 \mathrm{E}-07$ & $1.06 \mathrm{E}-01$ & $2.65 \mathrm{E}-02$ & $6.18 \mathrm{E}-03$ & $7.26 \mathrm{E}-05$ & $0.00 \mathrm{E}+00$ & $0.00 \mathrm{E}+00$ & $0.00 \mathrm{E}+00$ & $0.00 \mathrm{E}+00$ & $0.00 \mathrm{E}+00$ & $1.44 \mathrm{E}-01$ \\
\hline $40 \%$ & $6.02 \mathrm{E}-04$ & $2.17 \mathrm{E}-07$ & $1.27 \mathrm{E}-01$ & $3.17 \mathrm{E}-02$ & $7.39 \mathrm{E}-03$ & $8.34 \mathrm{E}-05$ & $0.00 \mathrm{E}+00$ & $0.00 \mathrm{E}+00$ & $0.00 \mathrm{E}+00$ & $0.00 \mathrm{E}+00$ & $0.00 \mathrm{E}+00$ & $1.70 \mathrm{E}-01$ \\
\hline $45 \%$ & $6.48 \mathrm{E}-04$ & $2.45 \mathrm{E}-07$ & $1.51 \mathrm{E}-01$ & 3.79E-02 & $8.78 \mathrm{E}-03$ & $9.28 \mathrm{E}-05$ & $0.00 \mathrm{E}+00$ & $0.00 \mathrm{E}+00$ & $0.00 \mathrm{E}+00$ & $0.00 \mathrm{E}+00$ & $0.00 \mathrm{E}+00$ & $2.04 \mathrm{E}-01$ \\
\hline $50 \%$ & $6.92 \mathrm{E}-04$ & $2.77 \mathrm{E}-07$ & $1.74 \mathrm{E}-01$ & 4.39E-02 & $1.04 \mathrm{E}-02$ & $1.02 \mathrm{E}-04$ & $0.00 \mathrm{E}+00$ & $0.00 \mathrm{E}+00$ & $0.00 \mathrm{E}+00$ & $0.00 \mathrm{E}+00$ & $0.00 \mathrm{E}+00$ & $2.35 \mathrm{E}-01$ \\
\hline $55 \%$ & $7.48 \mathrm{E}-04$ & $3.07 \mathrm{E}-07$ & $2.07 \mathrm{E}-01$ & $5.06 \mathrm{E}-02$ & $1.24 \mathrm{E}-02$ & $1.10 \mathrm{E}-04$ & $0.00 \mathrm{E}+00$ & $0.00 \mathrm{E}+00$ & $0.00 \mathrm{E}+00$ & $0.00 \mathrm{E}+00$ & $0.00 \mathrm{E}+00$ & $2.79 \mathrm{E}-01$ \\
\hline $60 \%$ & 7.99E-04 & $3.40 \mathrm{E}-07$ & 2.37E-01 & 5.92E-02 & $1.46 \mathrm{E}-02$ & $1.21 \mathrm{E}-04$ & $0.00 \mathrm{E}+00$ & $0.00 \mathrm{E}+00$ & $0.00 \mathrm{E}+00$ & $0.00 \mathrm{E}+00$ & $0.00 \mathrm{E}+00$ & $3.21 \mathrm{E}-01$ \\
\hline $65 \%$ & 8.54E-04 & 3.74E-07 & 2.77E-01 & $6.97 \mathrm{E}-02$ & $1.69 \mathrm{E}-02$ & $1.30 \mathrm{E}-04$ & $0.00 \mathrm{E}+00$ & $0.00 \mathrm{E}+00$ & $0.00 \mathrm{E}+00$ & $0.00 \mathrm{E}+00$ & $0.00 \mathrm{E}+00$ & $3.72 \mathrm{E}-01$ \\
\hline $70 \%$ & $9.23 \mathrm{E}-04$ & $4.16 \mathrm{E}-07$ & $3.21 \mathrm{E}-01$ & $8.25 \mathrm{E}-02$ & $2.00 \mathrm{E}-02$ & $1.39 \mathrm{E}-04$ & $0.00 \mathrm{E}+00$ & $0.00 \mathrm{E}+00$ & $0.00 \mathrm{E}+00$ & $0.00 \mathrm{E}+00$ & $0.00 \mathrm{E}+00$ & $4.25 \mathrm{E}-01$ \\
\hline $75 \%$ & $9.90 \mathrm{E}-04$ & 4.63E-07 & $3.75 \mathrm{E}-01$ & $9.87 \mathrm{E}-02$ & $2.40 \mathrm{E}-02$ & $1.49 \mathrm{E}-04$ & $0.00 \mathrm{E}+00$ & $0.00 \mathrm{E}+00$ & $0.00 \mathrm{E}+00$ & $0.00 \mathrm{E}+00$ & $0.00 \mathrm{E}+00$ & $5.03 \mathrm{E}-01$ \\
\hline $80 \%$ & $1.08 \mathrm{E}-03$ & $5.15 \mathrm{E}-07$ & $4.55 \mathrm{E}-01$ & $1.19 \mathrm{E}-01$ & $2.92 \mathrm{E}-02$ & $1.61 \mathrm{E}-04$ & $0.00 \mathrm{E}+00$ & $0.00 \mathrm{E}+00$ & $0.00 \mathrm{E}+00$ & $0.00 \mathrm{E}+00$ & $0.00 \mathrm{E}+00$ & $6.07 \mathrm{E}-01$ \\
\hline $85 \%$ & $1.18 \mathrm{E}-03$ & $5.83 \mathrm{E}-07$ & $5.69 \mathrm{E}-01$ & $1.50 \mathrm{E}-01$ & $3.67 \mathrm{E}-02$ & $1.76 \mathrm{E}-04$ & $0.00 \mathrm{E}+00$ & $0.00 \mathrm{E}+00$ & $0.00 \mathrm{E}+00$ & $0.00 \mathrm{E}+00$ & $0.00 \mathrm{E}+00$ & $7.50 \mathrm{E}-01$ \\
\hline $90 \%$ & $1.33 \mathrm{E}-03$ & $6.92 \mathrm{E}-07$ & $7.29 \mathrm{E}-01$ & $1.94 \mathrm{E}-01$ & $4.76 \mathrm{E}-02$ & $1.93 \mathrm{E}-04$ & $0.00 \mathrm{E}+00$ & $0.00 \mathrm{E}+00$ & $0.00 \mathrm{E}+00$ & $0.00 \mathrm{E}+00$ & $0.00 \mathrm{E}+00$ & $9.71 \mathrm{E}-01$ \\
\hline $95 \%$ & $1.59 \mathrm{E}-03$ & $8.96 \mathrm{E}-07$ & $1.06 \mathrm{E}+00$ & $2.99 \mathrm{E}-01$ & $7.58 \mathrm{E}-02$ & $2.15 \mathrm{E}-04$ & $0.00 \mathrm{E}+00$ & $0.00 \mathrm{E}+00$ & $0.00 \mathrm{E}+00$ & $0.00 \mathrm{E}+00$ & $0.00 \mathrm{E}+00$ & $1.44 \mathrm{E}+00$ \\
\hline Mean & 7.37E-04 & $3.39 \mathrm{E}-07$ & $3.11 \mathrm{E}-01$ & $8.42 \mathrm{E}-02$ & $2.08 \mathrm{E}-02$ & $1.03 \mathrm{E}-04$ & $2.75 \mathrm{E}-04$ & $3.89 \mathrm{E}-06$ & $1.15 \mathrm{E}-05$ & $2.19 \mathrm{E}-05$ & $1.15 \mathrm{E}-05$ & 4.17E-01 \\
\hline Min & $0.00 \mathrm{E}+00$ & $0.00 \mathrm{E}+00$ & $3.30 \mathrm{E}-29$ & $8.02 \mathrm{E}-30$ & $2.42 \mathrm{E}-30$ & $0.00 \mathrm{E}+00$ & $0.00 \mathrm{E}+00$ & $0.00 \mathrm{E}+00$ & $0.00 \mathrm{E}+00$ & $0.00 \mathrm{E}+00$ & $0.00 \mathrm{E}+00$ & 4.34E-29 \\
\hline Max & $2.57 \mathrm{E}-03$ & $2.91 \mathrm{E}-06$ & $5.45 \mathrm{E}+00$ & $1.95 \mathrm{E}+00$ & $4.71 \mathrm{E}-01$ & $2.85 \mathrm{E}-04$ & $1.93 \mathrm{E}-01$ & $3.40 \mathrm{E}-03$ & $7.74 \mathrm{E}-03$ & $1.77 \mathrm{E}-02$ & $8.91 \mathrm{E}-03$ & $7.38 \mathrm{E}+00$ \\
\hline
\end{tabular}




\section{TABLE C.53 Peak DSR Percentiles (mrem/yr per pCi/g) for Different Exposure Pathways for Tc-99 in Onsite Resident Scenario}

\begin{tabular}{|c|c|c|c|c|c|c|c|c|c|c|c|c|}
\hline Percentile & External & $\begin{array}{c}\text { Inhalation } \\
\text { w/o Radon }\end{array}$ & $\begin{array}{c}\text { Plant } \\
\text { Ingestion } \\
(\mathrm{WI})\end{array}$ & $\begin{array}{c}\text { Meat } \\
\text { Ingestion } \\
(\mathrm{WI})\end{array}$ & $\begin{array}{c}\text { Milk } \\
\text { Ingestion } \\
(\mathrm{WI})\end{array}$ & $\begin{array}{c}\text { Soil } \\
\text { Ingestion } \\
\end{array}$ & $\begin{array}{c}\text { Water } \\
\text { Ingestion }\end{array}$ & $\begin{array}{c}\text { Aquatic } \\
\text { Foods }\end{array}$ & $\begin{array}{c}\text { Plant } \\
\text { Ingestion } \\
\text { (WD) }\end{array}$ & $\begin{array}{c}\text { Meat } \\
\text { Ingestion } \\
\text { (WD) }\end{array}$ & $\begin{array}{c}\text { Milk } \\
\text { Ingestion } \\
\text { (WD) }\end{array}$ & $\begin{array}{c}\text { All } \\
\text { Pathways }\end{array}$ \\
\hline $5 \%$ & $6.58 \mathrm{E}-13$ & $7.02 \mathrm{E}-15$ & $2.00 \mathrm{E}-08$ & $4.44 \mathrm{E}-11$ & $5.21 \mathrm{E}-10$ & $6.19 \mathrm{E}-13$ & $0.00 \mathrm{E}+00$ & $0.00 \mathrm{E}+00$ & $0.00 \mathrm{E}+00$ & $0.00 \mathrm{E}+00$ & $0.00 \mathrm{E}+00$ & $1.60 \mathrm{E}-05$ \\
\hline $10 \%$ & $1.56 \mathrm{E}-12$ & $1.75 \mathrm{E}-14$ & 4.79E-08 & $1.12 \mathrm{E}-10$ & $1.26 \mathrm{E}-09$ & $1.56 \mathrm{E}-12$ & $0.00 \mathrm{E}+00$ & $0.00 \mathrm{E}+00$ & $0.00 \mathrm{E}+00$ & $0.00 \mathrm{E}+00$ & $0.00 \mathrm{E}+00$ & $4.02 \mathrm{E}-05$ \\
\hline $15 \%$ & $2.89 \mathrm{E}-12$ & $3.48 \mathrm{E}-14$ & $9.25 \mathrm{E}-08$ & $2.25 \mathrm{E}-10$ & $2.57 \mathrm{E}-09$ & $3.09 \mathrm{E}-12$ & $0.00 \mathrm{E}+00$ & $0.00 \mathrm{E}+00$ & $0.00 \mathrm{E}+00$ & $0.00 \mathrm{E}+00$ & $0.00 \mathrm{E}+00$ & $8.08 \mathrm{E}-05$ \\
\hline $20 \%$ & $5.07 \mathrm{E}-12$ & $5.84 \mathrm{E}-14$ & $1.58 \mathrm{E}-07$ & $3.97 \mathrm{E}-10$ & 4.45E-09 & $5.25 \mathrm{E}-12$ & $0.00 \mathrm{E}+00$ & $0.00 \mathrm{E}+00$ & $0.00 \mathrm{E}+00$ & $0.00 \mathrm{E}+00$ & $0.00 \mathrm{E}+00$ & $1.58 \mathrm{E}-04$ \\
\hline $25 \%$ & $7.82 \mathrm{E}-12$ & $9.38 \mathrm{E}-14$ & $2.69 \mathrm{E}-07$ & $6.72 \mathrm{E}-10$ & $7.29 \mathrm{E}-09$ & $8.72 \mathrm{E}-12$ & $0.00 \mathrm{E}+00$ & $0.00 \mathrm{E}+00$ & $0.00 \mathrm{E}+00$ & $0.00 \mathrm{E}+00$ & $0.00 \mathrm{E}+00$ & $5.40 \mathrm{E}-04$ \\
\hline $30 \%$ & $1.26 \mathrm{E}-11$ & $1.47 \mathrm{E}-13$ & $4.40 \mathrm{E}-07$ & $1.10 \mathrm{E}-09$ & $1.26 \mathrm{E}-08$ & $1.30 \mathrm{E}-11$ & $1.41 \mathrm{E}-03$ & $1.85 \mathrm{E}-06$ & $1.27 \mathrm{E}-04$ & 8.90E-07 & $2.34 \mathrm{E}-05$ & $1.68 \mathrm{E}-03$ \\
\hline $35 \%$ & $1.88 \mathrm{E}-11$ & $2.36 \mathrm{E}-13$ & 7.17E-07 & $1.71 \mathrm{E}-09$ & $2.06 \mathrm{E}-08$ & $2.02 \mathrm{E}-11$ & $2.91 \mathrm{E}-03$ & 5.19E-06 & 2.69E-04 & $2.00 \mathrm{E}-06$ & $5.30 \mathrm{E}-05$ & $3.40 \mathrm{E}-03$ \\
\hline $40 \%$ & $2.97 \mathrm{E}-11$ & $3.68 \mathrm{E}-13$ & $1.13 \mathrm{E}-06$ & $2.62 \mathrm{E}-09$ & $3.40 \mathrm{E}-08$ & $3.07 \mathrm{E}-11$ & $5.03 \mathrm{E}-03$ & $9.42 \mathrm{E}-06$ & $4.57 \mathrm{E}-04$ & $3.57 \mathrm{E}-06$ & $8.96 \mathrm{E}-05$ & $5.71 \mathrm{E}-03$ \\
\hline $45 \%$ & $4.53 \mathrm{E}-11$ & $5.64 \mathrm{E}-13$ & $1.71 \mathrm{E}-06$ & 4.10E-09 & $5.20 \mathrm{E}-08$ & $4.63 \mathrm{E}-11$ & $7.51 \mathrm{E}-03$ & $1.48 \mathrm{E}-05$ & 7.23E-04 & 5.83E-06 & $1.41 \mathrm{E}-04$ & $8.61 \mathrm{E}-03$ \\
\hline $50 \%$ & $7.16 \mathrm{E}-11$ & 8.30E-13 & $2.66 \mathrm{E}-06$ & $6.57 \mathrm{E}-09$ & $7.82 \mathrm{E}-08$ & $7.10 \mathrm{E}-11$ & $1.13 \mathrm{E}-02$ & $2.29 \mathrm{E}-05$ & $1.11 \mathrm{E}-03$ & $9.07 \mathrm{E}-06$ & $2.11 \mathrm{E}-04$ & $1.29 \mathrm{E}-02$ \\
\hline $55 \%$ & $1.11 \mathrm{E}-10$ & $1.32 \mathrm{E}-12$ & 4.27E-06 & $1.07 \mathrm{E}-08$ & $1.26 \mathrm{E}-07$ & $1.13 \mathrm{E}-10$ & $1.70 \mathrm{E}-02$ & 3.39E-05 & $1.59 \mathrm{E}-03$ & $1.32 \mathrm{E}-05$ & $3.23 \mathrm{E}-04$ & $1.95 \mathrm{E}-02$ \\
\hline $60 \%$ & $1.70 \mathrm{E}-10$ & $2.01 \mathrm{E}-12$ & $6.58 \mathrm{E}-06$ & $1.75 \mathrm{E}-08$ & $2.02 \mathrm{E}-07$ & $1.71 \mathrm{E}-10$ & $2.43 \mathrm{E}-02$ & $5.10 \mathrm{E}-05$ & $2.33 \mathrm{E}-03$ & $1.86 \mathrm{E}-05$ & $4.81 \mathrm{E}-04$ & $2.79 \mathrm{E}-02$ \\
\hline $65 \%$ & $2.69 \mathrm{E}-10$ & 3.34E-12 & $1.04 \mathrm{E}-05$ & $2.68 \mathrm{E}-08$ & $3.16 \mathrm{E}-07$ & $2.71 \mathrm{E}-10$ & $3.52 \mathrm{E}-02$ & 7.30E-05 & $3.34 \mathrm{E}-03$ & $2.56 \mathrm{E}-05$ & 7.03E-04 & $3.98 \mathrm{E}-02$ \\
\hline $70 \%$ & 4.23E-10 & $5.28 \mathrm{E}-12$ & $1.64 \mathrm{E}-05$ & 4.20E-08 & 5.09E-07 & 4.32E-10 & 4.95E-02 & $1.04 \mathrm{E}-04$ & 4.72E-03 & 3.60E-05 & $9.62 \mathrm{E}-04$ & $5.62 \mathrm{E}-02$ \\
\hline $75 \%$ & $6.36 \mathrm{E}-10$ & $8.24 \mathrm{E}-12$ & $2.55 \mathrm{E}-05$ & $6.56 \mathrm{E}-08$ & 7.92E-07 & $7.00 \mathrm{E}-10$ & $6.82 \mathrm{E}-02$ & $1.57 \mathrm{E}-04$ & $6.45 \mathrm{E}-03$ & 5.07E-05 & $1.33 \mathrm{E}-03$ & 7.78E-02 \\
\hline $80 \%$ & $1.00 \mathrm{E}-09$ & $1.24 \mathrm{E}-11$ & $4.15 \mathrm{E}-05$ & $1.10 \mathrm{E}-07$ & $1.29 \mathrm{E}-06$ & $1.08 \mathrm{E}-09$ & 8.97E-02 & 2.24E-04 & $8.70 \mathrm{E}-03$ & $6.87 \mathrm{E}-05$ & $1.82 \mathrm{E}-03$ & $1.03 \mathrm{E}-01$ \\
\hline $85 \%$ & $1.58 \mathrm{E}-09$ & $1.97 \mathrm{E}-11$ & $6.62 \mathrm{E}-05$ & $1.78 \mathrm{E}-07$ & $2.17 \mathrm{E}-06$ & $1.71 \mathrm{E}-09$ & $1.20 \mathrm{E}-01$ & 3.31E-04 & $1.15 \mathrm{E}-02$ & $9.34 \mathrm{E}-05$ & $2.52 \mathrm{E}-03$ & $1.36 \mathrm{E}-01$ \\
\hline $90 \%$ & 2.77E-09 & $3.49 \mathrm{E}-11$ & $1.16 \mathrm{E}-04$ & $3.40 \mathrm{E}-07$ & $3.80 \mathrm{E}-06$ & $2.95 \mathrm{E}-09$ & $1.61 \mathrm{E}-01$ & $5.07 \mathrm{E}-04$ & $1.55 \mathrm{E}-02$ & $1.36 \mathrm{E}-04$ & $3.72 \mathrm{E}-03$ & $1.83 \mathrm{E}-01$ \\
\hline $95 \%$ & $5.48 \mathrm{E}-09$ & $7.28 \mathrm{E}-11$ & 2.89E-04 & 8.67E-07 & $1.01 \mathrm{E}-05$ & $6.14 \mathrm{E}-09$ & $2.25 \mathrm{E}-01$ & $9.41 \mathrm{E}-04$ & $2.35 \mathrm{E}-02$ & 2.22E-04 & $6.02 \mathrm{E}-03$ & $2.54 \mathrm{E}-01$ \\
\hline Mean & 5.13E-07 & $7.46 \mathrm{E}-09$ & $3.53 \mathrm{E}-03$ & $1.11 \mathrm{E}-05$ & $1.20 \mathrm{E}-04$ & $5.76 \mathrm{E}-07$ & 4.98E-02 & $2.08 \mathrm{E}-04$ & $5.39 \mathrm{E}-03$ & $4.77 \mathrm{E}-05$ & $1.25 \mathrm{E}-03$ & $5.68 \mathrm{E}-02$ \\
\hline Min & $1.08 \mathrm{E}-14$ & $1.67 \mathrm{E}-16$ & $3.36 \mathrm{E}-10$ & $6.82 \mathrm{E}-13$ & $1.34 \mathrm{E}-11$ & $5.25 \mathrm{E}-15$ & $0.00 \mathrm{E}+00$ & $0.00 \mathrm{E}+00$ & $0.00 \mathrm{E}+00$ & $0.00 \mathrm{E}+00$ & $0.00 \mathrm{E}+00$ & $2.07 \mathrm{E}-07$ \\
\hline Max & 5.64E-05 & $1.82 \mathrm{E}-06$ & 4.67E-01 & $3.90 \mathrm{E}-03$ & $2.44 \mathrm{E}-02$ & 5.69E-05 & $5.53 \mathrm{E}-01$ & $1.38 \mathrm{E}-02$ & $1.60 \mathrm{E}-01$ & $1.58 \mathrm{E}-03$ & $3.84 \mathrm{E}-02$ & $6.38 \mathrm{E}-01$ \\
\hline
\end{tabular}


TABLE C.54 Peak DSR Percentiles (mrem/yr per pCi/g) for Different Exposure Pathways for U-234 in Onsite Resident Scenario

\begin{tabular}{|c|c|c|c|c|c|c|c|c|c|c|c|c|c|c|}
\hline Percentile & External & $\begin{array}{l}\text { Inhalation } \\
\text { w/o Radon }\end{array}$ & $\begin{array}{l}\text { Radon } \\
\text { (WI) }\end{array}$ & $\begin{array}{c}\text { Plant } \\
\text { Ingestion } \\
\text { (WI) }\end{array}$ & $\begin{array}{c}\text { Meat } \\
\text { Ingestion } \\
\text { (WI) }\end{array}$ & $\begin{array}{c}\text { Milk } \\
\text { Ingestion } \\
\text { (WI) }\end{array}$ & $\begin{array}{c}\text { Soil } \\
\text { Ingestion } \\
\end{array}$ & $\begin{array}{c}\text { Water } \\
\text { Ingestion }\end{array}$ & $\begin{array}{l}\text { Aquatic } \\
\text { Foods }\end{array}$ & $\begin{array}{l}\text { Radon } \\
\text { (WD) }\end{array}$ & $\begin{array}{c}\text { Plant } \\
\text { Ingestion } \\
\text { (WD) }\end{array}$ & $\begin{array}{c}\text { Meat } \\
\text { Ingestion } \\
\text { (WD) }\end{array}$ & $\begin{array}{c}\text { Milk } \\
\text { Ingestion } \\
\text { (WD) }\end{array}$ & $\begin{array}{c}\text { All } \\
\text { Pathways } \\
\end{array}$ \\
\hline $5 \%$ & 7.42E-05 & $4.28 \mathrm{E}-05$ & $1.27 \mathrm{E}-03$ & 7.75E-03 & $1.02 \mathrm{E}-03$ & $6.97 \mathrm{E}-04$ & 4.73E-04 & $0.00 \mathrm{E}+00$ & $0.00 \mathrm{E}+00$ & $0.00 \mathrm{E}+00$ & $0.00 \mathrm{E}+00$ & $0.00 \mathrm{E}+00$ & $0.00 \mathrm{E}+00$ & $2.74 \mathrm{E}-02$ \\
\hline $10 \%$ & $1.37 \mathrm{E}-03$ & $8.57 \mathrm{E}-05$ & $4.22 \mathrm{E}-02$ & $1.52 \mathrm{E}-02$ & $1.86 \mathrm{E}-03$ & $1.19 \mathrm{E}-03$ & $1.01 \mathrm{E}-03$ & $0.00 \mathrm{E}+00$ & $0.00 \mathrm{E}+00$ & $0.00 \mathrm{E}+00$ & $0.00 \mathrm{E}+00$ & $0.00 \mathrm{E}+00$ & $0.00 \mathrm{E}+00$ & $8.69 \mathrm{E}-02$ \\
\hline $15 \%$ & $3.19 \mathrm{E}-03$ & $1.09 \mathrm{E}-04$ & $9.93 \mathrm{E}-02$ & $1.95 \mathrm{E}-02$ & $2.48 \mathrm{E}-03$ & $1.49 \mathrm{E}-03$ & $1.38 \mathrm{E}-03$ & $0.00 \mathrm{E}+00$ & $0.00 \mathrm{E}+00$ & $0.00 \mathrm{E}+00$ & $0.00 \mathrm{E}+00$ & $0.00 \mathrm{E}+00$ & $0.00 \mathrm{E}+00$ & $1.50 \mathrm{E}-01$ \\
\hline $20 \%$ & $5.78 \mathrm{E}-03$ & $1.25 \mathrm{E}-04$ & $1.64 \mathrm{E}-01$ & $2.34 \mathrm{E}-02$ & 3.05E-03 & $1.76 \mathrm{E}-03$ & $1.69 \mathrm{E}-03$ & $0.00 \mathrm{E}+00$ & $0.00 \mathrm{E}+00$ & $0.00 \mathrm{E}+00$ & $0.00 \mathrm{E}+00$ & $0.00 \mathrm{E}+00$ & $0.00 \mathrm{E}+00$ & $2.30 \mathrm{E}-01$ \\
\hline $25 \%$ & $9.18 \mathrm{E}-03$ & $1.42 \mathrm{E}-04$ & $2.40 \mathrm{E}-01$ & $2.70 \mathrm{E}-02$ & 3.59E-03 & $2.01 \mathrm{E}-03$ & $1.99 \mathrm{E}-03$ & $0.00 \mathrm{E}+00$ & $0.00 \mathrm{E}+00$ & $0.00 \mathrm{E}+00$ & $0.00 \mathrm{E}+00$ & $0.00 \mathrm{E}+00$ & $0.00 \mathrm{E}+00$ & 3.38E-01 \\
\hline $30 \%$ & $1.28 \mathrm{E}-02$ & $1.59 \mathrm{E}-04$ & 3.38E-01 & $3.08 \mathrm{E}-02$ & 4.23E-03 & $2.28 \mathrm{E}-03$ & $2.22 \mathrm{E}-03$ & $0.00 \mathrm{E}+00$ & $0.00 \mathrm{E}+00$ & $0.00 \mathrm{E}+00$ & $0.00 \mathrm{E}+00$ & $0.00 \mathrm{E}+00$ & $0.00 \mathrm{E}+00$ & 4.40E-01 \\
\hline $35 \%$ & $1.73 \mathrm{E}-02$ & $1.76 \mathrm{E}-04$ & 4.33E-01 & $3.47 \mathrm{E}-02$ & $4.81 \mathrm{E}-03$ & $2.51 \mathrm{E}-03$ & $2.44 \mathrm{E}-03$ & $0.00 \mathrm{E}+00$ & $0.00 \mathrm{E}+00$ & $0.00 \mathrm{E}+00$ & $0.00 \mathrm{E}+00$ & $0.00 \mathrm{E}+00$ & $0.00 \mathrm{E}+00$ & $5.52 \mathrm{E}-01$ \\
\hline $40 \%$ & $2.20 \mathrm{E}-02$ & $1.90 \mathrm{E}-04$ & $5.46 \mathrm{E}-01$ & $3.83 \mathrm{E}-02$ & $5.47 \mathrm{E}-03$ & $2.73 \mathrm{E}-03$ & $2.64 \mathrm{E}-03$ & $0.00 \mathrm{E}+00$ & $0.00 \mathrm{E}+00$ & $0.00 \mathrm{E}+00$ & $0.00 \mathrm{E}+00$ & $0.00 \mathrm{E}+00$ & $0.00 \mathrm{E}+00$ & $6.50 \mathrm{E}-01$ \\
\hline $45 \%$ & 2.63E-02 & $2.09 \mathrm{E}-04$ & $6.39 \mathrm{E}-01$ & $4.23 \mathrm{E}-02$ & $6.15 \mathrm{E}-03$ & $2.93 \mathrm{E}-03$ & $2.83 \mathrm{E}-03$ & $0.00 \mathrm{E}+00$ & $0.00 \mathrm{E}+00$ & $0.00 \mathrm{E}+00$ & $0.00 \mathrm{E}+00$ & $0.00 \mathrm{E}+00$ & $0.00 \mathrm{E}+00$ & 7.48E-01 \\
\hline $50 \%$ & 3.08E-02 & $2.26 \mathrm{E}-04$ & 7.40E-01 & 4.65E-02 & $6.79 \mathrm{E}-03$ & $3.18 \mathrm{E}-03$ & $3.05 \mathrm{E}-03$ & $0.00 \mathrm{E}+00$ & $0.00 \mathrm{E}+00$ & $0.00 \mathrm{E}+00$ & $0.00 \mathrm{E}+00$ & $0.00 \mathrm{E}+00$ & $0.00 \mathrm{E}+00$ & $8.59 \mathrm{E}-01$ \\
\hline $55 \%$ & $3.55 \mathrm{E}-02$ & $2.43 \mathrm{E}-04$ & $8.35 \mathrm{E}-01$ & $5.10 \mathrm{E}-02$ & $7.48 \mathrm{E}-03$ & 3.43E-03 & $3.25 \mathrm{E}-03$ & $0.00 \mathrm{E}+00$ & $0.00 \mathrm{E}+00$ & $0.00 \mathrm{E}+00$ & $0.00 \mathrm{E}+00$ & $0.00 \mathrm{E}+00$ & $0.00 \mathrm{E}+00$ & $9.50 \mathrm{E}-01$ \\
\hline $60 \%$ & $3.93 \mathrm{E}-02$ & $2.60 \mathrm{E}-04$ & $9.16 \mathrm{E}-01$ & $5.62 \mathrm{E}-02$ & $8.29 \mathrm{E}-03$ & $3.68 \mathrm{E}-03$ & $3.51 \mathrm{E}-03$ & $0.00 \mathrm{E}+00$ & $0.00 \mathrm{E}+00$ & $0.00 \mathrm{E}+00$ & $0.00 \mathrm{E}+00$ & $0.00 \mathrm{E}+00$ & $0.00 \mathrm{E}+00$ & $1.04 \mathrm{E}+00$ \\
\hline $65 \%$ & $4.38 \mathrm{E}-02$ & $2.81 \mathrm{E}-04$ & $9.92 \mathrm{E}-01$ & $6.20 \mathrm{E}-02$ & $9.13 \mathrm{E}-03$ & $4.00 \mathrm{E}-03$ & $3.75 \mathrm{E}-03$ & $0.00 \mathrm{E}+00$ & $0.00 \mathrm{E}+00$ & $0.00 \mathrm{E}+00$ & $0.00 \mathrm{E}+00$ & $0.00 \mathrm{E}+00$ & $0.00 \mathrm{E}+00$ & $1.12 \mathrm{E}+00$ \\
\hline $70 \%$ & 4.79E-02 & 3.03E-04 & $1.08 \mathrm{E}+00$ & $6.87 \mathrm{E}-02$ & $1.01 \mathrm{E}-02$ & $4.36 \mathrm{E}-03$ & 4.02E- 03 & $0.00 \mathrm{E}+00$ & $0.00 \mathrm{E}+00$ & $0.00 \mathrm{E}+00$ & $0.00 \mathrm{E}+00$ & $0.00 \mathrm{E}+00$ & $0.00 \mathrm{E}+00$ & $1.21 \mathrm{E}+00$ \\
\hline $75 \%$ & $5.29 \mathrm{E}-02$ & $3.28 \mathrm{E}-04$ & $1.15 \mathrm{E}+00$ & $7.58 \mathrm{E}-02$ & $1.12 \mathrm{E}-02$ & $4.72 \mathrm{E}-03$ & $4.32 \mathrm{E}-03$ & $0.00 \mathrm{E}+00$ & $0.00 \mathrm{E}+00$ & $0.00 \mathrm{E}+00$ & $0.00 \mathrm{E}+00$ & $0.00 \mathrm{E}+00$ & $0.00 \mathrm{E}+00$ & $1.28 \mathrm{E}+00$ \\
\hline $80 \%$ & $5.87 \mathrm{E}-02$ & $3.57 \mathrm{E}-04$ & $1.23 \mathrm{E}+00$ & $8.58 \mathrm{E}-02$ & $1.25 \mathrm{E}-02$ & $5.18 \mathrm{E}-03$ & $4.70 \mathrm{E}-03$ & $2.17 \mathrm{E}-28$ & $6.49 \mathrm{E}-30$ & $0.00 \mathrm{E}+00$ & $8.96 \mathrm{E}-30$ & $2.87 \mathrm{E}-30$ & $2.91 \mathrm{E}-31$ & $1.37 \mathrm{E}+00$ \\
\hline $85 \%$ & $6.60 \mathrm{E}-02$ & $3.96 \mathrm{E}-04$ & $1.32 \mathrm{E}+00$ & $9.73 \mathrm{E}-02$ & $1.43 \mathrm{E}-02$ & $5.75 \mathrm{E}-03$ & $5.22 \mathrm{E}-03$ & $1.20 \mathrm{E}-04$ & $1.12 \mathrm{E}-06$ & $1.15 \mathrm{E}-09$ & 4.83E-06 & $8.08 \mathrm{E}-07$ & $8.90 \mathrm{E}-07$ & $1.46 \mathrm{E}+00$ \\
\hline $90 \%$ & $7.65 \mathrm{E}-02$ & 4.42E-04 & $1.42 \mathrm{E}+00$ & $1.16 \mathrm{E}-01$ & $1.67 \mathrm{E}-02$ & $6.55 \mathrm{E}-03$ & 5.93E-03 & $9.48 \mathrm{E}-03$ & $5.48 \mathrm{E}-05$ & $6.35 \mathrm{E}-07$ & 3.65E-04 & $5.37 \mathrm{E}-05$ & $6.25 \mathrm{E}-05$ & $1.57 \mathrm{E}+00$ \\
\hline $95 \%$ & $9.31 \mathrm{E}-02$ & $5.24 \mathrm{E}-04$ & $1.59 \mathrm{E}+00$ & $1.57 \mathrm{E}-01$ & $2.10 \mathrm{E}-02$ & $8.09 \mathrm{E}-03$ & $6.94 \mathrm{E}-03$ & $9.37 \mathrm{E}-02$ & 4.72E-04 & $5.82 \mathrm{E}-06$ & $3.60 \mathrm{E}-03$ & $5.32 \mathrm{E}-04$ & 7.14E-04 & $1.75 \mathrm{E}+00$ \\
\hline Mean & $3.54 \mathrm{E}-02$ & $2.50 \mathrm{E}-04$ & 7.37E-01 & $6.05 \mathrm{E}-02$ & $8.40 \mathrm{E}-03$ & 3.63E-03 & $3.28 \mathrm{E}-03$ & $4.18 \mathrm{E}-02$ & 1.60E-04 & $2.64 \mathrm{E}-06$ & $1.61 \mathrm{E}-03$ & $2.97 \mathrm{E}-04$ & $3.89 \mathrm{E}-04$ & $8.57 \mathrm{E}-01$ \\
\hline Min & $9.99 \mathrm{E}-07$ & $9.39 \mathrm{E}-09$ & 3.64E-05 & $1.52 \mathrm{E}-06$ & $1.60 \mathrm{E}-07$ & 3.42E-08 & $1.33 \mathrm{E}-07$ & $0.00 \mathrm{E}+00$ & $0.00 \mathrm{E}+00$ & $0.00 \mathrm{E}+00$ & $0.00 \mathrm{E}+00$ & $0.00 \mathrm{E}+00$ & $0.00 \mathrm{E}+00$ & 4.39E-05 \\
\hline Max & $1.87 \mathrm{E}-01$ & $1.46 \mathrm{E}-03$ & $2.32 \mathrm{E}+00$ & $8.59 \mathrm{E}-01$ & 7.03E-02 & $2.98 \mathrm{E}-02$ & $1.05 \mathrm{E}-02$ & $2.11 \mathrm{E}+01$ & $8.08 \mathrm{E}-02$ & $1.66 \mathrm{E}-03$ & 8.13E-01 & $1.90 \mathrm{E}-01$ & $1.41 \mathrm{E}-01$ & $2.23 \mathrm{E}+01$ \\
\hline
\end{tabular}


TABLE C.55 Peak DSR Percentiles (mrem/yr per pCi/g) for Different Exposure Pathways for U-235 in Onsite Resident Scenario

\begin{tabular}{|c|c|c|c|c|c|c|c|c|c|c|c|c|}
\hline Percentile & External & $\begin{array}{l}\text { Inhalation } \\
\text { w/o Radon }\end{array}$ & $\begin{array}{l}\text { Plant } \\
\text { Ingestion } \\
(\mathrm{WI})\end{array}$ & $\begin{array}{c}\text { Meat } \\
\text { Ingestion } \\
(\mathrm{WI})\end{array}$ & $\begin{array}{c}\text { Milk } \\
\text { Ingestion } \\
(\mathrm{WI})\end{array}$ & $\begin{array}{c}\text { Soil } \\
\text { Ingestion }\end{array}$ & $\begin{array}{c}\text { Water } \\
\text { Ingestion }\end{array}$ & $\begin{array}{l}\text { Aquatic } \\
\text { Foods }\end{array}$ & $\begin{array}{c}\text { Plant } \\
\text { Ingestion } \\
\text { (WD) }\end{array}$ & $\begin{array}{c}\text { Meat } \\
\text { Ingestion } \\
\text { (WD) }\end{array}$ & $\begin{array}{c}\text { Milk } \\
\text { Ingestion } \\
\text { (WD) }\end{array}$ & $\begin{array}{c}\text { All } \\
\text { Pathways }\end{array}$ \\
\hline $5 \%$ & $4.75 \mathrm{E}-02$ & $4.50 \mathrm{E}-05$ & $8.05 \mathrm{E}-03$ & $5.48 \mathrm{E}-04$ & $3.96 \mathrm{E}-04$ & $4.65 \mathrm{E}-04$ & $0.00 \mathrm{E}+00$ & $0.00 \mathrm{E}+00$ & $0.00 \mathrm{E}+00$ & $0.00 \mathrm{E}+00$ & $0.00 \mathrm{E}+00$ & $6.84 \mathrm{E}-02$ \\
\hline $10 \%$ & $9.38 \mathrm{E}-02$ & $8.64 \mathrm{E}-05$ & $1.45 \mathrm{E}-02$ & $9.10 \mathrm{E}-04$ & $6.95 \mathrm{E}-04$ & $9.39 \mathrm{E}-04$ & $0.00 \mathrm{E}+00$ & $0.00 \mathrm{E}+00$ & $0.00 \mathrm{E}+00$ & $0.00 \mathrm{E}+00$ & $0.00 \mathrm{E}+00$ & $1.35 \mathrm{E}-01$ \\
\hline $15 \%$ & $1.08 \mathrm{E}-01$ & $1.17 \mathrm{E}-04$ & $1.98 \mathrm{E}-02$ & $1.16 \mathrm{E}-03$ & 8.92E-04 & $1.35 \mathrm{E}-03$ & $0.00 \mathrm{E}+00$ & $0.00 \mathrm{E}+00$ & $0.00 \mathrm{E}+00$ & $0.00 \mathrm{E}+00$ & $0.00 \mathrm{E}+00$ & $1.61 \mathrm{E}-01$ \\
\hline $20 \%$ & $1.19 \mathrm{E}-01$ & $1.45 \mathrm{E}-04$ & $2.53 \mathrm{E}-02$ & $1.36 \mathrm{E}-03$ & $1.05 \mathrm{E}-03$ & $1.74 \mathrm{E}-03$ & $0.00 \mathrm{E}+00$ & $0.00 \mathrm{E}+00$ & $0.00 \mathrm{E}+00$ & $0.00 \mathrm{E}+00$ & $0.00 \mathrm{E}+00$ & $1.81 \mathrm{E}-01$ \\
\hline $25 \%$ & $1.26 \mathrm{E}-01$ & $1.75 \mathrm{E}-04$ & $3.11 \mathrm{E}-02$ & $1.55 \mathrm{E}-03$ & $1.18 \mathrm{E}-03$ & $2.04 \mathrm{E}-03$ & $0.00 \mathrm{E}+00$ & $0.00 \mathrm{E}+00$ & $0.00 \mathrm{E}+00$ & $0.00 \mathrm{E}+00$ & $0.00 \mathrm{E}+00$ & $1.97 \mathrm{E}-01$ \\
\hline $30 \%$ & $1.35 \mathrm{E}-01$ & $2.10 \mathrm{E}-04$ & $3.76 \mathrm{E}-02$ & $1.77 \mathrm{E}-03$ & $1.32 \mathrm{E}-03$ & $2.34 \mathrm{E}-03$ & $0.00 \mathrm{E}+00$ & $0.00 \mathrm{E}+00$ & $0.00 \mathrm{E}+00$ & $0.00 \mathrm{E}+00$ & $0.00 \mathrm{E}+00$ & $2.13 \mathrm{E}-01$ \\
\hline $35 \%$ & $1.44 \mathrm{E}-01$ & $2.43 \mathrm{E}-04$ & 4.44E-02 & $1.98 \mathrm{E}-03$ & $1.45 \mathrm{E}-03$ & $2.62 \mathrm{E}-03$ & $0.00 \mathrm{E}+00$ & $0.00 \mathrm{E}+00$ & $0.00 \mathrm{E}+00$ & $0.00 \mathrm{E}+00$ & $0.00 \mathrm{E}+00$ & $2.27 \mathrm{E}-01$ \\
\hline $40 \%$ & $1.53 \mathrm{E}-01$ & $2.82 \mathrm{E}-04$ & $5.18 \mathrm{E}-02$ & $2.22 \mathrm{E}-03$ & $1.60 \mathrm{E}-03$ & $2.91 \mathrm{E}-03$ & $0.00 \mathrm{E}+00$ & $0.00 \mathrm{E}+00$ & $0.00 \mathrm{E}+00$ & $0.00 \mathrm{E}+00$ & $0.00 \mathrm{E}+00$ & $2.41 \mathrm{E}-01$ \\
\hline $45 \%$ & $1.62 \mathrm{E}-01$ & $3.29 \mathrm{E}-04$ & $6.05 \mathrm{E}-02$ & $2.46 \mathrm{E}-03$ & $1.74 \mathrm{E}-03$ & $3.23 \mathrm{E}-03$ & $0.00 \mathrm{E}+00$ & $0.00 \mathrm{E}+00$ & $0.00 \mathrm{E}+00$ & $0.00 \mathrm{E}+00$ & $0.00 \mathrm{E}+00$ & $2.56 \mathrm{E}-01$ \\
\hline $50 \%$ & $1.71 \mathrm{E}-01$ & $3.97 \mathrm{E}-04$ & $6.99 \mathrm{E}-02$ & $2.70 \mathrm{E}-03$ & $1.90 \mathrm{E}-03$ & $3.59 \mathrm{E}-03$ & $0.00 \mathrm{E}+00$ & $0.00 \mathrm{E}+00$ & $0.00 \mathrm{E}+00$ & $0.00 \mathrm{E}+00$ & $0.00 \mathrm{E}+00$ & $2.72 \mathrm{E}-01$ \\
\hline $55 \%$ & $1.81 \mathrm{E}-01$ & $4.91 \mathrm{E}-04$ & $7.81 \mathrm{E}-02$ & $3.00 \mathrm{E}-03$ & $2.07 \mathrm{E}-03$ & $4.04 \mathrm{E}-03$ & $0.00 \mathrm{E}+00$ & $0.00 \mathrm{E}+00$ & $0.00 \mathrm{E}+00$ & $0.00 \mathrm{E}+00$ & $0.00 \mathrm{E}+00$ & $2.90 \mathrm{E}-01$ \\
\hline $60 \%$ & $1.92 \mathrm{E}-01$ & $6.08 \mathrm{E}-04$ & $8.89 \mathrm{E}-02$ & $3.32 \mathrm{E}-03$ & $2.23 \mathrm{E}-03$ & $4.62 \mathrm{E}-03$ & $0.00 \mathrm{E}+00$ & $0.00 \mathrm{E}+00$ & $0.00 \mathrm{E}+00$ & $0.00 \mathrm{E}+00$ & $0.00 \mathrm{E}+00$ & $3.08 \mathrm{E}-01$ \\
\hline $65 \%$ & $2.04 \mathrm{E}-01$ & $7.56 \mathrm{E}-04$ & $1.01 \mathrm{E}-01$ & $3.68 \mathrm{E}-03$ & $2.45 \mathrm{E}-03$ & $5.45 \mathrm{E}-03$ & $0.00 \mathrm{E}+00$ & $0.00 \mathrm{E}+00$ & $0.00 \mathrm{E}+00$ & $0.00 \mathrm{E}+00$ & $0.00 \mathrm{E}+00$ & $3.29 \mathrm{E}-01$ \\
\hline $70 \%$ & $2.16 \mathrm{E}-01$ & $9.03 \mathrm{E}-04$ & $1.17 \mathrm{E}-01$ & $4.10 \mathrm{E}-03$ & $2.70 \mathrm{E}-03$ & $6.40 \mathrm{E}-03$ & $0.00 \mathrm{E}+00$ & $0.00 \mathrm{E}+00$ & $0.00 \mathrm{E}+00$ & $0.00 \mathrm{E}+00$ & $0.00 \mathrm{E}+00$ & $3.52 \mathrm{E}-01$ \\
\hline $75 \%$ & $2.32 \mathrm{E}-01$ & $1.08 \mathrm{E}-03$ & $1.36 \mathrm{E}-01$ & $4.58 \mathrm{E}-03$ & $2.96 \mathrm{E}-03$ & $7.50 \mathrm{E}-03$ & $0.00 \mathrm{E}+00$ & $0.00 \mathrm{E}+00$ & $0.00 \mathrm{E}+00$ & $0.00 \mathrm{E}+00$ & $0.00 \mathrm{E}+00$ & $3.79 \mathrm{E}-01$ \\
\hline $80 \%$ & $2.51 \mathrm{E}-01$ & $1.30 \mathrm{E}-03$ & $1.61 \mathrm{E}-01$ & $5.18 \mathrm{E}-03$ & $3.29 \mathrm{E}-03$ & 8.61E-03 & $0.00 \mathrm{E}+00$ & $0.00 \mathrm{E}+00$ & $0.00 \mathrm{E}+00$ & $0.00 \mathrm{E}+00$ & $0.00 \mathrm{E}+00$ & $4.14 \mathrm{E}-01$ \\
\hline $85 \%$ & $2.77 \mathrm{E}-01$ & $1.56 \mathrm{E}-03$ & $1.97 \mathrm{E}-01$ & $6.01 \mathrm{E}-03$ & $3.78 \mathrm{E}-03$ & $1.00 \mathrm{E}-02$ & $4.02 \mathrm{E}-04$ & $1.25 \mathrm{E}-06$ & $1.54 \mathrm{E}-05$ & $1.97 \mathrm{E}-07$ & $2.49 \mathrm{E}-07$ & $4.59 \mathrm{E}-01$ \\
\hline $90 \%$ & $3.09 \mathrm{E}-01$ & $1.91 \mathrm{E}-03$ & $2.43 \mathrm{E}-01$ & $7.28 \mathrm{E}-03$ & $4.42 \mathrm{E}-03$ & $1.18 \mathrm{E}-02$ & $7.70 \mathrm{E}-03$ & $3.54 \mathrm{E}-05$ & $2.98 \mathrm{E}-04$ & $7.97 \mathrm{E}-06$ & $1.19 \mathrm{E}-05$ & $5.21 \mathrm{E}-01$ \\
\hline $95 \%$ & $3.64 \mathrm{E}-01$ & $2.42 \mathrm{E}-03$ & $3.48 \mathrm{E}-01$ & $9.50 \mathrm{E}-03$ & $5.70 \mathrm{E}-03$ & $1.43 \mathrm{E}-02$ & $5.29 \mathrm{E}-02$ & $2.21 \mathrm{E}-04$ & $2.04 \mathrm{E}-03$ & $1.84 \mathrm{E}-04$ & $2.54 \mathrm{E}-04$ & $6.49 \mathrm{E}-01$ \\
\hline Mean & $1.86 \mathrm{E}-01$ & $7.50 \mathrm{E}-04$ & $1.12 \mathrm{E}-01$ & $3.62 \mathrm{E}-03$ & $2.33 \mathrm{E}-03$ & $5.16 \mathrm{E}-03$ & $3.87 \mathrm{E}-02$ & $1.39 \mathrm{E}-04$ & $1.49 \mathrm{E}-03$ & $2.62 \mathrm{E}-04$ & $3.48 \mathrm{E}-04$ & $3.31 \mathrm{E}-01$ \\
\hline Min & $2.32 \mathrm{E}-06$ & $2.59 \mathrm{E}-08$ & $1.35 \mathrm{E}-06$ & $1.71 \mathrm{E}-09$ & $6.65 \mathrm{E}-10$ & $2.43 \mathrm{E}-07$ & $0.00 \mathrm{E}+00$ & $0.00 \mathrm{E}+00$ & $0.00 \mathrm{E}+00$ & $0.00 \mathrm{E}+00$ & $0.00 \mathrm{E}+00$ & $3.93 \mathrm{E}-06$ \\
\hline Max & $6.19 \mathrm{E}-01$ & $6.54 \mathrm{E}-03$ & $5.30 \mathrm{E}+00$ & $6.92 \mathrm{E}-02$ & $3.36 \mathrm{E}-02$ & $2.48 \mathrm{E}-02$ & $1.65 \mathrm{E}+01$ & $6.07 \mathrm{E}-02$ & $6.33 \mathrm{E}-01$ & $1.67 \mathrm{E}-01$ & $2.22 \mathrm{E}-01$ & $1.74 \mathrm{E}+01$ \\
\hline
\end{tabular}


TABLE C.56 Peak DSR Percentiles (mrem/yr per pCi/g) for Different Exposure Pathways for U-238 in Onsite Resident Scenario

\begin{tabular}{|c|c|c|c|c|c|c|c|c|c|c|c|c|c|c|}
\hline Percentile & External & $\begin{array}{c}\text { Inhalation } \\
\text { w/o Radon }\end{array}$ & $\begin{array}{c}\text { Radon } \\
\text { (WI) }\end{array}$ & $\begin{array}{c}\text { Plant } \\
\text { Ingestion } \\
\text { (WI) }\end{array}$ & $\begin{array}{c}\text { Meat } \\
\text { Ingestion } \\
\text { (WI) }\end{array}$ & $\begin{array}{c}\text { Milk } \\
\text { Ingestion } \\
\text { (WI) }\end{array}$ & $\begin{array}{c}\text { Soil } \\
\text { Ingestion }\end{array}$ & $\begin{array}{c}\text { Water } \\
\text { Ingestion }\end{array}$ & $\begin{array}{c}\text { Aquatic } \\
\text { Foods }\end{array}$ & $\begin{array}{c}\text { Radon } \\
(\mathrm{WD})\end{array}$ & $\begin{array}{c}\text { Plant } \\
\text { Ingestion } \\
\text { (WD) }\end{array}$ & $\begin{array}{c}\text { Meat } \\
\text { Ingestion } \\
\text { (WD) }\end{array}$ & $\begin{array}{c}\text { Milk } \\
\text { Ingestion } \\
\text { (WD) }\end{array}$ & $\begin{array}{c}\text { All } \\
\text { Pathways }\end{array}$ \\
\hline $5 \%$ & $1.15 \mathrm{E}-02$ & $3.19 \mathrm{E}-05$ & $8.51 \mathrm{E}-08$ & $3.86 \mathrm{E}-03$ & $5.09 \mathrm{E}-04$ & 4.43E-04 & 3.52E-04 & $0.00 \mathrm{E}+00$ & $0.00 \mathrm{E}+00$ & $0.00 \mathrm{E}+00$ & $0.00 \mathrm{E}+00$ & $0.00 \mathrm{E}+00$ & $0.00 \mathrm{E}+00$ & $2.41 \mathrm{E}-02$ \\
\hline $10 \%$ & $2.00 \mathrm{E}-02$ & $5.98 \mathrm{E}-05$ & $1.11 \mathrm{E}-07$ & $7.10 \mathrm{E}-03$ & $8.72 \mathrm{E}-04$ & 7.29E-04 & 7.68E-04 & $0.00 \mathrm{E}+00$ & $0.00 \mathrm{E}+00$ & $0.00 \mathrm{E}+00$ & $0.00 \mathrm{E}+00$ & $0.00 \mathrm{E}+00$ & $0.00 \mathrm{E}+00$ & $4.22 \mathrm{E}-02$ \\
\hline $15 \%$ & $2.31 \mathrm{E}-02$ & 7.70E-05 & $1.25 \mathrm{E}-07$ & $9.42 \mathrm{E}-03$ & $1.17 \mathrm{E}-03$ & $9.17 \mathrm{E}-04$ & $1.05 \mathrm{E}-03$ & $0.00 \mathrm{E}+00$ & $0.00 \mathrm{E}+00$ & $0.00 \mathrm{E}+00$ & $0.00 \mathrm{E}+00$ & $0.00 \mathrm{E}+00$ & $0.00 \mathrm{E}+00$ & $4.90 \mathrm{E}-02$ \\
\hline $20 \%$ & 2.49E-02 & $9.07 \mathrm{E}-05$ & $1.35 \mathrm{E}-07$ & $1.17 \mathrm{E}-02$ & $1.39 \mathrm{E}-03$ & $1.07 \mathrm{E}-03$ & $1.27 \mathrm{E}-03$ & $0.00 \mathrm{E}+00$ & $0.00 \mathrm{E}+00$ & $0.00 \mathrm{E}+00$ & $0.00 \mathrm{E}+00$ & $0.00 \mathrm{E}+00$ & $0.00 \mathrm{E}+00$ & $5.38 \mathrm{E}-02$ \\
\hline $25 \%$ & $2.68 \mathrm{E}-02$ & $1.03 \mathrm{E}-04$ & $1.43 \mathrm{E}-07$ & $1.40 \mathrm{E}-02$ & $1.58 \mathrm{E}-03$ & $1.20 \mathrm{E}-03$ & $1.49 \mathrm{E}-03$ & $0.00 \mathrm{E}+00$ & $0.00 \mathrm{E}+00$ & $0.00 \mathrm{E}+00$ & $0.00 \mathrm{E}+00$ & $0.00 \mathrm{E}+00$ & $0.00 \mathrm{E}+00$ & $5.86 \mathrm{E}-02$ \\
\hline $30 \%$ & $2.88 \mathrm{E}-02$ & $1.15 \mathrm{E}-04$ & $1.51 \mathrm{E}-07$ & $1.64 \mathrm{E}-02$ & $1.82 \mathrm{E}-03$ & $1.33 \mathrm{E}-03$ & $1.67 \mathrm{E}-03$ & $0.00 \mathrm{E}+00$ & $0.00 \mathrm{E}+00$ & $0.00 \mathrm{E}+00$ & $0.00 \mathrm{E}+00$ & $0.00 \mathrm{E}+00$ & $0.00 \mathrm{E}+00$ & $6.29 \mathrm{E}-02$ \\
\hline $35 \%$ & 3.06E-02 & $1.26 \mathrm{E}-04$ & $1.58 \mathrm{E}-07$ & $1.90 \mathrm{E}-02$ & $2.05 \mathrm{E}-03$ & $1.47 \mathrm{E}-03$ & $1.84 \mathrm{E}-03$ & $0.00 \mathrm{E}+00$ & $0.00 \mathrm{E}+00$ & $0.00 \mathrm{E}+00$ & $0.00 \mathrm{E}+00$ & $0.00 \mathrm{E}+00$ & $0.00 \mathrm{E}+00$ & $6.75 \mathrm{E}-02$ \\
\hline $40 \%$ & $3.25 \mathrm{E}-02$ & $1.39 \mathrm{E}-04$ & $1.65 \mathrm{E}-07$ & $2.16 \mathrm{E}-02$ & $2.29 \mathrm{E}-03$ & $1.62 \mathrm{E}-03$ & $2.01 \mathrm{E}-03$ & $0.00 \mathrm{E}+00$ & $0.00 \mathrm{E}+00$ & $0.00 \mathrm{E}+00$ & $0.00 \mathrm{E}+00$ & $0.00 \mathrm{E}+00$ & $0.00 \mathrm{E}+00$ & 7.21E-02 \\
\hline $45 \%$ & $3.44 \mathrm{E}-02$ & $1.50 \mathrm{E}-04$ & $1.73 \mathrm{E}-07$ & 2.43E-02 & $2.52 \mathrm{E}-03$ & $1.77 \mathrm{E}-03$ & $2.15 \mathrm{E}-03$ & $0.00 \mathrm{E}+00$ & $0.00 \mathrm{E}+00$ & $0.00 \mathrm{E}+00$ & $0.00 \mathrm{E}+00$ & $0.00 \mathrm{E}+00$ & $0.00 \mathrm{E}+00$ & 7.69E-02 \\
\hline $50 \%$ & $3.62 \mathrm{E}-02$ & $1.63 \mathrm{E}-04$ & $1.80 \mathrm{E}-07$ & $2.76 \mathrm{E}-02$ & $2.79 \mathrm{E}-03$ & $1.92 \mathrm{E}-03$ & $2.30 \mathrm{E}-03$ & $0.00 \mathrm{E}+00$ & $0.00 \mathrm{E}+00$ & $0.00 \mathrm{E}+00$ & $0.00 \mathrm{E}+00$ & $0.00 \mathrm{E}+00$ & $0.00 \mathrm{E}+00$ & 8.23E-02 \\
\hline $55 \%$ & $3.84 \mathrm{E}-02$ & $1.77 \mathrm{E}-04$ & $1.88 \mathrm{E}-07$ & $3.12 \mathrm{E}-02$ & $3.08 \mathrm{E}-03$ & $2.09 \mathrm{E}-03$ & 2.44E-03 & $0.00 \mathrm{E}+00$ & $0.00 \mathrm{E}+00$ & $0.00 \mathrm{E}+00$ & $0.00 \mathrm{E}+00$ & $0.00 \mathrm{E}+00$ & $0.00 \mathrm{E}+00$ & $8.74 \mathrm{E}-02$ \\
\hline $60 \%$ & $4.06 \mathrm{E}-02$ & $1.93 \mathrm{E}-04$ & $1.97 \mathrm{E}-07$ & $3.55 \mathrm{E}-02$ & $3.39 \mathrm{E}-03$ & $2.28 \mathrm{E}-03$ & $2.58 \mathrm{E}-03$ & $0.00 \mathrm{E}+00$ & $0.00 \mathrm{E}+00$ & $0.00 \mathrm{E}+00$ & $0.00 \mathrm{E}+00$ & $0.00 \mathrm{E}+00$ & $0.00 \mathrm{E}+00$ & $9.31 \mathrm{E}-02$ \\
\hline $65 \%$ & $4.29 \mathrm{E}-02$ & 2.07E-04 & 2.07E-07 & $4.01 \mathrm{E}-02$ & $3.74 \mathrm{E}-03$ & $2.49 \mathrm{E}-03$ & 2.73E- 03 & $0.00 \mathrm{E}+00$ & $0.00 \mathrm{E}+00$ & $0.00 \mathrm{E}+00$ & $0.00 \mathrm{E}+00$ & $0.00 \mathrm{E}+00$ & $0.00 \mathrm{E}+00$ & $9.99 \mathrm{E}-02$ \\
\hline $70 \%$ & $4.57 \mathrm{E}-02$ & $2.22 \mathrm{E}-04$ & $2.20 \mathrm{E}-07$ & $4.58 \mathrm{E}-02$ & $4.15 \mathrm{E}-03$ & $2.75 \mathrm{E}-03$ & $2.88 \mathrm{E}-03$ & $0.00 \mathrm{E}+00$ & $0.00 \mathrm{E}+00$ & $0.00 \mathrm{E}+00$ & $0.00 \mathrm{E}+00$ & $0.00 \mathrm{E}+00$ & $0.00 \mathrm{E}+00$ & $1.07 \mathrm{E}-01$ \\
\hline $75 \%$ & 4.90E-02 & 2.40E-04 & $2.36 \mathrm{E}-07$ & $5.28 \mathrm{E}-02$ & $4.61 \mathrm{E}-03$ & 3.03E-03 & 3.06E-03 & $0.00 \mathrm{E}+00$ & $0.00 \mathrm{E}+00$ & $0.00 \mathrm{E}+00$ & $0.00 \mathrm{E}+00$ & $0.00 \mathrm{E}+00$ & $0.00 \mathrm{E}+00$ & $1.16 \mathrm{E}-01$ \\
\hline $80 \%$ & $5.29 \mathrm{E}-02$ & 2.60E-04 & 2.69E-07 & $6.25 \mathrm{E}-02$ & $5.16 \mathrm{E}-03$ & $3.38 \mathrm{E}-03$ & $3.25 \mathrm{E}-03$ & $0.00 \mathrm{E}+00$ & $0.00 \mathrm{E}+00$ & $0.00 \mathrm{E}+00$ & $0.00 \mathrm{E}+00$ & $0.00 \mathrm{E}+00$ & $0.00 \mathrm{E}+00$ & $1.28 \mathrm{E}-01$ \\
\hline $85 \%$ & $5.79 \mathrm{E}-02$ & $2.87 \mathrm{E}-04$ & 4.78E-05 & 7.40E-02 & $5.97 \mathrm{E}-03$ & 3.83E-03 & $3.48 \mathrm{E}-03$ & $0.00 \mathrm{E}+00$ & $0.00 \mathrm{E}+00$ & $0.00 \mathrm{E}+00$ & $0.00 \mathrm{E}+00$ & $0.00 \mathrm{E}+00$ & $0.00 \mathrm{E}+00$ & $1.47 \mathrm{E}-01$ \\
\hline $90 \%$ & $6.43 \mathrm{E}-02$ & $3.19 \mathrm{E}-04$ & $3.54 \mathrm{E}-03$ & $9.31 \mathrm{E}-02$ & 7.35E-03 & 4.47E-03 & 3.75E-03 & $0.00 \mathrm{E}+00$ & $0.00 \mathrm{E}+00$ & $0.00 \mathrm{E}+00$ & $0.00 \mathrm{E}+00$ & $0.00 \mathrm{E}+00$ & $0.00 \mathrm{E}+00$ & $1.75 \mathrm{E}-01$ \\
\hline $95 \%$ & 7.54E-02 & 3.79E-04 & $6.48 \mathrm{E}-03$ & $1.29 \mathrm{E}-01$ & $9.70 \mathrm{E}-03$ & $5.73 \mathrm{E}-03$ & $4.09 \mathrm{E}-03$ & 7.02E-02 & $1.57 \mathrm{E}-04$ & 2.02E-08 & $2.70 \mathrm{E}-03$ & $2.93 \mathrm{E}-04$ & 4.02E-04 & 2.47E-01 \\
\hline Mean & 3.92E-02 & $1.81 \mathrm{E}-04$ & 7.26E-04 & 4.27E- 02 & $3.66 \mathrm{E}-03$ & $2.36 \mathrm{E}-03$ & $2.27 \mathrm{E}-03$ & $3.94 \mathrm{E}-02$ & $1.57 \mathrm{E}-04$ & $1.77 \mathrm{E}-07$ & $1.52 \mathrm{E}-03$ & $2.69 \mathrm{E}-04$ & $3.46 \mathrm{E}-04$ & $1.28 \mathrm{E}-01$ \\
\hline Min & $3.50 \mathrm{E}-12$ & $9.81 \mathrm{E}-14$ & 1.47E-10 & $6.74 \mathrm{E}-12$ & $1.08 \mathrm{E}-12$ & $3.18 \mathrm{E}-13$ & $6.14 \mathrm{E}-13$ & $0.00 \mathrm{E}+00$ & $0.00 \mathrm{E}+00$ & $0.00 \mathrm{E}+00$ & $0.00 \mathrm{E}+00$ & $0.00 \mathrm{E}+00$ & $0.00 \mathrm{E}+00$ & $1.60 \mathrm{E}-10$ \\
\hline $\operatorname{Max}$ & $1.12 \mathrm{E}-01$ & $1.10 \mathrm{E}-03$ & $1.16 \mathrm{E}-02$ & $6.09 \mathrm{E}-01$ & $5.67 \mathrm{E}-02$ & $1.55 \mathrm{E}-02$ & $5.00 \mathrm{E}-03$ & $1.62 \mathrm{E}+01$ & $2.35 \mathrm{E}-01$ & 3.87E-04 & $6.24 \mathrm{E}-01$ & 7.44E-02 & $1.17 \mathrm{E}-01$ & $1.73 \mathrm{E}+01$ \\
\hline
\end{tabular}





\section{Argonne}

\section{Environmental Science Division}

Argonne National Laboratory

9700 South Cass Avenue, Bldg. 240

Argonne, IL 60439-4847

www.anl.gov

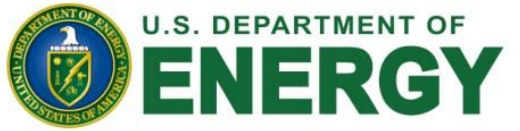

Argonne National Laboratory is a U.S. Department of Energy

laboratory managed by UChicago Argonne, LLC 